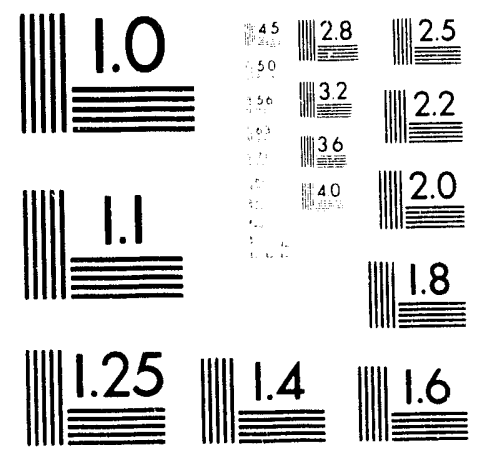



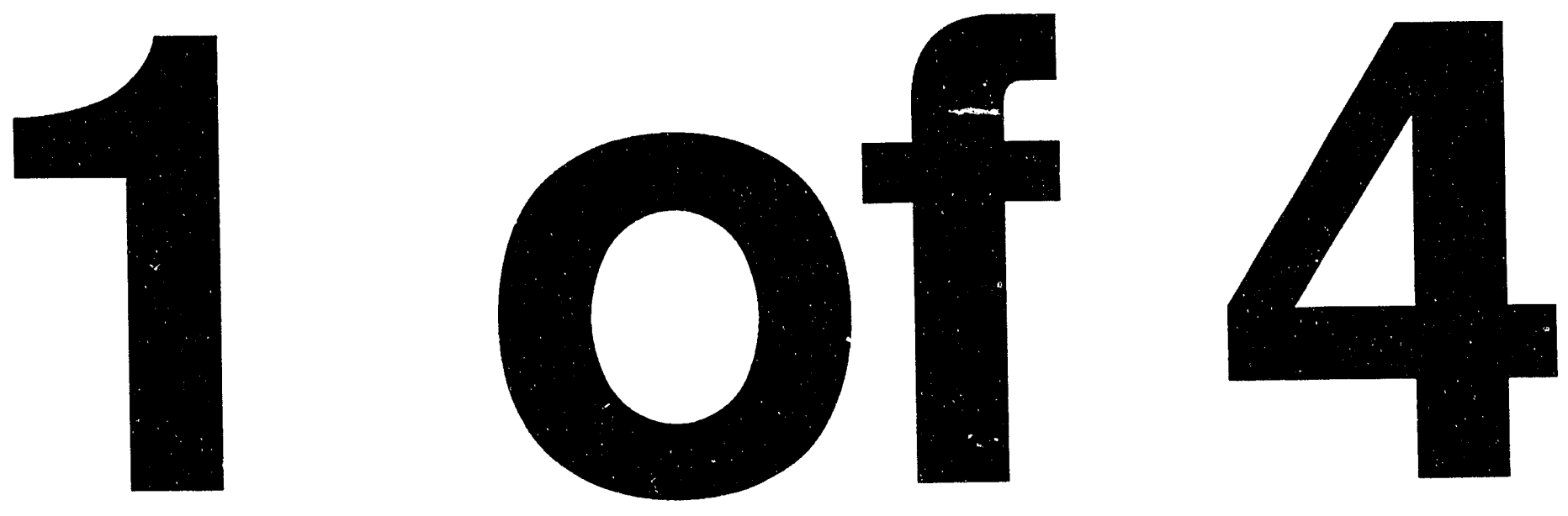


\section{EARLY-WARNING PROCESS/CONTROL FOR ANAEROBIC DIGESTION AND BIOLOGICAL NITROGEN TRANSFORMATION PROCESSES: BATCH, SEMI-CONTINUOUS, AND/OR CHEMOSTAT EXPERIMENTS}

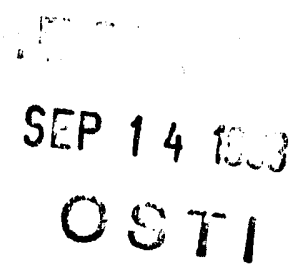


The New York State Energy Research and Development Authority (Energy A uthority) is responsible for the development and use of safe, dependable, renewable and economic energy sources and conservation technologies. It sponsors energy research, development and demonstration (RD\&D) projects and financing programs designed to help utilities and other private companies fund certain energy-related projects. The Energy Authority is a public benefit corporation which was created in 1975 by the New York State Legislature.

In working toward these goals, the Energy Authority sponsors RD\&D projects in two major program areas: Energy Efficiency and Economic Development, and Energy Resources and Environmental Research.

Under its financing program, the Energy Authority is authorized to issue tax-exempt bonds to finance certain electric or gas facilities and special energy projects for private companies.

The Energy Authority also has responsibility for constructing and then operating facilities for the disposal of low-level radioactive wastes produced in New York State. The generators of these wastes ultimately will bear the costs of the construction of these facilities.

A 13-member board of directors governs the Energy Authority, with Francis J. Murray, Jr., Commissioner of the State Energy Office, serving as Chairman of the Board and Chief Executive Officer. F. William Valentino, President of the Energy Authority and Chief Operating Officer, manages its programs, staff and facilities.

The Energy Authority derives its basic RD\&D revenues from an assessment levied on the intrastate sales of New York State's investor-owned electric and gas utilities. Additional RD\&D funds come from the investment of retained eamings, as well as from an annual contribution from the New York Power Authority.

The Energy Authority's RD\&D program is also supported by funds from a variety of cosponsors, including utilities, universities, industries, private engineering and scientific research firms, local governments, and State and Federal agencies.

For further information on the Energy Authority's programs, contact the New York State Energy Research and Development Authority, phone (518) 465-6251, ext. 272.

State of New York Mario M. Cuomo. Governor
Energy Research and Development Authority Francis J. Murray, Jr., Chairman

F. William Valentino, President 


\begin{abstract}
EARLY-WARNING PROCESS/CONTROL FOR ANAEROBIC DIGESTION AND

BIOLOGICAL NITROGEN TRANSFORMATION PROCESSES:

BATCH, SEMI-CONTINUOUS, AND/OR CHEMOSTAT EXPERIMENTS
\end{abstract}

Final Report

Prepared for

THE NEW YORK STATE

ENERGY RESEARCH AND DEVELOPMENT AUTHORITY

Project Manager

Barry Liebowitz

Prepared by

SCIENCE APPLICATIONS INTERNATIONAL CORPORATION

1710 Goodridge Drive

McLean, VA 22102

Principal Investigator

Robert Hickey

720-RIER-RER-85

Energy Authority

Report 93-9

September 1992
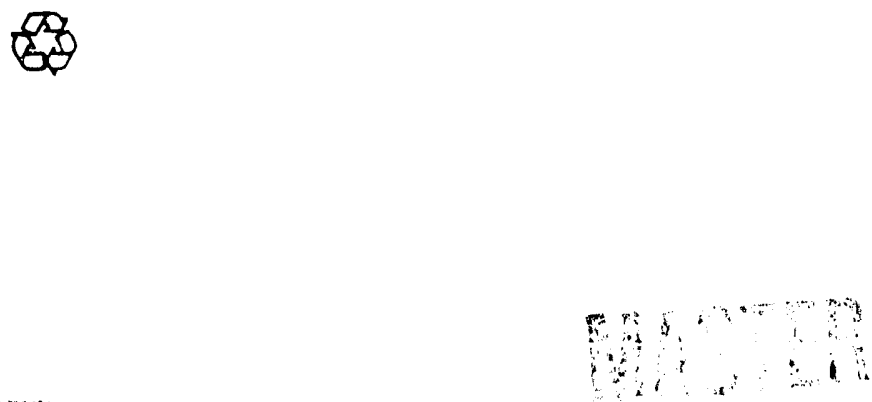


\section{NOTICE}

This report was prepared by Science Applications International Corporation in the course of performing work contracted for and sponsored by the New York State Energy Research and Development Authority (hereafter the "Energy Authority"). The opinions expressed in this report do not necessarily reflect those of the Energy Authority or the State of New York, and reference to any specific product, service, process, or method does not constitute an implied or expressed recommendation or endorsement of it. Further, the Energy Authority, the State of New York, and the contractor make no warranties or representations, expressed or implied, as to the fitness for particular purpose or merchantability of any product, apparatus, or service, or the usefulness, completeness, or accuracy of any processes, methods, or other information contained, described, disclosed, or referred to in this report. The Energy Authority, the State of New York, and the contractor make no representation that the use of any product, apparatus, process, method, or other information will not infringe privately owned rights and will assume no liability for any loss, injury, or damage resulting from, or occurring in connection with, the use of information contained, described, disclosed, or referred to in this report.

First Printing: July 1993 
EARLY-WARNING PROCESS/CONTROL FOR ANAEROBIC DIGESTERS:

SEMI-CONTINUOUSLY FED, AND BATCH EXPERIMENTS

PHASE 2: ON-LINE MONITORING OF THE RESPONSE OF ANAEROBIC DIGESTION TO ORGANIC AND HYDRAULIC LOAD INCREASES AND HEAVY METALINDUCED INHIBITION 


\section{ACKNOHLEDGEAENTS}

The author would like to thank Dr. Michael Switzenbaum for his advice and encouragement. Special thanks are also due to Ms. Juliana Vanderweilen for her tireless work in performing much of the experimental work reported herein. Thanks are also due to Mr. Howard Feiler of SAIC and Mr. Barry Liebowitz of the New York State Energy Research and Development Authority for their help in completing this project. Finally, the work of Ms. Susan Christians in helping prepare this document is gratefully acknowledged. 


\section{TABLE OF CONTENTS}

Section

Page

EXECUTIVE SUMMARY

$S-1$

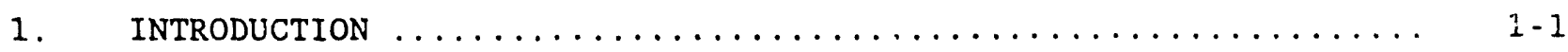

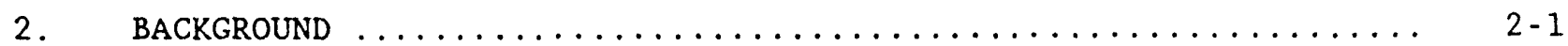

3. EXPERIMENTAL APPROACH AND DESIGN $\ldots \ldots \ldots \ldots \ldots \ldots \ldots \ldots \ldots \ldots \ldots \ldots$

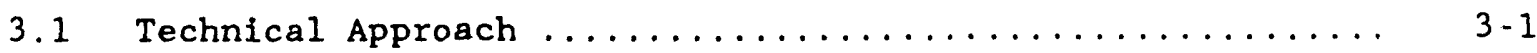

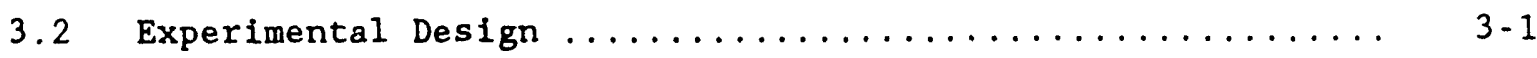

3.2.1 Reserve Digesters ..................... 3-2

3.2 .2 Organic Overload Assay .................... $3-4$

3.2.3 Supplemental Serum Bottle Assays ............. $3-4$

3.2 .4 Test Digester Operation .................. 3-6

4. EXPERIMENTAL RESULTS - THE EFFECT OF ORGANIC VARIATIONS

AND OVERLOADS ............................. $4-1$

4.1 Organic Overload Serum Bottle Assay .............. 4-1

4.2 Organic Overload of a 10 -Day HRT Digester ........... $4-4$

4.3 Organic Overload of a 20-Day HRT Digester .......... 4-16

4.4 Discussion and summary ................... $4-24$

5. EXPERIMENTAL RESULTS - THE EFFECTS OF HYDRAULIC LOAD INCREASES .. 5-1

5.1 Hydraulic Load Increase of $1.25 \times$ Normal ........... 5.1

5.2 Hydraulic Load Increase of $2 \times$ Normal ............ $5-1$

5.3 Hydraulic Load Increase of $4 \times$ Normal $\ldots \ldots \ldots \ldots \ldots \ldots$

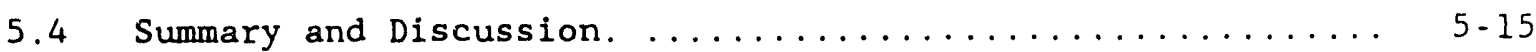

6. EFFECT OF COPPER ADDITION TO SEMI-CONTINUOUSLY

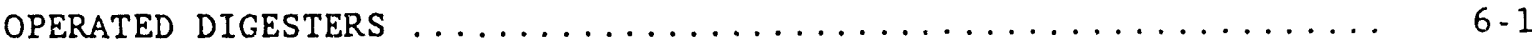

6.1 Effect of Copper Pulse to a Mesophilic Digester ......... 6.1

6.2 Response of a Thermophilic Digester to a Copper Pulse .... 6-5

6.3 Effect of Copper Addition on a Chronic Basis

to a Mesophilic Digester ..................... 6-5 
TABLE OF CONTENTS (Cont'd)

Section $\quad \underline{\text { Page }}$

6.4 Summary and Discussion $\ldots \ldots \ldots \ldots \ldots \ldots \ldots \ldots \ldots \ldots \ldots \ldots \ldots \ldots$

7. RESULTS OF TIME SERIES ANALYSIS $\ldots \ldots \ldots \ldots \ldots \ldots \ldots \ldots \ldots \ldots$

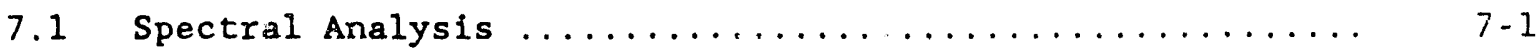

7.2 Box-Jenkins Time Series Analysis ................ $7-1$

8. EXPERIMENTAL RESULTS: FACTORS AFFECTING CO CONCENTRATION DURING ANAEROBIC DIGESTION AND THE INFLUENCE OF CO ON

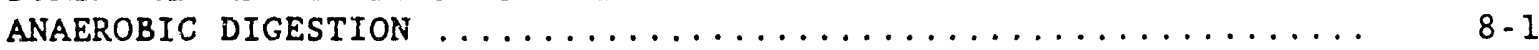

8.1 Effect of Acetate Concentration on $\mathrm{CO}$ and $\mathrm{H}_{2}$ Levels of

Digester Inocula in Defined Medium ............... $8-1$

8.2 CO Production By $M$, BARKERI During Methanogenesis on

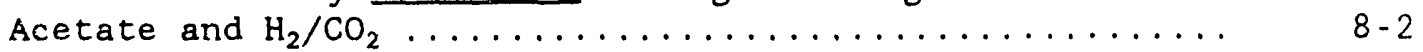

8.3 Development of a Thermodynamic Model ............. $8-5$

8.4 Evaluation of the Thermodynamic Relationship Using
Batch Assays $\ldots \ldots \ldots \ldots \ldots \ldots \ldots \ldots \ldots \ldots \ldots \ldots \ldots \ldots \ldots$

8.4.1 Digester Steady State Results .............. 8.7

8.4.2 Serum Bottle Assays ................... 8-10

8.4.3 Effect of Altering Methane and Hydrogen
Concentrations........................ 8-11

8.4.4 Effect of Supplementing Acetate ............ 8.13

8.5 Evaluation of the Thermodynamic Relationship Using Data from the On-line Data Acquisition System ............... 8-13

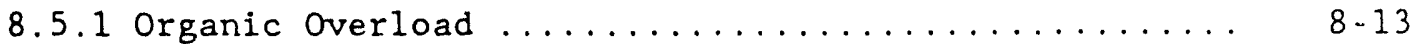

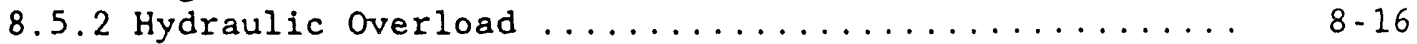

8.5.3 Copper-Induced Inhibition ............... 8-16

8.6 Effect of Oxygen on Co Concentrations ............ 8.19

8.6.1 Response of a Mesophilic Anaerobic Digester to

Exposure to Air ....................... 8-19

8.6.2 Effect of Oxygen on a Thermophilic Digester ....... 8-22

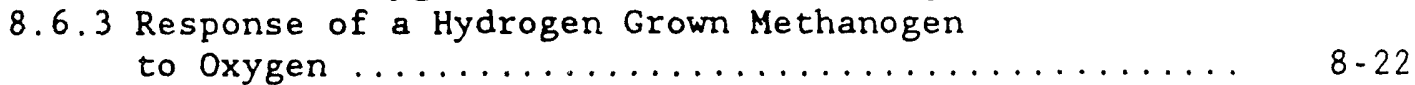

8.7 Discussion and Summary of Results $\ldots \ldots \ldots \ldots \ldots \ldots \ldots \ldots$ 8.24

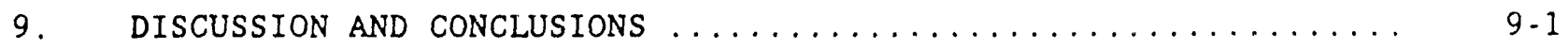


TABLE OF CONTENTS (Cont'd)

Section $\quad \underline{\text { Page }}$

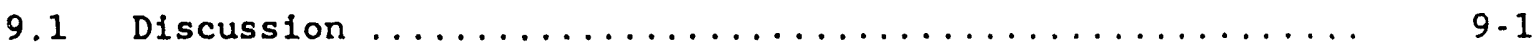

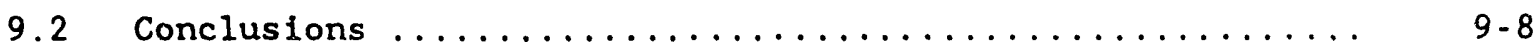

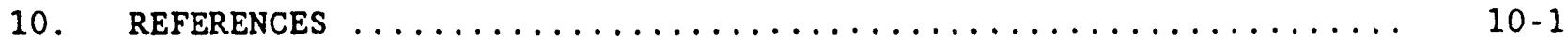

APPENDIX A. REAL-TIME DATA ACQUISITION SYSTEM $\ldots \ldots \ldots \ldots \ldots \ldots \ldots \ldots$ A-1 
3-1 Schematic of the 20 -day HRT reserve digester $\ldots \ldots \ldots \ldots \ldots \ldots \ldots$

3-2 Schematic of the 5-1iter test digester set-up used: 1) digester; 2) liquid recirculation pump; 3) in-line $\mathrm{pH}$ probe; 4) $\mathrm{pH}$ meter; 5) liquid flow distributor; 6) calibrated feed reservoir; 7) feed pump; 8) in-line gas filter and liquid trap; 9) gas recirculation pump; 10) dual infrared detectors $\left(\mathrm{CH}_{4}\right.$ and $\left.\mathrm{CO}_{2}\right) ; 11$ ) 10 -port gas sampling valve; 12) reduced gas chromatographic system; 13) $\mathrm{N}_{2}$ carrier gas; 14) gas production rate measurement system; 15) liquid trap; 16) computer interface system; 17) Apple II+ computer; 18) effluent port; 19) gas flow control

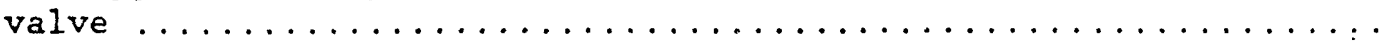

4-1 The effect of an organic overload on the hydrogen gas accumulation pattern - 10 -day HRT inoculum ................. $4-2$

4-2 The effect of an organic overioad on the carbon monoxide

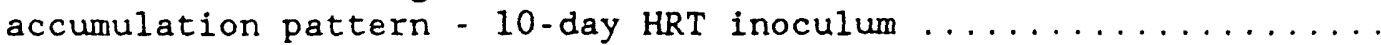

4-3 Comparison of the response of conventional and novel anaerobic digestion process indicators (measured at the end of each 24. hour feed cycle) during a 2.65 -fold organic load increase

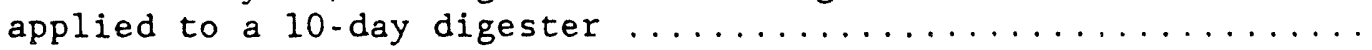

4-4 On-line measurements from a 5-liter test digester subjected to a 2.65-fold load increase for: a) gas production rate; b) $\mathrm{H}_{2}$;

c) $\mathrm{CO} ;$ and, d) off-line acetate determinations ............ 4-12

4-5 Measured total and volatile solids concentrations during a 2.5-fold load increase applied to a 20 -day $\mathrm{HR}^{\mathrm{T}}$ digester .........

4-6 Conventional and novel process indicators, measured at the end of each 24-hour feed cycle, during a 2.5-fold load

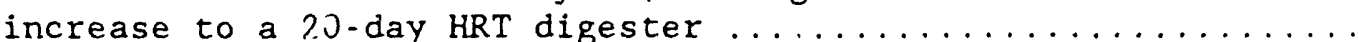

4-7 Gas production rate data for a 20-day HRT digester prior to and after a 2.5 -fold organic load increase ................

4-8 Change in values of initial gas production rate (A) and decay coefficient (b) as a result of a 2.5-fold organic

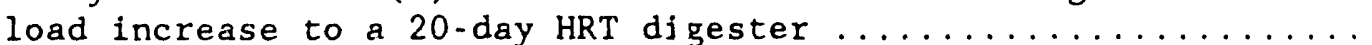

5-1 On-line measurements from a 5-1iter, 10-day HRT digester subjected to a 2.0-fold hydraulic load increase for a) gas production rate; b) $\mathrm{H}_{2}$; and c) $\mathrm{CO}$ 


\section{LIST OF FIGURES (Cont'd)}

No.

Page

5-2 Diurnal accumulation patterns of (a) acetate and b) propionate before, during and after a $\%$-day, 2.0-fold hydraulic load

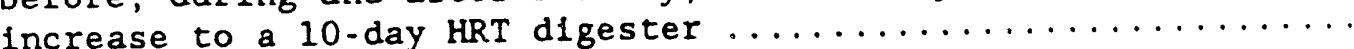

5-3 Effluent total and volatile solids and soluble COD concentra-
tions observed from a 10 -day HRT digester subjected to a 4.0 fold hydraulic load increase ..................... $5-9$

5-4 Gas production rate and VFA/TA ratio prior to and during a 4 fold hydraulic load increase to a 10-day HRT digester ........ 5-10

5-5 Response of (a) hydrogen and (b) carbon monoxide to a 4-fold hydraulic load increase to a 10 day HRT digester ........... 5-12

5-6 Accumulation patterns of a) acetate; b) propionate; and c) iso-valerate from a 10 -day HRT digester subjected to a 4.0.

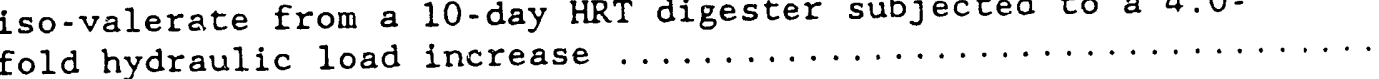

6-1 The effect of pulsing $7.85 \mathrm{mg}$ of copper per gram of VS on a) gas production, b) $\mathrm{H}_{2}$ and c) $\mathrm{CO}$ concentrations of a 10-day HRT

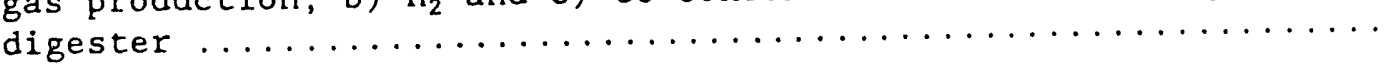

6-2 Response of a) $\mathrm{H}_{2}$ and b) $\mathrm{CO}$ of a 10-day HRT thermophilic digester to an $11 \mathrm{mg}$ of copper per gram of VS pulse .......... 6.6

6-3 Comparison of the response of conventional and novel anaerobic digester process indicators (end-of-cycle) for a 20-dey HRT digester subjected to chronic continuous copper

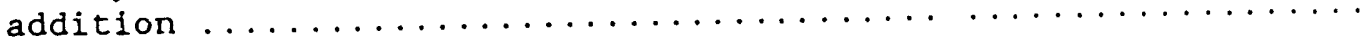

6-4 On-line (a) gas and (b) methane production rate data from a 20 day HRT digester subject to continual copper addition ......... 6-11

6-5 Fifteen minute interval total gas and methane production rates during the course of chronic copper addition to a 20-day HRT

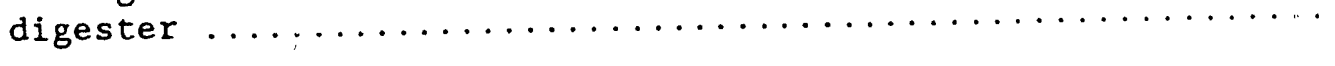

6-6 Graphical presentation of modeling of gas production data of a 20-day HRT digester subjected to continuous copper addition .....

6-7 Accumulation pattern of (a) acetate; (b) propionate; and (c) iso-valerate during copper induced inhibition of a 20 -day HRT

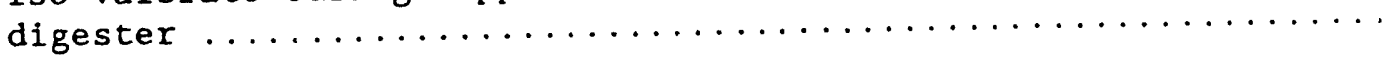

6-8 On-line measure of (a) hydrogen and (b) CO concentrations during copper induced (chronic addition) inhibition of a 20 -day HRT digester 


\section{LIST OF FIGUEES (Cont'd)}

№,

Page

8-1 Carbon monoxide concentration and methane production observed durlng growth of $M$. barkeri on acetate $\ldots \ldots \ldots \ldots \ldots \ldots \ldots$

8-2 Free energy evaluation for production and oxidation of carbon monoxide during methanogenesis from acetate under mesophilic

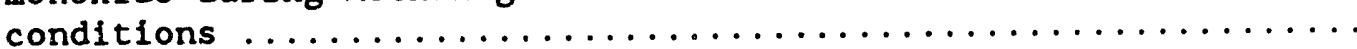

8-3 Effect of decreasing headspace methane content on the carbon monoxide concentrations of acesate fed digester inocula. At the times Ind: ated by the arrows the headspace was replaced with a nitrogen/carbon dioxide mixture $(70: 30) \ldots \ldots \ldots \ldots \ldots$

8-4 Effect of pulsing $10 \mathrm{mM}$ acetate, after 8.5 hours incubation, on $C O$ concentrations compared to controls receiving no added acetate for mesophilic digester inoculum fed vaste activated

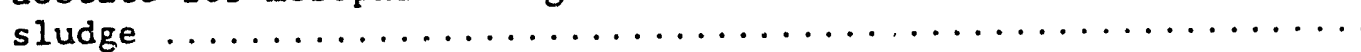

8-5 Corparison of measured and predicted acetate values for a batch daily fed 5-11ter mesophilic digester subjected to a

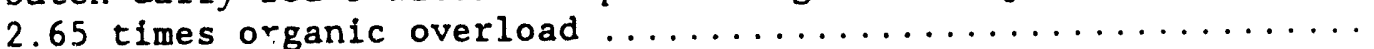

8-6 Comparison of predicted and measured acetate concentrations during the start of a two-fold increase in hydraulic loading

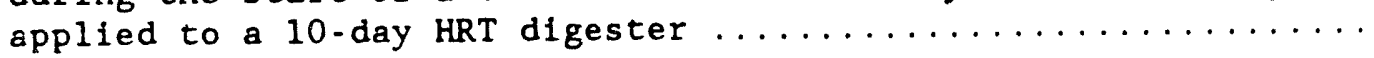

8-7 Comparison of the predicted and measured acetate concentrations during the initial 12 days of chronic addition of copper to a

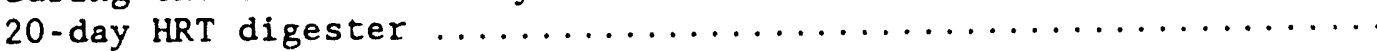

8-8 Response of carbon monoxide concentrations of a hydrogen grown culture of $M$. barkeri to addition of 1.0 percent oxygen to the

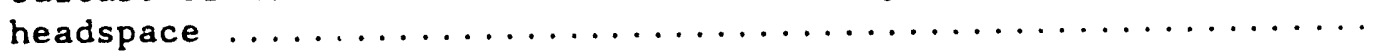

9-1 Three stage model of substrate and energy flow for the anaerobic

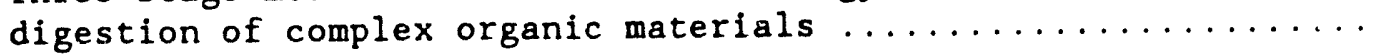

A-1 Schematic representation of the sampling control and data

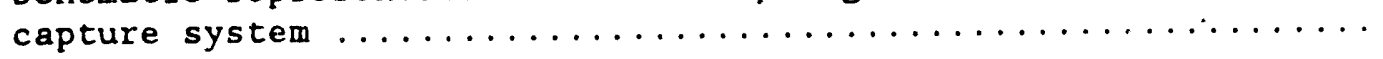

A-2 Schematic representation of the headspace composition measure-

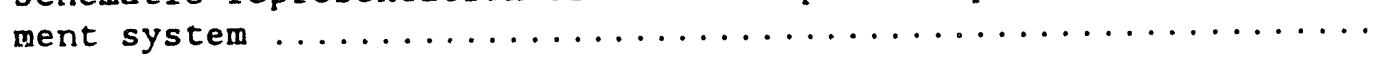

A-3 Schematic representation of the gas production measurement

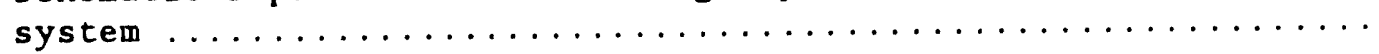

A-4 Effect of methane concentration on the ability to quantify trace levels of carbon monoxide using molesieve $5 \mathrm{~A}$ as a column packing 
LIST OF FIGURES (COnt'd)

№.

Page

A-5 Operation of 10-port sample valve operation during (a) sample inject and (b) backflush modes (sample loop - loading

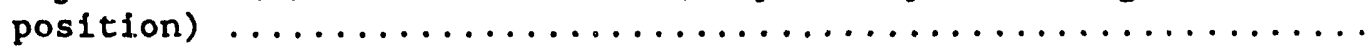

A -9 


\section{LIST OF TABLES}

No.

Page

3-1 Experimental Design for the Organic Overload Serum Bottle Assay $3-5$

3-2 Monitoring Frequency Schedule for Test Digesters ............

4-1 Concentrations of Volatile Fatty Acids and Selected Headspace Gases Produced as a Result of Batch Anaerobic Digestion of Waste Activated Sludge at Various Applied Organic Loadings ...........

4-2 Estimation of Extent of Hydrolysis During the Organic Overload

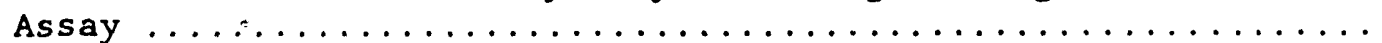

4-3 Summary of Feed and Effluent Total and Volatile Solids Concentrations Prior to and During a Continuous Organic

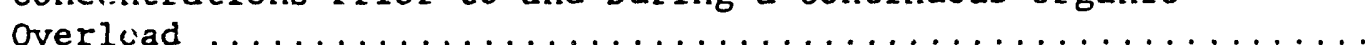

4-4 Comparison of the Response of Conventional and Novel Process Indicators of a 10-day HRT Anaerobic Digester in Response to a

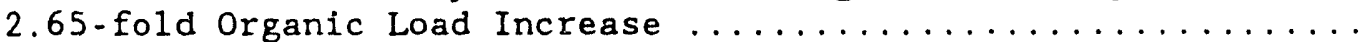

4-5 Results of Modeling Gas Production Rate Data of a 10 -day HRT Digester, Subjected to a 2.65-fold Organic Load Increase, as an

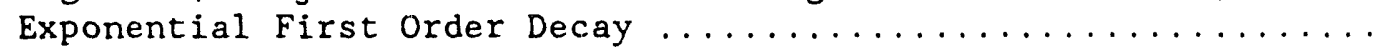

4-6 Results of Modeling Methane Production Rate Data as an

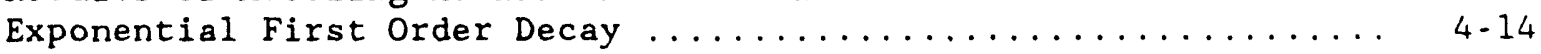

4-7 Daily Concentrations of Volatile Fatty Acids During a Continuous 2.65-fold Organic Overload of a 10-day HRT digester . . 4-17

4-8 Results of Modeling Gas Production Rate Data of a 20-day HRT Digester, Subjected to a 2.5-fold Organic Load Increase, as an Exponential First Order Decay ...................... 4-23

5-1 Daily Analysis of a 10-day HRT Test Digester Subjected to a 1.25 -fold Hydraulic Load Increase ..................... 5-2

5-2 Comparison of Conventional and Novel Trace Gas Indicators During a 2-fold Hydraulic Load Increase to a 10-day HRT Digester......

5-3 Results of Modeling Gas Production Rate Data for a 10-day HRT Digester Subjected to a 2 -fold Hydraulic Load Increase ......... 
No.

Page

5-4 Daily End-of-Cycle Volatile Fatty Acid Concentrations Observed During a 4-fold Hydraulic Load Increase Applied to a 10-day HRT

Digester .................................. $5-14$

6-1 Daily Analysis of a 10-day HRT Test Digester Overload Prior to

Receiving a Copper Fuise ........................ $6-2$

6-2 Dally Analysis of a 20-day HRT Digester Prior to and During Addition of Copper on a Chronic Basis ................... 6.8

6-3 Accumulation Pattern of Volatile Fatty Acids as a Result of Addition of Copper on a Chronic Basis to a 20-day HRT Digester .. 6-10

6-4 Accumulation of Copper in a 20-day HRT Digester Subjected to Chronic Addition of High Copper Feed ..................6.13

7-1 Standard Deviations of Indicator Parameters of Anaerobic Digestion ................................

8-1 Results of Serum Bottle Assay Demonstrating the Relationship Between $\mathrm{CO}$ and Acetate for Digester Inoculum ..............

8-2 Headspace Composition of Laboratory Anaerobic Digesters Treating Waste Activated Sludge ..................... 8 .

8-3 Response of a 20-day HRT Mesophilic Digester to Inadvertent Exposure to Air .............................8-20

8-4 Effect of Oxygen Addition to a 20-day HRT Inoculum on $\mathrm{CO}$ and $\mathrm{H}_{2}$

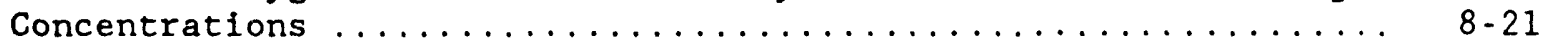

A-1 Gas Chromatograph Operating Conditions for Mercury Reduction Analysis of Hydrogen and Carbon Monoxide (On-Line Analysis) ..... A-10 


\section{EXECUTIVE SUMMARY}

An on-line, real-time data acquistion system was developed to monitor 5-1iter laboratory-scale anaerobic digesters for headspace $\mathrm{H}_{2}, \mathrm{CO}, \mathrm{CH}_{4}, \mathrm{CO}_{2}$ and gas production rate. Wet chemical analyses for VFA's, COD, SCOD, VS, TS and pH were used to augment the on-line information and provide complete data sets required to evaluate digester operation. Digesters operated at 10- and 20-day HRT's were subject to organic load increases of 2.65-and 2.5-fold, respectively. Hydraulic load increases of $1.25,2.0$ and 4.0 times the normal load were applied to 10 -day HRT digesters. Finally, 10- and 20-day HRT digesters were subject to copperinduced inhibition where the copper was added as a one-time pulse and added daily, respectively.

The response of novel trace gas indicators, $\mathrm{H}_{2}$ and $\mathrm{CO}$, were compared to the response of conventional process indicators to evaluate if $\mathrm{H}_{2}$ and $\mathrm{CO}$ could help improve process monitoring and control. The results indicate this may be so, especially for $\mathrm{CO}$.

Results from the experiment when the organic load to a 10-day HRT digester was increased 2.65 -fold indicated that the hydrogen utilizing population was not seriously challenged by the increase in loading. The results do indicate, however, that the hydrogen concentration, or diurnal profile, may indeed reflect the loading or level of stress imposed on the hydrogen-using population of the digester as suggested (Mosey, 1983).

Hydrogen monitoring, however, does not provide an indication of the level of stress being exerted on the acetate catabolizing population. Conventional wisdom stresses, and results generated during the course of this research support, that the acetate catabolizing population is more easily overloaded than the hydrogen utilizers. Substantial accumulation of acetate and higher molecular weight VFAs occurred without this being reflected in the $\mathrm{H}_{2}$ response. Even when the digestion process was under upset conditions (as judged by the VFA/TA ratio exceeding the 0.35 to 0.4 level), the hydrogen concentrations provided no indication this was so. 
Fortunately, the other trace gas monitored during the study, carbon monoxide, appears to have a strong relationship with the levels of VFAs in the system and thus provides a measure of the status of the acetate catabolizing population. During the batch organic overload experiment and the on-line experimentation, accumulation of acetate was always mirrored by a similar increase in $C O$.

The on-line monitoring of total gas and methane production during the organic overload did provide some indication that there was a potential problem. The responses observed were far less sensitive than observed for $C O$, however, and it is unlikely that total gas or methane production could provide adequate earlywarning of an upset. Variations in sludge composition, volume, and concentration normally found at treatment plants would provide sufficient background noise to preclude using gas production as an early warning indicator.

When a 20-day HRT system was subjected to an identical increase in organic loading, no perturbation in digester performance was observed. Acetate levels did not increase substantially and the CO response pattern before and after the organic load increase was applied, remained unchanged. Therefore, monitoring $C O$ can be used to predict acetate concentration reasonably well in conjunction with hypothesized thermodynamic relations of state, and thexeby allow detection of the accumulation of acetate due to organic overloading.

An hydraulic overload, applied at two times the normal loading, was insufficient to produce a response that could be considered an upset although the magnitude of the diurnal pattern of acetate accumulation and consumption was increased. This result was again clearly reflected in the $C O$ diurnal response pattern. Hydrogen gas concentrations also rose above normal steady-state levels. This moderate increase in hydrogen appears to indicate the increase in level of loading or stress on this population group.

When a four times hydraulic overload was applied to a 10-day HRT system, performance declined. A large accumulation of acetate, propionate or other VFAs did not occur, however. This may be because hydrolysis limits substrate flow. 
A pulse addition of copper on a continuously operated digester at a dosage sufficient to cause a temporary but significant, i.e., 15 percent, decrease in methane production resulted in dramatic responses of both hydrogen and $\mathrm{CO}$. The response of hydrogen was similar to that observed in prior batch studies with hydrogen initially decreasing from the normal "baseline" and then rising precipitously. The co response observed from the continuous system was similar but even more pronounced than observed for batch assays. The same type of "finger print" due to pulse addition of heavy metals was present for batch and continuously operated systems.

In a digester chronically dosed with copper, the responses of these two trace gases was somewhat muted. Hydrogen never displayed the drop in concentrations observed for pulse addition, but did show a steady rise above normal operating concentrations. Gaseous CO levels rose quite early and continued to rise, albeit slowly, during copper addition. When severe inhibition occurred, co dramatically rose several orders of magnitude.

A thermodynamic relationship was developed that allowed calculation of acetate concentration in the system based on headspace gas composition, particularly $\mathrm{CO}$, and $\mathrm{pH}$ only. Batch serum bottle assays were performed to verify the model, which was then used to evaluate data already collected for the hydraulic and organic load increases, and chronic copper addition experiments. The results of this model appeared to conform quite well for both batch and on-line digester systems in the absence of toxicant upsets. By simultaneously monitoring $\mathrm{H}_{2}$ and $\mathrm{CO}$, information on both of the major immediate precursors for methane production is available. Because $C O$ levels are apparently partially regulated by the $\mathrm{H}_{2}$ concentration, monitoring $\mathrm{CO}$ alone cannot provide early warning; $\mathrm{CO}$ and $\mathrm{H}_{2}$ need to be monitored together to yield the most information.

There are several caveats to using the thermodynamic relationship. First, the thermodynamic relationship is an equilibrium calculation, and using it on systems not close to or approaching equilibrium may produce erroneous results. Although the calculated acetate concentrations are presented as "predicted acetate" a better terminology might be "acetate potential" of the system at that point in 
time. That is the acetate concentration that would be in thermodynamic equilibrium with the measured headspace composition if the system is not kinetically or mass transfer limited.

An inherent assumption in the thermodynamic relationship is that equilibrium between the gaseous and aqueous phases exists. Experimental conditions were manipulated to foster equilibrium between the gaseous and aqueous phases in this study. In many natural ecosystems and engineered systems, this may not be true. Even during this research there was evidence of non-equilibrium between the two phases. The uptake of hydrogen occurs much more quickly than for $C O$. This is unlikely the result of low driving forces and low affinity for CO. Under normal conditions in the test digester, hydrogen concentration increased to approximately 40 ppin during the initial 3 to 4 hours after feeding and then declined to an apparent baseline concentration of approximately $20 \mathrm{ppm}$.

Mass transfer limitations will undoubtedly have an effect on the observed concentrations of both hydrogen and $\mathrm{CO}$. Whether integration of mass transfer kinetics with the thermodynamic model will be necessary for, or in certain cases even adequate to properly describe the system of interest (i.e. microenvironments in flocs, biofilms and sediments) requires further investigation.

A final concern is that $C O$ can be produced and uptaken by a number of bacterial types normally present in anaerobic consortia. Although the results generated herein support the hypothesis that acetoclastic methane producing activity controlled system CO levels, this may not be true in all instances. In systems where acetate metabolism accounts for most of the methane produced, such as industrial and municipal wastewater treatment systems and digesters, this is likely to be true.

Hydrogen and $\mathrm{CO}$ monitoring is promising, but according to this preliminary work, has limitations. Although a thermodynamic framework for explaining the $c 0$ evolution patterns observed in digester systems has been developed, support is circumstantial. Rigorous validation needs to be performed to obtain explanations why the model doesn't accurately describe the observed responses of trace gases during toxic upsets. 
Because the response of digester systems to different upset scenarios is markedly different, it may be possible to develop a monitoring strategy based on pattern recognition techniques that will allow detection of the onset of upsets and will identify the type of problem. This would allow a timely, better-focused remedial response to the problem. Additional research is needed to delineate how far this concept can be developed. 


\section{SECTION 1}

\section{INTRODUCTION}

Techniques used to monitor the anaerobic digestion process have been extensively reviewed in a previous report and will not be reported here. Preliminary work using batch serum bottle reactors to investigate the response of two trace gases produced during anaerobic digestion, hydrogen and carbon monoxide, found that both these gases have excellent potential as indicators of process upset due to organic and inorganic toxicant-induced inhibition. The object of this additional work was to extend the results to continuously operated systems. Upset scenarios are best examined in continuous, as opposed to batch, assay techniques such as hydraulic and organic overloads and are the focus of this effort. A comparison of batch assays to continuously operated systems for heavy metal-induced inhibition (copper) is also provided. Efforts to statistically model 15 -minute interval real-time, on-line, data from continuously operated digesters using Spectral and Box-Jenkins techniques are reported. Finally, the role of $C O$ and a thermodynamically based method of relating the observed $C O$ concentration to acetate levels in a digester system is presented. 


\section{SECTION 2}

\section{BACKGROUND}

The literature describing hydrogen and carbon monoxide in methanogenic systems and pure culture has been extensively reviewed in the previous report and will not be presented here. A quick review of the most relevant research articles is presented to provide the necessary background about the various experiments.

Hydrogen is an important intermediate for methanogerasis. It is produced during the fermentation of carbohydrates and other substrates and in the subsequent degradation of propionic acid and other higher molecular weight volatile fatty acids to acetate. In anaerobic digesters, hydrogen is primarily used by hydrotrophic methanogens to reduce carbon dioxide to methane.

Interspecies hydrogen transfer has iong been identified as playing a key role in regulating the fermentation of organic matter (Wolin and Miller, 1982). A buildup of hydrogen can inhibit the growth of hydrogen-producing organisms and alter electron flow, resulting in the formation of more reduced products such as lactate, butyrate or ethanol that serve as alternative sinks for the hydrogen produced, instead of acetate (Chung, 1976). Mosey (1983) has indicated that it may be simpler and more productive to monitor hydrogen as the prime indicator of system loading. Barnes and co-workers (1984) reported that an anaerobic fluidized-bed reactor treating a carbohydrate waste demonstrated an accumulation of hydrogen gas levels in response to severe organic overloading conditions. Archer and co-workers (1986) measured hydrogen concentration in a pilot-scale anaerobic contact system. Hydrogen concentration rose rapidly in response to volumetric shocks although no volatile acid accumulation was observed. They concluded that hydrogen is potentially a crucial parameter to monitor process control in operating industrial anaerobic digester systems. Harper and Pohland (1987) have shown that it may be feasible to enhance process stability of high rate anaerobic systems by manipulating the hydrogen level with various process configurations. Whitmore et al. (1987) were able to prevent upset of a glucosefed chemostat by controlling feed to the reactor based on dissolved hydrogen monitoring using membrane inlet mass spectrometry. By monitoring dissolved 
hydrogen levels at approximately 1.0 uM via a feedback on/off pump control, stable operation during a five-fold increase in influent COD concentration was possible. Hickey et al. (1987b) reported that for detecting the onset of inhibition due to organic toxicants such as chloroform or trichloracetic acid, hydrogen monitoring holds promise but probably should be used in conjunction with currently employed monitoring strategies. McCarty and Smith (1986), based on the result of monitoring laboratory CSTR's, anaerobic filters and baffled reactors under steady state operating conditions, reported that hydrogen monitoring could be an important tool for both process control and to diagnose process problems.

Conrad and Thauer (1983) first reported biological carbon monoxide (C0) formation under strictly anaerobic conditions. A bound $\mathrm{CO}$ is an intermediate in anabolic and catabolic pathways for both methanogens and acetogens (Zeikus et al., 1985). Eimanns and Thauer (1984) hypothesized that a bound CO moiety, with the CO most likely tightly bound to a metal, is probably an intermediate in the decarboxylation of acetate. Nelson and Ferry (1984) speculated that gaseous CO equilibrates with some component in the pathway that transforms the carboxyl group of acetate to $\mathrm{CO}_{2}$. Kohler and Zehnder (1984) reported that Methanothrix soehngenii, generally believed to be the principle acetate catabolizing methanogens in digester systems, possesses high levels of carbon monoxide dehydrogenase activity. Krzycki and Zeikus (1984a) reported that approximately 5 percent of the soluble protein in acetate grown $\underline{M}$. barkeri was Co dehydrogenase in acetate catabolism. Carbon monoxide has recently been reported to evolve from anaerobic digester systems during methanogenesis from acetate by an enrichment culture and pure culture of Methanosarcina barkeri (Hickey, et al., 1987a; Hickey, 1987)

In batch assays conducted during the initial phase of this research to examine the response of $C O$ to pulse addition of organic and inorganic toxicants, $C O$ showed promise as an indicator of process upset. It was, therefore, decided to examine the response of both $\mathrm{CO}$ and hydrogen to a variety of upset scenarios in continuously operated systems. 2 


\section{SECTION 3}

\section{EXPERIMENTAL APPROACH AND DESIGN}

\subsection{TECHNICAL APPROACH}

This part of the research effort was designed to monitor the behavior of model, WAS fed, laboratory anaerobic digester systems during steady state and in response to toxic shocks, organic overloads and hydraulic overloads. Shocks of both failure and sub-failure proportions were examined.

In this study semi-continuous (batch fed daily) digesters were used in conjunction with an on-line, real-time data acquisition system to assess the behavior of conventional and novel process indicators of the anaerobic digestion process in response to the previo:ss?y described perturbations.

\subsection{EXPERIMENTAL DESIGN}

There were three major components or tiers in the experimental design:

- Construction and steady state operation of reserve digesters (inocula source);

- Serum bottle assays (batch tests); and

- CSTR test digester on-line experimentation.

Two reserve digesters were constructed, brought to steady state operating conditions, and maintained at steady state for the duration of the experimental program. Portions of the reserve digester volume, less than or equal to one day's feed volume, 5.5 liters, were periodically removed to start the serum bottle assays. This approach allowed a continual supply of relatively uniform biomass to be avallable on demand throughout the study. Once a test reactor was subjected to a toxicant or significant organic or hydraulic overload and the necessary data obtained, the contents were discarded and after thorough cleaning replaced with fresh inocula from one of the reserve digesters. This approach not only streamlined data collection, but also provided better assurance that data collected in each individual run could be directly compared to other runs since 
the inocula for all tests came from the same source with no prior history of exposure to the toxicants, and were unaltered by prior testing (i.e., bacterial population changes due to changes in organic or hydraulic loading).

A detailed description of the complete experimental design and testing schedule follows.

\subsubsection{Reserve Digesters}

Two reserve digesters with working volumes of 55 and 110 liters, constructed from 12 inch schedule 80 PVC pipe and $3 / 4$ inch PVC stock, were used to supply inocula with 10 - and 20-day hydraulic retention times (HRT), respectively. A schematic of the 20-day HRT (110 liter) digester is presented in Figure 3-1. To avoid any influence due to temperature variation, reactors were housed in constant temperature incubators maintained at $35^{\circ} \mathrm{C}$, a near optimal temperature for mesophilic methanogenic cultures.

The seed for the reserve digesters was obtained from municipal digesters located in Northampton, Massachusetts. These digesters receive a combination of primary and waste activated sludge and were operating at a hydraulic retention time (HRT) of 15 to 20 days when the sludge was obtained.

A volume of digester sludge, equaling the working volume of the reserve digesters, was placed in the reactors after careful screening with $1 / 16$ to $1 / 32$ inch mesh to remove any large debris. Gas production rate was monitored over the next day to ensure that an active biomass had been obtained. The 10-and 20-day HRT reserve digesters were then fed the volume of sludge ( 5.5 liters) required to maintain the proper residence times. The reserve digesters were maintained mixed using a liquid recirculation system that withdrew sludge just below the 1iquid/gas interface and returned it to the bottom of the reactor through a three nozzle distributor. Mixing was performed with Manostat Varistaltic pumps operated on a timer system. Pumps were cycled in an on/off mode of 15 minute intervals except during daily feeding and 12 hours after feeding. The pumping system was timed to stay on continuously for 45 minutes prior to feeding sludge and for an additional 45 minutes after feeding. A 45 minute continuous mixing 


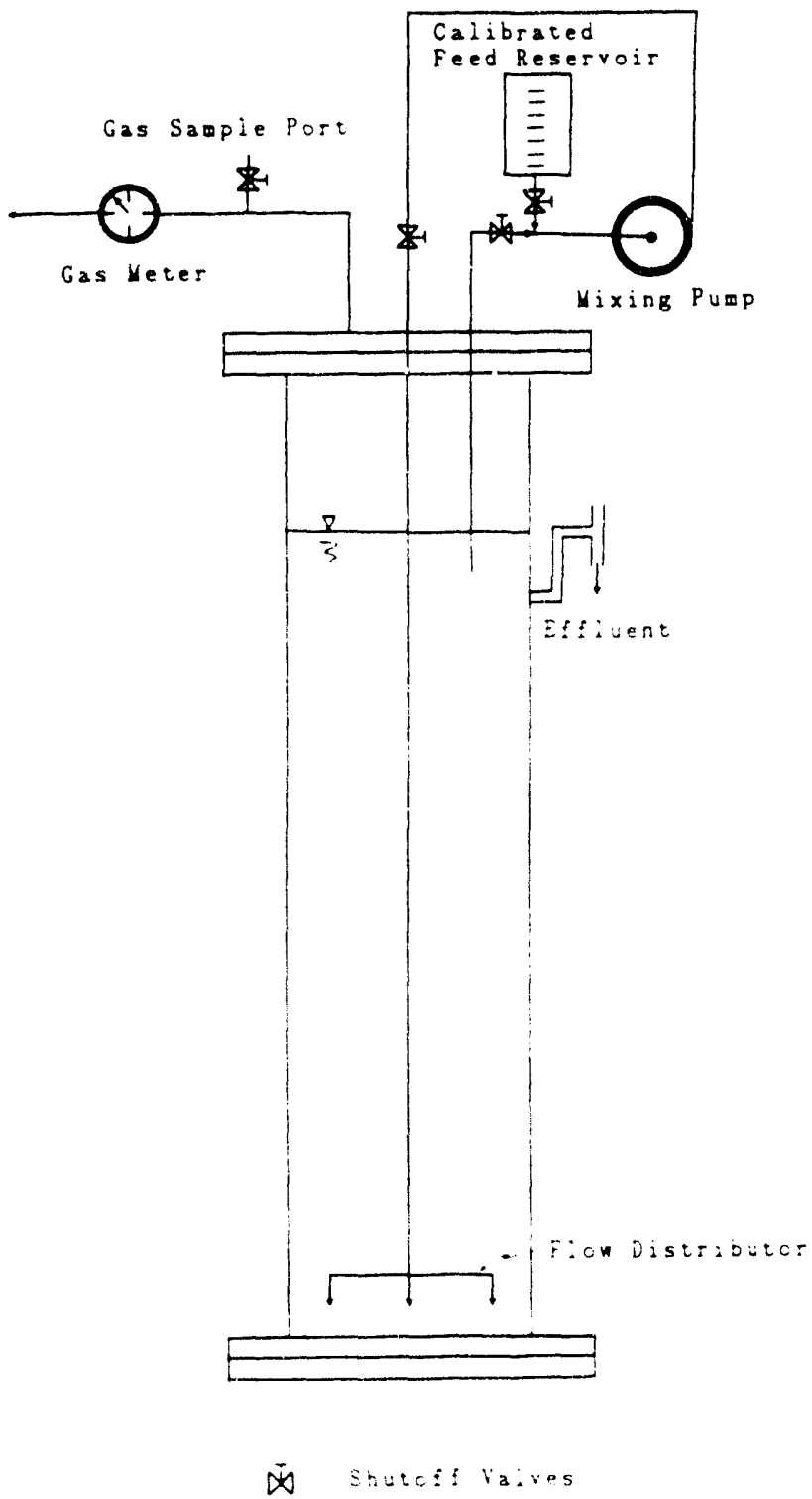

Figure 3-1. Schematic of the 20 day HRT reserve digester.

3- 3 
perlod was employed 12 hours after dally feeding. Flow through the reactors was maintained at approximately $80-90 \mathrm{~m}^{3} / \mathrm{m}^{2}-d$, which appeared sufficient to keep the reactor contents well mixed. Silicon tubing was initially used in the Manostat pumps; later a change was made to norprene tubing which had a longer life and much lower permeability to oxygen.

Gas production was monitored with Wet Tip Meters (Wet Tip Meter Co., Wayne, PA). Gas composition was assayed by gas chromatography procedures described later in this section.

\subsubsection{Organic Overload Assay}

The organic overload assay was conducted in a slightly different manner than the toxicant assays. The ability to obtain cuncentrated WAS from the Amherst POTW is limited. A 3.5 to 4 percent DAF float solids is the maximum concentration that could be attained. The experimental design used for the organic overload assay is presented in Table $3-1$.

Most organic and hydraulic overload experimentation is more appropriately conducted in continuous rather than batch tests. Therefore, these experimental runs were performed in 5-1iter test digesters connected to a real-time data acquisition system. One organic overload experiment was performed in a batch assay to obtain sume measure of the response pattern to expect in later experiments.

\subsubsection{Supplemental Serum Bottle Assays}

Several additional serum bottle assays were conducted to more thoroughly examine CO evolution during methogenesis from acetate. The defined medium of owens et at. (1979) was used in all assays. Inocula were obtained from the 110 liter, 20. day HRT reserve digester. For sisme assays, the inocula were first enriched for acetate utilizing populations.

In order to enrich for acetate utilizing methaneogenic populations, the following procedure was used. Sludge was anaerobically transferred from the digester and 
Table 3-1. Experimental Design for the Organic Overload Serum Bottle Assay.

Sample No.

1

2

3
Feed Strength

$1.0 \mathrm{X}$

$2.0 \mathrm{x}$

3. $0 \mathrm{x}$ ml Sludgeb

100

100

100

a) $X$ represents the normal feedstock solids concentration to which the reserve digesters have been acclimated.

b) Sludge removed from the reserve digesters was mixed with an appropriately concentrated amount of WAS. Total volume placed in each serum bottle will be $100 \mathrm{ml}$ in all cases. 
continually gassed with the $\mathrm{N}_{2}: \mathrm{CO}_{2}$ mixture. Serum bottles were filled with 95 $\mathrm{ml}$ of defined medium and $5 \mathrm{ml}$ of digester sludge anaerobically transferred with a syringe pump and repeating pipet, respectively. Sludge and defined medium reservoirs were maintained completely mixed with magnetically driven stir bars. Sludge, defined medium and serum bottles were continually gassed with the $\mathrm{O}_{2}$ free (<2 ppm) $\mathrm{N}_{2}: \mathrm{CO}_{2}$ gas during the entire transfer period. Upon completion of the transfers, serum bottles were capped with butyl rubber septa and sealed with aluminum crimp seals. Substrate (25 or $50 \mathrm{mM}$ acetate) was then added to the serum bottles via syringe injection and samples incubated at $35^{\circ} \mathrm{C}$. Gas headspace was monitored over time to track substrate utilization. Upon substrate depletion, these inocula were used in the serum bottle assays.

In one assay, digester inoculum (20-day HRT) was used to examine the relationship between acetate concentration and gaseous $C O$ levels without enriching for an acetate utilizing population. This assay used $95 \mathrm{ml}$ of defined medium and $5 \mathrm{ml}$ of inoculum to examine the relationship between $\mathrm{CO}$ and acetate from a thermodynamic perspective. Reaction rates were reduced to allow a greater probability that near equilibrium conditions between the gaseous and liquid phases could be obtained. In addition, the inoculum was starved for 3 days while being held in a $35^{\circ} \mathrm{C}$ incubator to reduce endogenous activity due to breakdown of undigested substrate in the sludge. This minimized interferences in the acetate and CO relationship.

\subsubsection{Test Digester Operation}

A schematic of a test digester is presented in Figure 3-2. The long narrow design served two purposes. First, the smaller diameter reactor had a small headspace and, therefore, changes in gas composition occurred quickly. Second, a smaller pump was required to provide sufficient mixing for the digester to be considered a completely mixed reactor (CSTR). Manostat Varistaltic pumps were used to mix the test digesters.

The mixing pumps were operated on a 15 -minute cycle of 8 minutes on, 7 minutes off. The system was designed so that an in-line $\mathrm{pH}$ probe could be inserted into 


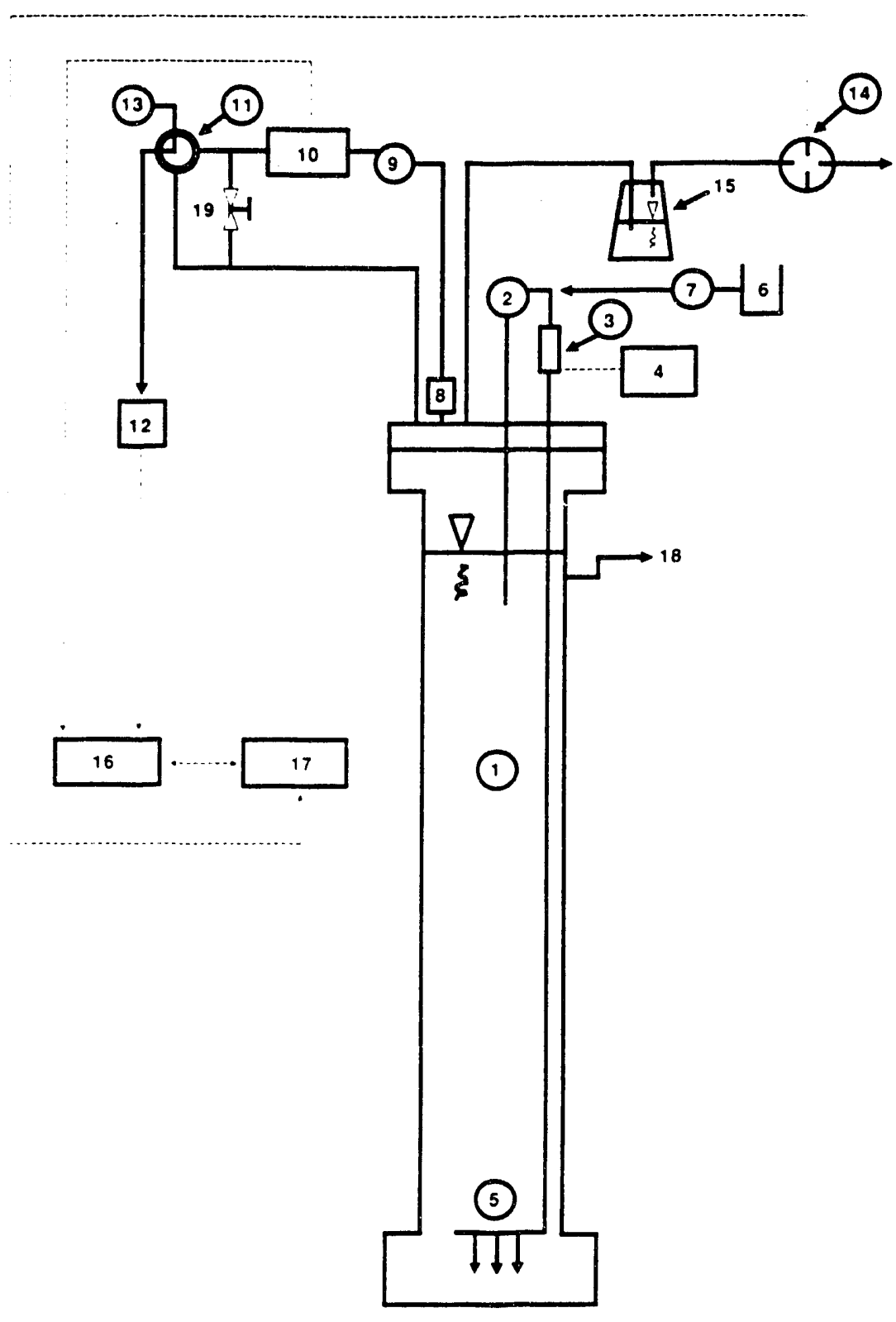

Figure 3-2. Schematic of the 5-liter test digester set-up used: 1) digester;

2) liquid recirculation pump; 3) in-line $\mathrm{pH}$ probe; 4) $\mathrm{pH}$ meter;

5) liquid flow distributor; 6) calibrated feed reservoir; 7)

feed pump; 8) in-line gas filter and liquid trap; 9) gas recirculation pump; 10) dual infrared detectors $\left(\mathrm{CH}_{4}\right.$ and $\left.\mathrm{CO}_{2}\right)$; 11) 10 -port gas sampling valve; 12) reduced gas chromatographic system; 13) $\mathrm{N}_{2}$ carrier gas; 14) gas production rate measurement system; 15) liquid trap; 16) computer interface system; 17) Apple II+ computer; 18) effluent port; 19) gas flow control valve. 
the recirculation line to allow continuous monitoring if desired. Gas analysis for $\mathrm{CO}_{2}$ and $\mathrm{CH}_{4}$ was performed on-line with non-destructive infrared (IR) detectors. Hydrogen and $C O$ were analyzed with a RGD2 mercury reduction type gas chromatograph (Trace Analytical, Stanford, CA) equipped for on-line, discrete sample analysis. A liquid trap, filled with an acidified saturated salt solution to prevent absorption of the off-gas into the liquid, was placed in the off-gas line between the reactor and gas measurement system. Gas production was continuously measured via a liquid displacement technique.

The general procedure for starting up a test digester follows. The reactor was thoroughly cleaned to remove any wall growth and adsorbed material from the previous run. Residual detergent was removed by thorough rinsing with tap and distilled water. The reactors were reassembled to an airtight condition. Oxygen was removed from the reactors by purging with nitrogen gas. The reactor was connected to the effluent drawoff of one of the reserve digesters and five liters withdrawn into the test digester. A final purge of the gas space was made with a methane-carbon dioxide gas mixture (70/30), typical of the off-gas composition observed in the reserve digesters during the initial portion of experimentation. The reactor(s) were then fed the appropriate volume of WAS to maintain a 10 - or 20-day HRT, and on-line instrumentation was callbrated and placed in service.

A minimum of one or two days normal draw and fill operations ensured that steady state was maintained. On the third day, or later, when the unit was deemed to be operating at steady state (as judged by gas production, TS, TVS, pH and alkalinity measurements) and ready to receive the toxic shock or organic overload conditions, the perturbation part of the experiment was initiated. Monitoring followed the monitoring frequency schedule (Table 3-2).

In addition to the mesophilic systems, a two-liter thermophilic digester was operated for approximately six months to compare mesophilic and thermophilic systems. The thermophilic digester was operated continuously and used primarily to observe the effect of minor variations in loading rate and substrate composition. This digester was monitored intensively for gas production and 
Table 3-2. Monitoring Frequency Schedule for Test Digesters.

Parameter
$\mathrm{CH}_{4}$
$\mathrm{CO}_{2}$
$\mathrm{H}_{2}$
$\mathrm{CO}$
$\mathrm{pH}$
Alkalinity
Volatile Fatty Acids
Total Solids
Volatile Solids
COD
SCOD

\section{Frequency}

4/hour

4/hour

4/hour

4/hour

1/day

1/day

$1 /$ day

1/day

$1 /$ day

1/day

$1 /$ day 
$\mathrm{H}_{2}$ and $\mathrm{CO}$ response patterns. $\mathrm{H}_{2}$ and $\mathrm{CO}$ were sampled manually while gas production was measured using a liquid displacement technique. 


\section{SECTION 4}

\section{EXPERTMENTAL RESULTS - THE EFFECT OF ORGANIC VARIATIONS AND OVERLOADS}

This section describes results from monitoring semi-continuously operated mesophilic digesters. The digesters were fed waste activated sludge (WAS) as the sole substrate on a daily draw and fill schedule. The real-time, on-line data acquisition system, augmented with appropriate wet chemical analyses, was used to investigate long-term events and the possible relationships of hydrogen $\left(\mathrm{H}_{2}\right)$ and carbon monoxide (CO) to process stability.

In order to obtain some measure of the response of total gas and methane production as well as trace gas responses to organic overloads, so that finalization of the on-line data acquisition system configuration could be made, a preliminary serum bottle assay was performed. These results are examined first.

\subsection{ORGANIC OVERLOAD SERUM BOTTLE ASSAY}

Organic loading rates of 1,2 , and 3 iimes the normal loading applied to a 10-day HRT digester $\left(1.0 \mathrm{~g}\right.$ VS $\left.1^{-1} \mathrm{~d}^{-1}\right)$ were simulated by varying the organic strength of WAS fed to serum bottle reactors. The hydrogen response patterns to the increases in organic loading are presented in Figure 4-1. The initial rate of hydrogen accumulation and peak concentrations attained were directly related to applied organic load. By the conclusion of a 24 -hour cycle, the average hydrogen concentrations for all three organic loading conditions were essentially the same. Significant accumulations of volatile fatty acids (VFAs) were evident at this time for samples receiving greater than normal strength feed (Table 4-1). No significant amounts of higher molecular weight VFAs greater than $C_{5}$ were found in any of the samples, although traces of iso-caporate were detected in the sample receiving three times normal loading.

Total gas production was increased by approximately 12 and 24 percent by the twoand three-fold organic overload conditions, respectively (Table 4-1). A substantial portion of this gas was carbon dioxide as methane production was increased by only 6 and 12 percent above controls for the two and three-fold 


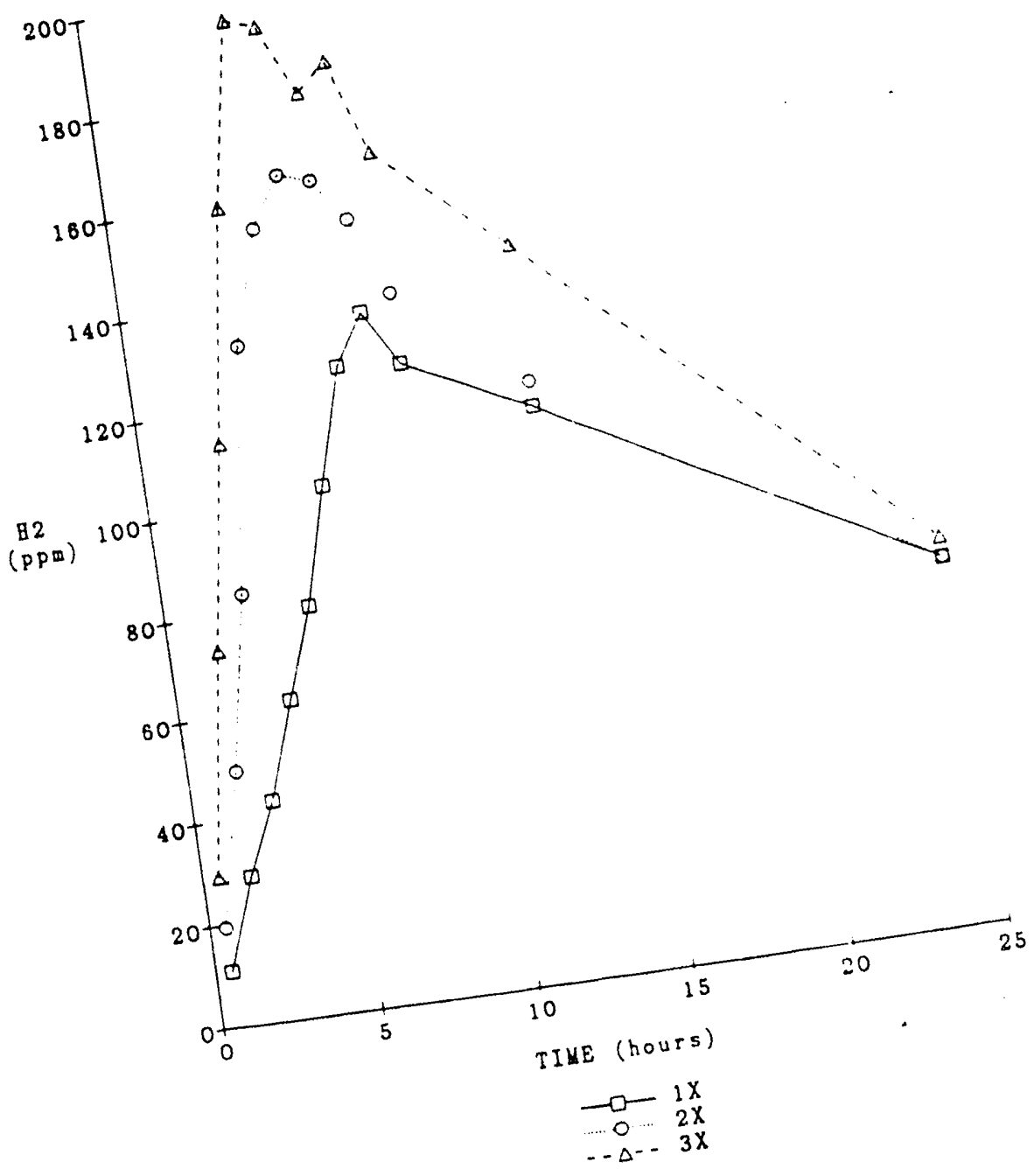

Figure 4-1. The effect of an organic overload 10 -day HRT inoculum.

the hydrogen gas accumu- 


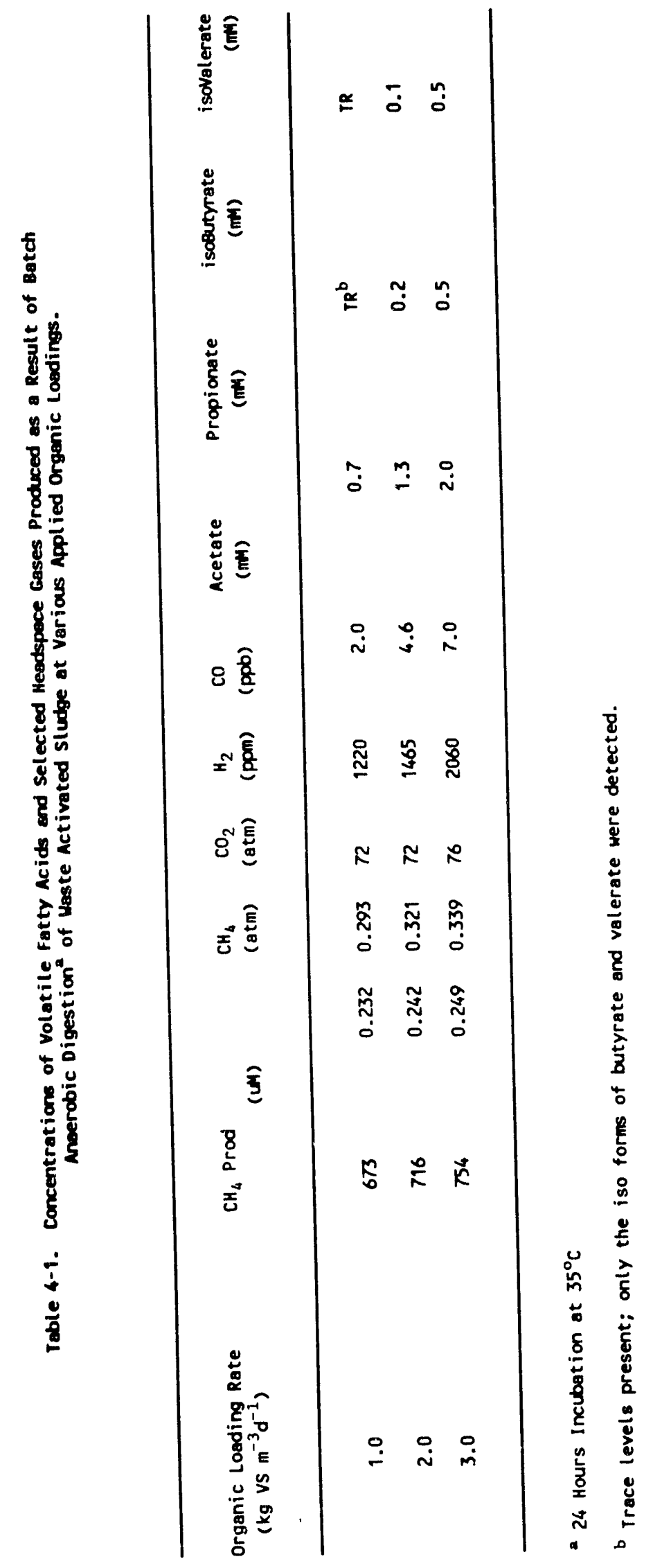


organic load increases, respectively. As seen in Table 4-2, significant additional solubilization of the WAS occurred in samples receiving the greater strength feed.

Results of monitoring the trace concentrations of carbon monoxide over the course of the assay are presented in Figure 4-2. Higher loading (increased organic strength) resulted in more rapid accumulation of $\mathrm{CO}$ in the headspace as well as higher final $C O$ concentrations. Gaseous $C O$ was observed to be still increasing, albeit slowly, for the three-fold organic overload at the conclusion of the assay.

\subsection{ORGANIC OVERLOAD OF A 10-DAY HRT DIGESTER}

A 10-day test digester was operated in a semi-continuous mode (batch daily feeding) for approximately two weeks after transfer of digester sludge from the 55 liter, 10-day HRT reserve digester. During this period, the feed sludge solids concentration was maintained at approximately 1.5 percent TS ( 1.2 percent VS). Daily monitoring of the digester was initiated nine days prior to commencement of the organic overload portion of the experiment. During this period a rather constant volatile solids destruction efficiency of 35.5 percent was observed (Table 4-3). After collection of this baseline information, the real-time data acquisition system was used to investigate the response of this digester to a continuous organic overload.

On the ninth day of data collection the digester feed was switched from the WAS/DAF mixture of 1.5 percent TS to the more concentrated DAF solids (4.0 percent TS). This increase in feed strength was made while maintaining a constant 10-day HRT. Daily data collected subsequent to this point in time will be referred to by the day after initiation of the overload. For example, day 1 refers to the on-line information collected for the 24-hour period immediately following the introduction of the first day of the DAF solids feed and to the wet chemical analyses performed at the conclusion of that 24 -hour cycle.

In order to reduce the extremely heterogeneous nature of the concentrated sludge, 
Table 4-2. Estimation of Extent of Hydrolysis During the Organic Overload Assay.

\begin{tabular}{lllll}
$\begin{array}{l}\text { Feed Strength } \\
(\mathrm{gVS} / 1)\end{array}$ & $\begin{array}{l}\text { COD of Methane } \\
\text { Produced } \\
(\mathrm{mg} / 1)\end{array}$ & $\begin{array}{l}\text { COD of Final } \\
\text { Volatile Acids } \\
(\mathrm{mg} / 1)\end{array}$ & $\begin{array}{l}\text { Total } \\
\text { Solubilized } \\
\text { COD } \\
(\mathrm{mg} / \mathrm{L})\end{array}$ & $\begin{array}{l}\text { Fraction of } \\
\text { Control }\end{array}$ \\
\hline & & & 430 & 1.0 \\
10.0 & 430 & - & 762 & 1.8 \\
20.0 & 458 & 304 & 1144 & $2: 7$ \\
\hline 30.0 & 482 & 662 & & \\
\hline
\end{tabular}

- Volatile acids above controls (or normal loading) samples. It was assumed that VFAs of controls were essentially the same at the start and conclusion of the 24 -hour assay. 


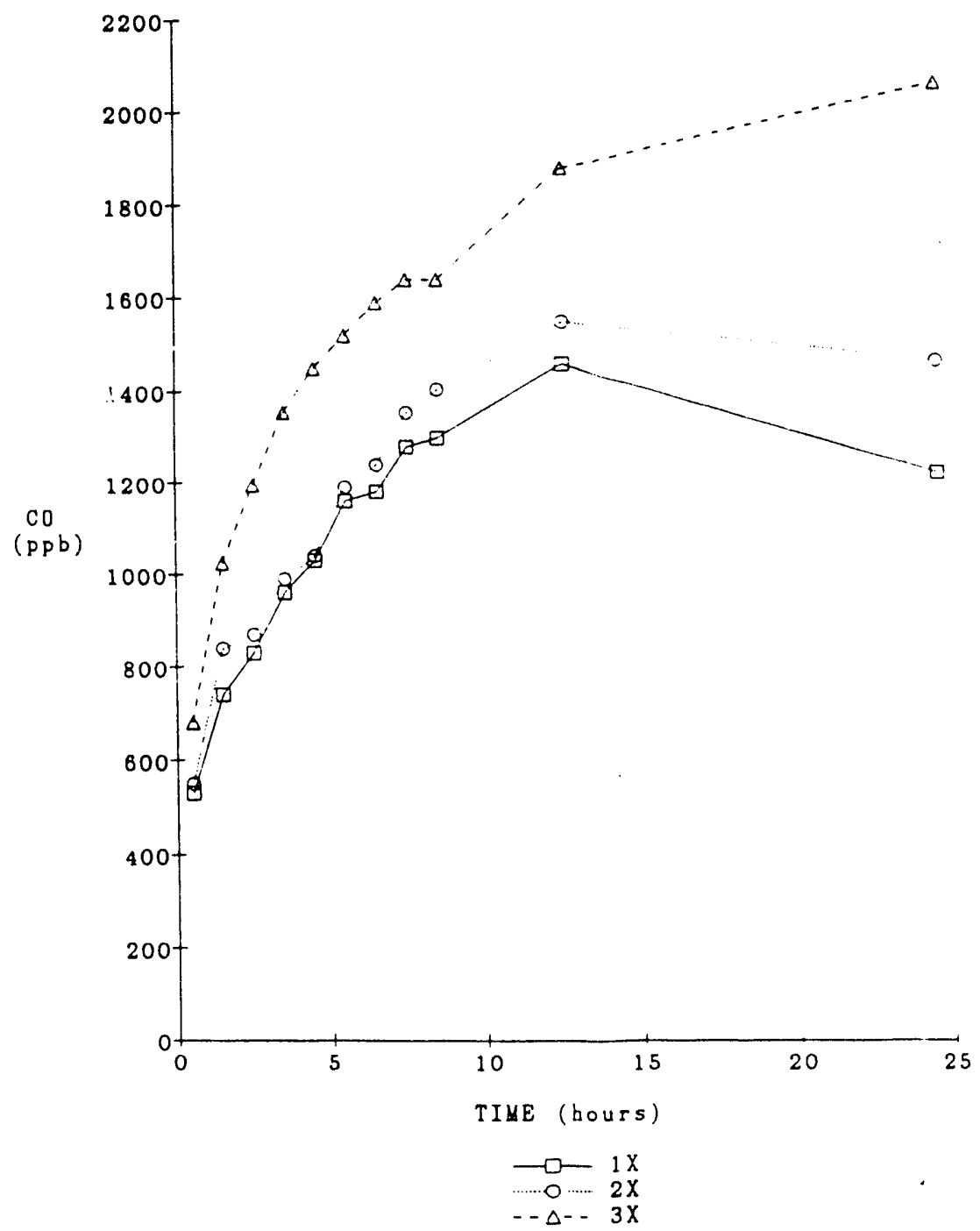

Figure 4-2. The effect of an organic overload on the carbon monoxide accumulation pattern - 10-day HRT inoculum. 
Table 4-3. Summary of Feed and Effluent Total and Volatile Solids Concentrations Prior to and During a Continuous Organic כverload.

Feed

TS VS

(mg/1)

$(\mathrm{mg} / \mathrm{L})$

11,400

11,700

11,500

11,600

11,500

12,400

11,300

12,100

33,200

30,500

38,900

38,600

30,600

39,900

32,400

38,400

30,300

41,300

33,400

38,800

31,000

36,000

28,600

35,100

27,400

41,000

31,800

40.000

32,800

-
Effluent

TS

$(\mathrm{mg} / \mathrm{L})$

10,900

$-, 760$

10,300

7,320

10,500

7,430

10,200

7,070

10,700

7,720

10,600

7,710

10,200

7,270

10,500

7,570

10,600

7,840

12,600

9,240

15,200

11,140

16,800

12,331

18,000

13,120

19,700

14,600

21,900

16,000

20,500

14,900

20,500

14,700

25,400

18,500

26,400

19,700

23,000

17,000 
the DAF solids were blended at low speed for several minutes and then screened to remove any remaining large debris.

Data for normal anaerobic digestion process indicators, measured at the conclusion of each dally feeding cycle, are compiled in Table 4-4. As can be seen, the continuous organic overload resulted in a slow deterioration in process performance. Trend plots of daily, end-of-cycle, data collected for conventional and trace gas process indicators are compared in Figure 4-3. As would be anticipated, the volatile fatty acid to total alkalinity (VFA/TA) ratio was the first of the conventional process indicators to register a possible upset situation. This occurred after six days. By day 8 , the VFA/TA ratio had increased to the 0.35 to 0.4 range, which is considered to signal an upset condition (Zickefoose and Hayes, 1976). Trend piots for gas production or $\mathrm{CO}_{2}$ and $\mathrm{CH}_{4}$ content did not show any definite indication of upset until day 7 or 8 when the upset condition, as judged by the VFA/TA ratio, existed.

Novel process indicators, $\mathrm{H}_{2}$ and $\mathrm{CO}$, both demonstrated a significant initial response to the increase in organic loading. The concentration of both gases more than doubled from the normal operating ranges observed during the pre-shock equilibrium period from day 0 to day 1 . Hydrogen gas concentration subsequently levelled off and remained at approximately $40 \mathrm{ppm}$ for the remainder of the experiment (Figure 4-3c). By contrast, CO was observed to increase each day much the same as the VFA/TA ratio (Figure 4-3b). As will be discussed later, this is believed to be due to the direct relationship of gaseous $C 0$ to the acetate concentration in anaerobic digesters. Evidence that strongly supports this hypothesis will be presented.

A clearer picture of the effect of the continuous organic overload can be seen from examining the real-time gas production and composition data as well as the supplemental analysis of the daily VFA accumulation patterns prior to and during the overload. For this experiment, real-time data was collected on a 15 -minute interval. 
Table 4-4. Comparison of the Response of Conventional and Novel Process Indicators of a 10-day HRT Anaerobic Digester In Response to a 2.65-fold Organic Load Increase.

\begin{tabular}{|c|c|c|c|c|c|c|c|c|}
\hline Day & $\begin{array}{c}\text { Gas Pro } \\
(1 / d)\end{array}$ & $\begin{array}{l}\mathrm{CH}_{4} \\
(8)\end{array}$ & $\begin{array}{l}\mathrm{CO}_{2} \\
(8)\end{array}$ & $\begin{array}{l}\mathrm{H}_{2} \\
(\mathrm{ppm})\end{array}$ & $\begin{array}{l}\mathrm{CO} \\
(\mathrm{ppb})\end{array}$ & $\begin{array}{l}\mathrm{pH} \\
(\mathrm{s} . \mathrm{u} .)\end{array}$ & $\begin{array}{c}\mathrm{TA}^{*} \\
(\mathrm{mg} / 1)\end{array}$ & $\mathrm{VFA} / \mathrm{TA}$ \\
\hline-8 & 1.87 & 63 & 30 & 19 & 610 & 7.0 & 2520 & 0.06 \\
\hline-7 & 1.85 & 63 & 30 & 18 & 460 & 7.0 & 2530 & 0.05 \\
\hline-6 & 1.88 & 63 & 30 & 12 & 380 & 7.0 & 2500 & 0.08 \\
\hline-5 & 1.87 & 63 & 29 & 8 & 380 & 7.0 & 2560 & 0.07 \\
\hline-4 & 1.69 & 64 & 30 & 17 & 530 & 7.0 & 2560 & 0.08 \\
\hline-3 & 1.68 & 64 & 29 & 24 & 690 & 7.0 & 2520 & 0.06 \\
\hline-2 & 1.68 & 64 & 29 & 15 & 530 & 7.0 & 2580 & 0.08 \\
\hline-1 & 1.80 & 65 & 29 & 18 & 610 & 7.0 & 2580 & 0.06 \\
\hline 0 & 1.89 & 64 & 29 & 25 & 610 & 7.0 & 2580 & 0.06 \\
\hline 1 & 4.18 & 63 & 32 & 54 & 1220 & 7.0 & 2840 & 0.11 \\
\hline 2 & 4.97 & 68 & 32 & 33 & 1220 & 7.1 & 3080 & 0.13 \\
\hline 3 & 4.99 & 66 & 31 & 42 & 1600 & 7.1 & 3320 & 0.14 \\
\hline 4 & 4.86 & 66 & 32 & 51 & 1990 & 7.1 & 3750 & 0.14 \\
\hline 5 & 4.76 & 65 & 31 & 42 & 2440 & 7.2 & 3780 & 0.17 \\
\hline 6 & 4.40 & 64 & 32 & 43 & 2900 & 7.0 & 4180 & 0.25 \\
\hline 7 & 4.07 & 62 & 33 & 37 & 2670 & 7.0 & $\begin{array}{c}4380 \\
.\end{array}$ & 0.27 \\
\hline 8 & 3.58 & 61 & 36 & 40 & 3210 & 7.0 & 4520 & 0.38 \\
\hline 9 & 3.41 & 58 & 37 & 34 & 3970 & 6.9 & 4780 & 0.43 \\
\hline 10 & 3.11 & 56 & 38 & 34 & 4580 & 6.9 & 4940 & 0.56 \\
\hline 11 & 2.93 & - & - & - & - & 6.9 & 5080 & 0.65 \\
\hline
\end{tabular}

a TA - Total Alkalinity (mg/L as $\mathrm{CaCO}_{3}$ ) 


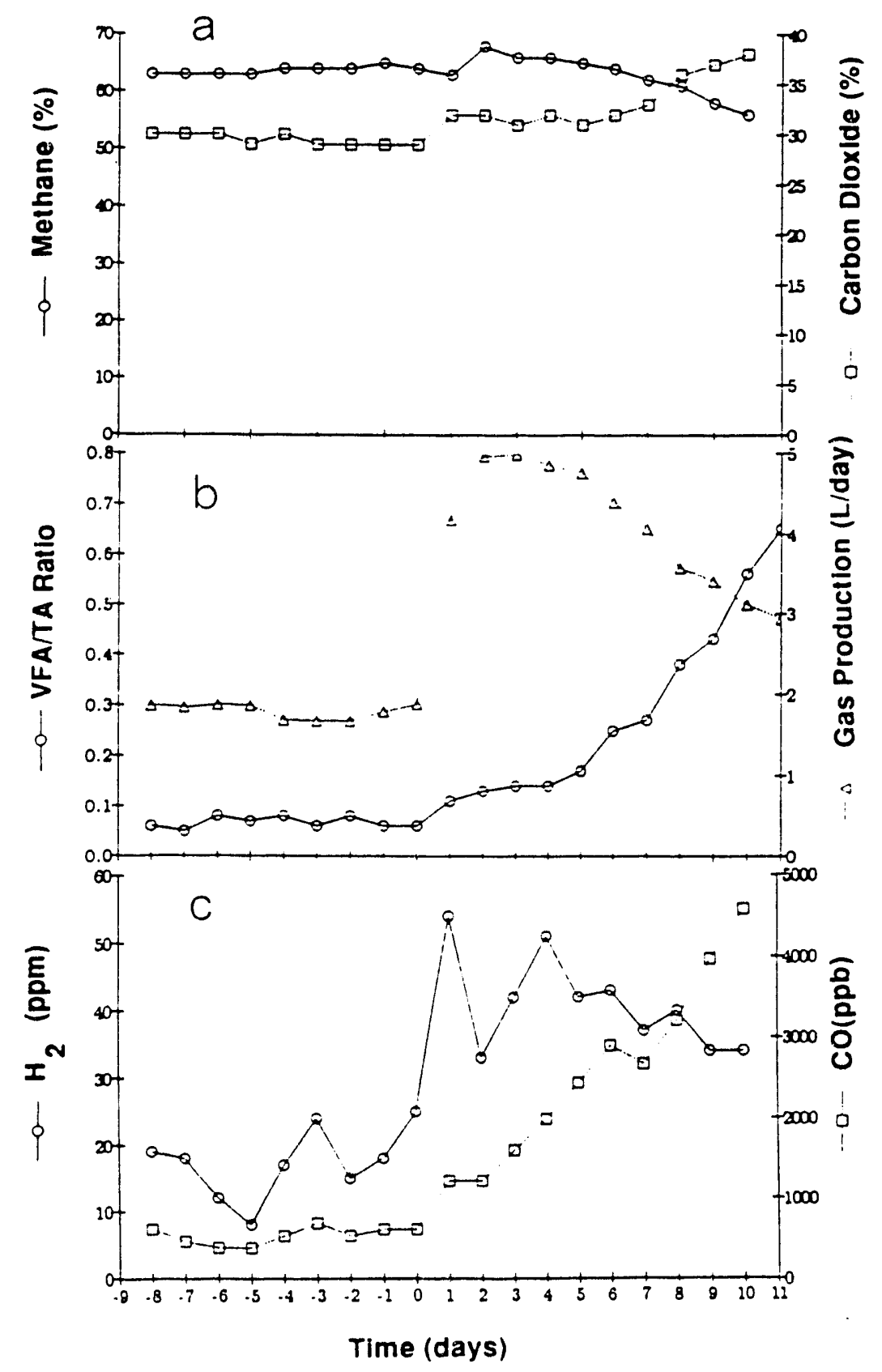

Figure 4-3. Comparison of the response of conventional and novel anaerobic digestion process indicators (measured at the end of each 24hour feed cycle) during a 2.65 -fold organic load increase applied to a 10-day digester. 
Total gas production rate data diurnal patterns are presented in Figure 4-4a. Total gas production increased by approximately 220 percent over the course of day 1 and an additional 20 percent on day 2, for approximately a 263 percent overall increase from the final day of the pre-shock period. Subsequent to day 2 , the diurnal gas production profile appeared to stabilize for the next 3-4 days and then decline.

Total gas and methane production data for all days was described with reasonable accuracy by an exponential first order decay of the form

$$
Q-A e^{-b t}
$$

where

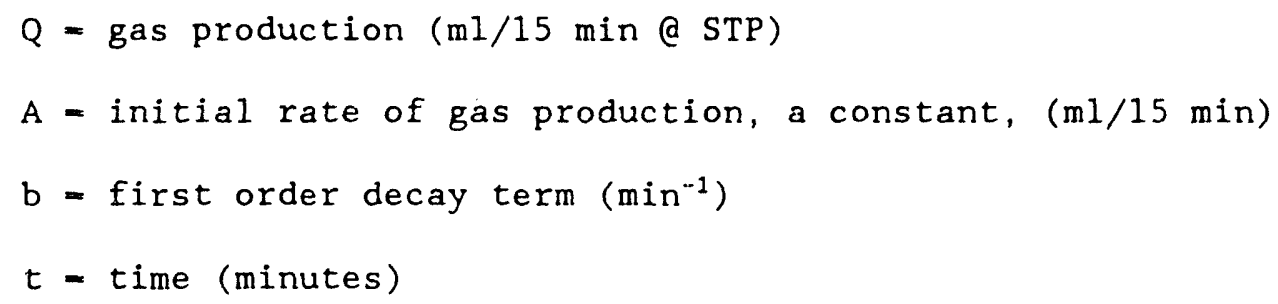

From this analysis it is evident that even during the apparent period of stabilized gas production (day 3 to day 7), changes in the digester performance were occurring. Table 4-5 summarizes results of modeling gas production data according to equation ( 1 ). Starting on day 6 , the decay coefficient showed an increase that indicated that although the initial rate of gas production was similar for all days, on days 6 and 7 the rate of gas production fell off much more quickly than during the three previous days.

Methane production rate information subjected to the same type analysis (Table 4-6) demonstrated essentially the same trend as gas production. An examination of methane and carbon dioxide content of the gaseous headspace during the course of each day gives more insight than methane production rate data.

Methane content, for selected days during the overload, demunstrated what can be termed a hysteresis in diurnal concentration profiles. The methane concentration diurnal pattern dipped slightly lower each day and did not completely recover to 

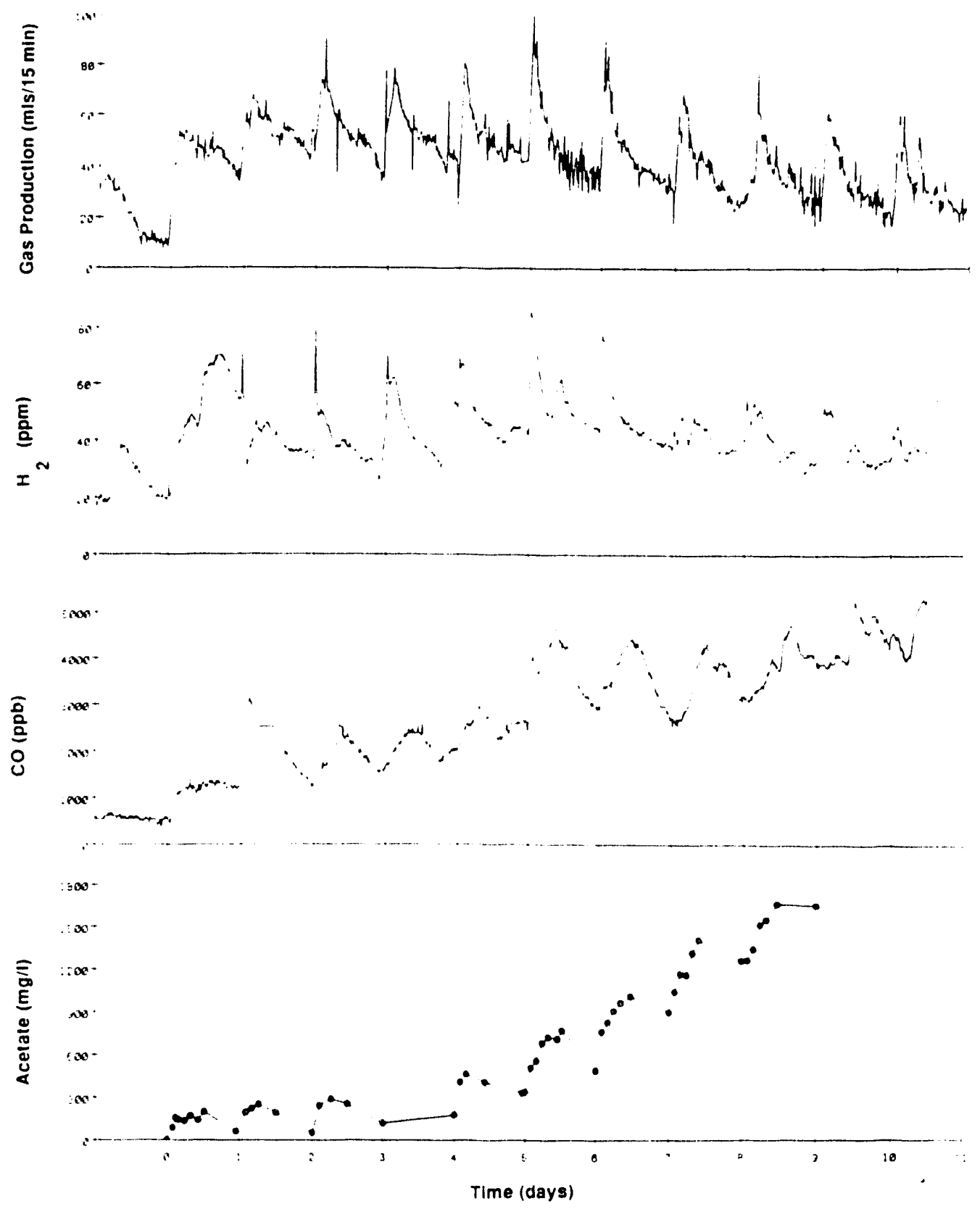

Figure 4-4. On-line measurements from a 5-1iter test digester subjected to a 2.65-fold load increase for: a) gas production rate; b) $\mathrm{H}_{2}$; c) $\mathrm{CO}$; and, d) off-line acetate determinations. 
Table 4-5. Results of Modeling Gas Production Rate Data of a 10-day HRT Digester, Subjected to a 2.65-fold Organic Load Increase, as an Exponential First Order Decay.

\begin{tabular}{llll} 
Day & $\mathrm{A}$ & $\mathrm{b}$ & $\mathrm{r}^{2}$ \\
\hline 0 & 37.9 & 0.060 & 0.949 \\
1 & 49.0 & 0.008 & 0.977 \\
2 & 60.4 & 0.011 & 0.981 \\
3 & 68.2 & 0.022 & 0.969 \\
4 & 66.3 & 0.023 & 0.972 \\
5 & 63.6 & 0.019 & 0.972 \\
6 & 67.8 & 0.036 & 0.926 \\
7 & 64.2 & 0.036 & 0.961 \\
8 & 56.9 & 0.035 & 0.952 \\
9 & 48.5 & 0.027 & 0.936 \\
10 & 51.2 & 0.039 & 0.949 \\
11 & 44.0 & 0.031 & 0.953 \\
\hline
\end{tabular}


Table 4-6. Results of Modeling Methane Production Rate Data as an Exponential First Order Decay.

\begin{tabular}{llll} 
Day & \multicolumn{3}{c}{ Decay } \\
\hline 0 & A & $\mathbf{b}$ & $\mathbf{r}^{2}$ \\
\hline 1 & 26.4 & 0.066 & 0.980 \\
2 & 31.8 & 0.012 & 0.991 \\
3 & 37.2 & 0.008 & 0.993 \\
4 & 44.3 & 0.025 & 0.982 \\
5 & 43.8 & 0.026 & 0.987 \\
6 & 41.0 & 0.021 & 0.985 \\
7 & 41.3 & 0.036 & 0.944 \\
8 & 39.3 & 0.038 & 0.978 \\
9 & 35.8 & 0.040 & 0.971 \\
10 & 29.9 & 0.033 & 0.953 \\
11 & 30.1 & 0.045 & 0.969 \\
& 25.2 & 0.039 & 0.970 \\
\hline
\end{tabular}


the daily starting concentration by the end of the 24-hour cycle. Carbon dioxide displayed the reverse trend ending each 24-hour cycle at a slightly higher $\mathrm{CO}_{2}$ concentration.

The response of hydrogen during the first day of the organic overload was clearly different from the typical diurnal response pattern (Figure 4-4b). Subsequent to this, the diurnal hydrogen profiles were quite similar for all days. The $\mathrm{H}_{2}$ response patterns during the overload are clearly different from those observed prior to the imposition of the organic overload. In all cases, immediately after feeding, hydrogen levels jumped above the days' end level and then quickly returned to the 40-50 ppm range. It is thought, because of the temporary nature of the $\mathrm{H}_{2}$ increase, that the rapid rise is an artifact of the feed or feeding technique employed. The feed sludge was generally placed in the $35^{\circ} \mathrm{C}$ incubator containing the test digester 30 to 90 minutes prior to feeding to prevent a temperature decrease from influencing the digester response. Some degradation probably occurred during this time. The rapid increase in $\mathrm{H}_{2}$ could be the result of small $\mathrm{H}_{2}$ bubbles contained in the sludge being introduced to the digester with the feed, or could be due to the addition of some highly degradable "hydrogen source" compounds in the feed that were produced during storage of the sludge or during the pre-feeding temperature equilibration period.

Just as was observed for hydrogen, carbon monoxide demonstrated a dramatic rise above normal levels during the first day of the overload. Unlike hydrogen, however, $C O$ did not establish a new equilibrium operating level. The carbon monoxide diurnal pattern continued to increase each successive day (Figure 4-4c).

This same hysteresis was also clearly evident in the diurnal VFA data. Acetate, propionate, butyrate, iso-butyrate and iso-valerate data all demonstrated a cyclical daily pattern with a hysteresis. This information is presented graphically for acetate in Figure 4-4d. With the exception of propionate, VFAs appeared to keep a reasonably constant diurnal profile for the first several days of the overload but thereafter increased on each successive day. Interestingly, 
iso-valerate accumulated more rapidly than either iso- or n-butyrate. Acetate and propionate accumulated most rapidly (Table $4-7$ ).

\subsection{ORGANIC OVERLOAD OF A 20-DAY HRT DIGESTER}

A 20-day HRT system was subjected to the exact same increase in organic loading as the 10-day HRT system. Unlike the previous experiment, an increase in organic strength did not result in an observable upset of process performance.

The effect of the increase in organic strength of the feed sludge on digester TS and VS is presented in Figure 4-5. The same approximate levels of TS and VS removal, 50 and 60 percent, respectively, were observed prior to increasing the organic load and subsequent to attaining a new steady state operating level. As can be seen in Figure 4-5, it took approximately 13 days of the overload, for the system to re-equilibrate at a new solids level. The response of other conventional process parameters are summarized in Figure 4-6. With the exception of a slight and gradual change in $\mathrm{CO}_{2}$ content, there was not a noticeable effect on the major gaseous products. Gas production appeared to stabilize at a new steady state level after approximately 13 days of the overload, which agrees well with the solids data. The increase in gas production, approximately 2.5 times the initial level, was almost exactly the same as the magnitude of the organic load increase (2.5 times). The VFA/TA ratio did not change appreciably but showed a random variation around a mean value of about 0.06 .

The two trace gases, $\mathrm{H}_{2}$ and $\mathrm{CO}$, when examined daily, did not demonstrate a significant response to the organic load increase during the first 12 days after the initiation of the increase in loading. On day 13, however, hydrogen dramatically increased to $135 \mathrm{ppm}$ from the apparent baseline value of $20-21 \mathrm{ppm}$. There was no apparent reason for this perturbation based on the routine analyses performed. Volatile fatty acids including acetate remained low. Ammonium concentration was measured from a sample of the effluent that day and was found to be $2000 \mathrm{mg} / \mathrm{L}$. Wiegant (1986) reported that hydrogen utilizing methanogens in a thermophilic system were more sensitive to ammonia inhibition than acetate catabolizing methanogens. Because there was no data base on the ammonia levels 
Table 4-7. Dally Concentrations of Volatile Fatty Acids During a Continuous 2.65-fold Organic Overload of a 10-day HRT Digester.

\section{Volatile Fatty Acids (mg/L)}

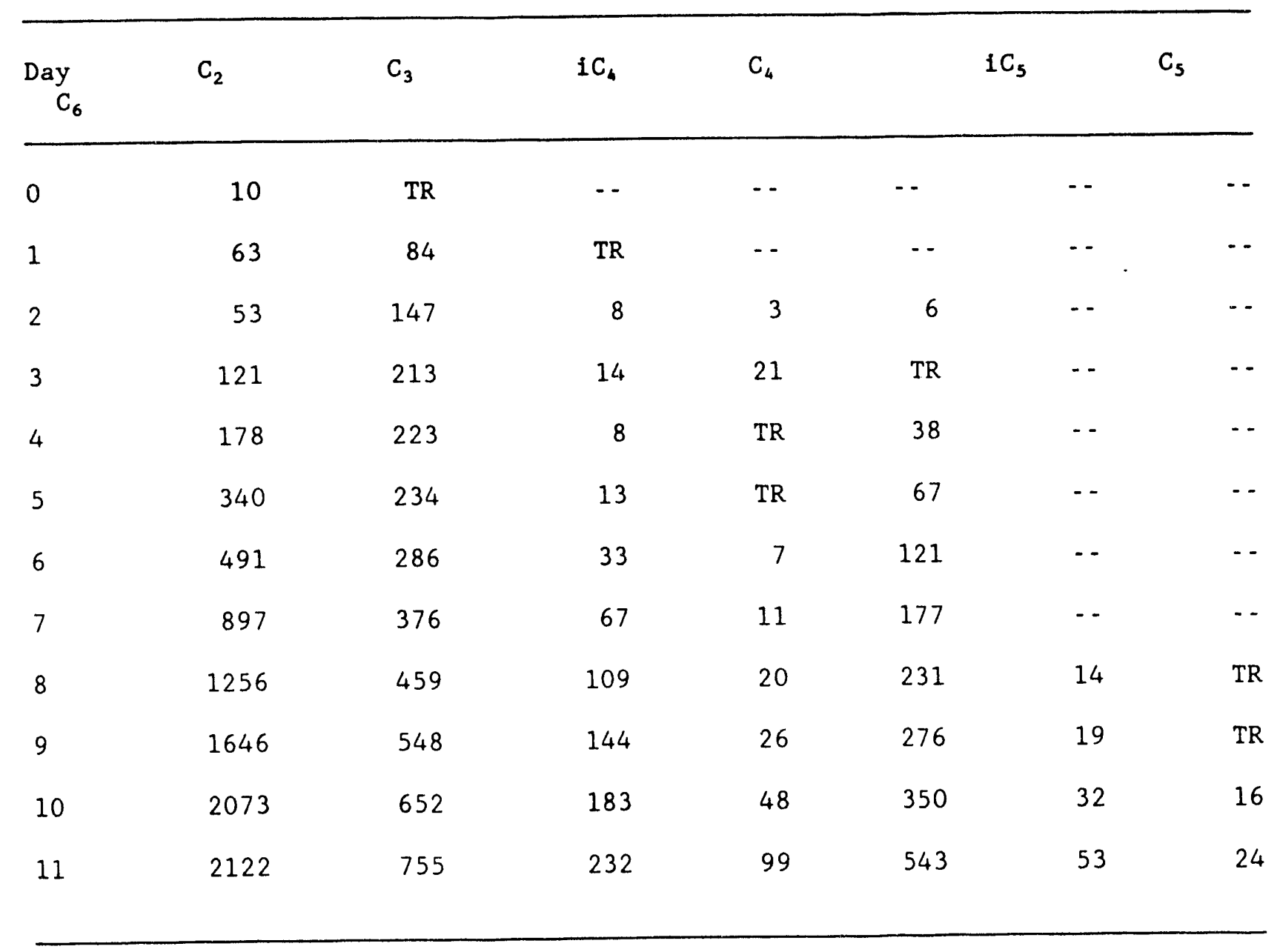

$\mathrm{TR}=$ trace levels

- - below detection limits 


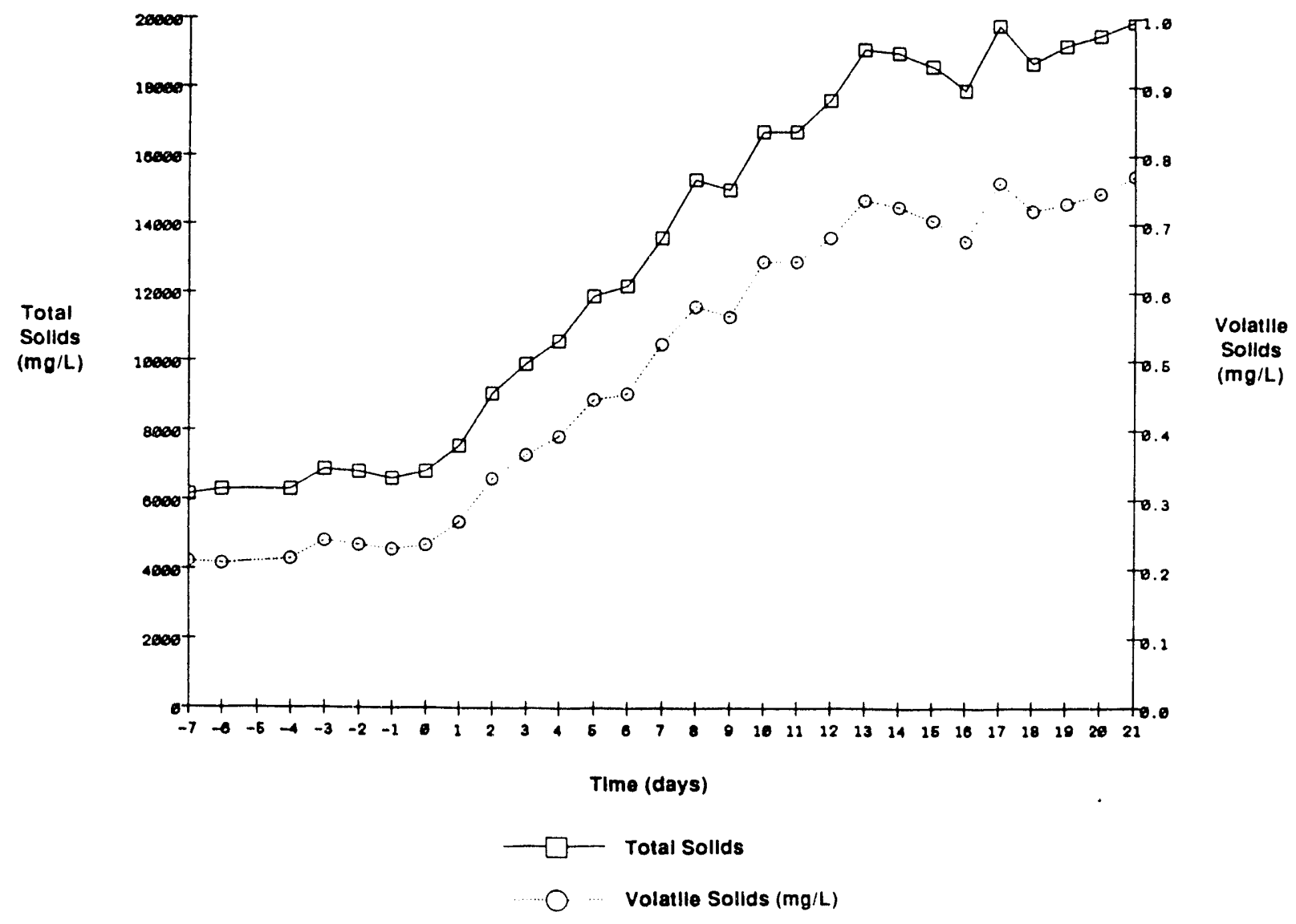

Figure 4-5. Measured total and volatile solids concentrations during a 2.5-fold load increase applied to a 20-day HRT digester. 


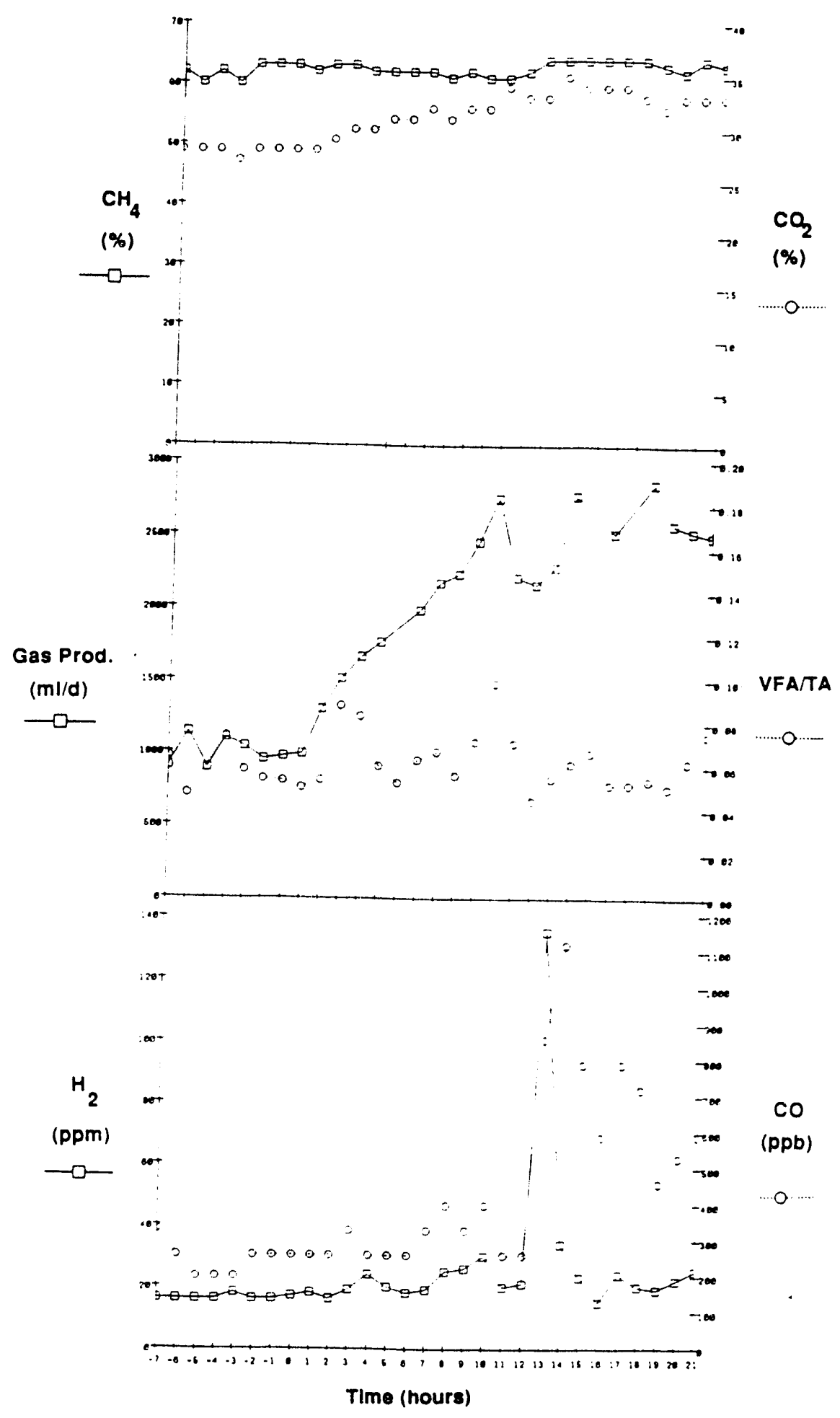

Figure 4-6. Conventional and novel process indicators, measured at the end of each 24-hour feed cycle, during a 2.5-fold load increase to a 20 -day HRT digester. 
to compare the ammonium measurement, it is impossible to make any conclusions. After this dramatic rise, hydrogen dropped back to the $20 \mathrm{ppm}$ range within 2 days. Carbon monoxide also increased on day 13 and continued upward until day 14. Co subsequently began a gradual decline towards pre-perturbation levels. A slow decline in $C O$ after rising well above the normal concentration appears to be the characteristic response, probably because co at trace levels is metabolized at a slow rate. This may be related to both mass transfer limitations and low substrate affinity. For CO levels to return to "baseline" values, the high $C O$ concentration in the gas phase needs to be washed or flushed out.

Gas production rate data is presented in Figure 4-7a. As was done for the prior organic overload experiment (10-day HRT), the daily gas production rate information was fit to the exponential equation;

$$
Q=A e^{-b t}
$$

The results of this statistical evaluation are presented in Figure $4-8$ and Table 4-8. As is evident from Table 4-8, an excellent fit was obtained for all days. One noticeable feature of the coefficients are the times required until they stabilized to new steady state levels. The maximum gas production rate coefficient stabilized at a new higher level by 8 days. The decay coefficient, however, did not stabilize until day 12 or 13, precisely the time that the solids concentrations stabilized. The 15 -minute interval hydrogen reveals a little more information than the daily data. As can be seen in Figure 4-7b, the diurnal hydrogen response pattern actually began to show signs of a mild perturhation between days 7 and 10 followed by the dramatic change that began after feeding on day 12 .

The co data (Figure 4-7c) shows a large peak on day 7 that began immediately after feeding. This was probably caused by inadvertent exposure to a small amount of oxygen. Co did not return to approximately the same daily value by 


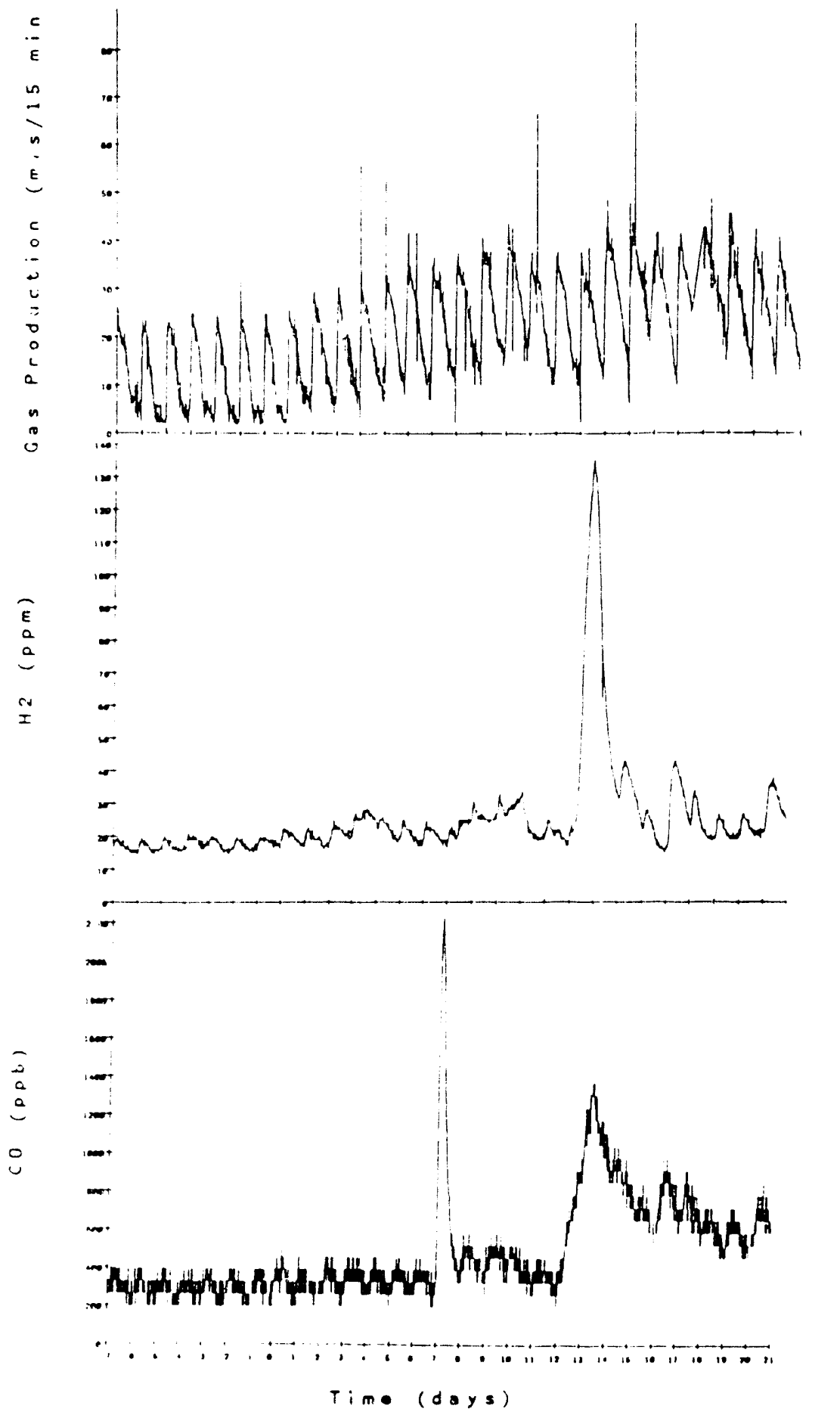

Figure 4-7. Gas production rate data for a 20 -day HRT digester prior to and after a 2.5-fold organic load increase.

$4-2 i$ 


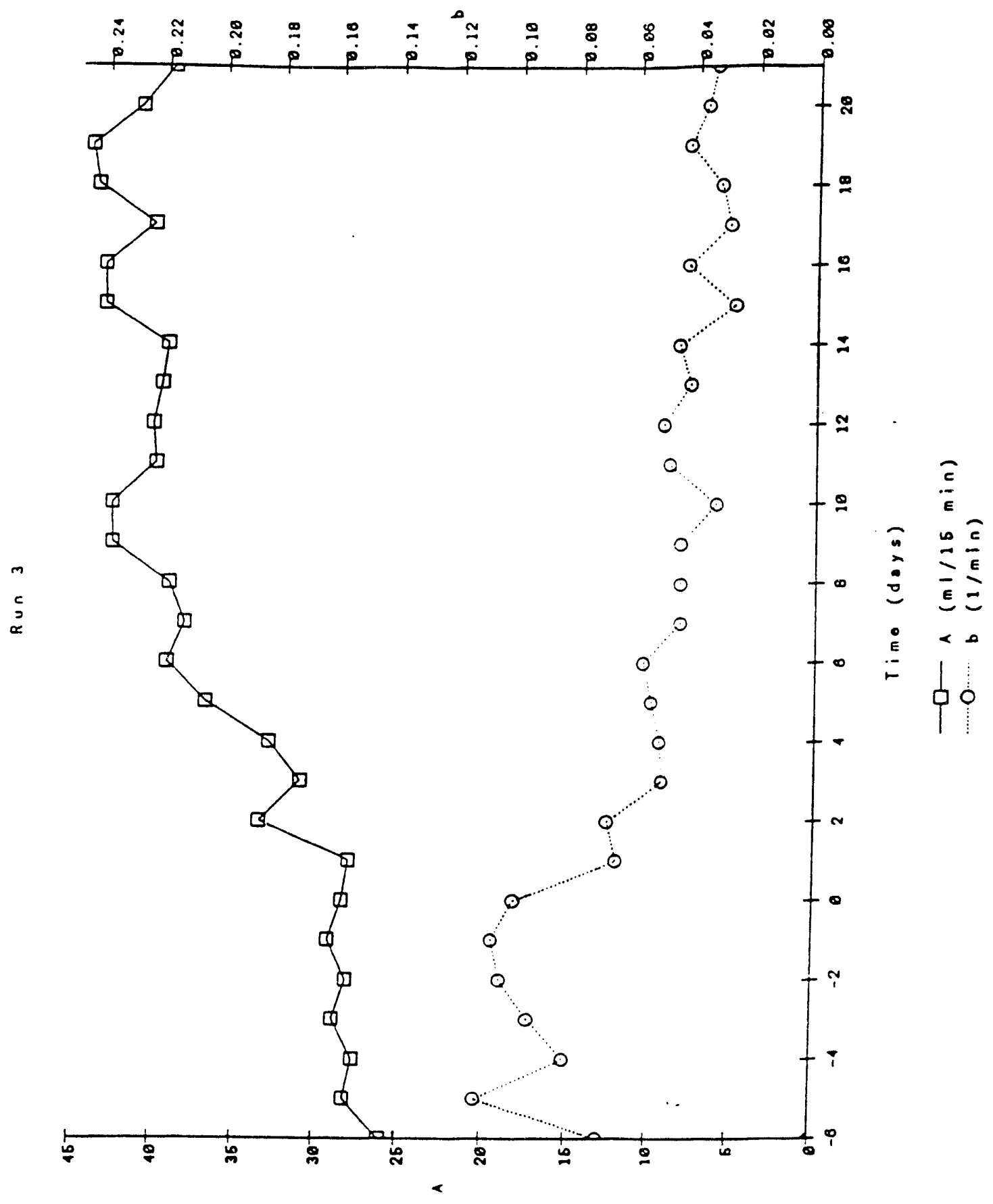

Figure 4-8. Change in values of initial gas production rate (A) and decay coefficient (b) as a result of a 2.5 -fold organic load increase to a 20 -day HRT digester. 
Table 4-8. Results of Modeling Gas Production Rate Data of a 20-day HRT Digester, Subjected to a 2.5-fold Organic Load

Increase, as an Exponential First Order Decay.

\begin{tabular}{|c|c|c|c|}
\hline Day & A & $\mathrm{b}$ & $\mathrm{r}^{2}$ \\
\hline-5 & 25.93 & 0.072 & 0.978 \\
\hline-4 & 28.10 & 0.113 & 0.963 \\
\hline-3 & 27.56 & 0.084 & 0.967 \\
\hline-2 & 28.87 & 0.096 & 0.969 \\
\hline-1 & 28.08 & 0.105 & 0.969 \\
\hline 0 & 29.23 & 0.108 & 0.969 \\
\hline 1 & 28.40 & 0.101 & 0.967 \\
\hline 2 & 28.01 & 0.067 & 0.968 \\
\hline 3 & 33.60 & 0.070 & 0.986 \\
\hline 4 & 31.06 & 0.051 & 0.979 \\
\hline j & 33.03 & 0.052 & 0.983 \\
\hline 6 & 36.93 & 0.055 & 0.988 \\
\hline 7 & 39.34 & 0.058 & 0.982 \\
\hline 8 & 38.28 & 0.045 & 0.984 \\
\hline 9 & 39.24 & 0.045 & 0.988 \\
\hline 10 & 42.79 & 0.045 & 0.994 \\
\hline 11 & 42.82 & 0.033 & 0.983 \\
\hline 12 & 40.07 & 0.049 & 0.987 \\
\hline 13 & 40.25 & 0.051 & 0.992 \\
\hline 14 & 39.76 & 0.042 & 0.989 \\
\hline 15 & 39.43 & 0.046 & 0.932 \\
\hline 16 & 43.34 & 0.027 & 0.949 \\
\hline 17 & 43.38 & 0.043 & 0.987 \\
\hline 18 & 40.29 & 0.029 & 0.995 \\
\hline 19 & 43.87 & 0.032 & 0.990 \\
\hline 20 & 44.26 & 0.043 & 0.989 \\
\hline 21 & 41.16 & 0.037 & 0.987 \\
\hline
\end{tabular}


day 8. The perturbation on the 12 th day appears to be linked to the hydrogen response. CO values remained elevated for the remainder of the experimental run.

All these rather dramatic changes in $\mathrm{H}_{2}$ and $\mathrm{CO}$ occurred without any apparent change in process performance or gas production rate.

\subsection{DISCUSSION AND SUMMARY}

An increase in organic loading during a one day batch assay with a 10-day HRT inoculum demonstrated that a significant but temporary increase in hydrogen gas occurred. In the latter experiments, hydrogen concentration quickly stabilized at a new apparent steady state diurnal pattern if monitored with the real-time data acquisition system. When the organic overload was continued past 5 days, the diurnal hydrogen profiles and 24-hour cycle ending hydrogen concentrations slightly declined which indicates that the hydrogen utilizing population was not challenged by the increases in organic loading during this experiment. This is not surprising since the hydrogen consuming population of digester sludge has been reported capable of utilizing 20-fold more hydrogen than is normally produced during the anaerobic digestion of sludge (Kasper and Wuhrmann, 1978).

The results do indicate, however, that the hydrogen concentration, or diurnal profile, may reflect the loading or level of stress being imposed on the hydrogen using population of the digester as has been suggested (Mosey, 1983).

Hydrogen monitoring, however, does not provide an indication of the level of stress being exerted on the acetate-catabolizing population. Conventional wisdom stresses, and results generated during the course of this research support, that the acetate-catabolizing population is more easily overloaded than the hydrogenutilizers. In both batch and continuous experiments, substantial accumulation of acetate and higher molecular weight VFAs occurred without a corresponding $\mathrm{H}_{2}$ response. Even when the digestion process was under upset conditions (as judged 
by the VFA/TA ratio exceeding the 0.35 to 0.4 level), the hydrogen concentrations provided no indication this was so.

It should be kept in mind that the organically overloaded system that was pushed to a state of severe upset was also highly buffered. A pH of greater than 6.8 was maintained throughout the experiment despite considerable build-up of both volatile fatty acids and the carbon dioxide partial pressure. It is possible that in less well buffered systems, accumulation of VFAs and an increase in $\mathrm{CO}_{2}$ on the same level as observed here would produce a greater pH depression. Hypothetically, the hydrogen response would be much greater since the depressed $\mathrm{pH}$ would result in reduced activity of the hydrogen consumers. Under these circumstances, hydrogen might provide an indication of the onset or occurrence of a process upset. Whether or not, in this situation, hydrogen would provide significantly more information than a simple $\mathrm{pH}$ measurement, however, needs to be examined.

Fortunately, the other trace gas monitored during this study, carbon monoxide, appears to have a strong relationship with the levels of VFAs in the system and thus provides a measure of the status of the acetate catabolizing population. During the batch organic overload experiment and the on-line experimentation, accumulation of acetate was always mirrored by a similar increase in CO. This relationship will be investigated later in this report (Chapter 8).

Assuming for the moment that $\mathrm{CO}$ and acetate are related, monitoring both $\mathrm{H}_{2}$ and CO should lend insight into the functioning of both major routes of methane production in anaerobic digesters and industrial wastewater treatment systems. CO response would appear to provide a convenient, surrogate on-line measure of the VFA level in a system, while hydrogen would supply information concerning the status of the carbon dioxide reduction to methane pathway.

The on-line monitoring of total gas and methane production during the organic overload did provide some indication that there was a potential problem. The responses observed were far less sensitive than observed for $C O$, however, and it 
is unlikely that total gas or methane production could provide adequate earlywarning of an upset. Variations in sludge composition, volume, and concentration normally encountered at treatment plants would superimpose a sufficient background noise to preclude using gas production as an early warning indicator.

Methane and carbon dioxide concentration will provide some information, and this is why they are generally measured. They are, however, not adequate early warning indicators and this is why they are monitored along with other process indicators such as the VFA/TA ratio (Zickefoose and Hayes, 1976).

As a final note of interest, results from the 10-day HRT organic overload experiment indicate that acetate accumulated most rapidly followed by propionate. This can be anticipated based on the thermodynamics of the propionate reaction (McCarty, 1982). The third VFA to accumulate was not butyrate (n or iso) as might be anticipated. Iso-valerate accumulated more quickly than the combined total of both $\mathrm{n}$ - and iso-butyrate. The accumulation of iso-valerate contrasted with results obtained by Smith (1980) who, based on $\mathrm{C}^{14}$ labelling studies, concluded iso-valerate was not an important intermediate in sludge digestion. This difference may reflect the different sludges used in the respective studies. Smith (1980) did not report what type of sludge he used. Here a high protein waste, WAS, was used. Iso-valerate is a product that has been reported to be primarily associated with amino acid degradation (Thauer et al., 1977). If Smith (1980) used primary or a combination of primary/WAS, the protein content of the sludge would be reduced, which may explain the differences between the two studies. 


\section{SECTION 5}

\section{EXPERIMENTAL RESULTS - THE EFFECTS OF HYDRAULIC LOAD INCREASES}

Hydraulic overloads of $1.25,2.0$ and 4.0 times normal were imposed on 10-day HRT test digesters for 6,7 , and 8 consecutive days, respectively. The same feed strength was maintained; therefore, the organic loading rate increased proportionately with the hydraulic load. In order to reduce any background interference due to variability in sludge solids concentration or composition, the same batch of feed sludge (held at $2-4^{\circ} \mathrm{C}$ ) was used for the duration of each experimental run. Daily analysis of the feed for COD, total solids, volatile solids, and volatile fatty acids over the course of the experiment indicated that storage of the sludge at $2-4^{\circ} \mathrm{C}$ was sufficient to prevent any significant change in the composition of the sludge over a several week time frame.

\subsection{HYDRAULIC LOAD INCREASE OF $1.25 \times$ NORMAL}

A 25 percent increase in the hydraulic loading on a 10-day HRT digester produced little observable response from conventionally monitored parameters (Table 5-1).

The hydrogen and CO diurnal profiles were slightly elevated above the baseline patterns obtained prior to the overload, but much less so during the two-fold hydraulic overload experiment and were not significantly different from a statistical basis.

\subsection{HYDRAULIC LOAD INCREASE OF $2 \times$ NORMAL}

A 7 -day hydraulic shock was imposed on a 10-day HRT digester operating at steadystate. The imposition of this hydraulic overload did not cause a system upset or deterioration in process performance. With the exception of a slight decrease in total alkalinity and increase in COD, gas production was the only conventional process indicator, monitored on a daily, end-of-cycle basis, that exhibited a response from reducing the HRT to 5 days (Table 5-2). Gas production almost doubled while gas composition in terms of the major components $\left(\mathrm{CH}_{4}\right.$ and $\left.\mathrm{CO}_{2}\right)$ remained essentially unchanged. The total gas production diurnal pattern, 
Table 5-1. Daily Analysis of 10-Day HRT Test Digester Subjected to a 1.25 -fold Hydraulic Load

Increase.

\begin{tabular}{cccccccc}
\multicolumn{7}{c}{ 10-Day HRT - Run \#1 - Effluent Data } \\
Day & $\mathrm{pH}$ & $\begin{array}{c}\text { TA } \\
(\mathrm{mg} / \mathrm{L})\end{array}$ & $\begin{array}{c}\text { VFA } \\
(\mathrm{mg} / \mathrm{L})\end{array}$ & $\begin{array}{c}\text { COD } \\
(\mathrm{mg} / \mathrm{L})\end{array}$ & $\begin{array}{c}\text { SCOD } \\
(\mathrm{mg} / \mathrm{L})\end{array}$ & $\begin{array}{c}\text { TS } \\
(\mathrm{mg} / \mathrm{L})\end{array}$ & $\begin{array}{c}\text { VS } \\
(\mathrm{mg} / \mathrm{L})\end{array}$ \\
\hline-2 & 7.0 & $\ldots$ & $\ldots$ & 16300 & 2060 & 12700 & 8960 \\
-1 & 7.1 & 2920 & 130 & 15200 & 2130 & 12200 & 8420 \\
0 & 7.1 & $\cdots$ & $\ldots$ & 14900 & 2110 & 11300 & 7950 \\
1 & 7.0 & 2920 & 140 & 11000 & 1260 & 10900 & 7710 \\
2 & 7.1 & 2880 & 230 & 14000 & 1130 & 10600 & 7450 \\
3 & 7.0 & 2700 & 410 & 10700 & 3140 & 11000 & 7990 \\
4 & 7.1 & 2920 & 210 & 15500 & 2190 & 10300 & 7310 \\
5 & 7.1 & 2940 & 140 & 11500 & 2120 & 10600 & 7410 \\
6 & 7.0 & 2790 & 120 & 11200 & 1950 & 10500 & 7350
\end{tabular}

Note:

$\mathrm{TA}$ - mg/L as $\mathrm{CaCO}_{3}$

VFA - $\mathrm{mg} / \mathrm{L}$ as $\mathrm{HAC}$ 
Table 5-2. Comparison of Conventional and Novel Trace Gas Indicators During a 2-fold Hydraulic Load Increase to a 10-day HRT Digester.

\begin{tabular}{lccccccccc} 
Day & $\begin{array}{c}\mathrm{TS} \\
(\mathrm{mg} / \mathrm{L})\end{array}$ & $\begin{array}{c}\mathrm{VS} \\
(\mathrm{mg} / \mathrm{L})\end{array}$ & $\begin{array}{c}\mathrm{pH} \\
(\mathrm{su})\end{array}$ & $\begin{array}{c}\mathrm{TA} \\
(\mathrm{mg} / \mathrm{L})\end{array}$ & $\mathrm{VFA} / \mathrm{TA}$ & $\begin{array}{c}\mathrm{CH}_{4} \\
(8)\end{array}$ & $\begin{array}{c}\mathrm{CO}_{2} \\
(8)\end{array}$ & $\begin{array}{c}\mathrm{H}_{2} \\
(\mathrm{ppm})\end{array}$ & $\begin{array}{c}\mathrm{CO} \\
(\mathrm{ppb})\end{array}$ \\
\hline-2 & 9030 & 6290 & 7.0 & 2660 & 0.05 & 69 & 31 & 12 & 220 \\
-1 & 11390 & 8210 & 7.0 & 2670 & 0.06 & 67 & 30 & 11 & 280 \\
\hline $0^{2}$ & 11290 & 8220 & 7.0 & 2700 & 0.06 & 68 & 31 & 15 & 330 \\
1 & 11220 & 8200 & 7.0 & 2620 & 0.06 & 66 & 31 & 35 & 550 \\
\hline 2 & 11330 & 8080 &.- & 2600 & 0.05 & 69 & 31 & 43 & 660 \\
3 & 10580 & 7700 & 7.0 & 2490 & 0.05 & 69 & 31 & 44 & 660 \\
4 & 10820 & 7750 & 7.0 & 2460 & 0.05 & 70 & 31 & 37 & 880 \\
5 & 10940 & 7480 & 7.1 & 2490 & 0.06 & 71 & 30 & 30 & 770 \\
\hline 6 & 10980 & 7950 & 7.0 & 2420 & 0.07 & 71 & 31 & 30 & 880 \\
\hline 7 & 11650 & 8460 & 7.0 & 2430 & 0.07 & 69 & 31 & 32 & 880 \\
\hline $8^{b}$ & 11730 & 8750 & 6.9 & 2480 & 0.08 & 66 & 30 & 35 & 660 \\
\hline 9 & 11200 & 7770 & 7.0 & 2590 & 0.05 & 68 & 30 & 19 & 440 \\
\hline & & & & & & & & & \\
\hline
\end{tabular}
a - start of hydraulic overload
b - return to normal hydraulic loading 
prior to and during the hydraulic overload sequence, is presented in Figure 5-1a. A gradual rise in gas production was noted over the 7-day overload. Results of modeling the gas production rate information as a first order exponential decay are summarized in Table 5-3. By day 2 or 3 of the overload, both the initial gas production rate and decay rate coefficients levelled off to reasonably steady values. A drop in the loading rate back to a 10 -day residence time produced an immediate drop in the decay coefficient back to pre-overload conditions. A different effect was observed for the initial gas production rate coefficient. On the day when a normal, 10-day hydraulic loading was resumed, total gas and methane production rates during the first several hours increased 15 percent above the levels reached during the overload and well above the pre-overload values. This phenomenon will be addressed in Section 5.4 .

Upon introduction of the hydraulic overload, the volatile fatty acids profile over the course of the day changed considerably. As seen in Figures 5-2a and $b$, acetate and propionate rose to higher concentrations and persisted at these elevated levels for a longer duration during the load increase. The amount of these acids present at the end of a 24 -hour cycle was, however, essentially the same during the overload period and prior to introduction of the overload. The diurnal profiles of acetate and propionate were essentially identical for all days during the overload period. As is shown in Figures 5-2a and b, the diurnal profiles for both these acids had returned to near pre-shock levels by the second day of resumption of a normal hydraulic loading. No other volatile fatty acids, i.e., butyrate and iso-valerate, accumulated during the study.

The change in hydraulic loading was also reflected in the hydrogen and carbon monoxide profiles. For several days, subsequent to initiating the overload period, the base level and peak $\mathrm{H}_{2}$ and $\mathrm{CO}$ levels increased daily (Figures 5-1b and $c)$. After 4 days, the hydrogen levels decreased slightly and appeared to stabilize, while the carbon monoxide concentration daily profile appeared to remain approximately the same after day 4. Following resumption of the normal 


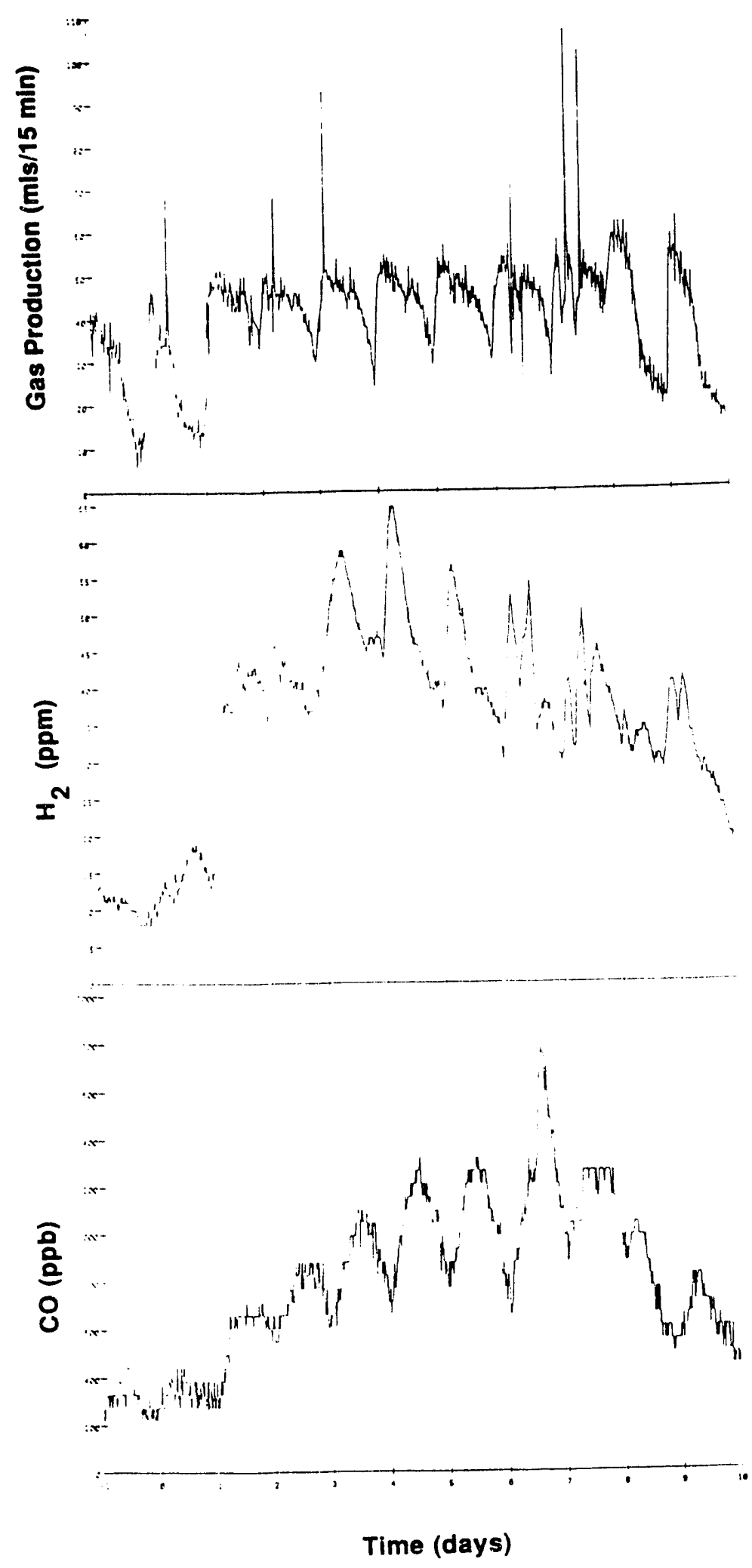

Figure 5-1. On-line measurements from a 5-liter, 10-day HRT digester subjected to a 2.0-fold hydraulic load increase for a) gas production rate; b) $\mathrm{H}_{2}$; and c) $\mathrm{CO}$. 
Table 5-3. Results of Modeling Gas Production Rate Data of a 10-day HRT Digester Subjected to a 2-fold Hydraulic Load

Increase.

\begin{tabular}{llll} 
Day & $\mathrm{A}$ & $\mathrm{b}$ & $\mathrm{r}^{2}$ \\
\hline-2 & 41.2 & 0.050 & 0.88 \\
-1 & 42.1 & 0.044 & 0.93 \\
0 & 42.7 & 0.049 & 0.84 \\
1 & 48.3 & 0.014 & 0.93 \\
2 & 49.5 & 0.013 & 0.97 \\
3 & 51.4 & 0.015 & 0.96 \\
4 & 52.0 & 0.014 & 0.98 \\
5 & 50.6 & 0.013 & 0.97 \\
6 & 50.1 & 0.009 & 0.97 \\
7 & 55.3 & 0.019 & 0.88 \\
$8^{2}$ & 64.2 & 0.049 & 0.96 \\
\hline
\end{tabular}

a day one of return to normal hydraulic loading rate 

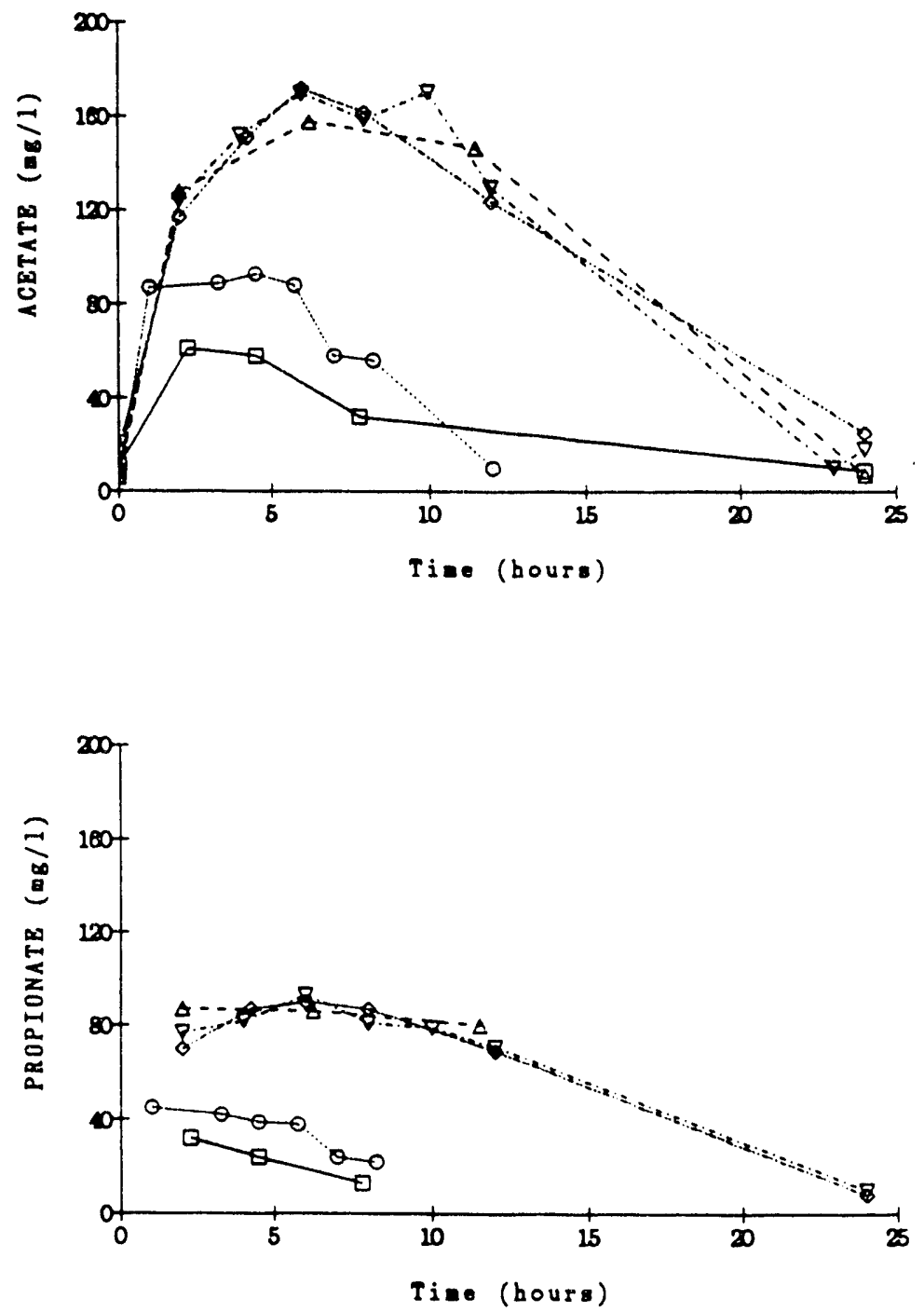

Figure 5-2. Diurnal accumulation patterns of (a) acetate and b) propionate before, during and after a 7-day, 2.0-fold hydraulic load increase to a 10-day HRT digester. 
10-day hydraulic loading, hydrogen and carbon monoxide levels gradually began to decline towards the original pre-overload patterns.

Gas and methane production also remained elevated above pre-overload levels during this period but decreased substantially each day, presumably as accumulated volatile solids or soluble intermediates were consumed.

\subsection{HYDRAULIC LOAD INCREASE OF $4 \times$ NORMAL}

A third attempt was made to perturb digester performance by increasing the hydraulic loading rate of a 10-day HRT system. While maintaining a constant feed strength the hydraulic retention time was decreased from 10 to 2.5 days for 8 consecutive days.

A hydraulic load increase of this magnitude produced an immediace effect on system performance and conventional process indicators. Effluent total and volatile solids and SCOD concentrations all dramatically increased (Figure 5-3). Gas production gradually increased by a small amount (Figure 5-4). Both major gaseous products, methane and carbon dioxide, and trace gases, hydrogen and $\mathrm{CO}$, displayed little change. Methane and carbon dioxide remained at approximately 68 and 27 percent, respectively. Hydrogen consistently remained at about $10 \mathrm{ppm}$ (on a daily basis) while CO remained between 300 and $500 \mathrm{ppb}$, both prior to and during the overload.

Based on the solids and SCOD information, the digester performance stabilized by day 3 or 4 of the overload at what would be considered "upset" conditions (i.e, VFA/TA ratio exceeded $0.35-0.4)$. The $\mathrm{pH}$ had decreased from approximately 6.8 to 6.4 by this time.

The performance of the 10-day HRT digester, when examined on a 15-minute sample interval basis, displayed less of a diurnal pattern for total gas and methane production than observed during the previous run for pre-overload conditions. After the first 3 days of overload, total gas and methane production rates were essentially constant during the course of each 24 -hour feed cycle. Methane and 


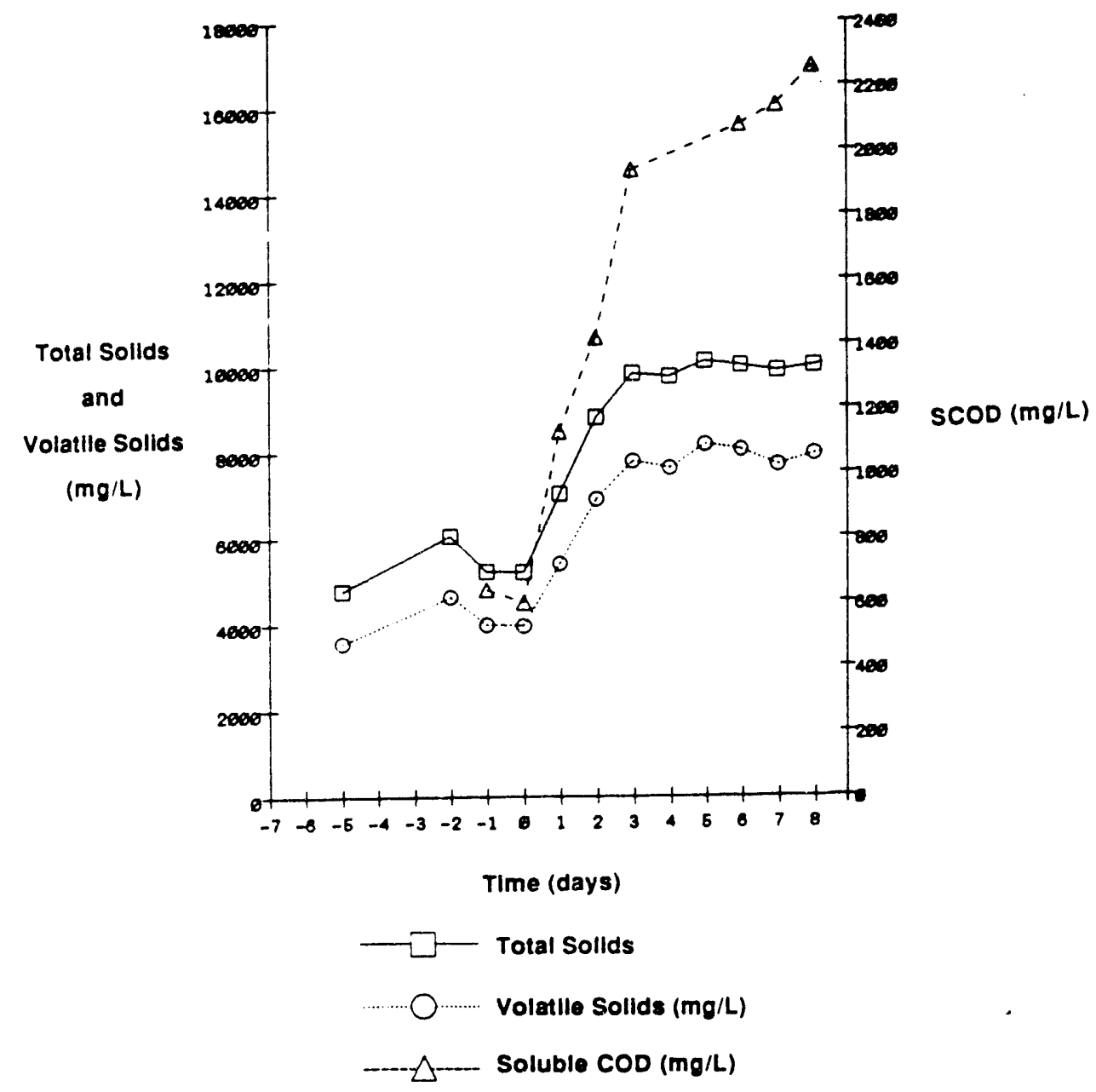

Figure 5-3. Effluent total and volatile solids and soluble COD concentrations observed from a 10 -day HRT digester subjected to a 4.0fold hydraulic load increase. 


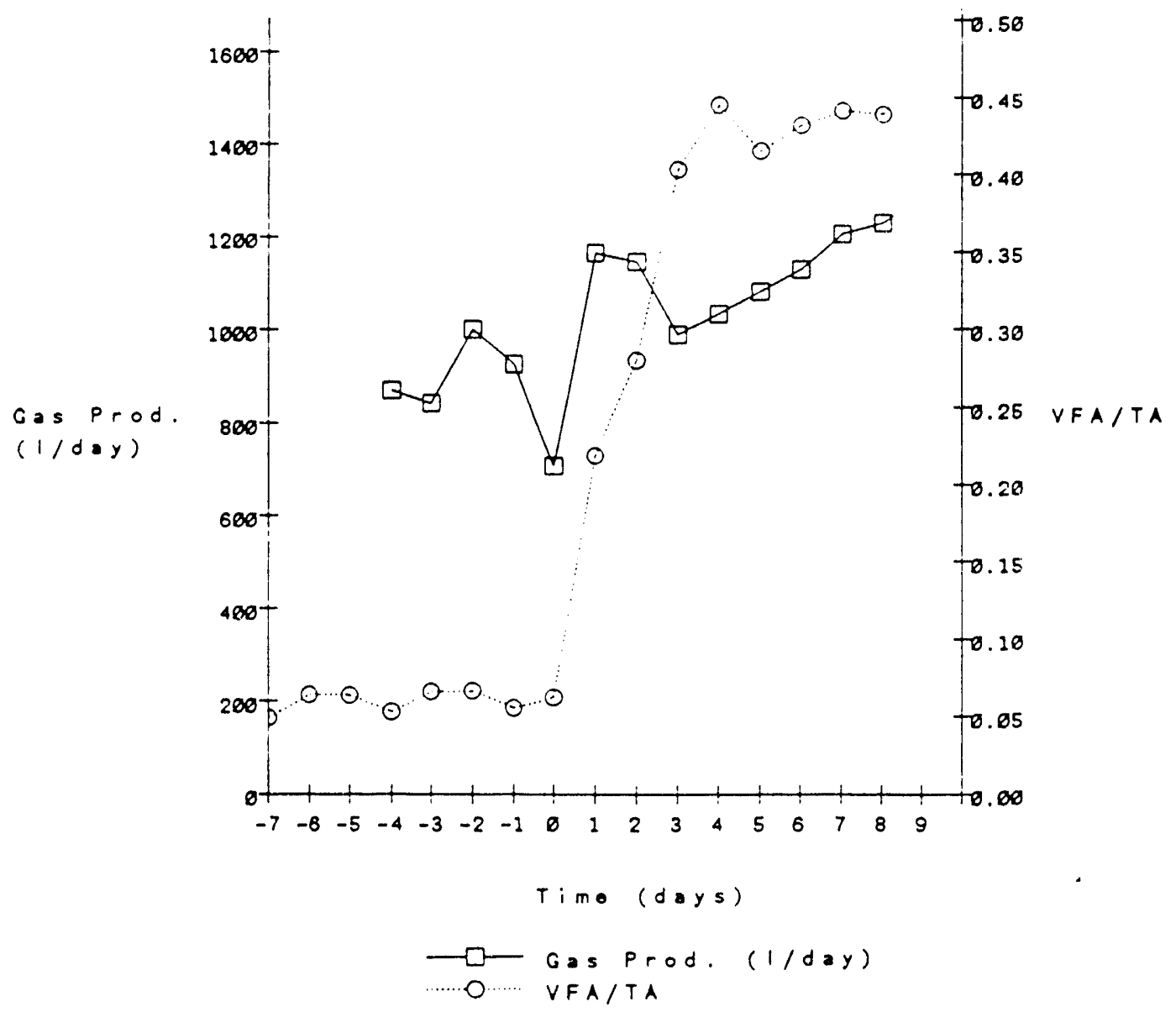

Figure 5-4. Gas production rate and VFA/TA ratio prior to and during a 4fold hydraulic load increase to a 10-day HRT digester. 
carbon dioxide levels also remained relatively constant during each day. Methane concentration generally decreased immediately after feeding by 2 or 3 percent but within several hours rebounded to the 68-70 percent concentration range. Carbon dioxide levels remained essentially constant for the entire 24-hour cycle (Figure $5-5 a)$.

The accumulations of acetate and propionate were somewhat different than observed for the prior 2-fold increase in organic load, experimental run. In addition, iso-valerate accumulated as previously seen during the organic overload experiment. The patterns of acetate, proportionate and iso-valerate accumulation for the first 4 days of the experiment are shown in Figure 5-6. Acetate displayed a diurnal accumulation pattern followed by consumption. Unlike in the 2 -fold increase of hydraulic load experiment, however, acetate levels did not return to the starting value of the 24 -hour cycle but remained elevated for the first 4 days. After this point, a constant acetate diurnal pattern was observed. Propionate and iso-valerate accumulated at near constant rates for the initial 4 days of the overload. Thereafter, propionate concentrations levelled off at a concentration in excess of acetate. This is shown in the daily end-of-cycle information collected (Table 5-4). Iso-valerate increased for the first 5 days and then levelled off at approximately $100 \mathrm{mg} / \mathrm{L}$.

The $\mathrm{C}_{2}$ through $\mathrm{C}_{5}$ VFAs $\left(\mathrm{C}_{6}\right.$ through $\mathrm{C}_{8}$ acids were present only at trace levels or below detection limits) do not account. for the rise in SCOD observed. For example, on day 7 the effective SCOD of the VFAs $\left(\mathrm{C}_{2}\right.$ through $\left.\mathrm{C}_{5}\right)$ was $750 \mathrm{mg} / \mathrm{L}$. The SCOD on this day was 2140 or $1520 \mathrm{mg} / \mathrm{L}$ above the pre-overload concentration. At least 50 percent of the SCOD produced, was present as intermediate products other than volatile fatty acids. Because of the proteinaceous nature of the substrate and lack of accumulation of hydrogen, these other soluble products may be primarily peptides and amino acids.

Hydrogen and CO displayed almost constant diurnal profiles, both prior to and after the start of the hydraulic overload (Figure 5-5b). The hydrogen concentration remained almost constant at values ranging from $8-12 \mathrm{ppm}$. The $\mathrm{CO}$ concentration increased slightly from the pre-load increase average of $372( \pm 45)$ ppb to $531( \pm 81) \mathrm{ppb}$ after the load increase. 


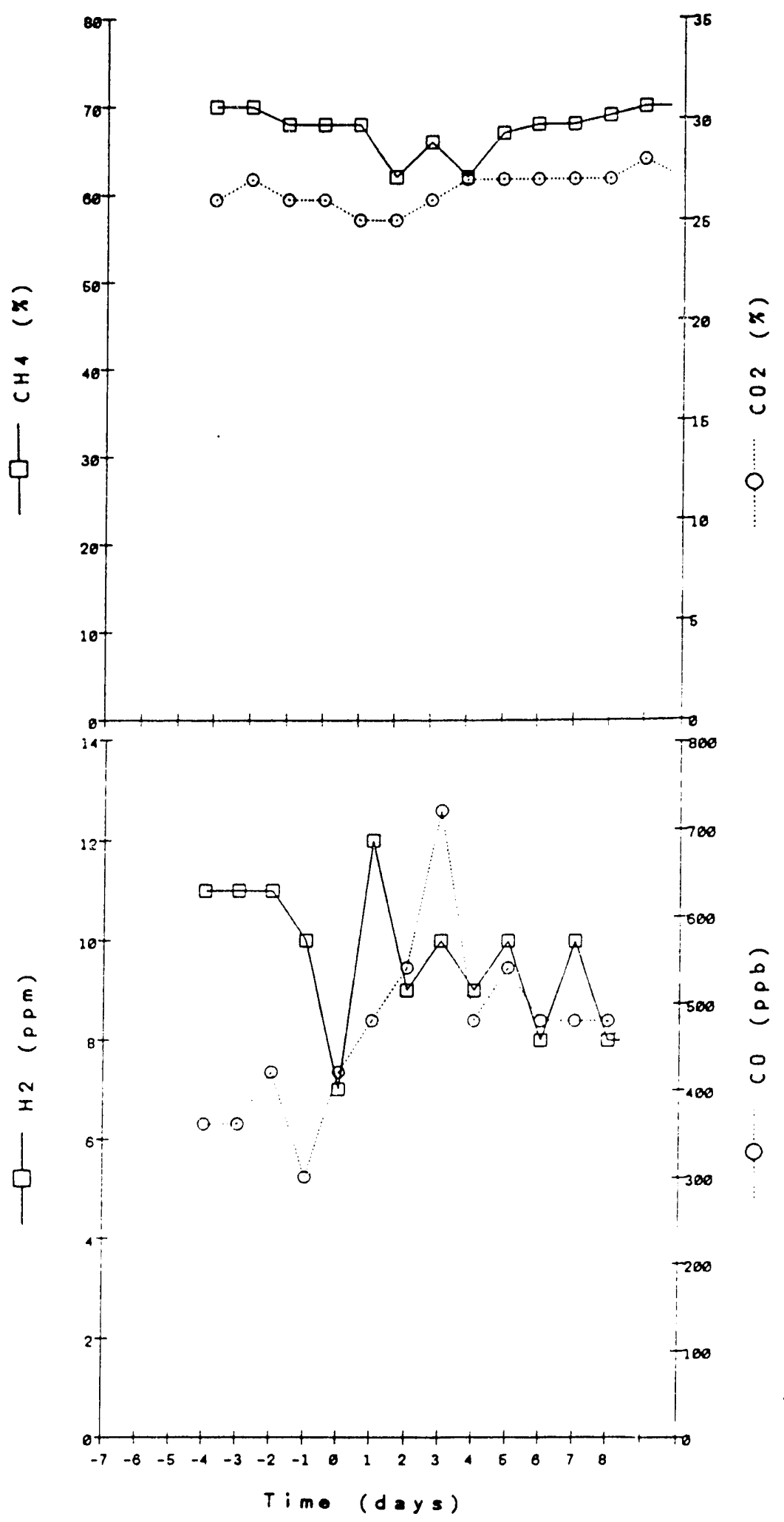

Figure 5-5. Response of (a) hydrogen and (b) carbon monoxide to a 4-fold hydraulic load increase to a 10-day HRT digester. 

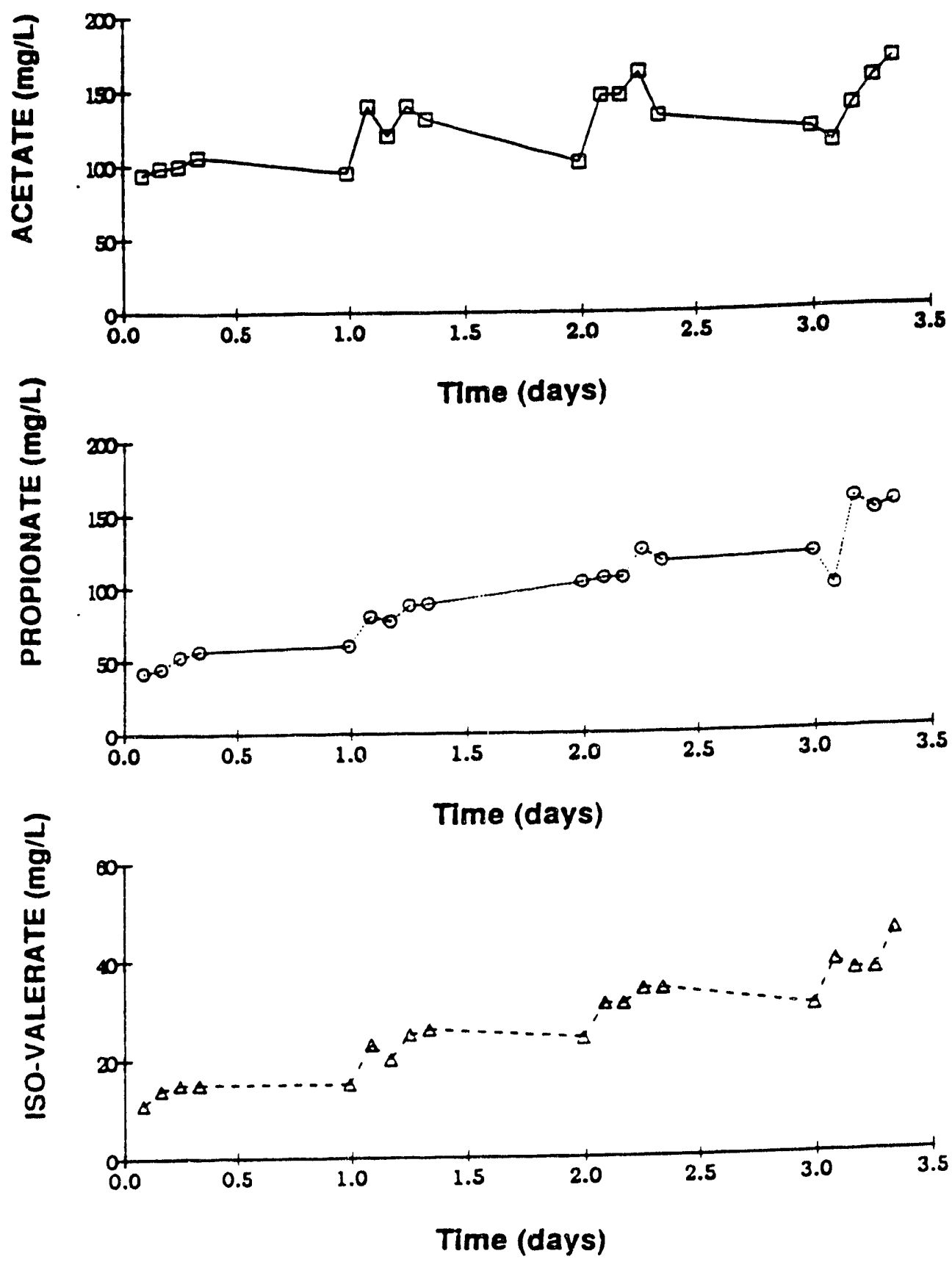

Figure 5-6. Accumulation patterns of a) acetate; b) propionate; and c)
iso-valerate from a 10 -day HRT digester subjected to a 4.0 -
fold hydraulic load increase. 
Table 5-4 End-of-Cycle Volatile Fatty Acid Concentrations Observed During a 4-fold Hydraulic Load Increase Applied to a 10day HRT Digester.

\begin{tabular}{|c|c|c|c|c|c|c|}
\hline Day No. & $c_{2}$ & $c_{3}$ & $1 C_{4}$ & $C_{4}$ & $1 C_{5}$ & $c_{5}$ \\
\hline 1 & 95 & 60 & 15 & 5 & 31 & - \\
\hline 2 & 101 & 103 & 24 & 6 & 53 & 7 \\
\hline 3 & 122 & 121 & 30 & 13 & 70 & 13 \\
\hline 4 & 75 & 101 & 37 & 10 & 78 & 7 \\
\hline 5 & 133 & 174 & 45 & 19 & 104 & 18 \\
\hline 6 & 78 & 141 & 38 & 9 & 85 & 11 \\
\hline 7 & 125 & 181 & 47 & 13 & 98 & 16 \\
\hline
\end{tabular}




\subsection{SUMMARY AND DISCUSSION}

After the 2-fold, 7-day hydraulic overload experiment was completed and the hydraulic load returned to the normal 10-day HRT rate, the initial rates of both total and methane gas production increased approximately 15 percent above what was observed during the overload and close to 50 percint above what was measured prior to the overload (Table 5-3). Considering that the applied organic loading rate had been reduced by half, this was initially confusing. Apparently this increase in gas production is a consequence of less wastage or "washout" of some key bacteria or exocellular enzyme upon reduction of the hydraulic ioading. This may indicate that a significant change of these "pool sizes" or alteration of the composition of the bacterial populations occurred over a short, 7 day, period. This indicates that antecedent conditions will significantly influence the behavior of a system.

Circumstantial support for this washout behavior is easily obtained by comparing the volume of sludge remaining after the daily feed under both hydraulic regimes. At a 5-day HRT loading, 80 percent of the system biomass (and enzyme pool) remained in the reactor after feeding. This assumes the amount of useful bacterial and/or enzymes entering with the feed is negligible compared to the digester populations. When the hydraulic loading was reduced to 10 days, 90 percent of the system biomass (and enzyme pool) remained. The expected activity, assuming the reduced loading does not alter initial kinetic rates substantially, would be $9 / 8$ or approximately a 12.5 percent increase. This compares well with observed results.

Since the diurnal gas production profiles stabilized after only several days of the overload, this indicates that substantial changes in the hydrolytic and/or fermentative populations may have occurred in response to changes in loading within this short time span, and therefore the reliability of using gas production, even measured in a real-time mode, as a process indicator is much diminished. 
The most reliable process indicator identified from this portion of the study appears to be $C O$ since it is related to the pool size of an intermediate and is not affected by changes in loading or substrate concentration.

Just as was observed during the organic overload experiments, the volatile acids that accumulated were acetate > propionate $>$ iso-valerate > iso-butyrate > butyrate. 


\section{SECTION 6}

\section{EFFECT OF COPPER ADDITION TO SEMI-CONTINUOUSLY OPERATED DIGESTERS}

Several experiments were conducted with semi-continuously operated digesters to compare the response of these systems and the results from the batch serum bottle assay segment of experimentation. Both mesophilic and thermophilic systems (10day HRT) were subjected to pulse addition of copper under relatively comparable conditions so that some sense of the similarities and differences of the responses between these two systems could be obtained. Copper was selected as the model inorganic toxicant based on results from the batch assays previously conducted. Chronic addition of copper was examined using a 20-day HRT system adapted to a higher strength solids feed ( 3.75 percent TS).

\subsection{EFFECT OF COPPER PULSE TO A MESOPHILIC DIGESTER}

A 10-day HRT test digester was operated at an 8-day hydraulic residence time for 5 consecutive days prior to copper addition as part of another experiment. The digester was pulsed with copper added as $\mathrm{CuCl}_{2}$ to the feed at a dose of 62.5 $\mathrm{mg} 1^{-1}$ on the sixth day while maintaining the 8 -day hydraulic retention time. The digester performance prior to introduction of the copper appeared to be quite stable (Table 6-1). The applied copper dose to the digester, taking into account the feed and digester volatile solids levels, was $7.85 \mathrm{mg} \mathrm{Cu} \mathrm{gVs}^{-1}$.

The impact of copper addition on total gas production is presented in Figure 6 1a, which compares the daily results of the 24 -hour periods preceding and subsequent to introducing copper to the system. Methane production paralleled total gas production during the entire experimental run (approximately 68 percent of the total gas production rate). Cumulative total gas and methane production (based on 16-hour results) decreased to 87 and 86 percent respectively of that observed prior to the copper pulse. Total gas and methane production were most severely inhibited during the first 4 to 6 hours and recovered to approximately the production rate observed the previous day subsequent to this time which compares favorably with methane inhibition data from the serum bottle assay 
Table 6-1. Dally Analysis of a 10-Day HRT Test Digester overload Prior to Recelving a Copper Pulse.

\begin{tabular}{cccccccc} 
Day & $\mathrm{pH}$ & $\begin{array}{c}\mathrm{TA} \\
(\mathrm{mg} / \mathrm{L})\end{array}$ & $\begin{array}{c}\mathrm{VFA} \\
(\mathrm{mg} / \mathrm{L})\end{array}$ & $\begin{array}{c}\mathrm{COD} \\
(\mathrm{mg} / \mathrm{L})\end{array}$ & $\begin{array}{c}\mathrm{SCOD} \\
(\mathrm{mg} / \mathrm{L})\end{array}$ & $\begin{array}{c}\mathrm{TS} \\
(\mathrm{mg} / \mathrm{L})\end{array}$ & $\begin{array}{c}\mathrm{VS} \\
(\mathrm{mg} / \mathrm{L})\end{array}$ \\
\hline-7 & 7.0 & $\cdots$ & $\ldots$ & 16300 & 2060 & 12700 & 8960 \\
-6 & 7.1 & 2920 & 130 & 15200 & 2130 & 12200 & 8420 \\
-5 & 7.1 &.- & $-\cdots$ & 14900 & 2110 & 11300 & 7950 \\
-4 & 7.0 & 2920 & 140 & 11000 & 1260 & 10900 & 7710 \\
-3 & 7.1 & 2880 & 230 & 14000 & 1130 & 10600 & 7450 \\
-2 & 7.0 & 2700 & 410 & 10700 & 3140 & 11000 & 7990 \\
-1 & 7.1 & 2920 & 210 & 15500 & 2190 & 10300 & 7310 \\
0 & 7.1 & 2940 & 140 & 11500 & 2120 & 10600 & 7410 \\
1 & 7.0 & 2790 & 120 & 11200 & 1950 & 10500 & 7350 \\
\hline
\end{tabular}

Note:

$\mathrm{TA}$ - mg/L as $\mathrm{CaCO}_{3}$

VFA - $\mathrm{mg} / \mathrm{L}$ as $\mathrm{HAC}$ 

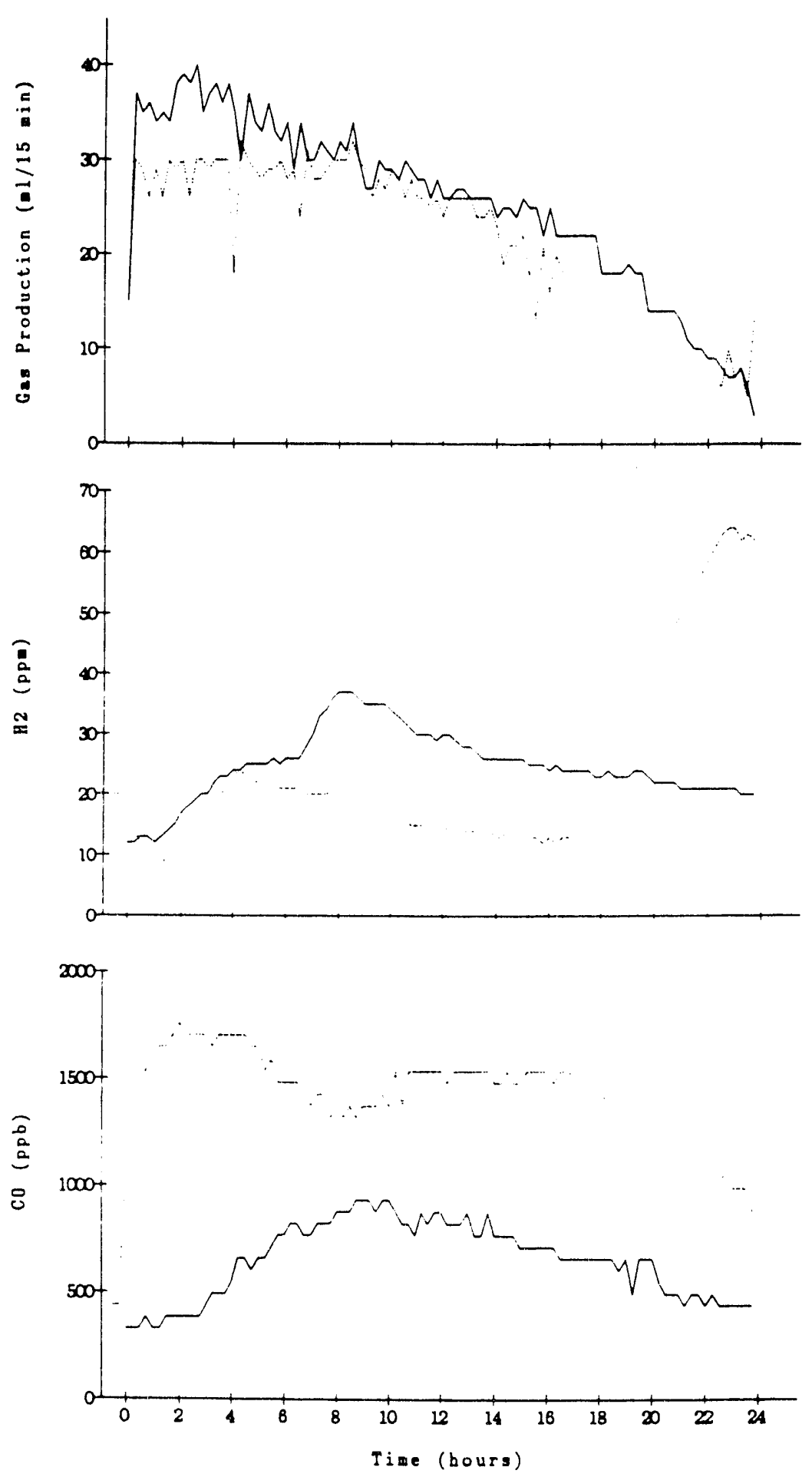

prior to copper pulse
after copper pulse

Figure 6-1. The effect of pulsing $7.85 \mathrm{mg}$ of copper per gram of VS on a) gas production, b) $\mathrm{H}_{2}$ and c) CO concentrations of a 10-day HRT digester. 
experiments. At a copper dose of $7.85 \mathrm{mg} \mathrm{gVS}^{-1}$, a reduction of methane production to approximately 84 percent of normal would be anticipated based upon the serum bottle data from the previous study.

The hydrogen and $C O$ diurnal patterns prior and subsequent to introduction of the copper are presented in Figures $6-1 b$ and $c$. During the first 90 minutes after feeding, the headspace $\mathrm{H}_{2}$ decreased from 20 to $9 \mathrm{ppm}$. The hydrogen concentration then gradually rose to a peak level of $24 \mathrm{ppm}$ at 255 minutes and subsequently declined to between 12 and 13 ppm by 900 minutes and remained there for the next several hours. A computer malfunction resulted in no data being collected between 1020 and 1310 minutes. The $\mathrm{H}_{2}$ concentration had increased to over $60 \mathrm{ppm}$ by this point in time. Just such a sharp rise in $\mathrm{H}_{2}$ was previously observed during batch serum bottle testing.

The response of $C O$ to introducing a slug of copper was more dramatic than observed during serum bottle assays but it followed the same general pattern. Within approximately 5 minutes after feeding, the co concentration jumped from a near constant (for the previous 90 minutes) level of $440 \mathrm{ppb}$ up to $1100 \mathrm{ppb}$. A peak CO concentration of $1760 \mathrm{ppm}$ was observed after 120 minutes. The CO concentration gradually declined to approximately 1400 to $1500 \mathrm{ppb}$ during the next several hours and remained at that level up to the 1.020 minute mark. The decrease in co coincided with the recovery of methane production to near uninhibited levels. This was also observed during the serum bottle assays when copper was used as a toxicant. During the ensuing period when $\mathrm{H}_{2}$ levels increased, the $C O$ concentration decreased to $880 \mathrm{ppb}$, where it remained at the conclusion of the 24 -hour cycle.

Methane and $\mathrm{CO}_{2}$ concentration profiles over the course of the 24-hour cycle after copper addition were essentially identical to the previous day's. Volatile acids and alkalinity were also similar before and after the addition of copper. With the exception of the temporary reduction in gas and methane production, conventional indicators of process stability did not change appreciably as a result of the pulse addition of copper at a dose of $7.85 \mathrm{mg} / \mathrm{gVS}$. Essentially a complete return to methane and total gas production rates observed for controls 
was also observed for samples receiving approximately the same copper dose during batch assays.

\subsection{RESPONSE OF A THERMOPHILIC DIGESTER TO A COPPER PULSE}

A copper pulse of approximately $11 \mathrm{mg} / \mathrm{gVS}\left(77.5 \mathrm{mg} 1^{-1}\right)$ was applied to a 10 -day HRT thermophilic digester that had been maintained at steady state for approximately 4.5 hydraulic residence times. The hydrogen and carbon monoxide diurnal profiles (Figures $6-2 a$ and $b$ ) closely resembled results generated during copper addition to the mesophilic system. Hydrogen levels declined during the first 90 minutes and then slowly increased to slightly above $100 \mathrm{ppm}$ by 12 hours. A large rise in $\mathrm{H}_{2}$ concentration occurred sometime during the final 12 hours of the feeding cycle reaching close to $200 \mathrm{ppm}$ by the end of 24 hours. This is precisely the same pattern of $\mathrm{H}_{2}$ response observed during monitoring of the mesophilic digester. The thermophilic digester displayed considerably higher $\mathrm{H}_{2}$ values both prior to and after the copper pulse. The average "baseline" hydrogen level measured in the thermophilic system (based on 10 days measurements) was 97 ppm.

Just as was observed for the mesophilic digester, co rose quickly following copper addition, peaked after several hours, and then gradually declined over the remainder of the day. The co concentration levels returned to near normal by the end of the 24-hour cycle.

A slight depression of methane concentration concurrent with a small rise in $\mathrm{CO}_{2}$ was observed as a result of copper addition. These two major gases reached close to normal levels by the end of 24 hours. A failure of the gas production measurement system precluded evaluation and comparison of gas and methane production prior to and during copper induced inhibition.

\subsection{EFFECT OF COPPER ADDITION ON A CHRONIC BASIS TO A MESOPHILIC DIGESTER}

A 20-day HRT digester that had been acclimated to degrading DAF concentrated Waste Activated Sludge (TS $=3.75$ percent) was used to examine the effect of chronic addition of copper as compared to pulse addition. Copper $\left(\mathrm{CuCl}_{2}\right)$ was 

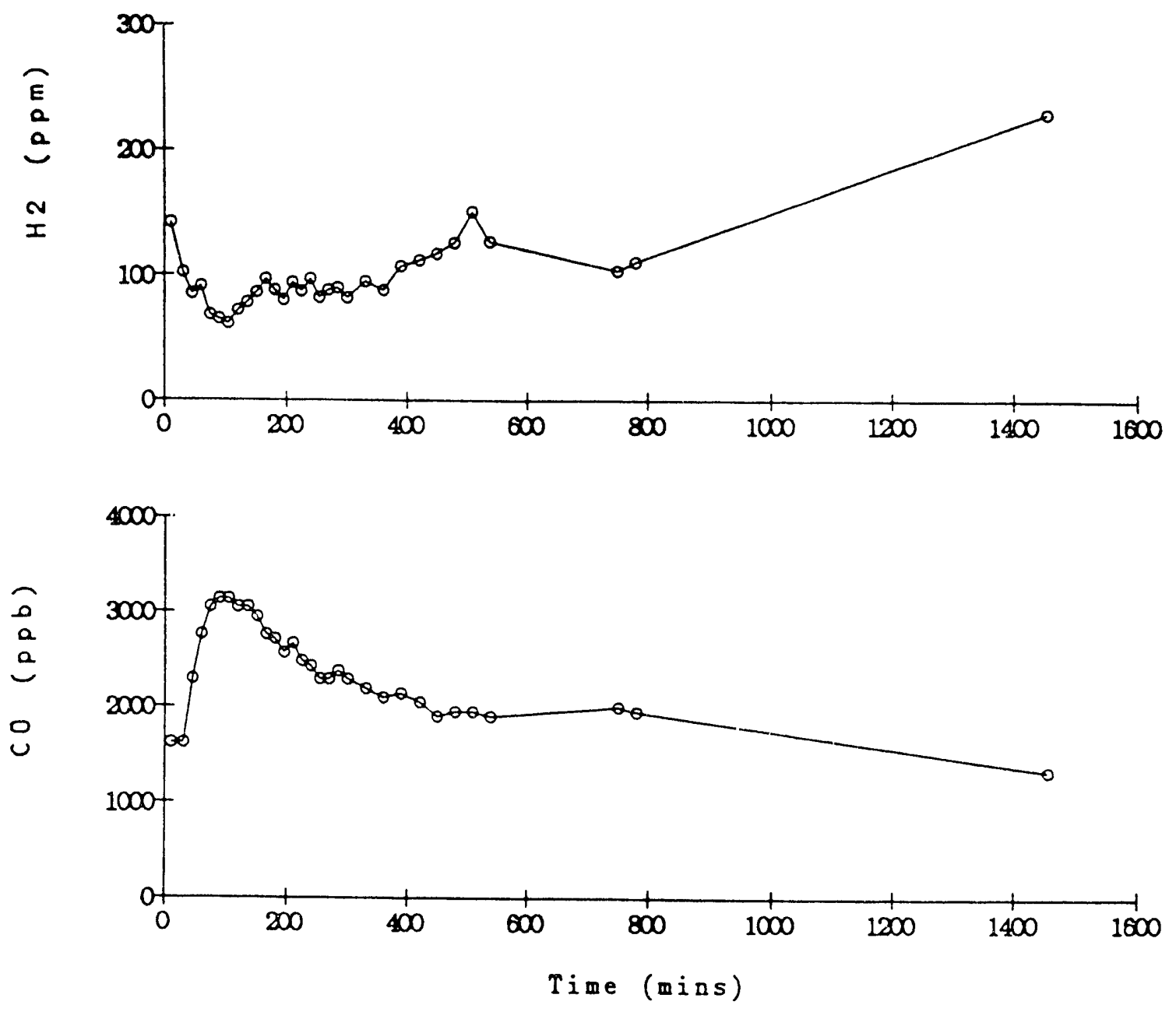

Figure 6-2. Response of a) $\mathrm{H}_{2}$ and b) $\mathrm{CO}$ of a 10-day HRT thermophilic digester to an $11 \mathrm{mg}$ of copper per gram of VS pulse. 
implemented to the daily feed at a dose of $750 \mathrm{mg} / \mathrm{L}$ for the first day and thereafter at $1500 \mathrm{mg} / \mathrm{L}$ for the next 14 days.

As anticipated, based on the dose used and the previous pulse addition of copper performed in the batch assays and in continuously operated systems, there was little notice sle effect due tc copper addition for the first several days. Total and volatile solids levels increased slightly after several days but not significantly until day 10 of the copper addition. A complete summary of process indicators, monitored on a daily basis during the experiment, is presented in Table 6-2. All of the conventional process indicators (Figures $6-3 a-c$ ) responded at about the same pace, showing stress in the system by day 7 or 8 . Of these indicators, the VFA/TA ratio appeared to give the most rapid and clearest indication of an oncoming upset. By day 11 of copper addition it was clear that an upset was occurring, despite the fact that the VFA/TA ratio had not reached the 0.35 to 0.4 level which is considered indicative of upset conditions.

CO was the first parameter monitored on a daily basis to demonstrate a significant variation for normal baseline conditions (Figure 6-3c), increasing approximately one and one-half times between days 2 and 3 . By day 5, co had more than doubled from the "baseline" value of 400-600 ppb. Hydrogen concurrently rose by a factor of close to two on this day as well. Both indicators remained at essentially these same levels until days 11 and 12 for $\mathrm{CO} a \mathrm{~d}_{2}$ respectively, when they both rose dramatically. Concurrent to this, gas production levels decined precipitously.

The accumulation of the individual volatile fatty acids (VFAs) was tracked on a daily basis (Table 6-3). The effective $C O D$ concentration of $C_{2}$ "hrough $C_{7}$ acids is plotted, together with the accumulation of SCOD in the system (Figure 6-4). As is evident, SCOD accumulated much more rapidly than did VFAs, increasing from a baseline value of approximately 4200 to $10,500 \mathrm{mg} / \mathrm{L}$ by day 15 . This increase of over $8000 \mathrm{mg} / \mathrm{L}$ occurred during a period when the COD equivalent due to VFA accumulation accounted for less than $2000 \mathrm{mg} / \mathrm{L}$ of COD increase. From solids information it is evident that the solubilization of particulate material 


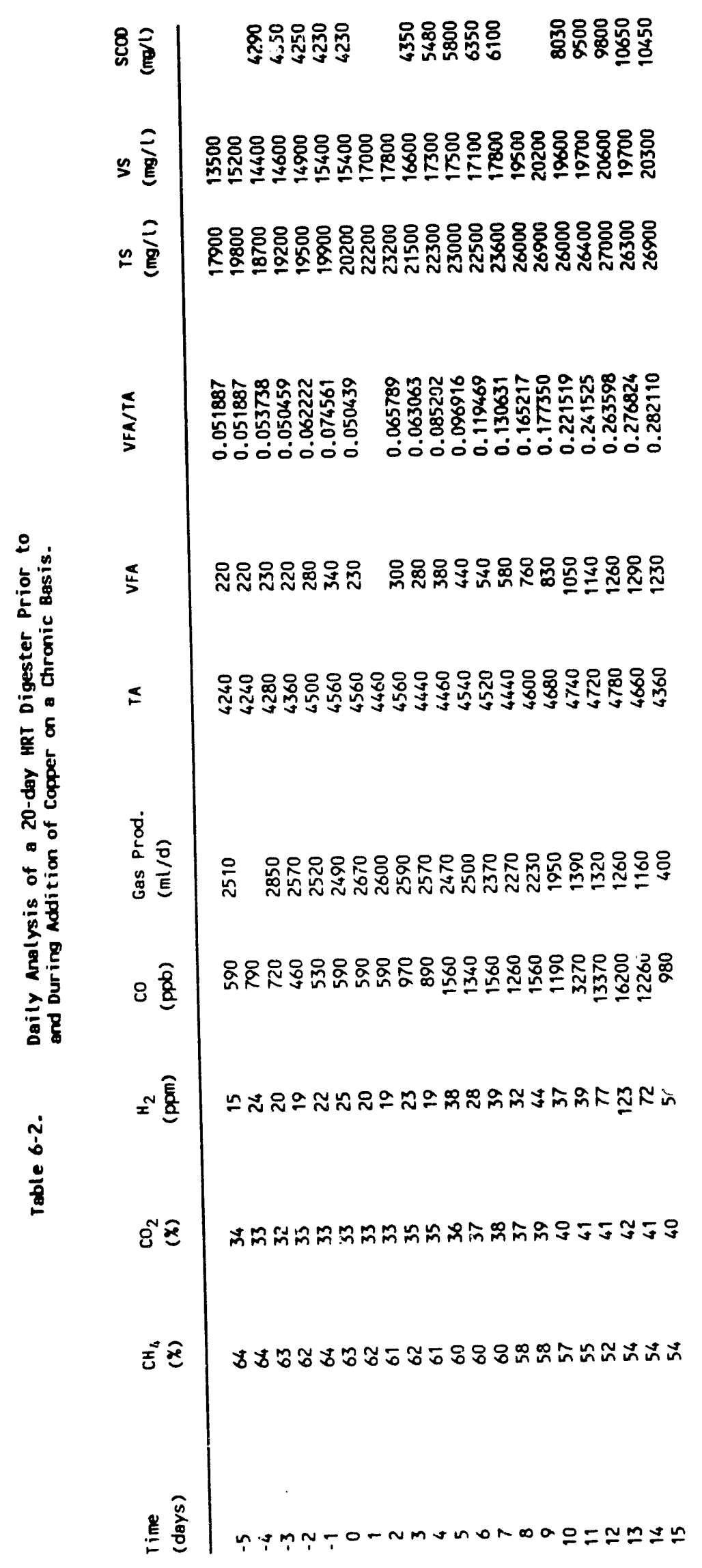




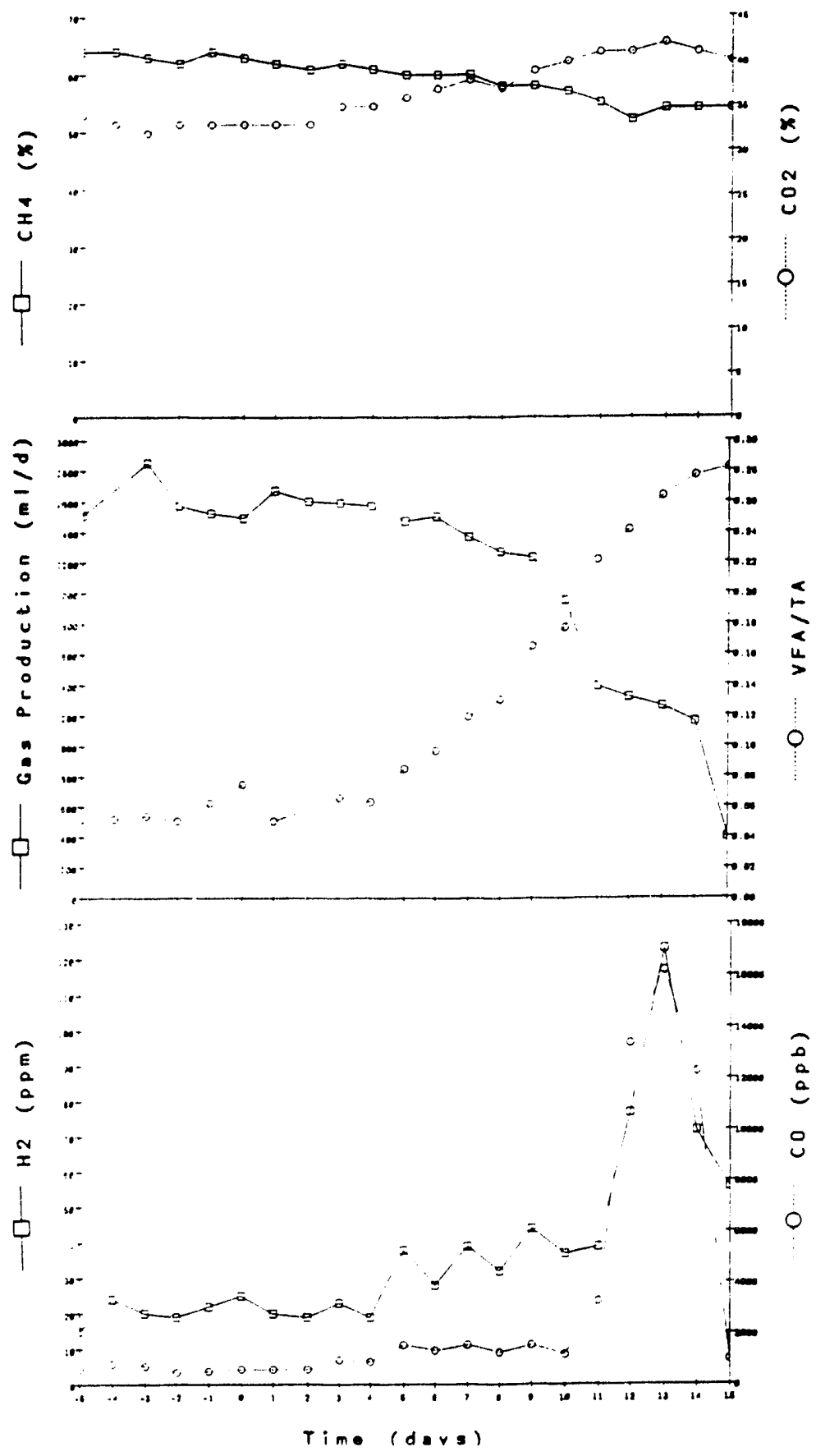

Figure 6-3. Comparison of the response of conventional and novel anaerobic digester process indicators (end-of-cycle) for a 20-day HRT digester subjected to chronic continuous copper addition. 
Table 6-3. Accumulation Pattern of Volatile Fatty
Acids as Result of Copper on a Chronic
Basis to 20-day HRT Digester.

\begin{tabular}{|c|c|c|c|c|c|c|c|c|}
\hline $\begin{array}{l}\text { Time } \\
\text { (days) }\end{array}$ & $\mathrm{C}_{2}$ & $C_{3}$ & $1 \mathrm{C}_{4}$ & C4 & $i C_{5}$ & C5 & $1 \mathrm{C}_{6}$ & C7 \\
\hline-1 & 14 & TR & $\cdots$ & -- & $-\cdot$ & $-\cdot$ & $-\cdot$ & $\cdots$ \\
\hline 0 & 16 & $\mathrm{TR}$ & $-\cdot$ & -- & $\cdots$ & -- & $\cdots$ & $\cdots$ \\
\hline 1 & 13 & TR & $-\cdot$ & $\cdots$ & -- & $\cdots$ & -- & $\cdots$ \\
\hline 2 & 14 & TR & $\cdots$ & $\cdots$ & $\cdots$ & $-\cdot$ & $\cdots$ & $\cdots$ \\
\hline 3 & 45 & $\mathrm{TR}$ & $\cdots$ & -- & $-\cdot$ & -- & $\cdots$ & $\cdots$ \\
\hline 4 & 60 & TR & $\cdots$ & $\cdots$ & $\cdots$ & $-\cdot$ & - & $\cdots$ \\
\hline 5 & 85 & 6 & $\cdots$ & $\cdots$ & $\mathrm{TR}$ & $\cdots$ & $-\cdot$ & $\cdots$ \\
\hline 6 & 172 & 27 & $\cdots$ & $\cdots$ & 5 & $\cdots$ & $\cdots$ & $\cdots$ \\
\hline 7 & 188 & 58 & $\cdots$ & $\cdots$ & 12 & $\cdots$ & $\cdots$ & $\cdots$ \\
\hline 8 & 307 & 100 & 5 & $\cdots$ & 23 & -- & $\cdots$ & $\cdots$ \\
\hline 9 & 366 & 150 & 12 & 3 & 45 & $\cdots$ & $\cdots$ & $\cdots$ \\
\hline 10 & 467 & 208 & 30 & 4 & 70 & -- & $\cdots$ & $\cdots$ \\
\hline 11 & 568 & 265 & 47 & 26 & 106 & 11 & $\cdots$ & $\cdots$ \\
\hline 12 & 480 & 289 & 44 & 28 & 104 & 19 & - - & $\cdots$ \\
\hline 13 & 551 & 381 & 67 & 45 & 145 & 27 & 10 & 15 \\
\hline 14 & 536 & 388 & 79 & 40 & 145 & 24 & 11 & 9 \\
\hline
\end{tabular}




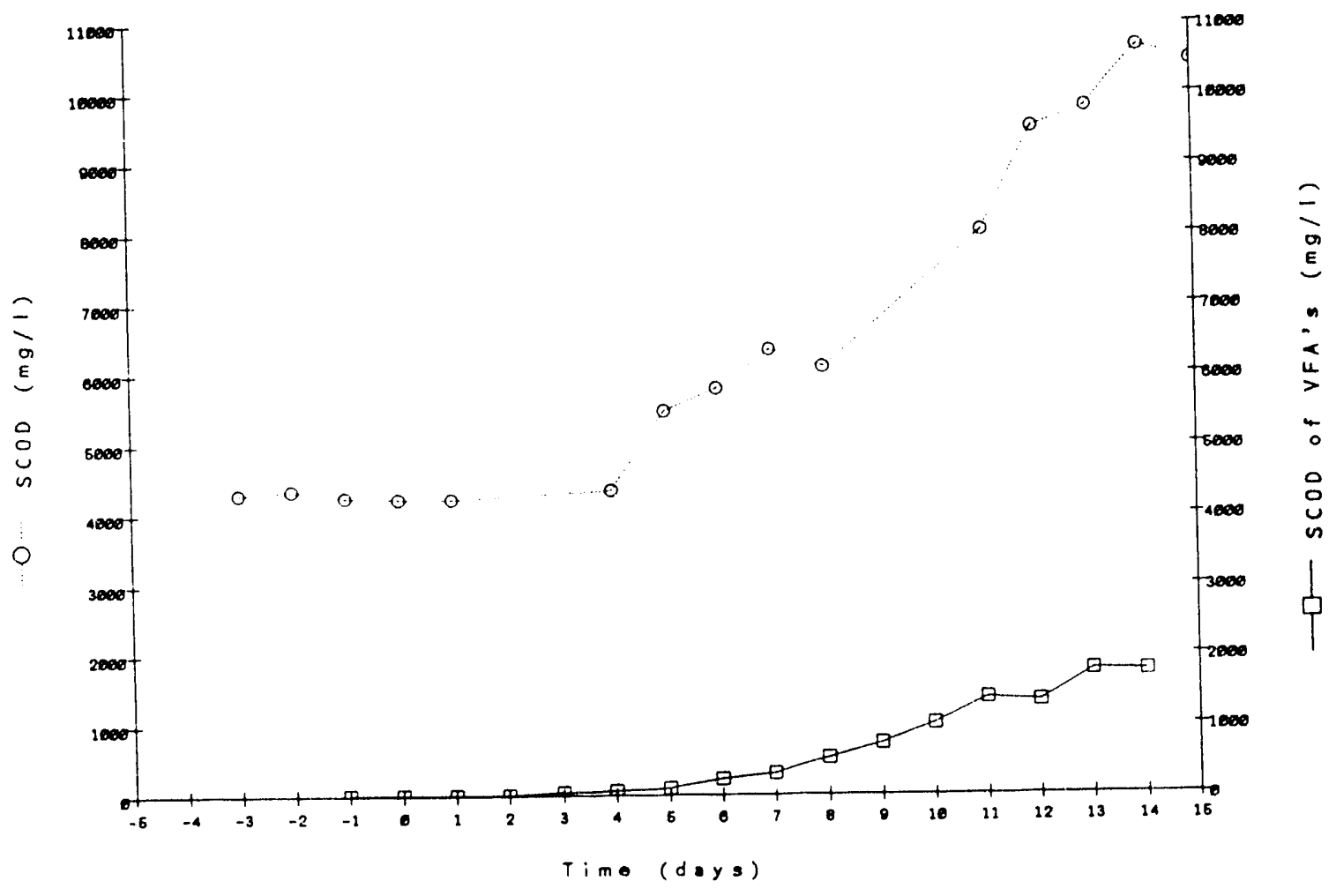

Figure 6-4. On-line (a) gas and (b) methane production rate data from a 20 day HRT digester subject to continual copper addition. 
was impaired. From the results it is apparent that conversion of soluble products to VFAs was also inhibited.

The accumulation of copper (reported in terms of both $\mathrm{mg} / \mathrm{L}$ and $\mathrm{mg} / \mathrm{g}$ VS) is reported in Table 6-4. Substantially greater copper dosages, on a $\mathrm{mg} / \mathrm{g}$ VS, are needed to produce the same levels of inhibition for chronic compared to pulse copper addition. To attain approximately 50 percent inhibition of methane production, a copper concentration of approximately $32.9 \mathrm{mg} / \mathrm{g}$ VS was necessary for a chronic gradual build-up compared to $13.9 \mathrm{mg} \mathrm{Cu} / \mathrm{g}$ VS for pulse addition. This is approximately a 2.4 times increase in dose required for 50 percent inhibition due to a one-time pulse addition. This is likely because the copper added to the anaerobic digester in a stepwise fashion was continually partitioned to the solids phase and precipitated with additional sulfide that was produced during the 12 days needed to reach a 50 percent inhibition level.

Total gas and methane production rates closely paralleled each other (Figures 6 $5 a$ and $b$ ). Each day's gas production rate data was modeled according to a first order decay equation of the form

$$
Q=A e^{-b t}
$$

as used in the hydraulic and organic overload portions of the experimental work. The calculated regression coefficients, for the 12 days prior to and 15 days after initiation of the copper addition, are graphically presented in Figure 6-6 along with the average values for data prior to copper addition as well as 2 and 3 standard deviations. Not until day 7 could a significant decrease in the initial rate of gas production from average values $(41.5 \pm 1.9)$ be detected, although for days 4 and 5 the value of A decreased to slightly less than two standard deviations from the average value. The decay coefficient was observed to be somewhat more variable $(0.0386 \pm 0.008)$ and did not drop below 2 standard deviations of the pre-copper addition data average until day 8 .

As observed in previous organic and hydraulic overload experiments, the only VFA's that accumulated to significant levels were acetate, propionate, and isovalerate. The accumulation of VFAs was much lower than previously observed for 

Table 6-4. Accumulation of Copper in a 20-day HRT Digester Subjected to Chronic Addition of High Copper Feed.

\section{Copper Concentration}

\begin{tabular}{ccc} 
Day & $\mathrm{mg} / \mathrm{L}$ & $\mathrm{mg} / \mathrm{g} \mathrm{VS}$ \\
\hline 3 & 185 & 10.4 \\
4 & $\cdots$ & - \\
5 & 283 & 16.3 \\
6 & 347 & 19.8 \\
7 & 403 & 23.6 \\
8 & 457 & 25.7 \\
9 & 525 & 26.9 \\
10 & 570 & 28.2 \\
11 & 645 & 32.9 \\
14 & 869 & 42.2 \\
\hline
\end{tabular}




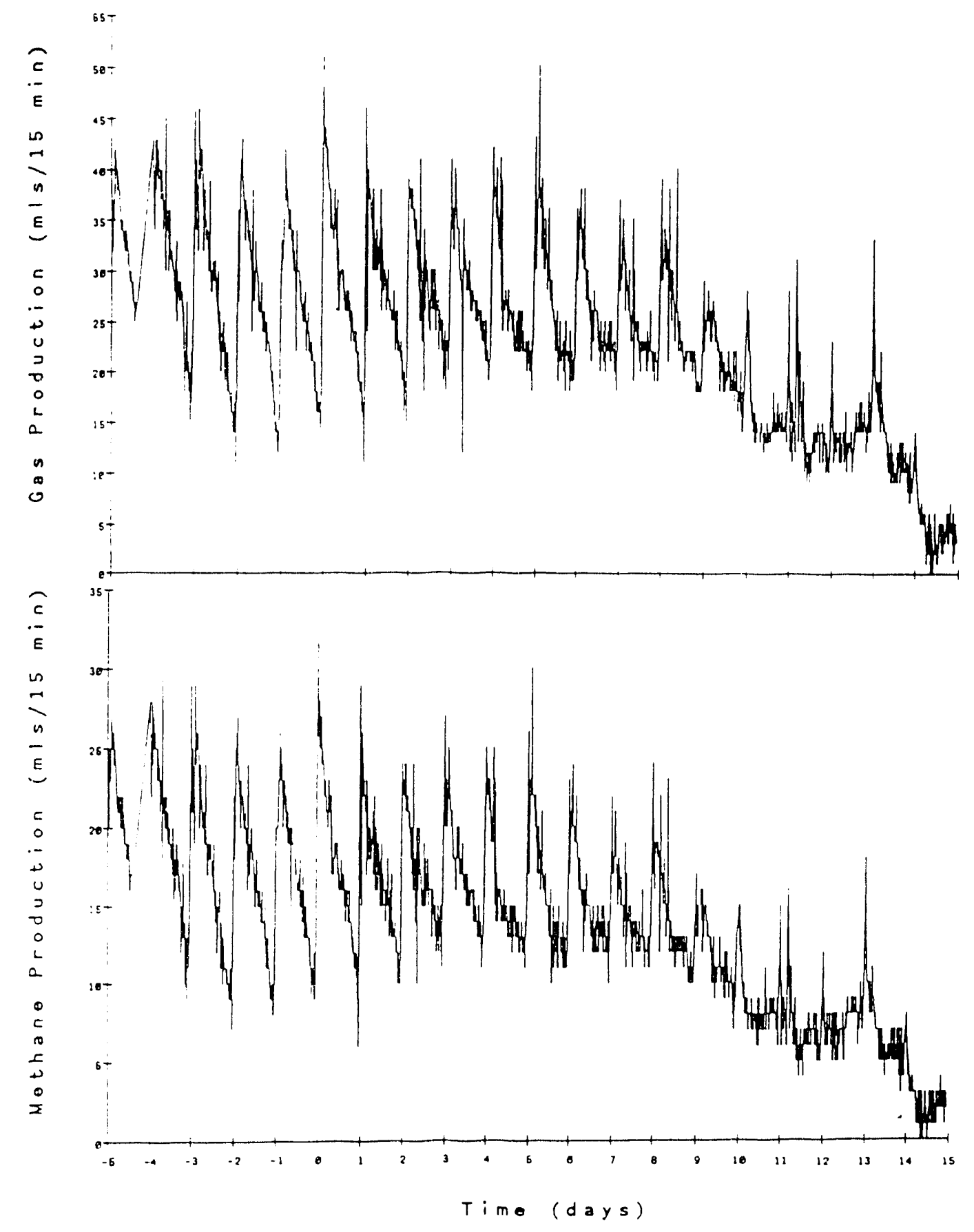

Figure 6-5. Fifteen minute interval total gas and methane production rates during the course of chronic copper addition to a 20 -day HRT digester. 


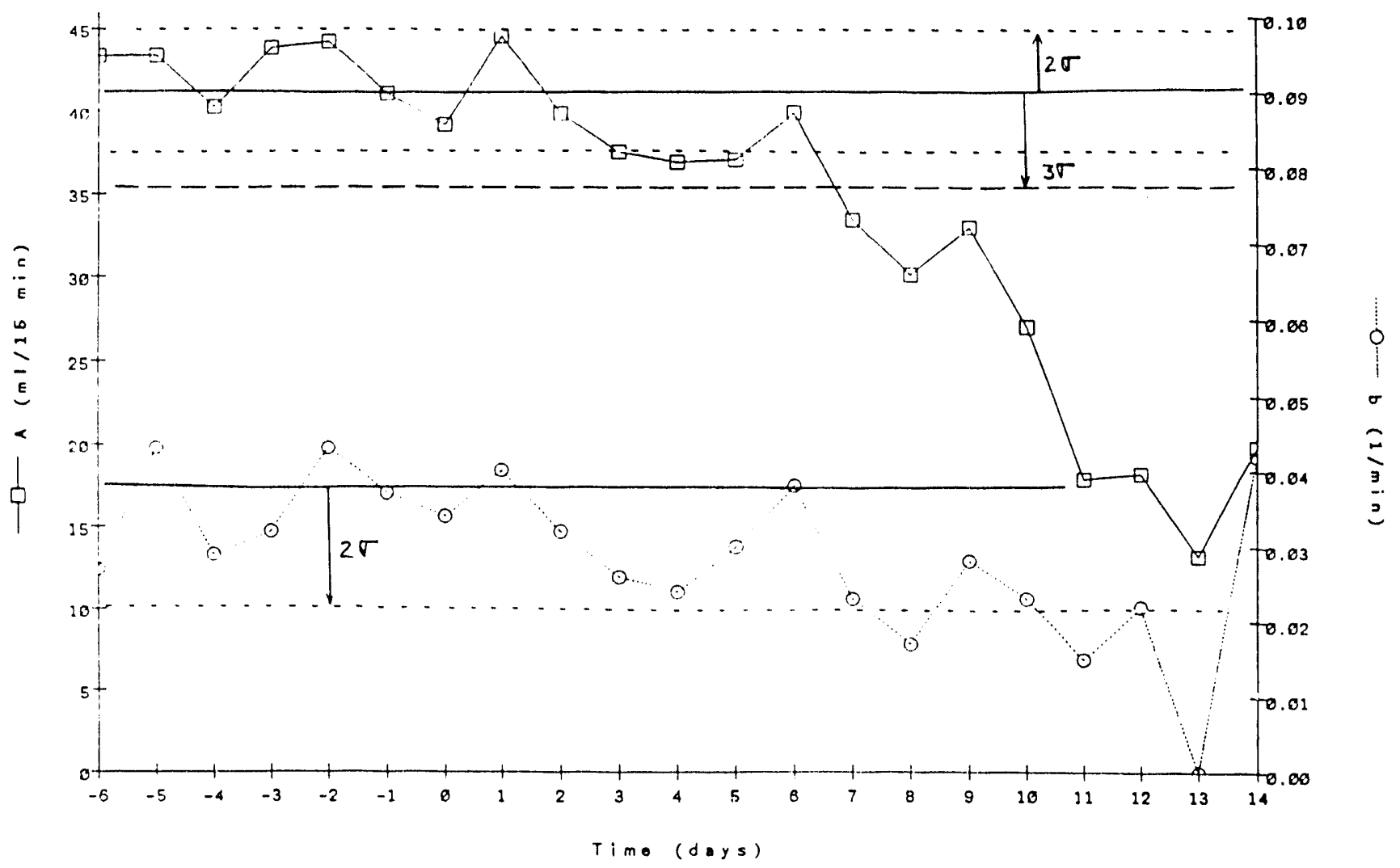

Figure 6-6. Graphical presentation of modeling of gas production data of a 20-day HRT digester subjected to continuous copper addition. 
an organically overloaded 10-day HRT digester for comparable levels of overall total gas and methane inhibition. The pattern of these three VFAs accumulation is shown in Figures 6-7a-c. Acetate displayed a somewhat steady increase over the entire period. Starting at day 8 , the rate of accumulation appeared to increase slightly. Propionate concentrations did not increase substantially until day 5 and increased steadily thereafter. Iso-valerate increased essentially in the same pattern as propionate but at a lower rate.

The real-time data for $\mathrm{H}_{2}$ and $\mathrm{CO}$ are presented in Figures 6-8a and $\mathrm{b}$, respectively. Starting on day 4 , the hydrogen concentration began to slowly increase until day 11, in contrast to pulse addition of copper that resulted in a shortterm decrease in hydrogen followed by a rise above the normal baseline. Starting on day 11, which was when digester performance began to significantly decline (as judged by conventional indicators such as gas production, VFA/TA ratio, VS and TS etc.), hydrogen levels quickly rose to almost $100 \mathrm{ppm}$, peaked during day 12 at approximately $130 \mathrm{ppm}$, and declined steadily thereafter.

The response pattern displayed by $\mathrm{CO}$ was somewhat similar to $\mathrm{H}_{2}$. By day 3 a significant increase in CO concentration was evident. Beginning on day 4 the CO baseline rose approximately 2.5-fold over the previous baseline of $600 \mathrm{ppb}$. Starting late in the day on day 10 of copper addition, co concentrations rose by over an order of magnitude in several hours, reaching a concentration of approximately $24 \mathrm{ppm}$ from a baseline value of $1500 \mathrm{ppb}$.

Clearly, hydrogen appears to be a reasonable indicator of process upset once the upset is underway. $\mathrm{CO}$, however, appears to give a better indication of oncoming upset. As early as day 3 the CO levels began to rise and by the beginning of day 5 were well above normal operating levels. Finally, as system performance began to seriously deteriorate, CO rose between one and two orders of magnitude above normal baseline values. 


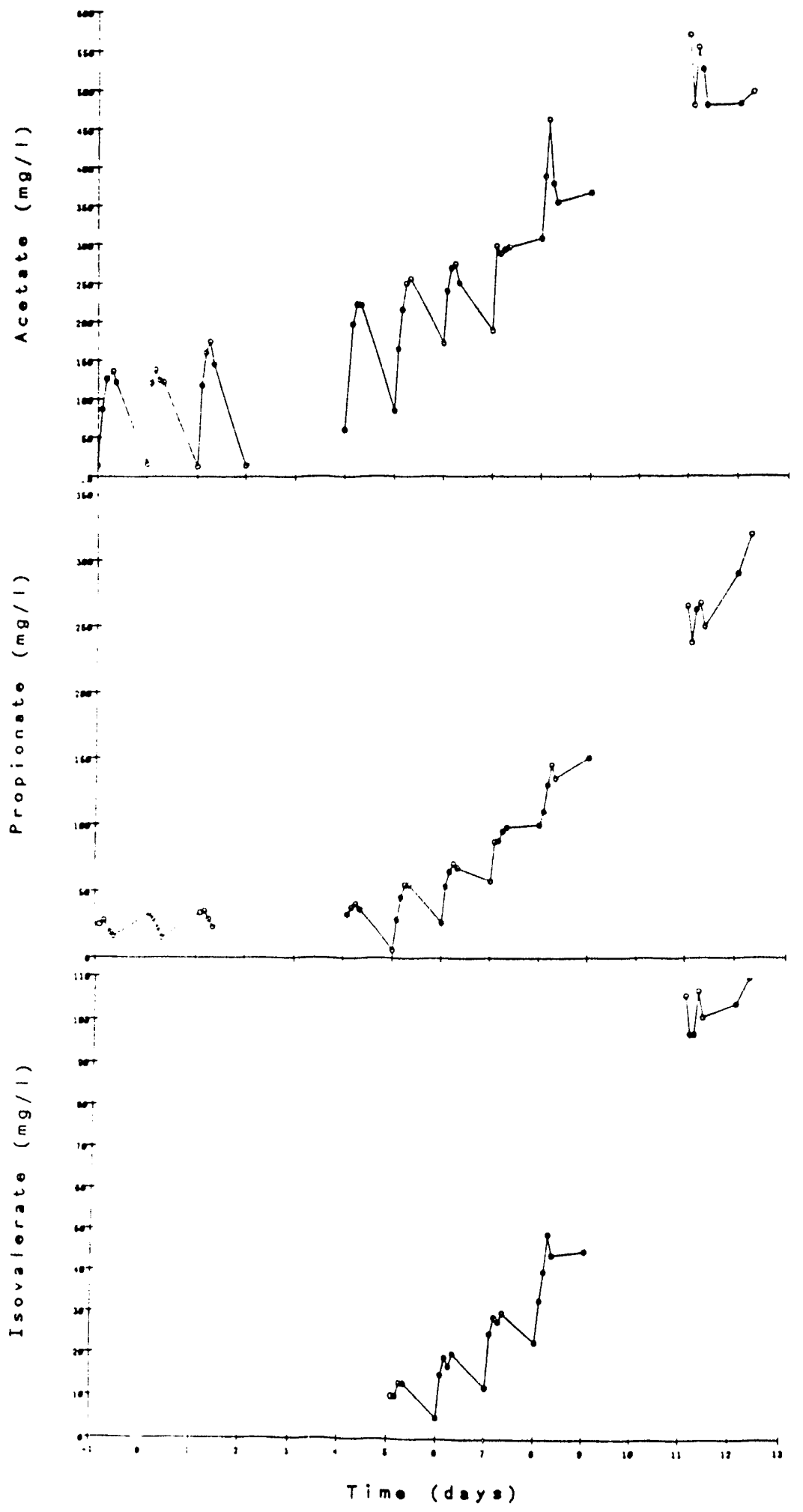

Figure 6-7. Arcumulation pattern of (a) acetate; (b) propionate; and (c) iso-valerate during copper induced inhibition of a 20 -day HRT digester.

$6-17$ 

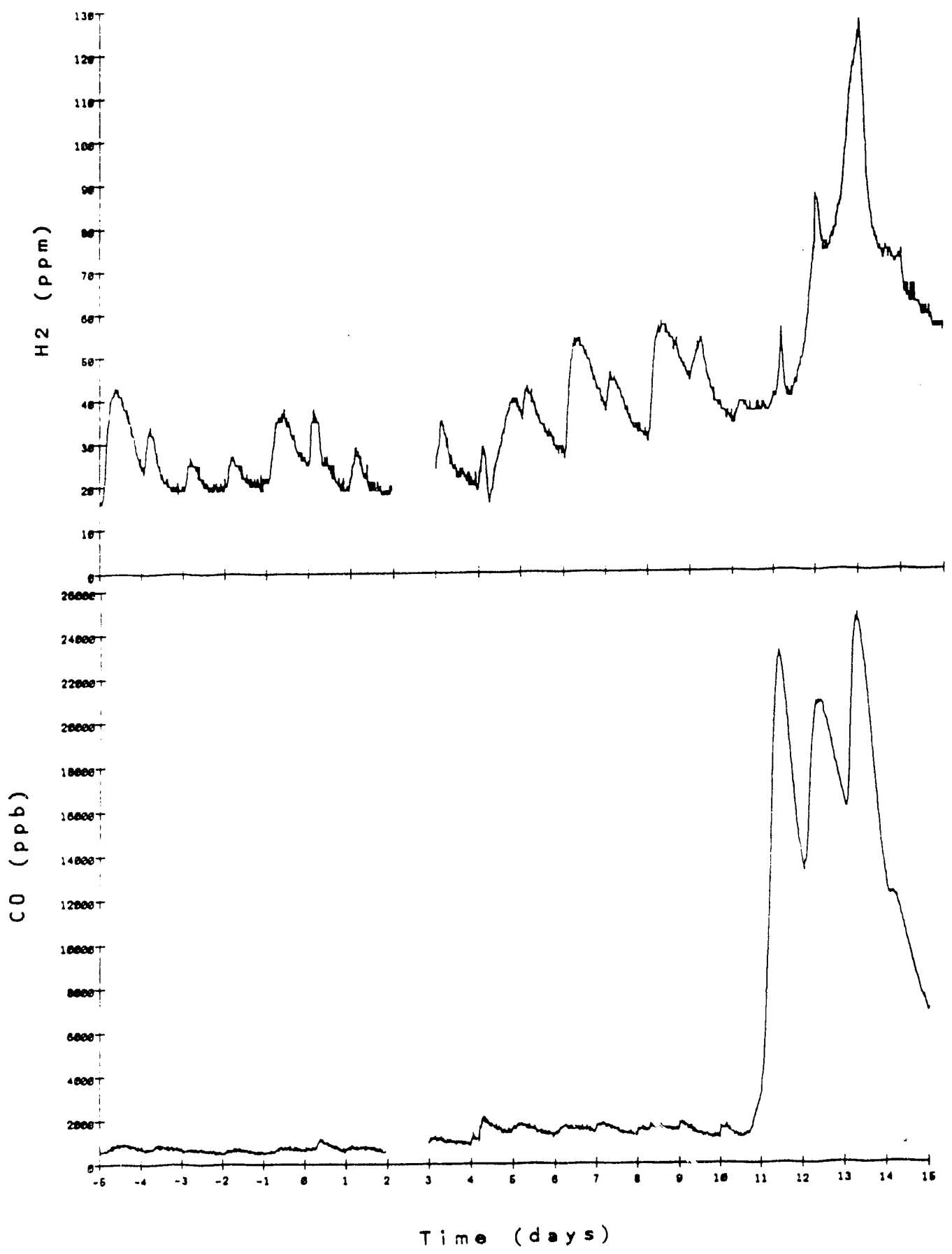

Figure 6-8. On-line measure of (a) hydrogen and (b) co concentratione during copper induced (chronic addition) inhibition of a 20 -day HRT digester. 


\subsection{SUMMARY AND DISCUSSION}

The pulse addition of copper (as $\mathrm{CuCl}_{2}$ ) to both mesophilic and thermophilic digesters resulted in the same characteristic response to metal induced inhibition that had been previously observed in batch serum bottle examples. A pulse of $7.85 \mathrm{mg} \mathrm{Cu} / \mathrm{gVS}$ produced the same level of inhibition of methane production, ca 15\%, that was predicted based on the batch studies. The hydrogen concentration immediately decreased upon addition of copper (20 to $9 \mathrm{ppm}$ ) while the CO concentration rose from 440 to $1100 \mathrm{ppb}$ in five minutes.

Although the relative concentrations of hydrogen and $C O$ were considerably higher for the thermophilic compared to mesophilic systems, the same response pattern was observed when $11 \mathrm{mg} \mathrm{Cu} / \mathrm{gVS}$ was pulsed to this reactor. Hydrogen concentration was immediately depressed with a concurrent increase in $\mathrm{CO}$.

The effect of chronic addition of copper to a mesophilic system receiving a 'ijk 3.75 percent, solids feed produced a considerably different response pattern than that of pulsed addition. Hydrogen values did not decrease but rather gradually increased from day 5 on, eventually reaching concentrations and diurnal profiles three to four times the normal steady state levels. This is similar to the concentration of hydrogen reached once it rebounded from the initial depression during pulse copper addition near the end of the 24 -hour period.

Co levels rose 2- to 3 -fold over normal values by day 5 of copper addition, the approximate time period (ca. day 6 or 7 ) when gas production began to show some effect and when the diurnal pattern was subject to modeling. When serious inhibition of gas production began to occur, the co concentration rose several orders of magnitude.

CO was the most sensitive indicator of system upset for this as well as the pulse addition experiments, and was far more reliable than gas production rate, VFA/TA ratio, $\mathrm{H}_{2}, \mathrm{CH}_{4}, \mathrm{CO}_{2}$ or $\mathrm{pH}$.

Although there was a definite decrease in solids reduction due to the chronic copper addition, VS increased from ca. 15,000 to $20,000 \mathrm{mg} / \mathrm{L}$, this was not the 
only point where substrate flow was inhibited. The soluble COD rise was fourfold greater than could be accounted for in the increase in VFAs. Conversion of soluble hydrolysis products to VFAs was obviously strongly inhibited.

Acetate consumption was also inhibited. The diurnal profile of acetate accumulation and consumption began increasing by day 4 or 5 .

The accumulation of VFAs followed the same order as the hydraulic and organic load increase with acetate $>$ propionate $>$ iso-valerate $>$ iso-butyrate $>$ butyrate $>$ valerate. This would be the approximate order if the build-up of these acids was related to the free energy available for their degradation, except for acetate. Whether the accumulation of these acids is related to inhibition of acetogenesis or is simply caused by the build-up of the products of their syntrophic degradation needs to be examined further. Results clearly show that hydrolysis and fermentative as well as acetate catabolism and eventually methanogenesis from hydrogen were all inhibited by copper addition. 


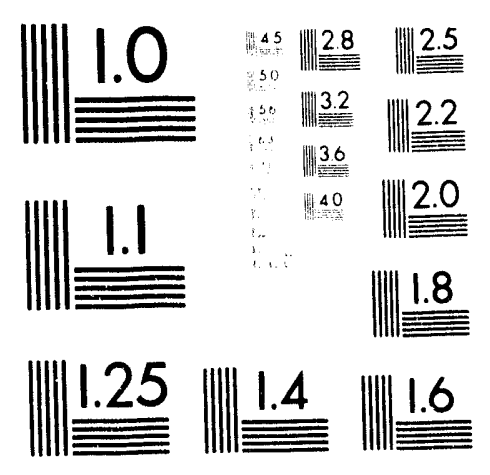



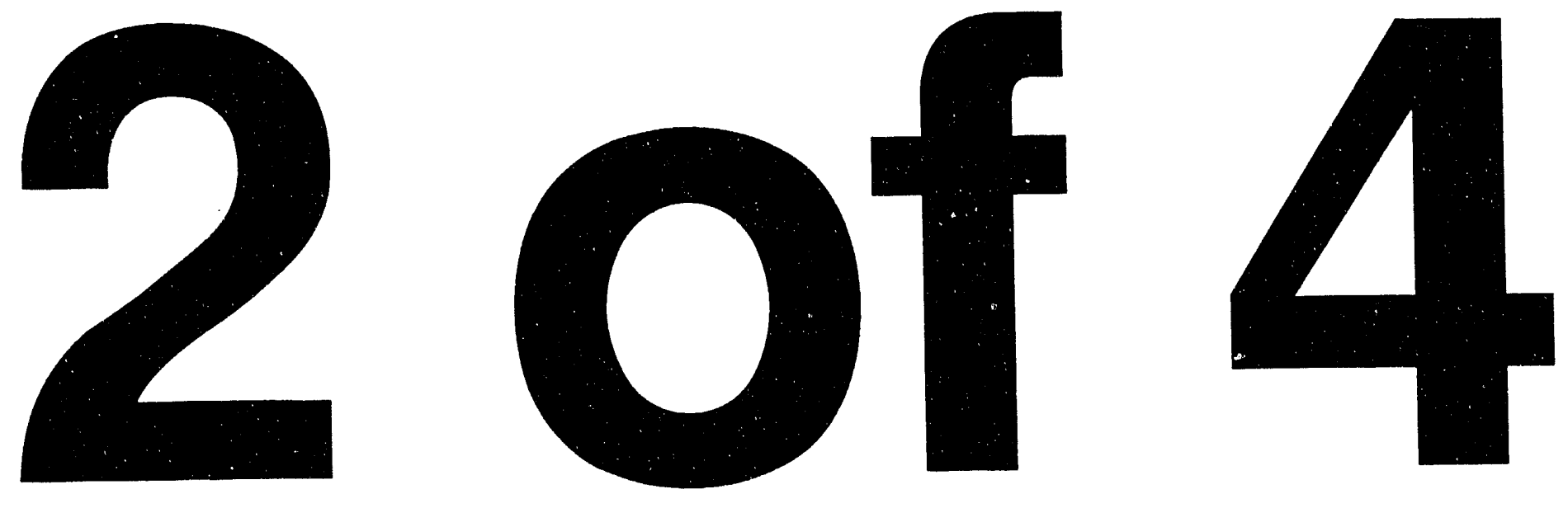


\section{SECTION 7}

\section{RESULTS OF TIME SERIES ANALYSIS}

Data from the five on-line experimental runs was subjected to time series analysis. The pre-shock or steady state data was analyzed via spectral analysis while attenpts were made to model both steady state and shock conditions with Box-Jenkins ARIMA models. A synopsis of results of these analyses follows.

\subsection{SPECTRAL ANALYSIS}

Spectral analysis was used to investigate pre-shock, steady state digester operation. Parameters investigated via univariate spectral analysis (using the BMDP statistical software package) were methane, carbon dioxide, hydrogen and carbon monoxide concentrations as well as total and methane gas production rates. For each run, the means, standard deviations, minimum and maximum values of each parameter were obtained. The standard deviation results, summarized in Table 7 1 , show that the parameter that had the most significant variation in each run was $C O$. This indicates, as has already been qualitatively discussed, that $C O$ is a good candidate to investigate for use as a process indicator/early warning monitoring tool. Total gas and methane production rates also demonstrated significant variation, indicating the potential usefulness of these parameters. By contrast, methane and carbon dioxide concentrations varied little and as such are not probably useful as parameters to investigate for Box-Jenkins time series analysis.

For all parameters investigated by spectral analysis only low frequencies appeared to be significant. This indicates that the only important cycle in the data, for these experiments, occurred at low frequencies (i.e., a 24-hour cycle). No significant other patterns could be discerned by spectral analysis for any parameter.

\subsection{BOX-JENKINS TIME SERIES ANALYSIS}

The Box-Jenkins time series approach was used to evaluate, steady state (preshock) and shock conditions separately and together. In general, there was 
Table 7-1. Standard Devlations of Indicator Parameters of Anaerobic Digestion.

\section{Standard Deviations}

Parameter

$1 \quad 2 \quad 3_{3}^{\text {Run No. }}$

\begin{tabular}{lrrrrr}
\hline Gas Production Rate & 9.85 & 11.11 & 7.52 & 7.63 & 3.37 \\
Methane Production Rate & 6.36 & 6.92 & 4.72 & 4.80 & 2.47 \\
Hydrogen & 6.57 & 1.51 & 1.5 & 6.68 & 1.42 \\
Carbon Monoxide & 63.20 & 58.97 & 53.83 & 112.57 & 69.52 \\
Methane & 0.65 & 2.17 & 1.02 & 0.76 & 0.70 \\
Carbon Dioxide & 0.44 & 0.91 & 0.23 & 0.61 & 0.39 \\
\end{tabular}

Note: Run's 1, 2 and 5 are for 10 -day HRT systems subject to 2.65 fold-organic, 2 -and 4-fold hydraulic load increases, respectively.

Run 3 is a 20 -day HRT system, subject to a 2.6 -fold organic load increase. Run 4 is a 20-day HRT system equilibrated to high solids feed and inhibited by a chronic application of copper. 
little differenc in the results regardless of the way the data was analyzed.

The time series of each parameter was investigated according to the following sequence. First, the raw data was analyzed for autocorrelation and partial autocorrelations. Second, the time series was differenced to exclude daily (diurnal) patterns and then differenced again to exclude the influence of the prior value. A number of autoregressive and/or moving average terms can then be selected to adequately describe the data. These differencing techniques are designed to take a time series and by addition of a series of furctions reduce the time series to a stationary series or "white noise". If this can be accomplished, the functions can be said to adequately describe the actual time series and can be used to model and predict performance of the parameter of interest.

Unfortunately, despite painstaking efforts to prevent controllable physical occurrences, i.e., feed solids level and time of feeding, from negatively influencing the time series, adequate ARIMA models could be developed for only a few cases. Transforming the original time series by taking logs did not improve results. In those cases when the times series of a parameter could be reduced to a stationary series by differencing and building ARIMA models, the models were dissimilar. The large member of terms (and different terms) were required for each data set. This led the statistical consultants to conclude that even if ARIMA models could be developed for each data set, by some esoteric transforms, the models would be so specific for that particular data set, they would not be robust enough for general application. The model would, therefore, be of little use except as an academic exercise.

Although no analyses were undertaken to verify the validity of this observation, it was concluded that the daily intervention in the system caused by batch feeding was at least in part responsible for the failure of the Box-Jenkins times series to describe the data sets.

A more fruitful line of investigation of techniques to effectively monitor and/or provide early warning of the anaerobic digestion process centered on developing a relationship to link acetate levels in the digester to gaseous $C O$ concentrations. This will be examined next. 


\section{SECTION 8}

\section{EXPERIMENTAL RESULTS: FACTORS AFFECTING CO CONCENTRATION DURING}

ANAEROBIC DIGESTION AND THE INFLUENCE OF CO ON ANAEROBIC DIGESTION

Due to the central role that acetate catabolism plays in anaerobic siudge digestion and the anaerobic treatment of high-strength industrial wastes, it was decided to further investigate the apparent relationship of acetate to $\mathrm{CO}$. In this chapter the results of a series of serum bottle assays using digester inocula in defined medium with acetate as the sole added organic carbon source are presented. Results generated with acetate and hydrogen grown pure cultures of Methanosarcina barkeri 227 are presented and compared to results generated using digester inocula. Based upon these results and the current knowledge concerning the pathway involved in acetate catabolism tc produce methane, a thermodynamic model was formulated to estimate steady state co levels. Monitoring results from the two mesophilic reserve digesters and a two-liter thermophilic digester provide circumstantial support for the model.

Results from the serum bottle assays listed above and information generated during both hydraulic and organic overloads, applied to continuously operated digesters, were evaluated with the thermodynamic model.

Finally, the effect of manipulating the gaseous headspace concentrations of methane, hydrogen and carbon monoxide, as well as the effect of oxygen addition were investigated on a preliminary basis.

8.1 EFFECT OF ACETATE CONCENTRATION ON CO AND $\mathrm{H}_{2}$ LEVELS OF DIGESTER INOCULA IN DEFINED MEDIUM

Although the samples in the assays described in previous sections were continually mixed, kinetic and mass transfer effects may have masked the relationships between the biochemical and chemical reactions in the samples and, therefore, the CO concentration in the gaseous headspace. A set of assay conditions were accordingly designed to slow the kinetic rates down and reduce interferences due to other reactions occurring becides acetate catabolism. 
This was accomplished by first starving the inoculum for several days, to reduce residual activity due to breakdown of undergraded organic material in the sludge, and then using a small inoculum of this sludge in defined medium to reduce the rate of the biochemical reactions. The samples were continually mixed to promote attainment of equilibrium between the gaseous and aqueous phases.

Gaseous headspace concentrations were analyzed at the 48, 72, 96, and 144 hour marks for samples fed $2.0 \mathrm{mM}(120 \mathrm{mg} / \mathrm{L})$ and $4.0 \mathrm{mM}(240 \mathrm{mg} / \mathrm{L})$ acetate. Acetate concentrations during the assay were calculated based on observed methane production. The methane produced was assumed to correspond stoichiometrically to acetate consumed. The amount of methane produced accounted for between 98 and 101 percent of the potential methane, based on the amount of acetate added, indicating calculated acetate concentrations were reasonably accurate. No measurable methane production was observed in controls not supplemented with acetate. Results for these samples are summarized in Table 8-1.

CO levels in samples fed with higher doses of acetate, regardless of the acetate level used (up to $40 \mathrm{mM}$ ), had peak observed $C O$ concentrations between 2500 and $2800 \mathrm{ppb}$. Co concentrations quickly rose to this level and remained there for a period apparently directly related to the starting acetate dose.

\subsection{CO PRODUCTION BY M. BARKERI DURING METHANOGENESIS ON ACETATE AND $\mathrm{H}_{2} / \mathrm{CO}_{2}$}

In order to test if acetate metabolism by methanogens might be involved in the production and regulation of $\mathrm{CO}$ in digester systems, a growing culture of acetate adapted Methanosarcina barkeri strain 227 was regassing with $\mathrm{N}_{2}: \mathrm{CO}_{2}(80: 20)$ and $\mathrm{H}_{2}$, $\mathrm{CO}$ and methane monitored over time. The CO concentration and methane production data are presented in Figure 8-1. CO levels quickly rose to a concentration of approximately 2500 to $2800 \mathrm{ppb}$ and remained at that level until late in the fermentation, the same $C O$ pattern observed when digester inoculum was fed acetate as the sole carbon source. Hydrogen concentrations were approximately an order of magnitude higher for the pure culture compared to the 

Table 8-1. Results of Serum Bottle Assay Demonstrating the
Relationship Between CO and Acetate for Digester Inoculum.

\begin{tabular}{|c|c|c|c|c|c|c|c|}
\hline $\begin{array}{l}\text { Initial Acetate } \\
\text { Concentration } \\
\qquad(\mathrm{mM})\end{array}$ & $\begin{array}{l}\text { Time } \\
\text { (hrs) }\end{array}$ & $\begin{array}{l}\mathrm{CH}_{4} \\
(8)\end{array}$ & $\begin{array}{l}\mathrm{CO} 2 \\
(8)\end{array}$ & $\begin{array}{l}\text { H2 } \\
\text { (ppm) }\end{array}$ & $\begin{array}{l}\mathrm{CO} \\
(\mathrm{ppb})\end{array}$ & $\begin{array}{c}\text { Acetate } \\
(\mathrm{mM})\end{array}$ & $\begin{array}{l}\text { Fraction of } \\
\text { Total Free } \\
\text { Energy } \\
\text { Associated } \\
\text { With Co } \\
\text { Oxidution }\end{array}$ \\
\hline \multirow[t]{4}{*}{2.0} & 48 & 0.9 & 33.9 & 16 & 2220 & 1.8 & 0.54 \\
\hline & 72 & 2.6 & 36.6 & 21 & 1925 & 1.4 & 0.56 \\
\hline & 96 & 3.8 & 33.4 & 16 & 980 & 1.2 & 0.55 \\
\hline & 144 & 5.1 & 32.5 & 14 & 530 & 0.8 & 0.54 \\
\hline \multirow[t]{4}{*}{4.0} & 48 & 1.0 & 33.6 & 14 & 2540 & 3.8 & 0.53 \\
\hline & 72 & 2.6 & 36.1 & 20 & 2450 & 3.4 & 0.54 \\
\hline & 96 & 3.9 & 33.8 & 17 & 1590 & 3.1 & 0.55 \\
\hline & 144 & 6.3 & 32.5 & 16 & 830 & 2.4 & 0.53 \\
\hline
\end{tabular}

Results are average values of replicate samples. Free energy calculations are based on measured values above at $\mathrm{pH}=7.0$ and $35^{\circ} \mathrm{C}$ for an activity of 1.0 according to Thauer et $\underline{\text { al }} .{ }^{42}$ 


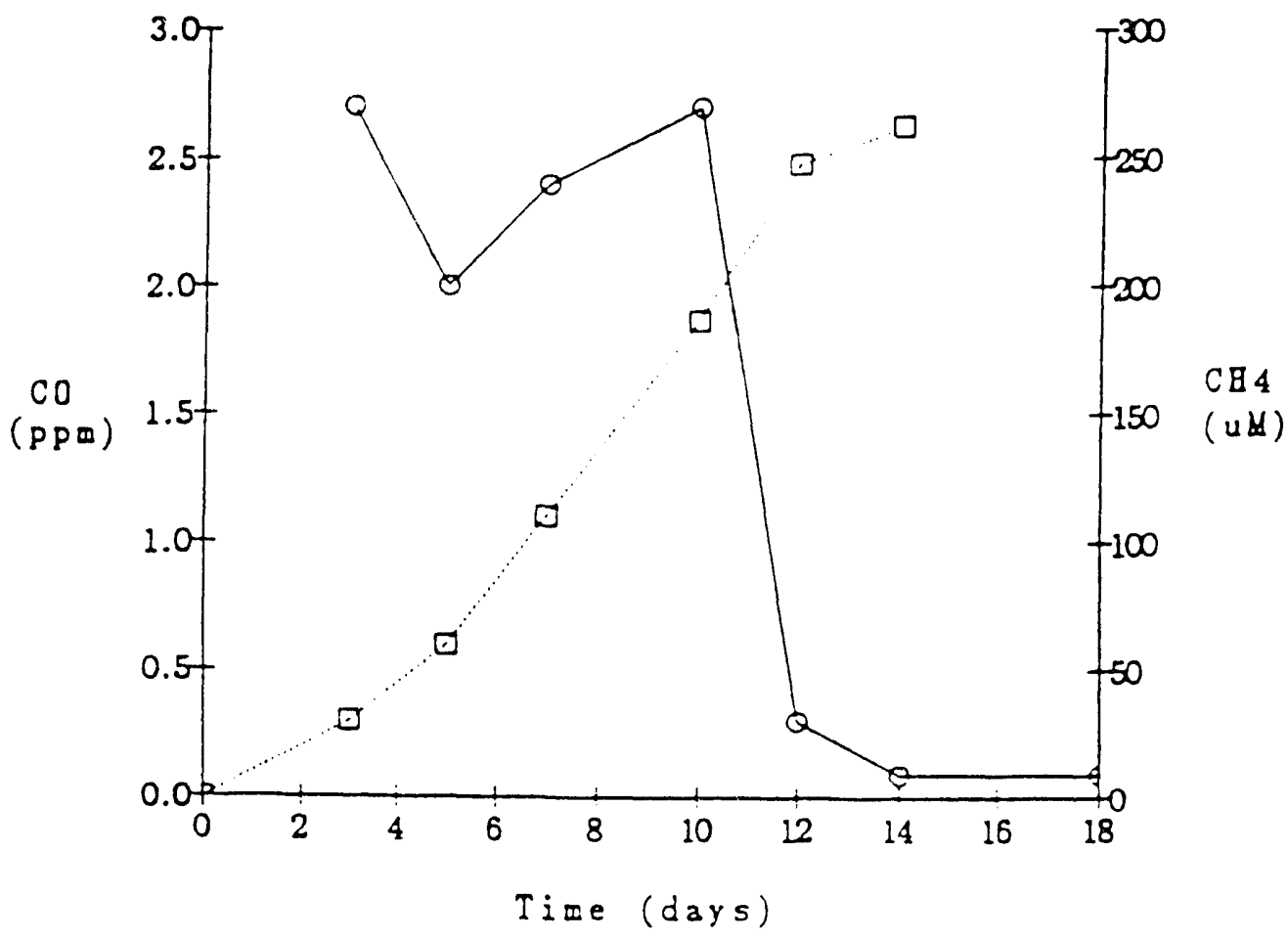

Figure 8-1. Carbon monoxide concentration and methane production observed during growth of $\mathrm{M}$. balceri on acetate.

$$
8-4
$$


digester sludge inocula. Hydrogen quickly accumulated to approximately 150-170 ppm and remained at that level until methane production essentially ceased.

Hydrogen grown $\underline{M}$ barkeri 227 was also assayed for $C 0$ production. After 24 hours of incubation, the headspace pressure decreased from an initial value of 2 atmospheres of $\mathrm{H}_{2}: \mathrm{CO}_{2}(80: 20)$ to approximately 1 atmosphere $(101 \mathrm{kPa})$ of pressure. CO concentrations of between 20-23 ppm were observed at this time. If the fermentation was allowed to continue and the headspace pressure maintained at 1 atmosphere, by replenishing the consumed gas with $\mathrm{N}_{2}: \mathrm{CO}_{2}(70: 30)$, the $\mathrm{CO}$ concentration dropped to below $100 \mathrm{ppb}$. This is despite the fact that the $\mathrm{N}_{2}: \mathrm{CO}_{2}$ gas contained approximately $500 \mathrm{ppb}$ of $\mathrm{CO}$. Hydrogen was still present at concentrations of approximately $800 \mathrm{ppm}$ at this time.

The observation that $C O$ concentrations from a hydrogen grown methanogen equilibrated at much lower levels than observed in digester systems with $\mathrm{H}_{2}$ concentrations considerably above those reported for anaerobic sludge digesters, here and elsewhere (McCarty and Smith, 1986), lends some circumstantial support to the hypothesis that the $\mathrm{CO}$ concentrations observed in digester systems may be directly related to acetate catabolism.

In an effort to understand this, it was decided to investigate co production from a thermodynamic perspective.

\subsection{DEVELOPMENT OF A THERMODYNAMIC MODEL}

The catabolism of acetate by methanogens can be written as a sequence of enzymatic redox reactions. Although a complete elucidation of the exact pathways used by acetate utilizing methanogens has yet to be delineated (and in fact could be somewhat different for different species and strains), the catabolism of acetate to methane and $\mathrm{CO}_{2}$ can be conveniently divided into two half cell reactions that represent the production of $a$ bound $C O$ and its subsequent oxidation to $\mathrm{CO}_{2}$. 


$$
\begin{aligned}
& \mathrm{CH}_{3} \mathrm{COO}^{-}+3 \mathrm{H}^{+}+2 e^{-}+\mathrm{Y}-\mathrm{CH}_{4}+\mathrm{CO}-\mathrm{Y}+\mathrm{H}_{2} \mathrm{O} \\
& \mathrm{CO}-\mathrm{Y}+\mathrm{H}_{2} \mathrm{O}-\mathrm{CO}_{2}+\mathrm{Y}+2 \mathrm{H}^{+}+2 e^{-}
\end{aligned}
$$

Where $Y$ and $C O-Y$ represent the unknown carbonyl carrier and the carrier with a bound $\mathrm{CO}$ attached, respectively.

Several steps not explicitly reflected in these equations but used to develop the sequence of reactions include: ( $i$ ) acetate is first activated and then split into bound methyl and bound carbon monoxide moieties; (ii) once split, the methyl group is bound to a yet unknown carrier that is not CoM but subsequently transfers the methyl group to CoM; (iii) reducing equivalents for the reduction of methyl-CoM to methane are supplied by the oxidation of the bound Co moiety either through $\mathrm{H}_{2}$ produced or a membrane bound electron transport chain that has a redox state in equilibrium with the $\mathrm{H}_{2}$ partial pressure of the system; and (iv) ATP for the activation of acetate is generated by a chemosmotic (electron transport) process via a membrane bound ATPase or a similar enzymatic reaction. It is possible to manipulate these equations in a fashion that allows a thermodynamic evaluation of the role of $\mathrm{CO}$ in acetate metabolism.

By further assuming that the bound $\mathrm{CO}(\mathrm{CO}-\mathrm{Y})$ equilibrates with the gaseous $\mathrm{CO}$ concentration, allows substitution of $\mathrm{CO}$ in equations (2) and (3) and yields two half cell reactions to which free energy values can be assigned to all substrates and products:

$$
\begin{aligned}
& \mathrm{CH}_{3} \mathrm{COO}^{-}+3 \mathrm{H}^{+}+2 \mathrm{e}^{-}-\mathrm{CH}_{4}+\mathrm{CO}+\mathrm{H}_{2} \mathrm{O} \\
& \mathrm{CO}+\mathrm{H}_{2} \mathrm{O}-\mathrm{CO}_{2}+2 \mathrm{H}^{+}+2 \mathrm{e}^{-}
\end{aligned}
$$

Further, assuming that the two half cells are in some type of dynamic equilibrium with the $\mathrm{H}_{2} / \mathrm{H}^{+}$redox couple allows the two reactions to be rewritten in a form that allows measurement of all reactants and products of both reactions. Free energy values were calculated according to Thauer et al. (1977). 


$$
\begin{array}{cc} 
& \begin{array}{c}
\Delta \mathrm{G}^{\prime} \\
\mathrm{KJ} / \mathrm{mole}
\end{array} \\
\mathrm{CH}_{3} \mathrm{COO}^{-}+\mathrm{H}^{+}+\mathrm{H}_{2} \rightarrow \mathrm{CH}_{4}+\mathrm{CO}+\mathrm{H}_{2} \mathrm{O} & -15.8 \\
\mathrm{CO}+\mathrm{H}_{2} \mathrm{O}-\mathrm{CO}_{2}+\mathrm{H}_{2} & -20.0
\end{array}
$$

The effect of acetate and methane on $\mathrm{CO}$ concentrations previously observed can be investigated using these equations in their Nernst form. For a pH of 7.0 and $35^{\circ} \mathrm{C}$ these equations $[(6)$ and (7) $]$ reduce to:

$$
\begin{aligned}
& \Delta G_{8}^{\prime}=-15.8+5.9 \log \left(\left[\mathrm{CH}_{4}\right][\mathrm{CO}] /\left[\mathrm{CH}_{3} \mathrm{COO}^{-}\right]\left[\mathrm{H}_{2}\right]\right) \\
& \Delta G_{9}^{\prime}=-20+5.9 \log \left(\left[\mathrm{CO}_{2}\right]\left[\mathrm{H}_{2}\right] /[\mathrm{CO}]\right)
\end{aligned}
$$

These equations can be rearranged to estimate $c 0$ production and its subsequent oxidation to $\mathrm{CO}_{2}$ using the other system parameters.

\subsection{EVALUATION OF THE THERMODYNAMIC RELATIONSHIP USING BATCH ASSAYS \\ 8.4.1 Digester Steady State Results}

The 10- and 20-day HRT mesophilic digesters were monitored daily over two months while steady state operation was maintained. Gas analyses performed at the end of a 24-hour fill and draw feeding schedule for the 10-day HRT mesophilic digester are presented together with results from a 10-day HRT thermophilic digester operated under identical hydraulic and organic loading rates in Table 8-2. Assuming that acetate concentrations were between 1.0 and $2.0 \mathrm{mM}$ (typical for anaerobic digesters and in agreement with the total volatile fatty acid concentrations of between 60 and $120 \mathrm{mg} / \mathrm{L}$ measured during this period) and substituting these values into equations (9) and (10) allows calculation of free energy as a function of $\mathrm{CO}$ concentration. The $\mathrm{CO}$ production and $\mathrm{CO}$ oxidation lines were plotted together for mesophilic conditions along with the system operating point (Figure 8-2). It was observed for the mesophilic system that measured $C O$ fell approximately at the point where the two lines intersected or the point where the free energy available for $C O$ production and oxidation was 


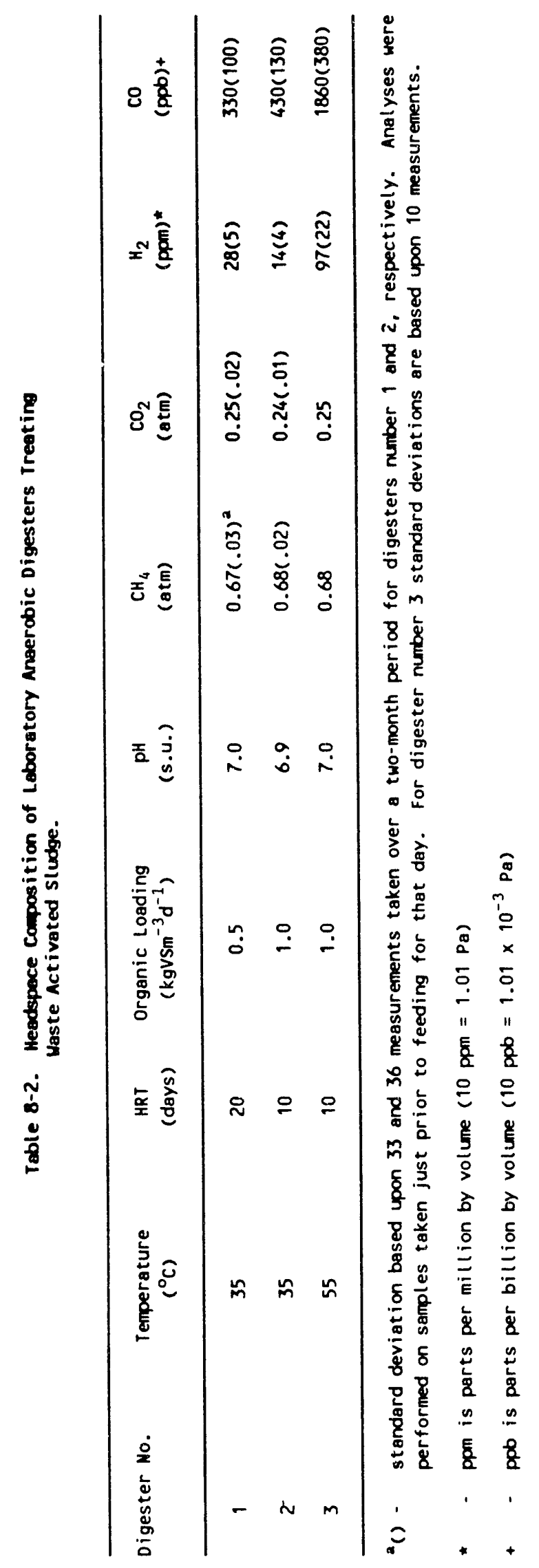




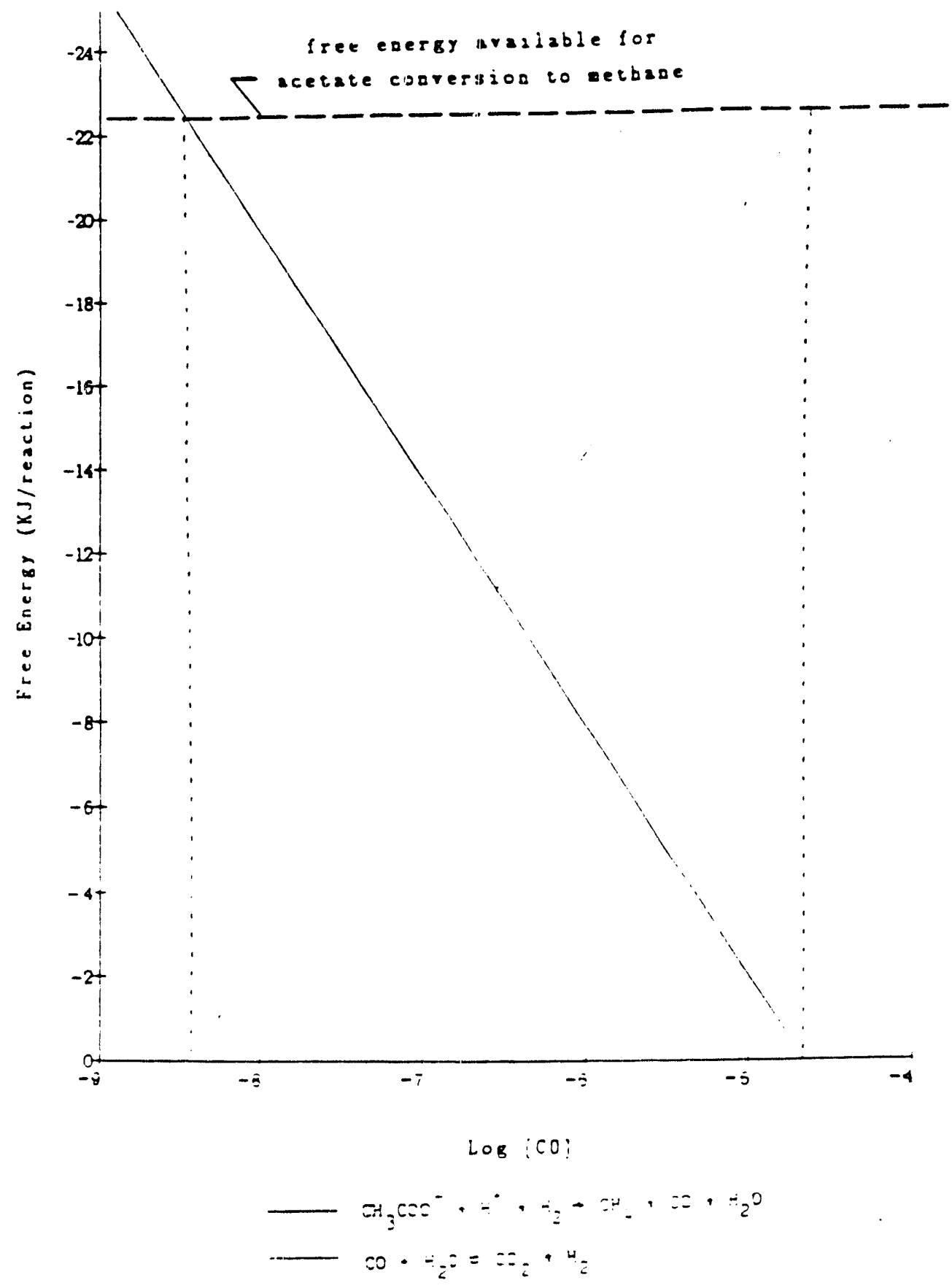

Figure 8-2. Free energy evaluation for production and oxidation of carbon monoxide during methanogenesis from acetate under mesophilic conditions. 
approximately equal. Although the absolute values for $\mathrm{CO}$ and $\mathrm{H}_{2}$ were considerably higher for the thermophilic system, the same trend of measured gaseous (free) co concentration equilibrating the point where free energy was approximately equal for the production and consumption of $C O$ was evident. Free energies were calculated assuming an activity of 1.0 and without making any corrections for effects from changes in entropy. Intersection of the equations (9) and (10) with $0.0 \mathrm{KJ}$ bounds the range of co values possible given the environmental conditions in the digesters and assuming there are no other reactions coupled to the equations. Many possible steps such as chemosmotic ATP generation are implicitly included in equations ( 9 ) and (10) and, therefore, do not affect the calculations. In an effort to further delineate the possible reasons for the observation that $C O$ concentrations equilibrated at the point where approximately the same amount of free energy was available for production and consumption, other data sets where all the parameters needed to compute the free energies associated with $C O$ production and oxidation were available and were examined.

\subsubsection{Serum Bottle Assays}

The next test of this observed trend was to review data collected from serum bottle assays previously performed during which experimental conditions were controlled to maximize the potential for samples attaining equilibrium between the baseous and liquid phases. For each observation of gas composition and acetate concentration, the free energy available for $C O$ production and oxidation was computed using equations (9) and (10).

The free energy available for oxidation of $\mathrm{CO}$ and $\mathrm{CO}_{2}$ averaged approximately 54 percent (53-56) of the overall free energy available for acetate conversion to methane and $\mathrm{CO}_{2}$ (Table 8-1). It is worthwhile to note that over the course of these experimental runs, the methane content of the sample headspaces varied from 0.01 atmospheres for the initial measurement to 0.063 atmospheres for the final measurement. Despite changing methane and acetate concentrations, especially methane where the highest content was an order of magnitude below the level observed in the digester systems, the relative energy split between the two half reactions remained essentially constant. 
By using equations (9) and (10) and based on empirical observations, (1.e., assuming the free energy associated with $C O$ oxidation to carbon dioxide is equal to 54 percent of the overall free energy available), it is possible to develop a predictive equation for equilibrium acetate concentration that requires information about the headspace composition only. This equation, presented below, can be tested by examining results from other systems.

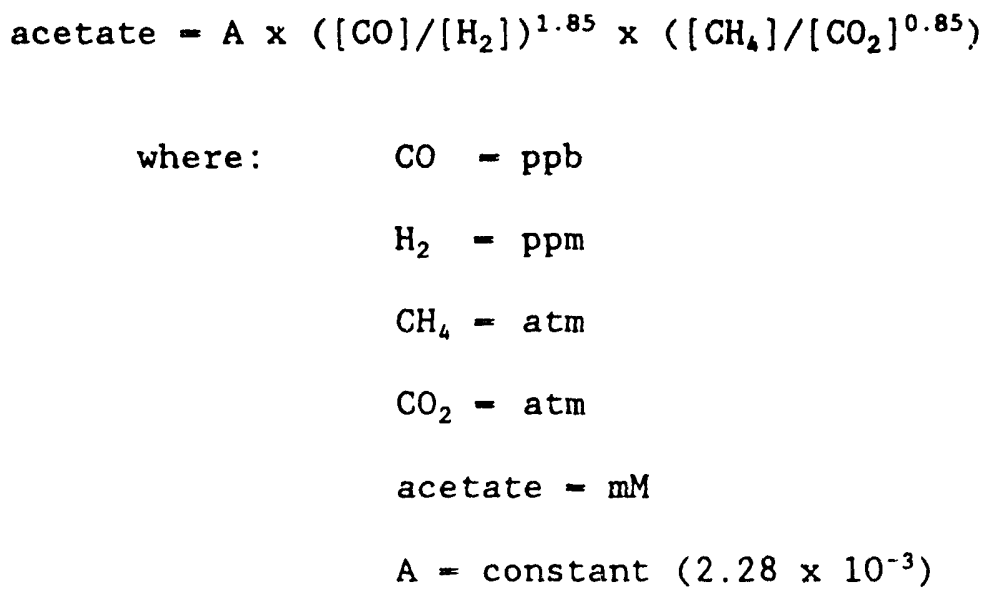

Equation (11) accounts for the direct relationship between acetate and $C O$ and the inverse relationship between $C O$ and methane that has been previously observed. If this relationship is valid, however, it should be possible to alter the concentration of hydrogen or $\mathrm{CO}$ by varying the concentration of the other.

\subsubsection{Effect of Altering Methane and Hydrogen Concentrations}

The influence of methane concentration on $\mathrm{CO}$ was examined near the conclusion of an experimental run using a culture, enriched for acetate utilizing methanogens, that was fed $40 \mathrm{mM}$ acetate. At the times indicated (Figure 8-3), the headspace was purged with $\mathrm{N}_{2}: \mathrm{CO}_{2}(70: 30)$ to remove methane from the headspace. $\mathrm{H}_{2}$ and $\mathrm{CO}$ levels were simultaneous considerably reduced to levels in the $\mathrm{N}_{2}: \mathrm{CO}_{2}$ gas (less than $1 \mathrm{ppm}$ and $500 \mathrm{ppb}$, respectively), while $\mathrm{CO}_{2}$ was slightly reduced from the mid-thirties to 30 percent. Hydrogen quickly returned to the same apparent equilibrium value, approximately $20 \mathrm{ppm}$, previously attained while the $C O$ concentration rose to a level higher than observed just prior to replacement of the headspace. 


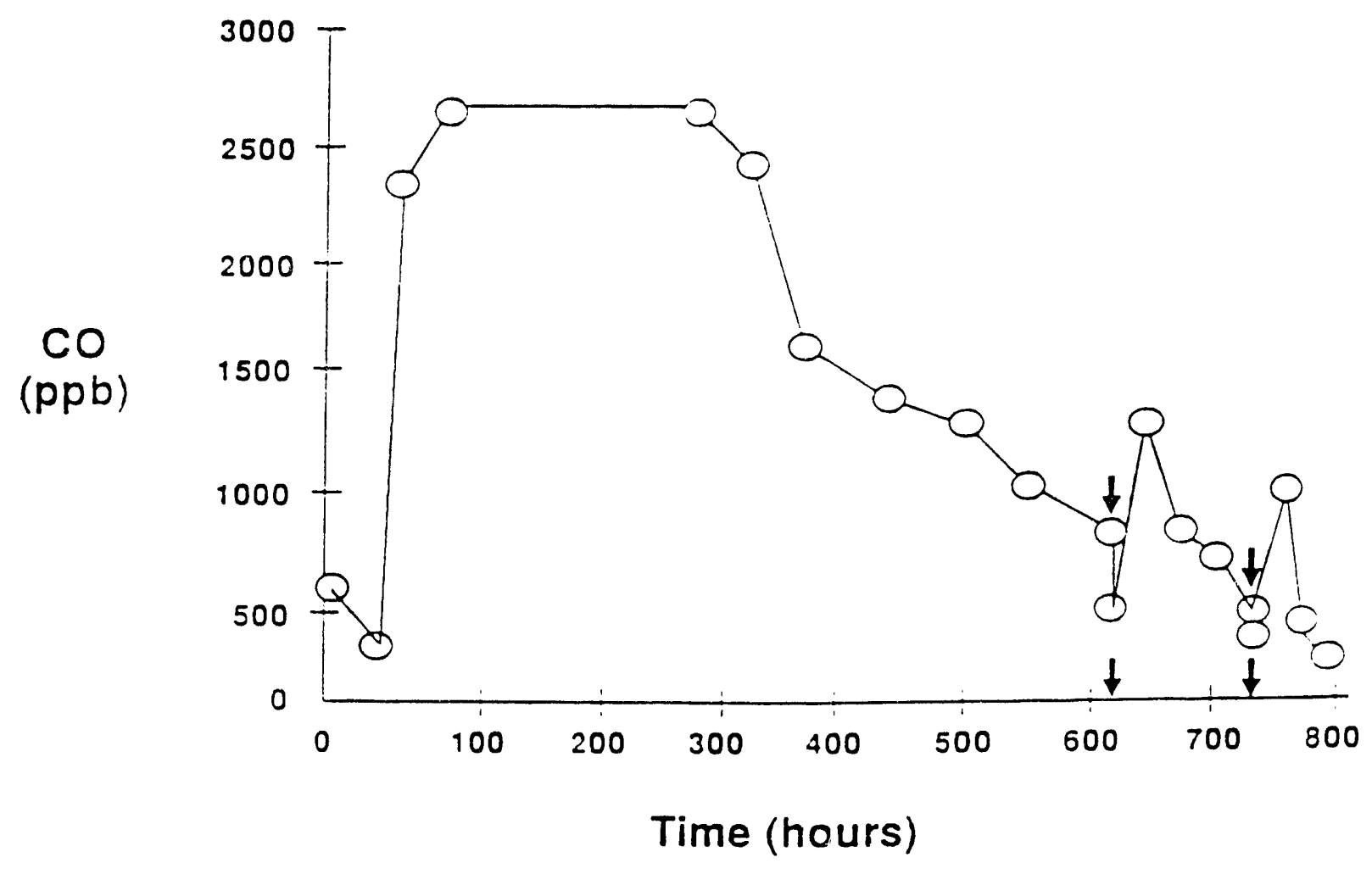

Figure 8-3. Effect of decreasing headspace methane content on the carbon monoxide concentrations of acetate fed digester inocula. At the times indicated by the arrows the headspace was replaced with a nitrogen/carbon dioxide mixture (70:30). 


\subsubsection{Effect of Supplementing Acetate}

Batch assays were performed to determine if there was a relationship between acetate and $C O$ concentration. Serum bottle digesters were inoculated with digester sludge from a 20 -day HRT reactor and fed WAS. Different concentrations of acetate (up to $20 \mathrm{mM}$ ) were pulsed at times ranging from 0 - to 8.5 hours after the WAS was added. In all cases, CO displayed a rapid rise to concentrations well above control. sample levels (Figure 8-4). There was little observed difference in the rate of methane production measured in samples pulsed with acetate compared to WAS-fed controls until late in the assay, greater than 12 hour incubation, when the rate of both total gas and methane production declined in the control samples as a result of substrate depletion. It was also noted that acetate pulsing was always accompanied by a concurrent slight depression in the gaseous concentrations of $\mathrm{H}_{2}$ compared to control samples.

\subsection{EVALUATION OF THE THERMODYNAMIC RELATIONSHIP USING DATA FROM THE ON-LINE DATA ACQUISITION SYSTEM}

A true test of the utility of the developed thermodynamic relationship was provided by examining the data of digester's subject to an organic overload, hydraulic overload and heavy metal inhibition.

\subsubsection{Organic Overload}

In this situation, headspace concentrations of methane, carbon dioxide, hydrogen and $C O$, measured on a 15 -minute interval basis, were used to calculate equilibrium acetate concentrations. The calculated equilibrium acetate and actual measured values are presented in Figure 8-5. Although there is some obvious difference between the calculated equilibrium acetate values and actual measured acetate concentrations, the trends of both are remarkably similar. It appears that the thermodynamic relationship can predict the actual acetate concentration of the system quite well despite diurnal and longer term variations in the headspace methane, $\mathrm{CO}_{2}, \mathrm{CO}$ and $\mathrm{H}_{2}$ values. It should be kept in mind that the acetate levels demonstrated a continual rise over the entire experimental run. 


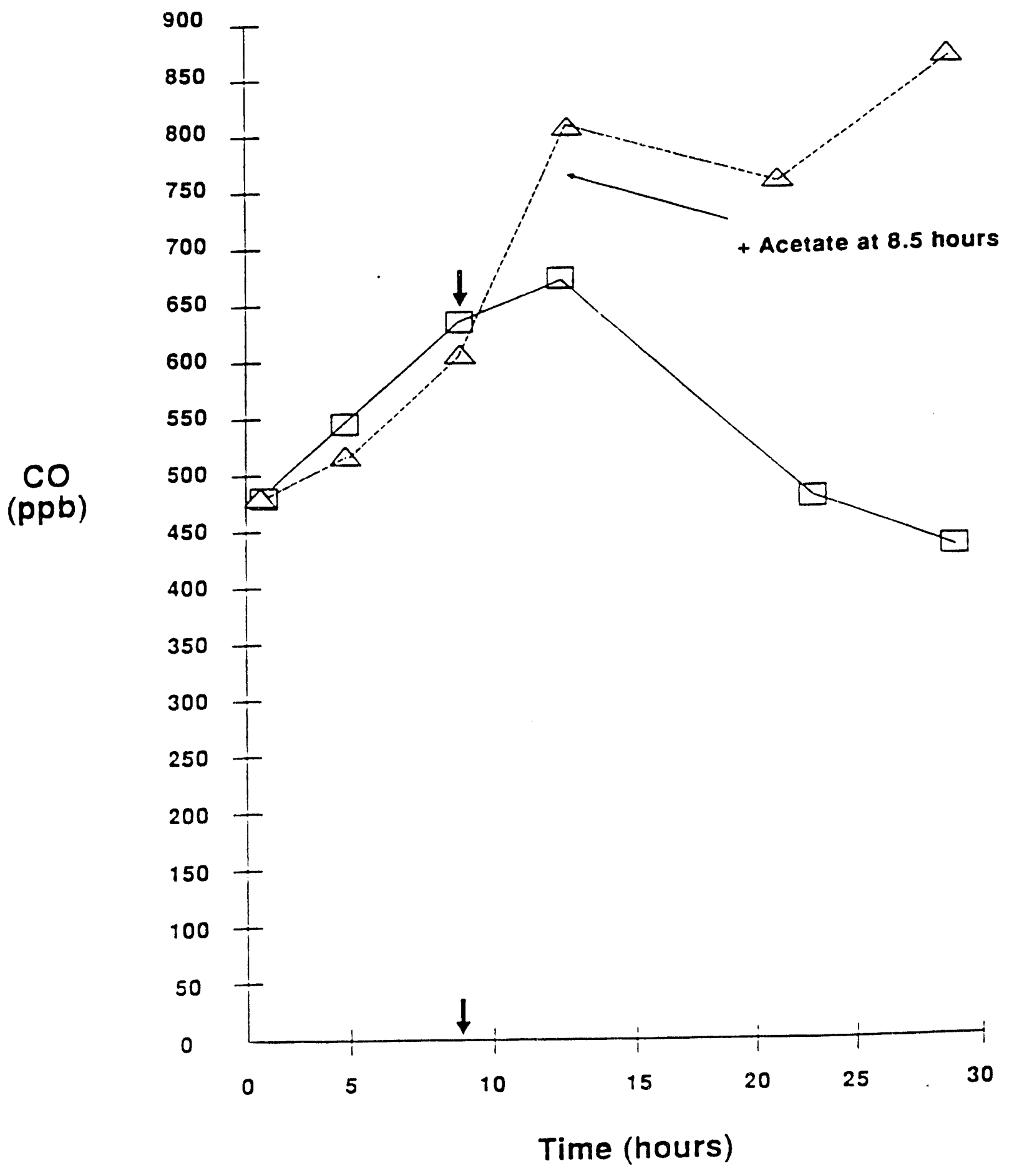

Figure 8-4. Effect of pulsing $10 \mathrm{mM}$ acetate, after 8.5 hours incubation, on $C O$ concentrations compared to controls receiving no added acetate for mesophilic digester inoculum fed waste activated sludge. 


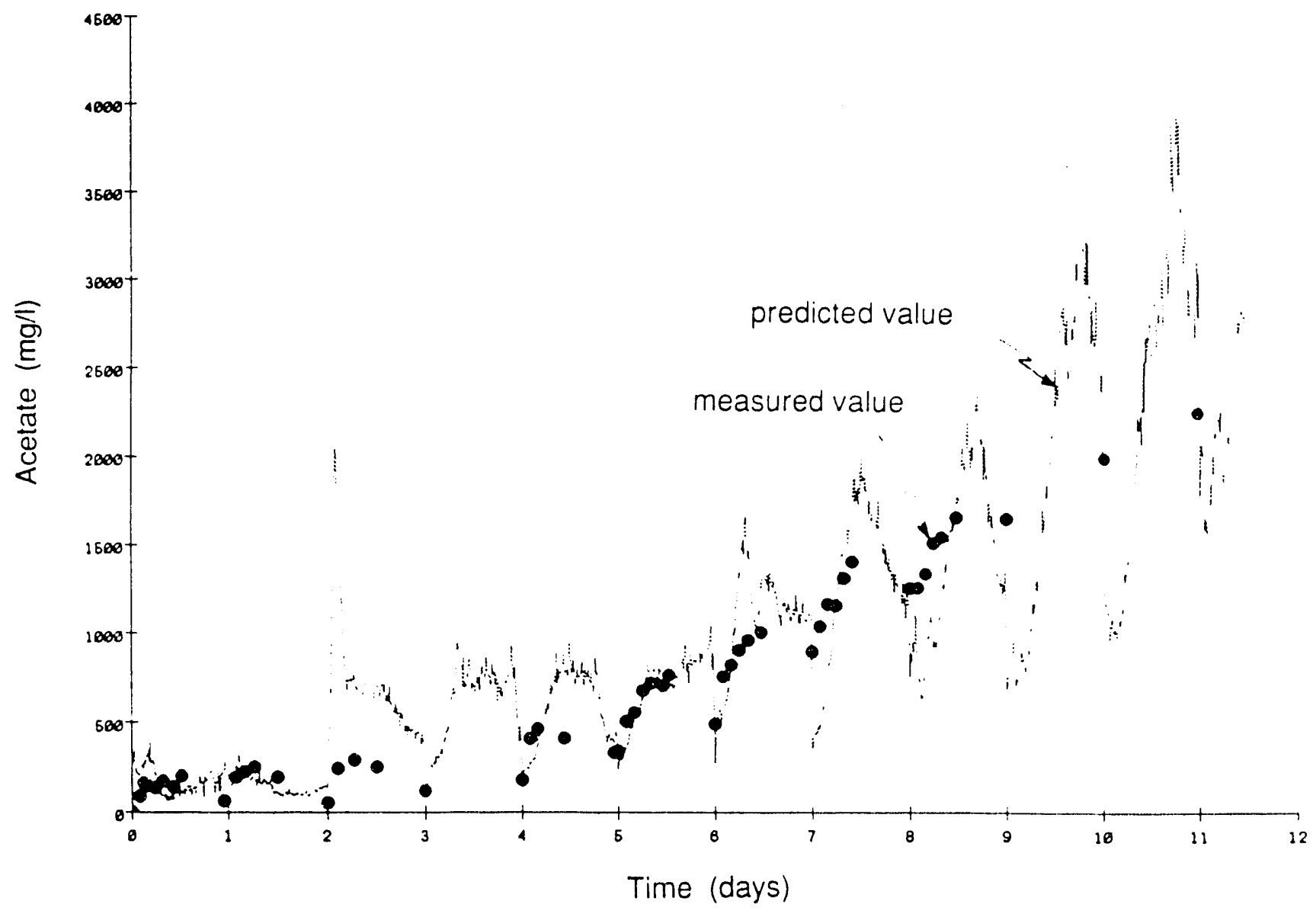

Figure 8-5. Comparison of measured and predicted acetate values for a batch daily fed 5 -liter mesophilic digester subjected to a 2.65 times organic overload. 


\subsubsection{Hydraulic Overload}

Data from the two times hydraulic overload experiment highlights one of the caveats of this thermodynamic relationship. Days 2 and 3 of the experiment show the drawback of using thermodynamics to predict the behavior of dynamic system (Figure 8-6). Fundamental to the calculation of the equilibrium acetate concentration is the ratio of $C O$ to hydrogen (equation (11)). As can be seen in Figure 5-1b, hydrogen rose rapidly for the first several hours and then declined by between 4 and 6 hours. Co by contrast rose for the first 12-14 hours and then declined (Figure 5-1b). Acetate concentrations increased much like $\mathrm{CO}$, peaking at about 10 hours and then declining to starting levels by the end of a 24 -hour cycle.

The more dynamic behavior of $\mathrm{H}_{2}$ results in some error in the calculated equilibrium acetate concentrations. Calculated acetate levels actually decreased during the first several hours due to the rise in $\mathrm{H}_{2}$ which decreases the $\mathrm{CO} / \mathrm{H}_{2}$ ratio. When the hydrogen concentration levelled off, the actual and calculated acetate values approach one another. Finally, when hydrogen decreases, the calculated acetate values overshoot the real values. By the conclusion of a 24 hour cycle, hydrogen values stabilized, but CO concentrations were still slowly decreasing, presumably the result of a slow uptake and washout of $C O$. At this point in time it is probable that $C O$ values are higher than the actual equilibrium value. This results in the $\mathrm{CO} / \mathrm{H}_{2}$ ratio being artificially high and consequently predicted acetate concentrations being high. The further a system is moved from steady state, the more severe this shortcoming becomes.

\subsubsection{Copper Induced Inhibition}

During the initial days of this experiment, when little or no inhibition was evident (Day 1 to Day 2), the same phenomenon of calculated acetate values lagging actual concentrations is evident. Once acetate values began to increase above normal levels, the match between calculated and actual values improved considerably (Figure 8-7). When the level of inhibition of the hydrogen consuming population reached the point where $\mathrm{H}_{2}$ levels rose to extremely high levels and CO levels concurrently rose (due to an as yet undetermined reason), the relationship ceases to accurately describe the behavior of the system. 


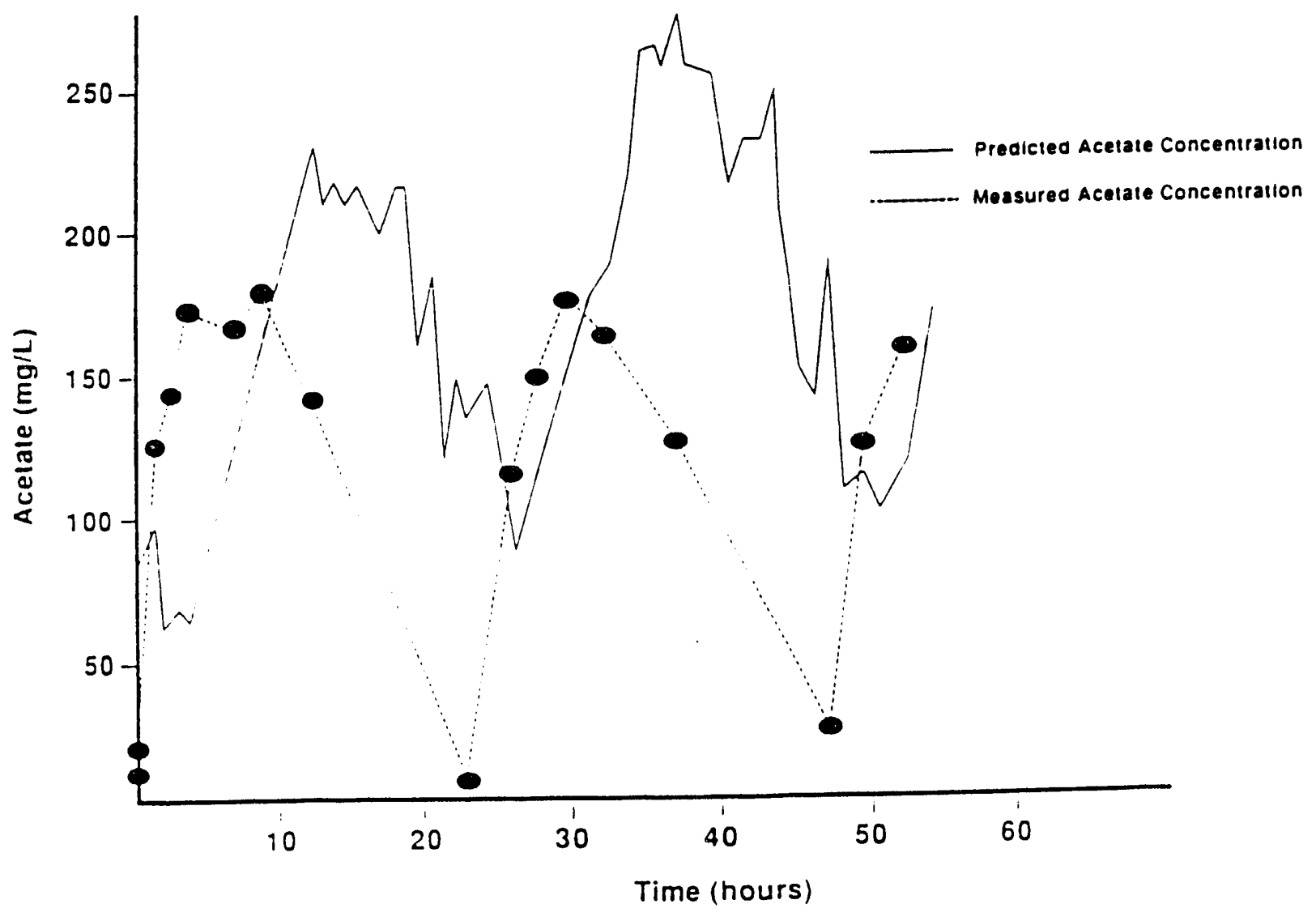

Figure 8-6. Comparison of predicted and measured acetate concentrations during the start of a two-fold increase in hydraulic loading applied to a 10-day HRT digester. 


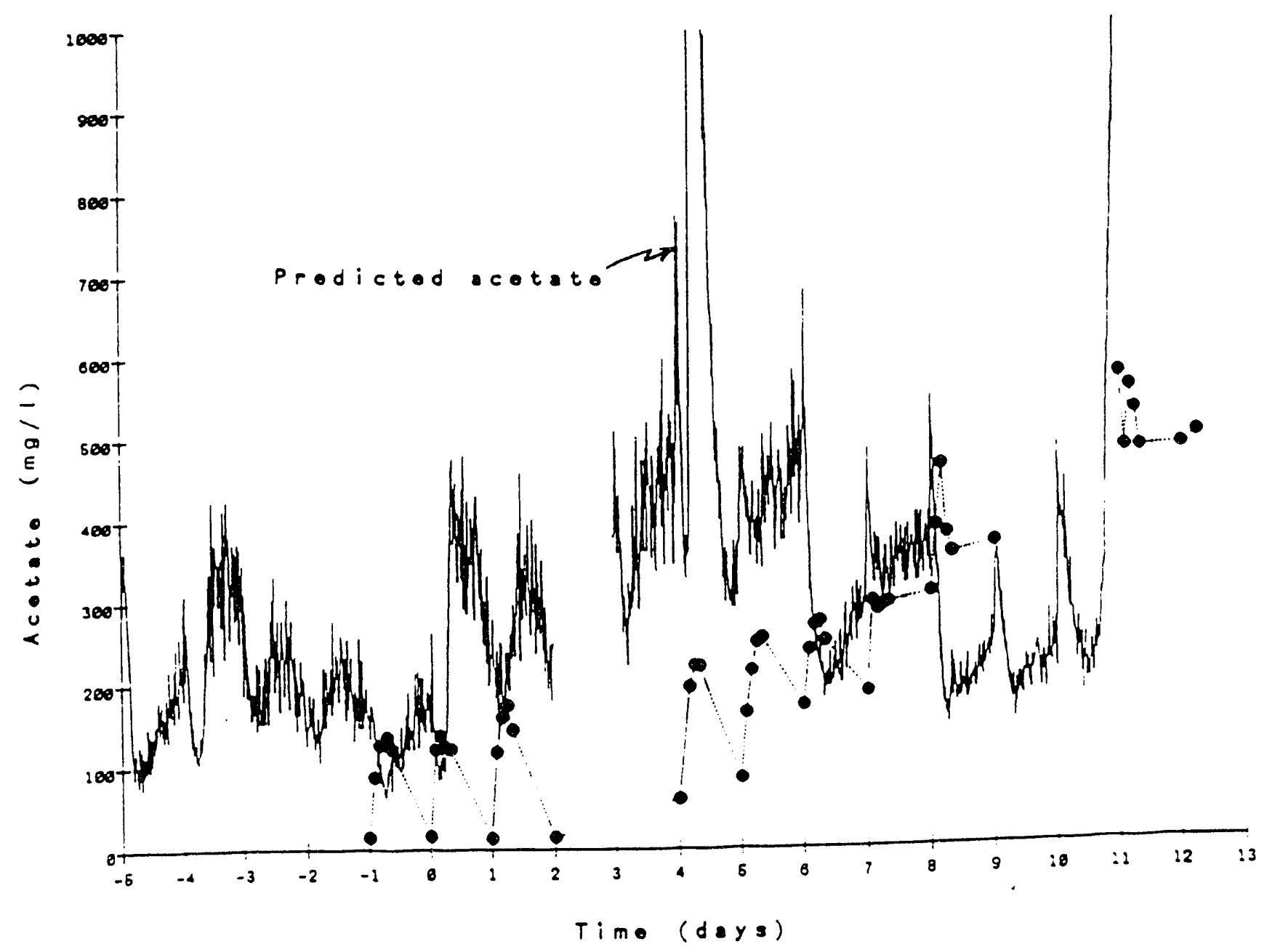

Figure 8-7. Comparison of the predicted and measured acetate concentrations
during the initial 12 days of chronic addition of copper to a
20-day HRT digester. 
Despite the shortcomings of using a thermodynamically based relationship to describe a dynamic system, the results are quite good and, if anything, acetate values calculated by this relationship are conservative in nature. There is one potential major interference with the use of this relationship that has been identified and is discussed below.

\subsection{EFFECT OF OXYGEN ON CO CONCENTRATIONS}

\subsubsection{Response of a Mesophilic Anaerobic Digester to Exposure to Air}

On several occasions when laboratory digesters were inadvertently exposed to relatively large quantities of air, the concentration of carbon monoxide in the gaseous headspace of the affected digesters dramatically increased. The results of one event for a 20-day HRT mesophilic digester are presented in Table 8-3. The introduction of air occurred overnight between 0ctober 6 and 7 and was due to the rupture of tubing on a mixing pump. The sharp decline in methane and carbon dioxide is believed to be the result of dilution with air. Under this assumption, approximately one-half the headspace can be estimated to be air. The oxygen concentration would, therefore, be on the order of 10 percent. Gas production as well as methane and carbon dioxide levels returned to normal within one day.

Carbon monoxide levels rose over 17 fold above the average observed concentrations for the preceding week due to the introduction of oxygen into the system. It took two days for $C O$ levels to return to normal baseline levels. While this was occurring, $\mathrm{H}_{2}$ levels rose to over two times the normal baseline and remained elevated for at least 3 days.

A more detailed picture of the response that $O_{2}$ produced on $C 0$ levels was obtained from a serum bottle assay and is presented in Table 8-4. After CO and $\mathrm{H}_{2}$ levels peaked ( 6 hours), $5 \mathrm{ml}$ of air was injected into the headspace of one of four samples. As can be seen, the introduction of approximately 1.7 percent $\mathrm{O}_{2}$ in the well-mixed samples caused a simultaneous rapid increase in $\mathrm{CO}$ and decrease in $\mathrm{H}_{2}$. Within 14 hours both gaseous components had returned to near normal levels. 
Table 8-3. Response of a 20-day HRT Mesophilic Digester to Inadvertent Exposure to Alr.

\begin{tabular}{lllll} 
Date & $\begin{array}{c}\mathrm{H}_{2} \\
(\mathrm{ppm})\end{array}$ & $\begin{array}{c}\mathrm{CO} \\
(\mathrm{ppb})\end{array}$ & $\begin{array}{c}\mathrm{CH}_{4} \\
(8)\end{array}$ & $\begin{array}{c}\mathrm{CO}_{2} \\
(8)\end{array}$ \\
\hline $9 / 30 / 85$ & 33 & 480 & $\cdots$ & $\cdots$ \\
$10 / 1$ & 38 & 530 & 69 & 20 \\
$10 / 2$ & 26 & 450 & 69 & 24 \\
$10 / 3$ & 28 & 480 & 67 & 21 \\
$10 / 7$ & 31 & & 33 & 11 \\
$10 / 8$ & 78 & 8340 & 65 & 19 \\
$10 / 9$ & 70 & 1310 & 66 & 24 \\
$10 / 10$ & 66 & 400 & 65 & 23 \\
$10 / 15$ & 500 & & 68 & 24 \\
$10 / 16$ & 38 & 300 & 68 & 26 \\
\hline
\end{tabular}


Table 8-4. Effect of Oxygen Addition to a 20-day HRT Inoculum on $\mathrm{CO}$ and $\mathrm{H}_{2}$ Concentrations.

\begin{tabular}{lrrrr} 
& \multicolumn{2}{c}{$\mathrm{CO}_{2}(\mathrm{ppb})$} & \multicolumn{2}{c}{$\mathrm{H}_{2}(\mathrm{ppm})$} \\
\cline { 2 - 5 } Time & $+\mathrm{O}_{2}$ & $-\mathrm{O}_{2}$ & $+_{2}$ & $-\mathrm{O}_{2}$ \\
\hline 300 & 970 & 1030 & 101 & 104 \\
410 & 1740 & 1030 & 76 & 104 \\
440 & 1970 & 1070 & 64 & 102 \\
1500 & 2260 & 1070 & 53 & 97 \\
& 1810 & 490 & 49 & 41 \\
\hline
\end{tabular}

$\mathrm{O}_{2}$ added at 370 minutes 


\subsubsection{Effect of Oxygen on a Thermophillc Digester}

When oxygen was inadvertently introduced into a thermophilic digester during feeding, the effect on $\mathrm{CO}$ and $\mathrm{H}_{2}$ headspace concentrations was followed at approximately 15 minute intervals. In the thermophilic system, co rose to close to $20 \mathrm{ppm}$ betore gradually returning to the baseline of approximately $1.2 \mathrm{ppm}$ (1200 ppb). Hydrogen levels declined concurrently with the rise in CO, in contrast to the normal observed diurnal hydrogen pattern of a slight rise in $\mathrm{H}_{2}$ following feeding. The hydrogen concentration eventually rose after co levels began to decline from the peak concentrations observed. The hydrogen concentration after approximately 700 minutes was observed to decrease to $50 \mathrm{ppm}$, well below the normal observed baseline value of 80-100 ppm for the thermophilic reactor.

\subsubsection{Response of a Hydrogen Grown Methanogen to Oxygen}

A culture of $\underline{M}$. barkeri 227 grown on $\mathrm{H}_{2} / \mathrm{CO}_{2}$ was injected with $5 \mathrm{~m} 1$ of air, the equivalent to approximately 1.08 oxygen in the headspace. The starting $\mathrm{H}_{2}$ concentration was $800 \mathrm{ppm}$, the measured threshold level for this bacterium below which consumption was not observed to occur. Almost immediately upon agitation, the resazsurin redox indicator turned dark pink indicating the redox potential had risen to above $-40 \mathrm{mV}$. Co levels quickly rose from $400 \mathrm{ppb}$ to over $6800 \mathrm{ppb}$ within 90 minutes (Figure 8-8). After, between 60 and 90 minutes, the redox indicator returned to being colorless, indicating the redox potential was $-40 \mathrm{mV}$ or lower (Hungate, 1966). The co concentration subsequently declined at a rate nearly equal to the rise previously observed, decreasing to $200 \mathrm{ppb}$ by the 400 minutes. During the period when $\mathrm{CO}$ levels were declining, the $\mathrm{H}_{2}$ level was observed to also drop precipitously, reaching 3 ppm by 400 minutes and ultimately falling below $1 \mathrm{ppm}$ by 1400 minutes. The culture was then regassed with $\mathrm{H}_{2} / \mathrm{CO}_{2}$ and methane production (rate not measured) resumed. Once the headspace pressure dropped below one atmosphere $(101 \mathrm{KPa})$, the pressure in the vial was returned to 1 atmosphere by allowing the headspace to equilibrate with a $50 \mathrm{ml}$ ground glass syringe filled with $\mathrm{N}_{2}: \mathrm{CO}_{2}(70: 30)$. After three days incubation, an $\mathrm{H}_{2}$ level of $820 \mathrm{ppm}$ (approximately the threshold value) was measured. 


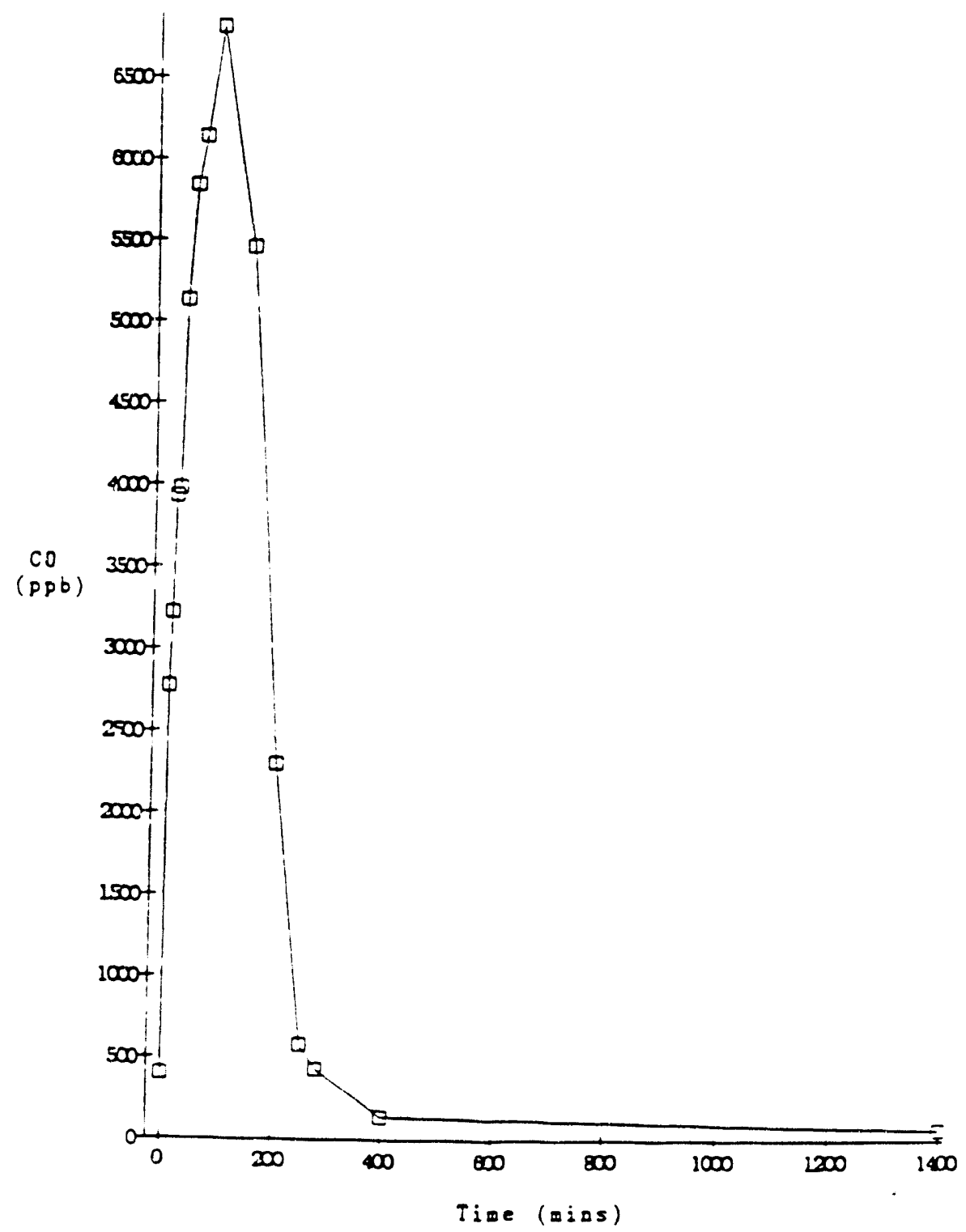

Figure 8-8. Response of carbon monoxide concentrations of a hydrogen grown culture of $M$. barkeri to addition of 1.0 percent oxygen to the headspace. 


\subsection{DISCUSSION AND SUMAYAY OF RESULTS}

A thermodynamic framework is presented that explains acetate metabolism by methanogens from a redox perspective. As more knowledge concerning the biochemistry is acquired, modification and extension of the relationship will undoubiedly be required. This relationship provides additional insight into the role of $\mathrm{CO}$ as an internal control parameter in acetate metabolism of methanogens. Rigorous evidence from pure culture assays is not presented, but there are no discrepancies between this relationship and the current knowledge concerning acetoclastic methanogenesis. In fact, the model offers plausible explanations for some unexplained observations in the 1iterature. Considerably more work with more defined systems will, however, be necessary to provide complete validation of the relationship.

Certain assumptions had to be made due to incomplete knowledge of the pathway of acetate catabolism by methanogens. The key assumptions in the analysis are that hydrogen and carbon monoxide are integrally related in methanogenesis from acetate and that the $C O$ concentration is controlled by the acetate catabolizing reactions of acetoclastic methanogens. In addition, prior work performed with pure cultures supports the validity of equations (9) and (10). The reverse of equation (10) has been proposed to occur curing fixation of $\mathrm{CO}_{2}$ by Methanobacterium thermoautotrophicum. Eikmanns et a1. (1985) observed that trace levels of $C O$ were produced by $\underline{M}$ thermoautotrophicum during growth on $\mathrm{H}_{2} / \mathrm{CO}_{2}$. The oxidation of $\mathrm{CO}$ by methanogens and acetogens possessing $\mathrm{CO}$ dehydrogenase activity produces $\mathrm{CO}_{2}$ and $\mathrm{H}_{2}$ (Diekert et a1., 1986). Acetobacterium woodii catalyzes fixation of $\mathrm{CO}_{2}$ and oxidation of $\mathrm{CO}$ by the action of CO-dehydrogenase enzyme activity (Diekext et al., 1986). Reported production of $\mathrm{CO}_{2}$ and $\mathrm{H}_{2}$ from $\mathrm{CO}$ in stoichiometric amounts by cell suspensions of acetate grown M. barkeri (Bott et al., 1986) and the observation that the COdehydrogenase of $\underline{M}$. barkeri catalyzed an exchange between $\mathrm{CO}_{2}$ and the carboxyl of acetate (Eikmanns and Thauer, 1984) further supports the validity of equation (10). 
The observation that increasing acetate levels resulted in increased co levels and that increasing methane concentrations caused a decrease in the observed $c 0$ levels lends support to validate equation (9).

Ragsdale and Wood (1985) reported that the CO-dehydrogenase of Clostridium thermoautotrophicum was responsible for not only catalyzing the fixation of $\mathrm{CO}_{2}$ to a bound $C O$, but was integral to the subsequent condensation of the bound $C O$ and a bound methyl with CoA to form acetyl-CoA. A reversal of this role for the CO-dehydrogenase enzyme of SRBs has been hypothesized (Schauder et al., 1986). They proposed that $C O$-dehydrogenase catalyzes the splitting of acetate into bound $\mathrm{CO}$ and methyl moieties and as well as catalyzing the oxidation of the bound $\mathrm{CO}$ to $\mathrm{CO}_{2}$. Wood et al. (1986), further speculated that the CO-dehydrogenase of acetate utilizing methanogens functions in both the cleavage of an activated form of acetate to bound $\mathrm{CO}$ and methyl groups as well as the subsequent oxidation of the bound $\mathrm{CO}$, the two reactions are not isolated and only one operating value of Y-CO and, therefore, $C O$ is possible. The observation that $C O$ is controlled at a concentration approximately equal to the point where the free energy for the oxidation of $C O$ is equal to approximately 54 percent of the overall energy available for acetate conversion to methane and carbon dioxide is surprising. This is almost equal to the free energy split at standard conditions for these two equations ( 55.6 percent). There is no apparent reason for this phenomena; however, it appears consistent and reproducible.

$\mathrm{CO}$ formation from $\mathrm{CO}_{2}$ has been linked to membrane energy producing reactions in both methanogens and acetogens. Interestingly, the acetogen, Acetobacterium woodii could also oxidize $\mathrm{CO}$ to $\mathrm{CO}_{2}$ in a reaction that was coupled to ATP generation. Schemes for chemiosotic (electron transport) energy generation for acetate utilizing methanogens based on $C O$ oxidation have been proposed (Krzycki et al., 1987). Bott et al. (1986) demonstrated a coupling of CO oxidation and ATP production for $\underline{M}$. barkeri, consistent with results presented.

Several researchers have observed that the concentration of $C O$ produced during CO-dehydrogenase mediated fixation of $\mathrm{CO}_{2}$ was well above that thermodynamically feasible according to the reverse of equation (10). The linkage of this $\mathrm{CO}_{2}$ fixation reaction to an exergonic membrane process was postulated to be the 
reason for this apparent anomaly (Eikmanns and Thaser, 1985). The development of a similar framework used herein might be useful in evaluating observed $c 0$ levels for their data from a thermodynamic perspective.

There are several caveats to applying the thermodynamic relationship. The thermodynamic relationship is by its nature an equilibrium calculation. Using the relationship on systems not close to or approaching equilibrium will likely produce erroneous results. Although the calculated acetate concentrations are presented as "predicted acetate" a better terminology might be "acetate potential" of the system at that point in time. That is the acetate concentration that would be in thermodynamic equilibrium with the measured headspace composition if the system is not kinetically or mass transfer limited.

An inherent assumption in the thermodynamic relationship is that equilibrium between the gaseous and aqueous phases exists. Experimental conditions were manipulated to foster equilibrium between the gaseous and aqueous phases in this study. In many natural ecosystems and engineered systems, this may not hold true. Even during this research there was evidence of non-equilibrium between the two phases. The uptake of hydrogen occurs much more quickly than for co. This is unlikely the result of low driving forces and low affinity for $C O$. Under normal conditions in the test digester, hydrogen concentration was observed to increase to approximately $40 \mathrm{ppm}$ during the initial 3 to 4 hours after feeding and then decline back to an apparent baseline concentration of approximately 20 ppm (Hickey, Ph.D. Dissertation, University of Massachusetts, Amherst, 1987). The increase in hydrogen caused $C O$ levels to also rise. When hydrogen levels subsequently declined, however, CO uptake progressed much more slowly, presumably due to the low affinity for $C O$ and lower driving force for mass transfer. This resulted in an increase in the $C O$ to hydrogen ratio, which is used in calculating equilibrium acetate concentration, and overestimating acetate values. In extremely dynamic systems, this could limit the utility of the thermodynamic relationship using gas monitoring. However, using techniques that allow a direct measure of dissolved gases, such as membrane inlet mass spectrometry (Whitmore et. al., 1985) would still allow use of the thermodynamic acetate calculation. Systems experiencing more even loading such as chemostats and continually fed digesters will be especially amenable to using the developed relationship using 
gas monitoring. The gaseous headspace volume to liquid volume ratio was high for the test digesters and serum bottle reactors used in these experiments. In systems with a lower ratio typical of full-scale reactors, the "washout" effect of $\mathrm{CO}$ will be greater and the problem of $\mathrm{CO}$ remaining elevated and not in equilibrium with acetate, somewhat mitigated.

The effect of microenvironments within sediments, sludge flocs and biofilms also needs to be addressed (Conrad et. al., 1985). The mass transfer limitations will undoubtedly affect the observed concentrations of both hydrogen and $c 0$. Whether integration of mass transfer kinetics with the thermodynamic model will be necessary for, or in certain cases even adequate to properly describe the system of interest requires thorough investigation.

The final concern is whether $C O$ can be produced and uptaken by a number of bacterial types normally present in anaerobic consortia. Interestingly, the hydrogen using methanogens typically found in digester systems have been deficient in CO-dehydrogenase enzyme activity (Bott et. al., 1985). Although the results support the hypothesis that acetoclastic methane producing activity controlled system CO levels, this may not be true in all instances. In systems where acetate metabolism accounts for most of the methane produced such as industrial and municipal wastewater treatment systems and digesters, this is likely to be true. 


\section{SECTION 9}

\section{DISCUSSION AND CONCLUSIONS}

\subsection{DISCUSSION}

In terms of substrate and energy flow, the anaerobic digestion of sludge and other particulate substrates can be envisioned to occur as a three step process (McCarty, 1982). In Figure 9-1, a schematic representation of this three step process, $C O$ is shown to be related to acetate conversion. This is an essential consideration that was used in examining the results generated during this research. It must be emphasized that although a significant amount of evidence has been collected to support this hypothesis, it is all circumstantial in nature. Rigorous proof still needs to be obtained. For the purposes of this discussion, however, it will be assumed that co concentrations measured in anaerobic digester systems are the result of acetate conversion to methane, and monitoring the two trace gases, hydrogen and carbon monoxide, supplies information on both the major terminal reactions in anaerobic digester systems (See Figure 9-1). There is the potential to use monitoring of these gases, in concert with major gas components methane and carbon dioxide, to better control the digestion process and possibly provide an early warning of oncoming trouble situations and upsets. A working knowledge of the anaerobic degradation sequence is required to interpret the monitoring results.

There are three basic causes of anaerobic digester upset or failure; hydraulic overload, organic overload and inhibition due to the presence of toxicants.

Hydraulic overload occurs when a key organism cannot reproduce fast enough to avoid being "washed out" of the digester. Although generally associated with the slow growing acetate catabolizing methanogens, other organisms may also be sensitive to this phenomenon. Hydraulic overload can occur as a result of reduction of effective digester volume due to scum and/or grit accumulation, poor mixing, over pumping or dilute sludge (inability to adequately concentrate sludge 
FIGURE 9.1

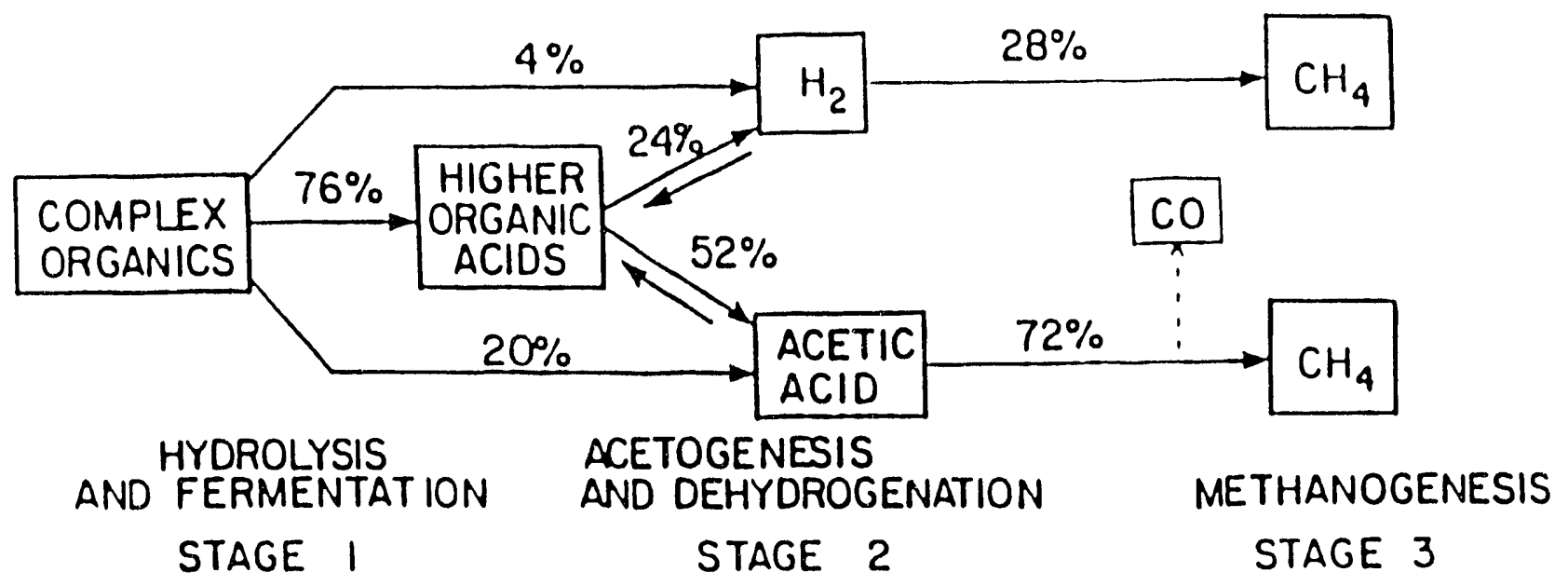

Figure 9-1. Three stage model of substrate and energy flow for the anaerobic digestion of complex organic materials. 
in the thickening procoss) or excessive sludge production. This latter case represents a combined hydraulic and organic overload.

Organic overload occurs when the substrate loading exceeds the rate at which the system micro-organisms can degrade the organic material under balanced conditions. Organic overload can result from a sudden increase in feed solids concentration or excessive loading on an infrequent basis.

Toxic inhibition occurs when organic or inorganic toxicants enter the system with the feed sludge at a concentration high enough to inhibit one or more of the major groups of organisms responsible for degrading the sludge, or the production of compounds such as ammonia or hydrogen sulfide occurs at concentrations high enough to inhibit the process.

The response of an anaerobic digester to a particular type of upset appears to revolve around where the interruption and/or backup in substrate flow occurs.

Applying an organic overload to a 10-day HRT mesophilic system caused a build-up of acetate and subsequently, higher molecular weight VFAs. This is due to the inability of the acetate catabolizing population to consume acetate as quickly as it was produced. Because these organisms are slow growing, with generation times of 3 to 9 days (Taylor, 1982), enough additional cells could not be produced to help ameliorate this situation. The build-up of acetate was reflected in the simultaneous build-up of gaseous $C O$, which increased in accordance with the thermodynamic relationship developed in Chapter 8 .

Hydrogen concentrations also rose during the first day of this overload, most likely due to the increase in amount of hydrogen produced. Because the hydrogen utilizing population normally operates at an activity level far below its potential (Kasper and Wuhrmann, 1978; Winfrey and Zeikus, 1979) and due to the fact that this population has a much faster generation time than the acetate utilizers (Taylor, 1982), the hydrogen population quickly established a new baseline level and were able to keep the hydrogen concentration at that level for the remainder of the experiment, even when by conventional indicators an upset was occurring. Evidence from solids and VFAs data indicated that hydrolysis or 
solubilization did not retard substrate flow to the extent it could be considered the rate-limiting step. The only backup or interruption in substrate flow due to the overload was at the acetate consumption step. This is why the upset is reflected in only the $C O$ and VFA data.

When a 20-day HRT system was subjected to an identical increase in organic loading, no perturbation in digester performance was observed. Acetate levels did not increase substantially and accordingly the response pattern observed for CO, before and after the organic load increase was applied, remained unchanged. It can be concluded, therefore, that monitoring $C O$ will allow detection of accumulation of acetate due to organic overloading and predict within a reasonable level the actual concentration of acetate when used in conjunction with the thermodynamic relationship.

A hydraulic overload was applied at two times the normal loading. This was insufficient to produce a response that could be considered an upset although the magnitude of the diurnal pattern of acetate accumulation and consumption was increased. This result was again clearly reflected in the co diurnal response pattern. Hydrogen gas concentrations also rose above normal steady-state levels. This moderate increase in hydrogen appears to indicate the increased level of loading or stress on this population group.

When a four-times hydraulic overload was applied to a 10-day HRT system, performance declined. A large accumulation of acetate, propionate or other VFA's did not occur, however. This is believed due to the fact that hydrolysis limited substrate flow. As would be anticipated hydrogen and $C O$ did not change appreciably.

A pulse addition of copper on a continuously operated digester at a dosage sufficient to cause a temporary but significant, i.e., 15 percent, decrease in methane production resulted in dramatic responses of both hydrogen and $C O$. The response of hydrogen was similar to that observed in prior batch studies with hydrogen initially decreasing from the normal "baseline" and then rising precipitously. The CO response was similar but even more pronounced than 
observed for batch assays. The same type of "finger print" due to pulse addition of heavy metals was present for batch and continuously operated systems.

In a digester chronically dosed with copper, the responses of these two trace gases was somewhat muted. Hydrogen never displayed the drop in concentrations observed for pulse addition, but did show a steady rise above normal operating concentrations. Gaseous CO levels rose quite early and continued to rise, albeit slowly, during copper addition. When severe inhibition occurred, co dramatically rose several orders of magnitude.

It was observed that inadvertent addition of oxygen to digester systems, particularly continuously operated systems, produced essentially the same type hydrogen and carbon monoxide response patterns as for pulse addition of heavy metals. Possible explanations are that both oxygen and copper (and possibly other heavy metals) interfere with substrate flow at the same point in the degradation sequence or interfere with some enzyme or enzymatic reaction in the same manner or change the redox of the system in a comparable manner. Whether the CO response in heavy metal-inhibited samples is related to the methanogenic population, as would appear to be the case for the samples inhibited by organic compounds, cannot be determined from the data collected during this study. The response exhibited by a digester subjected to chronic application of copper was clearly different from the response due to oxygen and other inhibitory events studied.

The thermodynamic relationship established between $C O$ and acetate applies to both batch and on-line digester systems in the absence of toxicant upsets. By simultaneously monitoring $\mathrm{H}_{2}$ and $\mathrm{CO}$, information on both of the major immediate precursors for methane production is available. Because co levels are apparently partially regulated by the $\mathrm{H}_{2}$ concentration, monitoring $\mathrm{CO}$ alone cannot be relied upon to provide early warning; both need to be monitored together to gain the fullest amount of information.

Predicted and measured $C O$ values agreed for both normal operating and stress conditions for the on-1ine digesters. Together with gas production and major gas $\left(\mathrm{CH}_{4}\right.$ and $\left.\mathrm{CO}_{2}\right)$ concentrations a more focused snapshot of digester status can be $9-5$ 
obtained than is currently possible with wet chemistry based monitoring strategies normally used. The ability to obtain a "status report" on anaerobic digesters by monitoring the headspace composition, particularly trace gases related to metabolic activity, is a tantalizing prospect that may be someday possible. A considerable level of extended research will be necessary to develop the data base and breadth of experience to determine if this will be possible.

Variations in substrate composition and minor variations in applied loading rate that will typically occur in any digester system, may have a significant effect on the hydrogen response pattern, and $c 0$ to a lesser extent, over the course of the day. It will be necessary to develop the capability of reliably differentiating these normal background variations from those being imposed by organic or hydraulic overloads and toxic inhibition before the potential of monitoring these two trace gases can be realized.

Hydrogen and carbon monoxide are not the only trace gases evolved in anaerobic ecosystems. Oremland (1984) reported that traces of ethane were produced concurrent with methane in a lake sediment. Inhibition of methane production with BES caused a simultaneous cessation in ethane production. A more complete "status report" of anaerobic ecosystems could possibly be obtained by identifying and characterizing the behavior of other trace gases evolved during normal functioning and under stress or inhibition.

Hydrogen and CO monitoring holds promise, but has been shown, in this preliminary work, to have limitations. Although a thermodynamic framework for explaining the co evolution patterns observed in digester systems has been developed, support for this is circumstantial. Rigorous validation needs to be performed as well as explanations as to why the model doesn't accurately describe the observed responses of trace gases during toxic upsets.

Oxygen has been shown to be present in many gas samples taken from digester systems (Leininger et al., 1983). Whether this is due to improper sampling and, if not, whether interference in the viability of an $\mathrm{H}_{2}$ and $\mathrm{CO}$ based monitoring system will result needs to be fully explored. 
The response of digester systems to various upset scenarios is markedly different. Because of this, it may be possible to develop a monitoring strategy based upon some pattern recognition technique that will allow detection of the onset of upsets as well as identification of the type of problem. This would lead to more timely and better focused remedial response to the problem. More research is needed to better delineate how far this concept can be advanced.

Many bacterial trophic groups in addition to methanogens possess carbon monoxide dehydrogenase enzyme activity. These other groups need to be examined to determine when and at what concentrations $C O$ is evolved from these bacteria. Experimental work aimed at defining the circumstances when these other groups may control or influence $C O$ concentrations in complex ecosystems needs to be performed.

Experimental work should also be performed using soluble substrates in CSTR and fixed-film systems. It will likely be necessary to determine how diffusion of substrate into the biofilm and the subsequent diffusion of gas back out of the biofilm affects the levels of hydrogen and carbon monoxide in the gases.

\subsection{CONCLUSIONS}

1. The concentration of gaseous $C O$ is directly related to the acetate concentration in digester systems.

2. Acetate levels can be described, in the absence of toxicants, by a thermodynamic model that is based on dividing the acetate conversion to methane reaction into two half-cell reactions describing the production and subsequent oxidation of $\mathrm{CO}$. Accordingly, the levels of methane, carbon dioxide, hydrogen and carbon monoxide can be used to estimate acetate concentration.

3. The thermodynamic model does not accurately describe acetate concentration when inorganic toxicants are added to systems at dosages sufficient to severely interfere with methane production. 
4. Both $\mathrm{H}_{2}$ and $\mathrm{CO}$ demonstrated more sensitive responses to heavy metal-induced inhibition than did conventional process indicators. Monitoring these two trace gases may allow not only an earlier indication of upset, but also fingerprint the event as a heavy metal inhibition.

5. The addition of oxygen to an anaerobic digester system produced similar response patterns in terms of $\mathrm{CO}$ and $\mathrm{H}_{2}$ as did pulse addition of heavy metals. This may impair trace gas monitoring as a method to diagnose the source of an upset, but does not reduce the efficacy of detecting upset events, as oxygen can inhibit methane production as well.

6. A reasonable estimate of the loading on a system and the stress placed on an anaerobic digester system can be obtained from monitoring $\mathrm{H}_{2}$ and $\mathrm{CO}$ under non-inhibition conditions. Information on both major precursors of methane in digester and industrial wastewater treatment systems is generated by monitoring these two gases. Hydrogen levels reflect the balance between the $\mathrm{H}_{2}$ producing and using populations, while $\mathrm{CO}$ levels reflect the pool size of an intermediate in acetate catabolism to methane and, therefore, indirectly reflects acetate concentrations.

7. Hydrogen monitoring of well buffered systems (and presumably systems employing $\mathrm{pH}$ control) does not provide any indication of overload of acetate conversion to methane, which is the most labile of the two methane producing pathways. The utility of $\mathrm{H}_{2}$ as a stand alone indicator of anaerobic system status, therefore, is minimal.

8. A considerably greater data base, using different feedstocks with variable composition and strength, needs to be collected before $\mathrm{H}_{2}$ and/or $\mathrm{CO}$ monitoring can be confidently implemented in the field. Field-durable, less sophisticated systems for measuring $\mathrm{CO}$ and $\mathrm{H}_{2}$ need to be developed before monitoring programs and strategies based on and $\mathrm{CO}$ and $\mathrm{H}_{2}$ can be reduced to practice. 


\section{SECTION 10}

\section{REFERENCES}

Archer, D.B., Hilton, M.G., Adams, P. and Weicko, H. (1986) "Hydrogen as a process control index in a pilot scale anaerobic digester," Biotechnol. Lett., 8: $197-202$.

Baresi, L., Mah, R.A., Ward, D.M. and Kaplan, I.R. (1978) "Methanogenesis from acetate: enrichment studies," Appl. Environ. Microbiol., 36:186-197.

Barnes, D., Bliss, P.J., Grauer, B. and Robins, K. (1984) "Pretreatment of high strength wastewater by an anaerobic fluidized bed process. Part II - response to organic load transients," Environ. Technol. Lett., 6:73-78.

Bott, M., Eikmanns, B. and Thauer, R.K. (1986) "Coupling of carbon monoxide oxidation and $\mathrm{CO}_{2}$ and $\mathrm{H}_{2}$ with the phosphorylation of $\mathrm{ADP}$ in acetate grown Methanosarcina barkeri, " Eur. J. of Biochem., 159:393-398.

Bott, M.H., Eikmanns, B. and Thauer, R.K. (1985) "Defective formation and/or utilization of carbon monoxide in $\mathrm{H}_{2} / \mathrm{CO}_{2}$ fermenting methanogenes dependent on acetate as carbon source," Arch. Microbiol., 143:266-269.

Chung, T. K. (1976) "Inhibitory effects of $\mathrm{H}_{2}$ on growth of Clostridium cellobioparum," App1. Environ. Microbio1., 31:342-348.

Conrad, R., Phelps, T.J. and Zeikus, J. G. (1985) "Gas metabolism evidence in support of the juxtaposition of hydrogen-producing and methanogenic bacteria in sewage sludge and lake sediments," Appl. Environ. Microbiol., 50:595-601.

Conrad, R. and Thauer, R.K. (1983) "Carbon monoxide production by Methanobacterium thermoautotrophicum, " FEMS Microbiol. Lett., 20:229-232.

Eikmanns, B., and Fuchs, G. and Thauer, R.K. (1985) "Formation of carbon monoxide from $\mathrm{CO}_{2}$ and $\mathrm{H}_{2}$ by Methanobacterium thermoautotrophicum, "Eur. J. Biochem., 146:149-154.

Eikmanns, B. and Thauer, R. K. (1984) "Catalysis of an isotopic exchange between $\mathrm{CO}_{2}$ and carboxyl group of acetate by Methanosarcina barkeri grown on acetate," Arch. Microbio1., 138:365-370.

Harper, S.R. and Pohland, F.G. (1986) "Recent developments in hydrogen management during anaerobic biological wastewater treatment," Biotechnol. Bioeng., 28:585602 .

Hickey, R. F., et al. (1987a) "Production of trace levels of carbon monoxide during methanogenesis on acetate and methanol, "Blotechnol. Lett., 9:63.

Hickey, R. F., et al. (1987b) "The effect of organic toxicant on methane production and hydrogen gas levels during the anaerobic digestions of waste activated sludge," Water Res., 11:1417. 
Hickey, R.F., et al. (1989) "The effect of heavy metals on methane $:$ oduction and hyärogen and carbon monoxide levels during batch anaerobic sludge digestions," Water Res, 23: 207-218.

Kasper, H.F. and Wuhrmann, K. (1978a) "Kinetic parameters and relative turnovers of some important catabolic reactions in digesting sludge," Appl. Environ. Microbiol. , 36:1-7.

Kohler, H.E. and Zehnder, A.J.B. (1984) "Carbon monoxide dehydrogenase and acetate thiokinase in Methanothrix soehngeni1," FEMS Microbiol. Lett., 21:287294.

Koster I.W. (1986) "Characteristics of the pH-influenced Adaptation of Methanogenic Sludge to Ammonium Toxicity." J.Chem. Tech. Biotechnol, 36:445.

Krzycki, J.A., Morgan, J.B., Conrad, R. and Zeikus, J.G. (1987) "Hydrogen metabolism during methanogenesis from acetate by Methanogenesis barkeri, " FEMS Microbiol. Lett., 40:193-198.

Krzycki, J. A and Zeikus, J. G. (1984a) "Characterization and purification of carbon monoxi ie dehydrogenase from Methanosarcina barkeri," J. Bacteriol., $158: 231-237$.

Leininger, K. V., et al. (1983) A Survey of Anaerobic Digester Operations, ASCE Task Committee Report.

Lupton, F.S., Conrad, R. and Zeikus, J.G. (1984) "Physiological function of hydrogen methabolism during growth of sulfidogenic bacteria on organic substrates," J. Bacteriol., 159:843-849.

McCarty, P.L. and Smith, D.P. (1986) "Anaerobic wastewater treatment," Environ. Sci. Technol. , 20:1200-1206.

McCarty, P.L. (1982) "One hundred years of anaerobic treatment," in Anaerobic Digestion. 1.981, D. E. Huges et al. eds., E. sevier Biomedical, Amsterdam.

Mosey, F.E. (1983) "Mathematical modelling of the anaerobic digestion process: regulatory mechanisms for the formation of short-chain volatile acids from glucose," Water Sci. and Technol., 15:209-232.

Nelson, M. J. K. and Ferry, J. G. (1984) "Carbon monoxide-dependent methyl coenzyme $M$ methylreductase in acetotrophic Methanosarcina spp. " J. Bacteriol., $160: 529-532$.

Owen, W. F., Stuckey, D. C., Healey, J. B., Young, L. Y. and McCarty, P. L. (1979) "Bioassay for monitoring biochemical methane potential and anaerobic toxicity," Water Res., 13:485-492.

Ragsdale, S.W. and Wood, H.G. (1985) "Acetate biosynthesis by acetogenic bacteria," J. Biol. Chem., 260:3970-3977. 
Schauder, R., Eikmanns, B., Thauer, R.K., Widde1, F. and Fuchs, G. (1986) "Acetate oxidation to $\mathrm{CO}_{2}$ in anaerobic bacteria via a novel pathway not involving reactions of the citric acid cycle," Arch. Microbiol., 145:162-172.

Smith, P.H. (1980) Studies of Methanogenic Bacteria in Sludge, EPA-600/2-80-093.

Taylor, G. T. (1982) "The methanogenic bacteria," Progress in Industrial Microbiology, 16:231-329.

Thauer, R.K., Jungerman, K. and Decker, K. (1977) "Energy conservation in chemotrophic anaerobic bacteria," Bacteriol. Rev., 41:100-180.

Whitmore, T.N., et al. (1987) "Hydrogen-dependent control of the continuous aerobic digestion process," Appl. Microblol, Blotechnol, 26:383.

Whitmore, T.N., Lazzari, M. and Lloyd, D. (1985) "Comparative studies in thermophilic and mesophilic anaerobic digesters using membrane inlet mass spectrometry," Biotechnol. Lett., I:283-288.

Wiegant, W.M. and Freeman, G. (1986) "The mechanism of ammonia inhibition in the thermophilic digestion of livestock waste," Agricultural Waste, 16:243.

Winfrey, M. R. and Zeikus, J. G. (1979) "Anaerobic metabolism of immediate methane precursors in Lake Medota," Appl. Environ. Microbiol., 37:244-253.

Wolin, M.J. and Miller, T.L. (1982) "Interspecies hydrogen transfer: 15 years later," ASM News, 48:561-565.

Wood, H.G., Ragsdale, S.W. and Pezacka, E. (1986) "The acetyl-CoA pathway of autrotrophic growth," FEMS Microbiol. Review, 39:345-362.

Zeikus, J.G., Kerby, R. and Krzycki, J.A. (1985) "Single-carbon chemistry of acetogenic and methanogenic bacteria," Science, 227:1167-1173.

Zickefoose, C. and Hayes, R. B. J. (1976) Anaerobic Sludge Digestion Operations Manual, EPA 430/9-76-001. 


\section{APPENDIX A}

REAL-TIME DATA ACQUISITION SYSTEM

$\underline{\text { Scope }}$

Presented in this section are details of construction and operation of the realtime data acquisition and control system that was assembled to allow on-line measurement of test digester gas production rate as well as quantification of headspace methane, carbon dioxide, hydrogen and carbon monoxide. The system includes three subsystems: (1) sampling control and data capture; measurement of headspace composition; and (3) gas production rate quantification. These three subsystems will be discussed separately.

\section{Apparatus Description}

\section{A. Sampling Control and Data Capture System}

This system is the control center that initiates and coordinates sampling, data collection and interpretation, and data storage. The main components are an Apple II+ computer (with associated dual disk drive, Epson printer, a cathode ray tube monitor, and software) and a Cyborg (Newton, MA) ISAAC 91-A data acquisition system (with Apple interface, clock and digital/analog (D/A) ard A/D converters). A schematic of this system is presented in Figure A-1. Essentially, the ISAAC 91-A translates low voltage (0-5 volts) or milliamp signals from the monitoring instruments into quantitative values that the Apple computer can recognize. The ISAAC accepts signals from the two infrared (IR) analyzers for $\mathrm{CH}_{4}$ and $\mathrm{CO}_{2}$ analyses, the reduced gas chromatographic system for $\mathrm{CO}$ and $\mathrm{H}_{2}$ analyses with capability for monitoring a pH meter as well. The balance that was used to measure gas production via displaced liquid was linked directly to the Apple IIt.

\section{B. Headspace Composition Measurement System}

Digester headspace composition was measured by two instruments; major product gases $\mathrm{CH}_{4}$ and $\mathrm{CO}_{2}$ are measured by a dual IR analyzer (Analytical Development Company, Limited, Hoddesdon, England) and a RGD2 mercury oxide based reduced gas chromatographic system (Trace Analytical, Standford, CA) was used to quantify trace levels of $\mathrm{H}_{2}$ and $\mathrm{CO}$. A schematic of this system is presented in Figure A2. Briefly, gas was withdrawn from the headspace of the digester on the suction 


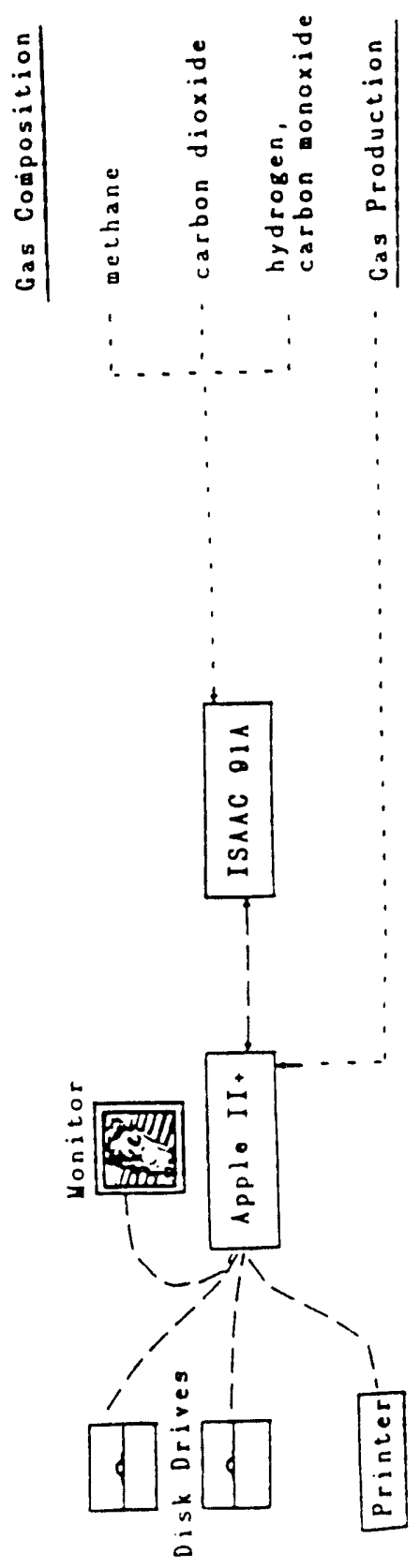

Figure A-1. Schematic representation of the sampling control and data capture system.

$$
\text { A- } 2
$$




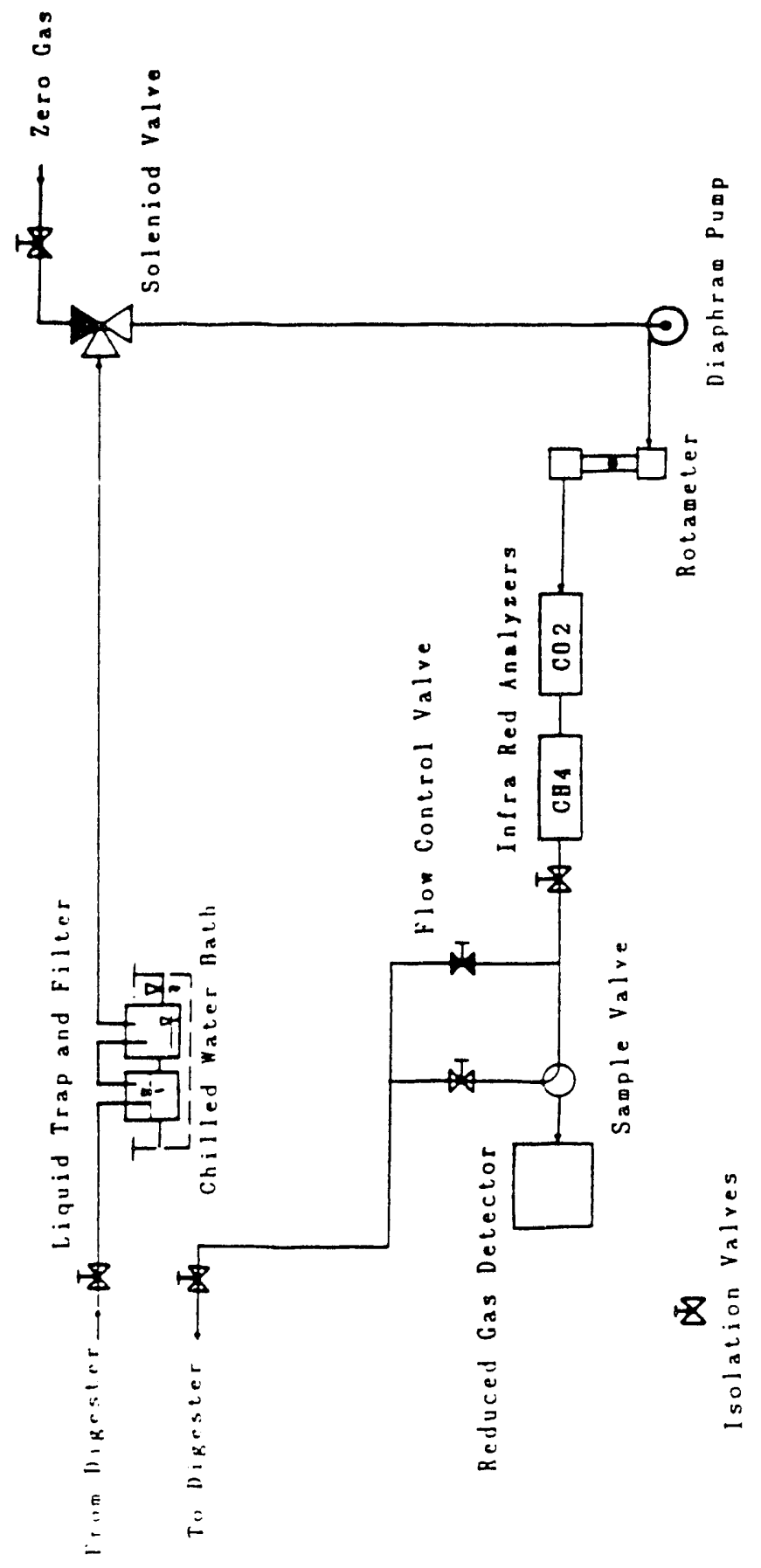


side of a diaphragm pump contained in the dual IR unit at a rate of approximately $450 \mathrm{ml} \mathrm{min} \mathrm{m}^{-1}$. The gas was first conditioned in a combined moisture trap and particulate filter. The trap was maintained in a chilled water bath ( $40-50^{\circ}$ F) to reduce the moisture level of the gas to the point where significant further condensation did not occur in the gas lines subsequent to this point in the system. The gas was filtered once more in the IR unit and then sequentially analyzed for $\mathrm{CO}_{2}$ and methane. Upon exit from the IR unit, the flow was split with approximately 20 to $30 \mathrm{ml} \mathrm{min}^{-1}$ shunted continually through the $0.1 \mathrm{cc}$ sample loop for the RGD2 reduced gas chromatographic system. The two flows were rejoined and sent back to the reactor headspace.

The total gas volume of the system, including the test digester headspace, liquid traps, gas trap sample cells, etc., was calculated by measuring dilution of headspace methane concentration by sequential injections of air into the system. A gas volume of approximately $1075 \mathrm{ml}$ was calculated. This value was used to calculate methane production rates, along with gas production and methane concentration.

\section{Gas Production Measurement System}

The gas production rate was monitored using a liquid displacement technique (Figure A-3). The gas produced, maintained at essentially 1 atmosphere (approximately 1" of water column), was forced to displace an acidified salt solution into a container mounted on a computer linked Ohaus balance (Saddlebrook, New Jersey). During each sample time, the total mass of liquid displaced was measured, converted into gas produced at STP, and production over the last interval obtained by subtracting the previous cumulative gas production value from the new total.

More detail on the rationale for selecting the specific requirements for the components used and the operating characteristics of each sub-system follows.

\section{OPERATION}

A. Sampling Control and Data Capture

The Cyborg ISAAC $91-A$ allowed the Apple IIt to access both analog and digital

$$
A-4
$$




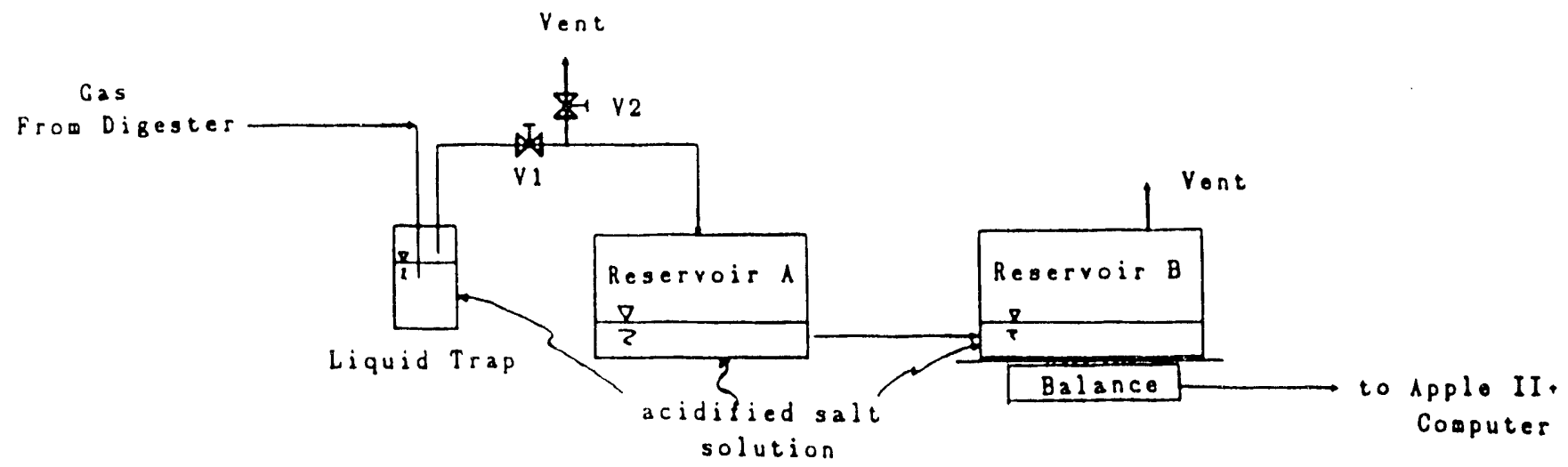

Figure A-3. Schematic representation of the gas production measurement system.

$$
\text { A- } 5
$$


signals under the direct control of Labsoft, an extension of Applesoft BASIC. Once the Labsoft software is loaded, programs are written just as with Applesoft. The system identified all Labsoft commands by the \& (ampersand) which must precede all such commands. The software as written provides for sampling initiation on a user determined time interval, from 1 minute up. Data was collected, stored on disk as a sequential file, displayed on the monitor and a hard copy printed for each sample time. For the experimental runs conducted in conjunction with this study, a 15 minute sampling interval was used.

\section{B. Headspace Composition Measurement System}

It was observed, during the serum bottle assay portion of the experimental plan, that as the methane concentrations increased and $c 0$ concentrations decreased it became increasingly more difficult to accurately quantify $C O$ as graphically displayed in Figure A-4, where the order of elution is $\mathrm{H}_{2}<\mathrm{CH}_{4}<\mathrm{CO}$. Obviously for the on-line portion of experimentation when methane concentration would be between 60 and 70 percent and $C 0$ concentration in the $200-600 \mathrm{ppb}$ range during normal operation, modification of the chromatographic system was in order.

To avoid the interference that high methane concentrations imposed on accurately quantifying trace ( $\mathrm{ppb}$ ) levels of carbon monoxide, modification of the separation procedure, including a different packing that would elute co prior to methane, was needed. In addition, it was observed during the serum bottle assays that moisture and $\mathrm{CO}_{2}$ as well as higher molecular weight compounds tended to accumulate on the packing in a relatively short time and would cause an excessively noisy baseline and shifting (shortening) of elution times which resulted in a relatively short operating period before column reconditioning was required. Therefore, the additional constraints of not irreversibly adsorbing $\mathrm{CO}_{2}$ or $\mathrm{H}_{2} \mathrm{O}$ was also required of the new packing material. Arso, the packing itself had to be extremely clean so that leakage of monomers, even at a very small rate, did not occur as this would upset the baseline because any reduced compounds leached into the carrier gas flow would react with the mercury oxide catalyst of the RGD2 detector.

Polymer resins, such as Poropak, worked well in eluting $\mathrm{CO}$ before $\mathrm{CH}_{4}$, but 


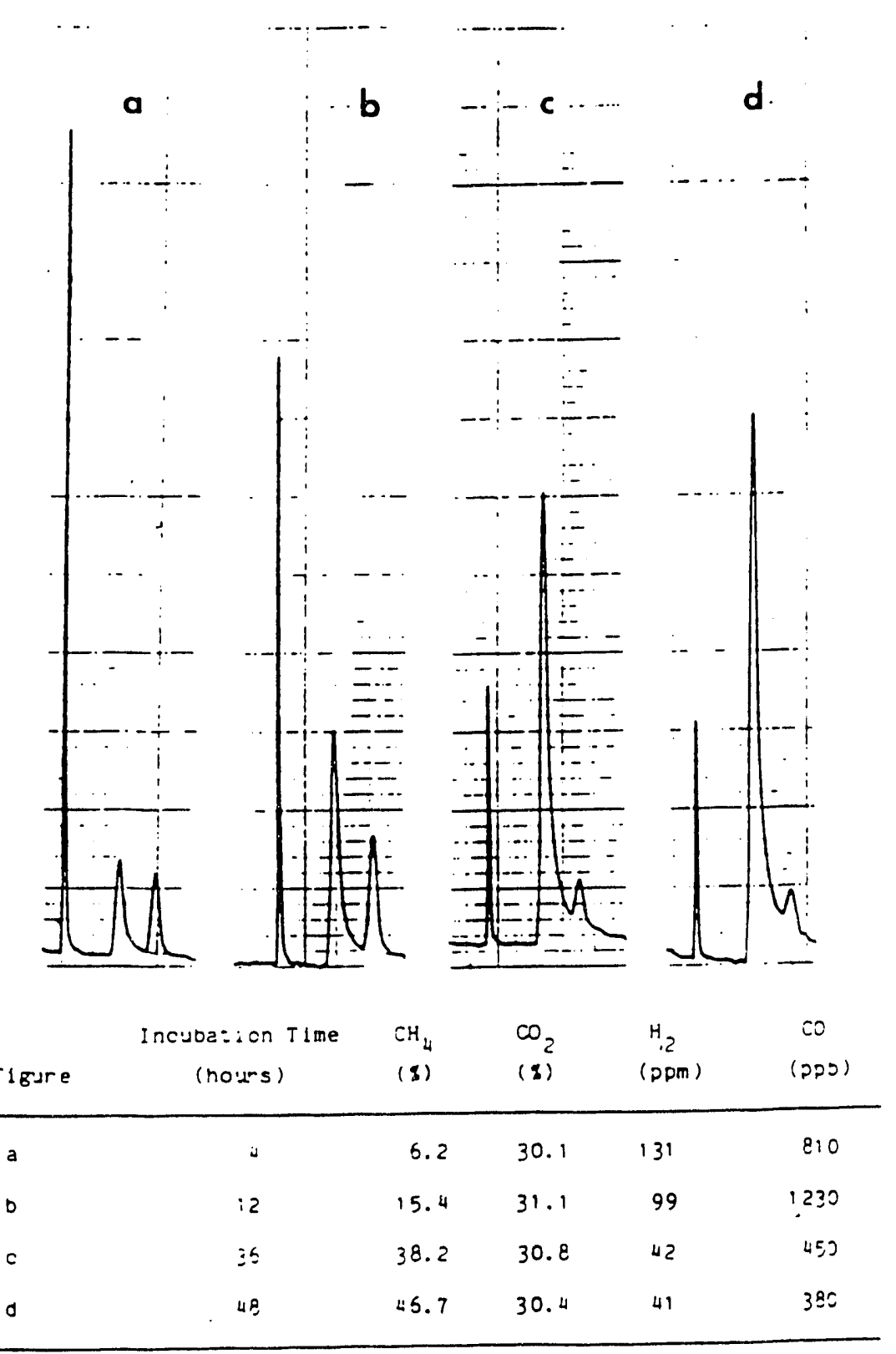

Figure A-4. Effect of methane concentration on the ability to quantify trace levels of carbon monoxide using molesieve $5 \mathrm{~A}$ as a column packing.

A-7 
momoner leaching occurred to the extent that excessive baseline noise was observed. Silica gels could also perform the necessary separation but irreversibly bound water. Nitric acid treated glass beads were also tested, but suffered excessive water retention. Finally, an activated carbon packing, Spherocarb (Analabs), was selected. As long as the column temperature was maintained above $120^{\circ} \mathrm{C}$, no permanent capture of the $\mathrm{H}_{2} \mathrm{O}$ occurred. Water vapor, $\mathrm{CO}_{2}$, and other higher molecular weight compounds were easily backflushed from the packing and long experimental runs were, therefore, feasible.

The final chromatographic set-up included a design that allowed backflush of the heavier fraction, i.e., $\mathrm{CH}_{4}, \mathrm{CO}_{2}, \mathrm{H}_{2} \mathrm{O}$, and continual flow to the detector to be accomplished using only one carrier gas flow. This required the using a 10-port sampling valve (Valco), several needle valves (for flow control), and flow restrictors. The configuration of this set up is schematically shown in Figures A-5a and $b$ for the sample inject and backflash modes, respectively.

Rules of thumb for backflushing chromatographic columns advocate using 1.5 to 2.0 times the sample inject time. The excessively high moisture content of the digester gas, approximately saturated at room temperature, and the extremely polar nature of the water molecule, however, required a longer backflush time. It appeared, based on qualitative results from observing chromatograms with different sample inject and backflush times, that, closer to two and one-half to three times the sample inject time was needed to properly backflush the spherocarb column when operated at approximately $120^{\circ} \mathrm{C}$. Operating conditions and flow rates at the three "vent" points are presented in Table A-1.

\section{Gas Production Measurement System}

The liquid displacement technique has the potential disadvantage of variable and increasing gaseous headspace pressure. In order to minimize this, so it did not affect measured gas production values, two approaches were feasible. Approach one was to measure the gas pressure continually and adjust the gas reading accordingly. Although precise, it is difficult and expensive to continually measure low pressure. A second approach was to reduce the pressure change to an acceptable level and neglect the pressure changes that occur.

$$
\text { A- } 8
$$



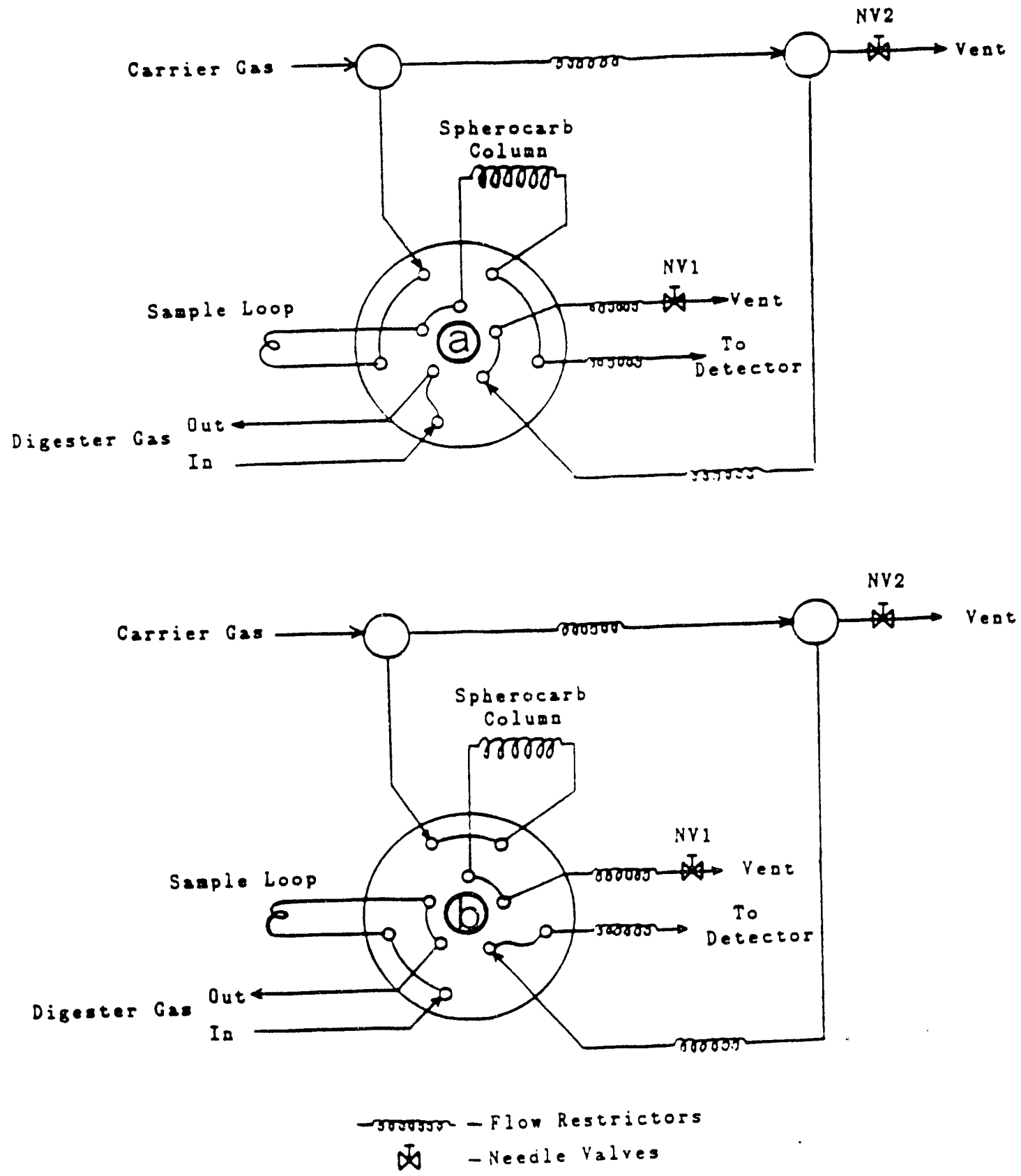
Figure A-5. Operation of 10 -port sample valve operation during (a) sample
inject and (b) backflush modes (sample loop - loading position).

A - 9 

Table A-1. Gas Chromatograph Operating Conditions for Mercury Reduction Analysis of Hydrogen and Carbon Monoxide (On-Line Analysis).

\begin{tabular}{lcc}
\hline Carrier Gas: & Nitrogen (pre-purified, catalytically cleaned) \\
Gas Pressure: & 30 psig & \\
Flow Rates (ml/min) & Sample Inject Position & 30 \\
Detector & 31 & 22 \\
NV1 & 21 & 26 \\
NV2 & 25 & 78 \\
Total & 77 & \\
Column Temperature & $120^{\circ} \mathrm{C}$ & \\
Detector Temperature & $280^{\circ} \mathrm{C}$ & \\
Column Packing & Spherocarb $(60 / 80$ mesh)
\end{tabular}

NV - needle valve 
This is less precise but infinitely more practical. This second approach was chosen.

Consideration must be given to the liquid volume to be displaced, the surface area of the liquid reservoirs, and the volume of gas contained in Reservoir $A$ (Figure $A-3$ ). It is desirable to maintain a nearly constant liquid level in Reservoir $A$ and a minimal headspace.

By selecting reservoirs with large cross-sectional areas, the depth of fluid displacement was minimized. For the 5-1iter test digesters, the 1.5 percent TS feed, a 10-day HRT inoculum and a 3 -fold organic overload, a maximum of approximately 4 liters of gas was anticipated, based on serun bottle results. Reservoir A was selected with a cross-sectional area such that 4 liters equalled 2.5 inches. The acidified salt solution is displaced into the headspace of Reservoir $B$ to avoid additional pressure build-up on this side of the measurement system. Enough salt solution was added to Reservoir A so that the maximum headspace in the reservoir was not less than 10 liters with 4 liters of liquid displaced. Using this scenario, the final milliliter of gas measured of the total of four liters was less than one half of one percent in error. Resetting the balance and returning the displaced salt solution from Reservoir $B$ to Reservoir A once or more during the day lowered this error even further.

It was necessary to choose a computer compatible balance that had the capacity to measure grams and it was, unfortunately, not possible to obtain a balance that displayed the sensitivity to measure in one gram intervals, the sensitivity needed for some experiments, and also have the weight capacity to measure a complete day's gas production in all situations. Therefore, the gas-measuring system was designed to allow rapid reset by transfer of displaced liquid to Reservoir A from Reservoir B. This was accomplished by the following procedure:

- The computer was set in the balance calibration mode and instructed balance adjustment was to occur.

- The current balance reading was then automatically recorded and the balance tared to zero after completion of the acidified salt solution transfer. 
To transfer the displaced salt solution from Reservoir B to Reservoir A, the following procedure was used:

- After setting the computer monitoring system in the calibration mode, V1 was closed. Valve V2 was then opened and Reservoir B elevated to force the salt solution back to Reservoir A. Reservoir $B$ was then repositioned on the balance, and V2 closed. After a short equilibration period necessary for the liquid levels in Reservoirs A and B to reach starting levels, VI reopened to allow accumulated gas to be released. Approximately 1-2 minutes were required to reset the balance. 
EARLY-WARNING PROCESS/CONTROL FOR ANAEROBIC DIGESTERS:

BATCH EXPERIMENTS 


\section{ACKNOWLEDGEMENTS}

There are a great number of individuals who helped to make this research a successful and rewarding experience. The author would like to extend his sincere thanks and gratitude to all who were involved. Special thanks and gratitude are due to Dr. Michael S. Switzenbaum for his continual encouragement, support, friendship and inexhaustible supply of baseball anecdotes. The comments, advice and gift of several bacterial cultures used in this study by Dr. Terry L. Miller and Dr. Meyer J. Wolin are greatly appreciated. The advice and comentary of Dr. James $K$. Edzwald and Dr. Richard $E$. Mudgett are also greatly appreciated.

Special thanks are due to Ms. Juliana Vanderwielen for her skill and tireless dedication in contributing to virtually every facet of this research. Without her diligent effort the value of this work would be much diminished. The author would also like to extend thanks to Ms. Doris Atkinson and Ms. Kajsa Norgen for their assistance in the lab. The advice and insightful comments offered by Dr. Steve Goodwin during the latter stages of the project are greatly appreciated. Many thanks are also extended to Ms. Carolyn o'Grady for typing the manuscript.

This research was supported in part by the New York State Energy Research and Development Authority under contract number 720-RIER-RER-85 to science Applications International Corporation (SAIC). The assistance of Mr. Barry Liebowitz and Mr. Howard Feiler are appreciated. 


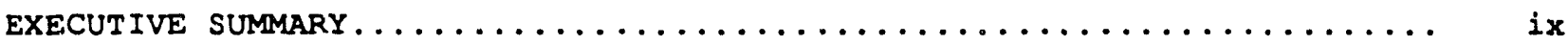

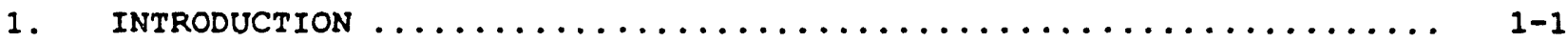

1.1 RESEARCH OBJECTIVES ..........................

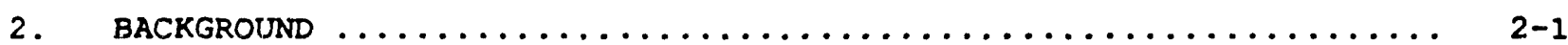

2.1 MONITORING OF ANAEROBIC DIGESTION .................. 2-1

2.1.1 Conventional Monitoring strategy............... 2-1

2.1.2 Enzyme Activity Monitoring..................... 2-2

2.1.3 Electron Carriers and Co-factor Monitoring........ 2-3

2.1 .4 DNA Monitoring.......................... 2-3

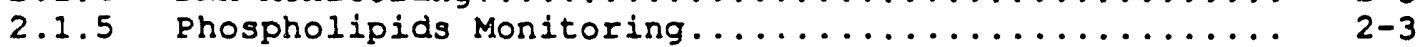

2.1 .6 sumary............................ 2-4

2.2 HYDROGEN IN ANAEROBIC FERMENTATION ................. 2-4

2.3 CARBON MONOXIDE IN METHANOGENIC ECOSYSTEMS ............ 2-10

2.4 TOXICANTS IN ANAEROBIC SYSTEMS ................... $2-14$

2.4 .1 organic Toxicants...................... 2-15

2.4 .2 Inorganic Toxicants....................... 2-16

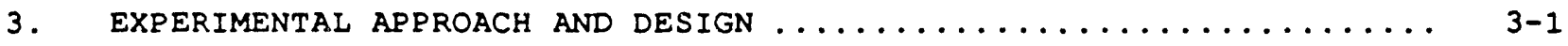

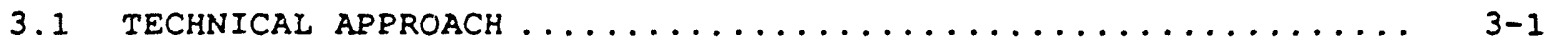

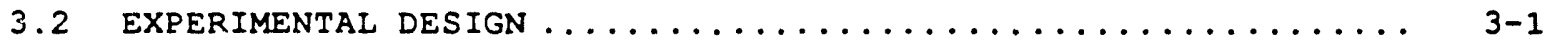

3.2 .1 Reserve Digesters....................... 3-2

3.2 .1 .1 Eeedstock ...................... 3-2

3.2 .1 .2 start-Up and Acclimation ................... $3-4$

3.2.1.3 Daily schedule .................. 3-5

3.2 .2 Batch Serum Bottle Assays.................. 3-5

3.2 .2 .1 Inorganic Toxicants ............... 3-5

3.2 .2 .2 Organic Toxicants .................. 3-6

3.3 METHODS .................................

3.3 .1 Serum Bottle Assays...................... 3-7

3.3 .2 Gas Analyses............................ $3-8$

3.3.3 Volatile Fatty Acids Determination............... 3-9

3.3 .4 Additional Analyses....................... 3-9

3.3 .5 Quality Control of Gas Analysis................ 3-9

4. EXPERIMENTAL RESULTS - THE EFEECTS OE ORGANIC TOXICANTS ON METHANE PRODUCTION AND, TRACE GAS CONCENTRATIONS .................. 
4.1 RESUlts of batch assays ......................... $4-1$

4.1 .1 chloroform............................ 4-1

4.1 .2 Bromoethanesulfonic Acid................... 4-4

4.1 .3 Trichloroacetic Acid....................... 4-13

4.1 .4 Formaldehyde........................... 4-13

4.2 SUMMARY AND DISCUSSION OF RESUITS FROM ORGANIC TOXICANT ASSAYS 4-22

5. EXPERIMENTAL RESUITS - THE EFEECTS OF PULSE ADDITION HEAVY METALS ON ANAEROBIC DIGESTION ............................... 5-1

5.1 RESULTS OF SERUM BOTTLE ASSAYS ..................... 5-1

$5.1 .1 \quad$ Copper............................... 5-1

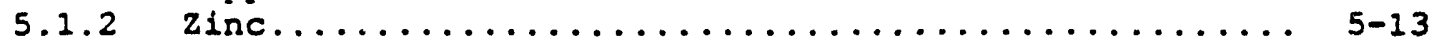

$5.1 .3 \quad$ Cadmium.............................. 5-18

5.2 SUMMARY OF RESULTS FROM METALS INHIBI IION ASSAYS ........ 5-18

5.3 SUBSTRATE PULSE EXPERIMENTS ..................... 5-20

$5.3 .1 \quad$ Acetate............................. 5-20

5.3 .2 sucrose............................. 5-26

$5.3 .3 \quad$ Formate............................... 5-29

5.4 SUMMARY OF RESULTS AND DISCUSSION ................. 5-32

6. DISCUSSION AND CONCLUSIONS .........................6-1

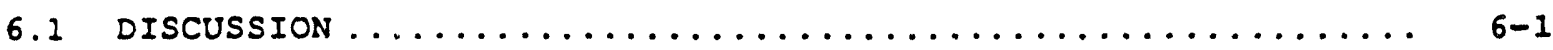

6.2 concLUSIONS $\ldots \ldots \ldots \ldots \ldots \ldots \ldots \ldots \ldots \ldots \ldots \ldots \ldots \ldots \ldots \ldots \ldots$

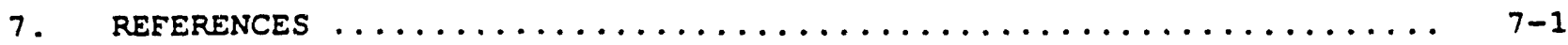

APPENDIX A. Serum Bottle Assays.............................

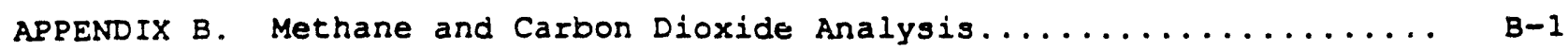

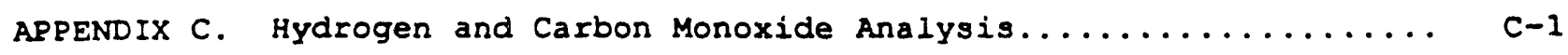

APPENDIX D. Volatile Fatty Acid (VEA) Analysis................. D . 
2-1 Hypothetical model for carbon and electron transformation and ATP synthesis during acetate catabolism by methanogens.... $2-8$

3-1 Schematic representation of the 20-day HRT reserve digester.... 3-3

4-1 Effect of chloroform on cumulative 24-hour methane production.. 4-2

4-2 Methane production rate as a function of time and applied chloroform dose.................................4-3

4-3 Hydrogen accumulation as a function of time and chloroform dose 4-5

4-4 Carbon monoxide accumulation as a function of time and applied chloroform dose...............................4 4-7

4-5 Methane production rate as a function of time and BES dose.... 4-10

4-6 Hydrogen concentration as a function of time and BES dose..... 4-11

4-7 Carbon monoxide concentration as a function of time and BES dose 4-12

4-8 Methane production rate as a function of time and TCAA dose... 4-15

4-9 Hydrogen concentration as a function of TCAA dose and time.... 4-16

4-10 Carbon monoxide concentration as a function of time and TCAA dose...................................... 4-17

4-11 Methane production as a function of time and formaldehyde dose. 4-19

4-12 Hydrogen concentration as a function of time and formaldehyde dose........................................ 4-20

4-13 Carbon monoxide concentration as a function of time and formaldehyde dose............................... 4-21

4-14 Accumulation of (a) acetate; (b) propionate; (c) butyrate as a function of the level of inhibition of methane production induced by BES, chloroform and TCAA....................... 4-25

4-15 Hydrogen concentration as a function of time and applied chloroform dose 20 -day HRT inoculum.................... 4-29

4-16 Hydrogen concentration as a function of time and applied formaldehyde dose 20 -day HRT inoculum.................. 4-30

5-1 Cumulative methane production (24 hours) as a function of copper

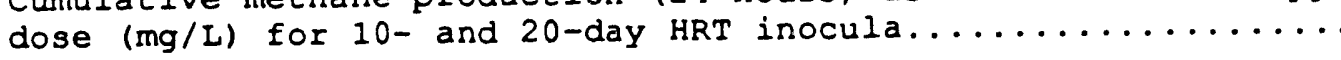

5-2 Cumulative methane production (24 hours) as a function of copper dose (mg/gVs) for $10-$ and 20 -day HRT inocula.............. 5-3

5-3 Inhibition of methane production (24 hours) as a function of filtered copper concentration...................... 5-6 
5-4 Acetate accumulation as a function of filtered copper concentration $-20-$ day HRT inoculum.................... 5-9

5-5 Effect of pulse addition of copper on (a) four hour interval methane production rates, (b) hydrogen response pattern, and (c) Co reponse pattern as a function of time and copper dose for

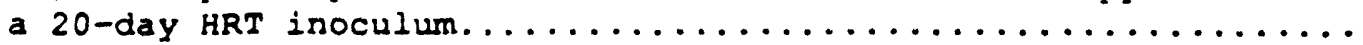

5-6 Carbon monoxide response pattern as a function of copper dose 20-day HRT inoculum............................. 5-12

5-7 Cumulative 24-hour methane production as a function of zinc dose (mg/gVs) for $10-$ and $20-$ day HRT inoculum................ 5-14

5-8 Effect of pulse addition of zinc on (a) four hour interval methane production rates, (b) hydrogen response pattern, and (c) Co response pattern as a function of time and zinc dose for a 10 -day HRT ionoculum.........................

5-9 Co response pattern as a Eunction of zinc dose - 10-day HRT

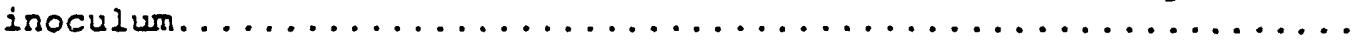

5-10 Relationship between level of inhibition of methane production rate and initial accumulation rate of hydrogen for copper inhibited samples.............................

5-11 Relationship between level of inhibition of methane production rate and initial accumulation rate of hydrogen for zinc

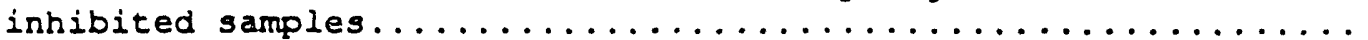

5-12 Correlation between level of inhibition of methane production, induced by heavy metals addition, and carbon monoxide

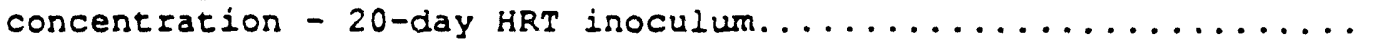

5-13 Correlation between level of inhibition of methane production, induced by heavy metal addition, and carbon monoxide

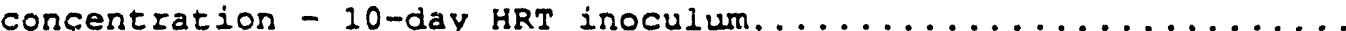

5-14 Comparison of the effect of pulse addition of acetate on

(a) methane production rate, (b) hydrogen response pattern, and (c) Co response pattern for 20-day HRT samples and 20-day HRT samples inhibited by $75 \mathrm{mg} / \mathrm{L}$ of copper..................

5-15 Comparison of the effect of pulse addition of sucrose on

(a) methane production rate, (b) hydrogen response pattern, and

(c) CO response pattern for 20-day HRT samples and 20-day HRT

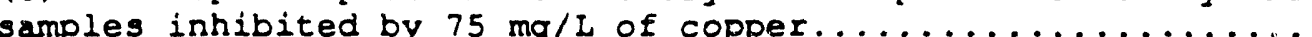

5-16a Effect of formate supplementation on carbon monoxide

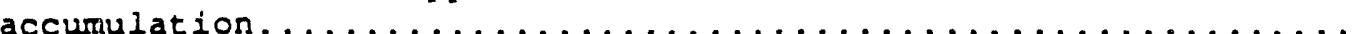




\section{IIST OF TIGURES (Continued)}

Eigure

Rage

5-17 Comparison of acetate accumulatation pattern as a function of inhibition of methane production for copper and BES inhibited sample..................................... 5-34

6-1 Three stage model of substrate and energy flow for the anaerobic digestion of complex organic materials...................6-2

A-1 Effect of mixing on (a) methane production, and (b) hydrogen accumulation patterns.............................A-2

A-2 Experimental setup for preparing serum bottle assays........ A-3 
2-1 Limiting Influent Metal Concentrations................ 2-18

3-1 Monitoring Frequency for Reserve Digesters..............

3-2 Typical Experimental Design for Serum Bottle Assays......... 3-8

4-1 Cumulative Gas and Methane Production and Volatile Fatty Acid Concentrations at Conclusion of Chloroform Inhibition Assay... 4-6

4-2 Effect of BES on Cumulative Methane, and Total Gas Production and Volatile Fatty Acids Concentrations After 24-Hour

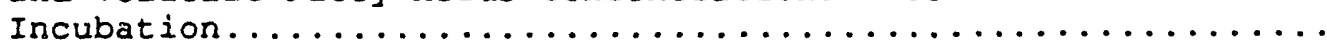

4-9

4-3 Effect of TCAA on Methane Volatile Fatty Acid Concentrations

After 24 -Hour Incubation with TCAA....................4-14

4-4 Estimated Inhibition of Acetate-Splitting Methanogens........ 4-24

5-1 Statistical Analysis of Copper Inhibition of Anaerobic Digestion 5-5

5-2. Filtered Copper Concentrations Versus Applied Dose for 10- and 20-Day HRT Inocula............................. 5-5

5-3 Volatile Fatty Acid Concentrations at Conclusion of 48-Hour Incubation with Copper - 10-Day HRT Inoculum............. 5-8

5-4 Filtered Zinc Concentrations versus Applied Dose for a 10-Day HRT Inoculum. ............................... 5-16

A-1 Methane and Total Gas Production with Associated Relative Standard Deviation for WAS fed 20-Day HRT Inoculum.......... A-8

A-2 Methane and Total Gas production with Associated Relative Standard Deviation for 20-Day HRT Inoculum fed French-

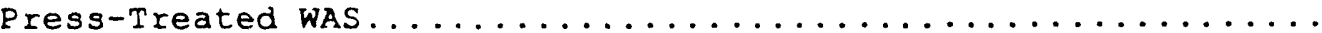

B-1 Gas Chromatograph operating Conditions for Thermal Conductivity Analysis of Methane and Carbon Dioxide Quantification.........

C-1 Gas Chromatograph operating Conditions for Mercuric Oxide Reduction Analysis for Hydrogen and Carbon Monoxide.......... C-2

D-1 Gas Chromatograph operating Conditions for FID Analysis for

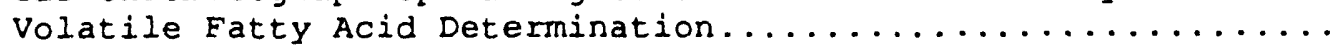


EXECUTIVE SUMMARY

The overall goal of this study was to develop an early-warning process control system for anaerobic digesters. To reach this goal the study was divided into two phases. In Phase 1, experiments with batch anaerobic digesters were used to collect data on the effects of inorganic and organic toxicants on conventional and unconventional anaerobic digester parameters. The conventional parameters of anaerobic digestion studied were: gas production, gas composition, i.e., methane $\left(\mathrm{CH}_{4}\right)$ and carbon dioxide $\left(\mathrm{CO}_{2}\right)$, volatile fatty acids (VFA), alkalinity (ALK) and $\mathrm{pH}$. The unconventional parameters were hydrogen $\left(\mathrm{H}_{2}\right)$ and carbon monoxide $(\mathrm{CO})$. In phase 2 of this study, experiments with daily draw-and-fill, constantly stirred tank reactors (CSTRs) were used to collect real-time data on the effects of organic and hydraulic overload and toxic organic and inorganic shocks on the unconventional digester parameters mentioned while conventional parameters were measured at discrete final intervals.

In this report, the results of experiments to ascertain the response of anaerobic digestion to toxic compounds by batch bioassay techniques (serum bottles) are reported and evaluated. Two reserve sludge digesters (10 and 20 day hydraulic retention time (HRT)), operated on a fill-and-draw basis and fed waste-activated sludge, were used to supply inocula for the serum bottle assays. The batch assays were conducted to determine the concentrations of the toxicants necessary to impose inhibitory and lethal effects on the 10- and 20-day HRT inocula, and evaluate the response patterns of the two trace gases $\left(\mathrm{H}_{2}\right.$ and $\left.\mathrm{CO}\right)$ of interest in addition to the conventional parameters mentioned. This allowed a comparison of the various parameters.

Co was found to be a normal trace component of gases evolved during anaerobic digestion. Both $C O$ and hydrogen displayed characteristic response patterns to inhibition of methane production by organic and inorganic toxicants. When organic toxicants were used, hydrogen and co rose to levels well above noninhibited controls. The rate of increase in these two trace gases was observed to be directly related to the severity of inhibition. The pulse addition of heavy metals produced a very different and more complex response. The rate of hydroger accumulation was retarded in direct relation to the 
inhibition with methanogenesis (i.e., lower rates of $\mathrm{H}_{2}$ accumulation with increasing levels of inhibition of methane production). This was determined to be, in part, due to interruption in the substrate degradation sequence at a point prior to the major hydrogen-producing step(s). Only minor VFA accumulation was observed as a consequence of inhibition as well. CO displayed a characteristic response that was clearly different from that observed for the case when organic toxicants were used. The response was the same for all three metals (copper, zinc and cadmium) used.

Because of the characteristic responses of both hydrogen and co to these two classes of toxicants, it may be possible not only to discern more quickly than is currently possible when an upset is under way, but also to identify the type or nature of the upset due to the characteristic "fingerprint" of the compiled hydrogen and co responses. 


\section{CHAPTER 1}

INTRODUCTION

Anaerobic digestion is a biologically catalyzed process in which organic matter is decomposed, in the absence of oxygen, primarily to the gaseous end products of carbon dioxide $\left(\mathrm{CO}_{2}\right)$ and methane $\left(\mathrm{CH}_{4}\right)$. Naturally occurring habitats for the unique consortium of microbes that carry sut this sequence of fermentative reactions include marshes, rice paddies, benthic deposits and ruminants (such as cattle, sheep and buffaloes). For over 100 years this process has been used as the workhorse for the stabilization and volumetricreduction of municipal wastewater sludges (McCarty, 1982).

Towards the end of the late 1960's, anaerobic digestion began to fall from favor with design engineers and wastewater treatment plant operators alike due to a general perception of unrealiability of the process when compared to the more robust aerobic digestion. Aerobic digestion was observed to display reduced temperature sensitivity and require less operator skill and attention, particularly for small to medium-sized facilities. Aerobic digestion is, however, an energy-intensive process. The rise in the cost of energy experienced during the latter part of the 1970 's spurred renewed interest in alternative sludge stabilization processes, including a reexamination of anaerobic digestion. The Environmental Protection Agency (EPA) now estimates that the number of municipal wastewater treatment facilities that employ anaerobic sludge digestion will increase from the 1984 level of approximately 3850 to close to 5000 by the year 2000 (EPA, 1984).

Accelerated activity in the harnessing of anaerobic systems for the treatment of industrial wastewaters and in the bioconversion of specialty-grown crops and agricultural residues to pipeline-quality methane has occurred during the past decade (Speece, 1983; Hall, 1983). A variety of novel process modifications have been developed to reduce equipment size and cost and increase the efficiency of anaerobic treatment systems (Lettinga et al., 1980; Switzenbaum and Jewell, 1980; Young and McCarty, 1969; Hickey and Owens, 1981). These advances, coupled with an enhanced understanding of the complex microbiology and biochemistry of anaerobic ecosystems obtained during the past 
two de les, tue significantly advancer the state of the art and maje anaerobic treatment systems competitive and in many situations superior to aerobic treatmeat options on both efficiency and cost bases. The last remaining hurdles to widespread application of anaerobic treatment co industrial wastewaters are improved process-reliability and enhanced userconfidence.

The greatest unknowns in anaerobic treatment are now these systems respond to organic overloads and the addition of toxicants on an intermittent or chronic basis. Recent efforts have focused on ascertaining the impact these events impart and the capability of the system biomass to adapt and recover (Yang and Speece, 1985: Speece, 1983; Parkin and Speece, 1982).

Unfortunately, despite the great strides that have been made towards increasing the fundamental understanding of how anaerobic systems func:ion, ittle of this information has been integrated into process-control s:rategies and te ing procedures. The same analyses and pruiess control techniques develop̣ed almost two decades aso are st.. utilized today.

Attempts, to date, to advance the state is the art cor mon.coring axdatoblc treatment systems through analysis of t.se liquid phase have suffered trom tue nwieldly and time-consuming analytical sucedures requirea and the $j$ eat number $:$ interferences that the physical and chen al composition us the iquia phase can impose.

Analyzir.g parameters in the gaseous headspace offers several distinct - dvantares over liquid or slurry phase monitori.y. First, process -nstrumentation in the field of gas chromatography (and related fielas) has advancea much more quickly than its liquid counterfart. Second, the gas phase of an anaerobic treatment system of any type, althuugh not optimal by any means, is considerably more amenable to un-line montcoring than the liquid phase. Sampling conditions are much less severe with respect to the potent-al for chemical or physical fouling. Third, the headspace of an anaerobla system reflects a composite estimate of system status. Gas composition wili be the same throughout the system heaspace. liquid phase, particularly for sludge ot slurry-tyfe, vstems where inádequate 
mixing has been often reported as a significant problem (Leininger et al. 1983).

Analysis of the gas phase of anaerobic systems is a vital part of current process-control strategies. Monitoring has been, however, confined to the primary gaseous components methane $\left(\mathrm{CH}_{4}\right)$, carbon dioxide $\left(\mathrm{CO}_{2}\right)$ and occasionally hydrogen sulfide $\left(\mathrm{H}_{2} \mathrm{~S}\right)$. Recent experimental results have demonstrated that several gas species are important intermediates in the biochemical pathways of methanogenic and acetogenic organisms. Hydrogen (H2) gas has been identified as an important intermediate product in the functioning of anaerobic ecosystems (Bryant et al. 1967). Several researchers have recently postulated that hydrogen may prove to be an excellent process indicator for anaerobic digesters under normal and upset conditions (Mosey, 1983; McCarty, 1982).

More recently, research conducted to elucidate the biochemical pathways involved in methane formation from immediate precursors $\left(\mathrm{H}_{2} / \mathrm{CO}_{2}\right.$ and acetate) as well as the anabolic pathways of the various methanogens, has found that trace quantities of carbon monoxide are produced by actively growing cultures (Conrad and Thauer, 1983). Although not experimentally demonstrated, it has been hypothesized that trace quantities of carbon monoxide (CO) could be evolved during acetate catabolism as well (Nelson and Ferry, 1984).

\subsection{RESEARCH OBJECTIVES}

The goals of this research were to monitor the behavior of the two intermediate product gases, $\mathrm{H}_{2}$ and $\mathrm{CO}$, in anaerobic systems operating under steady state conditions, in response to organic overloads, and in response to toxic or inhibitory shocks induced through the application of organic and inorganic toxicants.

The literature was reviewed to determine: the most commonly encountered toxicants; their median and range of concentrations observed; and documented cases of identification of these species as the causative agent in an anaerobic system upset or failure. Based upon this review, a tiered experimental plan was developed to examine the effect a variety of these potential inhibitory and/or toxic agents have on total gas and methane 
production, volatile fatty acid (VFA) accumulation, and the concurrent impact on the concentration of the intermediate product gases $\mathrm{CO}$ and $\mathrm{H}_{2}$. Following a preliminary experimental phase of this study, several upset situations were chosen for more in-depth examination.

One goal of chis research effort was to gather a sufficient data base concerning the response of anaerobic systems, particularly the two trace gas species of interest, to a variety of system perturbations. Based upon these data, the use of these gases as indicators of anaeroblc biological process status could be evaluated, both as stand-alone indicators or as one of several indicators to be used in concert with one another or with conventional control parameters (i.e., gas production, methane content, etc.). The second goal of this research was to develop a more sensitive and effective process control strategy to maximize the warning-time between when an upset is about to occur and when in fact it does occur. This would allow more timely remedial measures to be executed and help to eliminate or alleviate the severity of an oncoming upset. 
CHAPTER 2

BACKGROUND

\subsection{MONITORING $\because$ : ANAEROBIC DIGESTION}

madit inul anaerobic digester monitoring involves analyzing a combination of indicator parameters. There is no ont single indicator that can reliably signal a process imbalance or upset. Even the combination of parameters currently used for process control cannot adequately detect the onset of a process upset until it is well under way, particularly for :apidly occurring events such as toxic shocks. Alternate monitoring parameters have been proposed that have their basis in approximating viable biomass concentratıons and metabolic activities. The alternatives examined to date, however, suffer severe deficiencies themselves.

\section{1 conventional Monitering Strategy}

Iraditional monitoring consists of concurrently measuring chemical parameters in the liquid and gaseous phase. A combination of pH, alkalinity, volatile acids, gaseous methane and carbon dioxide content as well as gas production are typically recommended for monitoring purposes (zickefoose and Hayes, 1976). In general, these parameters describe the system operational efficiency as well as the chemical and to a lesser extent, physical environment within a digester. They do not, however, reflect the current metabolic status of the system biomass. This monitoring strategy is useful in detecting process upsets once they are under way. In many instances this may be adequate to avoid system failure for slow-to-develop difficulties such as gradual organic or hydraulic overloads. Toxic or inhibitory events, however, may occur much more rapidly and cause more damage to the system's viability. These events cannot be adequately diagnosed by current process control strutegies in time to avoid significant process deterioration and possible failure.

In the treatment of many industrial wastes, the potential for toxicity has been a concern and impediment towards application of anaerobic processes (Speece, 1983). An effective and sensitive early-warning system would be 
especially useful in enhancing system reliability and improving user confidence.

The information required to detect the onset of a potential process upset must be integrally related to the bacterial metabolic activity of the system. There have been attempts to relate digester performance with parameters associated with metabolic activity such as enzyme activity levels, genetic material and specific electron carriers.

\subsubsection{Enzyme Activity Monitoring}

Virtually all metabolic activity within a cell is mediated by enzymes. The oxidation-reduction reactions that regulate substrate utilization are catalyzed by oxido-reductases (Patterson, 1969). Dehydrogenase enzymes are the major group responsible for the oxidation of substrates in anaerobic environments (Taylor, 1982). They function by transferring hydrogen atoms (or electrons) from reactants to cyclic intermediate carriers (or acceptors).

Lenhard (1967) related dehydrogenase levels to activity levels of digester sludge samples. The utility using dehydrogenase activity to detect toxic shocks or organic overloads was not examined. A chemical extraction following a 15- to 60-minute incubation was required to determine dehydrogenase activity.

Ashley and Hurst (1981) examined the use of acid and alkaline phosphatase levels to determine anaerobic digester stability. They found that phosphatase activity rose with increasing volatile fatty acid concentrations, and that activity reflected changes in the population of non-methanogenic degradative organisms. They concluded phosphatase activity could be used to give warning of instability in advance of conventional process control monitoring analysis which examines end products of metabolism. Organic overloads and toxic shock (monochloroacetic acid) events were examined. A cumbersome chemical analysis not well suited for on-line measurement is, however, required for determination of phosphatase activity. 


\subsubsection{Electron Carriers and co-factor Monitoring}

Factor $F_{420}$ is a low molecular weight electron carrier that is specific to methanogens (Edwards and MCBride, 1975). The oxidized form of this compound fluoresces blue-green when excited by light at $420 \mathrm{~nm}$ (Cheeseman et al., 1972). Factor $F_{420}$ is a major electron (hydrogen) carrier in methanogens being involved in both catabolic and anabolic oxidation-reduction reactions. Taylor (1982) reported $F_{420}$ as being a co-factor for hydrogenase, NADP+ reductase and dehydrogenases. Mink and Dugan (1977) demonstrated that $\mathbf{F}_{420}$ could be used to identify and enumerate methanogens in mixed populations. Delafontaine et al. (1979) developed an extraction procedure to quantify $F_{420}$. Pause and Switzenbaum (1984) used this procedure to monitor $F_{420}$ in laboratory-scale digesters. They observed that $F_{420}$ content was a function of the physiological growth conditions, type of substrate being degraded (possibly a reflection of the system ecology), and the particular methanogenic species present (all do not fluoresce equally). In addition, $F_{420}$ appears to be a heat-stable compound that does not significantly degrade after cell death. The use of $F_{420}$ appears to be of limited utility as an early-warning indicator because of these reasons.

\section{1 .4 DNA MOnitering}

The DNA ratio with respect to volatile organic matter for pure cultures was found to be relatively constant at approximately 3.8 percent (Webb and Levy, 1956). Agardy and Shepherd (1965) found that DNA levels for anaerobic digesters averaged close to this value as well. Although DNA does reflect cell numbers, it cannot differentiate between functional and dead or inactive cells (Pohland and Kang, 1975). Its use as an early-warning indicator is, therefore, minimal.

\section{1 .5 Phospholipids Monitoring}

Phospholipids are found in the membranes of all cells (White, 1983). They have a relatively rapid turnover in both living and dead cells (White, 1983) and can be related to active biomass concentrations (White et al., 1979). Phospholipids levels were determined in thermophilic digesters fed a variety of supplements (Henson et al., 1985). The digesters receiving different supplements could be distinguished from the controls and each other. The 
methods of analysis, however, are destructive and require chemical analysis, not currently suitable for on-line measurement.

\section{1 .6 Summary}

All of the above attempts to monitor digester activity, performance or ecology have met with limited success. They require rather sophisticated and timeconsuming wet chemical techniques that do not lend themselves to on-line measurement. Conclusive evidence that any of the above techniques, even if practical assay techniques suitable for on-line measurement can be developed, can accurately function as early-warning indicators has not been demonstrated.

There does exist, however, the potential to obtain some measure of the metabolic status of anaerobic systems by monitoring the concentration of certain intermediate product gases (hydrogen and carbon monoxide) that have been reported to be present at trace levels. In contrast to liquid phase sampling, where sample preparation, extraction procedures, etc., are not well adapted for on-line data acquisition, gas analyses are amenable to real-time data acquisition.

\subsection{HYDROGEN IN ANAEROBIC FERMENTATION}

Hydrogen is an important intermediate for methanogenesis in the rumen and anaerobic digester environments. In the rumen, virtually all the methane produced is derived from $\mathrm{H}_{2}$ (Hungate, 1966) while approximately 28 to 30 percent of methane from a typical anaerobic digester is produced via the reduction of $\mathrm{CO}_{2}$ by hydrogen (Jeris and McCarty, 1965; Kasper and Wurhman, 1978a; Smith, 1980). Hydrogen is produced during the anaerobic fermentation of carbohydrates and other substrates degraded via the Embden-Meyerhof or pentose phosphate pathways and in the subsequent degradation of pyruvic acid and higher weight volatile fatty acids to acetate. In methanogenically active environments, this hydrogen is utilized by hydrotrophic methanogens to reduce carbon dioxide to methane gas.

Interspecies hydrogen transfer was initially identified as playing a key role in regulating anaerobic fermentation of organic matter nearly 20 years ago (Bryant et ded., 1967; Hungate, 1967). A culturc known as Methanobacillus omelianskii was discovered to be a syntrophic co-culture of a methanogen 
(Methanobacterium bryantii) and an ethanol-oxidizing organism. The ethanoloxidizing " $S$ " organism produces acetate and $\mathrm{H}_{2}$ as its major end products. M. bryantil utilizes the $\mathrm{H}_{2}$ to reduce $\mathrm{CO}_{2}$ and form methane. In doing this, the M. bryantii maintains an extremely low partial pressure of hydrogen and allows the " $S$ " organism to carry out a reaction that is thermodynamically unfavorable at higher hydrogen partial pressures. Other syntrophic associations of obligate proton-reducing bacteria and methanogens have been isolated that degrade butyrate (Tomei et al., 1985; Shelton and Tiedje, 1984) and propionate (Boone and Bryant, 1980). The degradation of important methanogenic precursors butyrate and propionate was shown by McCarty (1982) to be even more sensitive to in-situ hydrogen levels than ethanol oxidation. Interspecies hydrogen transfer can markedly affect the rate and extent of anaerobic fermentation as well as the products formed (scheifinger et al., 1975).

A buildup of hydrogen can inhibit the growth of hydrogen-producing organisms such as clestridium cellobioparum and alter electron flow resulting in the formation of more reduced products such as lactate, butyrate or ethanol (which serve as alternate sinks for the hydrogen produced) instead of acetate (Chung, $1976)$.

In anaerobic habitats, the in-situ concentration of hydrogen, under normal environmental conditions, is well below reported $\mathrm{K}_{\mathrm{s}}$ values. There is a surplus capacity to consume hydrogen in most methanogenic environments (Winfrey and zeikus, 1979). Kasper and wurman (1978a) reported that in a typical digester, there is the capacity to remove 20 fold the amount of $H_{2}$ actually consumed. Tomei (1985), after observing that in a syntrophic butyrate degrading culture the methanogen and proton reducer grew attached, hypothesized that attachment may be an important strategy that allows the syntrophic association to outcompete other organisms which may have a greater substrate affinity. Conrad (1985) and co-workers observed that $\mathrm{H}_{2}$ turnover rates, as determined by using in-situ pool sizes and temperatures, accounted for only 5 to 6 percent of the expected $\mathrm{H}_{2} / \mathrm{CO}_{2}$ dependent methan production in sewage sludge and lake sediments. This discrepancy they claimed, supports the contention that $h_{2}$-dependent methanogenesis occurs as a consequence of direct interspecies $\mathrm{H}_{2}$ transfer between juxtapositioned microbial associations within flocs or consortia. 
Methanogenic bacteria have been reported to have a minimum threshold for hydrogen consumption. Lovley (1985) reported that three hydrogen-utilizing methanogens Methanobacterium formicicum, Methanobacterium byantii M.O.H., and Methanospillum hungatei JF-1 had hydrogen thresholds of approximately 6.5, 6.9 and $9.5 \mathrm{~Pa}$, respectively. In another study Lovley et al. (1984) 1 and that five rumen isolates could not metabolize hydrogen below 7.7 to $12 \mathrm{~Pa}$. A syntrophic association such as described above would prove an excellent survival strategy to allow the methanogens to obtain energy when ambient $\mathrm{H}_{2}$ levels are below this threshold utilization value.

Methanogens are not the only organisms that can utilize hydrogen as an energy source. Hydrogen can also be a substrate for sulfate reduction (Abram and Nedwell, 1978). Sulfate reducers can, in fact, outcompete methanogens for hydrogen in most natural environments, provided sulfate is not limiting (Lovley et al., 1982; Oremland and Polcin, 1982). This is believed to be due to the greater substrate affinity and growth rate displayed by the sulfatereducing bacteria (Kristjansson, 1982) or a lower threshold utilization value (Lovley et al., 1982). Sulfate-reducing bacteria (SRB) and methanogens can also function in a syntrophic fashion. When sulfate is limiting, SRB's can ferment intermediates such as lactate to hydrogen, $\mathrm{CO}_{2}$ and acetate which are subsequentially utilized by the methanogens (McInerney and Bryant, 1981).

Methanogens have recently been observed to produce hydrogen when growing on acetate (Nelson and Ferry, 1984). Lovley and Ferry (1985) reported that two thermophilic Methanesarcina species maintained $\mathrm{H}_{2}$ partial pressures of 16 to $98 \mathrm{~Pa}$ (160-910 ppm) during growth. Consumption of hydrogen was observed during latter stages of growth. Hydrogen was also produced when methanol or trimethylamine were employed as substrates for methanogenesis. The net rates of hydrogen production and consumption were less than 1 percent of the rates of methane production. Boone et al. (1987) reported that $\mathrm{H}_{2}$ levels appeared to be related to the redox potential of some electron carrier(s) involved in acetate catabolism.

Recently, Phelps et al. (1985) observed a kind of interspecies hydrogen transfer between $a \mathrm{SRB}$ and methanogen based on $\mathrm{H}_{2}$ production by the 
methanogen. In pure culture $M$ barkeri was observed to produce up to 10 uM of $\mathrm{H}_{2}$ in the headspace. When Methanesarcina barkeri was co-cultured with Desulfovibrie vulgaris using methanol or acetate as an energy source the $D$. yulgaris grew on $\mathrm{H}_{2}$ produced by the methanogen. The net result was hardly a syntrophic association but actually caused a reduction of almost one-third the amount of methane formed. R. vulgaris could not grow on methanol or acetate in pure culture. Another interesting example of obligate interspecies hydrogen transfer has recently been reported. Zinder and Koch (1984) isolated a syntrophic association of a thermophilic acetate oxidizias organisms and a thermophilic Methanobacterium. One member of the coculture "oxidizes" acetate to $\mathrm{CO}_{2}$ and reduces protons to $\mathrm{H}_{2}$. The methanogen reduces the $\mathrm{CO}_{2}$ to methane using $\mathrm{H}_{2}$.

The physiological role of hydrogen production in methanogenesis from acetate is not well understood. Baresi and wolfe (1981) found that hydrogenases are apparently constitutive. Acetate-grown Methanesarcina has approximately the same levels of hydrogenase, CoM, and methylcoenzyme $M$ methylreductase as those grown on hydrogen and $\mathrm{CO}_{2}$. Lovley and Ferry (1985) suggested that $\mathrm{H}_{2}$ could be produced within the cytoplasm from the oxidative cleavage of acetate. After diffusion across the cell membrane, $\mathrm{H}_{2}$ could be oxidized on the outside of the cell membrane and the reducing equivalents used for reduction of methyl coenzyme $M$ or the cytoplasmic side of the membrane.

Krzycki and zeikus (1984b) proposed a comparable model of carbon and electron flow for conversion of acetate to methane and $\mathrm{CO}_{2}$ (Figure 2-1). Hydrogen cycling as proposed by Lovley and Ferry (1985) is accomplished by electron carriers in the membrane. This hydrogen cycling could also drive ATP generation in the cell. Their scheme relates the physiological roles of hydrogen and carbon monoxide in acetate catabolism. Krzycki et al. (1987) observed that although hydrogen and methane production of $\boldsymbol{M}$. barkeri was inhibited by chloroform or potassium cyanide, hydrogenase activity was not affected by these inhibitors at levels that completely arrested methanogenesis. They speculated that inhibition of hydrogen formation by $\mathrm{KCN}$ was possibly due to inhibition of acetate cleavage. 


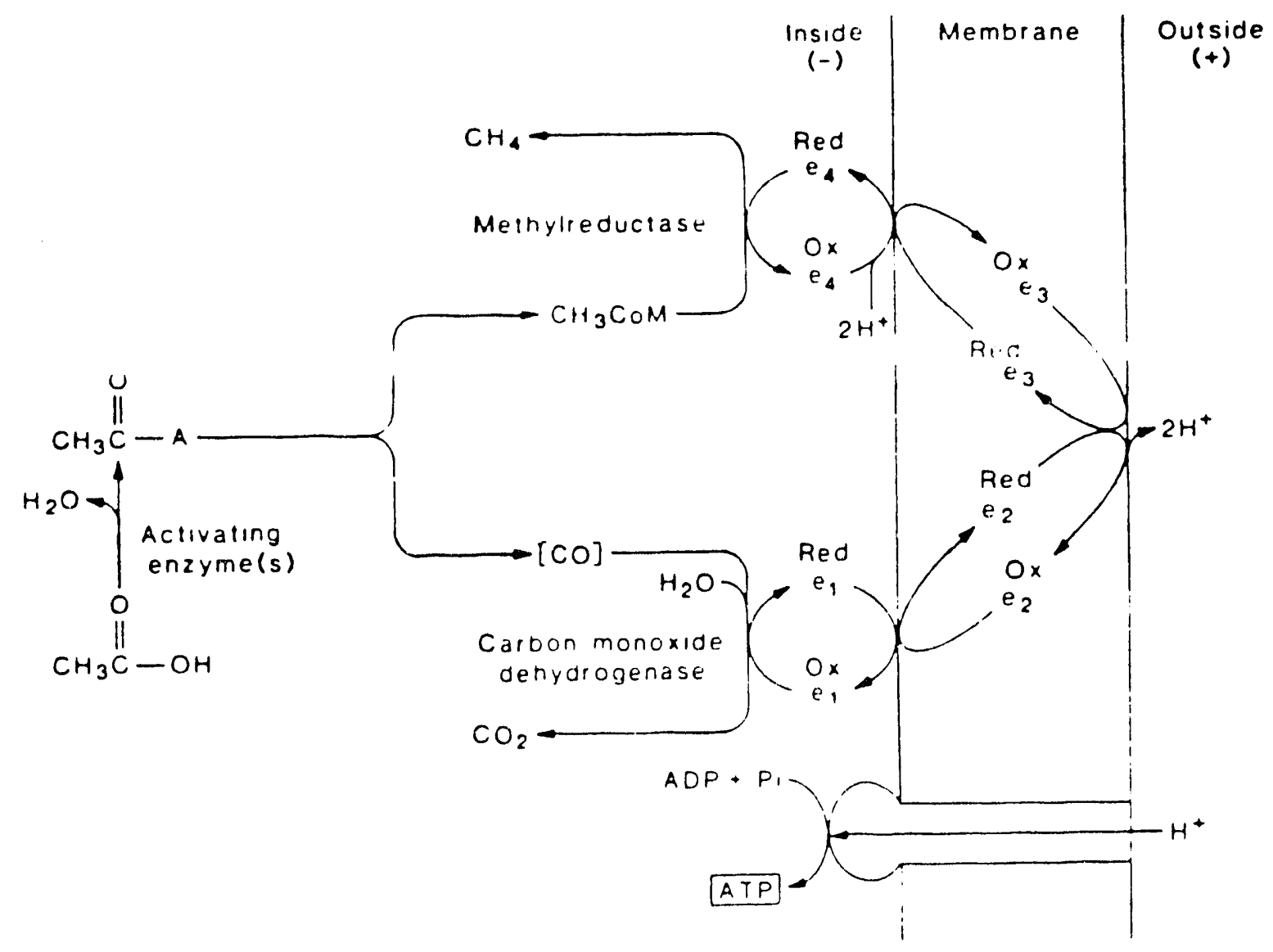

Figure 2-1. Bypothetical model for carbon and electron
transformation and ATP synthesis during
acetate catabolism by methanogens. 
There also exists some acidogenic hydrogen-consuming organisms in methanogenic habitats. They have the ability to synthesize acetate from hydrogen and carbon dioxide (Ohwaki and Hungate, 1977; Adamse, 1981). Their activity in one study of mesophilic and thermophilic cattle manure digesters indicated that $\mathrm{CO}_{2}$ reduction accounted for less than 5 percent of the acetate formed (Mackie and Bryant, 1981).

Mosey (1983) has indicated that it may be simpler and more informative to monitor hydrogen as an indicator of anaerobic system status in place of the currently used process control indicators. Severe organic overloading conditions imposed on several high-rate industrial anaerobic systems have, in fact, resulted in quantifiable hydrogen accumulation in the gaseous headspace (Barnes, et al., 1984, and Archer et al., 1986). Scott et al., (1983), using membrane inlet mass spectrometry, observed that chloroform addition to a digester sludge sample produced a quantifiable increase in dissolved $\mathrm{H}_{2}$ concomitant with the arrest of methane production. Whitmore et al. (1985) reported that spiking glucose, propionate or butyrate to mesophilic and thermophilic digester systems all caused a rise in dissolved $\mathrm{H}_{2}$. In these cases an increase in methane production accompanied the rise in $\mathrm{H}_{2}$. McCarty and Smith (1986), based on the results of monitoring laboratory CSTRs, anaerobic filters and baffled reactors under steady state operating conditions, reported that hydrogen monitoring could be an important tool for both process control and in diagnosis of process problems.

The improvement of the digestion process through hydrogen control has also been advocated. Podolak et al. (1984) reported that operating an anaerobic rotating biological contactor under vaccuum to lower the $\mathrm{H}_{2}$ partial pressure, measurably improved process performance. Poels et al. (1985) found that stripping hydrogen in an auxiliary vessel to lower the $\mathrm{H}_{2}$ partial pressure resulted in an improvement in COD removal of up to 10 percent. Harper and Pohland (1986) presented several processing options for maximizing the effectiveness of high-strength industrial treatment systems based on hydrogen management.

Clearly, hydrogen serves an important role in anaerobic degradation. Its dynamics in anaerobic ecosystems, however, appear convoluted and variable 
depending upon substrate concentration and strength and operating conditions; in short, difficult to assess. This may limit the use of hydrogen as a stand-alone indicator of the anaerobic digestion process. Monitoring of a second, sensitive and metabolically controlled indicator gaseous intermediate in concert with hydrogen, however, may significantly improve the capability to detect the onset of potential upsets. Carbon monoxide may be such an indicator.

\subsection{CARBON MONOXIDE IN METHANOGENIC ECOSYSTEMS}

Recent evidence indicates that carbon monoxide dehydrogenase activity is relatively common among some of the major trophic groups in anaerobic ecosystems. Daniels et al. (1977) reported that many methanogens possess COdehydrogenase enzyme activity. High levels of co-dehydrogenase activity have also been found in acetogens (Diekert and Thauer, 1978; Clark et al., 1982; Lynd et al., 1982; Diekert and Ritter, 1983; Drake et al., 1981; Hu et al., 1982). In the case of hydrogen-utilizing methanogens (Stupperich et al., 1983; Stupperich and Fuchs, $1984 \mathrm{a} \& \mathrm{~b}$ ) and acetogenic bacteria (Pezacka and wood, 1984) this co-dehydrogenase activity is believed to be an essential element for the anabolic fixation of $\mathrm{CO}_{2}$ into cell carbon. Schauder et al. (1986) found that sulfate-reducing bacteria (SRB) not possessing an operative citric acid cycle had high levels of co-dehydrogenase enzyme activity. In SRBs, the function is thought to be essentially a reverse of the reaction of forming $2 \mathrm{CO}_{2}$ from acetate (Schauder et al., 1986).

Baross et al. (1982) reported that Co was evolved in incubated samples of extreme thermophiles $\left(100^{\circ} \mathrm{C}\right)$ from submarine hydrothermal vents. Methane and hydrogen were also observed to be evolved from this anaerobic consortium. Uffen (1983) reported that Rhodopseudemonas gelatinesa has CO dehydrogenase enzyme activity that leads to production of $c 0$ when this bacterium is incubated anaerobically in the dark.

Conrad and Thauer (1983) were the first to report biological carbon monoxide formation under strictly anaerobic conditions. They observed between 76 and $121 \mathrm{ppm}$ of $\mathrm{CO}$ in growing gassed $\left(80 \% \mathrm{H}_{2} / 20 \% \mathrm{CO}_{2}\right)$ cultures of Methanobacterium 
thermoautotrophicum maintained at $65^{\circ} \mathrm{C}$. They proposed the reaction occurring was:

$$
\mathrm{CO}_{2}+\mathrm{H}_{2} \Leftrightarrow \mathrm{CO}+\mathrm{H}_{2} \mathrm{O} ; \Delta \mathrm{G}^{\circ}=+20 \mathrm{~kJ} / \mathrm{mole}
$$

Eikmanns et al. (1985) later demonstrated that the co concentration in the gas phase could exceed the equilibrium concentration by a factor of more than 15 . They postulated, based on this and the observation that co formation occurred only when methane was also generated, that $\mathrm{CO}$ formation from $\mathrm{H}_{2}$ and $\mathrm{CO}_{2}$ was energy-driven by linkage to an exergonic process. This was substantiated by using uncoupling agents to inhibit $c 0$ formation.

Bott et al. (1985) reported that the absence of co formation by hydrogenutilizing methanogens requiring acetate for growth correlated with the absence of carbon monoxide dehydrogenase activity.

Diekert et al. (1984) reported that an acetogenic bacterium produced trace levels of co during carbon dioxide fixation. Lupton et al. (1984a) found that the SRB Desulferibrie vulgaris produced and subsequently consumed trace amounts of carbon monoxide during growth.

Stupperich and Fuchs (1984a\&b) proposed a pathway for autotrophic synthesis of activated acetic acid from carbon dioxide by Methanobacterium thermoautotrophicum. Acetate synthesis was completely arrested by $0.2 \geq \mathrm{M} \mathrm{KCN}$ while methanogenesis was unaffected. Addition of $\mathrm{CO}$ (108) to the gas headspace restored acetate synthesis to approximately 70 percent of its original level. Other inhibitors examined (iodopropane, $\mathrm{CCl}_{4}, \mathrm{BES}$ and $\mathrm{N}_{2} \mathrm{O}$ ) all simultaneously inhibited methanogenesis and acetate synthesis to the same extent. Results indicate the activity of a carbon monoxide dehydrogenase in $\mathrm{CO}_{2}$ fixation. These researchers further showed that the methyl and carboxyl groups of synthesized acetate were formed via two distinct pathways; the carboxyl group being formed via a carbon monoxide dehydrogenase mediated pathway.

Evans and co-worker's (1986), using high field carbon-13 nuclear magnetic resonance (NMR) spectroscopy, demonstrated that the co dehydrogenase activity of $M$. thermoautetrophicum is reversible. They observed a specific exchange of 
the $\mathrm{C}_{1}$ of acetate and $\mathrm{CO}_{2}$ before its incorporation into the metabolite 2,3 cyclopyrophosphoglycerate (CCP). CCP is a novel intermediate in cell carbohydrate synthesis.

O'Brien and co-workers (1984) reported that Methanosarcina barkeri MS could grow and produce methane fromi $c o$. In these experiments, co served both as carbon and energy source. If the gaseous co concentration exceeded 20 percent, net hydrogen production was observed. Conversely, net hydrogen consumption was observed if $C O$ was present at concentrations of less than 20 percent. $\mathrm{H}_{2}$ and $\mathrm{CO}$ metabolism, they proposed, may be interrelated.

Eikmanns and Thauer (1984) found that KCN at low concentrations (2.5 to $10 \mu \mathrm{M})$ inhibited methane production from acetate, but not from $\mathrm{H}_{2}$ and $\mathrm{CO}_{2}$ for Methanosarcina barkeri (strain Eusavo). The isotopic exchange between ${ }^{14} \mathrm{CO}_{2}$ and the $\mathrm{Cl}$ of acetate was simultaneously inhibited with methanogenesis. They postulated that conversion of acetyl $\mathrm{COA}$ to methyl-CoM plus $\mathrm{CO}_{2}$ has a positive free energy under standard conditions $(+24 \mathrm{~kJ} / \mathrm{mole})$, indicating that the formation of methyl $\mathrm{COM}$ and $\mathrm{CO}_{2}$ from acetyl $\mathrm{COA}$ is a reversible redox reaction. This was supported by the observation that cells which ferment acetate to $\mathrm{CH}_{4}$ and $\mathrm{CO}_{2}$ also catalyze an isotopic exchange between $\mathrm{CO}_{2}$ and the carboxyl group of acetate $\left(C_{1}\right)$. Hydrogen $(0.5 \%)$ completely inhibited methanogenesis (from acetate) and the isotopic exchange reaction. Carbon monoxide, however, at 1.08 caused a virtual complete cessation of methanogenesis but only partially inhibited the exchange reaction. The rate of isotopic exchange was still 25 percent, the normal rate, when the co concentration was increased to 6.0 percent. $M$. barkeri did not mediate, at detectable rates, an isotopic exchange between formate or $C O$ and the $C_{1}$ of acetate. This observation excludes free formate or $\mathrm{CO}$ as intermediates. They proposed that a bound CO moiety, with the co most likely tightly bound to a metal, is probably an intermediate in the decarboxylation of acetate during methanogenesis.

Kryzcki and zeikus (1984a) extracted and purified Co dehydrogenase from acetate-grown Methansarcina barkeri MS. Oxygen deactivated CO dehydrogenase activity by 908 in 30 minutes. Addition of $\mathrm{CO}, \mathrm{H}_{2}$, or dithionite to the

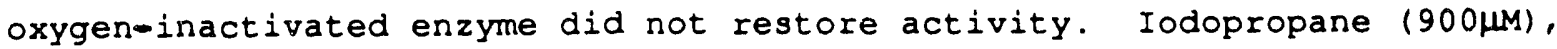


sodium arsenite $(10 \mathrm{mM})$, and $\mathrm{N}_{2} \mathrm{O}(0.5 \mathrm{~atm})$ did not produce significant inhibition. Addition of $10 \mathrm{mM} \mathrm{Na}{ }_{2} \mathrm{SO}_{3}$ produced approximately 408 inhibition.

Kryzcki and zeikus (1984a) further reported that up to 58 of soluble cellular protein of acetate-grown cells is $C O$ dehydrogenase. The enzyme was apparently nickel bound and had low affinity for $C O(\mathrm{~km} \sim 5 \mathrm{mM}$ for dissolved $\mathrm{CO})$. This suggests that activity is in fact related to acetate metabolism. $O^{\prime} B r i e n$ and coworkers (1984) made a similar observation based on the fact that co dehydrogenase activity appeared to be constitutive and has a low affinity for co.

Kohler and zehnder (1984) demonstrated that acetate is activated by an acetate thiokinase rather than a phosphotransacetylase in Methanethrix soehngenii. The tentative pathway of acetate metabolism they proposed is similar to that reported for Methanesarcina species. M. seehngenii has high co hydrogenase activity suggesting a physiological role in production of methane from acetate (Kohler and zehnder, 1984).

Nelson and Ferry (1984) used two acetotrophic methanogens Methanosarcina strain TM-1 and Methanosaraina acetiverans, which are unable to utilize $\mathrm{H}_{2}$ and $\mathrm{CO}_{2}$ when grown on acetate, to investigate the presence of a $\mathrm{CO}$-dependent $\mathrm{CH}_{3}-$ s-CoM methylreductase system in the acetate-to-methane pathway. Both strains contained high amounts of co dehydrogenase activity. Co dehydrogenase activity for acetate-grown cells wer 96 and 40-fold greater than $\mathrm{CO}$ dehydrogenase activity in extracts of $\mathrm{H}_{2}-\mathrm{CO}_{2}$ grown Methanobacterium formicicum. No formate dehydrogenase activity was found in either acetotroph.

When acetotroph TM- 1 was cultured on $\mathrm{CH}_{3} \mathrm{OH}$, $\mathrm{CO}$ dehydrogenase activity was reduced to a level to $5 \%$ of that found in acetate-grown cells. More than one co dehydrogenase was observed present in $\mathrm{TM}-1$ indicating multiple physiological functions for this enzyme.

Eikmanns and Thauer (1985) demonstrated that low concentrations of isopropyl iodide inhibited all suspensions of $\mathrm{M}$. barkeri from forming $\mathrm{CH}_{4}$ from acetate. Inhibition was abolished by short exposure to light. This strongly indicates the involvement of a corrinoid enzyme in methanogenesis from acetate. The 
proposet role this erizyme is involvemer. in the methly grup transt. to CoM

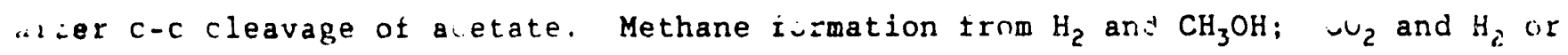
$\mathrm{CH}_{3} \mathrm{OH}$ was not inhibited by isopropyl iodide, even at 20 times the amount that Inhlbited wethanogenesis from acetate. They proposed acetate metabolisa to occur as follows:

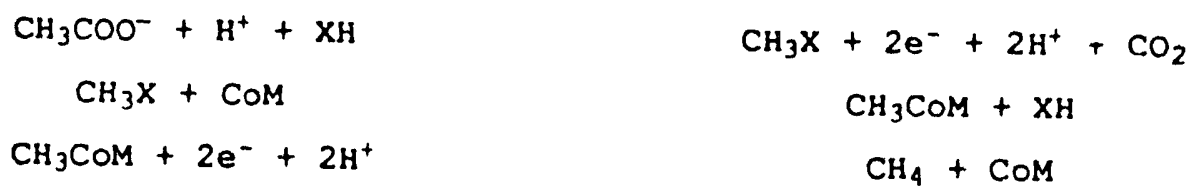

Equation (2) they concluded, must be reversible and the sum of several reactions:

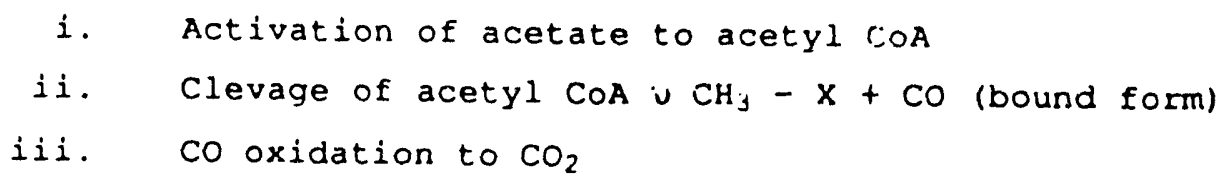

Smith, Lequerican and Hart (1985) reported that low concentrations if vaCN, 10 HM) sperifically inhibited methanogenesis from act ate, oxidation of. setat ethyl groups to $\mathrm{CO}_{2}$ and assimilation of one carbon units sn Methanczacina backexi 227. Oxidation of the methyl group from mishanol was not afiected by NaCN. Incorporation of methanol into cell cartinn was, lw,wever, inh-oited, by $\therefore$ aCN. It was suggested, based on these ubservatil, that :o dehydrogenase r. 39 a dual metabolic function. Namely, exidation nf the methyl group of acetate (but not $\mathrm{CH}_{3} \mathrm{OH}$ ) to methane, and tre synthesis of acetyl CoA for carbon asimilation from methanol and $\mathrm{CO}_{2}$.

\subsection{TOXICANTS IN ANAEROBIC SYSTEMS}

One of the major unknowns ir anaerobic treatment is how these systems respond to toxic or inhibitory shocks imposed by the presence of irganic or inorganic toxicants (Parkin et al., 1983). Most of the investigat Lons to date have examined the impact of toxicants on the : aerobic argestion of sewage 31 udges. Infortunately, little of this work has fald significant attention to she 
speciation and partitioning of these toxicants in the anaerobic environment to which they are applied.

\subsubsection{Organic Texicants}

The most frevalent identified organic toxicants repoxted as inhibiting anaerobic dijester performance are chlorinated compounds and formaldehyde (Swanwick et al., 1969). Swanwick and Foulkes (1971) found that like metals, chloroform can be concentrated in the sludges produced by wastewater treatment operations. Stickley (1970) reported that inhibition of digestion could occur as a result of influent sewage chloroform concentrations of $0.01 \mathrm{mg} / \mathrm{L}$.

of the chlorinated compounds found in sludge, chloroform is the one usually found at the greatest concentration (Stickley, 1970). Chloroform and carbon tetrachloride are the most toxic of the chlorinated methane analogs for both digester and rumen systems (Swanwick and Foulkes, 1971; Thiel, 1969; Rufener and wolin, 1968; Bauchop, 1967). Chloroform toxicity was observed to be a function of mass per mass loading (mg $\mathrm{CHCl}_{3} / \mathrm{kg} \mathrm{TS}$ ). Above $10 \mathrm{mg} / \mathrm{kg}$ severe inhibition was reported (Swanwick and Foulkes, 1971). Recent experimental work with chloroform has demonstrated that toxicity to an acetate enrichment culture is a function of toxicant dose, biomass concentration, sludge age, temperature, toxiciant exposure time and pattern of administration (Yang and Speece, 1986).

Inhibition of methanogenesis by chloroform was observed to be accompanied by a concomitant accumulation of hydrogen gas in both digester and rumen environments (Thiel, 1968; Bauchop, 1967). Scott et al. (1983) reported that when chloroform was added at dosages that resulted in arrest of methane production, quantifiable increases in dissolved $\mathrm{H}_{2}$ occurred. These results indicate that the toxic action is probably selective to the methanogenic population.

The only other organic compounds reported present in sludges at concentrutions that could potentially inhibit methanogensis, in addition to chloroform, are toluene (U.S.EPA, 1982) and formaldehyde (Swanwick et al., 1969). 
There are, of course, a variety of toxic compounds germane to certain industrial wastewater streams that could potentially cause inhibition or failure of a high rate in anaerobic treatment process. An excellent review of many of these compounds and their inhibitory or toxic levels was provided by Henze and Harremoes (1983).

\subsubsection{Inorganic Toxicants.}

Heavy metals can be present in significant concentrations in municipal sewage. They may originate from industrial, commercial and domestic sources (Davis and Jackson, 1975; Klein et al., 1974). In areas where combined sewers exist, stormwater can represent a significant source of heavy metals (Collins and Ridgeway, 1980). Metals are removed from municipal wastewater in both the primary and secondary treatment proce ses (Petrasek and Kugelman, 1983; Lester, 1983; Nielsen and hrudey, 1983). Significant concentration of these metals occurs in the sludges produced. Petrasek and Kugelman (1983) found that most metals were concentrated 10 to 30 fold in the sludges produced. High heavy metal concentrations in sludge could lead to upset of the sludge stabilization process (Russell et al., 1982) and limit the options available for the ultimate disposal of these sludges (Coker and Matthews, 1983; Spiegel et al., 1985). Anaerobic digestion will, in general, be the first process to exhibit a deterioration in performance due to heavy metals (Lester et al., 1983). Barth et al. (1965) folind that 89 percent of the zinc, 75 percent of the copper and 28 percent of the nickel entering a secondary wastewater treatment facility reached the anaerobic digesters.

In one extensive study of difficulties in anaerobic digester performance, Swanwick and co-workers (1969) found that heavy metal inhibition was the second leading cause behind inadequate design ur operation. zinc and copper were the only metals specifically cited as the causative agent of digester upset or failure.

One of the most important features which distinguishes heavy metals from other toxic pollutants is that they are not biodegradable (sterritt and Lester, 1980). Their potential toxicity is controiled, to a large extent, by the physical and chemical nature of the environment (Callander and Barrord, $1983 a$ b; Mosey et al., 1971). As for all ions, heavy metals can be 
stimulatory, inhibitory or toxic, depending upon the concentration (Kugelman and Chin, 1971). A trace level of many metals is required for activation or function of many enzymes and co-enzymes. Excessive amounts, however, can result in inhibition or toxicity. Heavy metal toxicity is believed to occur due to disruption of enzyme function and structure by binding with thiol and othe: groups on protein molecules or replacing naturally occurring metals in enzyme prosthetic groups (Vallee and Ellmer, 1982).

Bacteria can display two types of resistance to heavy metal inhibition: nonspecific resistance arising from differences in the physiological state of the organism and inheritable specific resistance factors (Sterritt and Lester, 1980). Immobilization of metals by extracellular polymers is an example of a nonspecific resistance.

Some bacteria have been shown to have active heavy metal detoxification mechanisms. Peterson et al. (1979) demonstrated that Micrococaus luteus produced 5 to 6 times the amount of lipids when exposed to lead. Lead was found to be predominantly partitioned into this lipid material. Klebsiella aerogenes accumulated significantly more intercellular sulfide as a response to exposure to heavy metals (Aiking et al.. 1985). Some bacteria can transform metals. Methanogenic bacteria, for example, have been found to be capable of methylating mercury as a method of protection (Wood et al., 1968).

A resistant stra:n or species can have an ameliorating effect on the inhibitory action of metals on a more sensitive organism. A strain of $\mathbf{E}$. coli, which had much greater resistance to mercuric chloride than $s$ aureus, reduced the effect of mercury on $S$. aureus by 50 percent when the two organisms were co-cultured in the presence of mercury (Stuzenberger and Bennet, 1965). In general these mechanisms are believed to be important only in cases in chronic presence of these toxicants.

Studies on the toxicity of heavy metals to anaerobic digestion have indicated considerable variation in the effect of individual metals based on total metal concentration (Kugelman and Chin, 1971). A major factor influencing the toxicity of heavy metals is the manner in which they are speciated and partitioned (Gould and Genetelli, 1975; Hayes and Theis, 1978). In general, the soluble form is thought to be the toxic species (Kugelman and chin, 1971). 
Insoluble forms are thought to be of much less toxicological significance (Hayes and Theis, 1978; Sterritt and Lester 1980).

Heavy metals may be precipitated in anaerobic environments as sulfides (except chromium (CR)), carbonates or hydroxides (Mosey et al., 1971; Callander and Barford, 1983b). They may also be bound to the sludge via ligands on the sludge surfaces. Metals may also be chelated and maintained in solution.

Chelation of metals can make them more accessible to cells in some instances and less available in other cases (Callander and Barford, 1983b). The degradation of a chelator or sludge ligands in the anaerobic digester environment could lead to mobilization or resolubilization of bound metals.

Hayes and Theis (1978) ranked toxicity of heavy metals to anaerobic digestion to be: $\mathrm{Ni}>\mathrm{Cu} \gg \mathrm{Cr}>\mathrm{Pb}>\mathrm{Zn}$. Based upon a literature survey, Anthony and Breimhurst (1981) recommended that the influent concentrations of metals in sewage entering a wastewater treatment plant be limited to the values detailed in Table 2-1 to avoid inhibition of the anaerobic sludge digestion process. In all cases, the level of metals in the influent required to produce inhibition was significantly lower for anaerobic digestion than for secondary treatment process.

\section{Limiting Influent Metal Concentrations (mg/I)}

\begin{tabular}{lcc}
\hline \multicolumn{1}{c}{ Metal } & $\begin{array}{c}\text { Inhibition of } \\
\text { Secondary Treatment }\end{array}$ & $\begin{array}{c}\text { Inhibition of } \\
\text { Anaerobic Digestion }\end{array}$ \\
\hline Cadmium & 2.1 & 0.63 \\
Chromium (total) & 12.5 & 1.1 \\
Copper & 1.3 & 0.42 \\
Lead & 3.8 & 2.8 \\
Nickel & 2.1 & 0.28 \\
Zinc & 4.0 & 2.4
\end{tabular}

Hobson and Shä $\{1376\}$ found that Methanobacterium formicicum. the primary hydrogen-utilizing methanogen isolated from an anaerobic digester treating 
piggery waste, was severely inhibited by total copper concentrations in excess of $200 \mathrm{mg} / \mathrm{L}$. The culture contained sulfide as a reducing agent at a calculated value of $66 \mathrm{mg} / \mathrm{L}$ (as S). Pankhania and Robinson (1984) cultured Methanospirillum hungatei CRI in the absence of reducing agents in TES buffer. Cadmium at $15 \geq M$ and $50 \geq M$ of copper, zinc or mercury completely inhibited methane production from $\mathrm{H}_{2} / \mathrm{CO}_{2}$. The activity of hydrogenase and formate dehydrogenase was significantly reduced after incubation with these metals. Formate dehydrogenase activity was inhibited more severely than hydrogenase activity. The relative reduction in enzyme activity produced by the various metals was $\mathrm{Cu} \simeq \mathrm{Hg}>\mathrm{Cd}>\mathrm{Zn}>\mathrm{Co}$.

Ashley et al. (1982) found that $1 \mathrm{mg} / \mathrm{L}$ of dissolved nickel in a digester severely inhibited methanogenesis. Increasing the dissolved nickel concentrations to $12 \mathrm{mg} / \mathrm{L}$ resulted in inhibition of many of the hydrolytic bacteria. Dosing with nickel was observed to result in a build-up of higher molecular weight acids. The proteolytic bacteria appeared to be the most resistant population to nickel.

Mosey et al. (1971) postulated that digestion failure due to heavy metals occurs only when their free ion exceeds some threshold value. Mosey and Hughes (1975) proposed it is possible to express the toxicity of heavy metal ions in terms of the pS of digesting sludge (pS $=-\log _{10} \mathrm{AS}^{-2}$, where $\mathrm{A}$ is the activity). A pS value of 14.0 was shown to indicate the presence of inhibitory concentrations of the heavy metals $\mathrm{zn}, \mathrm{Cu}, \mathrm{Fe}$, and $\mathrm{Cd}$. Mosey (1976) further proposed that toxicity of metals is related to an adsorptive/ binding constant $K$ that can be expressed as an equivalent weight of metal per mass of solids (meq/kg TS). When the metal load exceeded 400 meq/ $\mathrm{kg}$ digester failure was reported possible. At $800 \mathrm{meq} / \mathrm{kg}$ digester failure is almost certain. 
CHAPTER 3

EXPERIMENTAL APPROACH AND DESIGN

\subsection{TECHNICAL APPROACH}

This research effort was designed to monitor the belavior of model, Waste Activated Sludge (WAS) fed, laboratory anaerobic digester systems during steady state and in response to toxic shocks, organic overloads and hydraulic overloads. Shocks of both failure and sub-failure proportions were examined.

Both continuous (or semicontinuous) and batch techniques have been used to evaluate toxicity and biodegradability. The continuous procedures closely simulate full-scale and anaerobic operation. However, they are more costly in terms of facilities, time, equipment and personnel than batch bioassay techniques. Batch bioassay techniques, by contrast, can be used to evaluate the influence of various parameters, but in general, do not simulate the response of full-scale systems as well as continuous assays. Nonetheless, batch tests are useful for sorting out the important variables. In this study, therefore, a combination of semi-continuous (batch fed daily) digesters in conjunction with batch serum bottle assays were utilized. The results of the batch assays are the subject of this report. In an effort to ensure that results from each individual experimental run were comparable, the following approach was taken.

\subsection{EXPERIMENTAL DESIGN}

Briefly, there were three major components or tiers in the experimental design, they were:

- Construction and steady state operation of reserve digesters (inocula source)

- Serum bottle assays (batch tests)

- CSTR test digester on-line experimentation

Two reserve digesters were constructed and brought to steady state operating conditions. These digesters were maintained at steady state throughout the duration of the experimental program. Portions of the reserve digester volume, less than or equal to one day's feed volume (5.5 liters), were 
periodically removed to start the serum bottle assays. This approach allowed a continual supply of relatively uniform biomass to be available on demand throughout the study. Once the inocula was subjected to a toxicant and the necessary data obtained, the contents were discarded and after thorough cleaning replaced with fresh inocula from one of the reserve digesters. This approach not only streamlined data collection, but also provided better assurance that data collected in each individual run could be directly compared to other runs since the inocula for all tests came from the same source that had no prior history of exposure to the toxicants or were in any way altered by prior testing (i.e., bacterial population changes).

A more detailed description of the entire experimental design and testing schedule follows.

\subsubsection{Reserve Digesters}

Two reserve digesters with working volumes of 55 and 110 liters, constructed from 12 inch schedule 80 PVC pipe and $3 / 4$ inch PVC stock, were used to supply inocula with 10- and 20-day hydraulic retention times (HRT), respectively. A schematic of the 20-day HRT (110 liter) digester is presented in Figure 3-1. To avoid any influence due to temperature variation, reactors were housed in constant temperature incubators maintained at $35 \pm 0.5^{\circ} \mathrm{C}$. This is near optimal temperature for mesophilic methanogenic cultures.

3.2.1.1 Feedstock. WAS was obtained from the return sludge line at the Amherst, MA, Publicly Owned Treatment works (POTW). The total solids concentrations varied from 0.6 to 0.9 percent. The volatile fraction generally varied from 80 to 85 percent. This was mixed with the more concentrated sludge from the dissolved air flotation (DAF) unit $(3.0$ to 4. percent solids) to obtain a constant feed solids concentration of 1.25 to 1.5 percent total solids. In addition to the thickened WAS, the DAF-sludge contained skimmings from the primary and secondary clarifiers.

Sludge samples were analyzed for Total Solids (TS) and Volatile solids (VS) concentrations, mixed in the proper portions in a 150 liter mixing tak and then stored in 13.25 litex plastic containers. The 13.25 liters represented enough feedstock for any one day's experimentation. By providing a separate 


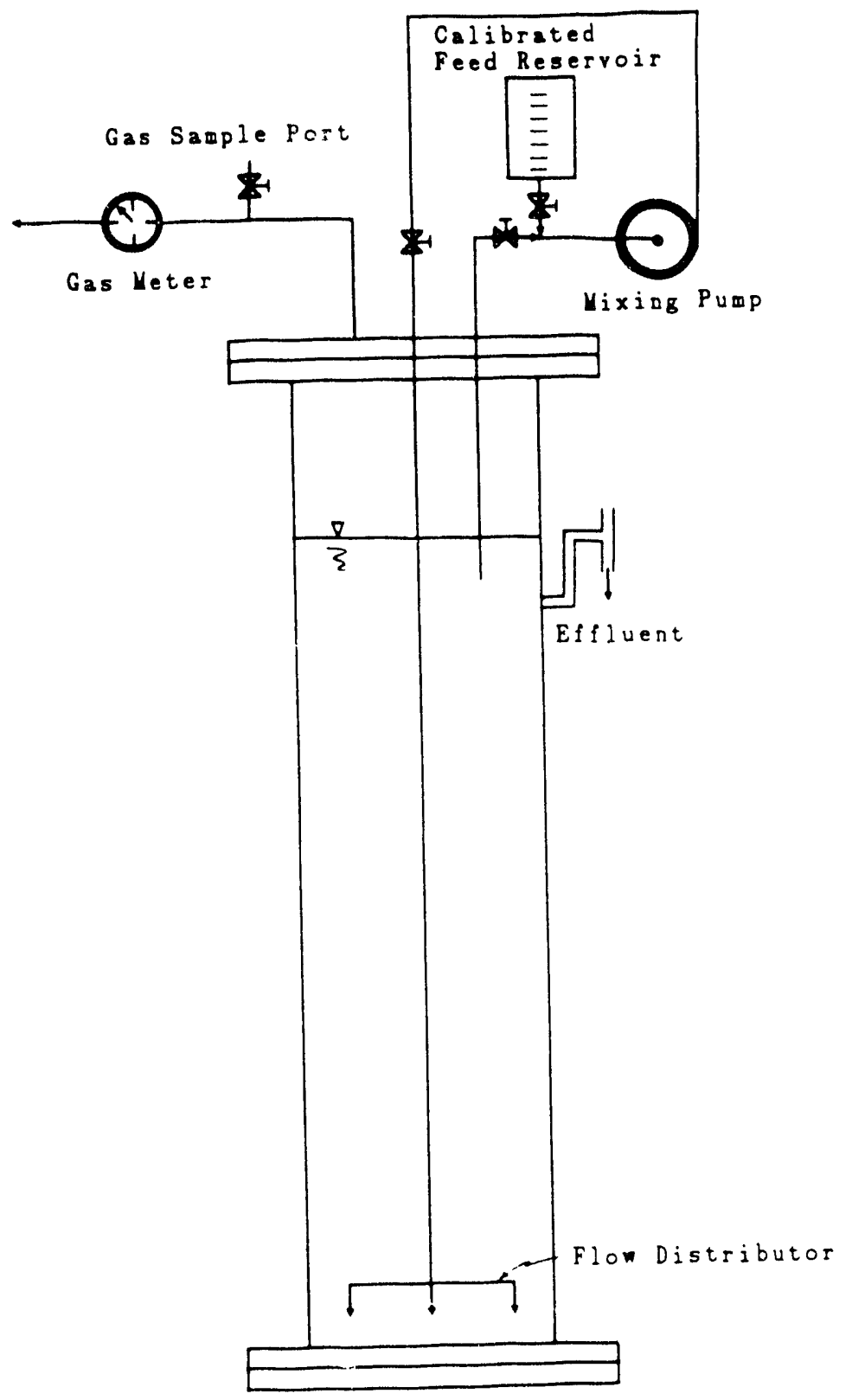

化 - Shutoff Valves

Figure 3-1. Schematic representation of the 20-day HRT reserve digester. 
storage container for each day's feed, the consistency of the feeds was assumed to be more uniformly maintained. The mixed sludge was screened through $1 / 16$ th inch mesh to remove any large particles that could potentially interfere with the digester mixing equipment.

Fresh WAS was collected at weekly intervals while DAF-solids were, on the average, collected every two weeks. WAS and DAF-sludge and the mixture of the two were stored in a $2-4^{\circ} \mathrm{C}$ incubator. Prior to feeding, the mixed sludge was removed from the digester and allowed to equilibrate to room temperature. In those instances when not even a slight change in temperature could be tolerated, the feed sludge was placed in a $35^{\circ} \mathrm{C}$ incubator for approximately 30 minutes prior to feeding.

3.2.1.2 Start-Up and Acclimation. The seed for the reserve digesters was obtained from municipal digesters located in Northampton, MA. These digesters receive a combination of primary and waste activated sludge and were operating at an HRT of between 15 to 20 days at the time the sludge was obtained.

A volume of digester sludge, equaling the working volume of the reserve digesters, was placed in the reactors after careful screening with $1 / 16$ to $1 / 32$ inch mesh to remove any large debris. Gas production rate was monitored over the next day to ensure an active biomass had been obtained. The 10- and 20-day HRT reserve digesters were then fed the volume of sludge ( 5.5 liters) required to maintain the proper residence times. The reserve digesters were maintained mixed by a liquid recirculation system that withdrew sludge just below the liquid/gas interface and returned it to the bottom of the reactor through a three nozzle distributor. Mixing was performed with Manostat Varistaltic pumps operated on a timer system. Pumps were cycled in an on/off mode of 15-minute intervals except during daily feeding and 12 hours after feeding. The pumping system was timed to stay on continuously for 45 minutes prior to feeding sludge and for an additional 45 minutes subsequent to feeding. A 45-minute continuous mixing period was employed 12 hours after daily feeding. Flow through the reactors was maintained at approximately 80$90 \mathrm{~m}^{3} / \mathrm{m}^{2}-\mathrm{d}$, which appeared sufficient to keep the reactor contents well mixed. Initially, silicon tubing was used in the Manostat pumps. Later a 
change was made to norprene tubing which had a longer life and much lower permeability to oxygen.

Gas production was monitored with wet Tip Meters (Wet Tip Meter Co., Wayne, PA). Gas composition was assayed by gas chromotography procedures which are detailed later in this chapter and in Appendix $A$.

\subsubsection{Daily Schedule.}

Briefly, the schedule followed the framework listed below:

A. Sludge was be removed from the $2^{\circ}-4^{\circ} \mathrm{C}$ storage refrigerator and equilibrated to room temperature.

B. An equipment survey followed, during which the operational status of digester and analytical equipment was checked, readings taken, maintenance performed (if required) and gas samples taken for analysis.

C. The digesters were then batch fed room-temperature-equilibrated sludge. The waste aliquot drawn from the digesters was assayed for parameters listed in the monitoring frequency schedule (Table 3-1).

\section{2 .2 Batch Sexum Bettle Assays}

Batch serum bottle assays were used to determine the concentrations of the organic and inorganic toxicants necessary to impose inhibitory and lethal effects on the 10- and 20-day HRT inocula. The impact that the toxicants had on the gas indicators of interest (Co and $\mathrm{H}_{2}$ ) was also preliminarily assessed at this time.

\section{2 .21 Inerganic Toxicants. Three heavy metals were tested for their} inhibition potential. These metals were zinc, copper and cadmium. They were selected based on their typically being fresent in the highest concentrations in sludges (Lester, 1983; Fetrasek and Kugelman, 1983; Nielsen and Hrudey, 1983: Spiegel et al., 1985), their relative solubility in comparison to most transition heavy metals in the reduced anaerobic environment present in digesters, as well as their relative reported incidence of inhibiting full scale digesters (Swanwick, 1969). Literature values (where available) and preliminary assays were used to estimate the dosage of a particular toxicant that was required to induce inhibition and lethal effects on the inocula. 
Table 3-1 Monitoring rrequency for Reserve Digesters

$\begin{array}{ll}\mathrm{CH}_{4} & 5 / \text { week } \\ \mathrm{CO}_{2} & 5 / \text { week } \\ \mathrm{H}_{2} & 5 / \text { week } \\ \mathrm{CO} & 5 / \text { week } \\ \mathrm{pH} & 7 / \text { week } \\ \text { Alkalinity } & 5 / \text { week } \\ \text { Volatile Fatty Acids } & 5 / \text { week } \\ \text { Total Solids } & 1 / \text { week } \\ \text { Volatile Solids } & 1 / \text { week } \\ \text { CoD } & 1 / \text { week } \\ \text { SCOD } & 1 / \text { week }\end{array}$

Using these values, the experimental design presented in Table 3-2 was employed to define the actual effect of the respective metals for the 10- and 20-day HRT digester sludge utilized in this study. The experimental design allowed over a 10 fold range of toxicant concentrations to be examined while maintaining the number of samples to a manageable level for the frequent sampling schedule employed. In those cases deemed appropriate, volatile fatty acids were analyzed at or near the conclusion of the assay. Total solids (TS) and volatile solids (VS) of the inoculum were analyzed at the start of each assay run so that all runs could be compared on a rational basis $\mathrm{kml}$ gas produced/mg Vs-time). In general, controls were run in triplicate and all other samples in duplicate.

$3.2,2,2$ erganic Texicants. The inhibitory action of organic toxicants tends to be selective towards one or more population in the anaerobic consortium. 
It is possible, therefore, to select compounds that are specific for the methanogenic population. Chlorinated methane analogs are known to be potent inhibitors of methanogenesis (Bauchop, 1967; Thiel, 1969). From this group, chloroform was selected.

One recent study demonstrated that monochloroacetate significantly destabilized laboratory anaerobic digesters at applied dosages of 200 and 500 $\mathrm{mg} / \mathrm{L}$ (Ashley and Hurst, 1981). Trichloracetic acid can, under certain conditions, be produced by chlorination of humics and lignin (Reckhow and Singer, 1984; Lindstrom and Ostenberg, 1986). Although there is no substantial body of literature to support the contention that chlorinated acetate analogs may be selective toxicants toward the acetate utilizers, trichloroacetic acid was selected as a toxicant under the premise that it might do this.

Other organic toxicants selected for study in the batch serum bottle assay segment of the experimental program were 2-bromoethanesulfonic acid (BES) and formaldehyde. BES is a structural analog of coenzyme $M$ and specific inhibitor of methanogenesis (Balch and Wolfe, 1979a). It was selected as a benchmark to compare the results of the less specific inhibitors. Formaldehyde was selected based on its widespread industrial use and demonstrated ability to inhibit methanogenesis (Parkin and Miller, 1982). Organic toxicant assays were conducted according to the experimental design presented in Table 3-2.

\subsection{METHODS}

\section{3 .1 Sexum Bottle Assays}

The protocol used was an adaptation of the serum bottle technique developed by Mi:ler and Wolin (1974) and later modified by Owen et al. (1979). Due ts differences in the physical and chemical nature of the various organic and inorganic toxicants that were examined, the manner in which the various toxicants were administered varied. In all cases, the experimental procedure was designed to simulate as closely as possible the real world situicion. A complete description of the serum bottle assay protocol is provided in Appendix A. 


\section{Typical Expeximental Design for serum Bottle Asarys}

\begin{tabular}{ccc}
\hline Serum Bottle Series & Toxicant Dosage \\
& $(\mathrm{mg} / \mathrm{L})$ & \\
\hline 1 & $0.25 \mathrm{x}$ & \\
2 & $0.50 \mathrm{x}$ & 100 \\
3 & $0.75 \mathrm{x}$ & 100 \\
4 & $1.0 \mathrm{x}$ & 100 \\
5 & $1.5 \mathrm{x}$ & 100 \\
6 & $3.0 \mathrm{x}$ & 100 \\
7 & 0 & 100 \\
\hline
\end{tabular}

a/X - represents dosage obtained from literature or preliminary assays that is likely to cause approximately $50 z$ inhibition of methane production.

$b / s l u d g e$ removed from the seed digesters and mixed with the appropriate amount of WAS (i.e., for 10-day HRT digester samples $90 \mathrm{ml}$ of inoculum and $10 \mathrm{ml}$ of WAS was used).

\section{3 .2 Gas Analyses}

Methane and carbon dioxide concentrations were routinely measured via thermal conductivity gas chromatography (Gow-Mac model 550). Separation was accomplished with a $2 \mathrm{~m} \times 4 \mathrm{~mm}$ I.D. porapak $Q$ column using helium as a carrier. operating conditions and procedures are detailed in Appendix $B$. Area integration, provided by an HP-3390A integrator, was used to quantify $\mathrm{CO}_{2}$ and $\mathrm{CH}$, concentrations.

Trace concentrations of hydrogen and carbon monoxide were analyzed with a RGD2 mercury reduction chromatographic system (Trace Analytical, Stanford, CA) equipped with a dual $0.1 \mathrm{ml}$ sample loop. Separation was achieved with a molecular sieve $5 \mathrm{~A}$ column using catalytically purified nitrogen as a carrier. Operating conditions and procedures are detailed in Appendix $\mathrm{C} . \mathrm{H}_{2}$ and $\mathrm{CO}$ were quantified by peak height analysis. 
Reserve digester gas production was measured with Wet Tip Meters (Wet Tip Meter Co., Wayne, PA). These meters functins on a liquid displacement principle.

\section{3 .3 Volatile Eatty Acids Determination}

Daily tracking of reserve digester volatile fatty acids was performed using the boiling technique of O'Brien and Donlan (1977). Speciation of the volatile acids at the conclusion of serum bottle assays and during test digester operation was accomplished with gas chromotography. A Varian 3700 equipped with a Flame Ionization Detector (FID) was used for the analysis. Operating conditions and procedures are detailed in Appendix $D$.

Samples analyzed for volatile fatty acids were centrifuged (2000g) and frozen immediately after they were removed from the serum bottles or test digesters if they were not to be analyzed that day.

\section{3 .4 Additional Analyses}

Chemical oxygen Demand (COD) determinations were performed according to the technique developed by Knetchel (1978) and now accepted by Standard Metheds (1985). Solids determinations (TS and TVS) were performed according to Standard Metheds, 26th edition, (1985). A Fisher Accumet pH meter equipped with a combination electrode was used to determine $\mathrm{pH}$ of the reserve and test digesters as well as serum bottle samples. All wet chemical analyses were run in duplicate with standards curves (when approprizte) being developed for each batch of reagants prepared.

Metal analyses were performed with a Perkin-Elmer Model 403 atomic adsorption flame ionization unit, optimized according to the standard operating guide. Samples were centrifuged at $2000 \mathrm{~g}$ for 15 minutes and then filtered through 0.45 um pore filters prior to analysis.

\section{3 .5 evality centrol of Gas Analysis}

External gas staidards were used to daily calibrate both the Gow-Mac and Trace Analytical gas chromatograpis. Experieace has shown that methane and carbon dioxide response is extremely linear for the operating conditions chosen. 


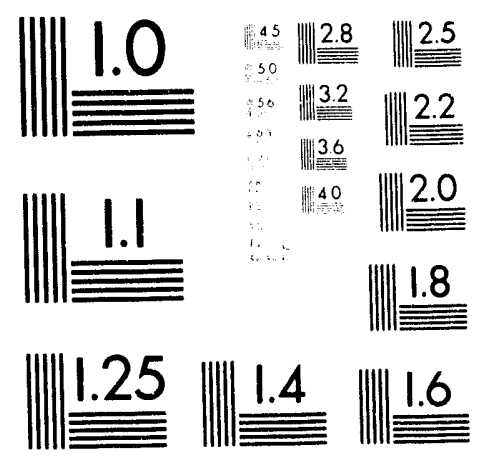



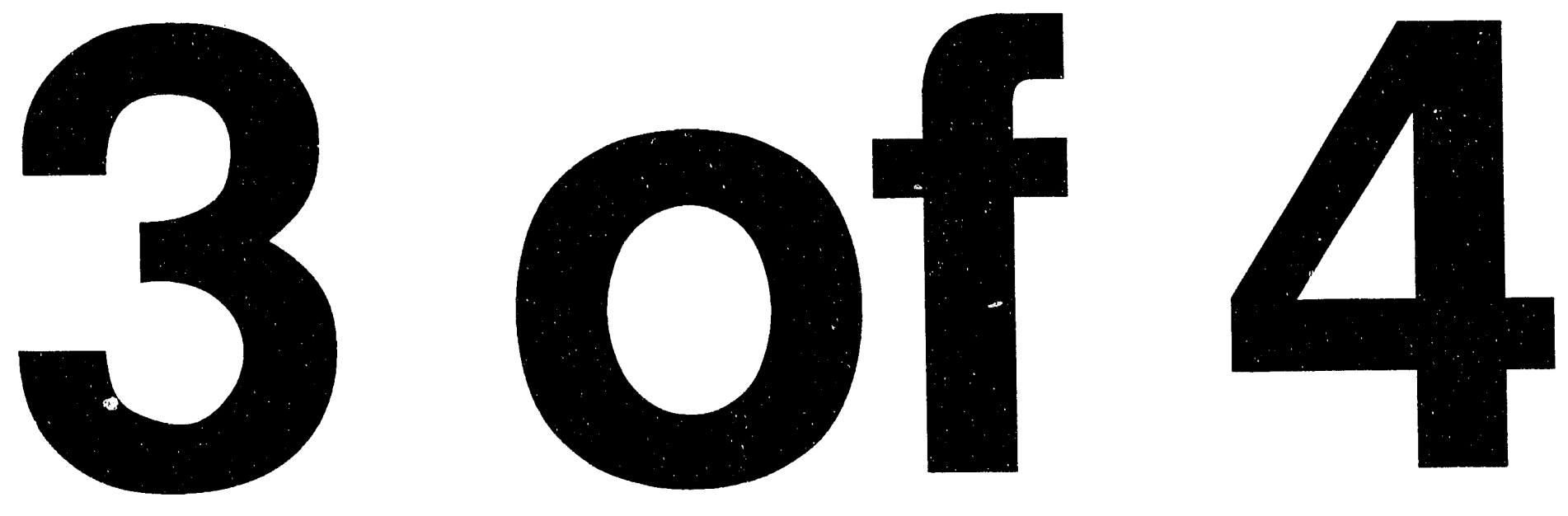
Area integration of a minimum of three sequential injections of pure $\mathrm{CH}_{4}$ and $\mathrm{CO}_{2}$ gas was used to define the area/count ratio for each gas. Periodic checks to verify the linear response were made.

Similarly, the Trace Analytical system was calibrated on a daily basis using primary standard mixtures of the respectire gases. Linearity was periodically checked using two standards with a five fold difference in gas concentration as recomended by the manufacturer. Details of the precision and quality control procedures are presented in Appendices $B$ and $C$. 


\section{CHAPTER 4}

EXPERIMENTAL RESUITS - THE EFFECTS OF ORGANIC TOXICANTS ON METHANE PRODUCTION AND TRACE GAS CONCENTRATIONS

Presented in this chapter are results generated while investigating the impact of pulse addition of four organic toxicants (chloroform, trichloroacetic acid (TCAA), bromoethanesulfonic acid (BES) and formaldehyde) on anaerobic sludge digestion. BES was selected for study since it selectively inhibits methanogens (Balch and Wolfe, 1979a) and would serve as a benchmark by which to compare the results generated with less specific inhibitors. Chloroform and formaldehyde were chosen based on their widespread industrial use and demonstrated ability to inhibit methane production (Parkin and Miller, 1982 ). TCAA was chosen to investigate the possibility that it would selectively inhibit acetate-catabolism as well as the recent findings that it may be one of the major halogenated products formed during chlorination of humic and lignin materials (Reckhow and Singer, 1984; Lindstrom and Osterberg, 1986).

\subsection{RESULTS OF BATCH ASSAYS}

\subsection{1 chloroform}

Chloroform dosages ranging from 0.15 to $2.0 \mathrm{mg} 1^{-1}$ were examined. The actual concentration of chloroform in a sample is different from the dose applied due to volatilization and/or possibly biodegradation. As will be discussed later, this is not believed to have altered results significantly in this study. Since the concentrations of chloroform and the other toxicants were not monitored during the assays, data will be discussed in relation to applied dose.

When chloroform was dosed at $1.0 \mathrm{mg} 1^{-1}$ or greater, methanogenesis was completely inhibited (Figure 4-1). At applied dosages of 0.15 and $0.50 \mathrm{mg}$ 1-1, methane production was also significantly but not completely inhibited. The $0.5 \mathrm{mg} 1^{-1}$ dose resulted in methane production being retarded by 85 to 90 percent compared to controls. Methane production quickly dropped to this rate and remained there for the remainder of the 24-hour assay (Figure 4-2). 


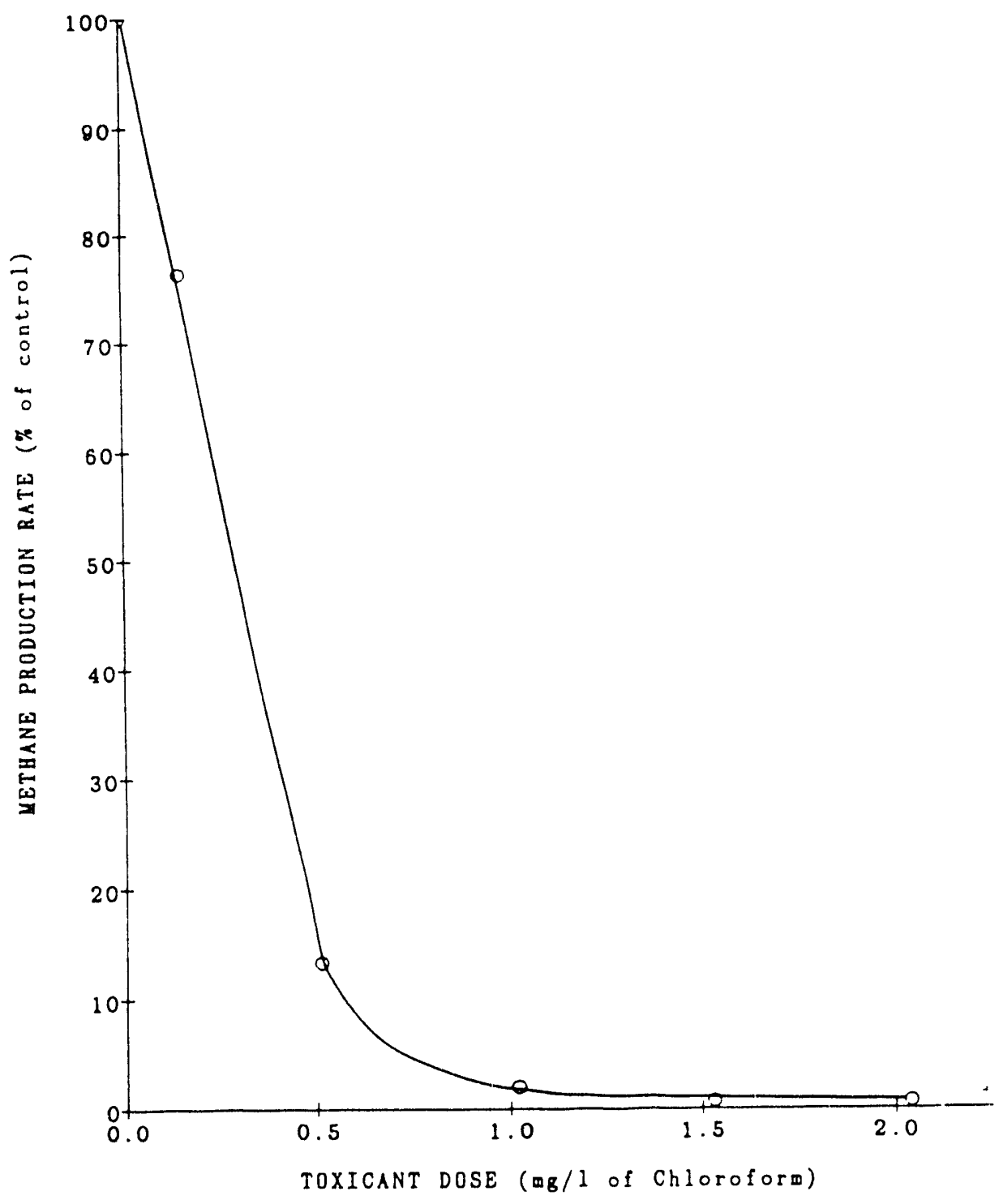

Figure 4-1. Effect of chloroform on cumulative 24-hour methane production. 


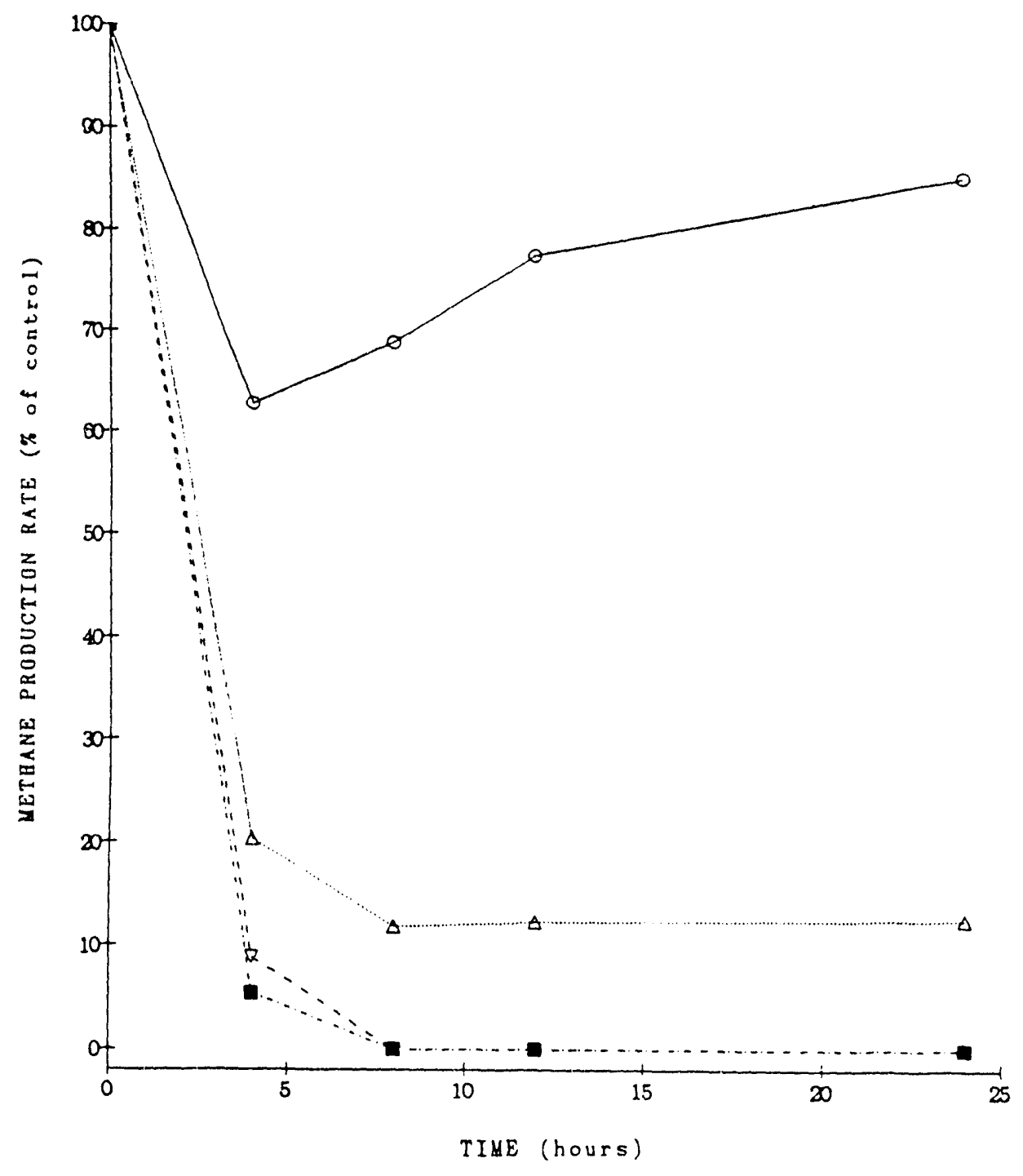

$\begin{array}{ccc}-\infty & 0.15 \mathrm{mg} / 1 & C h l o r o f o r m \\ \cdots \cdots \cdots & 0.51 \mathrm{mg} / 1 & C h l o r o f o r m\end{array}$

$-\nabla-1.02 \mathrm{~m} / 1$ Chloroform

…... $2.04 \mathrm{mg} / 1 \mathrm{Chloroform}$

Figure 4-2. Methane production rate as a function of time and appliad chloroform dose. 
Partial recovery was observed for the lowest dose applied (0.15 $\left.\mathrm{mg} 1^{-1}\right)$ after the initial 4 hours of incubation. During the final 12 hours of the assay, methane production averaged 85 percent of that observed for control samples.

Two distinct responses in headspace hydrogen gas concentrations were observed as a result of chloroform addition. The response was apparently dose dependent (Eigure 4-3). When chloroform was applied at $1.0 \mathrm{mg} 1^{-1}$ or more, a rapid accumulation of hydrogen in the headspace was observed. Within 4 hours, hydrogen exceeded $1000 \mathrm{ppm}(0.1 \%)$ for samples dosed with $1.0,1.5$, and $2.0 \mathrm{mg}$ $1^{-1}$ chloroform. Although no attempt was made to accurately quantify hydrogen levels once they exceeded this level, chromatograms taken past this point in time indicated hydrogen continued to rise substantially during the entire 24-hour assay in all three sets of samples. Samples receiving chloroform at dosages of 0.15 and $0.5 \mathrm{mg} 1^{-1}$ exhibited a hydrogen pattern similar to the control samples but at higher concentrations.

Volatile fatty acids were analyzed for samples sacrificed at the conclusion of the 24-hour assay. The analyses showed accumulation of acetate and propionate in all samples except in controls and samples dosed with $0.15 \mathrm{mg} 1^{-1}$. Lesser amounts $\left(\zeta 20 \mathrm{mg} 1^{-1}\right)$ of $n$-butyrate, iso-butyrate and iso-valerate were also detected in inhibited samples (Table 4-1).

The addition of chloroform produced a rapid increase in headspace co concentration. Measured Co concentrations rose well into the ppm range within 30 minutes for samples dosed $1.0 \mathrm{mg} 1^{-1}$ chloroform or more (Figure 4-4). The co rise appeared to be directly related to applied dose. Subsequent to the initial rapid increase in $c 0$, the headspace concentration leveled off, and in some cases decreased slightly over the next few hours. co concentrations subsequently rose in all except control samples at a slow, steady pace. Samples dosed with $0.15 \mathrm{mg} 1^{-1}$ chloroform displayed a steady increase in co over the initial 12 hours of the assay followed by a leveling off during the final 12 hours.

\section{1 .2 Bromoethanesulfonic Acid}

Bromoethanesulfonic acid (BES) is a structural analog of coenzyme $M$ and a specific inhibitor of methanogenesis (Balch and Wolfe, 1979a). Applied 


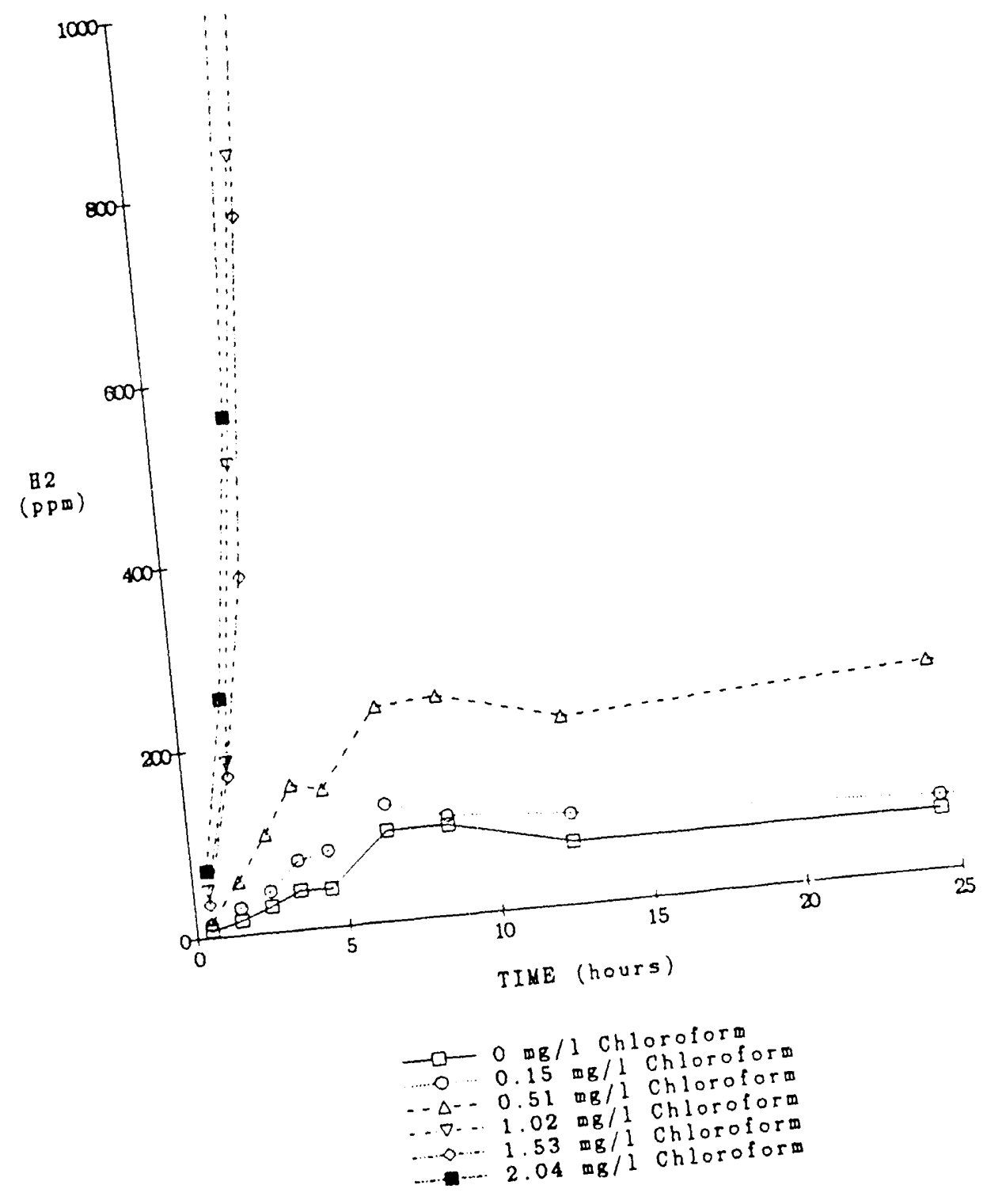

Figure 4-3. Hydrogen accumulation as a function of time
and chloroform dose. 


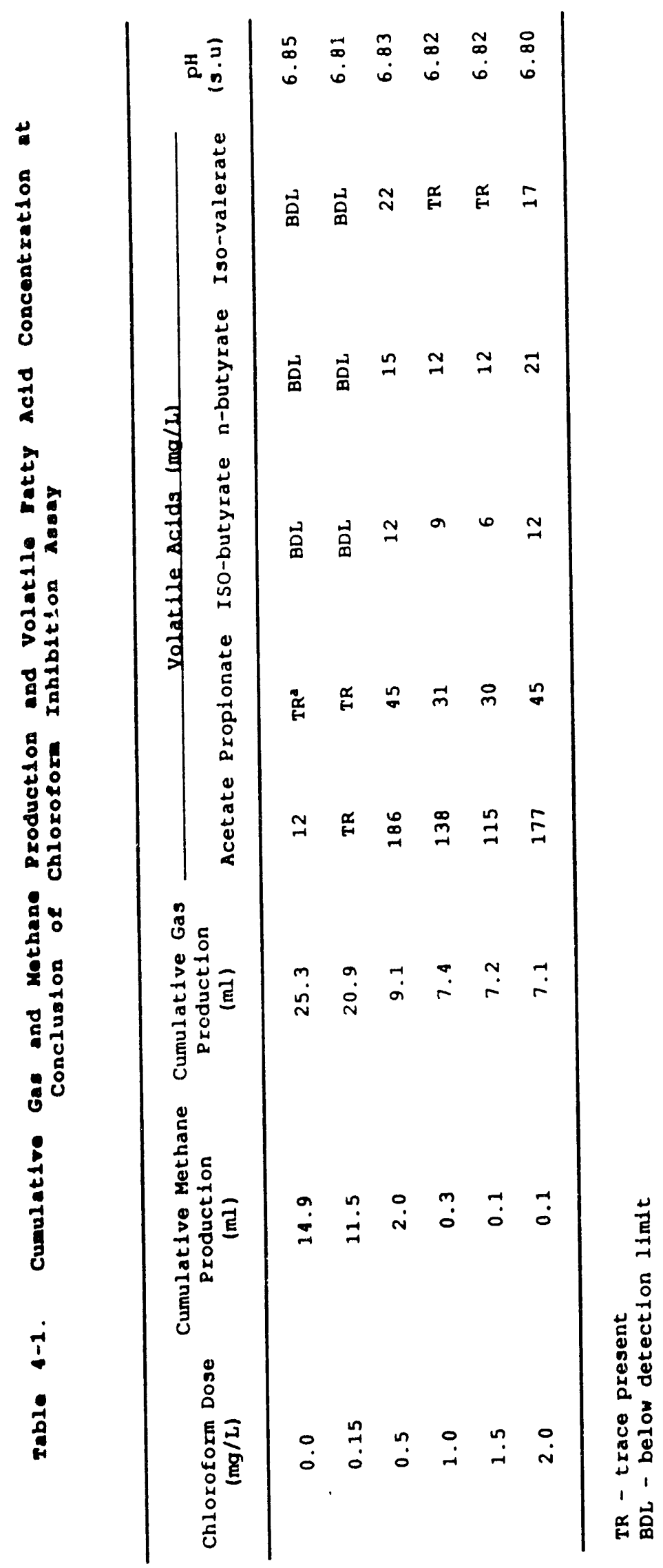




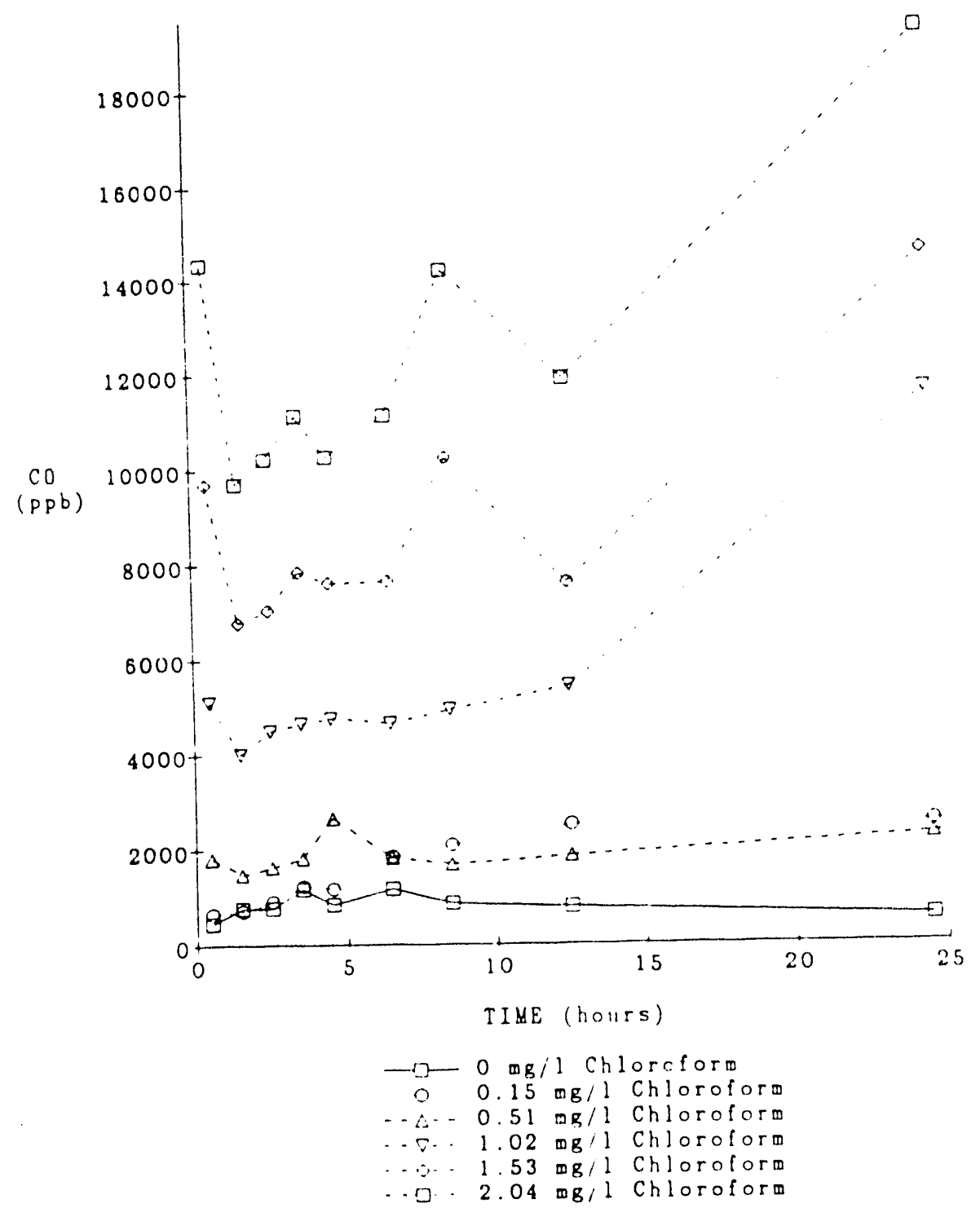
Figure 4-4. Carbon monoxide accumulation as a function
of time and applied chloroform dose. 
dosages of between 1.0 and $50.0 \mathrm{mM}$ BES wexe examined. The effect of this inhibitor on cumulative 24 -hour methane and total gas production, as a function of dose, is summarized in Table 4-2. Methane production rates, examined on a 4-hour interval basis (Eigure 4-5), declined continually during the assay. At the conclusion of 24 hours, samples receiving BES at dosages 5 $\mathrm{mM}$ or greater exhibited essentially complete inhibition of methanogenesis. No recovery was observed for any dosage over the 24-hour assay observation period.

The two distinct, dose dependent hydrogen gas responses, observed during the chloroform assay, were also observed for BES (Figure 4-6). Samples where methane

production was severely inhibited (< 30 percent the rate of controls) demonstrated a rapid rise in headspace $\mathrm{H}_{2}$ up to the percent range while samples that were inhibited less than 70 percent exhibited a response pattern similar to but higher in concentration than control samples. The higher the BES dose, the greater the hydrogen accumulation in the headspace.

Acetate accumulation appeared to be directly related to level of inhibition of methanogenesis (Table 4-2). Accumulation of butytate or other higher molecular weight VFAs in any of the samples remained restricted to levels below $20 \mathrm{mg} 1^{-1}$.

Co did not accumulate significantly above control values for samples dosed with $5.0 \mathrm{mM}$ BES or less. A steady, dose related increase in co for samples receiving greater than $5.0 \mathrm{mM}$ BES was, however, evident (Figure 4-7). Samples receiving $50 \mathrm{mM}$ BES demonstrated the most rapid increase in gaseous $\mathrm{CO}$. Within 12 hours co concentrations had reached $2800 \mathrm{ppb}$. Co did not increase further during the final 12 hours of the assay. This phenomenon will be addressed later. Other samples dosed with between 5.0 and $20.0 \mathrm{mM}$ BES were observed to have a steady, slow rise in gaseous co over time. In no sample was co observed to accumulate above the $2800 \mathrm{ppb}$ level. 


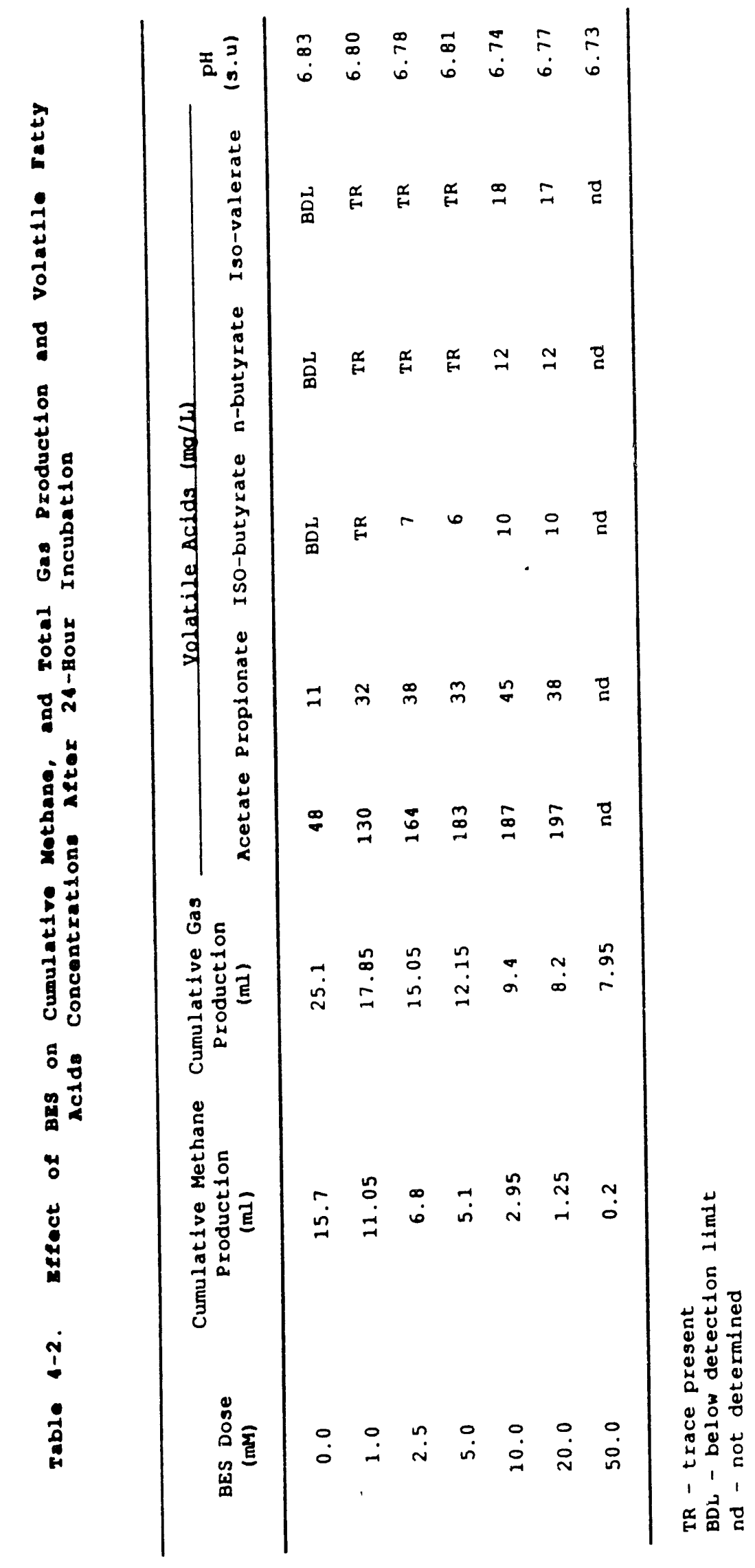




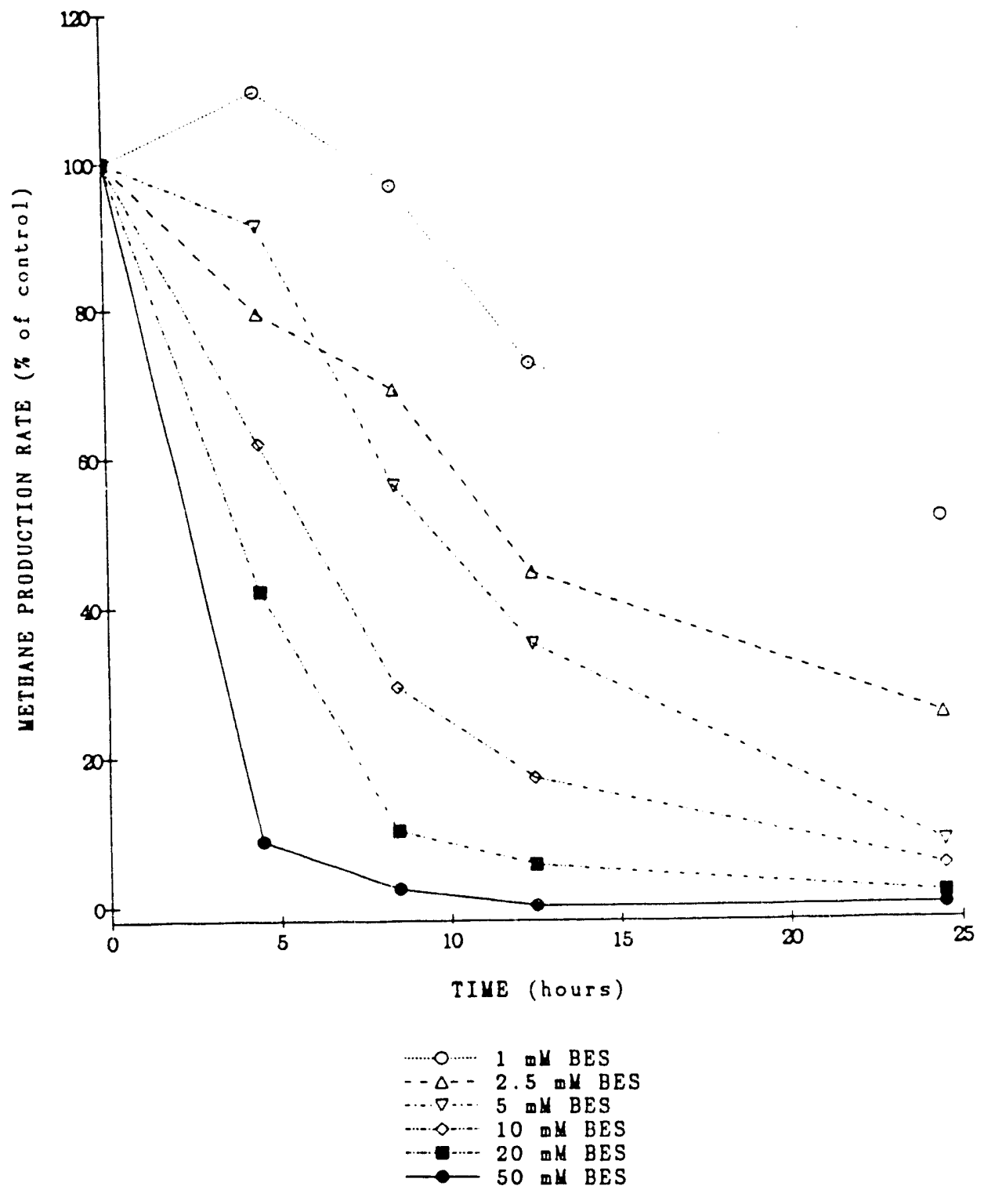

Figure 4-5. Methane production rate as a function of time and BES dose. 


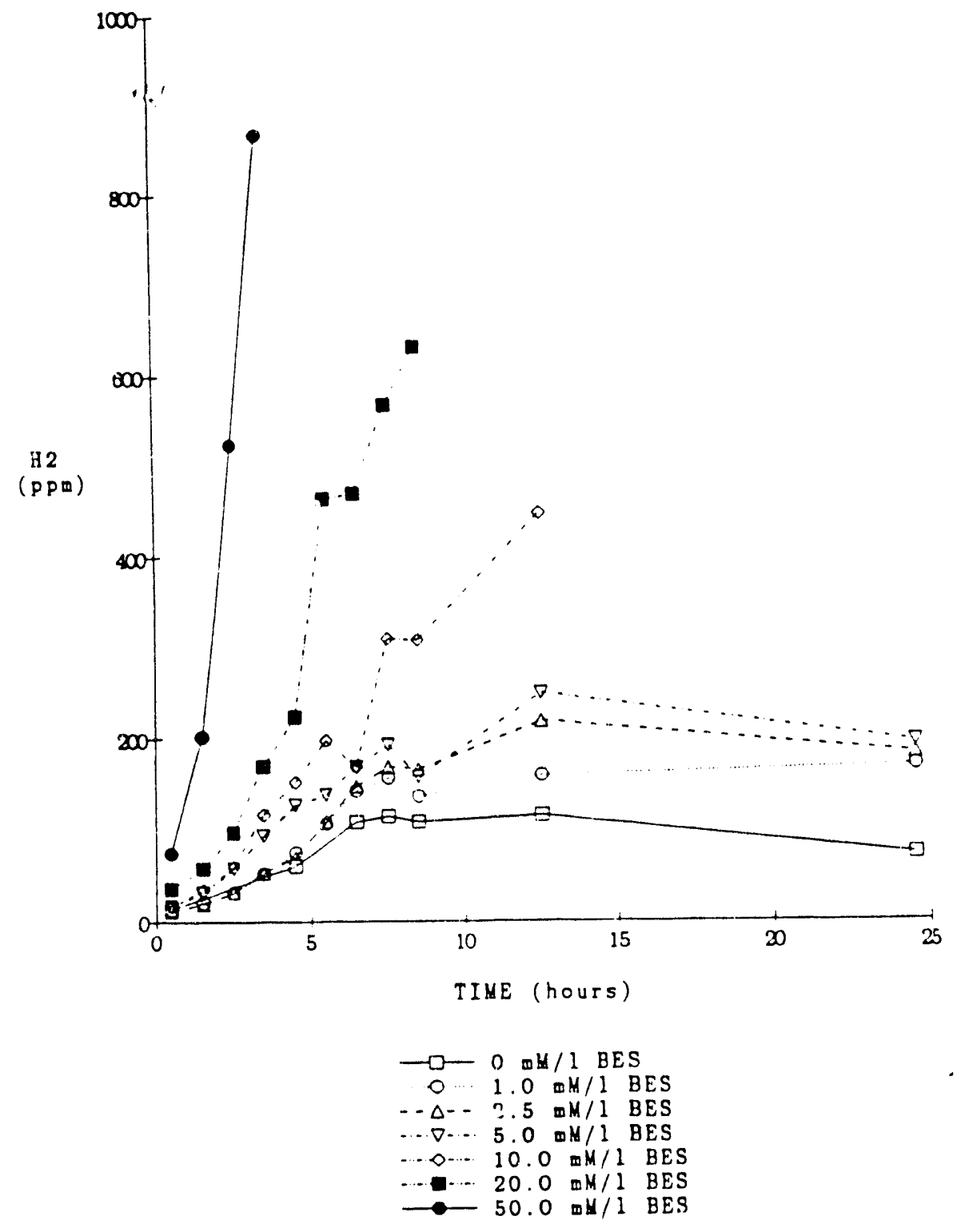

Figure 4-6. Hydrogen concentration as a function of time
apd BES dose. 


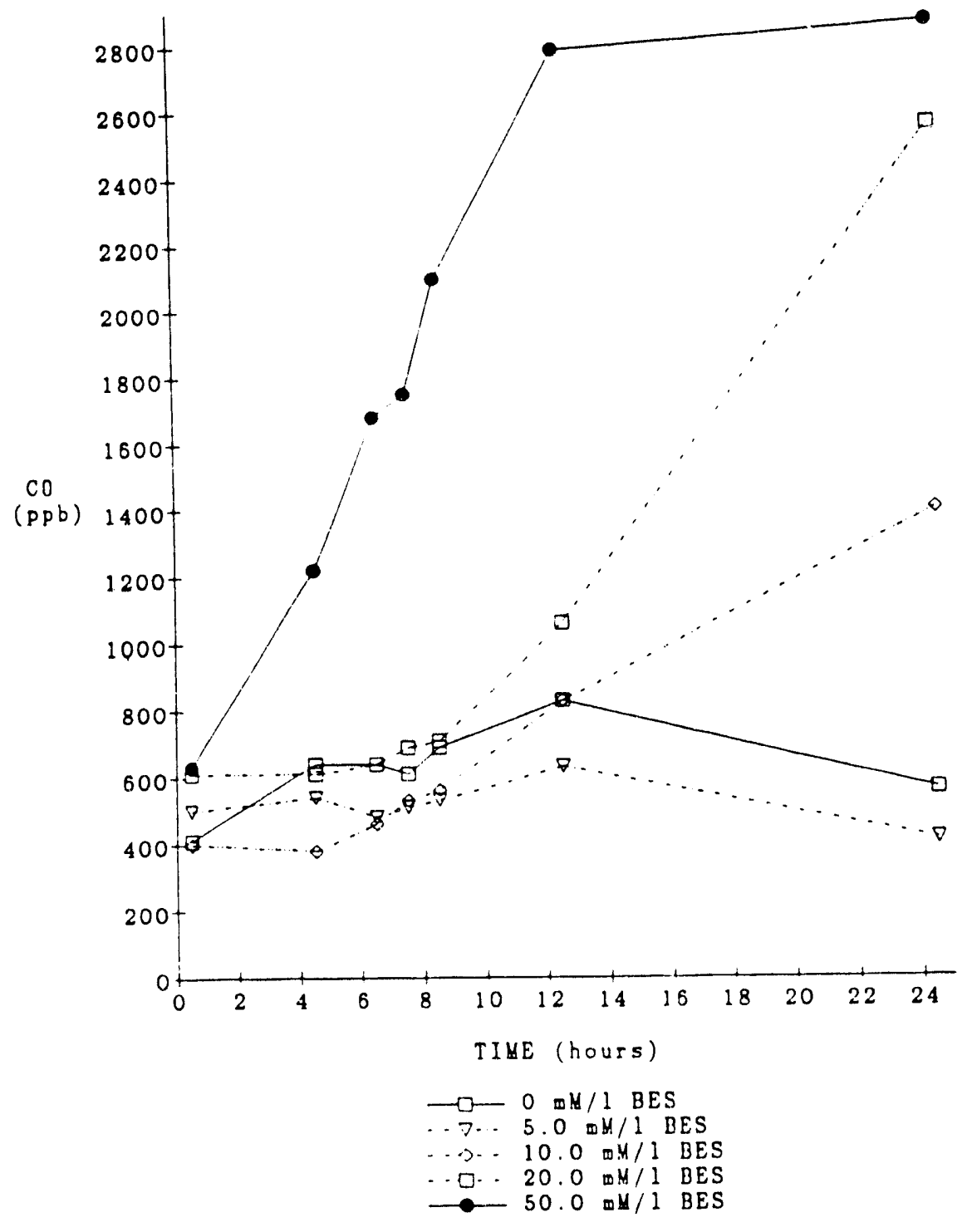
Figure 4-7. Carbon monoxide concentration as a function
of time and BES dose. 


\section{1 .3 Irichloroacetic Acid}

The effect of trichloroacetic acid (TCAA) was examined at concentrations ranging from 6 to $300 \mathrm{mg} \mathrm{1^{-1 }}$. The effect of TCAA on cumulative and 4-hour interval methane production is presented in Table 4-3 and Figure 4-8, respectively. Doses of $150 \mathrm{mg} 1^{-1}$ or greater completely inhibited methane production. Lower doses caused a rapid decrease in methane production rates followed by equilibration at a near constant rate for the remainder of the assay. A TCAA dose of $60 \mathrm{mg} 1^{-1}$ produced approximately a 90 percent reduction in methane production while doses of 6 and $30 \mathrm{mg} 1^{-1}$ resulted in approximately 12 and 65 percent inhibition, respectively.

Hydrogen response for TCA inhibited samples was essentially the same as for samples inhibited by chloroform and BES. Samples receiving the 6 and $30 \mathrm{mg}$ $1^{-1}$ dosages exhibited peak $\mathrm{H}_{2}$ headspace concentrations of 121 and $146 \mathrm{ppm}$, respectively, compared to the controls which averaged $110 \mathrm{ppm}$ (Figure 4-9). Samples that displayed essentially complete inhibition of methane production were observed to have a rapid and continual increase in hydrogen levels up to the percent range by the conclusion of the assay.

Volatile fatty acid levels at the conclusion of the 24-hour assay showed increasing accumulation as inhibition of methane production increased up to 60 mg $1^{-1}$ TCAA (Table 4-3). Above $60 \mathrm{mg} 1^{-1}$ of TCAA, accumulation of acids decreased with increasing dose.

TCAA addition produced a dose related response of gaseous $C O$. Unlike the rapid rise in co concentrations observed for chloroform, a several hour lagperiod was found to occur before co levels accumulated above control sample levels for all but the completely inhibited samples $\left(150\right.$ and $300 \mathrm{mg} 1^{-1}$ TCAA applied). The CO concentrations eventually attained, however, were well into the ppm range reaching $15 \mathrm{ppm}$ or greater in some samples (Figure 4-10).

\subsubsection{Eormaldebyde}

Formaldehyde was the least toxic of the organic compounds investigated. concentrations ranging from 10 to $100 \mathrm{mg} 1^{-1}$ were examined. The effect of 


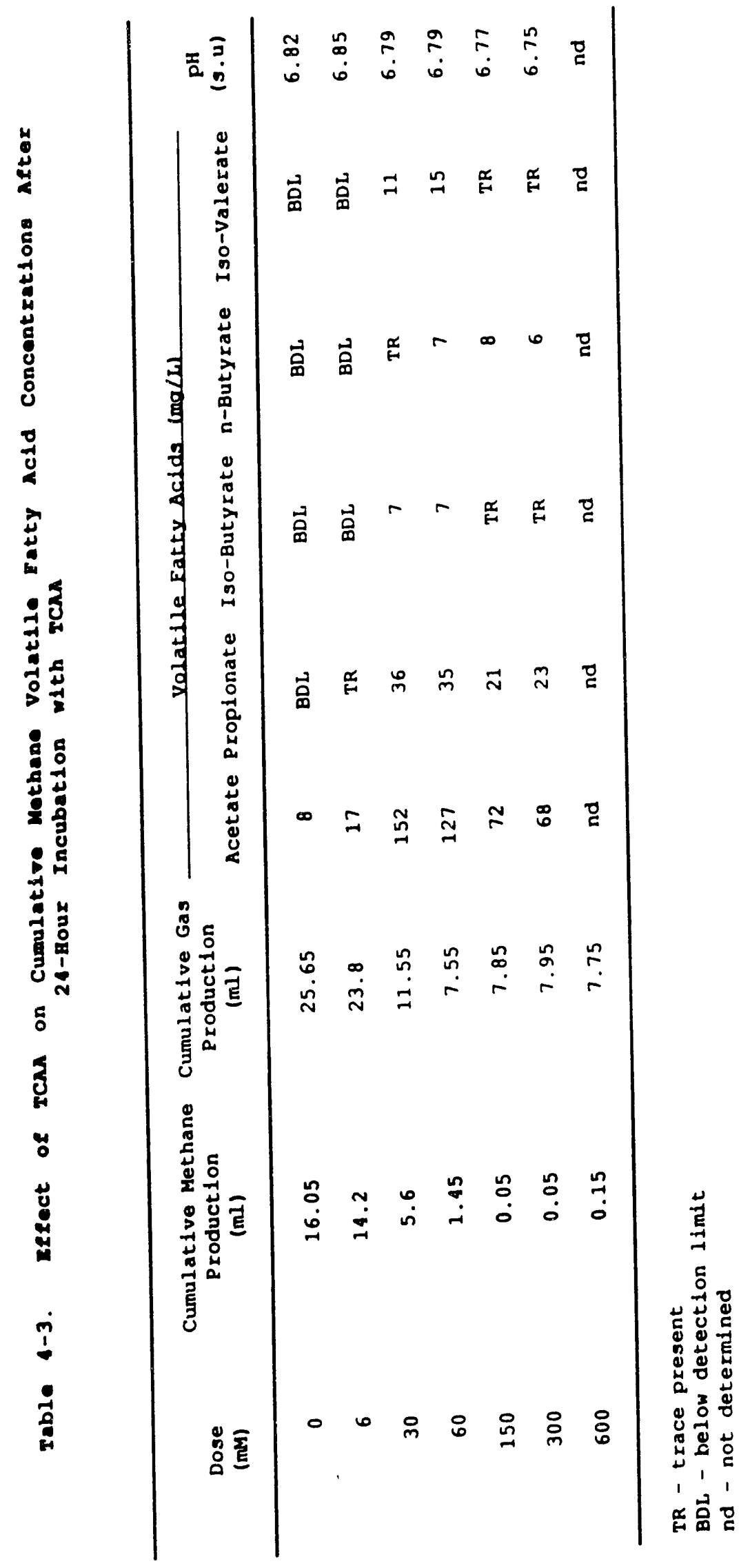




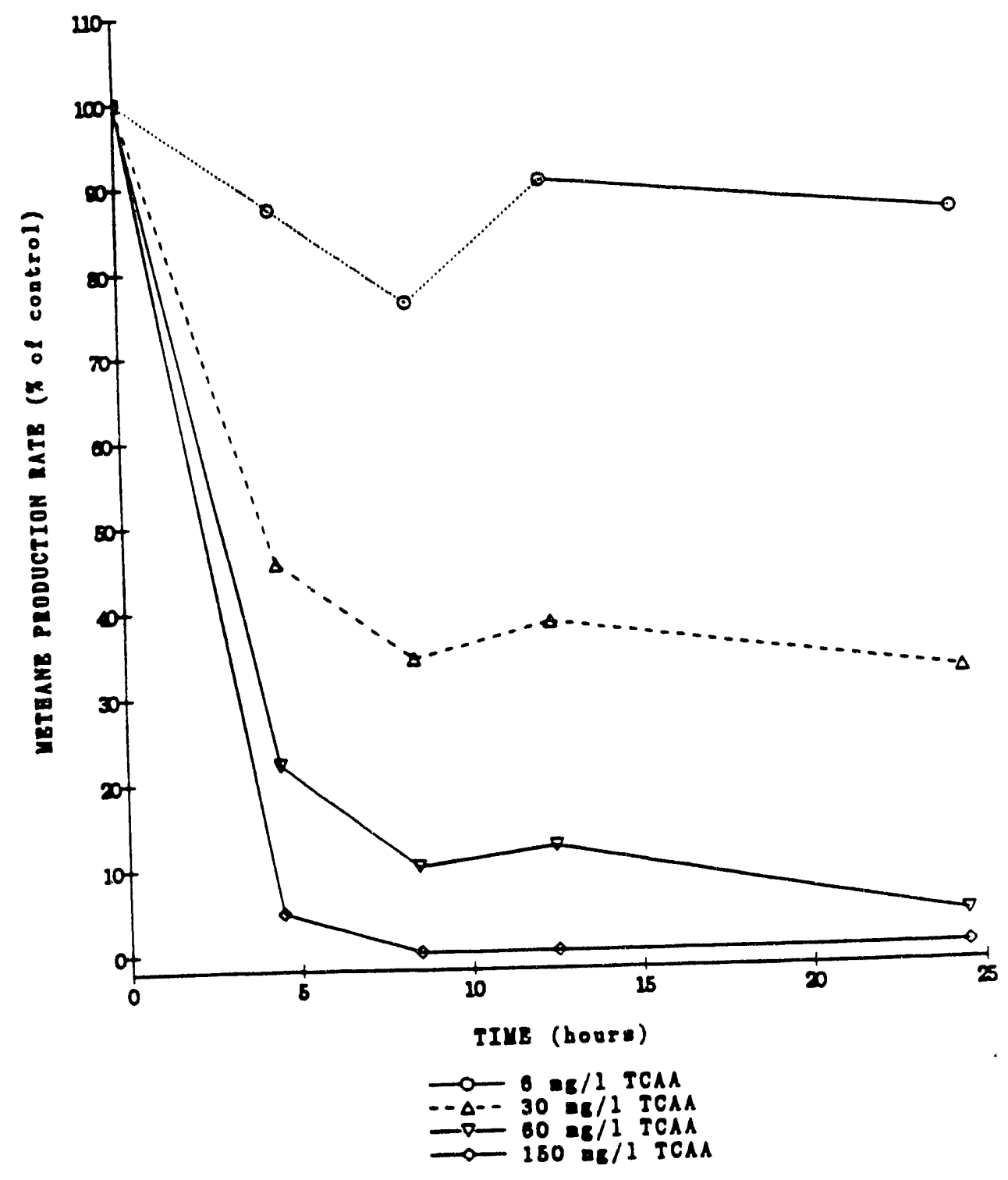

Figure 4-8. Methane production rate as a function of
'time and TCAA dose. 


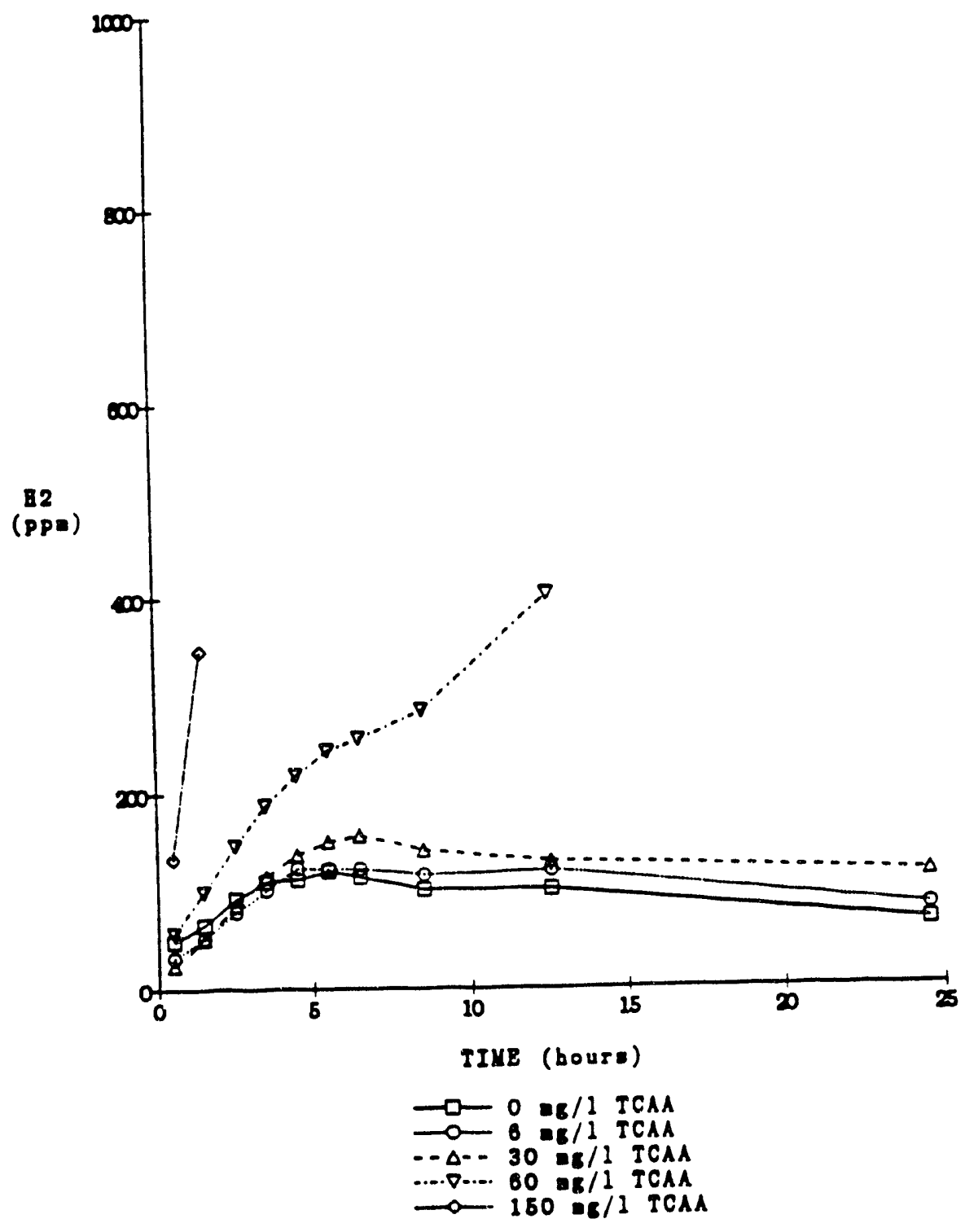

Figure 4-9. Hydrogen concentration as a function of TCAA
dose and time. 


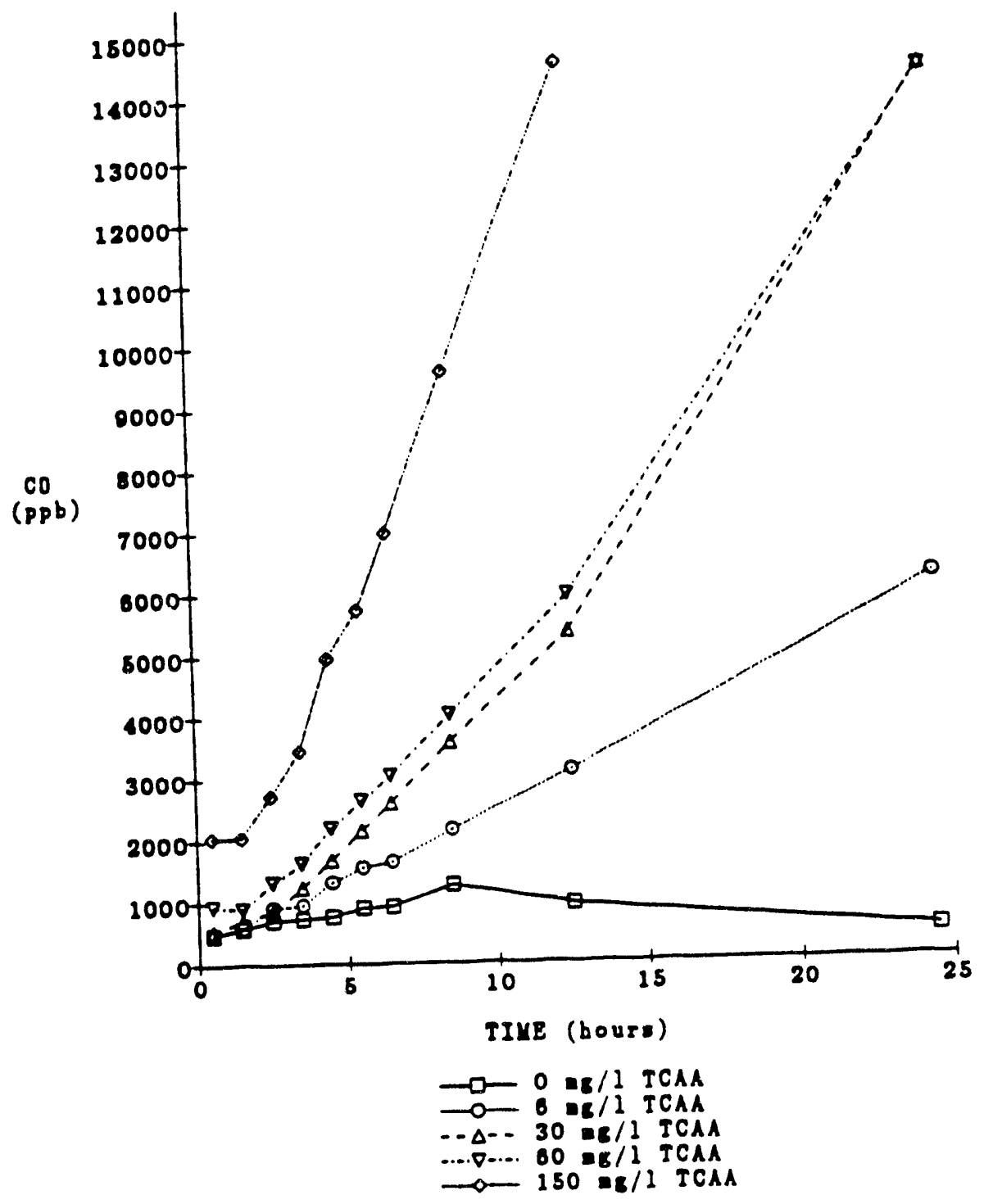

F1gure 4-10. Carbon monoxide concentration as a function
of time and TCA dose. 
formaldehyde on methane production rates as a function of dose and time is presented in Figure 4-11. Recovery was observed for all doses except $100 \mathrm{mg} 1^{-1}$. Even the $100 \mathrm{mg}^{-1}$ dosed samples displayed the beginnings of recovery by 24 hours. Time required for recovery seemed to be directly related to dose.

Hydrogen gas levels, as a function of time and dose, are presented in Figure 4-12 for controls and formaldehyde doses between 10 and $50 \mathrm{mg} 1^{-1}$. A dose of $100 \mathrm{mg} 1^{-1}$ produced a rapid accumulation of hydrogen well above the range that could be accurately quantified by the technique employed, within several hours of incubation, and remained at these elevated levels throughout the duration of the assay. The observed hydrogen response for other samples was dose dependent, with higher formaldehyde doses producing higher peak $\mathrm{H}_{2}$ concentrations. Interestingly, hydrogen levels were observed to decline in most cases slightly before recovery of methanogenesis was evident.

No accumulation of VFAs was observed in samples dosed with $30 \mathrm{mg} 1^{-1}$ of formaldehyde or less. Samples receiving $50 \mathrm{mg} 1^{-1}$ had final acetate, propionate and butyrate concentrations of 112,27 , and $6 \mathrm{mg} 1^{-1}$, respectively. Samples dosed with $100 \mathrm{mg} 1^{-1}$ of formaldehyde had less acetate $\left(72 \mathrm{mg} 1^{-1}\right)$ but more propionate $\left(40 \mathrm{mg} 1^{-1}\right)$.

Formaldehyde addition resulted in relatively rapid increases in $c 0$. The rate of increase and peak $C O$ concentrations reached were apparently directly related to formaldehyde dosage. For those samples demonstrating recovery of methane production (compared to the initial 4-hour rate), co concentrations were observed to decrease concurrent with the onset of recovery of methane production (Figure 4-13). By the conclusion of the 24-hour assay, the CO concentrations of samples dosed with $10 \mathrm{mg} 1^{-1}$ of formaldehyde or less had returned to the levels exhibited by control samples. Samples dosed with higher concentrations of formaldehyde displayed a precipitous decrease in $c O$ during the final 12 hours of the assay. 


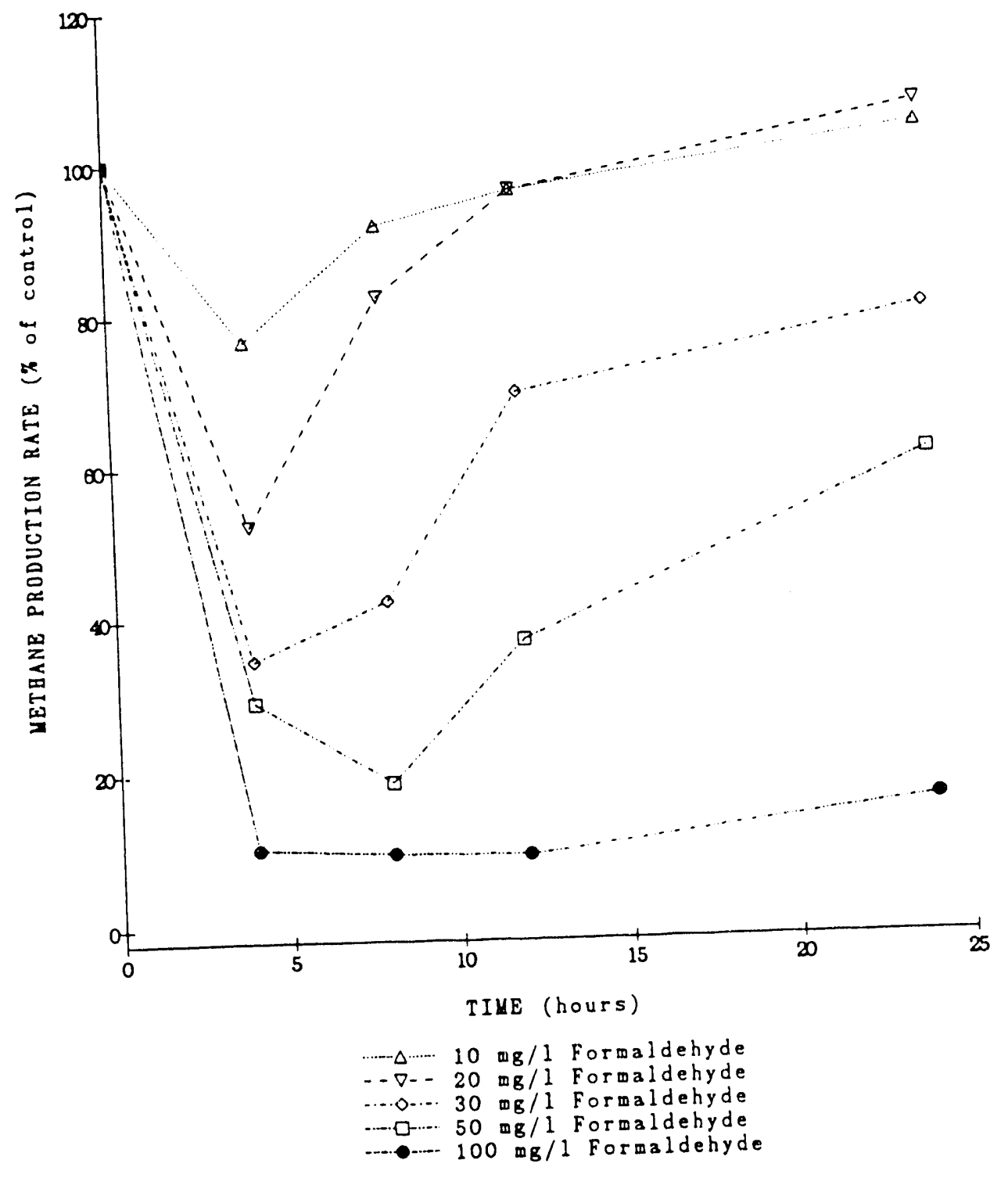

Figure 4-11. Methane production as a function of timo 


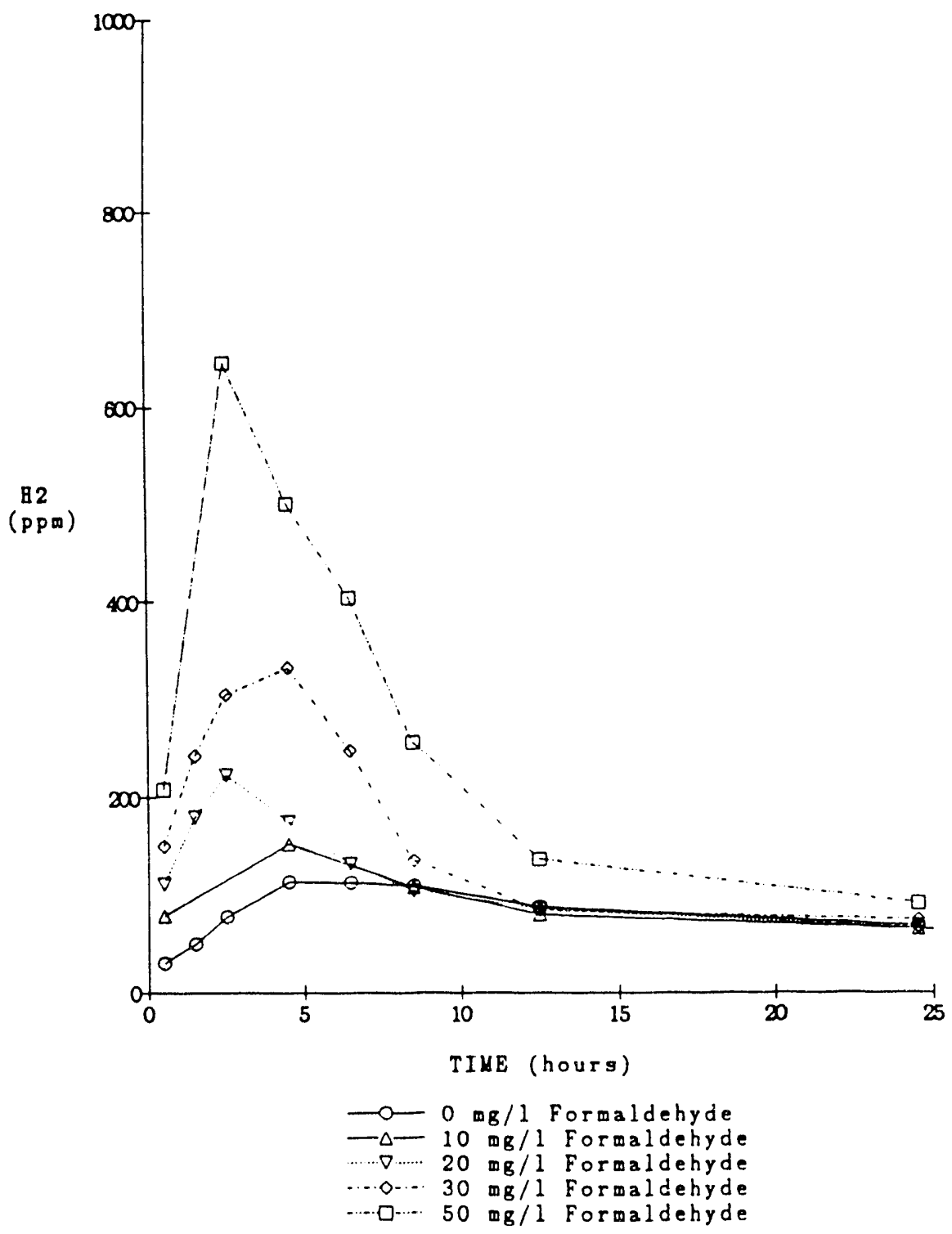

Figure 4-12. Hydrogen concentration as a function of
time and formaldehyde dose. 


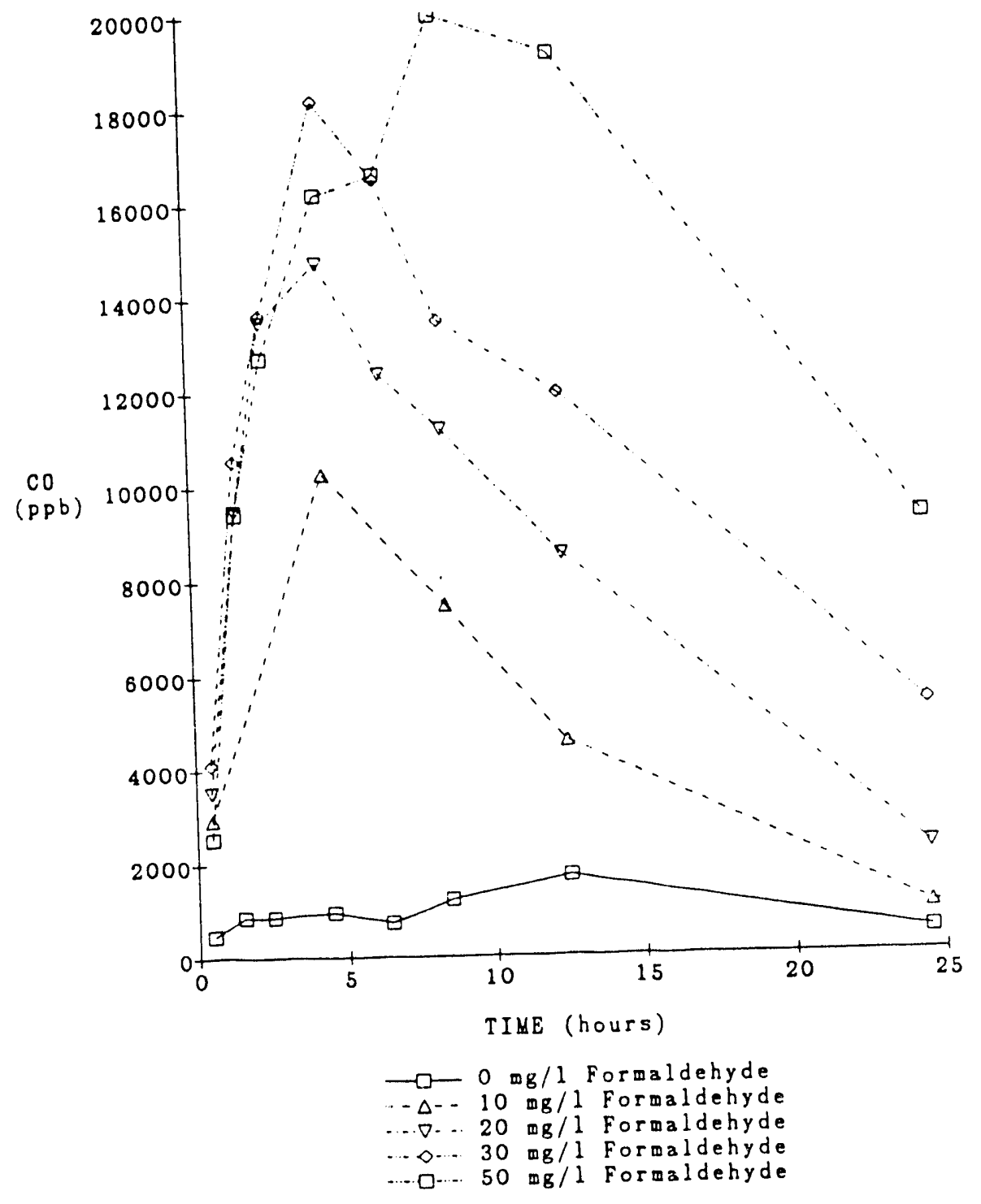
Figure 4-13. Carbon monoxide concentration as a function
of time and formaldehyde dose. 


\subsection{SUMMARY AND DISCUSSION OF RESULTS FROM ORGANIC TOXICANT ASSAYS}

with the exception of formaldehyde, each of the organic toxicants examined in this study displayed two distinct, dose related hydrogen response patterns. When inhibition of methane production was severe (greater than a 70 to 75 percent reduction compared to controls), hydrogen rapidly accumulated in the headspace of the serum bottles and continued to rise into the percent range during the course of the assay. If inhibition was less severe, the 24-hour hydrogen response pattern was observed to be similar to controls but at somewhat elevated levels. At first glance this can be construed as greater sensitivity of the acetate-splitting methanogens than the hydrogen utilizers to the organic toxicants employed. Just such a selective inhibition has been reported for BES in a thermophilic system (zinder et al., 1984a). This research team found that $1 \mathrm{mM}$ BES was sufficient to completely inhibit methane production from acetate while $50 \mathrm{mM}$ BES was required to inhibit methanogenesis from $\mathrm{H}_{2} / \mathrm{CO}_{2}$. The principle hydrogen-utilizing methanogen observed in this thermophilic system was a Methanobacterium se. that resembled $M$.

thermoautotrophicum (zinder et al., 1984b). A high tolerance for BES has been reported for $M$. themoautotrophicum (Zinder et al., 1984a; Eikmanns et al.., 1985). This is believed to be related to the permeability of the cell membrane to BES (Eikmanns et al., 1985) since low levels were observed to be inhibitory to the reduction of methyl call in cell extracts (Gunsalus et al., 1978).

Other investigators have reported that BES inhibited methane production from acetate and $\mathrm{H}_{2} / \mathrm{CO}_{2}$ equally (Healy et al., 1980) or that acetate catabolism was less sensitive to BES induced inhibition (zehrider et al., 1980).

BES has been shown to competitively inhibit the reduction of methyl CoM to methane (Balch and Wolfe, 1979a; Smith and Mah, 1978). Therefore, despite the fact that methyl COM is a common intermediate in methanogenesis from acetate and $\mathrm{H}_{2} / \mathrm{CO}_{2}$ (Lovley et al., 1984), different amounts of CoM in different species of methanogens or the same species cultured on different substrates could result in different levels of inhibition at the same BES dose.

The accumulation of propionate and other alternate electron sink products in the less severely inhibited samples was observed in all cases. It is possible 
that inhibition of the hydrogen-utilizers is, to a large extent, masked in the less severely inhibited samples by the accumulation of hydrogen in reduced products rather than the gaseous headspace. Once a certain threshold is reached where there is simply no additional soluble substrate being produced that can be used to dump reducing equivalents (i.e., hydrogen), hydrogen accumulates rapidly in the gaseous headspace. The following exercise was used to investigate this possibility.

The total amount of methane produced by the controls during the three assays was between 15 and $16 \mathrm{ml}$. Assuming 72 percent of the methane is derived through the acetate pathway (Jeris and McCarty, 1965), the inhibition of acetate metabolism can be estimated. This is done by summing the amount of potential methane (as COD), that could be generated through the acetate pathway, in the accumulated VFAs and comparing these values to controls. Results for the BES inhibition assay are presented in Table $4-4$ with the potential methane from acetate calculated as COD. BES is an analog of coenzyme $M$ (which has only been detected in methanogens, Balch and Wolfe, 1979a) and specific inhibitor of methanogenesis. Inhibition or toxicity to other groups in the consortium should not, therefore, affect results. As can be seen i.r Table 4-4, the calculated inhibition of acetate utilization is essentialiy the same as the inhibition of total methane production by the consortium. This indicates that the acetate and hydrogen utilizing methanogens are most lixely inhibited to approximately the same extent.

Using the BES results as a "bench mark," the more complex results observed for TCAA and chloroform can be analyzed. The build up of acetate, propionate and butyrate as a function of inhibition level of the consortium (measured as methane produced compared to controls) is presented in Figure 4-14. Both TCAA and chloroform displayed the same build up pattern of VFAs (for up to 80 percent inhibition) as BES inhibited samples when placed on this rational comparative basis. Because of the similarity of the VFA accumulation pattern observed for the BES, TCAA and chloroform assays, it appears that all three organic toxicants affect both groups of methanogens equally. At higher levels 


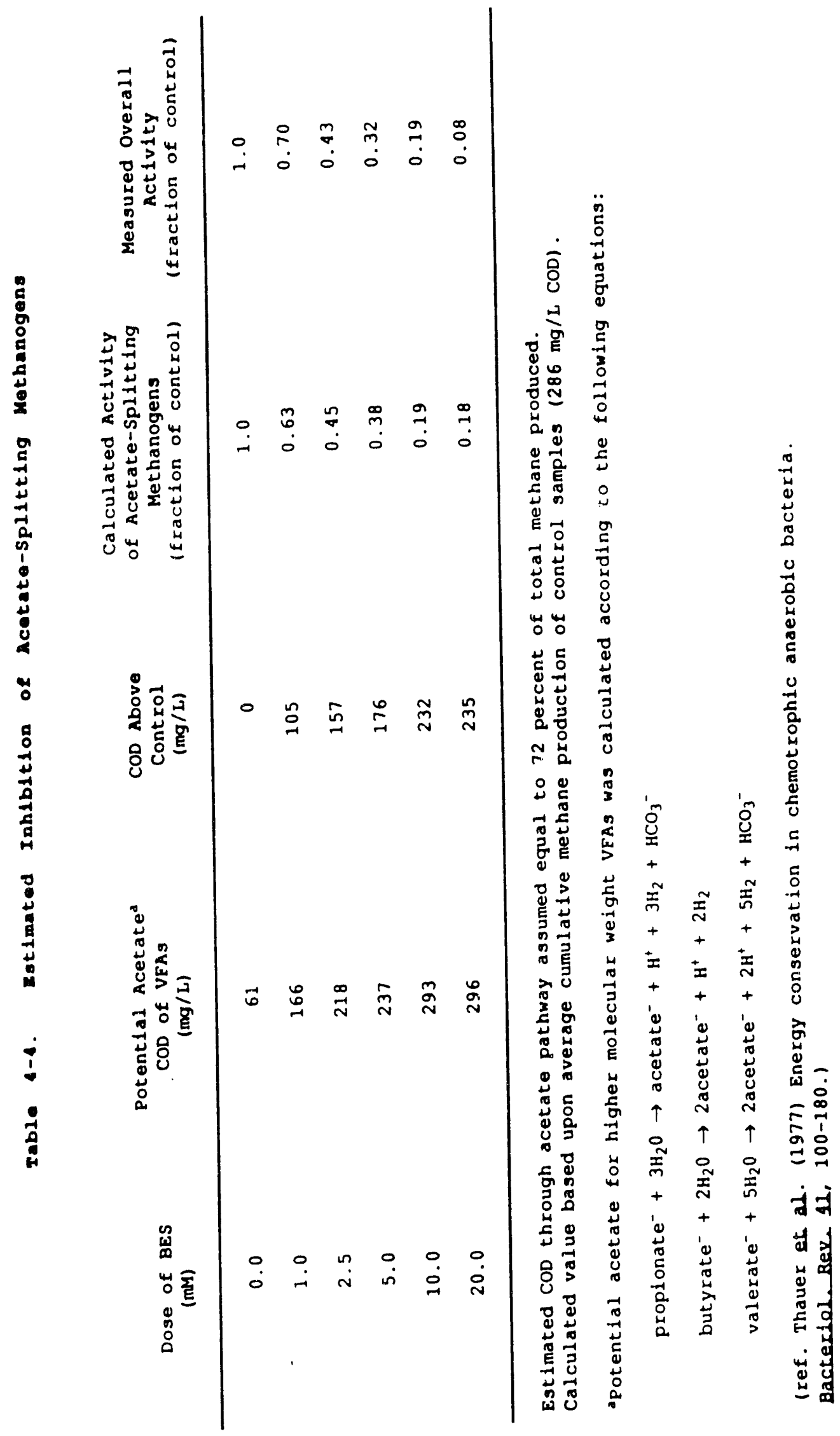



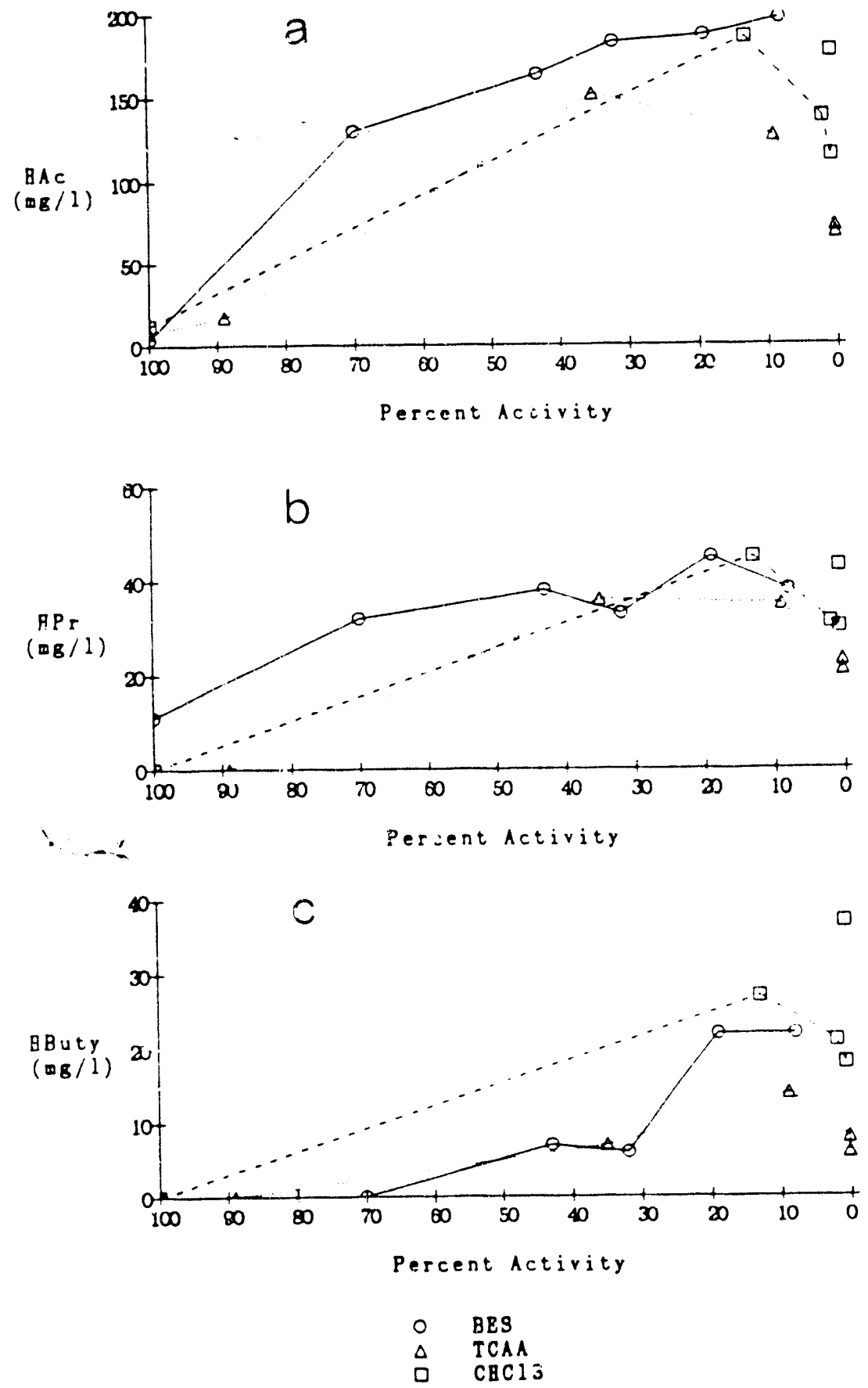

Figure 4-14. Accumulation of a) acetate; (b) propionate: (c) butyrate as e function of the level of inhtbition of mothane production induced by BES, chloroform and TCMA. 
of inhibition, however, TCAA and chloroform both displayed a precipitous drop in VFA accumulation. This indicates that some other reaction, in addition to methanogenesis, may have been inhibited at these high toxicant dosages.

Russell and Martin (1984) observed that when chloroform was used to inhibit rumen bacteria in-vitre, at a dose sufficient to essentially arrest methane production, amino acid degradation was also partially inhibited. Nagase and Matsuo (1984) reported the same results for chloroform inhibited digester inocula fermenting certain amino acids. The fermentation of some proteins and other amino acids was apparently not affected by chloroform at levels that completely arrested methane production. Hino and Russell (1985) found that the ratio of NADH to NAD in whole cells and cell extracts of rumen bacteria had a marked effect on the deamination of amino acids, in particular branched chain amino acids. As the amount of NADH relative to NAD increased, the rate and extent of amino acid degradation decreased. Sykes (1970) reported that purging carbon tetrachloride-inhibited digester samples (pulsed with protein) with nitrogen gas to remove accumulated $\mathrm{H}_{2}$ resulted in increased accumulation Of VFAs.

The effect of formaldehyde was different from the results observed with the other three organic toxicants used in this study. Recently it has been reported that formaldehyde may react with a specific component (fu-aldehyde activating factor (FAF!) of the hydrogen to methane pathway in $M$.

themoautetrophicum (Escalante-Semerena et al., 1984). Under a nitrogenatmosphere the resulting compound, $\left(\mathrm{CH}_{2}\right)=\mathrm{FAF}$, was observed to be oxidized to (HCO)-FAF with 1:1 stoichiometric liberation of $\mathrm{H}_{2}$. Assuming the hydrogenutilizing methanogenic population in the inoculum used in these assays responded in a fashion similar to $M$. themoautotrophicum, this could, in part, account for the rapid accumulation of hydrogen observed as a result of formaldehyde addition. It is possible, that at lower doses of formaldehyde, the hydrogen to methane pathway could be selectively inhibited. The observation that no VFAs accumulated in samples inhibited 36 percent or less lends some circumstantial support to this hypothesis. However, since vFAs were analyzed only at the conclusion of the assay and recovery occurred in these samples, accumulatin of VFAs followed by subsequent utilization during the recovery period couli have taken place. Several researchers have reported 
inhibition of methanogenic acetate-metabolism by formaldehyde. Huser et al. (1982) found that $10 \mathrm{mM}\left(300 \mathrm{mg} \mathrm{1^{-1 } )}\right.$ formaldehyde completely inhibited acetate-metabolism of Methanethrix seehagenii. Parkin and Speece (1982) reported that an acetate enrichment culture was severely inhibited by $100 \mathrm{mg}$ $1^{-1}$ of formaldehyde. Recovery time was observed to be related to formaldehyde dose just as was found in this study. It is probable, therefore, that higher formaldehyde doses did inhibit methanogenesis from acetate. Once more is known concerning the inhibitory action of formaldehyde on methanogenesis, more meaningful conclusions about the observed results can be made.

Besides samples from the formaldehyde inhibition assay, the only other samples that demonstrated recovery of methane production, above the rate exhibited during the initial four hours of incubation, were the samples dosed with $0.15 \mathrm{mg} 1^{-1}$ chloroform. Using a partitioning coefficient of 2.9 and the approach used by Yang and Speece (1986), the initial dissolved chloroform concentration can be approximated. Chloroform would have been reduced to approximately $0.12 \mathrm{mg} 1^{-1}$ by volatilization in these samples. Bouwer and McCarty (1983) reported that chloroform at $0.1 \mathrm{mg} 1^{-1}$ was anaerobically metabolized. Yang and speece (1986) reported that an acetate-enrichment culture acclimated to higher concentrations of chloroform $\left(1.0 \mathrm{mg} 1^{-1}\right.$ and greater) without any apparent biodegradation occurring. Whether recovery of methane production in this study was due to acclimation or biodegradation cannot be ascertained from the results. The protocol used in these assays, of removing gas produced at 4-hour intervals, would certainly have tended to enhance stripping of additional chloroform from the liquid to gaseous phase and thereby further reduced the concentration of chloroform in the liquid.

The influence of the four organic toxicants employed in this study was examined within the narrow constraints of a single inoculum HRT and an essentially constant volatile solids (cell) concentration. Recent studies have demonstrated that inhibition of methanogenesis in an enrichment culture was influenced by cell age (HRT) and solids concentration (Yang and Speece, 1985 and Yang and speece, 1986). The effect of varying these two parameters, however, was a change of degree rather than a substantive change in response patterns. Because of this, it is likely that response patterns of methane production and hydrogen gas concentration presented herein will be 
representative of a variety of experimental conditions that could be affected by varying these two parameters, although the absolute values will undoubtedly be altered.

It would appear that hydrogen holds potential as an indicator of process upset and recovery from upset as a result of the organic toxicants used in this study. This is evident in the chloroform (Figure 4-15) and formaldehyde (Figure 4-16) data where both upset and recovery were observed to occur in some of the samples.

In all cases, severe inhibition of methane production was accompanied by a substantial rise in gaseous $c 0$ levels. The response of $\mathrm{CO}$, to inhibition of methane production, induced by the four organic toxicants used in this study, did not follow a generic type pattern as was observed for hydrogen. This is not surprising since organic toxicants tend to be specific in the manner in which they inhibit an organism. Often, this fact is used as a first step in probing a pure culture of an organism to elucidate a metabolic pathway. In mixed culture systems, such as anaerobic digesters, this cause and effect relationship is obfuscated by the occurrence of many undefined reactions. Never the less, several interesting observations concerning the response patterns observed for co can be made.

BES is known to attack a specific site, the CoM methyl reducatase enzymatic system, in methanogens (Balch and Wolfe, 1979a) and in mixed culture has not been reported to interfere with other trophic groups (Healy et al., 1980). BES would, therefore, not be expected to directly interfere with the co dehydrogenase enzymatic system or the carbonyl carrier that equilibrates with Co (Nelson and Ferry, 1984). In fact, Co levels did not rise above controls in any samples except those where methane production was completely inhibited. Only when methane production rate decreased to less than 10 percent of controls during a time interval did co rise above control levels. In no circumstances did co levels rise above $2800 \mathrm{ppb}$. It would appear, based upon these observations, that $C O$ was affected indirectly by BES. The influence is likely the result of blocking up methanogenic pathway at the CoM methyl reductase reaction with the resulting subsequent build up of other intermediates in the pathway. 


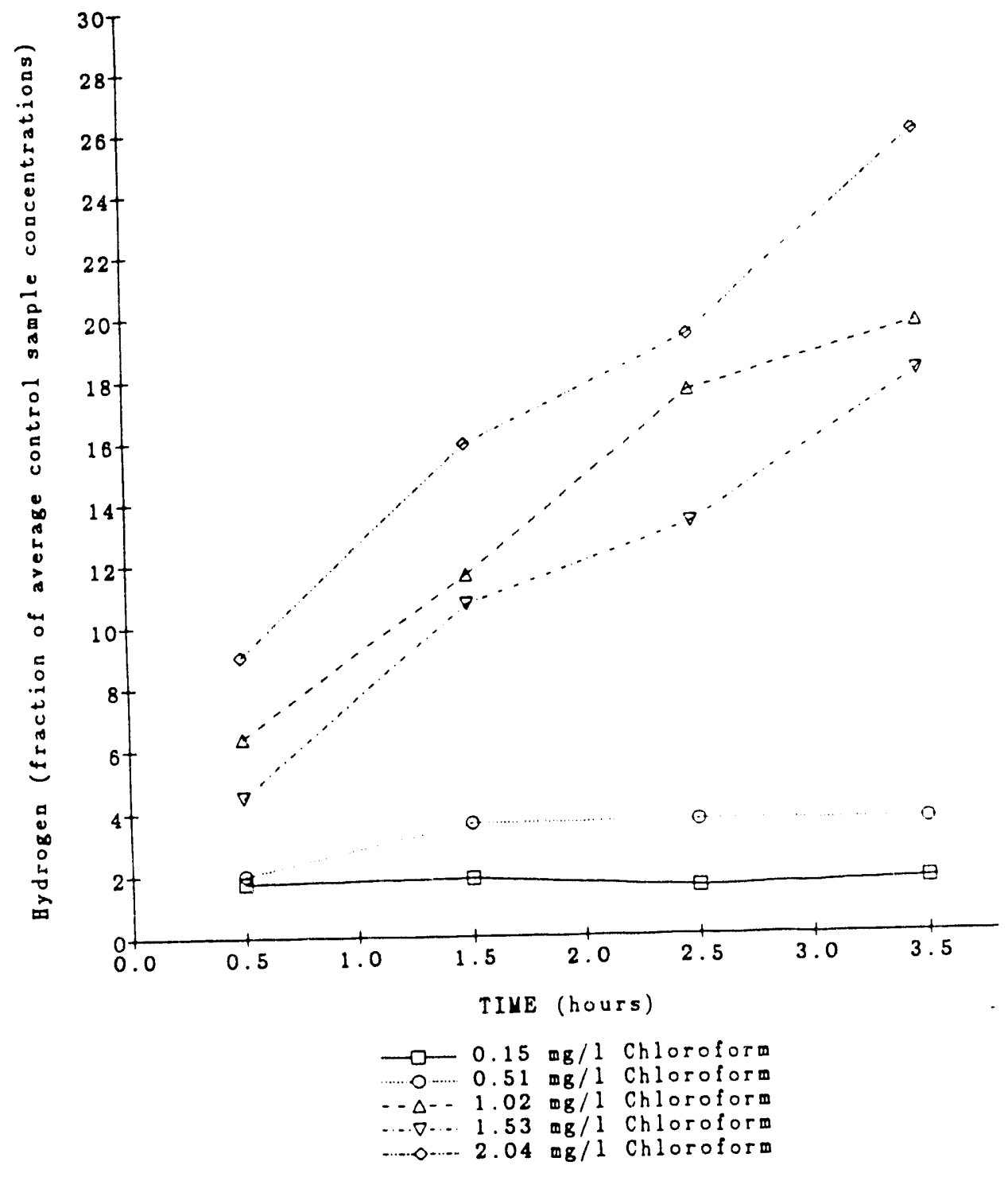
Figure 4-15. Hydrogen concentration as a function of
time and applied chloroform dose 20-day HRT
inoculum. 


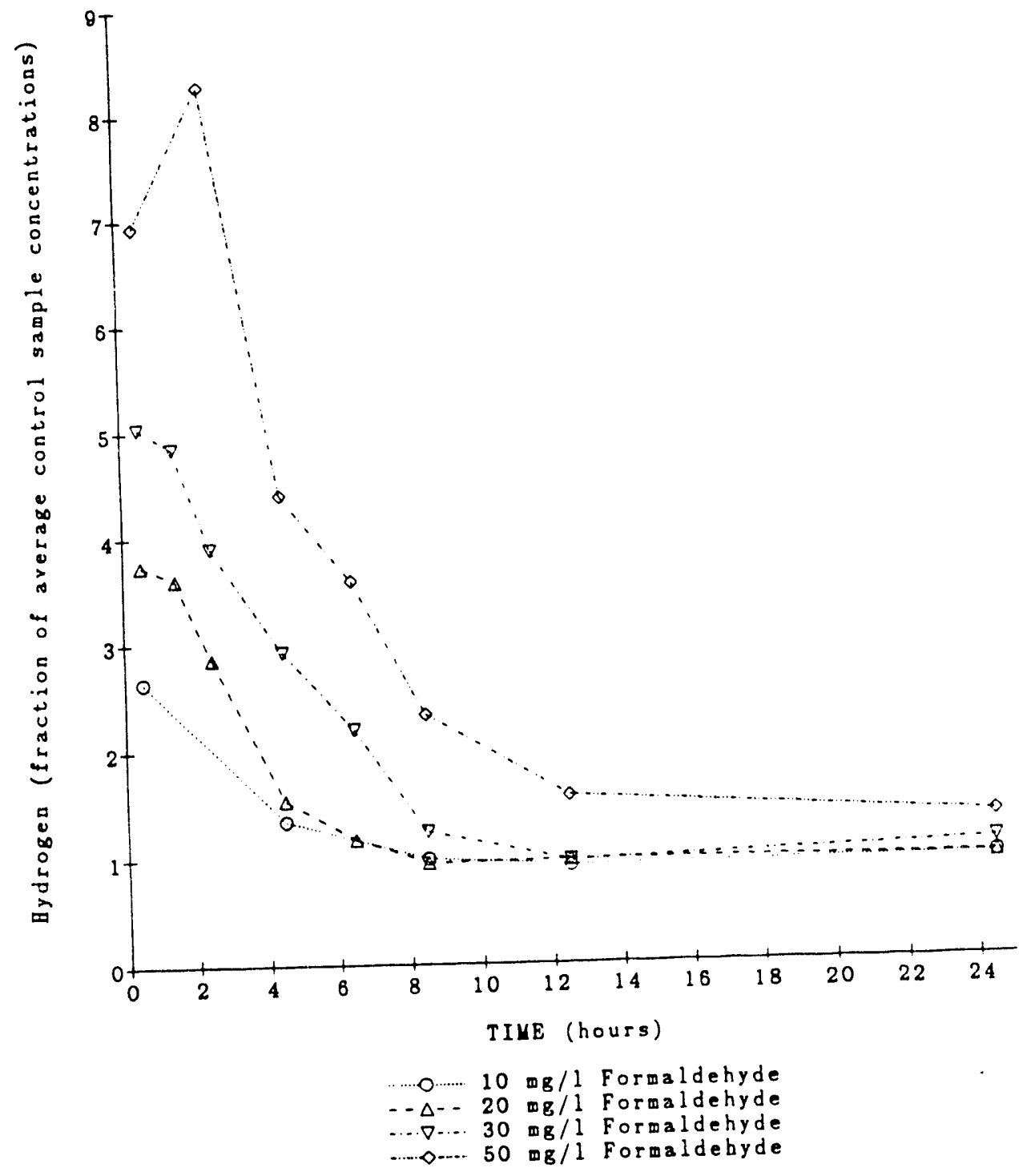

Figure 4-16. Hydrogen concentration as a function of
time and applied formaldehyde dose 20-day
HRT inoculum. 
By contrast, samples that were severely inhibited by chloroform addition $\left(1.0 \mathrm{mg} 1^{-1}\right.$ or greater) displayed an extremely rapid accumulation of $\mathrm{co}$. Even sampes dosed with $0.15 \mathrm{mg} 1^{-1}$ had a gradual rise in co over time, reaching a level four fold higher than controls by the conclusion of the assay. Eikmanns et al. (1985) observed that another chlorinated methane analog, carbon tetrachloride, inhibited both methane and $C O$ production by the autotrophic methanogen $M$. themoautotrophicum. Co in this organism has been shown to be associated with fixation of $\mathrm{CO}_{2}$ into cell carbon (Eikmanns et al.. 1985). These researchers also observed that the specific activity of the $c 0$ dehydrogenase in cell extracts was not affected by carbon tetrachloride.

Samples inhibited with TCAA had a response similar in many ways to samples inhibited by chloroform. One exception was that the accumulation during the initial 30 minute period was much less dramatic. The co concentrations after 30 minutes of incubation was directly related to dose, as was observed for chloroform. Samples receiving $600 \mathrm{mg} 1^{-1}$ TCAA had an average CO of $8.6 \mathrm{ppm}$ after only 30 minutes.

Formaldehyde-inhibited samples had the most unusual co response pattern of the four toxicants. This response resembled an accumulation and subsequent depletion of a metabolic intermediate. A rapid rise in $C O$, that was dose related, was initially observed in inhibited samples. As recovery from inhibition occurred, co levels declined towards control sample levels.

Despite the fact that co displayed a different response pattern to each of the four organic toxicants examined, the fact that a large increase in co was always concurrent with inhibition of methane production indicates it may still be a useful early warning indicator of process-upset as a consequence of organic toxic shocks.

It must be kept in mind that these results were generated from batch assays. Although the assays were conducted in a manner designed to simulate, as closely as possible, the real world situation of a pulse addition of organics as a result of draw and fill feeding, differences exist. In a typical digester, hydrogen would initially be at a certain baseline or operating level at time zero and not at near zero ppm (the $\mathrm{N}_{2} / \mathrm{CO}_{2}$ gas mixture had $<1$ ppm $\mathrm{H}_{2}$ ) 


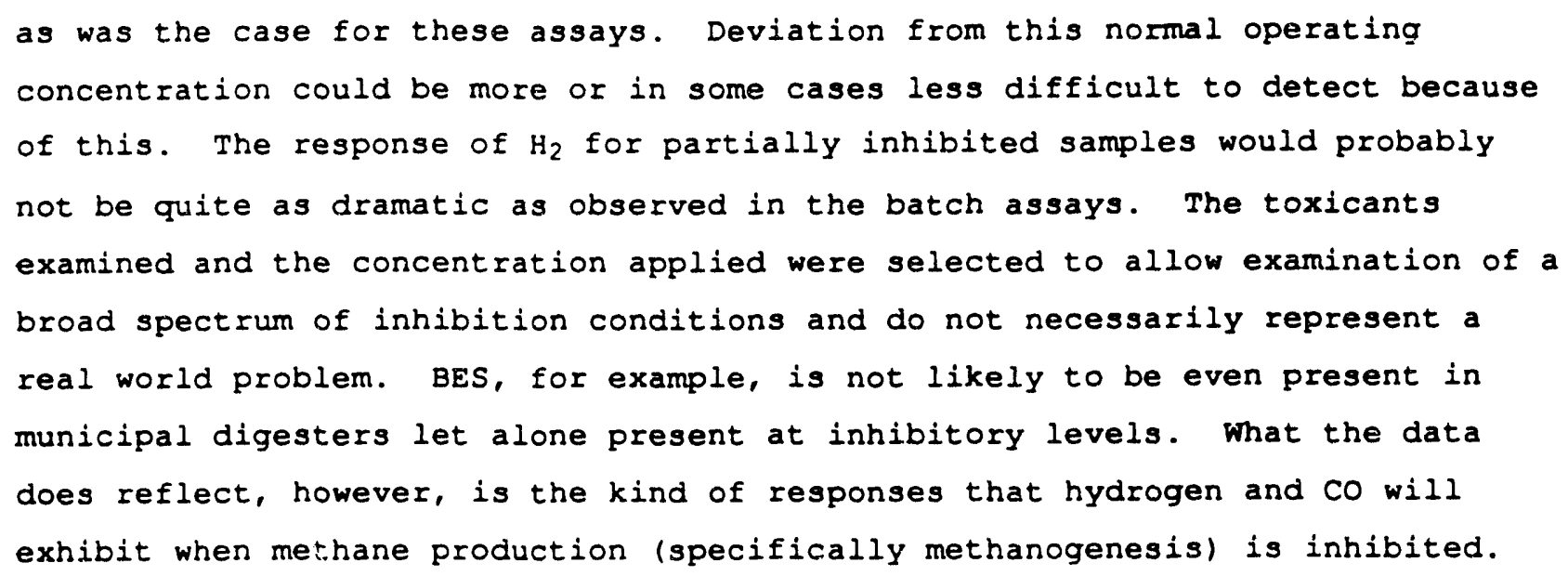




\section{CHAPTER 5}

EXPERIMENTAL RESULTS -- THE EFEECTS OF PULSE ADDITION HEAVY METALS ON ANAEROBIC DIGESTION

The experimental data presented in this chapter consists primarily of results generated during a series of inhibition assays conducted using serum bottle reactors and inocula from the 10 - and 20 -day HRT reserve digesters.

Additional data collected during serum bottle assays using pulses of various substrates to the digester inocula are also presented. It is worthwhile at this point to emphasize that although the serum bottle assays were designed to simulate, as closely as possible, the operation of a typical digester system operated on a semi-continuous (batch draw and fill) schedule, differences exist. Therefore, the similarities and differences in observed trends and response patterns between controls and perturbed samples are of greater consequence than the absolute values obtained during the assays.

\subsection{RESULTS OF SERUM BOTTLE ASSAYS}

The effect that heavy metal addition, added as pulses to the WAS-fed serum bottle reactors, had on gas and methane production and hydrogen and carbon monoxide response patterns was examined using three transition heavy metals, copper, zinc and cadmium.

\section{1 copper}

The effect of copper (added as $\mathrm{CuCl}_{2}$ ) was examined for both 20- and 10-day HRT inocula. Figure 5-1 presents the 24-hour results for cumulative methane production as a function of $C \mathrm{CH}^{2}$ dose (in $\mathrm{mg} \mathrm{1^{-1 }}$ ). The 10-day HRT inoculum displayed an apparent greater tolerance for copper than the 20-day HRT samples. The dosage of copper required to produce a 50 percent inhibition in methane production was determined to be approximately 75 and $90 \mathrm{mg} 1^{-1}$ for $20-$ and 10-day HRT samples, respectively. When the results are analyzed on a mass-applied/mass-of-biomass (mg gVs-1) basis, (Figure 5-2), both data sets converge to form one line. A 50 percent inhibition of methanogenesis occurred at a copper dosage of approximately $13.5 \mathrm{mg} \mathrm{Cu} \mathrm{CVS}^{-1}$. 


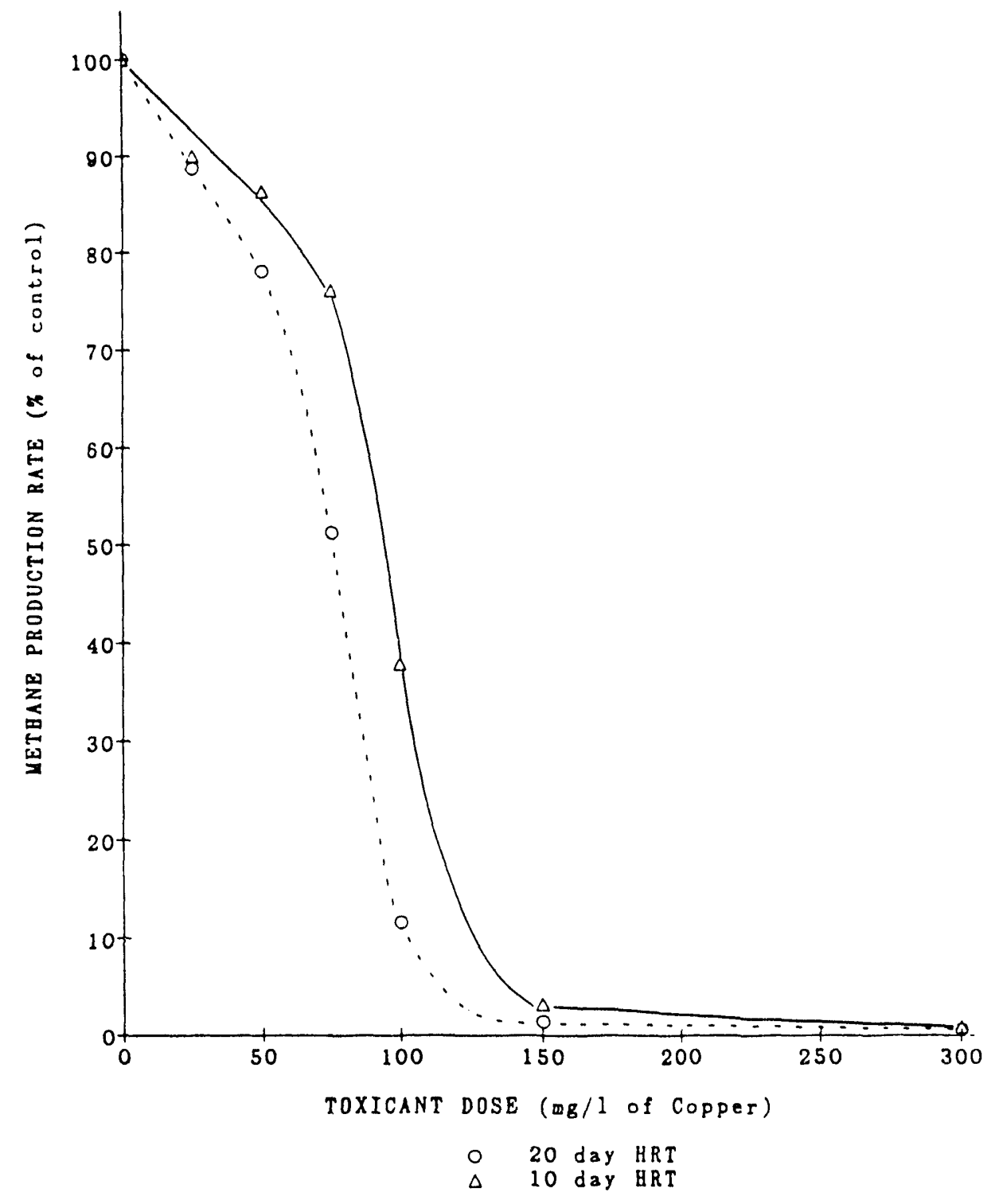

Figure 5-1. Cumulative methane production (24 hours) as a function of copper dose (mg/I) for 10 - and 20-day BRT inocula. 


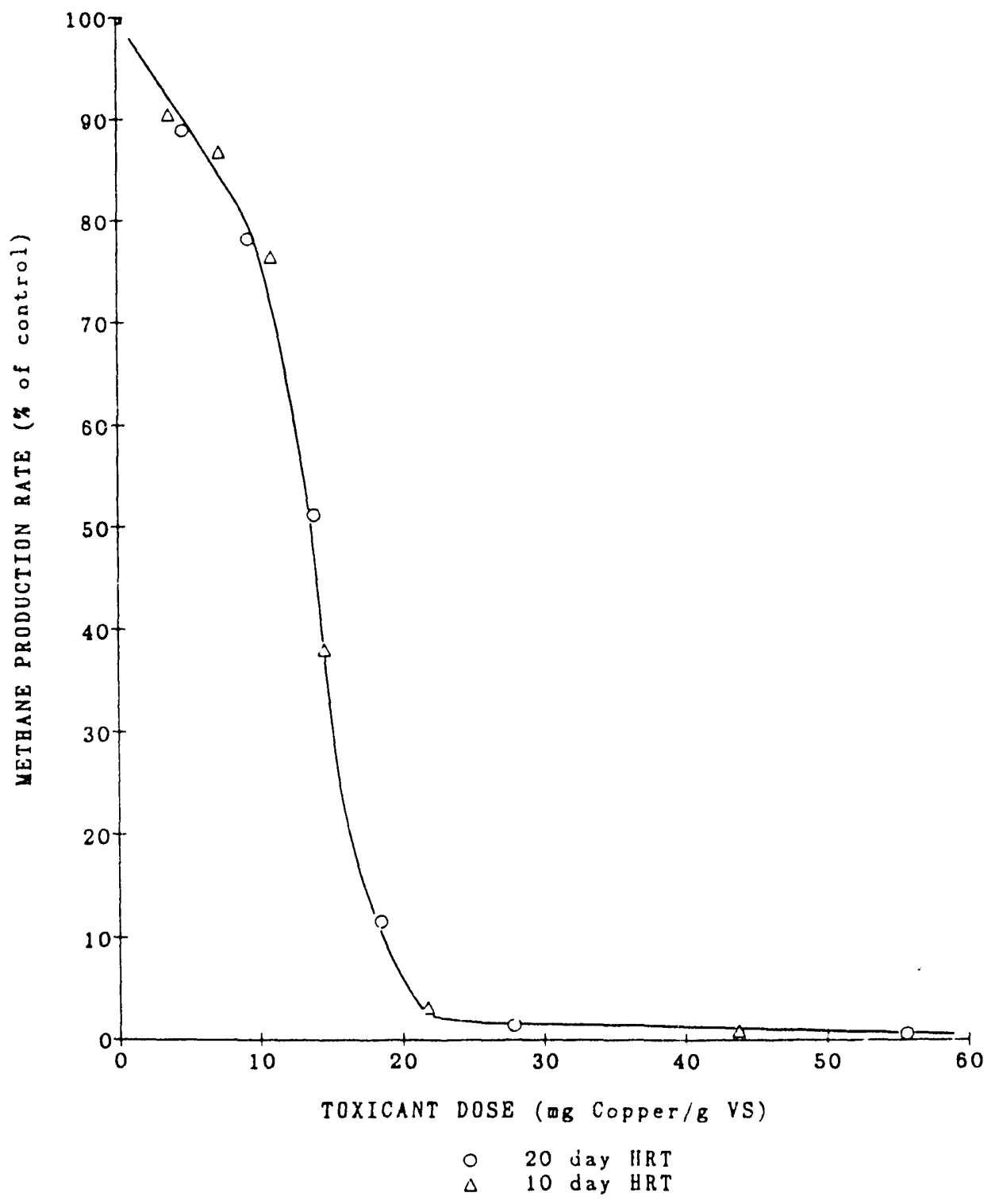

Figure 5-2. Cumulative methane production (24 hours) as a
function of copper dose (mg/gvs) for 10- and 20-
day HRT inocula. 
The data were fit to a third-order polynomial in order to allow statistical evaluation of the results. A modified $F$ test (Neter and Wasserman, 1974) was employed. The analysis verified that there was a statistical difference between the 10- and 20-day HRT inocula when copper dosage was analyzed on a mg $1^{-1}$ basis. There was no statistical difference between the two inocula when copper dose was analyzed on a mg gVs-1 basis. The results are presented in Table 5-1. Both conclusions are significant to greater than the 0.01 level.

Filtered copper concentrations were measured on samples sacrificed after 48 hours of incubation. These results (Table 5-2) demonstrate a rather strong sequestering of copper in the sludge phase. Much of the literature data for metals uptake by sludge demonstrates that. little change in filtered concentrations occurs after 24 hours. In fact, over 90 percent of the uptake occurs within minutes (Cheng et al., 1975). Therefore, although not precisely correct, a reasonable estimate of methane inhibition as a function of filtered copper concentration can be sbtained by comparing the cumulative 24-hour methane production to filtered copper concentrations on samples analyzed after 48 hours of incubation. The cumulative after 48-hour total-gas and methane production data showed a substantial drop in methane production by the control samples presumably due to substrate depletion. This tends to mask the level of inhibition of the copper dosed samples if cumulative 48-hour methane inhibition was used and would be less accurate than using 24-hour methane production and 48-hour filtered copper data.

The level of inhibition as a function of filtered copper concentration for the 20- and 10-day inoculum HRT assays are presented in Figure 5-3. The 20-day HRT inoculum demonstrated less inhibition at the same concentration of filtered copper compared to the 10 day HRT inoculum. This is believed due to the higher soluble-COD and, therefore, higher soluble liquid concentration associated with the 20-day inoculum. Soluble-COD concentrations for the reserve digesters during the investigation period averaged 1080 and $620 \mathrm{mg} 1^{-1}$ for the 20-and 10-day HRT systems, respectively. A high affinity for copper by anaerobic digester supernatant has been previously reported (Patterson and HaO, 1980). 


\section{Table 5-1. Statistical nalysis of Copper Inhibition of Anarobic Digestion}

Fit of 3 rd Order Polynomial

\begin{tabular}{lcccc} 
Data Set & $\mathrm{r}^{2}$ & $\mathrm{n}$ & residuals & $\mathrm{F}^{* 2,3}$ \\
\hline & & & & - \\
20-day HRT & 0.975 & 13 & 0.0469 & - \\
10-day HRT & 0.953 & 26 & 0.0717 & 5.19 \\
$\begin{array}{l}\text { 10+20-day HRT } \\
\text { (mg/L) }\end{array}$ & 0.927 & 0.2552 & 0.123 \\
$\begin{array}{l}\text { 10+20-day HRT } \\
\text { (as mg/gVS) }\end{array}$ & 0.965 & 26 & 0.1218 & \\
\end{tabular}

1 Data normalized as fraction of methanogenic activity of controls with all data points except highest dose $\left(300 \mathrm{mgl}^{-1}\right)$ used.

$2 F^{\star}=\frac{S S E_{R}-S S E_{F}}{(n-4)-(n-8)}+\frac{S S F_{F}}{(n-8)}$

$3 F_{0.99,4,18}=4.58$

If $F^{\star} \geq F$ lines are different

If $F^{\star} \leqq F$ lines are same.

SSF - the sum of residuals for fit of individual experimental runs.

$S S R$ - residuals for fit of combined experimental runs.

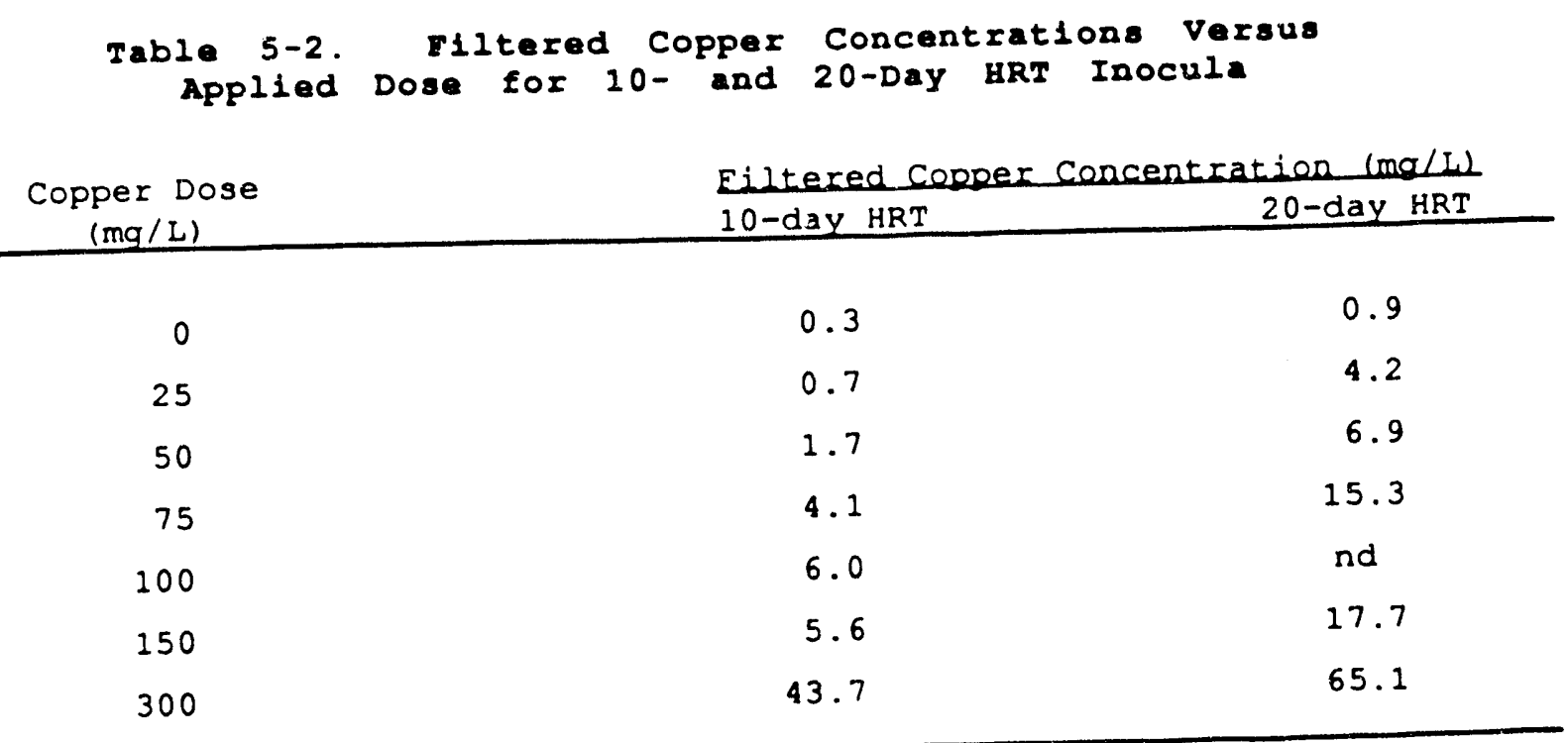

nd - not determined 


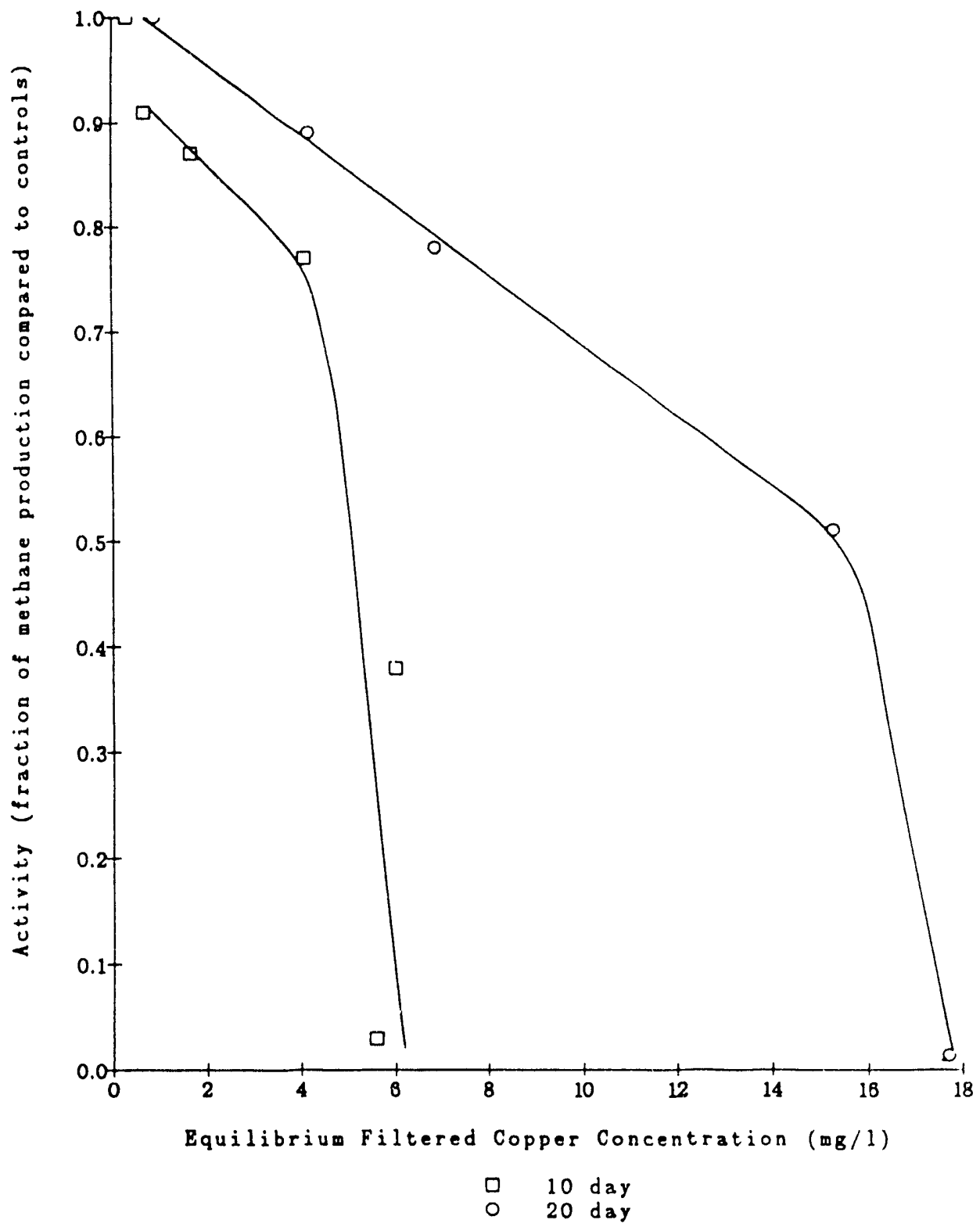

Figure 5-3 Inhibition of methane production (24 hours) as a function of filtered copper concentration. 
Volatile fatty acid (VFA) analyses performed on samples taken at the conclusion of the 48-hour assay for the 10-day HRT inoculum demonstrated a rather interesting trend (Table 5-3). For copper dosages of $75 \mathrm{mg} 1^{-1}$ or less, no volatile acid accumulation was observed despite significant inhibition in methane production. Acid accumulation did occur at copper dosages of 100 and

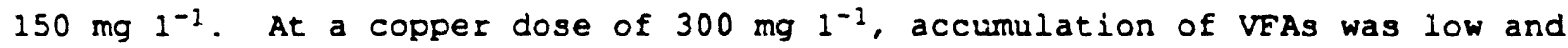
methane production was completely arrested. VFAs for the 20-day HRT inoculum assay, analyzed for after 48 hours of íncubation, exhibited essentially the same pattern. Ace:te accumulation as a function of filtered copper concentration ( 48 hours incubation time) is presented in Figure 5-4 for the 20-day HRT inocula.

The addition of copper produced some rather intriguing results when methane prodiction was observed in a four-hour basis. The four hour interval methane production rates for the 20-day HRT inoculum assay are plotted as a percentage of control samples in Figure 5-5a. Note that during the first interval, for

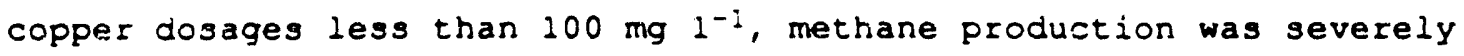
inhibited. Over the remainder of the 24-hour assay, methane production recovered and leveled off to what appears to be a constant value. Total recovery was observed for a copper dosage of $25 \mathrm{mg} 1^{-1}$. Higher dosages did not allow complete recovery within the 24-hour observation period. For the $150 \mathrm{mg} 1^{-1}$ dose, some evidence of recovery of methane production was noted after prolonged ( 5 days) incubation. No recovery was apparent in the samples dosed with $300 \mathrm{mg} 1^{-1}$ for either inoculum.

The effect of copper induced inhibition on hydrogen concentration, as a function of time, is graphically displayed in Figure 5-5b for the 20-day HRT samples. Increasing copper dosages retarded the accumulation of hydrogen in the serum tottle headspace. In samples where methane production was completely inhibited, no hydrogen accumulation in the headspace was observed during the first 24 hours of the assay. Samples inhibited with $300 \mathrm{mg} 1^{-1}$ copper displayed average hydrogen concentrations of 32 and 107 ppm after 2 and 5 days of incubation, respectively. Recovery of methane production was not evident even at 5 days. Samples inhibited with $150 \mathrm{mg} 1^{-1}$ of copper had average observed hydrogen concentrations of over 2000 ppm after 2 days incubation. These values decreased to approximately 150 ppm by day five 


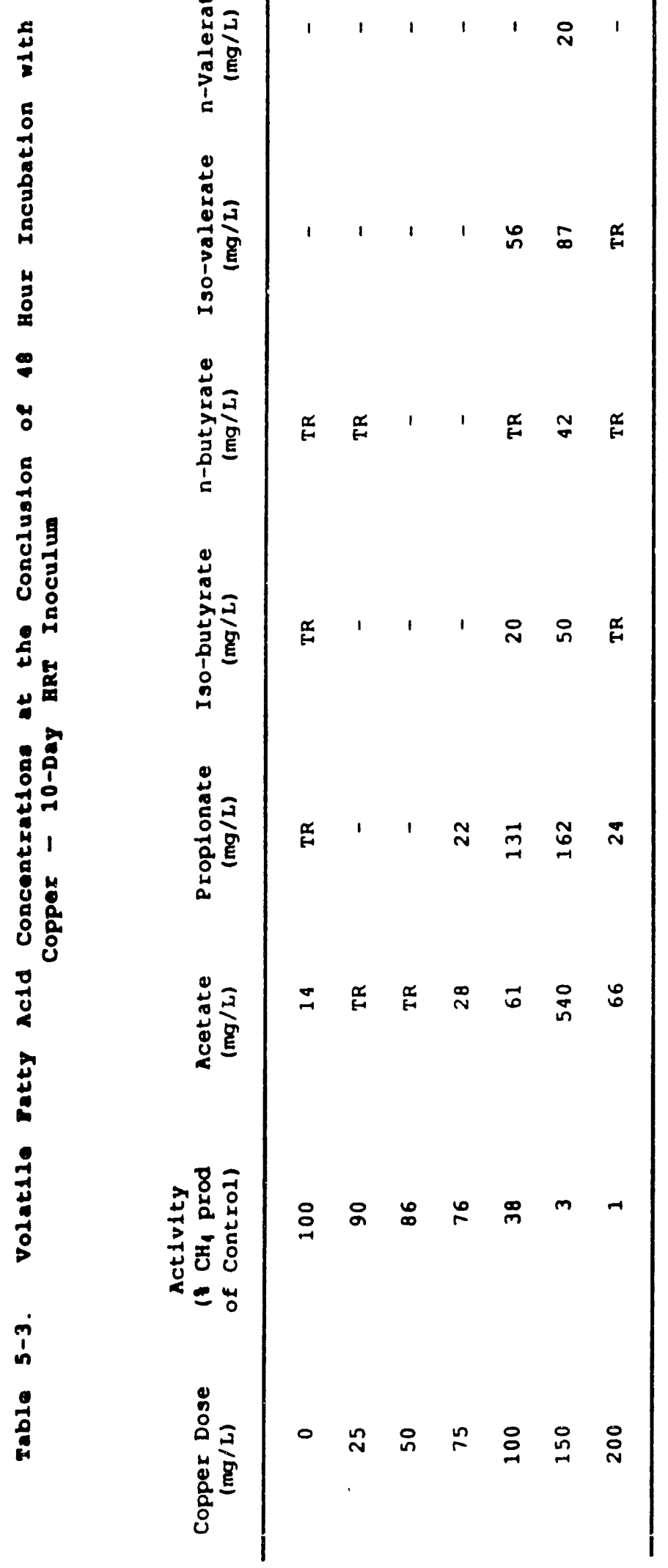




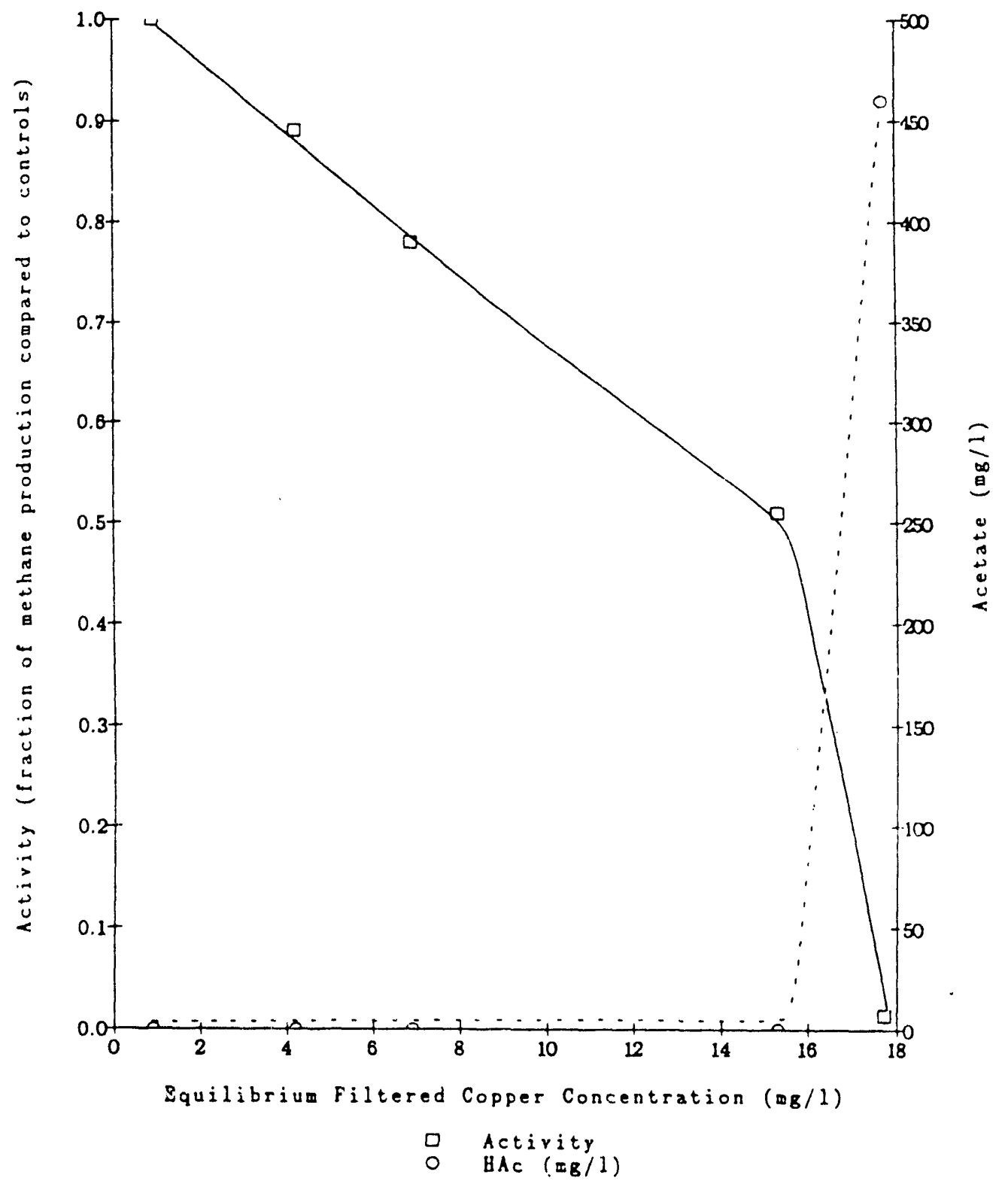

Figure 5-4. Acetate accumulation as a function of filtered copper concentration for 20-day HRT inoculum. 

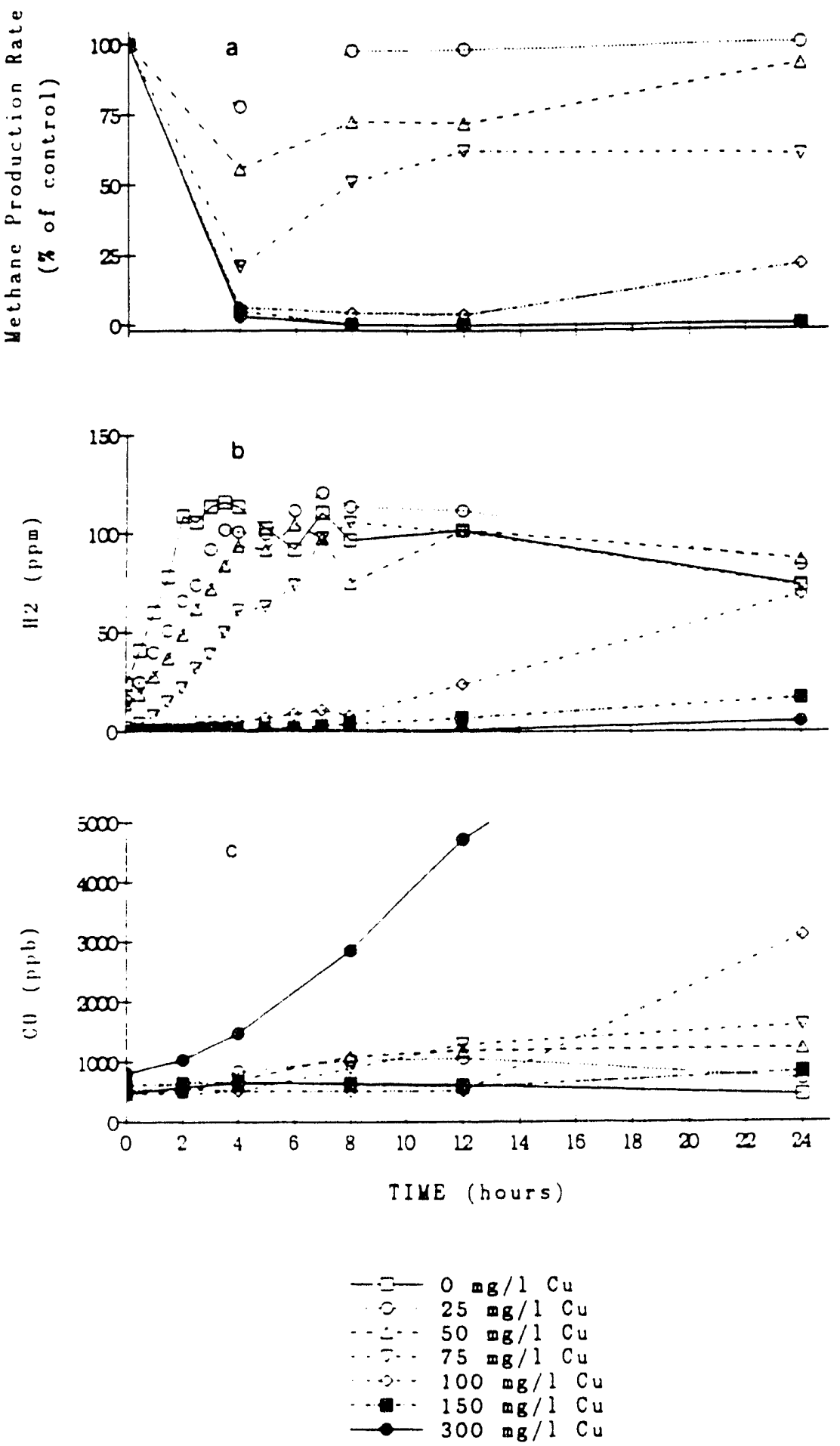

Figure 5-5. Effect of pulse addition of copper on (a) four hour interval mothane production rates, (b) hydrogen response pattern, and (c) CO response pattern as a function of time and copper dose for a 20-day HRT inoculum. 
concurrent with initiation methane production at a low rate. Results for the 10-day HRT inoculum were virtually identical to the 20-day HRT results.

These results, coupled with the VFA-data, indicate that other organisms within the anaerobic consortia may be more inhibited than or as severely inhibited as the methanogens by copper. Whether this is the obligate hydrogen-producing organisms, hydorlytic organisms or other trophic groups cannot be ascertained from the experiments as run.

The corresponding co response to the hydrogen data (20-day HRT inocula) above is presented in Figure 5-5c. Samples displayed three apparent dose related response patterns. Partially inhibited samples that displayed recovery of methanogenesis after the initial 4 hours (25 to $75 \mathrm{mg} 1^{-1}$ ), exhibited a definite increase in CO with time to values well above controls within the initial 12 hours of the assay. Samples where gas and methane production was severely inhibited $\left(100\right.$ and $150 \mathrm{mg} \mathrm{1^{-1 } )}$ exhibited no apparent response; Co remained essentially constant. Samples receiving $300 \mathrm{mg}^{-1}$ (approximately double the dose required to completely arrest methane production) displayed a rapid and continual increase co concentration over time. These samples exhibited no methane production or accumulation of hydrogen during the same time period.

When the co response is presented as a function of copper dose (Figure 5-6), several trends are apparent. The recovery of methane production in samples dosed with $25 \mathrm{mgl}^{-1}$ to control sample rates between 12 - and 24-hour marks was accompanied by a decrease in co concentration to near control levels. The co concentration of the $50 \mathrm{mg} 1^{-1}$ dosed samples was the same at the 12- and 24hour marks as was methane production rate. The $75 \mathrm{mg} 1^{-1}$ dosed samples displayed a continued rise in $C O$ as methane production rate continued to recover. Concurrent with the start of recovery of methane production in samples dosed with $100 \mathrm{mg} 1^{-1}$ copper, between the 12 - and 24-hour time interval, co levels increased for the first time since initiation of the assay. The start of methane production from samples dosed with $150 \mathrm{mg} 1^{-1}$ copper was observed at the 48 hour mark. This, too, was accompanied by a rise in headspace $C O(860 \mathrm{ppb})$. After 120 hours of incubation, methane production wag underway, albeit slowly, and the co concentration had climbed to 


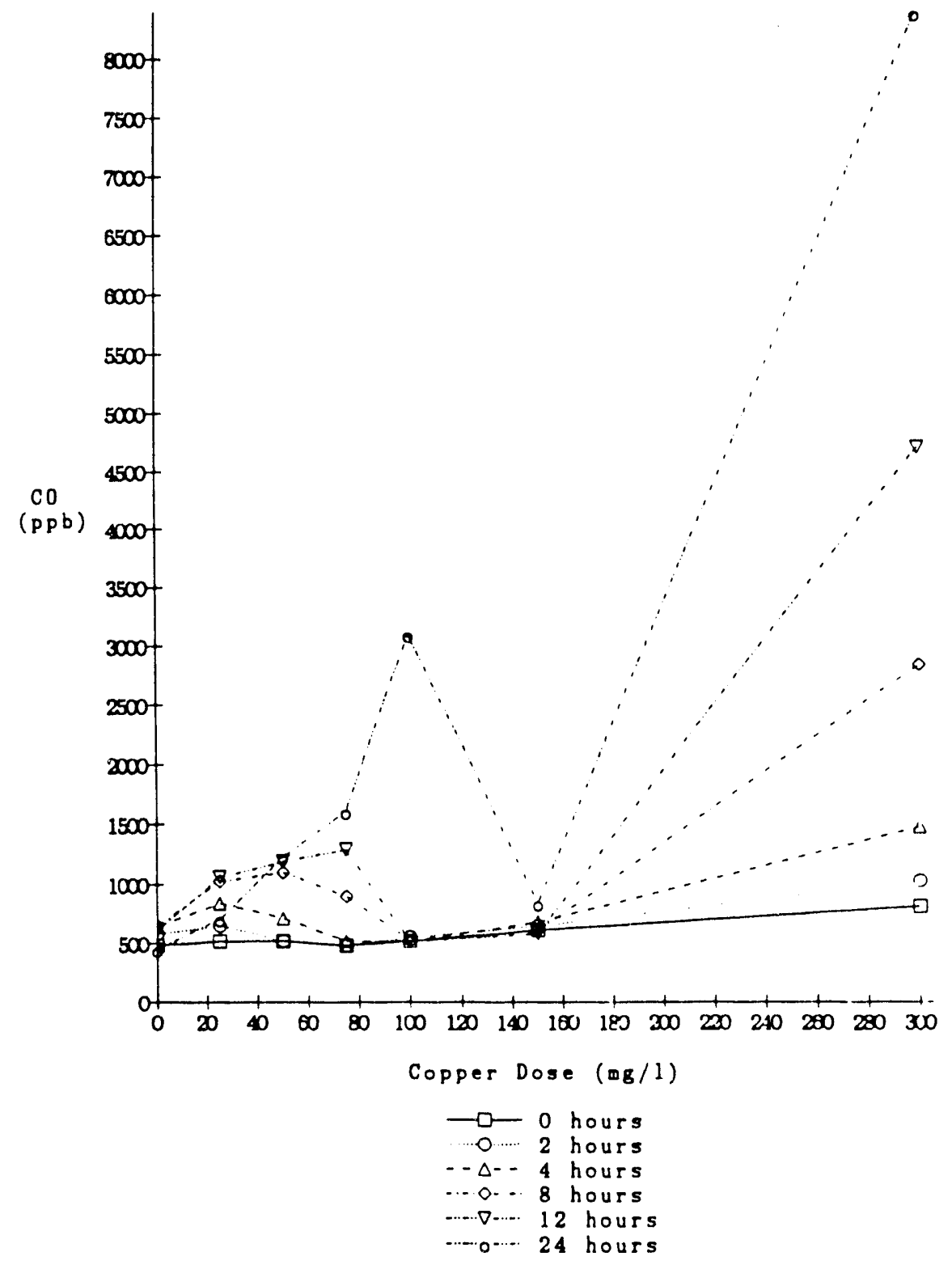

Figure 5-6. Carbon monoxide response pattern as a function of copper dnse - 20-day HRT inoculum. 
approximately $5300 \mathrm{ppb}$ in these samples. Samples receiving the highest copper dose, $300 \mathrm{mg} \mathrm{1-1}$, displayed a continued rise in co concentration over the 5day incubation period reaching $17,700 \mathrm{ppb}$ by this point in time. The results for the 10-day HRT inoculum (data not shown) was similar to the 20-day HRT results. For example, after 48 hours of incubation, samples dosed with $300 \mathrm{mg}$ $1^{-1}$ had average $C O$ concentrations of 10,700 and $9970 \mathrm{ppb}$ for the 10 - and 20day HRT inocula, respectively.

$5.1 .2 \quad 2$ عمن

Results of methane inhibition by zinc for a 24-hour period displayed a similar trend to copper results. Methane inhibition due to zinc, when analyzed on a mg $1^{-1}$ basis, demonstrated a perceptible difference between 10 - and 20-day inocula. When the data were analyzed on a $\mathrm{mg} \mathrm{zn}$ per gvs basis, there was no statistical difference between the 10 - and 20-day results, significant again to greater than the 0.01 level. Results are graphically displayed in Figure $5-7$.

A dose of approximately $65 \mathrm{mg} 2 n+2 \mathrm{gVs}^{-1}$ was necessary to cause a 50 percent inhibition of methane production. Zinc inhibited samples were observed to display a continual decrease in methane production rate with each successive four-hour time interval for all concentrations dosed. The results from the 10-day HRT inoculum are presented in Figure 5-8a. The results using a 20-day HRT inoculum (not shown) were virtually identical. No recovery of methane production was observed for any samples during the entire 48-hour incubation period used in both assays. This is in sharp contrast to copper results where, for lower doses, recovery was observed after the initial four hours of the assay.

Filtered zinc concentrations were measured on samples taken at the conclusion of the 48-hour assay using the 10-day HRT inoculum (Table 5-4). Samples preserved after the assay using the 20-day HRT inoculum were inadvertently destroyed during storage. Zinc appears to be partitioned to the sludge (solid) phase with even greater affinity than copper.

Volatile fatty acid analyses were also performed on samples taken at the conclusion of the 48-hour, 10-day HRT inoculum assay. These results displayed 


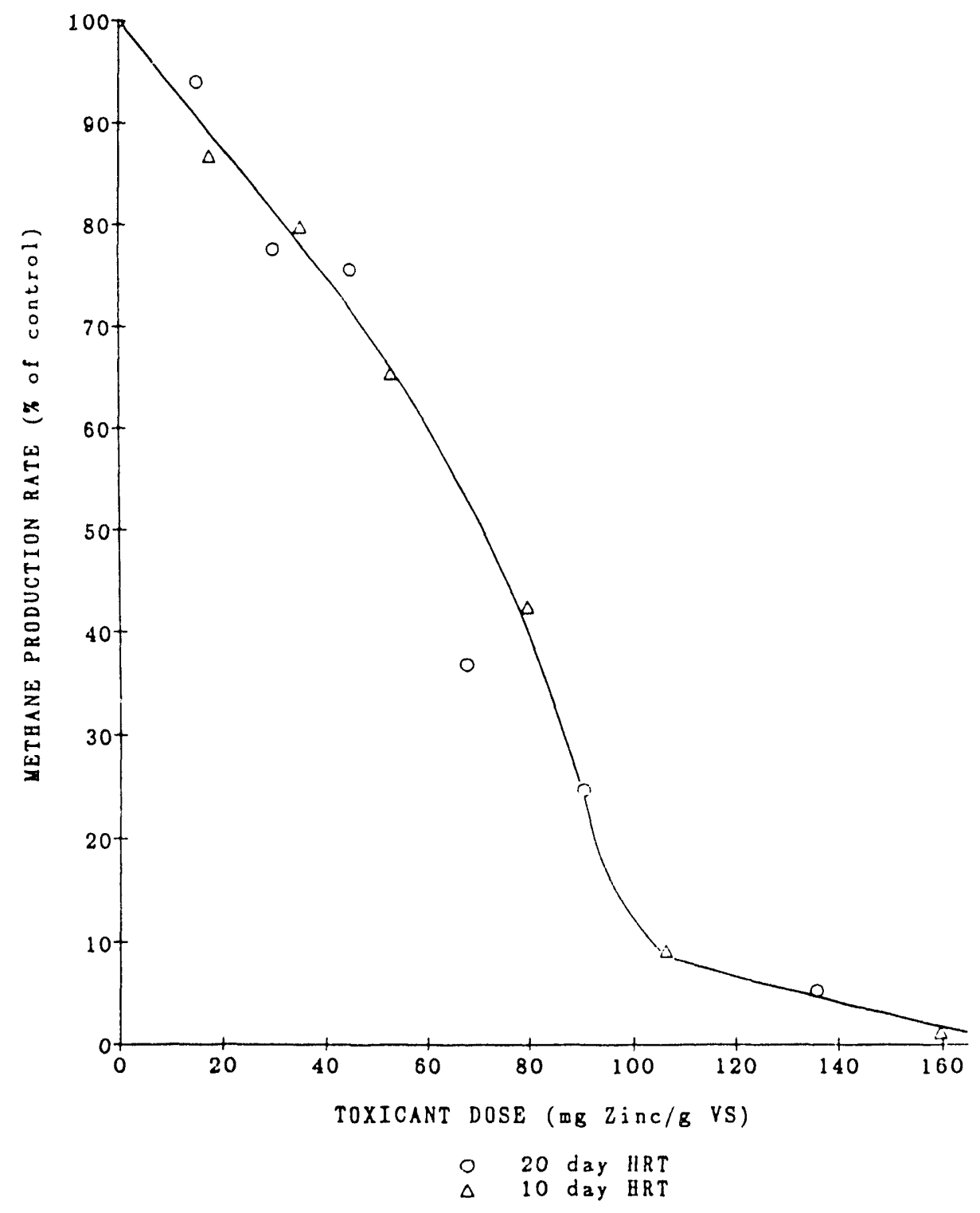

Figure 5-7. Cumulative 24-hour methane production as a function of zinc dose (mg/gVS) for 10- and 20-day HRT inoculum. 

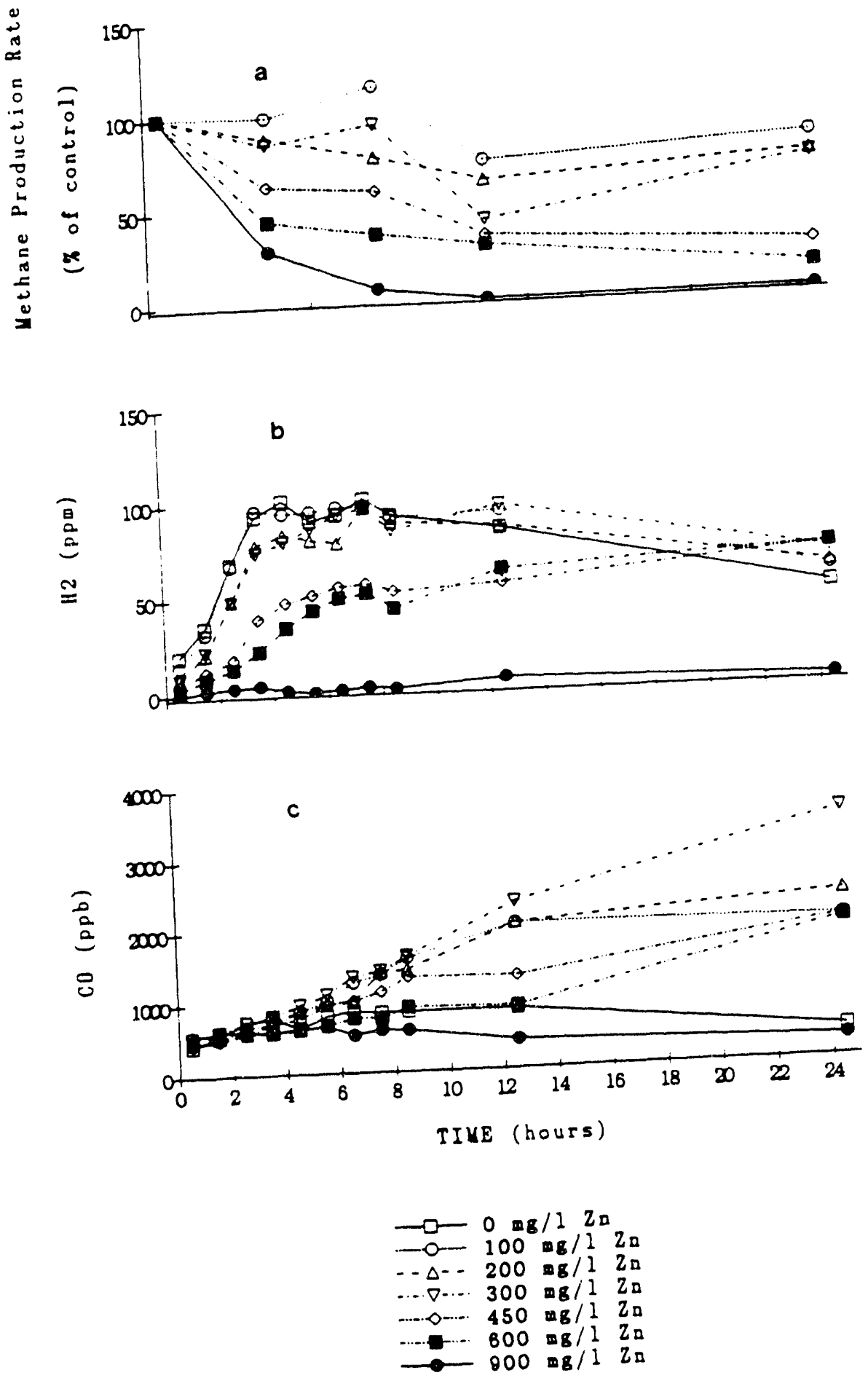

Figure 5-8. Effect of pulse addition of zinc on (a) four hour
interval methane production rates, (b) bydrogen
response pattern, and (c) co response pattern as
defuction of time and zinc dose for a 10 -day HRT
inoculum. 
Table 5-4. Filtered 2inc Concentrations rersus Applied Dose for 10-Day RRT Inoculum

Zinc Dose $(\mathrm{mg} / \mathrm{L})$

100

200

300

450

900
Filtered $\mathrm{zinc}$ Concentration

$(\mathrm{mg} / \mathrm{L})$

0.4

0.3

0.4

3.9

6.4

38.4

a similar trend to that observed for the copper inhibition assays. Accumulation of higher molecular weight VFAs did not occur in any samples.

Hydrogen concentrations in the gas headspace as a function of time and dosage for the 10-day HRT inoculum are presented in Figure 5-8b. Hydrogen accumulation rates were depressed with increased $z$ inc dose and concurrent reduced methane production rates (Figure 5-8a). This is similar to results generated using copper as a toxicant with one exception. Samples that displayed partial inhibition in the copper inhibition assays (generally the 25 to $100 \mathrm{mg} 1^{-1}$ dosages) had peak hydrogen levels in excess of those attained by the controls. This did not occur for zinc inhibited samples. Both hydrogen accumulation as well as peak hydrogen levels reached were depressed by $z$ inc induced inhibition.

The Co-response pattern exhibited by zinc inhibited samples (10-day HRT inoculum) is presented in Figures $5-8 \mathrm{c}$ and 5-9 as a function of time and dose respectively. Partially inhibited samples displayed the same co accumulation pattern that was observed for copper inhibited samples. With the exception of the samples dosed with $900 \mathrm{mg} 1^{-1}$ zinc (highest dose applied), all displayed a continual dose related rise in $c 0$ with time. Samples dosed with $900 \mathrm{mg} 1^{-1}$ displayed no apparent change in co concentration during the assay. The CO concentrations for the least severely inhibited samples 1100 and $200 \mathrm{mg} 1^{-1}$ of zinc) appeared to stabilize between the 12 and 24 hours as did the methane 


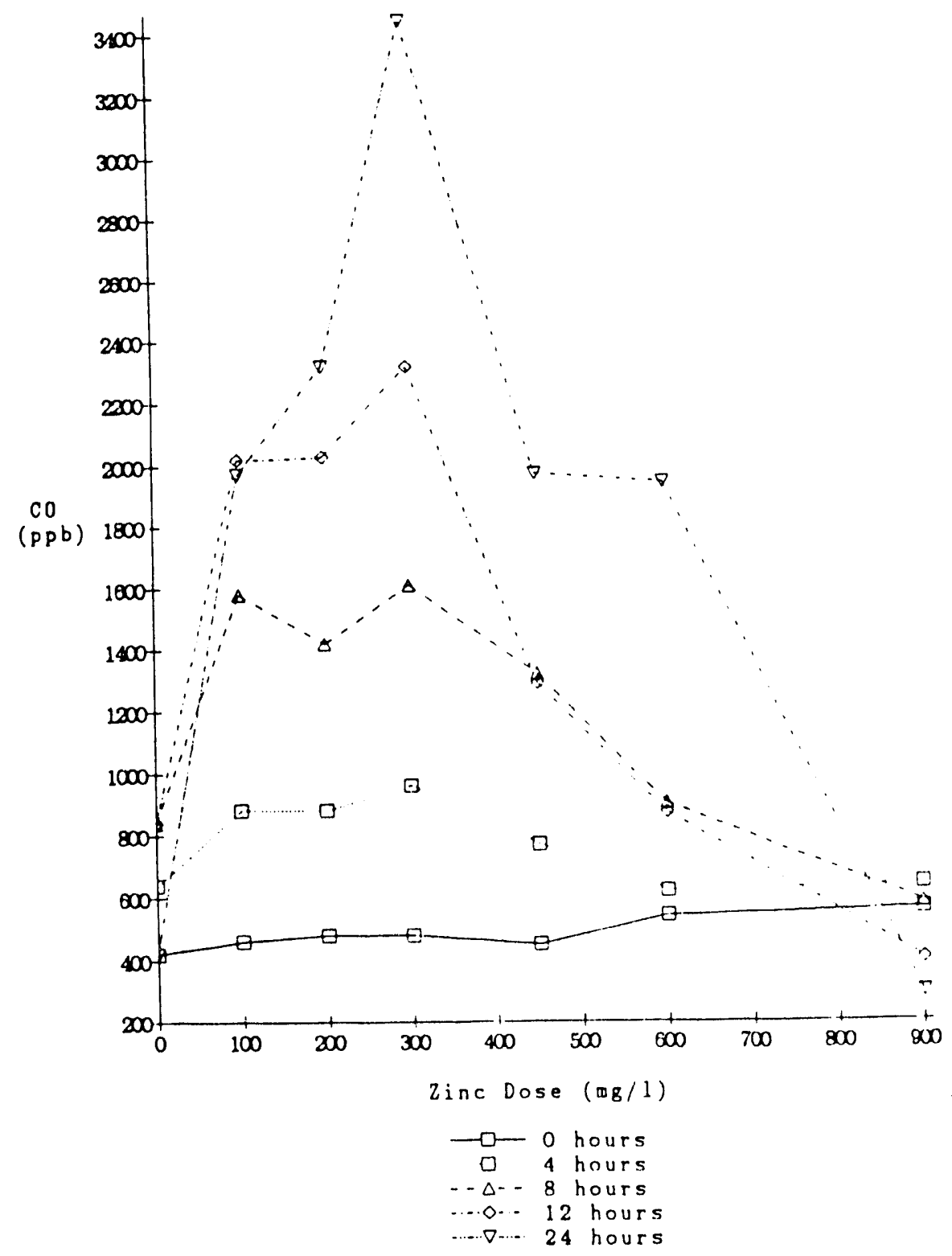

Figure 5-9. Co response pattern as a function of zinc dose -
10-day HRT inoculum. 
production rate. The rapid and continual rise in co to well into the ppm range exhibited by the $300 \mathrm{mg} 1^{-1}$ copper dosed samples was not observed for any samples in either zinc inhibition assay (10- or 20-day HRT inocula). However, no samples were dosed with zinc well above what was apparently required to completely inhibit methane production during either zinc inhibition assay. In fact, the co-response pattern for zinc closely resembled the Co-response pattern for copper if the copper results are truncated at a dose $150 \mathrm{mg} 1^{-1}$. The co-response for the 20 -day HRT inoculum were similar to the 10-day HRT results shown above.

\section{1 .3 Sadmiun}

The effect of cadmium was investigated for the 20-day inoculum only since results with zinc and copper indicated that the mass of toxicant per mass of volatile solids was more important than the difference between inoculum HRT. A dosage of approximately $27.5 \mathrm{mg} \mathrm{Cd}+2 \mathrm{gVS}^{-1}$ was required to inhibit cumulative 24-hour methane production rates by 50 percent. Methane production, when examined on a 4-hour time-interval basis, essentially resembles the results obtained using zinc as a toxicant. Little if any recovery was observed even at lower dosages.

Hydrogen response was similar to the results observed for copper and zinc, with the rate of hydrogen accumulation decreasing with increasing cadmium dose. The peak hydrogen concentrations reached for partially inhibited samples (25 to $100 \mathrm{mg} 1^{-1}$ cadmium) were slightly higher than the maximum levels observed in control samples just as for coppex inhibited samples.

The co-response patterns displayed by cadmium inhibited samples closely mirrored results reported for copper and zinc. Again, a strong apparent correlation between co concentration response pattern and methane production rate was observed.

\subsection{SUMMARY OF RESULTS FROM METALS INHIBITION ASSAYS}

All three metals examined inhibited the production of methane from waste activated sludge. Copper dosed samples that were not completely inhibited displayed partial recovery (and at the lowest dose complete recovery) after 4 
hours of incubation. Zinc and cadmium did not demonstrate any apparent recovery, even after 48 hours incubation.

VFAs were not observed to accumulate in samples that displayed partial inhibition of methane production for copper and zinc dosed samples until a threshold level was reached. In those samples where no VFA accumulation occurred, methane production exhibited what appeared to approximate an inverse, linear relationship to filtered metal concentration.

Samples inhibited with each of the three heavy metals tested displayed the same general trend of reduction in the rate of $\mathrm{H}_{2}$ accumulation in the headspace as a function of increased inhibition of methane production. The peak $\mathrm{H}_{2}$ levels eventually observed in copper and cadmium samples that were only partially inhibited, however, were observed to be up to 120 percent above controls in some instances. This did not occur for zinc inhibited samples indicating a difference in the toxic action between these metals may exist. When complete inhibition of methanogenesis was observed, no hydrogen accumulated in the headspace of any of the serum bottles during a 24 hour period. This together with VFA results indicates that other organisms or trophic groups within the anaerobic consortia involved in the degradation of the sludge to methanogenic precursors are at ieast as severely inhibited as the methanogens. This is in discord with "conventional wisdom" which stresses the fact that the methanogens are the most sensitive organisms in anaerobic ecosysterns.

During most experimental runs hydrogen was observed to accumulate at close to a linear rate during the first 4 hours of the assay. An attempt was made to correlate this rate of hydrogen accumulation to the rate of methane production observed. Since the first time methane production was measured occurred at the 4-hour mark, the rate of $\mathrm{H}_{2}$ accumulation during this period was matched to the methane production rate. Both $\mathrm{H}_{2}$ accumulation and methane production were normalized according to control values. Exceptions to using 4-hour hydrogen accumulation rates were made for those samples where $H_{2}$ peaked at less than 4 hours into the assay. The rate of accumulation in those cases was calculated for the time period during which approximately linear accumulation was observed. 
Reduced accumulation of $\mathrm{H}_{2}$ directly correlated with inhibition of methane production. Results for copper and zinc data sets 110- and 20-day HRT inocula) are presented in Figures 5-10 and 5-11, respectively. For both metals, the 10-day inoculum data had a steeper slope. It is presumed this is simply a function of the greater amount of feed supplied to the 10-versus 20day inocula and, therefore, more rapid accumulation of $\mathrm{H}_{2}$ in controls.

Co demonstrated a strong correlation to methane production. This relationship for the three 20-day HRT inoculum assays is presented in Figure 5-12. This figure shows the $C O$ concentrations and methane production rates (both normalized on control values) after 4 hours incubation. The 10-day HRT results for copper and zinc demonstrated the same exact trend (Figure 5-13) with a slightly more exaggerated response of $C O$ than was observed during the 20-day HRT inoculum assays.

\subsection{SUBSTRATE PULSE EXPERIMENTS}

In an effort to confirm the above hypothesis and to generate additional information to help assess at which stage of the anaerobic process substrate flow was being interrupted by heavy metal pulse addition, three additional experimental runs were conducted. In these experiments, a $2 \times 2$ matrix of controls and copper toxified samples (75 $\mathrm{mg} 1^{-1}$ of copper) were pulsed with various substrates and the gas production and composition was compared to controls for each group. The substrates were selected to investigate specific bacterial groups in the 20-day HRT consortium. Acetate was selected to investigate the acetate-utilizing methanogens, formate for the hydrogenutilizing methanogens, and sucrose to examine the acidogenic population.

\section{3 .1 Acetate}

Controls and samples receiving an acetate pulse (1200 $\mathrm{mg} 1^{-1}$ ) demonstrated essentially the same methane production rate for the first 12 hours. After this period, methane production from the acetate-pulsed samples began to exceed that of the uninhibited controls (Figure 5-14a). This was primarily due to a decrease in the gas production rate in the non-pulsed controls. Whereas the acetate-pulsed samples produced gas (and methane) at a near constant rate for the entire assay, the rate of gas and methane pruduction of 


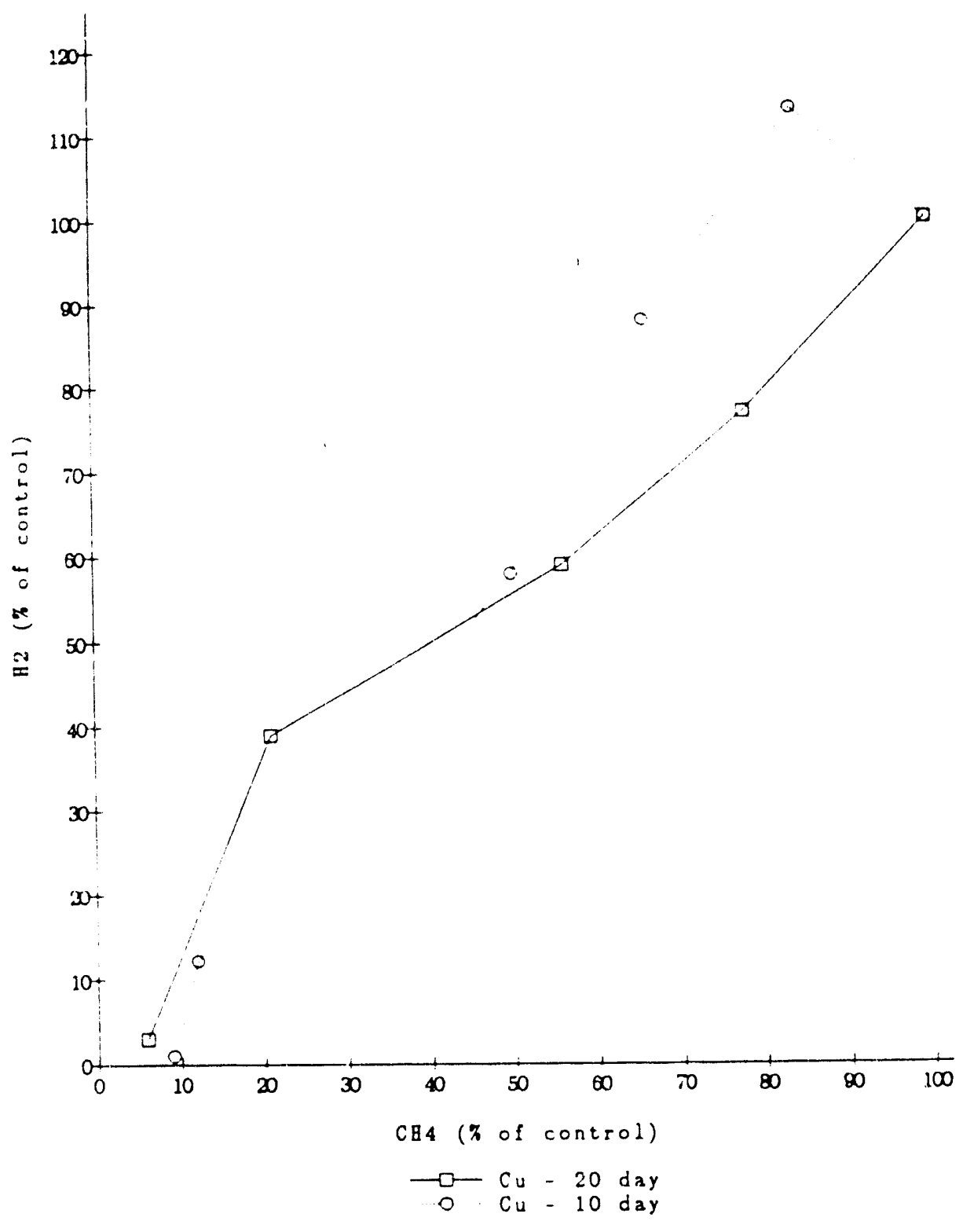

Figure 5-10. Relationship between level of inhibition of
methane production rate and initial accumulation
rate of hydrogen for copper inhibited samples. 


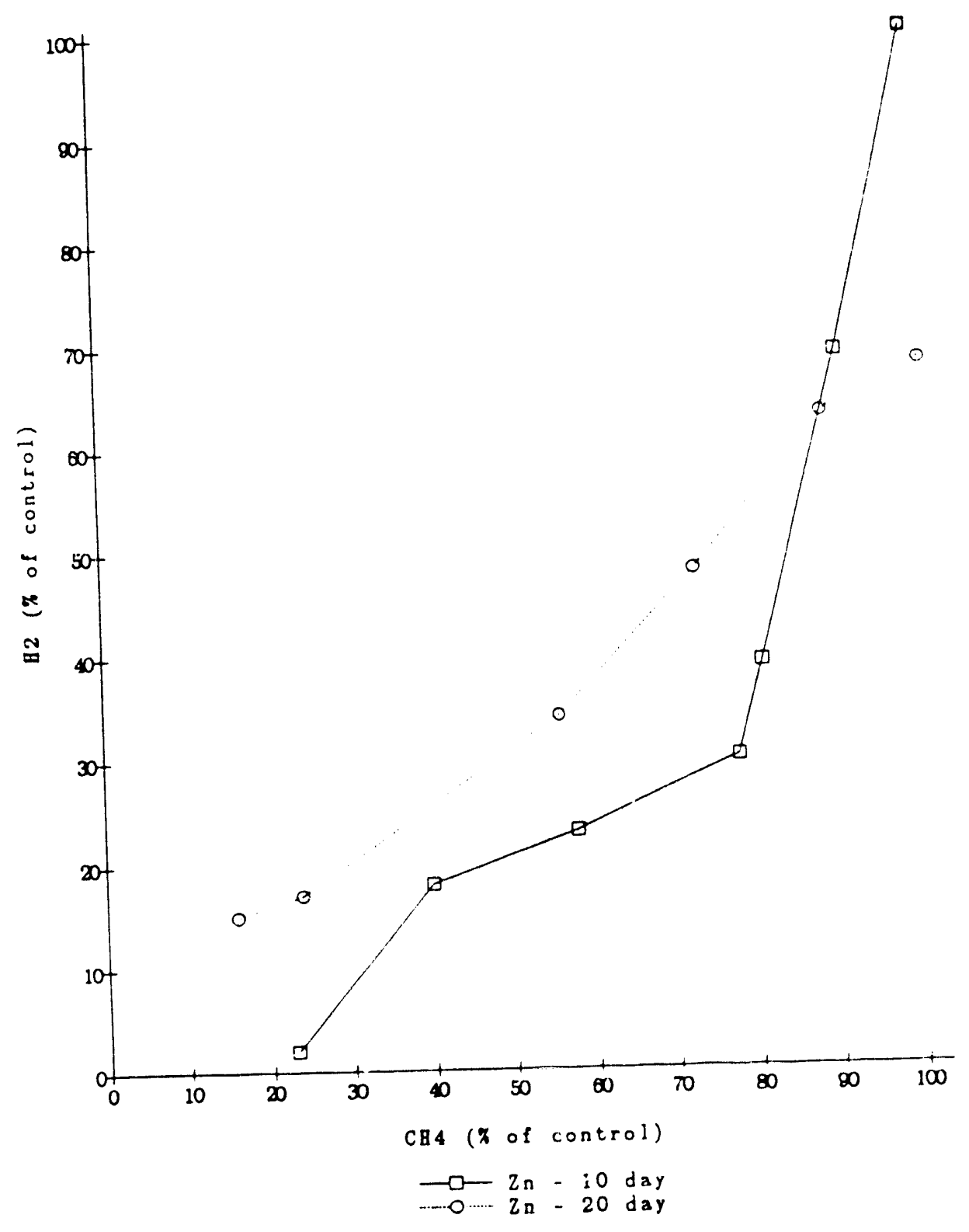

Figure 5-11. Relationship between level of inbibition of
methane production rate and initial accumulation
rate of hydrogen for zinc inbibited samples. 


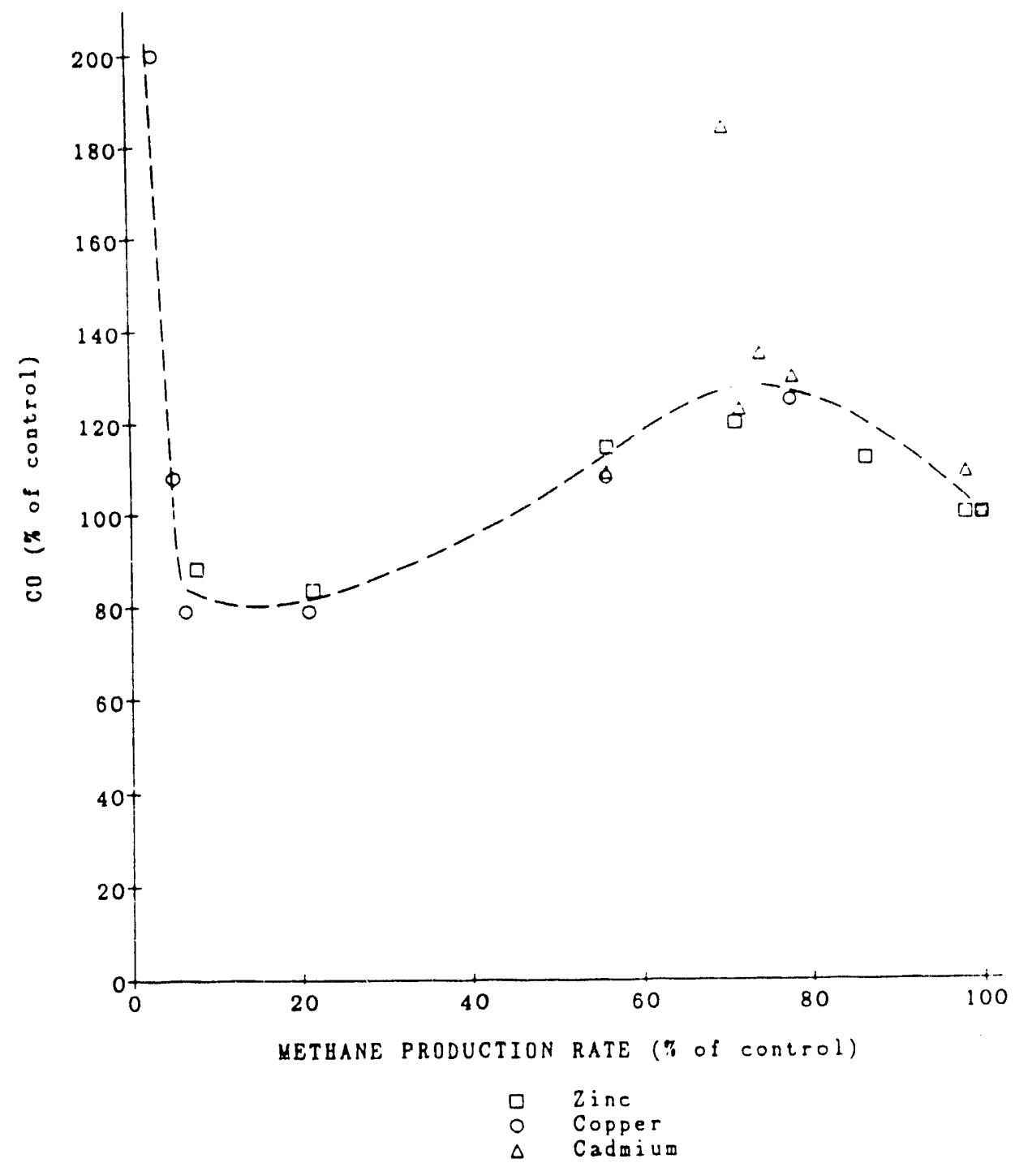

Figure 5-12. Correlation between level of inhibition of methane production, induced by heary metals addition, and carbon monoxide concentration 20-day HRT inoculum. 


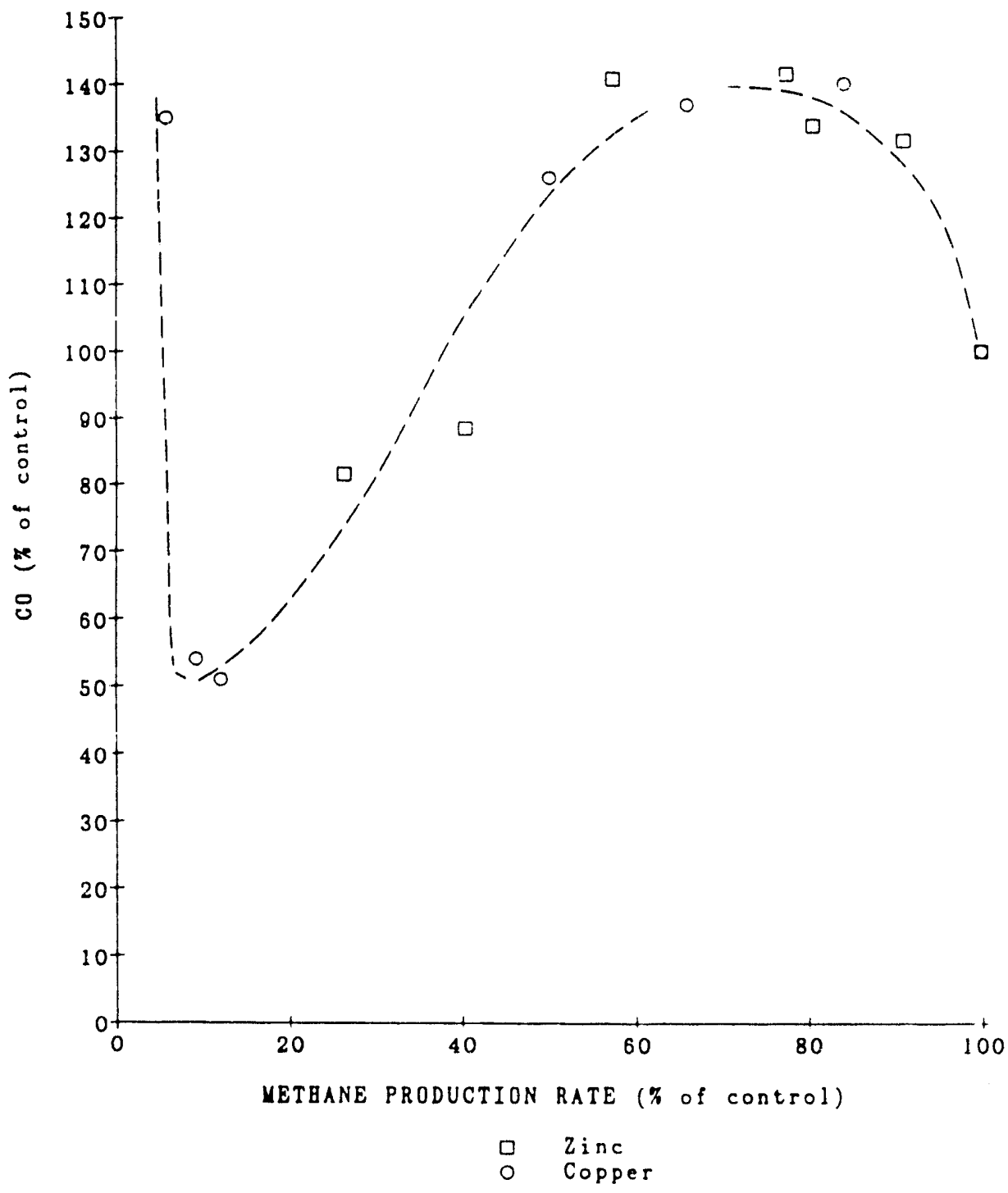

Figure 5-13. Correlation between level of inhibition of methane production, induced by heavy metal addition, and carbon monoxide concentration 10-day HRT inoculum. 

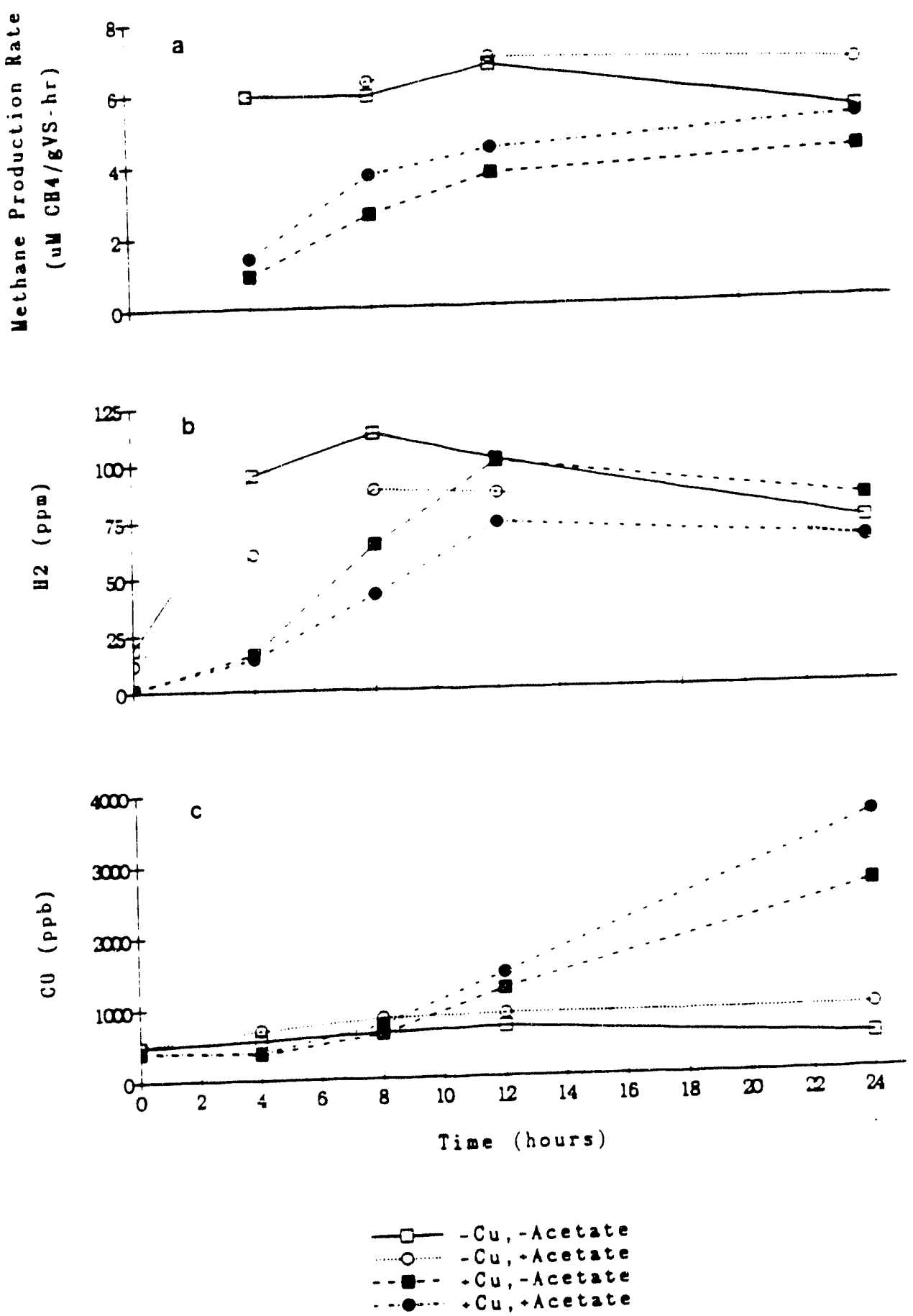

19ure 5-14. Comparison of the effect of pulse addition of acetate on (a) methane production rate, (b) hydrogen response pattern, and (c) CO response pattern for 20-day BRT samples and 20-day RRT samples labibited by $75 \mathrm{mg} / \mathrm{I}$ of copper. 
controls not receiving additional acetate declined to approximately 40 percent of the initial rate by 48 hours. Acetate-pulsed samples that were inhibited with $75 \mathrm{mg}^{-1}\left(15.9 \mathrm{mg} \mathrm{Cu} \mathrm{gVS}^{-1}\right)$ of copper demonstrated higher methane production rates compared to copper toxified samples without supplemented acetate but considerably lower than WAS-fed-only controls. Whether the increase in methane production observed in inhibited samples to which acetate was added is a function of increased substrate or a result of partial binding of the copper by acetate is not known. Results demonstrated that methanogenesis from acetate was inhibited by $75 \mathrm{mg} 1^{-1}$ of copper.

The hydrogen-response patterns for all four sample sets are presented in Figure 5-14b. Pulsing acetate actually reduced the magnitude of the $\mathrm{H}_{2}$ response for both controls and copper inhibited samples. The rice of hydrogen accumulation in copper inhibited samples (with or without added acetate) was lower than for the control samples. The rate of accumulation of hydrogen in the copper inhibited samples (no added acetate) is somewhat lower for this experiment than for the $75 \mathrm{mg} 1^{-1}$ dose samples presented earlier (Figure 5-5b). The hydrogen-response pattern, in fact, is somewhere between the 75 and $100 \mathrm{mg} 1^{-1}$ copper dosed samples (Figure 5-5b). This is because the copper dose in this experiment was somewhat higher $(15.9 \mathrm{mg} \mathrm{Cu}$ - gVs-1) compared to $13.9 \mathrm{mg} \mathrm{Cu}-\mathrm{gVs}^{-1}$ for the $75 \mathrm{mg} 1^{-1}$ dosed samples presented in the early data set.

The addition of acetate to controls resulted in a rise in co levels compared to controls not receiving supplemental acetate (Figure 5-14c). Copper addition resulted in much greater co concentrations being attained for samples with and without added acetate. Copper inhibited samples receiving supplemental acetate, however, demonstrated higher co levels than those samples receiving no added acetate.

\section{3 .2 Sucrose}

The effect of adding $500 \mathrm{mg} 1^{-1}$ of sucrose to a 20-day HRT inoculum, along with the normal WAS, on total gas and methane production rates (measured on 4-hour intervals) is presented in Figure 5-15a. Gas production was significañtiy incressed by sucrose addition, averaging slightly less than 70 percent above controls for the 4- through 12-hour time-interval. Gas 

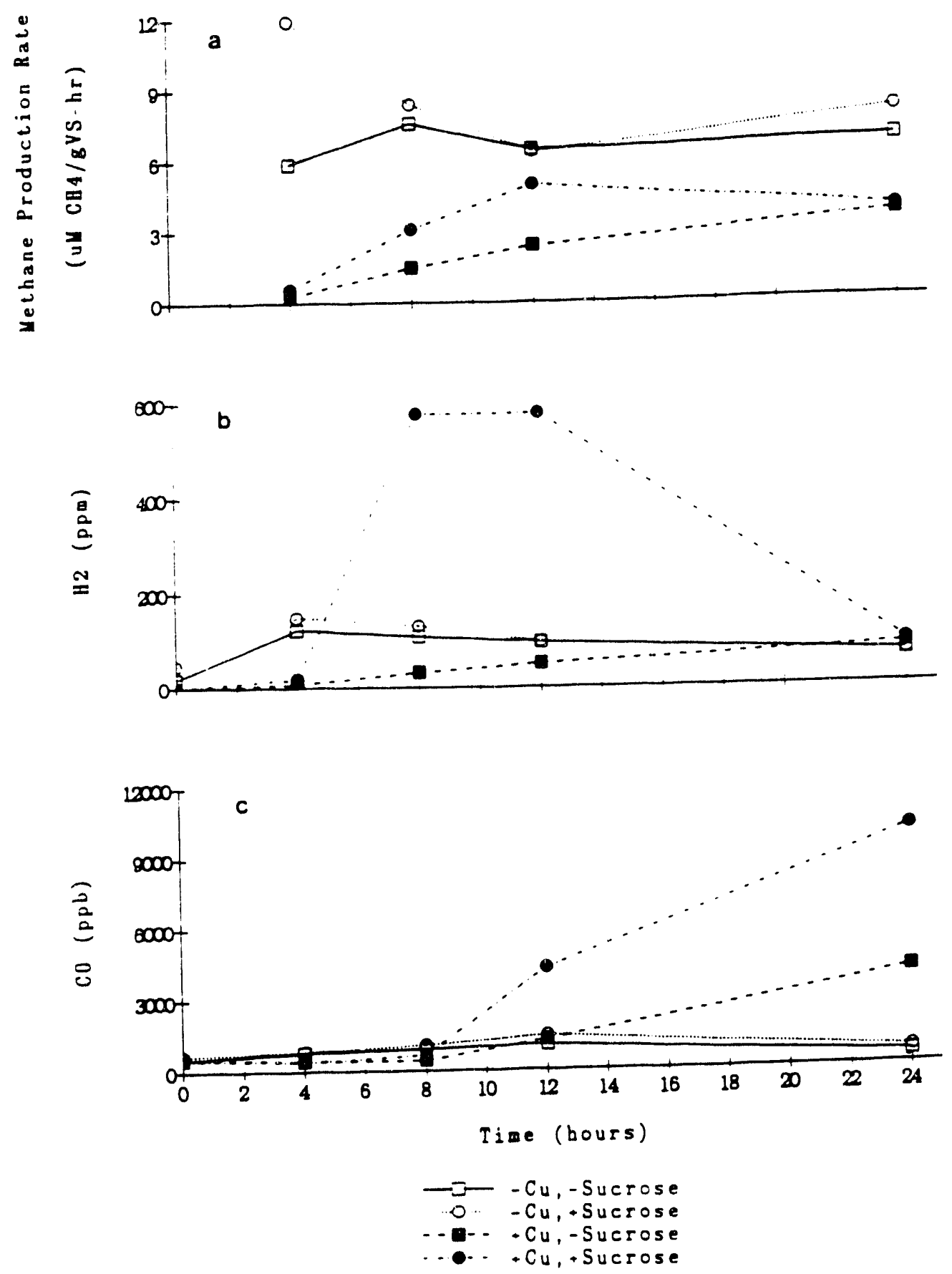

F1gure 5-15. Comparison of the effect of pulse addition of
sucrose on (a) methane production rate, (b)
hydrogen response pattern, and (c) co response
pattern for $20-$ day HRT samples and 20 -day HRT
samples inhibited by $75 \mathrm{mg} / \mathrm{I}$ of copper. 
production rates in sucrose supplemented samples subsequently decreased to 119 percent of controls for the final 12 hours of the assay. Methane production in sucrose supplemented controls was highest during the initial four hours of the assay and then declined to near control rates by the 8- to 12-hour time interval.

Sucrose addition resulted in a considerable increase in gas production from copper inhibited samples. This was principally a consequence of additional $\mathrm{CO}_{2}$ production for both controls and copper inhibited samples. Methane production increased by only 22 and 12 percent of the potential amount (based upon amount of sucrose added) for sucrose amended controls and copper inhibited samples, respectively.

only small amounts of acetate, propionate or other VFA's were detected in samples analyzed at the conclusion of the 24-hour assay. Shunting of some of the carbon and energy, associated with the added sucrose, to additional cell synthesis undoubtedly occurred, but this does not come close to accounting for all of the sucrose added. Eng (1986) observed that lactate accumulated in an anaerobic-upflow-sludge-blanket system fed pulses of sucrose. The concentrations of sucrose used were, however, greatly in excess of the $500 \mathrm{mg} 1^{-1}$ used herein.

Addition of sucrose produced a rapid accumulation of hydrogen in the headspace compared to controls fed WAS only. Hydrogen accumulation during the first two hours was approximately four times greater in samples supplemented with sucrose. By contrast, very little difference in carbon monoxide concentrations between controls and sucrose supplemented samples was evident after two hours. Slowly, the co concentrations in the sucrose supplemented samples began accumulating at a rate greater than controls. It appears that co levels peaked sometime shortly after 12 hours for both controls and sucrose-spiked samples. After this, co levels declined precipitously for both sample sets. Co concentrations averaged just below 400 and $700 \mathrm{ppb}$ for controls and sucrose supplemented samples, respectively, after 24 hours.

Hydrogen production was initially retarded for copper inhibited samples (both sucrose amended and not). Samples with sucrose eventually displayed a rapid 
accumulation of hydrogen during the seventh- and eighth-hour of incubation. Values decreased to those of copper inhibited (no sucrose added) samples by the conclusion of the assay (Eigure 5-15b).

Copper inhibited samples supplemented with sucrose displayed a rapid increase in $C O$ after 7 hours of incubation (Figure 5-15c). Co concentration was continuing to rise at the conclusion of the assay. Results for copper inhibited samples receiving no sucrose were the same as observed for samples receiving the same treatment $\left(75 \mathrm{mg} 1^{-1}\right.$ copper) in the acetate-pulse assay except the time period before co concentration began to rise above starting levels was slightly protracted. This is likely to be related to the copper dose being slightly higher $\left(16.2\right.$ versus $\left.35.9 \mathrm{mg} \mathrm{Cu}-\mathrm{gVs}^{-1}\right)$ on a mass per mass of volatile solids basis. For both the sucrose supplemented and control samples inhibited by copper, the rise in co was concurrent with a sharp rise in hydrogen concentrations.

\section{3 .3 Eermate}

A $600 \mathrm{mg} 1^{-1}$ pulse of formate stimulated methane production from control samples and copper inhibited samples. Formate was quickly converted to $\mathrm{H}_{2}$ and $\mathrm{CO}_{2}$ by the inoculum. It was evident that all samples had a surplus capacity to produce methane from hydrogen. Within 12 hours, all of the added formate was apparently consumed (as judged by additional methane production) in both sample sets (+copper and-copper). Based upon qualitative analysis of the sample chromatograms $\left(\mathrm{H}_{2}\right.$ levels were considerably greater than the concentrations that could be accurately quantified by the instrument used) hydrogen buildup in the copper-inhibited formate-pulsed controls was less than formate-spiked controls.

Formate addition resulted in a rapid accumulation of co in both controls and copper inhibited samples (Figures 5-16a and b). The observed co in controls pulsed with formate peaked at approximately $13,000 \mathrm{ppb}$ after four hours and declined steadily thereafter reaching the level observed in non-pulsed samples compared to formate pulsed controls much like the hydrogen accumulation. co concentrations peaked at between 11,000 and $12,000 \mathrm{ppb}$ at 12 rours and declined to about half that level by 24 hours. The response of the copper inhibited samples was identical to that observed in prior experimental runs. 


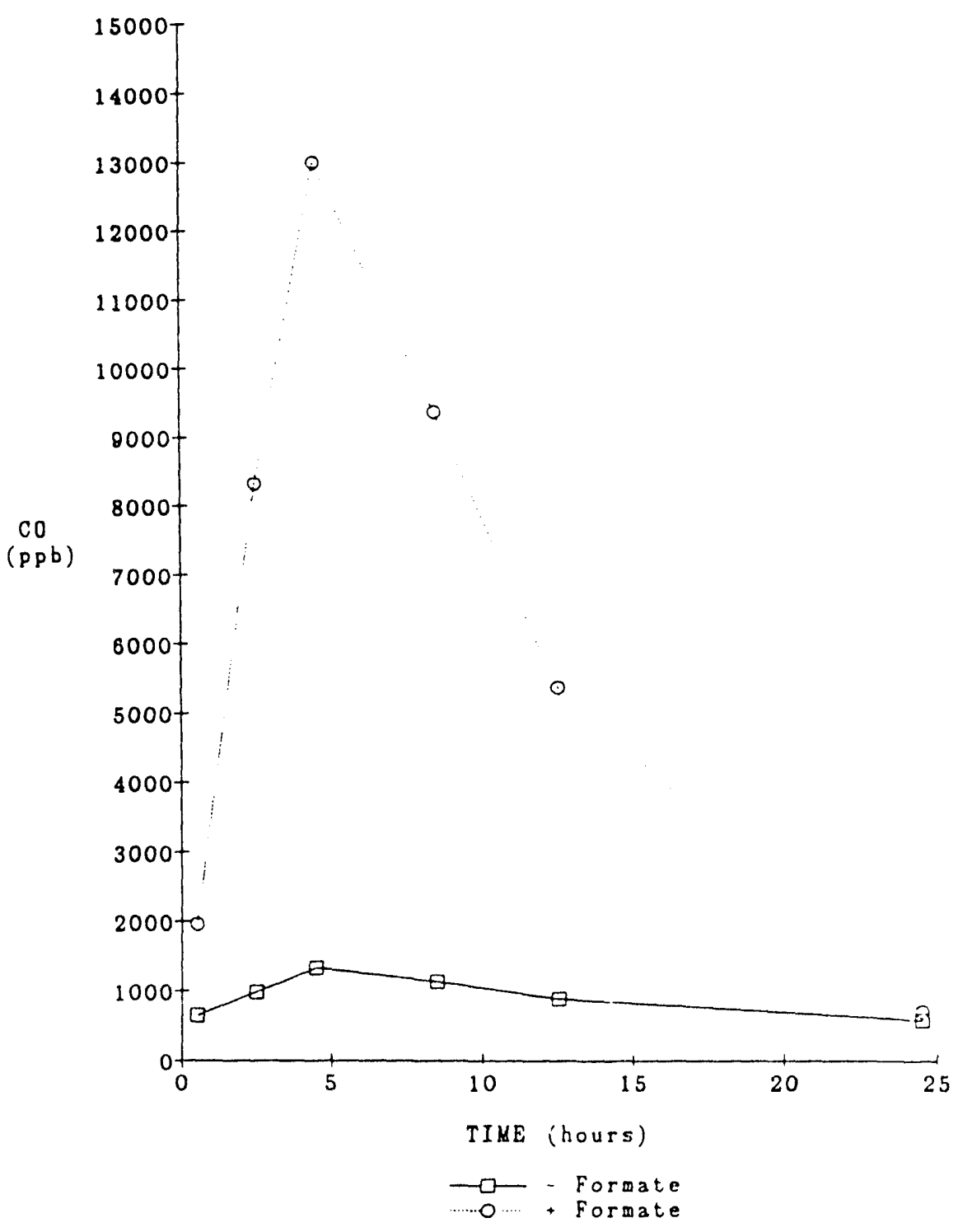

Figure 5-16a. Effect of formate supplementation on carbon monoxide accumulation. 


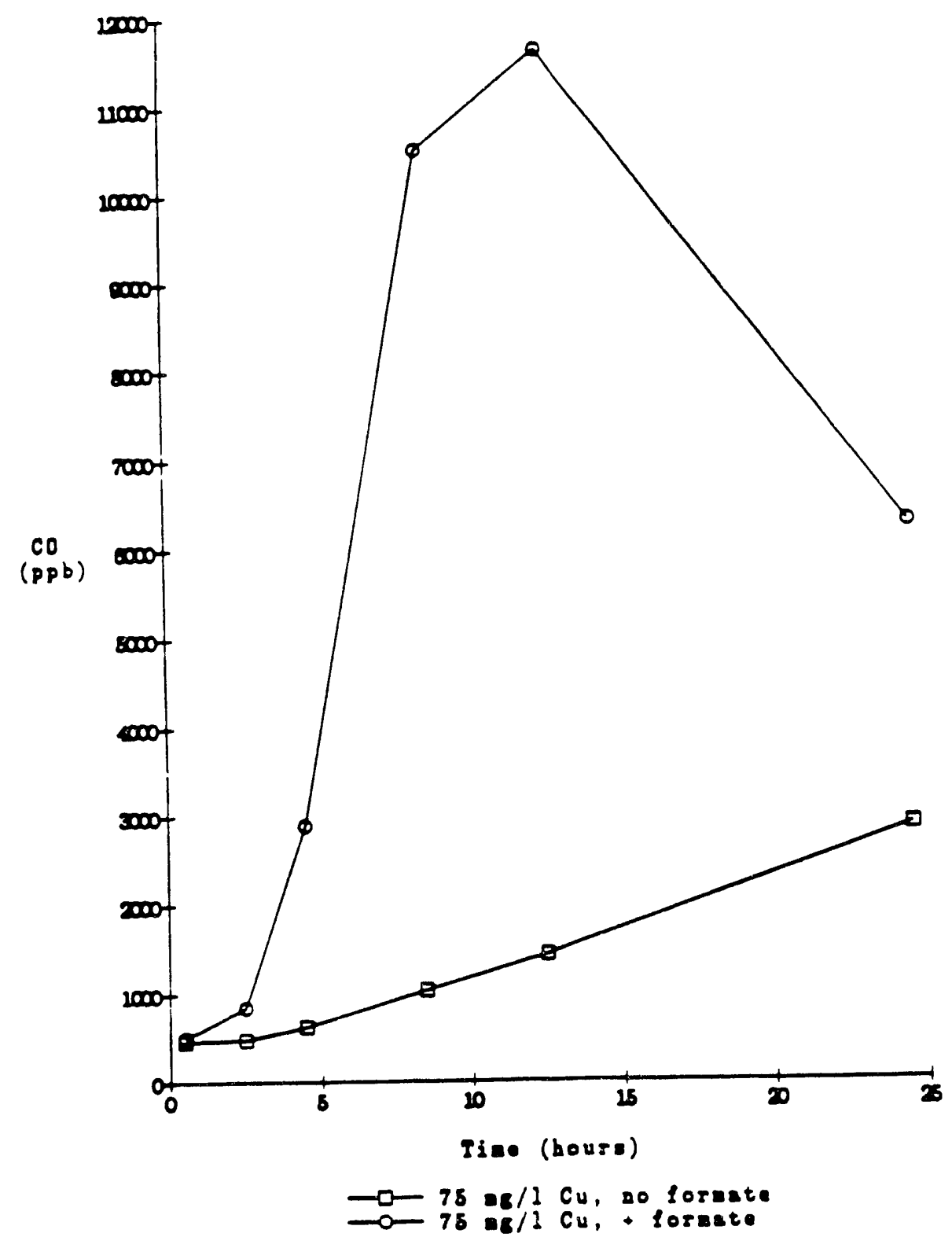

\footnotetext{
Figure 5-16b. Effect of formate supplementation to copper
inhibited samples on carbon monoxide accumulation.
} 


\subsection{SUMMARY OF RESULTS AND DISCUSSION}

It appears that the most significant consideration with respect to heavy metal induced toxicity in anasrobic digester systems is the operating solids level in the system. Higher TS or VS levels (the 10-day HRT digester) afforded better protection from metal inhibition, presumably through binding of the added heavy metals by ligands (and possibly ion exchange sites) contained on the cell membrane and extracellular polymer matrix.

Inhibition due to heavy metals can best be analyzed when metal dosage is expressed as $\mathrm{mg}$ (or milifequivalents) of toxicant per gram of volatile solids. When this was done, there was no discernable difference between the response of the 10-day and 20-day HRT digesters when either copper or zinc were employed as toxicants. This makes sense in light of the above discussion on metals partitioning and agrees with results of Mosey (1976) generated for the digestion of primary sludge. Parkin and speece (1982) also noted that both threshold and toxic concentrations for copper were related to the solids level of their system. This relationship may hold in general for biological systems. Lamb and Tollefson (1973) observed that by increasing the solids concentration of activated sludge, the toxic effect of metals could be alleviated. The higher concentration of soluble refractory CoD in the 20-day HRT system would appear to result in greater concentrations of copper (and other metals) exiting the digester in the supernatant rather than sludge phase. These metals are not likely to be readily bioavailable and will probably not influence the performance of the secondary treatment system if recycled to the head end of the plant. Greater mertals-escape from the treatment plan could result from a recycle of chelated metals from anaerobic digesters supernatant.

Samples inhibited with each of the three heavy metals tested displayed the same general trend of reduction in the rate of $\mathrm{H}_{2}$ accumulation in the headspace as a function of increased inhibition of methane production. The peak $\mathrm{H}_{2}$ levels eventually observed in copper and cadmium samples that were only partially inhibited, however, were observed to be up to 20 percent above controls in some instances. This did not occur for zinc inhibited samples indicating a difference in the toxic action between these metals may exist. 
When complete inhibition of methanogenesis was observed, no hydrogen accumulated in the headspace of any of the serum bottles during a 24-hour period. This indicates that other organisms or trophic groups within the anaerobic consortia involved in the degradation of the sludge to methanogenic precursors are at least as severely inhibited as the methanogens. This is in discord with "conventional wisdom" which stresses the fact that the methanogens are the most sensitive organisms in the anaerobic ecosystems. Acetate accumulation as a function of filtered copper concentration for the 10-day HRT inoculum, provides some strong circumstantial evidence for inhibition of other trophic groups in the consortium. Despite the fact that methane production decreased at a linear rate as filtered copper levels increased, no acetate accumulated (or other VFAs). When the filtered copper concentration increased further, the methane production dropped to near zero and substantial acetate accumulation was observed. When a specific inhibitor of methanogenesis, bromoethanesulfonic acid, was used to inhibit methanogenesis, acetate and higher molecular weight acids accumulated as did gaseous hydrogen (see chapter 4). Acetate accumulation as a function of the level of inhibition of methane production for copper- and BES-inhibited samples ( 20 -day HRT inocula) after 24- and 48-hours of incubation, respectively, is presented in Figure 5-17.

Results from substrate-pulsing experiments indicate that both the acetate- and hydrogen-utilizing populations are inhibited by heavy metals. The fact that added acetate did appear to stimulate methane production from copper-inhibited samples lends circumstantial support to the hypothesis that other groups in the consortium are significantly impaired by the copper (and presumably other metals). Samples pulsed with copper plus acetate would have produced methane at a rate close to the acetate-spiked controls, however, if the acetotrophicmethanogens were not at all inhibited. This did not occur, indicating the acetotrophic-methanogens were also partially inhibited by copper-addition.

Removal capacities for hydrogen over the first 4 hours were estimated to be 200-fold greater for the copper-inhibited, formate-spiked samples than for copper-inhibited samples without supplemental formate. This indicates that the inhibited controls were not producing hydrogen at any substantial rate and 


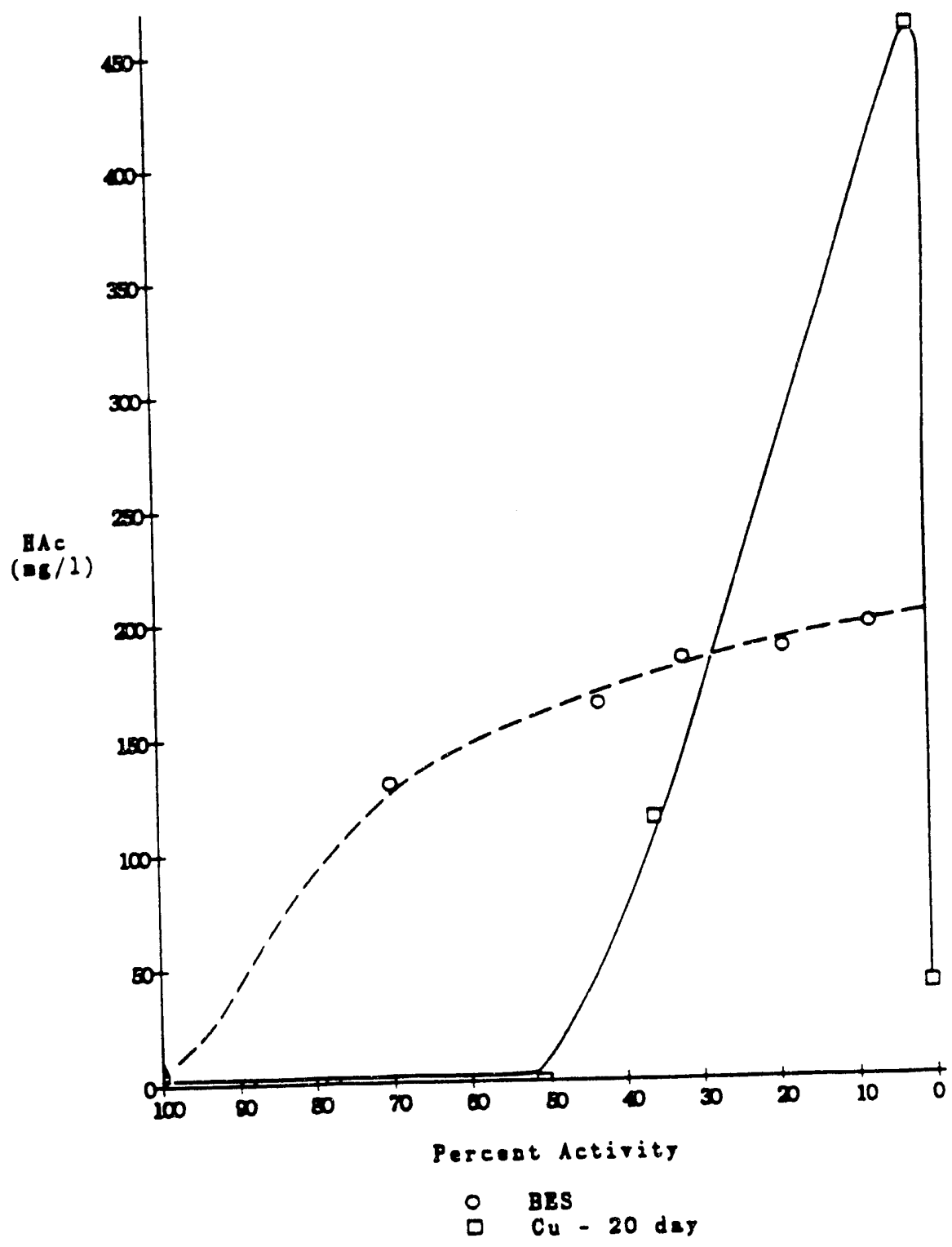
Figure 5-17. Comparison of acetate accumulation pattern as a
function of inhibition of mothan production for
copper and Bes labibited sample. 
further supports the contention that apparently some other group(s) of organisms are inhibited to an extent greater than or equal to the methanogens.

Methane production data frrin copper-inhibited controls and formate-spiked samples indicated that the hydrogen-utilizing methanogens may have been partially inhibited by the applied copper dose. This is, however, difficult to ascertain due to the different levels of hydrogen present in the two sample sets. It was observed that in formate-spiked, copper-inhibited samples, hydrogen accumulated at a rate of approximately half the rate of formatespiked controls. This is in agreement with results of pankhania and Robinson (1984) who observed that the formate dehydrogenase of Methanespirid m hungatei CPI was severely inhibited by heavy metals.

Extremely high concentrations of $C O$ were observed in samples supplemented with formate. A delay in accumulation of $C O$, n the copper-inhibited sample set appeared to coincide with the retardation in $\mathrm{H}_{2}$ accumulation compared to noninhibited controls that received formate. The high $\mathrm{H}_{2}$ levels reached, due to formate addition, caused a substantial increase in methane production from $\mathrm{CO}_{2}$ reduction. Presumably this was accompanied by a spurt in growth as well. It would appear, therefore, that the rise in co was probably associated with the anabolic fixation of $\mathrm{CO}_{2}$ in these instances. Under high growth rate conditions, high levels of co production have been reported for autotrophic me thanogens (Conrad and Thauer, 1983; Eikmanns et al., 1985).

The lag in co accumulation observed for the samples receiving copper, in addition to the formate fulse, can be explained in several ways. It raild be the retardation in $\mathrm{H}_{2}$ evolution observed retarded the fixing of $\mathrm{CO}_{2}$ by $\mathrm{simp}_{\mathrm{y}}$ reducing the driving force for methane production and therefore cell growth. Cell growti has been shown to be integrally related to methane production for one autotrophic methanogen, $M$. themoautotrophicum (Eikmanns et al., 1985). isternately, the copper could ha;e interfered with $\mathrm{CO}_{2}$ assimilation reducing the rate cf methane production or by directly inhibiting the formation of the bound co moiety. Co could also be related to fixation of $\mathrm{CO}_{2}$ by acetogens. Increased methane production was, however, approximately what would be theoretically anticipated based on the amount of formate added. 
Addition of sucrose resulted in a relatively small increase in methane production. $\mathrm{H}_{2}$ levels for the sucrose-spiked, copper-inhibited samples were observed to climb at a much greater rate than copper-inhibited samples receiving no sucrose, during the initial 8 hours of the assay. The $H_{2}$ concentrations, after peaking above $650 \mathrm{ppm}$, subsequently returned to the levels exhibited by non-sucrose spiked controls. The large peak of $\mathrm{H}_{2}$ indicates hydrogen was temporarily produced at a rate in excess of the hydrogen-utilizing population's ability to consume it and demonstrates that the hydrogen-utilizers were also inhibited. Hobson and Shaw (1976) reported that a copper dose of $300 \mathrm{mg} 1^{-1}$ completely inhibited the hydrogen-utilizing methanogen Methanobacterium formicicum while dosages of 100 and $200 \mathrm{mg} 1^{-1}$ caused a lag in hydrogen uptake. Capone et al. (1983) reported that $\mathrm{CO}_{2}$ production was less severely inhibited by heavy metals than methanogenesis, but that sulfate-reduction was more severely suppressed. Sarbon dioxide production was considerable in the samples inhibited by heavy metal addition in this study as well. Ashley et al. (1982) observed that dosing nickel to a sludge digester resulted in a build-up of higher molecular weight VFAs including some (i.e., iso-valerate and iso-caproate) that are believed to be breakdown products specifically related to amino acid degradation. They concluded that for nickel induced inhibition in their system methanogenesis was most severely inhibited and hydrolytic activity less affected.

In this study, circumstantial evidence strongly suggests that both trophic groups of methanogens are inhibited by heavy metals, but not as severely as other bacterial groups that play an important role in the degradation process. Because substrate flow is apparently interrupted at the same point by the heavy metals used in this study, there appears to be a generic hydrogenresponse to heavy metal related inhibition.

The relative toxicity of the three metals examined was $\mathrm{Cu}>\mathrm{Cd}>\mathrm{Zn}$, which is in agreement with the results from other researchers (Hayes and Theis, 1978; Capone et al. 19831 .

In this study, partial recovery from copper inhibition was observed after 4 hours for some samples. Thí3 is believed to be the consequence of copper (II) being reduced to copper (I) during the assay. The reduction of copper could 
decrease its toxicity or simply free more sulfide to precipitate additional copper. Mosey and Hughes (1975) reported that copper (II) was slowly reduced to copper (I) in anaerobic systems.

Recovery from heavy metal inhibition does not apparently occur in the short term due to adaptation or acclimation (Parkin and Speece, 1982).

CO also displayed a characteristic response pattern to heavy metal induced inhibition. Subsequent experiments conducted with the intent of specifically examining $C O$ and its relationship to acetate metabolism strongly indicated that $c o$ concentrations are redox controlled under non-inhibited conditions (Hickey and switzenbaum, 1990). In the batch assays reported later on in this chapter, it is likely that the co response was affected by the changes in the methane content of the gaseous headspace over time. Therefore, the results obtained from the batch systems are not directly comparable to a system operating with a 60 to 70 percent methane content before inhibition occurs. co-monitoring appears to have potential to be an excellent indicator to detect the onset of heavy metal induced inhibition since the magnitude of the coresponse should be easily identified against any normal background variations in concentration. Whether a similar response will be detectable upon chronic or gradual addition of a heavy metal to a system remains to be determined, as does the reason for this characteristic response pattern observed.

The investigations of the effect of heavy metals on anaerobic sludge digestion were carried out with two inocula and three heavy metals. The data cannot be considered as generalized results. Subtle but perceptible differences in the response of the digester systems to the three toxicants were observed. The rather strong similarities observed in the hydrogen response patterns for all experimental runs does, however, suggest they represent more than isolated case histories and may in fact represent a generic response to heavy metal induced inhibition.

The on-line data sets both demonstrated a characteristic response pattern due to pulse addition of mildly inhibitory doses of copper. Co was observed to rise sharpiy concomitant with a decrease in $\mathrm{H}_{2}$ concentrations. These responses are easily differentiated from the normal diurnal patterns for these 
two trace gases, particularly when the responses of the two trace gases are considered together. With the exception of total gas and methane production, conventionally employed process indicators did not show any indication of process upset. It would appear that hydrogen- and Co-response patterns could be used to detect upsets as a result of heavy metals and possibly be employed as a monitoring technique designed to provide early warning for upsets as a result of heavy metals. 
CHAPTER 6

DISCUSSION AND CONCLUSIONS

\subsection{DISCUSSION}

The results from the organic and heavy metal inhibition assays demonstrated that both groups of toxicants inhibited the acetate- and hydrogen-utilizing methanogenic populations. The responses observed from the trace gases, hydrogen and carbon monoxide, were significantly different for the two types of toxicants, however. In the case of organic toxicants, inhibition was accompanied by increased hydrogen concentrations with the concentration of hydrogen increasing with increased inhibition of methane production. By contrast, hydrogen accumulated less rapidly than normal for heavy metal inhibited systems. The rate of hydrogen accumulation decreased with reductions in the rate of methane production.

These seemingly contradictory results can be explained by examining the substrate flow (Figure 6-1) and identifying where the major interruption in this sequence of reactions occurred. In the case of organic toxicants, the data indicated other groups within the anaerobic consortium were not affected at all by the toxicants used but only at the highest concentrations. The point of interruption in normal substrate flow, therefore, was at the two methanogenic steps. Accordingly, both acetate and hydrogen accumulated. When heavy metals were used as toxicants, however, results indicated that some other group(s) within the consortium were more severely inhibited than the methanogens. Substrate flow was, therefore, interrupted at a point prior to the methanogenic step and accordingly, hydrogen and acetate did not accumulate despite the fact that the populations that consume these intermediates were inhibited.

The response of $c 0$ to both these toxicants is more difficult to interpret in terms of substrate flow. When organic toxicants were used to inhibit the digester inocula, co concentrations went up as did acetate concentrations. The concentrations of $c 0$ attained, however, did not appear to be closely related to the acetate concentration. The response of co to organic-toxicantinduced inhibition appeared to be specific to the compound employed. In all 


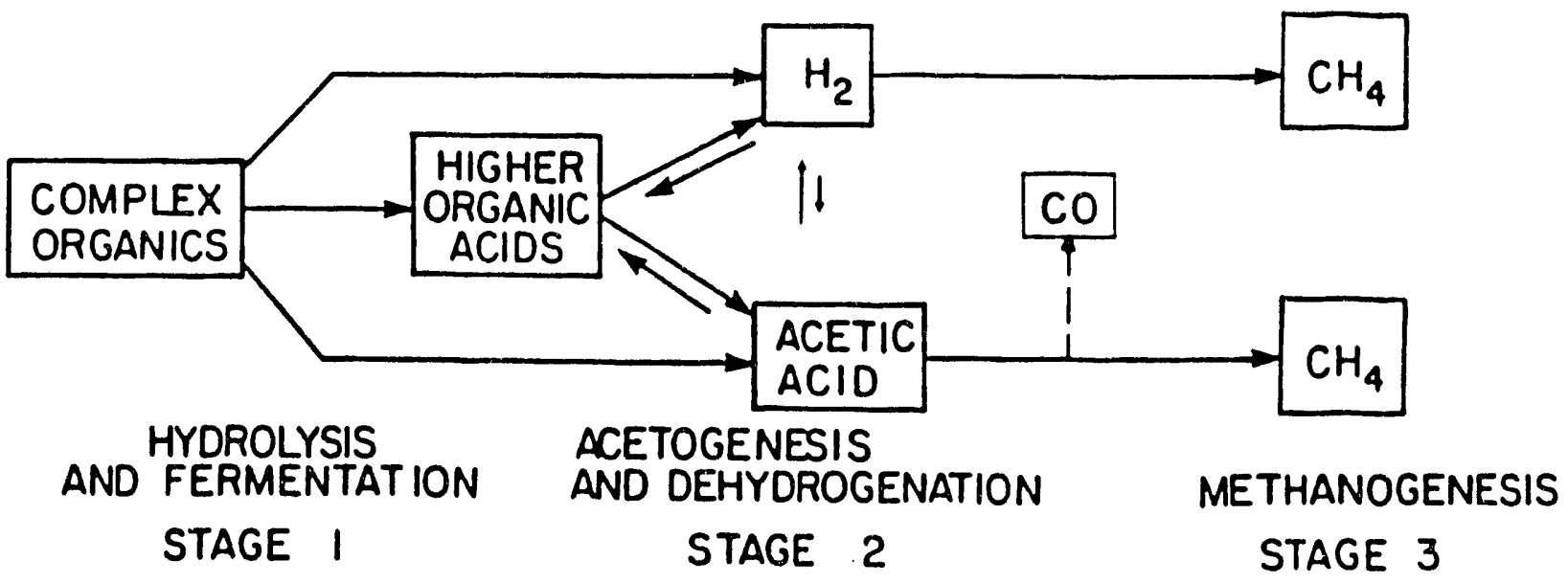

Figure 6-1. Three stage model of substrate and energy
flow for the anaeroblc digestion of complex
organic materials. 
cases, however, carbon monoxide was observed to accumulate well above control sample concentrations when severe inhibition of methane production occurred. co could, therefore, be used to provide confirming information, if monitored along with $\mathrm{H}_{2}$, for organic-toxicant-induced inhibition. Co-response was not readily predictable and no clear-cut relationship between so and the level of inhibition of methane production cculd be drawn from the linited data base collected. Since the site(s) where the various organic toxicants interfere with the normal metabolic process are probably different, this is not unexpected. When heavy metals were used as toxicants, the concentration of co went up initially for moderate (less than 20 percent) inhibition of methane production despite no accumulation of acetate and low hydrogen concentrations. It would appear that the heavy metals had a direct effect on at least one group of bacteria that are able to produce gaseous co. Carbon monoxide did, however, display a characteristic response pattern to heavy-metal-induced inhibition of methane production. Although the reasons for this observation are unclear, it indicates that monitoring $c o$ in conjunction with hydrogen may be an effective means not only to diagnose oncoming upsets, but also to allow a fingerprint as to the type of event occurring. A much larger data base will be required to determine if this is possible. Only a limited number of toxicants and only one administration pattern (pulse dose) were employed during this work, so sweeping generalizations are not valid. Whether the coresponse in heavy meta]. inhibited samples is related to the methanogenic population, as would appear to be the case for the samples inhibited by organic compounds, canrot be determined from the data collected during this study.

Most of the hydrogen-response information and the general framework for theoretically analyzing hydrogen in anaerobic systems has been done from the perspective of carbohydrate degradation. When examining the hydrogen-response patterns exhibited by anaerobic digester systems it is necessary to bear in mind that proteinaceous materials rather than carbohydrates may be the primary substrates, especially for biological sludges. Factors regulating the hydrolysis of proteins and subsequent degradation and fermentation of the polypeptides and amino acids produced are not well understood (Breure and van Andel, 1984; Breure et al., 1985). Accordingly, hydrogen-response patterns are difficult to predict from a theoreticai basis. 
Recently, Hino and Russell (1985) concluded that sequential transamination, via glutamatic dehydrogenase, is the predominant method of fermentation of branched chain amino acids such as leucine which leads to the formation of branched volatile acids such as iso-valerate. A build-up of hydrogen, therefore, could negatively affect the fermentative step in sludge digestion as well as the subsequent degradation of the VFAs produced. The role that this phenomenon may play in affecting $\mathrm{H}_{2}$ - and $\mathrm{CO}$-responses needs to be examined. It would appear this type of phenomenon would tend to reduce substrate flow at the fermentation stage and may in part account for the relatively low hydrogen concentrations observed when experimental results indicated the $\mathrm{H}_{2}$-consuming population was partially inhibited by organic toxicants.

Egsential information regarding toxicity and inhibition can be obtained from pure and enrichment culture studies. It is important to observe and understand the behavior of individual bacterial populations in response to toxic or inhibiting episodes, particularly for bacterial groups occupying such a central position and playing such a key metabolic role in an ecosystem as methanogens. Still, we must not lose sight of the fact that these organisms function in a complex consortium where many subtle but important interactions among different trophic groups occur. Results presented herein demonstrate the importance and need to compliment pure and enrichment culture studies with assay techniques that examine the response of the entire bacterial ecosystem of interest. The effects of interrupting or altering the synergistic, competitive and mutualistic relationships between trophic groups can only be determined by studying the system as a whole. Only by understanding where normal substrate flow is being interrupted or a key pathway-overload is occurring can monitoring results be readily interpreted.

As energy costs varied over the past decade, there has been renewed interest in anaerobic digestion for industrial wastewater treatment. A surge in research activities has led to significant advances in the basic understanding of the biochemical and microbiological processes involved. This in turn has allowed the development of a new generation of vastly improved process reactor configurations. As a result, there is now considerable interest in harnessing 
anaerobic processes for the treatment of industrial wastewater and in the bioconversion of specialty crop-grown biomass and agricultural residues to pipeline quality methane.

The largest potential growth market for the next 20 years for anaerobic biotechnology is in industrial wastewater treatment. The market is in a highly dynamic state. Within the past decade, over 60 anaerobic industrial wastewater treatment systems have been constructed worldwide (Speece, 1983 ). Most of these have been commissioned within the past five years.

The major impediment to accelerated activity in construction of new industrial anerobic treatment systems is a lack of proven reliability. There are a variety of large water usage industries with wastewaters that are quite amenable to anaerobic processing but can from time-to-time contain inhibitory or toxic concentrations of certain compounds or elements. The possibility of having a significant upset and loss of biological capability for an extended amount of time (anaerobic systems tend to be slow to start up and slow to recover from toxic events due to the low organism growth rate) has all but closed the door for application of anaerobic processes in these market areas. Reliability has been consistently chosen over cost effectiveness if there is reasonable doubt as to the performance capability of a treatment option. The increased reliability that an improved monitoring and potentially an early warning system would afford could serve as a catalyst for the installation of anaerobic treatment systems in these areas.

It should also be pointed out that there are significant differences associated with the anaerobic treatment of wastewaters bearing solids, such as municipal sludge, and wastewaters carrying soluble wastes that are more typical of industrial effluents. Because of these differences, supplemental research is required to confidently extend the utility of work performed with sludge digesters to industrial waste treatment. A brief review of the biochemistry will demonstrate why.

In methane fermentation of complex particulate organic materials, the rate limiting step during balanced fermentation is the hydrolysis of particulate material to soluble substrates such as simple sugars, amino acids, and 
volatile fatty acids (Eastman and Ferguson, 1981; Ghosh, 1981). These substrates are then fermented to intermediate products and eventually converted to methane and carbon dioxide.

The majority of industrial wastewaters that appear to be well suited to anaerobic treatment contain primarily soluble organic carbonaceous material. The rate limiting steps for conversion of these substrates to methane and carbon dioxide will be, in most cases, the terminal or methanogenic steps, particularly the conversion of acetate to methane. The response of a high rate, soluble substrate system to organic overloads or toxic shocks should be quite different than the response observed for a hydrolysis limited system. In general, soluble compounds are rapidly fermented to organic acids which can accumulate quickly. Because of this, it is anticipated that $\mathrm{H}_{2}$ - and $\mathrm{CO}-$ response may be much more dramatic and dynamic for high-rate systems treating wastewaters bearing soluble wastes during overloads, inhibition episodes and possibly for baseline or normal operating conditions than has been observed for sludge digesters. Several research teams have, in fact, reported large swings in gaseous hydrogen concentrations due to severe organic overloads (Archer et al., 1986; Eng et al., 1986; Barnes et al., 1984). More work will be necessary to even qualitatively determine if $\mathrm{CO}$ - and $\mathrm{H}_{2}$-monitoring will be appropriate for these type systems. Even if hydrogen and carbon dioxide demonstrate the same relationships observed for sludge digesters, it will be important to understand precisely how this relates to the stability of these high-rate processes. The effect that mass transfer limitations for substrate penetration into the biofilm and release of gas produced back to the bulk fluid needs to be investigated.

The concept of growing and harvesting specialty crops with expressed intent of producing pipeline quality gas is currently in the developmental stage. presently, there is no market in the biomass to methane area, but there is activity in the anaerobic digestion of agricultural residues, primarily cattle feed lot waste solids.

Obviously, when energy production is the primary goal of an anaerobic system, reliability will be a high priority. An early warning system of some type will undoubtedly be standard equipment on biomasg-to-methane systems. Current 
developmental efforts are now focusing on improving p:ocess stability, ease of operation and reliability. It would appear that a $\mathrm{H}_{2}$ - and CO-based monitoriag system might allow digester systems of this type be operated near their full capacity and thereby reduce the cost associated with methane production by permitting smaller or fewer digesters to be used.

Recent research has shown that anaerobic systems may be capable of treating wastewaters containing compounds that are either toxic or recalcitrant to aerobic systems. In the case of treatment of toxic or hazardous wastewaters, or possibly in the clean up of contaminated groundwater, close monitoring of any anaerobic process option will be necessary as a means to ensure system efficiency while maximizing throughput and processing efficiency. A monitoring system based upon $\mathrm{H}_{2}$ and $\mathrm{CO}$ (along with complementary conventional indicator parametersl may hold promise in these instances.

In certain respects, this research can be considered as an exploratory effort in that a wide variety of conditions, some practical real-world situations, and other more basic information-gathering experiments, were examined. Because of this, some of the information may appear more pertinent from an engineering perspective than other segments. Taken together, it represents the foundation upon which more focused problem solving oriented work can be launched.

\subsection{CONCLUSIONS}

- Carbon monoxide (CO) is evolved in trace amounts during the anaerobic digestion of waste activated sludge.

- Hydrogen gas accumulates rapidly when methanogenesis is severely inhibited by organic toxicants. When samples were less severely inhibited, $\mathrm{H}_{2}$ accumulated at a rate slightly above control levels but the inhibition of the hydrogen-utilizing methanogens was masked to a degree by the buildup of hydrogen-sink (reduced) soluble products such as propionate. Because of this there are limits on the utility of using $\mathrm{H}_{2}$ as an early warning indicator of impending process upset due to pulse addition of organic toxicants. Hydrogen should, however, give a more rapid indication of process upsets of this type than conventional process indicators.

- Carbon monoxide concentrations were also effected by organic toxicant addition. The variable responses observed indicated that more must be learned before co can be confidently used as a 
process indicator for upsets of this type. Clearly, during severe inhibition of methanogenesis, Co-levels will rise.

Monitoring both $\mathrm{H}_{2}$ and $\mathrm{CO}$ together, therefore, will give a more rapid indication of organic toxicant induced inhibition than conventional monitoring strategies can afford.

Heavy metals inhibited other members of the anaerobic consortia used in these studies more severely than methanogens. Substrate flow was interrupted at a point in the degradation sequence such that $\mathrm{H}_{2}$-levels declined upon heavy metal addition to a continuously operated system and accumulated less rapidly in batch assays (when $\mathrm{H}_{2}$ started out at

$<1 \mathrm{ppm}$ ).

Both $\mathrm{H}_{2}$ and $\mathrm{CO}$ demonstrated characteristic or generic response patterns to heavy-metal induced inhibition in batch serum bottle experiments. Results indicate that monitoring these two trace gases may allow not only an indication of upset, but also fingerprint the event as a heavy-metal inhibition.

A considerably greater data base, using different feedstocks with variable composition and strength with continuously operated systems needs to be collected before $\mathrm{H}_{2}$ and/or $\mathrm{CO}$ monitoring can be confidently implemented in the field. Development of field-durable, less sophisticated systems for measuring $\mathrm{CO}$ and $\mathrm{H}_{2}$ also needs to be realized before monitoring programs and strategies based upon $\mathrm{CO}$ and $\mathrm{H}_{2}$ Can be implemented.

Extension of the results generated for sludge digester systems may be applicable in biomass-to-energy systems, but are not likely to be directly applied for use in describing the operation of high-rate anaerobic wastewater treatment systems. This should not be construed to indicate that hydrogen and carbon monoxide monitoring is not appropriate. It simply means that responses are anticipated to be quite different for the two areas of anaerobic process application due to the different rate limiting steps. 


\section{REFERENCES}

Abram, J.W. and Newell, D.B. (1978) "Hydrogen as a substrate for methanogenesis and sulphate reduction in anaerobic saltmarsh sediment," Arch. Micrebiel. 217:93-97.

Adamse, A.D. and Velzeboer, C.T.M. (1982) "Features of a clestridium, strain CV-AAl, an obligatory anaerobic bacterium producing acetic acid from methanol," Antonie van Leeuwenheek., 48:305-313.

Agardy, F.J. and Shepherd, W.C. (1965) "DNA - a rational basis for digester loadings," I. Water Peld Cont. Fed., 37:1236-1242.

Aiking, H., Govers, H., and van't Riet, J. (1985) "Detoxification of mercury, cadmium, and lead in Klebsiella aerogenes NCTC 418 growing in continuous culture," Apol. Environ. Microbiel., 50:1262-1267.

Anthony, R.M. and Breimhurst, L.H. (1981) "Determining the maximum influent concentrations of priority pollutants for treatment plants, " I. Hater Poll. cont. Fed. 53:1457-1468.

Archer, D.B., Hilton, M.G., Adams, P. and Weicko, H. (1986) "Hydrogen as a process control index in a pilot scale anaerobic digester," Bietechnel. Iett., $8: 197-202$.

Ashley, N.V., Davies, M. and Hurst, T.J. (1982) "The effect of increased nickel ion concentrations on microbial populations in the anaerobic digestion of sewage sludge," Water Res., 16:963-971.

Ashley, N.V. and Hurst, T.J. (1981) "Acid and alkaline phosphatase activity in anaerobic digested sludge: a biochemical predictor of digester failure," Water Res. , 15:633-638.

Balch, W.E. and Wolfe, R.S. (1979a) "Specificity and biological distribution of coenzyme M (2-mercaptoethane-sulfonic acid)," J. Bacteriol., 137:256-263.

Balch, W.E. and Wolfe, R.S. (1979b) "Transport of coenzyme M (2mercaptoethanesulfonic acid) in Methaمobactrium suminantium, " Iacteriel. , 137: $264-273$.

Baresi, L. and Wolfe, R.S. (1981) "Levels of coenzyme $F_{420}$, coenzyme M, hydrogenase, and methylcoenzyme $M$. methylreductase in acetate-grown Methanesarcina," Aopl. Enviren_Microbiel. 41:388-391.

Baresi, I. (1984) "Methanogenic cleavage of acetate by lysates of Methanesarcina backeri," I. Bacteriel. " 260:365-370.

Baresi, I., Mah, R.A., Ward, D.M. and Kaplan, I.R. (1978) "Methanogenesis from acetate: enrichment studies," Apol. Environ.Microbiel., 36:186-197.

Barnes, D., Bliss, P.J., Grauer, B. and Robins, K. (1984) "Pretreatment of high strength wastewater by an anaerobic fluidized bed process. Part II response to organic load transients," Environ. Techoel. Lett., 6:73-78.

Baross, J.A., Lilley, M.D. and Gordon, L.I. (1982) "Is the $\mathrm{CH}_{4}$, $\mathrm{H}_{2}$ and $\mathrm{CO}$ venting from submarine hydrothermal systems produced by thermophilic bacteria," Nature, 298:366-368. 
Barth, E.E., English, J.N., Salotto, B.V., Jackson, B.N. and Eitinger, M.B. (1965) "Field survey of four municipal wastewater treatnient plants receiving metallic wastes," I. Water Pold_cont. Fed. 37:1101-1117.

Bauchop, T. (1967) "Inhibition of rumen methanogenesis by methane analogues," J. Bacteriel. , 24:171-175.

Boone, D.R., Menaia, J.A.G.E., Boone, J.E., and Mah, R.A. (1987) "Effects of hydrogen pressure during growth and effects of pregrowth with hydrogen on acetate degradation by Methanesarcina species," Apel. Enviren. Microbiel.", 53: 83-87.

Boone, D.R. and Bryant, M.P. (1980) "Propionate-degrading bacterium, Syntrephobacter welinii sp. nov. gen. nov., from methanogenic ecosystems," Apol. Enyiran. Microbial., 40:626-632.

Bott, M., Eikmanns, B. and Thauer, R.K. (1986) "Coupling of carbon monoxide oxidation to $\mathrm{CO}_{2}$ and $\mathrm{H}_{2}$ with the phosphorylation of $\mathrm{ADP}$ in acetate grown Methanesarcina barkeri," Eur. J. of Biechem., 259:393-398.

Bott, M.H., Eikmanns, B. and Thauer, R.K. (1985) "Defective formation and/or utilization of carbon monoxide in $\mathrm{H}_{2} / \mathrm{CO}_{2}$ fermenting methanogenes dependent on acetate as carbon source," Arch. Microbiel. 143:266-269.

Bouwer, E.J. and McCarty, P.I. (1983) "Transformations of 1- and 2-carbon halogenated aliphatic organic compounds under methanogenic conditions," Apel. Environ_Microbiel., 45:1286-1297.

Bryant, M.P., wolin, E.A., wolin, M.J. and Wolfe, R.S. (1967) "Methanebacillus emelianskifi, a symbiotic association of two species of bacteria," arch. Microbiol., 52:20-31.

Callander, I.J. and Barford, J.P. (1983a) "Precipitation, chelation, and the availability of metals as nutrients in anaerobic digestion. I. Methodology," Biotechool. Bioeng., 25:1947-1957.

Callander, I.J. and Barford, J.P. (1983b) "Precipitation, chelation, and the availability of metals as nutrients in anaerobic digestion. II. Applications," Bietechnel. Bioeng., 25:1959-1972.

Cheeseman, P.A., Toms-Wood, A. and Wolfe, R.S. (1972) "Isolation and properties of a fluorescent compound, Factor 420, from Methanobacterium ruminantium," I. Bacteriol., 122:527-531.

Cheng, M.H., Patterson, J.W. and Minear, R.A. (1975) "Heavy metal uptake by activated sludge," I_ Water Pold. Contrel_Eed, 43:362-376.

Chung, T.K. (1976) "Inhibitory effects of $\mathrm{H}_{2}$ on growth of clestridium cellobioparum," Aopl. Environ. Microbiel. 31:342-348.

Clark, J.E., Ragsdale, S.W., Ljungdahl, L.G. and Wiegel, J. (1982) "Levels of enzymes involved in the synthesis of acetate from $\mathrm{CO}_{2}$ in clestridium themoautotrophicum," I. Bacteriel. 151:507-509.

Coker, E.G. and Matthews, P.J. (1983) "Metals in sewage sludge and their potential effects in agriculture," Wat. Sci. Technel., 15:209-225. 
Collins, P.G. and Ridgeway, J.W. (1980) "Urban storm runoff quality in

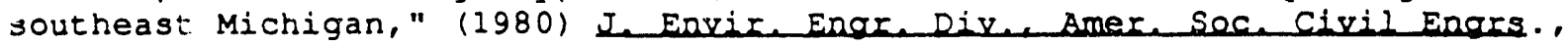
206: 153-162.

Conrad, R., Phelps, T.J. and Zeikus, J.G. (1985) "Gas metabolism evidence in support of the juxtaposition of hydrogen-producing and methanogenic bacteria in sewage sludge and lake sediments," Apol. Enviros. Microbiol., 50:595-601.

Conrad, R. and Thruer, R.K. (1983) "Carbon monoxide production by Methanobacterium thermoautotrophicum," EEMS Microbiol Iett. 20:229-232.

Daniels, L., Sparling, R. and Sprott, G.D. (1984) "The bioenergetics of methanogenesis," Biechem. et Biephys. Acta, 268:113-163.

Daniels, L., Eulton, G., Spencer, R.W. and Orme-Johnson, W.H. (1980) "Origin of hydrogen in methane produced by Methaمebacterium themoautotrophicum," In Bacteriel., 141:694-698.

Daniels, L.J., Fuchs, G., Thaver, R.K. and Zeikus, J.G. (1977) "Carbon monoxide oxidation by methanogenic bacteria," J. Bacteriel., 132:118-126.

Davis, J.A. III, and Jackson, J. (1975) "Heavy metals in wastewater in three urban areas," I. Water Pell. Cont. Eed., 47:2292-2297.

Delafontaine, M.J., Naveau, H.P. and Nyes, E.J. (1979) "Fluoremetric monitoring of methanogenesis in anaerobic digesters," Bietechnel. Jett. , 1: $71-74$.

Diekert, G., Schrader, E. and Harden, W. (1986) "Energetics of co formation and $C O$ oxidation in cell suspensions of acetobacterium weedii, "Arch. vicrobiol. 144:386-392.

Diekert, G., Hansch, M. and Conrad, F. (1984) "Acetate synthesis from $2 \mathrm{CO}_{2}$ in acetogenic bacteria: is carbon monoxide an intermediate?," arch. Microbiel., $138: 224-228$.

Diekert, G. and Ritter, M. (1983) "Carbon monoxide fixation into the carboxyl group of acetate during growth of Acetobacterium weodit on $\mathrm{H}_{2}$ and $\mathrm{CO}_{2}$, $\mathrm{EEMS}$ vicrobiol Let., 27:299-302.

Diekert, G.B. and Thaver, R.K. (1978) "Carbon monoxide oxidation by clestridium thempaceticum and clestridium formicoaceticum. I. Bacteriol., 136:597-606.

Drake, H.L., Hu, S., and Wood, N.H. (1980) "Purification of carbon monoxide dehydrogenase, a nickel enzyme from clestridium tbermoaceticum. I. Bis chem. 255:7174-7180.

Edwards, T. and MCBride, B.C. (1975) "New method for the isolation of methanogenic bacteria," Apel. Miarobiel. " 29:540-545.

Eikmanns, B. and Thauer, R.K. (1985) "Evidence for the involvement and role cl a corrinoid enzyme in methane formation from acetate in Mathanesarcina barkeri," Arch. Microbiol. 142:175-179. 
Eikmanns, B., Fuchs, f. and Thaver, R.K. (1985) "Formation of carbon monoxide from $\mathrm{CO}_{2}$ and $\mathrm{H}_{2}$ by Methanobacterium thermoautotrophicum, "Eur. J. Biochem." 146:149-154.

Eikmanns, B. and Thauer, R.K. (1984) "Catalysis of an isotopic exchange between $\mathrm{CO}_{2}$ and the carboxyl group of acetate by Methanesarcina barkeri grown on acetate," Arch. Microbiol., 138:365-370.

Ekiel, I., Sprott, G.D. and Patel, G.B. (1985) Acetate and $\mathrm{CO}_{2}$ assimilation by Methanethrix conciliz," I. Bacteriol., 162:905-908.

Eng, S.C., Fe:nandese, X.A. and Paskins, A.R. (1986) "Biochemical effects of admi. -stering shock loads of sucrose to a laboratory-scale anaerobic (UASB) efflcent treatment plant," Water Bes., 20:789-794.

Escalante-Semerena, J.C., Leigh, J.A. and wolfe, R.S. (1984) "New insights into the biochemistry of methanogenesis from $\mathrm{H}_{2}$ and $\mathrm{CO}_{2}$, " pp. 191-198 in Rrec. of the Fourth Intern. Symo. on Microbiel. Growth on $c=1$ compounds, R.S. Hanson and R.A. Crawford, eds., Amer. Soc. Microbiol., Washington, D.C.

Evans, J.N.S., Tolman, C.J. and Roberts, M.F (1986) "Indirect observation by ${ }^{13} \mathrm{C}$ NMR spectroscopy of a novel $\mathrm{CO}_{2}$ fixation pathway in methanogens," Science. $231: 488-491$.

Fuchs, G., Stupperich, E. and Thauer, R.K. (1978) "Acetate assimilation and the synthesis of alanine aspartate and glutamate in Methanobacterium thermoautotrophicum," Arch. Microbiel., 117:61-66.

Gould, M.S. and Genetelli, E.J. (1975) "Heavy metal distribution in anaerobical+y digested sludges," in Rroc. of 30th Purdue Industrial Waste conf., Lafayette, Ind., pp. 689-699.

Hall, E.R. (1983) "The Development and Utilization of Anaerobic Technology in Canadä," in Rrec. of Third Internat. Syme. on Anaerobic Digestion, Boston, MA.

Harper, S.R. and Pohland, F.G. (1986) "Recent tevelopments in hydrogen management during anaerobic biological wastewater treatment, "Bietechnel. Bieend., 28:585-602.

Hayes, T.D. and Theis, I.L. (1978) "The distribut 0 of of heavy metals in anaerobic digestion," I. Water Poll. Cont. Fed., 50:61-72.

Healy, J.B., Jr., Young, L.Y. and Reinhard, J. (1980) "Methanogenic decomposition of ferulic acid, a model lignin derivative," Appl. Enviren. Micrehiel., 39:436-444.

Henson, J.M, Smith, P.H. and White, D.C. (1985) "Examination of thermophilic methane-producing digesters by analysis of bacterial lipids," Apel. Environ. Miarobiel., 50:1428-1433.

Henze, M. and Hazremoes, P. (1983) "Review paper: anaerobic treatment in fluid film reactors," Water Sci. and Technoloox, 15:1-90.

Hickey, R.F. and Owens, R.W. (1981) "Methane generation from high strength industrial wastes with the anaerubic biological fluidized bed, "Bietecinai. Biceng. Symo., 12:399-413. 
Hickey, R.F. and Switzenbaum, M.S. (1990) "Some Observations on the Behavior and Thermodynamics of Carbon Monoxide as a Trace Component of Anaerobic Digester Gases and Methanogenesis from Acetate," Enviromental Science and Technology, in press.

Hobson, P.N. and Shaw, B.G. (1976) "Inhibition of methane production by Methanobacterium formicicum," Water Res.. 10:849-852.

Holder, U., Schmidt, D-E, Stupperich, E. and Fuchs, G. (1985) "Autotrophic synthesis of activated acetic acid from two $\mathrm{CO}_{2}$ in Methanobacterium thempautetropicum III. Evidence for common one-carbon precursor pool and the role of corrinoid," Arch. Microbiel. 141:229-238.

H.1, S.I., Drake, H.L. and wood, H.G. (1982) "Synthesis of acetyl coenzyme A fiom carbon monoxide, methyl tetrahydrofolate, and coenzyme $A$ by enzymes from clestridium thermoaceticum., I. Bacteriol. 149:440-448.

Hungate, R.E. (1967) "Hydrogen as an intermediate in the rumen fermentation," Arch Microbiel. 59:158-164.

Hungate, R.E. (1966) The Rumen and Its Microbes, Academic Press Inc., N.Y.

Huser, B.A., Wuhrmann, K. and zehnder, A.J.B. (1982) "Methanethrix soebngenij gen. nov. sp. nov., a new acetotrophic non-hydrogen-oxidizing methane bacterium," Arch. Microbiel. 132:1-9.

Jeris, J.S. and McCarty, P.L. (1965) "The biochemistry of methane fermentation using C 14 tracers," I. Water Pold Cent. Fed., 37:178-192.

Kasper, H.F. and Wuhrmann, K. (1978a) "Kinetic parameters and relative turnovers of some important catabolic reactions in digesting 3 ludge," Apele Environ. Microbiel., 36:1-7.

Kasper, H.F. and Wuhrmann, K. (1978b) "Product inhibition in sludge digestion," Microbial Ecelogy, 4:241-248.

Kenealy, W.R. and Zeikus, J.G. (1982) "One-carbon metabolism in methanogens: evidence for synthesis if a two-carbon cellular intermediate and unification of catabolism and anabolism in Methanesarcina barkeri," I. Bacteriel." 251: $932-941$.

Kerby, R., Niemczura, W. and zeikus, J.G. (1983) "Single-carbon catabolism in acetogens: analysis of carbon flow in Acetobacterium weodii and Butyribacterium methylotrophicum by fermentation and ${ }^{13} \mathrm{C}$-nuclear magnet: reasonance measurement," I. Bacteriol., 155:1208-1218.

Klein, L.A., Lang, M., Nash, N. and Kirschner, S.L. (1974) "Sources of metals in New York City wastewater," In Water Pel1. Cont. , 46:2653-2662.

Kluyver, A.J. and Schnellen, C.G. (1947) "on the fermentation of carbon monoxide by pure cultures of methane bacteria," Arch. Biechem., 14:57-70.

Knetchel, J.R. (1978) "A more economical method for the determination of chemical oxygen demand," Water and Rellution control, 25:May/June.

Kohler, H.E. and Zehnder, A.J.B. (1984) "Carbon monoxide dehydrogenase and acetate thiokinase in Methanothrix seebngenii," EEMS Microbiel. Iett." 21: 287-294. 
Kohler, H.E. and Zehnder, A.J.B. (1984) "Carbon monoxide dehydrogenase and acetate thiokinase in Methanothrix seehngenii," EEMS Microbiel. Lett." 21: 287-294.

Kristjansson, J.K., Schonheit, P. and Thauer, R.K. (1982) "Different Ks values for hydrogen of methanogenic bacteria and sulfate reducing bacteria: an explanation for the apparent inhibition of methanogenesis by sulfate, "Arch. Microbial., 231:278-282.

Krzycki, J.A., Morgan, J.B., Conrad, R. and zeikus, J.G. (1987) "Hydrogen metabolism during methanogenesis from acetate by Methanogenesis barkeri, "EEMS Microbiol. Lett., 40:193-198.

Krzycki, J.A., Lehman, L.J. and Zeikus, J.G. (1985) "Acetate catabolism by Methanosarcina barkeri: evidence for involvement of carbon monoxide dehydrogenase, methy 1 Coenzyme $M$, and methylreductase," I. Bacteriol.., 163:1000-1006.

Kryzcki, J.A. and zeikus, J.G. (1984a) "Characterization and purification of carbon monoxide dehydrogenase from Methanesarcina barkeri," I. Bacteriel., 158:231-237.

Krzycki, J.A. and zeikus, J.G. (1984b) "Acetate catabolism by Methanesarcina barkeri: hydrogen-dependent methane production from acetate by a soluble cell protein fraction," EEMS Microbiel_Lett., 25:27-32.

Krzycki, J.A., Wolkin, R.H. and zeikus, J.G. (1982) "Comparison of unitrophic and mixotrophic substrate metabolism by an acetate-adapted strain of Methanosarcina barkeri," I. Bacteriol." 249:247-254.

Kugelman, I.J. and Chin, K.K. (1971) "Toxicity, synergism, and antagonism in anaerobic waste treatment processes," pp. 53-90 in Anaerobic Biolegical Ireatment Precesses, ASChE, Adv. in Chem., F.G. Pohland, ed., Vol. 105.

Lamb, A. and Tollefson, E.L. (1973) "Toxic effects of cupric chromate and chromic ions on biological oxidation," water Res., 1:599-605.

Lawrence, A.W. and McCarty, P.L. (1965) "The role of sulfide in preventing heavy metal toxicity in anaerobic treatment," I. Wat. Poll. control Eed., 37: 392-406.

Leininger, K.V., et al. (1983) A Suryey of Anaerobic Digester operations, ASCE Task Committee Report.

Lenhard, G. (1967) "A standardized procedure for the determination of dehydrogenase activity in samples from anaerobic treatment systems, " hater Res., 2:161-167.

Lester, J.N. (1983) "Significance and behavior of heavy metals in wastewater treatment processes. I. Sewage treatment and effluent discharge," The Science of Total Enviren., 30:1-44.

Lester, J.N., Sterritt, R.M. and Kirk, P.W.W. (1983) "Significance and behavior of heavy metals in wastewater treatment processes. II. Sludge treatment and disposai," The sci. of rotal Environ.: 30:45-83. 
Lettinga, G., et al. (1980) "Use of the upflow sludge blanket (USB) reactor concept for biological wastewater treatment, especially anaerobic treatment," Bietechnol. Bioeng., 22:699-734.

Lovley, D.R. (1985) "Minimum threshold for hydrogen metabolism in methanogenic bacteria," Apel. Environ. Microbiol., 42:1530-1531.

Lovley, D.R. and Eerry, J.G. (1985) "Production and consumption of $\mathrm{H}_{2}$ during growth of Methanosarcina spe. on acetate," Apol. Enviren. Microbiol., 49:247249.

Lovley, D.R., white. R.C., and Eerry, J.G. (1984) "Identification of methyl coenzyme $M$ as an intermediate in methanogenesis from acetate in Methanosarcina spe. " I. Bacteriol., 160:521-525.

Lovley, D.R., Greening, R.C. and Ferry, J.G. (1984) "Rapidly growing rumen methanogenic organism that synthesizes coenzyme $M$ and has a high affinity for formate," Apel. Environ. Microbiel. , 48:81-87.

Lovley, D.R., Dwyer, D.F. and Klug, M.J. (1982) "Kinetic analysis of competition between sulfate reducers and methanogens for hydrogen in sediments," Apol. Environ. Microbiol., 43:1373-1379.

Lupton, F.S, Conrad, R. and zeikus, J.G. (1984a) "CO metabolism of Desulfovibrie vulgaris strain Madisen: physiological function in the absence or presence of exogeneous substrates," EEMS Microbiel. Lett., 23:263-268.

Lupton, F.S., Conrad, R. and zeikus, J.G. (1984b) "Physiological function of hydrogen metabolism during growth of sulfidogenic bacteria on organic substrates," I. Bacteriel., 159:843-849.

Lynd, L., Kerby, R. and zeikus, J.G. (1982) "Carbon monoxide metabolism of the methylotrophic acidogen Butyribacterium methyletrophicum," I. Bacteriel.", 249:255-263.

Mackie, R.I. and Bryant, M.P. (1981) "Metabolic activity of fatty acid oxidizing bacteria and the contribution of acetate, propionate, butyrate, and $\mathrm{CO}_{2}$ to methanogenesis in cattle waste at $40^{\circ}$ and $60^{\circ} \mathrm{C}$, "Apel. Environ. Microbial., 41:1363.

Macario, A.J.L. and Conway de Macario, E. (1983) "Antigenic fingerprinting of methanogenic bacteria with polyclonal antibody probes," Syst. Apel.

Microbiel., 4:451-458.

Martin, D.P., Misra, A. and Drake, H.K. (1985) "Dissimilation of carbon monoxide to acetic acid by glucose-limited cultures of clestridium thermoaceticum," Appl. Envir 20. Microbiel., 42:1412-1417.

McCarty, P.L. and Smith, D.P. (1986) "Anaerobic wastewater treatment," Enyiron. Sci. Techol. 20:1200-1206.

McCarty, P.L. (1982) "One hundred years of anaerobic treatment," in anaecobic Digestion, 1981, D.E. Hughes et al. eds., Elsevier Biomedical, Amsterdam.

McInerney, M.J., Mackit, R.I. and Bryant, M.P. (1981) "Anaerobic syntrophic associution of a butyrate-degrading bacterium and Methanesarcina eñIiched from bovine rumen fluid," Apel. Environ. Microbiel., 41:826-828. 
Miller, T.L. and Wolin, M.J. (1974) "A serum bottle modification of the Hungate tecinnique for cultivating obligate anaerobes," Aped. Enviren. Micrabiel., 22:985-987.

Mink, R.W. and Dugan, P.R. (1977) "Tentative identification of methanogenic bacteria by fluorescence microscopy," Apol. Enviren. Miarobiel., 33:713-717.

Mosey, F.E. (1983) "Mathematical modelling of the andexobic digestion process: regulatory mechanisms for the formation of short-chain volatile acids from glucose," Water Sei. and Technol., 25:209-232.

Mosey, F.E. (1976) "Assessment of the maximum concentration of heavy metals in crude sewage which will not inhibit the anaerobic digestion of sludge, "Water. Rellut. control, 25:10-18.

Mosey, F.E. and Hughes, D.A. (1975) "The toxicity of heavy metal ions to anaerobic digestion," Water Pollut. Control, 74:18-39.

Mosey, F.E., Swanwick, J.D. and Hughes, D.A. (1971) "Factors affecting the availability of heavy metals to inhibit anaerobic digestion, " water pellut. contral, 20:668-680.

Nelson, M.J.K. and Ferry, J.G. (1984) "Carbon monoxide-dependent methyl coenzyme $M$ methylreductase in acetotrophic Methanesarcina sop.," I.

Bacteriel., 160:526-532.

Neter, J. and wasserman, w. (1974) dpelied Linear Statistical Medels, Richard D. Irwin, Inc., Homewood, Illinois, pp. 160-167.

Nielsen, J.S, and Hrudey, S.E. (1983) Metal loadings and removal at a municipal activated sludge plant," Water Res., 27:1041-1052.

O'Brien, J.E. and Donlan, R.J. (1977) "A direct method for differentiating bicarbonate and acetate in digester control," presented meeting of Div.

Environ. Chem., American Chem. Soc., New Orleans.

Ohwaki, K. and Hungate, R.E. (1977) "Hydrogen utilization by clestridia in sewage sludge," Agol. Environ. Microbiel., 33:1270-1274.

Oremland, R.S. and Polcin, S. (1982) "Methanogenesis and sulfate reduction: competitive and noncompetitive substrates in estuarine sediments," Appl. Environ. Microbiol., 44:1270-1276.

Owen, W.E., Stuckey, D.C., Healey, J.B., Young, L.Y. and McCarty, P.L. (1979) "Bioassay for monitoring biochemical methane potential and anaerobic toxicity," water Res., 13:485-492.

Pankhania, I.P. and Robinson, J.P. (1984) "Heavy metal inhibition of methanogenesis by Methanospirilium buagatei GP1," EEMS Microbiol Lett., 22:277-281.

Parkin, G.F., Speece, R.E., Yang, C.H.J. and Kocher, W.M. (1983) "Response of methane fermentation systems to industrial toxicants," I. Water Pell. Cent. Eed. , 55:44-53.

Pause, S.M. and Switzenbaum, M.S. (i984; "in investigation of the use of fluorescence to monitor activity in anaerobic digesters, "Bietechoed. Lett., $6: 77-80$. 
Peterson, S.L., Bennett, L.G. and Tornabene, T.G. (1975) "Effects of lead in the lipid composition of Micrececeus Luteas cells," Appl. Environ. Microbiol." 29: $664-679$.

Petrasek, A.C. and Kugelman, I.J. (1983) "Metals removal and partitioning in conventional wastewater treatment plants," J. Water Rell. Cent. Fed., 55:11831190.

Pezacka, E. and Wood, H.G. (1984) "The synthesis of acetyl CoA by clestridium thermeaceticum from carbon dioxide, hydrogen, coenzyme A and methyletetrahydrofolate," Arch. Microbiol., 137:63-69.

Phelps, T.J., Conrad, R. and Zeikus, J.G. (1985) "Sulfate-dependent interspecies $\mathrm{H}_{2}$ transfer between Methanesarcial barkeri and Desulforibrie yulgaris during coculture metabolism of acetate or methanol," Bol. Enviren. vicrobiel., 5e:589-594.

Podolak, P.L., Friedman, A.A. and LaGrega, M.D. (1984) "Effects of reduced partial pressure on an anaerobic rotating biological contactor," pp. 1050-1076 in Rroce of second International conf on Fixed-Film Biological processes, J.T. Bandy, et al., eds., Arlington, VA.

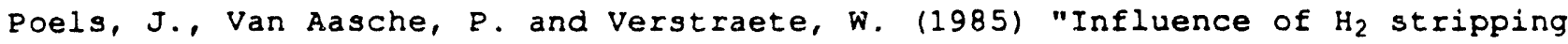
on methane production in conventional digesters," Bietechnel. Bieeng., 27: $1692-1698$.

Pohland, F.G. and Kang, S.J. (1975) Measurements of active biomass in biological waste treatment precesses, U.S.E.P.A. 600/2-75-029.

Ragsdale, S.W. and wood, H.G. (1985) "Acetate biosynthesis by acetogenic bacteria," I. Biol. Chem., 260:3970-3977.

Ragsdale, S.W., Clark, J.E., Ljungdahl, L.G., Lundie, L.L. and Drake, H.L. (1983) "Properties of purified carbon monoxide dehydrogenase from clestridium themoaceticum, a nickel, iron-sulfur protein," I. Biol. chem., 258:2364-2369.

Ruhlemann, M., Ziegler, K., Stupperich, E. and Fuchs, G. (1985) "Detection of acetyl coenzyme $A$ as an early $\mathrm{CO}_{2}$ assimilation intermediate in Methanobacterium," Arch. Microbiel., 141:399-406.

Rufener, W.H. and Wolin, M.J. (1968) "Effect of $\mathrm{CCl}_{4}$ on $\mathrm{CH}_{4}$ and volatile acid production in continuous cultures of rumen organisms and in a sheep rumen," Appl. Micrebiel. 16:1955-1956.

Russell, J.B. and Martin, S.A. (1984) "Effects of various methane inhibitors on the fermentation of amino acids by mixed rumen microorganisms in vitren ${ }^{2}$ Animal sein, 52:1329-1338.

Russell, L.L., Cain, C.B. and Jenkins, D.I. (1982) "Impact of priority pollutants on publicly owned treated works processes: A literature review," Preced. 37th Rurdue Indust. Waste conf., Lafayette, IN, Pp. 871-883.

Schauder, R., Eikmanns, B., Thauer, K.K., Widdel, F. and Fuchs, G. (1986) "Acetate oxidation $\mathrm{CO}_{2}$ in anaerobic bacteria via a novel pathway not involving reactions of the citric acid cycle," Arch Microbiel., 145:162-172. 
Scheifinger, C.C., Linehan, B. and wolin, M.J. (1975) "H2 production by Selenomonas cuminantium in the absence and presence of methanogenic bacteria," Appl. Microbiol., 22:480-483.

Scott, R.I., Williams, T.N., Whitmore, T.N. and Lloyd, D. (1983) "Direct measurement of methanogenesis in anderobic digesters by membrane inlet mass spectrometry, " Eur. J. Appl. Microbiol. Biotechnol. , 18:236-241.

Shelton, D.R. and Tiedje, J.M. (1984) "Isolation and partial characterization of bacteria in an anaerobic consortium that mineralizes 3-chlorobenzoic acid," Appl. Environ. Microbiel., 48:840-848.

Smith, M.R., Lequerica, J.L. and Hart, M. (1985) "Inhibition of methanogenesis and carbon metabolism in Methanosarcina sp. by cyanide.," I. Bacteriel., 262:67-71.

Smith, M.R. and Mah, R.A. (1978) "Growth and methanogenesis by Methanesarcina strain 227 on acetate and methanol," Apel. Enviren. Microbiel., 36:870-879.

Smith, P.H. (1980) Studies of Methanogenic Bacteria in Sludge, EPA-600/2$80-093$.

Speece, R.E. (1983) "Anaerobic biotechnology for industrial wastewater treatment," Environ. Sci. Techool., 17:416A-427A.

Spiegel, S.J., Farmer, J.K. and Garver, S.R. (1985) "Heavy metal concentrations in municipal wastewater treatment plant sludge," Bull. Environ. contam. Toxicel., 35:38-43.

Standard Methods for the Examination of Water and Wastewater, $16 t h$ Edition, (1985) APHA-AWWA-WPCF.

Sterritt, R.M. and Lester, J.N. (1980) "Interactions of heavy metals with bacteria," Sci, ef Tetal Enviren., 14:5-17.

Stickley, D.P. (1970) "The effect of chloroform in sewage on the production of gas from laboratory digesters," water peldut centrel, 69:585-592.

Stupperich, E. and Fuchs, G. (1984a) "Autotrophic synthesis of activated acetic from two $\mathrm{CO}_{2}$ in Methanebacterium thermoautotrophicum. I. Properties of in vitre system," Arch. Miarobiel., 139:8-13.

Stupperich, E. and Euchs, G. (1984b) "Autotrophic synthesis of activated acetic acid from two $\mathrm{CO}_{2}$ in Methanobacterium thermoautotroohicum. II. Evidence for different origins of acetate carbon atoms," Arah. Microbiel., 132:14-20.

Stupperich, E., Hammel, K.E., Fuchs, G. and Thauer, R.K. (1983) "Carbon monoxide fixation into the carbonyl group of acetyl coenzyme $A$ during autotrophic growth of Methanohacterium," EEMS Lett., 152:21-23.

Stuzenberger, F.J. and Bennet, E.O. (1965) "Sensitivity of mixed populations of Staphylecoceus aureus and Escherichia celi to mercurials," Apel. Enviren. Miarobiel., 23:570.

Swanwick, J.D. and Foulkes, M. (1971) "Inhibition of anaerobic digestion of sewage sludge by chlorinated hydrocarbons," Water peliut. centrei, i0:58-70. 
Swanwick, J.D., Shurben, D.G. and Jackson, S. (1969) "A survey of the performance of sewage sludge digesters in Great Britain," Wat. Pollut. centrol, 68:639-653.

Switzenbaum, M.S. and Grady, C.P.L. Jr. (1986) "Anaerobic treatment of domestic wastewater," I. Wat.Pellut, Control Fed., 58:102-106.

Switzenbaum, M.S. and Jewell, W.J. (1980) "Anaerobic attached-film expandedbed reactor treatment," I. Water pold cent. Fed. " 52:1953-1965.

Sykes, R.M. (1970) "Hydrogen production in the anaerobic digestion of sewage sludge," Ph.D. Dissertation, Purdue University, Lafayette, IN.

Taylor, G.T. (1982) "The methanogenic bacteria," Regress in Industrial Microbielooy, 26:231-329.

Thaver, R.K. Jungerman, K. and Decker, K. (1977) "Energy conservation in chemotrophic anaerobic bacteria," Bacteriel. Bey., 41:100-180.

Thiel, P.G. (1969) "The effect of methane analogs on methanogenesis in anaerobic digestion," Water Bes., 3:215-223.

Tomei, F.A., Maki, J.S. and Mitchell, R. (1985) "Interactions in syntrophic associations of endospore-forming, butyrate-degrading bacteria and Hzconsuming bacteria," Appl. Enviren. Micrebiel. , 50:1244-1250.

Uffen, R.L. (1983) "Metabolism of carbon monoxide by Rhodopseudomonas gelatinosa cell growth and properties of the oxidation system," I. Bacteriol.", 155: $956-965$.

USEPA, (1984) Needs Survey of U.S. and Territeries Treatment Capacity.

USEPA, (1982) Eate of Prierity Pollutants in Publicly owned Treatment werks, Yol.1. EPA $440 / 1-82 / 303$.

Vallee, B.L. and Ulmer, D.D. (1972) "Biochemical effects of mercury, cadmium and lead," Bna. Rev. Biochem., 41:91-128.

Webb, J.M. and Levy, M.B. (1955) "A sensitive method for the determination of DNA in tissues and microorganisms," I. Biolegical chem., 213:107.

White, D.C. (1983) "Analysis of microorganisms in terms of quantity and activity in natural environments," pp. 37-66 in Microbes in their Natiral Enviconments, J.H. Slater, R. Whittenbury, and J.W.T. Wimpenny, eds., Cambridge University Press, New York.

White, D.C., et al. (1979) "Biochemical measurements of microbial mass and activity from environmental samples," pp. 69-81 in Native aquatic bacteria. enumeration. activity and ecolegy, Costerton, J.W. and Colwell, R.R., eds., ASTM STP 695, Philadelphia.

Whitmore, T.N., Lazzari, M. and Lloyd, D. (1985) "Comparative studies in thermophilic and mesophilic anaerobic digesters using membrane inlet mass spectrometry," Bietechoel. Lett., 1:283-288.

Winfrey, M.R. and z'eikus, J.G. (1979) "Anaerobic metabolism of immediate methane precursors in Lake Mendota," Apel. Enviren. Microbiel., 37:244-253. 
Wolin, M.J. and Miller, T.L. (1982) "Interspecies hydrogen transfer: 15 years later," ASM News, 48:561-565.

Wood, H.G., Ragsdale, S.W. and Pezacka, E. (1986) "The acetyl-CoA pathway of autrotrophic growth," EEMS Microbiol. Beyiew, 39:345-362.

Yagi, T. (1958) "Enzymatic oxidation of carbon monoxide," Biochem. Biophys. Act $=$, 30:194-195.

Yang, $J$. and Speece, R.E. (1985) "Effects of engineering controls on methane fermentation toxicity response," I. Wat. Pell. control Fed., 57:1134-1141.

Zeikus, J.G., Kerby, R. and Krzycki, J.A. (1985) "Single-carbun chemistry of acetogenic and methanogenic bacteria," Science, 227:1167-1173.

Zehnder, A.J.B., Huser, B.A., Brock, T.D. and wuhrman, K. (1980) "Characterization of an acetate-decarboxylating non-hydrogen oxidizing methane bacterium," Arch. Microbiel. , 124:1-11.

Zickefoose, C. and Hayes, R.B.J. (1976) Anaerobic Sludge Digestion operations Manual, EPA 430/9-76-001.

Zinder, S.H. and Koch, M. (1984) "Non-aceticlastic methanogenesis from acetate: acetate oxidation by a thermophilic synrophic coculture," Arch. vicrobiol., 138:263-272.

Zinder, S.H., Anguish, T. and Cardwell, S.C. (1984a) "Selective inhibition by 2-bromoethanesulfonate of methanogenesis from acetate in a thermophilic anaerobic digester," Apol. Enviren. Microbiel., 47:1343-1345. 


\section{APPENDIX B}

METHANE AND CARBON DIOXIDE ANALYSIS (FOr Serum Bottle Asgays and Reserve Digesters)

Methane and carbon dioxide levels were analyzed via thermal conductivity (GowMac 550, Bridgewater, NJ). Separation was accomplished with porapak $Q$ ( $2 \mathrm{~m} x$ $4 \mathrm{~mm}$ ID stainless steel column) using ultra high priority (UHP) helium as a carrier. Area integration was performed with a HP-3390A integrator.

Calibration was with external standards of methane and $\mathrm{CO}_{2}$. Prior to use, the chromatograph was calibrated by minimum of three successive injections of gas standards. To preclude even a slight drift in the baseline affecting measured methane production during the serum bottle assays, twice-daily calibration was normally followed.

The operating conditions used are summarized in Table $B-1$.

Table B-I

Gas Chromatograph Operating Conditions for Thermal Conductivity Analysia of Methane and Carbon Dioxide Quantification

\begin{tabular}{ll}
\hline & \\
\hline Carrier Gas: & Helium (UHP) \\
Gas Pressure: & $30 \mathrm{psi}$ \\
Flow Rate: & $30 \mathrm{ml} / \mathrm{min}$ \\
Injection Port Temp.: & $110^{\circ} \mathrm{C}$ \\
Column Temp.: & $80^{\circ} \mathrm{C}$ \\
Detector Temp: & $70^{\circ} \mathrm{C}$ \\
Column Packing & Poropack $Q(60 / 80 \mathrm{mesh})$ \\
Bridge Current: & $6 \mathrm{ma}$ \\
Sample Volume: & $1 \mathrm{ml}$ \\
\hline
\end{tabular}


Rrecision of Methane and $\mathrm{CO}_{2}$ Analyses

The relative standard deviation of the methane analysis of headspace gas was obtained by sampling the 20-day HRT digester over the course of an hour with 12 successive injections. The average measured methane concentration was 64.4 $\pm 0.26 \%$ with a relative standard deviation of 0.4 percent. A similar analysis was performed for $\mathrm{CO}_{2}$. The average $\mathrm{CO}_{2}$ concentration was $28.56 \pm 0.378$ which produced a relative standard deviation of $1.28 \%$.

The major error associated with the analysis is likely to be associated with variability in the amount of sample injected. One disadvantage of using the tuberculin syringes is that the samples were not protected as well as if pressure-lock-type syringes were used. Better precision in withdrawing $1.0 \mathrm{ml}$ would be possible with the pressure-lock-type syringes. An adaptation of the activity-test of Dolfing and Bloemen (1985) might be a more appropriate technique for headspace sampling in the future. If this is done, however, the increase in pressure of the samples must be accurately quantified to allow calculation of the hydrogen and carbon monoxide partial pressures. 
APRENDIX C

HYDROGEN AND CARBON MONOXIDE ANALYSIS (FOr Serum Bottle Assays and Reserve Digesters)

Hydrogen concentrations were analyzed with a RGD2 mercury reduction based chromatographic system (Trace Analytical, stanford, CA). Separation was accomplished with molecular sieve $5 \mathrm{~A}(2 \mathrm{~m} \times 4 \mathrm{~mm}$ ID) using catalytically purified nitrogen as a carrier. Hydrogen was quantified by peak height analysis as recommended by the manufacturer.

Calibration was performed daily with standard gas mixtures (Scientific Gas Products). Operating conditions are presented in Table C-1. Linearity was checked on a periodic basis using gas standards with a 5 - or 10-fold difference in concentration as recomended by the manufacturer. Hydrogen gas standards of 100,500 and $1000 \mathrm{ppm}$ were normally kept on hand. With the 0.1 cc sample loop and the operating conditions described in Table A-4, hydrogenresponse was linear up to approximately $250 \mathrm{ppm}$. Co-response was linear up to 15 to $20 \mathrm{ppm}$. In those instances when $\mathrm{H}_{2}$ or $\mathrm{CO}$ were above the linear range of the instrument, a calibration curve using standard gas mixtures was constructed. This allowed accurate hydrogen quantification up to 1000 to 1200 ppm $\mathrm{H}_{2}$ to be made.

When excessive noise in the chromatograph baseline was observed, due to adsorption of $\mathrm{CO}_{2}$, water vapor, and higher molecular weight contaminants, the molesieve $5 \mathrm{~A}$ was regenerated by heating the column to $250^{\circ} \mathrm{C}$ for 24 hours while maintaining an approximate nitrogen flow rate of approximately $80 \mathrm{ml} / \mathrm{min}$. Bypass of th: detector is required during column regeneration.

The hydrogen analysis had a measured relative standard deviation of 0.48 percent. Using digester gas in place of the gas standards (based on 20 sequential injections of a digester gas headspacel this increased to 1.2 percent. Sexum bottle samples subject to toxicant addition generally had a standard deviation of 2 to $3 \mathrm{ppm}$. Because hydrogen gas concentration started at zero for all assays due to the gas used to purge the samples, relative standard deviations were initially high but after eight hours of incubation 
Table $c-1$

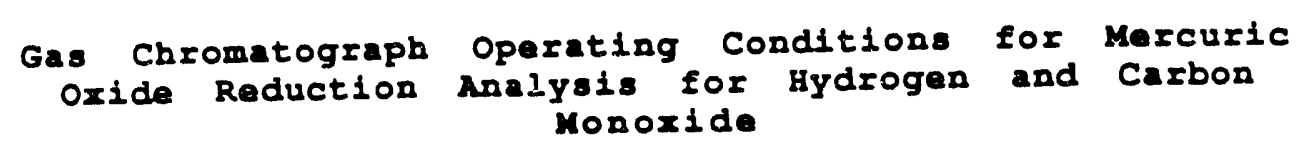

\begin{tabular}{ll}
\hline Carrier Gas: & $\begin{array}{l}\text { Nitrogen (catalytically } \\
\text { purified) }\end{array}$ \\
Gas Pressure: & $25 \mathrm{psig}$ \\
Flow Rate: & $40 \mathrm{ml} / \mathrm{min}$ \\
Column Temperature: & $125^{\circ} \mathrm{C}$ \\
Column Packing: & Molesieve $5 \mathrm{~A}(60 / 80 \mathrm{mesh})$ \\
Detector Temperature: & $280^{\circ} \mathrm{C}$ \\
Sample loop volume: & $0.1 \mathrm{ml}$ \\
\hline
\end{tabular}

averaged 2.7 and 8.3 percent for controls and inhibited samples respectively (based upon analysis of all organic toxicant assays).

Carbon monoxide measurements had a relative standard deviation of 0.38 percent based upon 15 injections of $11 \mathrm{ppm}$ standards. Serum bottle assay results (summation of 5 runs using copper as a toxicant) displayed average standard deviations of 10.5 and 13.6 percent, respectively, fo: controls and inhibited samples. 


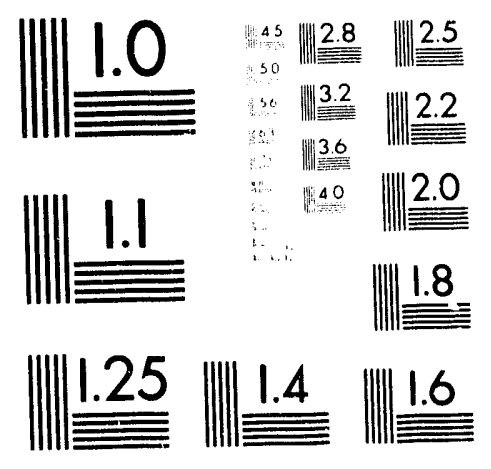



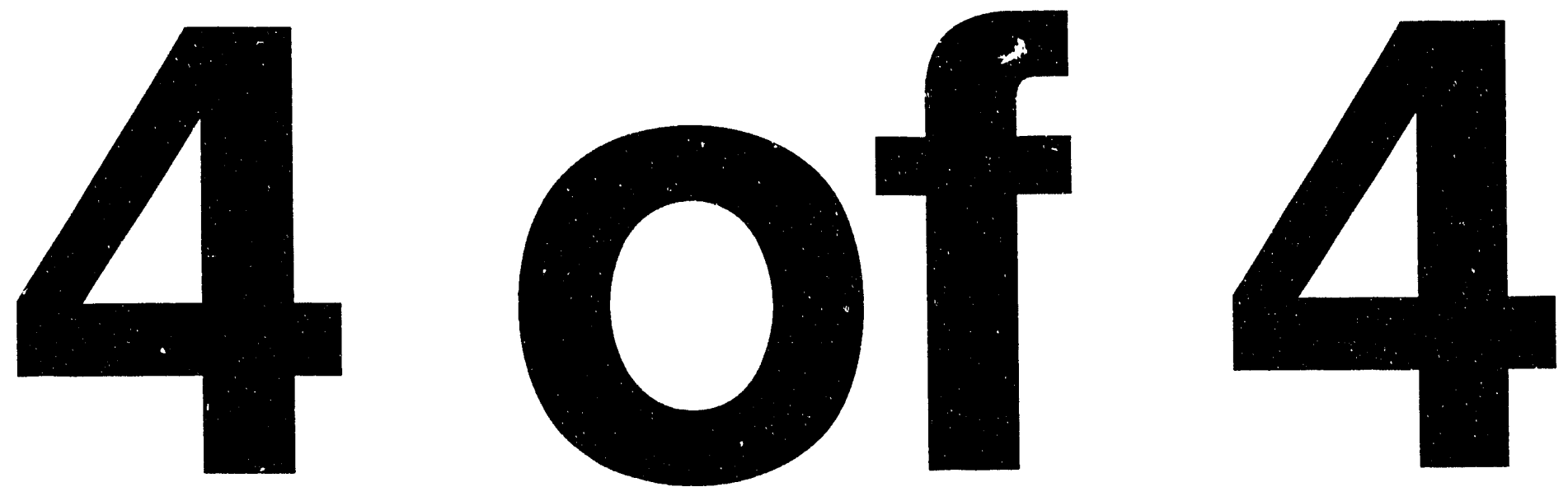


\section{APPENDIX D}

VOLATILE :ATTY ACID (VEA) ANALYSIS

Frozen samples were thawed, mixed and immediately analyzed for VFAs. Acids were speciated at $135^{\circ} \mathrm{C}$ on 158 SP $1220 / 18 \mathrm{H}_{3} \mathrm{PO}_{4}$ on $100 / 120$ chromosorb W AW $12 \mathrm{~m}$ $x 4 \mathrm{~mm}$ ID) using ultra high priority (UHP) nitrogen as a carrier. Analysis was performed with a Varian 3700 chromatograph equipped with a flame-ionization detector. A complete listing of operating conditions are detailed in Table D-1. Calibration was accomplished with external standards, $10 \mathrm{mM}$ of $\mathrm{C}_{2}$ through $C_{8}$ VFAs purchased from supelco, Inc. (Belfontaine, PA). These standards were diluted if necessary to bring them into the range observed for the samples being analyzed. Daily calibration was used and quantification was by area integration (Spectro Physics 4270 integrator).

Samples were prepared by centrifugation at $2000 \mathrm{x} g$ for 15 minutes. Samples were either analyzed immediately or frozen for later analysis. A 1.0 ul sample was injected with a Hamilton 7001 syringe equipped with a chaney guide. The normal procedure used was to flush the syringe 6 times with deionized water after injection and then waste 3 successive $1.0 \mathrm{ul}$ aliquots of the next sample before the subsequent injection.

A Iinear response of acid concentration to area count was observed for up to $10 \mathrm{mM}$ of all acids of interest $\left(C_{2}\right.$ to $\left.C_{6}\right)$. Based upon 3 successive injections of $10 \mathrm{mM}$ standards, the relative standard deviations were calculated to range from a low of 0.88 percent for propionate and butyrate to a peak of 1.93 percent for isocaproate. The relative standard deviation of digester samples was determined using 6 consecutive injections of a test digester sample. Acetate and propionate were the only acids present at concentrations greater than $15 \mathrm{mg} 1^{-1}$ in this sample. The relative standard deviations were determined to be 3.9 and 2.4 percent for acetate and propionate, respectively. 
Table $D-1$ Gas Chromatograph operating Conditions for rID Analysis
for volatile Fatty Acid Determination

\begin{tabular}{ll}
\hline & \\
\hline Carrier Gas: & Nitrogen (UHP) \\
Gas Pressure: & $60 \mathrm{psig}$ \\
Flow Rate: & $40 \mathrm{ml} / \mathrm{min}$ \\
Air (Zero Grade) Pressure: & $60 \mathrm{psig}$ \\
Flow Rate: & $270 \mathrm{ml} / \mathrm{min}$ \\
Hydrogen (Zero Grade) Pressure: & $40 \mathrm{psig}$ \\
Flow Rate: & $30 \mathrm{ml} / \mathrm{min}$ \\
Injection Port Temperature: & $200^{\circ} \mathrm{C}$ \\
Column Ternperature: & $135^{\circ} \mathrm{C}$ \\
Detector Temperature: & $200^{\circ} \mathrm{C}$ \\
Column Packing: & $158 \mathrm{Sp}-1220 / 1 \mathrm{z} \mathrm{H}_{3} \mathrm{PO}_{4}$ \\
& on $100 / 120 \mathrm{mesh}$ \\
& $\mathrm{Chromosorb} \mathrm{W-AW}$ \\
Sample Size: & $1.0 \mathrm{ul}$ \\
\hline
\end{tabular}


APPENDIX A

SERUM BOTTIE ASSAYS

\section{scepe}

The protocol used is an adaptation of the serum bottle technique developed by Miller and wolin (1974) and later modified by Owen et al. (1979). The assay, as run, was designed to simulate a daily draw-and-fill feed schedule used at many wastewater treatment plants. A preliminary serum bottle assay was performed to investigate the effect of continuously mixing samples versus the static incubation technique used by owen et al. (1979). Proper mixing of full-scale anaerobic sludge digesters is crucial to proper operation (Leininger et al., 1983) and essential in helping to keep gaseous and aqueous phases near equilibrium. This latter consideration is important for studies designed to investigate the response patterns displayed by trace gases such as hydrogen and carbor monoxide. The assay demonstrated that gas and methane production rates (compared to static controls) were accelerated approximately 2-fold as a consequence of continual mixing. The methane- and hydrogenresponse patterns observed for a 20-day HRT inoculum for mixed and non-mixed samples are presented in Figure A-1. Based on these results, all subsequent. assays employed continuous mixing.

\section{Apparatus}

The experimental set-up for rapidly preparing a series of serum-bottle reactors, schematically depicted in Figure A-2, is a modification of that described by owen et al. (1979). The use of a gassing manifold, a syringe pump and repeating pipet (or repeating hand syringe pump) to anaerobically transfer the digester inocula and WAS, respectively, allowed more rapid preparation of the serum bottles. Up to 20 serum bottles could be prepared for monitoring in approximately 10 to 15 minutes.

The serum-bottle reactors were continually mixed by teflon stir-bars energized by a gang-pulley system. The gang-pulley system was used to prevent an inadvertent heating of samples that could occur with direct contact between the serum bottles and a mixer. The gang-pulley systems were modified manometric BOD apparatus (Hach Model 2173A). The speed of the mixers varied 

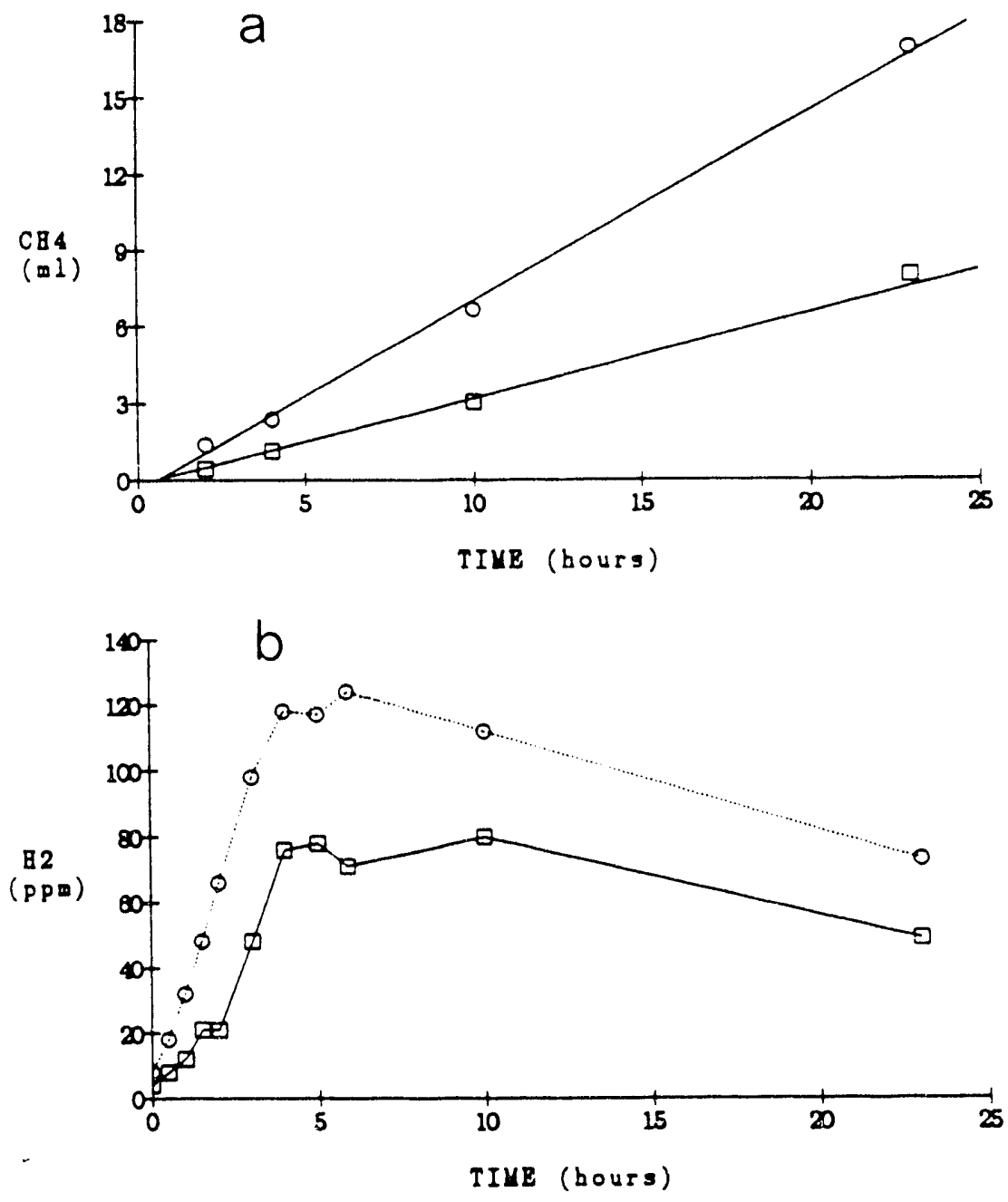

$$
\text { C Non-mixed }
$$

rigure A-1. Effect of mixing on (a) methane production and (b) bydrogen accumulation patterns. 


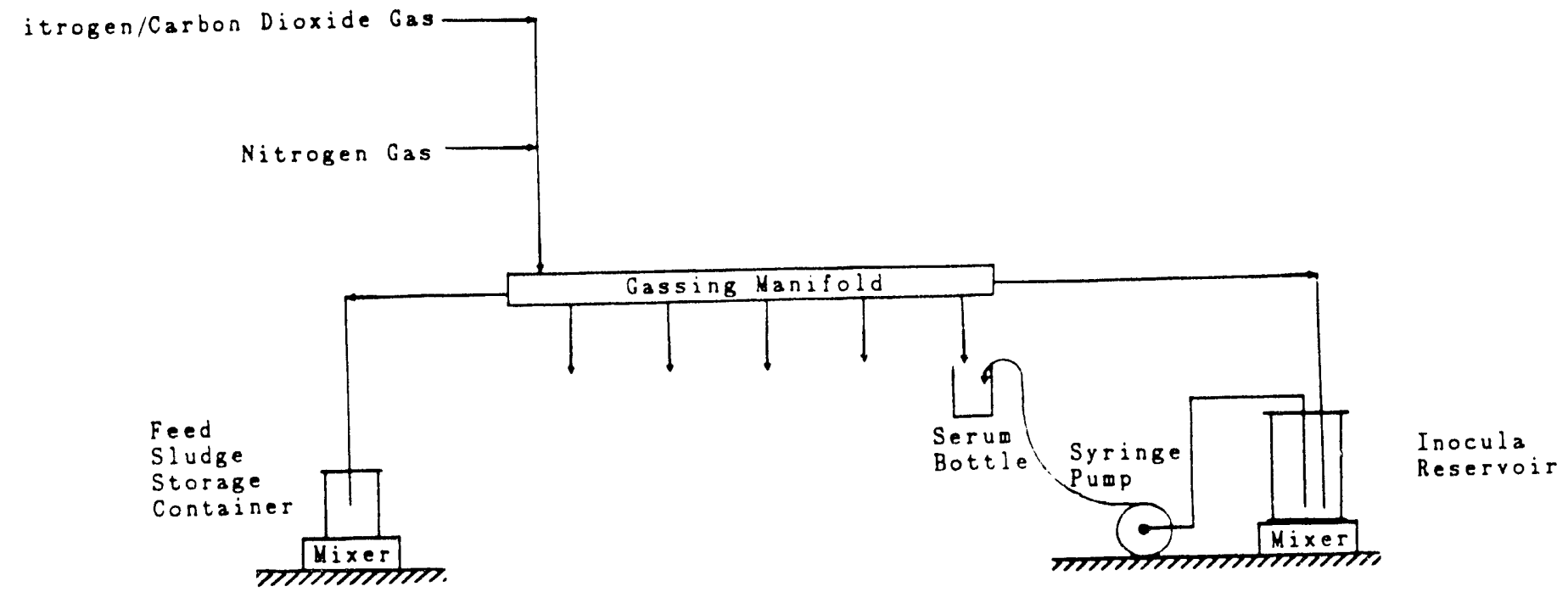

77777777

Figure A-2. Experimental set-up for preparing serum bottle assays. 
somewhat from unit to unit but was measured to be between 380 to $400=\mathrm{pm} 3 \mathrm{for}$ all units used.

\section{Assay Protecel}

0

Inocula: Two reserve digeaters, with working volunes of 55- and 110-liters, were used to supply inocula with 10- and 20-day hydraulic retention times (HRT) respectively, for the serum bottle assays. The digesters were housed in a walk in constanttemperature incubator maintained at $35 \pm 0.5^{\circ} \mathrm{C}$. The reactors were operated in a fill-and-draw mode. The units were fed daily a mix of waste activated sludge (WAS)

and dissolved air flotation waste activated sludge from the Amherst, MA POTW. The feed to each unit was maintained at a constant level of 1.25 to $1.50 \%$ total solids. On the day of an assay, sludge was anaerobically transferred from one of tine reserve digesters to a sealed, nitrogen gassed, reservoir either just prior to or during feeding of the digester.

Set-up: In general, samples were run in duplicate and controls in triplicate. On several occasions, when fewer experimentai conditions were examined, samples were also run in triplicate. For a typical serum bottle assay, the following procedure was used:

- Serum bottles $\left(160 \mathrm{ml}\right.$ total volume) were purged with a $\mathrm{O}_{2}-$ free $(<2 \mathrm{ppm})$ mixture of $\mathrm{N}_{2} / \mathrm{CO}_{2}(70 / 30)$ gas. An acid washed teflon-coated stir-bar was placed in each bottle.

- A 5 or $10 \mathrm{ml}$ aliquot of homogenized waste activated sludge (WAS) was pipeted into the serum bottles. The samples were continuously gassed with the same $\mathrm{N}_{2} / \mathrm{CO}_{2}$ mixture for several minutes to ensure no oxygen was present.

- A 90 or $95 \mathrm{ml}$ volume of anaerobic digester sludge for the 10and 20-day HRT inocula, respectively, was then anaerobically transferred to the sample bottle with a syringe pump. Both the inocula and the serum bottles were gassed during this entire procedure. The inoculum reservoir was maintained completely mixed with a magnetically driven stirring apparatus.

- The serum bottles were capped with butyl-rubber septas and crimp-sealed with aluminum seals. After sealing, the serum bottles were incubated for 30 minutes at $35^{\circ} \mathrm{C}$ on a gang pulley mixer. Gas pressure was then equilibrated to atmospheric with water lubricated ground glass syringes (equipped with 24 or 25 gauge needles) and samples reincubated at $35^{\circ} \mathrm{C}$ to start the assay.

Toxicant Addition: Due to differences in the physical and chemical nature of the various toxicants examined, the manner in which they were administered varied.

- The heavy-metal toxicants were added in microliter quantities and allowed to contact the was for several minutes before 
adding the digester inocula so that ligand binding of the metals by sludge would occur prior to initiating the assay, thereby simulating, within constraints of the batch

technique, a real world situation. Cheng et al. (1975) demonstrated the binding of heavy-metals to was is extremely rapid, occurring in 3 to 10 minutes. All three metals were added as divalent chlorides.

- Organic toxicants were added either just prior to sealing sample bottles or immediately after by syringe injection. Chloroform and trichloroacetic acid (TCAA) were added by syringe injection after sealing the serum bottles to preclude loss of toxicant by volatilization. Bromoethanesulfonic acid and formaldehyde were added just prior to sealing samples.

- If additional substrates were to be used in the assay (i.e., added acetate, formate or sucrosel these were adided by syringe injection of concentrated stock solutions immediately after sealing the serum bottles.

Nete: In retrospect, it would in fact have been better to wait until after the 30 minute preincubation period to add the organic toy cants and supplemental substrates. In that way, it would have veen possible to observe the immediate effect of these compounds if desired.

Monitoring: After the 30-minute pre-incubation period, when the samples were equilibrated to atmospheric pressure, the starting values for headspace gas composition were obtained. Gas chromatography was used to analyze serum bottle headspace for $\mathrm{CO}_{2}, \mathrm{CH}_{4}, \mathrm{CO}$ and $\mathrm{H}_{2}$ concentrations. Gas production, as well as methane and $\mathrm{CO}_{2}$ concentrations, were determined at $4,8,12$ and 24 hours. Hydrogen and carbon monoxide gas concentrations were monitored on an hourly basis for the first eight hours of the assay and then at the 12 and 24 hour marks. In general, assays were run for a 24-hour period.

For hourly tracking of headspace hydrogen and co concentrations, $0.5 \mathrm{ml}$ of gas was removed from one of the two or three replicate samples with nitrogen flushed $1.0 \mathrm{ml}$ disposable tuberculin syringes lequipped with 23 or 25 gauge needles). Samples were immediately injected into a $0.1 \mathrm{ml}$ sample loop, connected to the chromatograph used to mcasure hydrogen and co, and analyzed. During the sample times when gas production and methane content were measured $(0,4,8,12$ and 24 hour marks) nitrogen-flushed, water-lubricated, groundglass syringes (24 or 25 gauge needles) were used to measure the gas produced by displacement (Miller and Wolin, 1974). All of the gas produced was removed from the headspace at this time. A portion of this gas (minimum of $0.5 \mathrm{ml}$ ) was immediately injected into the $0.1 \mathrm{ml}$ sample loop of the reduced gas chromatograph and analyzed. In those instances when gas displacement was less than $0.5 \mathrm{ml}$, after measuring the amount of production the gas was reinjected 
into the headspace. If hydrogen or co analysis was deemed necessary, a $0.5 \mathrm{ml}$ sample was removed as described above. At the 0, 4, 8, 12 and 24 hour marks, all replicates were analyzed for $\mathrm{H}_{2}, \mathrm{CO}, \mathrm{CO}_{2}$, and $\mathrm{CH}_{4}$.

Methane was analyzed on a $1.0 \mathrm{ml}$ gas sample removed from the headspace of the serum bottles immediately after equilibrating headspace gas pressure to atmospheric.

Total and volatile solids concentrations of the inocula were determined for each experimental run. At the conclusion of each assay, pH was determined. Samples from each assay were centrifuged at $2000 \times \mathrm{g}$ for 15 minutes and the supernatant frozen for later analysis of volatile fatty acids. For organic toxicants and the organic overload experiment, this was done at the conclusion of the 24 hour assays. For heavy metal toxicants, samples were sacrificed after 48 hours incubation.

Calculation of Methane Production

Methane production for a discrete time interval (nomally 4 hours) is calculated by summing: 1) the methane content of wasted gas times the volume wasted, and 2) the change in serum bottle headspace concentration over the time interval times the headspace volume.

With the slotted-style butyl-rubber septa used in this study, nominal $125 \mathrm{ml}$ serum bottles have a total volume of $162 \mathrm{ml}$. For all assays, a total liquid volume of $100 \mathrm{ml}$ was used. The teflon coated stir bars used to mix the serum bottles had a measured (by liquid displacement in $25 \mathrm{ml}$ graduated cylinders) volume of approximately $2.0 \mathrm{ml}$. Headspace-volume, therefore, was $60 \mathrm{ml}$ for this study.

\section{Rrecision and Reproducibility of Assay Techoigue}

The heterogeneous nature of both the digester inoculum and the was feed, coupled with inherent errors in transfer of the exact volume to each serum bottle, as well as measurement errors for gas production, and methane concentration could potentially lead to significant error in the overall results. A serum bottle assay was run using six replicates and a 20-day HRT inoculum. The relative standard deviation of cumulative 24-hour results were 
observed to be 5.0 and 8.5 percent for total gas and methane production, respectively. A complete breakdown on a 4 -hour interval is presented in Table A-1. In an effort to obtain some measure as to what influence the heterogeneous nature of WAS had on the results, 3 replicate samples using WAS that had been essentially homogenized by passing the sludge through a frenchpress 3 times were assayed simultaneously. Extensive cell breakage was confirmed through microscopic observation. The cumulative 24-hour standard deviations measured for total gas and methane production were 2.5 and 2.1 percent, respectively. A complete 4 -hour interval analysis is presented in Table $A-2$.

\section{Beproducibility of Gas and Methane Production Between Experinental Buns}

A total of 11 assays were conducted using the 20-day HRT inoculum. Results of total gas and methane production for control samples were compared on a rationale basis $\mathrm{lml} \mathrm{CH}_{4}$ produced per day per gram of volatile solids of inocula added). The methane production was observed to be $35.1 \pm 2.1 \mathrm{ml} \mathrm{gVS}^{-1}$ day ${ }^{-1}$, giving a relative standard deviation of 5.0 percent.

Gas production, calculated as $\mathrm{ml}$ gas per gram of volatile solids of inocula added per dal, for the 11 runs averaged $55.1 \mathrm{ml} \mathrm{gVS}^{-1} \mathrm{day}^{-1}$ with a relative standara deviation of 9.8 percent.

These values are reasonable especially when the variations in the feed was are considered.

Rejection of Data and Quality control

Controls are always run in triplicate so that if one replicate behaves in a fashion unlike the other samples (which is not uncommon for biological tests), it can be rejected and not used in the data analysis. Normal procedure for rejecting data is to eliminate those samples that fall outside the 95 percent confidence intervals (Standard Methods (sect 801) 1985). This was done on three occasions to one of the three control sample replicates. 
Table A-1

Methane and Total Gas Production with Masociated

Relative standard Deriation for was fed 20-Day HRT Inoculum

\begin{tabular}{|c|c|c|c|c|}
\hline $\begin{array}{c}\text { Time } \\
\text { Interval } \\
\text { (hours) }\end{array}$ & 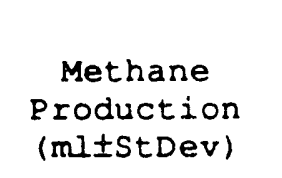 & $\begin{array}{c}\text { Relative } \\
\text { Standard } \\
\text { Deviation } \\
(\xi)\end{array}$ & $\begin{array}{c}\text { Gas } \\
\text { Production } \\
\text { (mltstDev) }\end{array}$ & $\begin{array}{c}\text { Relative } \\
\text { Standard } \\
\text { Deviation } \\
\text { ( }(\text { ) }\end{array}$ \\
\hline $0-4$ & $3.80 \pm 0.37$ & 9.8 & $7.92 \pm 0.15$ & 1.9 \\
\hline $4-8$ & $4.88 \pm 0.33$ & 6.8 & $7.25 \pm 0.15$ & 2.1 \\
\hline $8-12$ & $4.12 \pm 0.27$ & 6.8 & $6.73 \pm 0.29$ & 4.3 \\
\hline $12-24$ & $11.20 \pm 1.27$ & 11.3 & $12.72 \pm 1.42$ & 11.2 \\
\hline Overall & $24.1 \pm 2.06$ & 8.5 & $35.0 \pm 1.76$ & 5.0 \\
\hline
\end{tabular}

rable A-2

Metbane and Total Gas Production with Masociated Relative standard Deviation for 20-Day HRT Inoculum fed French-Press-Treated waS

\begin{tabular}{|c|c|c|c|c|}
\hline $\begin{array}{l}\text { Time - } \\
\text { Incerval } \\
\text { (hours) }\end{array}$ & $\begin{array}{l}\text { Methane } \\
\text { Production } \\
\text { (mltstDev) }\end{array}$ & $\begin{array}{c}\text { Relative } \\
\text { Standard } \\
\text { Deviation } \\
\text { (z) }\end{array}$ & $\begin{array}{c}\text { Gas } \\
\text { Production } \\
\text { (ml } 1 \text { StDev) }\end{array}$ & $\begin{array}{c}\text { Relative } \\
\text { Standard } \\
\text { Deviation } \\
\text { ( } 8)\end{array}$ \\
\hline $0-4$ & $4.00 \pm 0.27$ & 6.6 & $8.90 \pm 0.17$ & 1.9 \\
\hline $4-8$ & $5.03 \pm 0.21$ & 4.1 & $8.00 \pm 0.17$ & 2.2 \\
\hline $8-12$ & $4.00 \pm 0.0$ & 0.0 & $7.23 \pm 0.12$ & 1.6 \\
\hline $12-24$ & $12.57 \pm 0.208$ & 1.7 & $14.37 \pm 0.55$ & 3.8 \\
\hline Overall & $25.60 \pm 0.56$ & 2.2 & $38.50 \pm 0.95$ & 2.5 \\
\hline
\end{tabular}




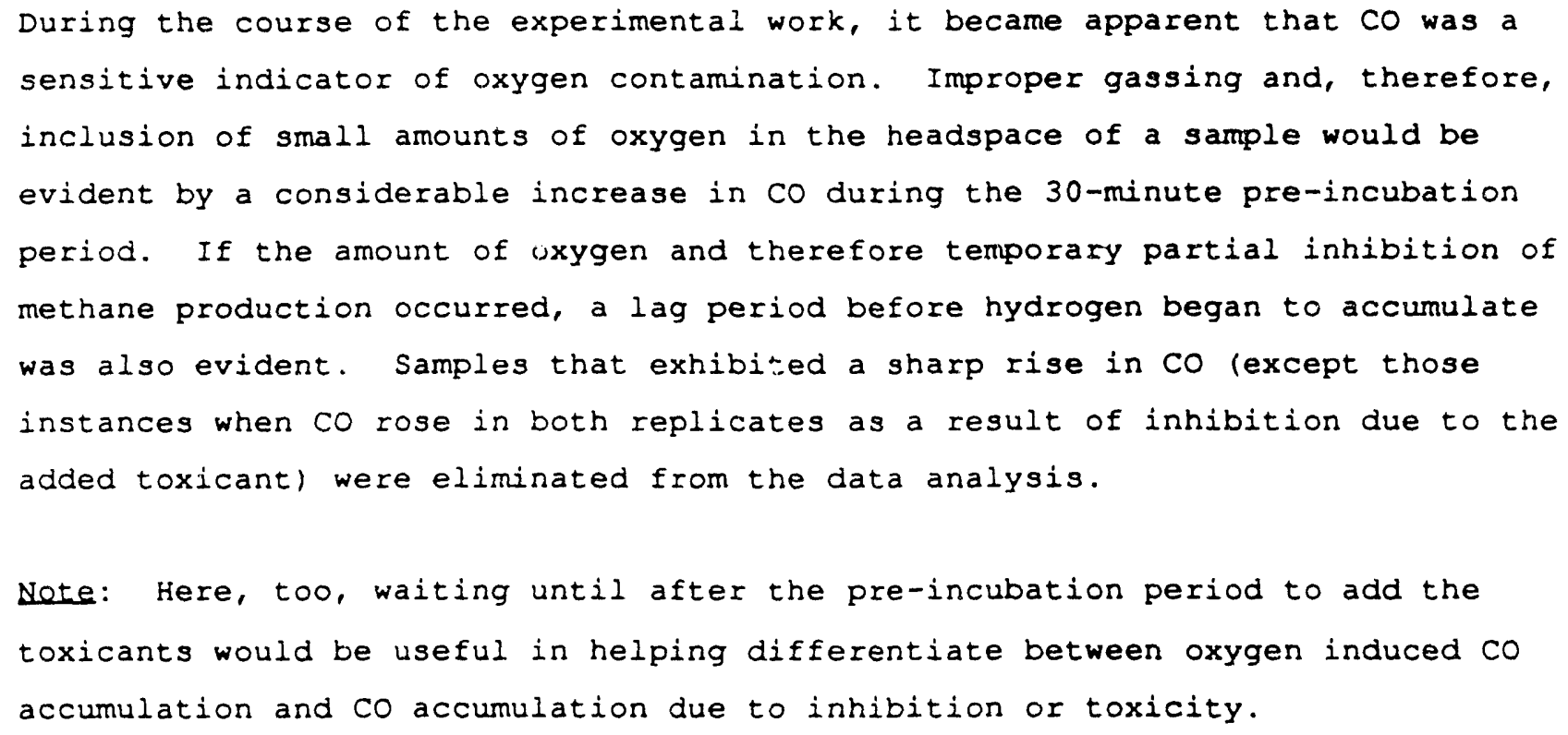


EARLY-WARNING PROCESS CONTROL FOR BIOLOGICAL NITROGEN TRANSFORMATION PROCESSES:

BATCH AND CHEMOSTAT EXPERIMENTS 


\section{ACKNOWLEDGEMENTS}

The author would like to thank Dr. Michael Switzenbaum for his support and guidance. Special thanks are due to Ms. Juliana Vanderwielen for her assistance in the laboratory. The support of Mr. Barry Liebowitz from NYSERDA and Mr. Yoward Feiler, SAIC are also gratefully acknowledged. 
CONTENTS

Section

Page

EXECUTTVE SUMMARY $\ldots \ldots \ldots \ldots \ldots \ldots \ldots \ldots \ldots \ldots \ldots \ldots \ldots \ldots$

1. INTRODUCTION $\ldots \ldots \ldots \ldots \ldots \ldots \ldots \ldots \ldots \ldots \ldots \ldots \ldots \ldots \ldots$

2. NITROGEN TRANSFORMATION PROCESSES $\ldots \ldots \ldots \ldots \ldots \ldots \ldots \ldots \ldots$

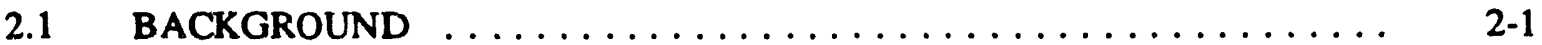

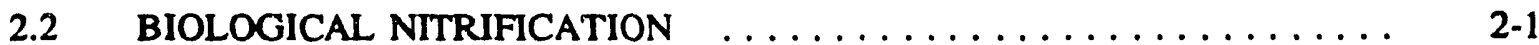

$2.3 \quad$ NITRATE IN GROUNDWATER $\ldots \ldots \ldots \ldots \ldots \ldots \ldots \ldots \ldots \ldots$

2.4 BIOLOGICAL DENITRIFICATION $\ldots \ldots \ldots \ldots \ldots \ldots \ldots \ldots \ldots \ldots \ldots$

2.5 TRACE GASES ASSOCIATED WITH NITROGEN

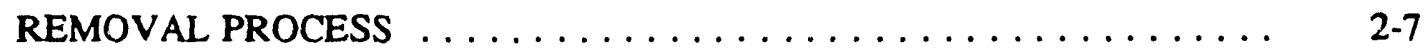

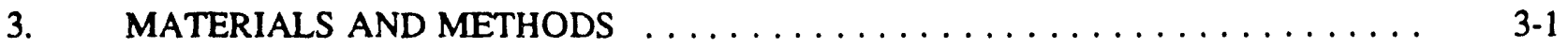

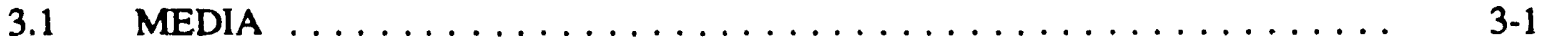

$3.2 \quad$ NITRIFYING INOCULUM PREPARATION $\ldots \ldots \ldots \ldots \ldots \ldots \ldots \ldots, 3-2$

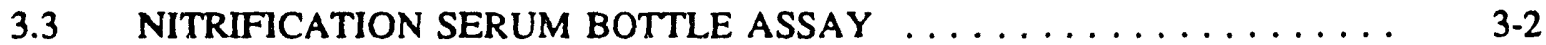

3.4 DENITRIFYING INOCULUM PREPARATION $\ldots \ldots \ldots \ldots \ldots \ldots \ldots . \ldots \ldots$

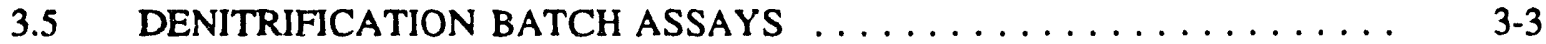

3.6 DENITRIFICATION CHEMOSTAT EXPERIMENTS $\ldots \ldots \ldots \ldots \ldots \ldots$.

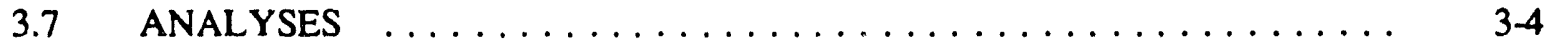

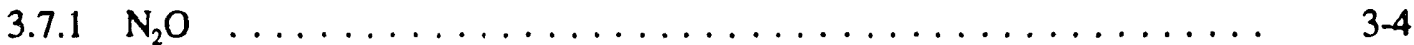

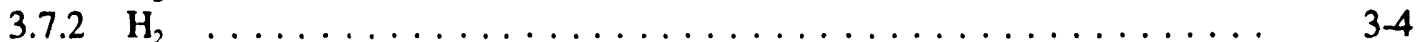

3.7.3 Ammonium ....................... 3.4

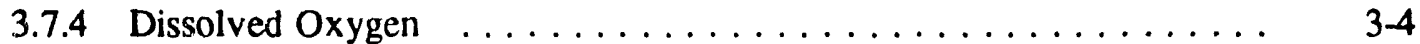

3.7 .5 Nitrate . . . . . . . . . . . . . . . . . . . . . . . . 3.5

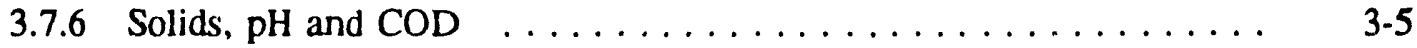

4. RESULTS OF NITRIFICATION EXPERIMENTS $\ldots \ldots \ldots \ldots \ldots \ldots \ldots$

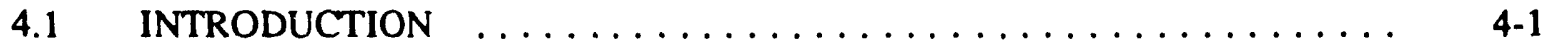

4.2 BATCH SERUM BOTTLE TESTING $\ldots \ldots \ldots \ldots \ldots \ldots \ldots \ldots \ldots$ 4-1

4.3 THERMODYNAMIC ANALYSIS $\ldots \ldots \ldots \ldots \ldots \ldots \ldots \ldots \ldots \ldots$ 4-2

5. RESULTS OF DENITRIFICATION STUDIES $\ldots \ldots \ldots \ldots \ldots \ldots \ldots \ldots \ldots$

5.1 BATCH ASSAY USING ACETATE AS THE ELECTRON DONOR $\ldots \ldots$ 5-1

5.2 CHEMOSTAT STUDIES USING ACETATE AS THE

5.3 CHEMOSTAT STUDIES USING METHANOL AS THE
ELECTRON DONOR FOR DENITRIFICATION $\ldots \ldots \ldots \ldots \ldots \ldots \ldots$

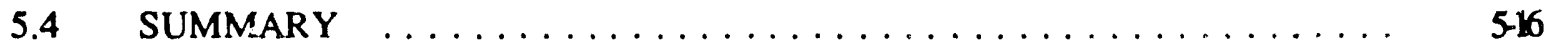




\section{CONTENTS (Cont'd)}

Section

Page

6. CONCLUSIONS AND DISCUSSIONS $\ldots \ldots \ldots \ldots \ldots \ldots \ldots \ldots \ldots \ldots$

$6.1 \quad$ NITRIFICATION $\ldots \ldots \ldots \ldots \ldots \ldots \ldots \ldots \ldots \ldots \ldots \ldots \ldots \ldots$

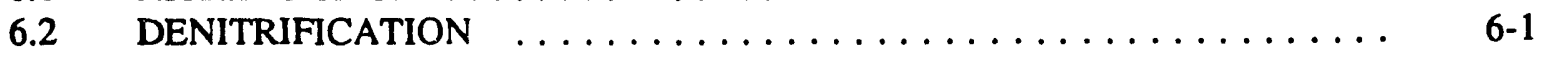

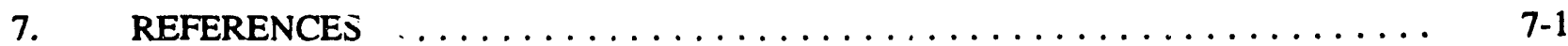




\section{FIGURES}

Figure

Page

5-1 Concentrations of Nitrate, Nitrite and $\mathrm{N}_{2} \mathrm{O}$ During Batch

Denitrification Assay Using Acetate as the Electron Donor $\ldots \ldots \ldots \ldots \ldots \ldots$

5-2 Concentrations of Nitrate, Nitrite and $\mathrm{H}_{2}$ During Batch

Denitrification Assay Using Acetate (Acetate $/ \mathrm{NO}_{3}-\mathrm{N}=4.5$ ) as

the Electron Donor. At 400 Minutes a Pulse of Nitrate was Added . . . . . . . . . .

5-3 Comparison of Nitrate Conversation, Nitrite and Nitrous Oxide

Accumulation Pattems During Experimental Run 4 (Excess Acetate -

Filled Triangles) and Run 5 (Excess Nitrate - Open Triangles) $\ldots \ldots \ldots \ldots \ldots \ldots$

5-4 Concentrations of Nitrah, Nitrite, $\mathrm{N}_{2} \mathrm{O}$ and $\mathrm{H}_{2}$ During Denitrification

Using Acetate as the Electron Donor (Acetate $/ \mathrm{NO}_{3}-\mathrm{N}=4.5$ ) in a Chemostat.

At Time $\mathrm{t}=0$, Feed was Switched to a $200 \mathrm{mg} / \mathrm{L}$ Nitrate Only $\ldots \ldots \ldots \ldots \ldots$

5-5 Concentrations of Nitrate, Nitrite, $\mathrm{N}_{2} \mathrm{O}$ and $\mathrm{H}_{2}$ in an Acetate-Fed

(Excess Acetate) Denitrifying Chemostat. At Time $\mathrm{t}=0$, Feed was Switched

to a $200 \mathrm{mg} \mathrm{NO}-\mathrm{N} / \mathrm{L}$ Only. At Time $\mathrm{t}-270$ Minutes, the Feed was Changed to

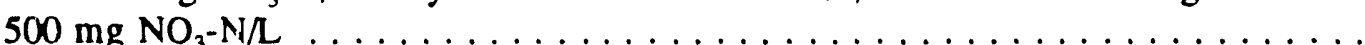

5-6 Concentrations of Nitrate, Nitrite $\mathrm{N}_{2} \mathrm{O}$ and $\mathrm{H}_{2}$ in a Methanol-Fed

Chemostat (Methanol/ $\mathrm{NO}_{3}-\mathrm{N}=3 / 1$ ). At Time Zero, a Feed Containing

$200 \mathrm{mg} \mathrm{NO}{ }_{3}-\mathrm{N} / \mathrm{L}$ Only was Used to Replace the Normal Feed

5-7 Effect of Reducing the HRT of a Methanol-Fed Chemostat

(Methanol/Nitrate- $N=3 / 1$ ) from 1.25 to 0.28 Days $(t=0)$. At

$\mathrm{t}=90$ Minutes, the HRT was Increased Back to 1.25 Days $\ldots \ldots \ldots \ldots$

5-8 Concentrations of Nitrate, Nitrite, $\mathrm{N}_{2} \mathrm{O}$ and $\mathrm{H}_{2}$ in a Methanol-Fed

(Methanol/ $\mathrm{NO}_{3}-\mathrm{N}=3.5 / 1$ ) Chemostat. At Time Zero, the Feed was Switched

to a Nitrate Only $(200 \mathrm{mg} \mathrm{N} / \mathrm{L})$ Feed. At $\mathrm{t}=60$ Minutes the Feed

Solution was Spiked with Methanol to Obtain a Concentration of

Ca $8000 \mathrm{mg} / \mathrm{L}$

5-9 Concentrations of Nitrite and $\mathrm{N}_{2} \mathrm{O}$ in a Nitrite-Fed Chemostat. At Time

Zero, the Feed was Switched to Nitrite (200 mg N/L) Only, No Methanol . . . . . . 


\section{TABLES}

Table

Page

2-1 Full-Scale Experience with Nitrification of Drinking Water

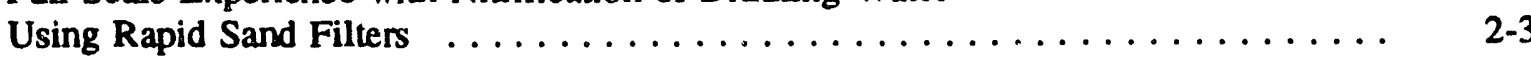

2-2 Full-Scale Experience with Nitrification in Biologically

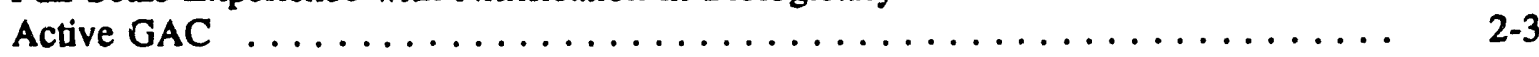

2-3 Reported Genera of Denitrifying Bacteria $\ldots \ldots \ldots \ldots \ldots \ldots \ldots$

2-4 Full- and Pilot-Scale Plants for Denitrification of

Drinking Water ............................ $2-8$

3-1 Composition of Media Used in Nitrification and Denitrifi-

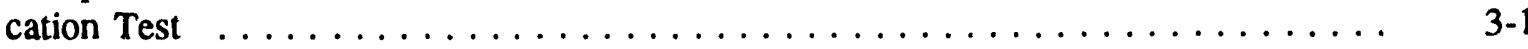

3-2 Starting Conditions for Nitrification Serum Bottle Assay $\ldots \ldots \ldots \ldots \ldots \ldots$ 3-2

4-1 Thermodynamics of Ammonium Oxidation (After Thauer et.al.

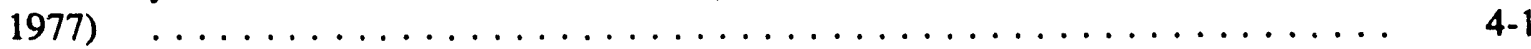

4-2 Results of Batch Serum Bottle Testing for $\mathrm{N}_{2} \mathrm{O}$ Production

During Nitrification $\ldots \ldots \ldots \ldots \ldots \ldots \ldots \ldots \ldots \ldots \ldots \ldots \ldots \ldots$ 4.2

4-3 Free Energy Calculations for Serum Bottle Nitrification Assay $\ldots \ldots \ldots \ldots \ldots$ 4-3

4-4 Effect of Changes in Other System Variables on Equilibrium $\mathrm{N}_{2} \mathrm{O}$

Concentration $\ldots \ldots \ldots \ldots \ldots \ldots \ldots \ldots \ldots \ldots \ldots \ldots \ldots \ldots \ldots \ldots$

4-5 Free Energy Available for Ammonium Oxidation $\ldots \ldots \ldots \ldots \ldots \ldots$

5-1 Concentrations of Nitrogen Species During Batch Denitrification

Assay (Room 2) Using Acetate as the Electron Donor . . . . . . . . . . . . $5-3$

5-2 Concentrations of Nitrogen Species During Batch Denitrification

Assay (Room 3) Using Acetate as the Electron Donor . . . . . . . . . . . 5-5

5-3 Concentrations of Nitrogen Species During Batch Denitrification

Assay (Room 4) Using Acetate as the Electron Donor . . . . . . . . . . . 5 5-5

5-4 Concentrations of Nitrogen Species During Batch Denitrification

Assay (Room 5) Using Acetate as the Electron Donor $\ldots \ldots \ldots \ldots \ldots \ldots$

5-5 Concentration of Nitrite and $\mathrm{N}_{2} \mathrm{O}$ During Reduction of Nitrite

Using Acetate as an Electron Donor (Room 6) $\ldots \ldots \ldots \ldots \ldots \ldots$

5-6 Summary of Chemostat Experiment Using Methanol as Electron

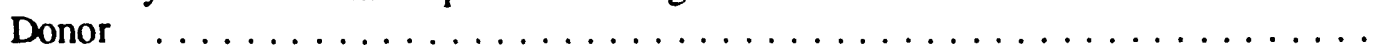




\section{EXECUTIVE SUMMARY}

The potential of using trace gases as indicators of biologically medicated nitrogen transformation processes was examined during this effort. The production of nitrous oxide $\left(\mathrm{N}_{2} \mathrm{O}\right)$ during both nitrification (oxidation of ammonium to nitrite and nitrate) and denitrification (reduction of nitrite and nitrate to nitrogen dioxide $\left(\mathrm{N}_{2}\right)$ ) has been reported in the literature. Therefore, $\mathrm{N}_{2} \mathrm{O}$ was selected as the trace gas to monitor during batch and continuous flow experiments.

The concentration of $\mathrm{N}_{2} \mathrm{O}$ was observed to rise concurrent with accumulation of nitrite and to a lesser extent to an increase in ammonium concentration. Based upon a thermodynamic evaluation of the data, it was observed that an increase in nitrite will result in an increase in $\mathrm{N}_{2} \mathrm{O}$ to the $3 / 2$ 's power. Monitoring $\mathrm{N}_{2} \mathrm{O}$ levels should, therefore, be a reasonably sensitive indicator of an increase in nitrite, which is generally observed to increase rapidly during perturbations in nitrification efficiency.

During denitrification studies, using acetate as the electron donor, $\mathrm{N}_{2} \mathrm{O}$ was observed to increase rapidly when insufficient acetate was present for complete reduction of the nitrate present or during an overload to the denitrifying system. Similar results were observed when nitrite was used as the oxidized nitrogen feed or when methanol was used as the electron donor.

It was also observed that hydrogen gas could be used as a measure of denitrification. During periods when excess electron donor was present, $\mathrm{H}_{2}$ rose rapidly, well above neutral concentration levels, particularly when acetate was used as the electron donor and to a much lesser extent when methanol was used as the electron donor. The concurrent monitoring of both $\mathrm{N}_{2} \mathrm{O}$ and $\mathrm{H}_{2}$ appears to hold much promise as a means of controlling electron donor addition, and presenting both under- or over-feeding of the added electron donor.

Further work on developing monitoring and control strategies and systems for nitrogen transformation processes based on $\mathrm{N}_{2} \mathrm{O}$ (and $\mathrm{H}_{2}$ ) monitoring would appear to be warranted. 


\section{SECTION 1}

\section{INTRODUCTION}

Although the nation is not running out of water, there is an increasing scarcity of high quality water in some areas. This is often due to contamination of surface and ground water reserves with nitrates, pesticides, volatile organic carbon compounds, and ammonium. Many of these problems are related to agricultural activities and, in the case of some surface water supplies, municipal wastewater treatment plant discharges (U.S. Water Resources Council, 1979). The degradation of water quality, combined with an increasing awareness of the adverse health effects of many pollutants, even at trace levels, has substantially increased the difficulty in providing a product water that is free from pathogens, other health concems (i.e. carcinogens) as well as taste, odor, and color. In the case of nitrogen transformation or removal, the use of biological treatment processes is an economical option.

Control of the different nitrogen species is important in water and wastewater maragement programs due to the many effects nitrogenous materials can have. Nitrogen, in its various forms, can deplete dissolved oxygen levels in receiving waters, stimulate aquatic growth, can be toxic to aquatic life, adversely affect chlorine disinfection efficiency, can present a public health hazard, and can affect the suitability of wastewater for reuse. Biological and chemical processes which occur in water and wastewater treatment plants and in the natural environment can change the nitrogen's chemical form which may eliminate one deleterious effect while producing or leaving unchanged another. For example, by converting ammonia to nitrate (nitrification) the oxygen-depleting and toxic effects of ammonia are eliminated, but is biostimulatory effects on algae growth may not be significantly changed. Incomplete nitrification can produce an accumulation of nitrite that represents a more serious toxic threat to fish populations.

As the treatment limits for nitrogen contaminants decrease, there will be a concomitant need for better process monitoring and control. New applications of biological nitrogen transformation processes such as in high density fish farming (aquaculture) represent applications where proper control is essential for the success of the entire enterprise (i.e. ammonium removal and low nitrite concentrations are essential for the health of the "product" fish).

In anaerobic digestion, certain trace gases offer great potential for simple, reliable monitoring and control of the process. In anaerobic systems, $\mathrm{H}_{2}$ and $\mathrm{CO}$ are the trace gases that lend insight into the health and stability of the treatment system (Hickey and Switzenbaum, 1991). 
In nitrogen removal processes, certain trace gases are related to the principal biological reactions involved. The major thrust of this research was, therefore, to determine if in fact these trace gases, principally $\mathrm{N}_{2} \mathrm{O}$, could be related to the performance of the nitrification and denitrification processes, and if there is potential to then use these gases for process monitoring and/or control. 


\section{SECTION 2}

\section{NITROGEN TRANSFORMATION PROCESSES}

\subsection{BACKGROUND}

This section briefly reviews the biological processes of nitrification and denitrification and describes the evolution of the trace gas nitrous $\left(\mathrm{N}_{2} \mathrm{O}\right)$ in conjunction with these processes.

Biological processes for control of nitrogenous compounds can be classified in two broad areas. First, a process designed to produce an effluent where influent nitrogen (ammonia and organic nitrogen) is substantially converted to nitrate nitrogen is referred to as nitrification. This process is performed by bacterial populations that sequentially oxidize ammonia to nitrate with the intermediate formation of nitrite. Nitrification will satisfy effluent or receiving water standards where reduction of residual nitrogenous oxygen demand, due to ammonia, is mandated or where ammonia reduction for other reasons is required. The second process, denitrification, involves the reduction of nitrate to nitrogen gas. The process is often used following nitrification when the total nitrogenous content of the effluent must be reauced.

\subsection{BIOLOGICAL NITRIFICATION}

Nitrification is the oxidation of ammonium to nitrate via a two-stage biological process as indicated below:

$$
\begin{aligned}
& \mathrm{NH}_{4}^{+}+3 / 2 \mathrm{O}_{2} \ldots \mathrm{NO}_{2}^{-}+\mathrm{H}_{2} \mathrm{O}+2 \mathrm{H}^{+} \\
& \mathrm{NO}_{2}^{-}+1 / 2 \mathrm{O}_{2} \cdots>\mathrm{NO}_{3}^{-}
\end{aligned}
$$

NET: $\quad \mathrm{NH}_{4}^{+}+2 \mathrm{O}_{2}-->\mathrm{NO}_{3}^{-}+\mathrm{H}_{2} \mathrm{O}+2 \mathrm{H}^{+}$

The two principal genera of importance in biological nitrification processes are Nitrosomonas and Nitrobacter, which are responsible for ammonium and nitrite oxidation, respectively. Both of these groups are classified as autotrophic organisms. These organisms are distinguished from heterotrophic bacteria because they derive energy for growth from the oxidation of inorganic nitrogen compounds, rather than from the oxidation of organic matter. Another feature of these organisms is that inorganic carbon (carbon dioxide) is used for synthesis rather than organic carbon. Each group oxidizes specific species of nitrogen compounds. Nitrosomonas can oxidize ammonia to nitrite, but cannot complete the oxidation to nitrate. On the other hand, Nitrobacter is limited to the oxidation of nitrite to nitrate. Since complete nitrification 
is a sequential reaction, treatment processes must be designed to provide an environment suitable to the growth of both groups of bacteria.

In addition to the above sited problems, ammonium can exert a significant chlorine demand and reduce the efficiency of disinfection through the formation of chloramines. Because of this, the World Health Organization Standards and National Academy of Science (1972) recommend the upper limit for ammonia to be 0.05 and $0.5 \mathrm{mg} / \mathrm{L}$, respectively.

In addition, sufficient amounts of ammonium ion present in the treated drinking water supply can cause bacterial regrowth in the distribution system, even if the water is heavily chlorinated (Olsen, 1982). This regrowth is considered to be responsible for taste, odor and color problems in water supply systems (Cullimore and Meann, 1977; Goodall, 1979; Hovel e․ al.. 1982).

Ritumann and Snoeyink (1984) proposed the concept of producing biologically stable water, that is water that does not readily support the growth of microorganisms to a significant extent, to reduce biological growth in the water distribution systems and the associated problems that can result. This approach advocated incorporating a biological treatment step prior to the normal treatment train to reduce the assimilable organic carbon (AOC) content and ammonium levels. The subsequent treatment processes provide the double-barrier treatment recommended to prevent bacterial contamination.

Nitrification, often employed for wastewater treatment to reduce the oxygen demand on receiving bodies of water, can be achieved by biological filters, fluidized beds, rapid sand filters and granular activated carbon (GAC) beds. In the latter two processes used for treating drinking water, nitrification often occurs fortuitously rather than by design, although many European countries are now attempting to design these systems to provide nitrification. Recent work by Bablon and co-workers (1986) demonstrated that dual media filters (sand/GAC) provided essentially complete nitrification at temperatures as low as $2^{\circ} \mathrm{C}$ in the presence of sufficient oxygen and phosphorous. Considerable experience operating rapid sand filters and GAC contactors in Germany and France has been described in detail. (Bouwer and Crowe 1988; Rittmann and Snoeyink, 1984) Full-scale operations that have been reported in the literature are summarized in Tables 2-1 and 2-2 for sand filters and GAC contactors.

While ammonium in drinking water poses no immediate health hazard, it can negatively affect the quality of water reaching the consumer. As previously mentioned, the presence of ammonium can enhance 
bacterial growth in the distribution system and consequently exert a significant oxygen demand (Gauntlett, 1981). This can result in depletion of oxygen in the distribution system and release of soluble iron (Larson, 1939). As little as 0.02 to $0.03 \mathrm{mg} / \mathrm{L}$ of ammonium is needed to allow formation of a nitrifying biofilm (Rittriann and Snoeyink, 1984). The levels of ammonium in many surface water supplies in the U.S. and abroad are often well above these values (Gauntlett, 1981).

Table 2-1. Full-Scale Experience with Nitrification of Drinking Water Using Rapid Sand Filters

\begin{tabular}{||l|c|c|c|}
\hline \multicolumn{1}{|c|}{ Location } & $\begin{array}{c}\text { Influent Ammonium } \\
\left.\left(\mathrm{mg} \mathrm{NH}_{4}^{+}\right)-\mathrm{N} / \mathrm{L}\right)\end{array}$ & \% Removal & Reference \\
\hline \hline Choisy-le-Roi, France & 0.5 & 100 & 17 \\
\hline $\begin{array}{l}\text { Mery-sun-Orse, } \\
\text { France }\end{array}$ & 0.34 & $\sim 100$ & 18 \\
\hline Mulheim, FRG & 1.0 & $\sim 100$ & 19 \\
\hline Rantoul, IL & 1.3 & 50 & 9 \\
\hline $\begin{array}{l}\text { Neuilly-sur-Marne, } \\
\text { France }\end{array}$ & 0.2 & $\sim 100^{\mathrm{b}}$ & 16 \\
\hline
\end{tabular}

a) Dual Media Filter (Sand/GAC)

b) $\quad 100 \%$ Removal at $2^{\circ} \mathrm{C}$, Parallel Sand Filter Removed ca. $80 \% \mathrm{NH}_{4}{ }^{+} \mathrm{N}$ (After Bouwer and Crowe, 1988)

Table 2-2. Full-Scale Experience with Nitrification in Biologically Active GAC

\begin{tabular}{|l|c|c|c|}
\hline \multicolumn{1}{|c|}{ Location } & $\begin{array}{c}\text { Influent Ammonium } \\
\left.\left(\mathrm{mg} \mathrm{NH}_{4}^{+}\right)-\mathrm{N} / \mathrm{L}\right)\end{array}$ & \% Removal & Reference \\
\hline Mulheim, FRG & 0.33 & 94 & 19 \\
\hline Choisy-le-Roi, France & 0.17 & $>70$ & 20 \\
\hline $\begin{array}{l}\text { Rouen la Chapelle, } \\
\text { France }\end{array}$ & 1.36 & 78 & 21 \\
\hline
\end{tabular}

(After Bouwer and Crowe, 1988) 


\subsection{NITRATE IN GROUNDWATER}

From 40 to 50 percent of the population in the U.S. depends on ground water for the sole source of potable water. (Pyl et. al.,1983) Nitrate and pesticide contamination is becoming more prevalent in agricultural regions (Asano, 1985). While high nitrate levels are found in both surface and ground waters, the concentrations in ground water are generally greater. The major concem about excessive nitrate in drinking water is methanoglobinemia or "blue baby syndrome", where in infants the nitrate is converted to nitrite which interferes with the respiratory system. There is also concern that nitrate and nitrite can be transformed into nitrosamines, which are carcinogenic, in the body.

\subsection{BIOLOGICAL DENITRIFICATION}

There are two ways that microorganisms use nitrate, assimilation and dissimilation. Nitrate assimilation is the overall process whereby nitrate is reduced to ammonia with the subsequent formation of nitrogenous cell constituents. Dissimilatory nitrate reduction is a process in which organisms use nitrate as a terminal electron acceptor instead of molecular oxygen. Nitrate is sequentially reduced through a series of steps believed to follow the sequence:

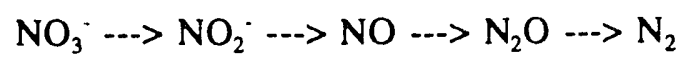

Both $\mathrm{NO}$ and $\mathrm{N}_{2} \mathrm{O}$ appear in the off-gases from denitrifying systems.

Denitrifying bacteria are ubiquitous in most soils and waters (Payne, 1981). This large number of bacteria includes heterotrophic and autotrophic species as shown in Table 2-3.

Table 2-3. Reported Genera of Denitrifying Bacteria

\section{GENUS}

\begin{tabular}{|l|l|l||}
\hline Acinetobacter & Flavobacterium & Pseudomonas \\
\hline Alcaligenes & Hyphomicrobium & Rhizobium \\
\hline Agrobacterium & Micrococcus & Rhodopseudomonas \\
\hline Azospirillum & Moarxella & Spirillum \\
\hline Bacillus & Paracoccus & Thiobacillus \\
\hline Cytophaga & Propionobacterium & Thermotrix \\
\hline
\end{tabular}

NOTE: a) Payne, 1981 
Heterotrophic bacteria require a source of organic carbon to denitrify. In wastewater treatment systems this is usually provided by adding methanol, acetate or by using the carbon contained in the raw waste. The stoichiometric relationships describing dissimilatory nitrate reduction for several different electron donors (carbon sources) are written as follows:

- Methanol: $\quad \mathrm{NO}_{3}^{-}+5 / 6 \mathrm{CH}_{3} \mathrm{OH}-->1 / 2 \mathrm{~N}_{2}+5 / 6 \mathrm{CO}+7 / 8 \mathrm{H}_{2} \mathrm{O}+\mathrm{OH}^{-}$

- Acetic Acid: $\mathrm{NO}_{3}{ }^{-}+5 / 8 \mathrm{CH}_{3} \mathrm{COOH}-->1 / 2 \mathrm{~N}_{2}+3 / 4 \mathrm{H}_{2} \mathrm{O}+5 / 4 \mathrm{CO}_{2}+\mathrm{OH}^{-}$

- Raw Waste: $\mathrm{NO}_{3}^{-}+1 / 10 \mathrm{C}_{10} \mathrm{H}_{19} \mathrm{NO}_{3} \cdots$

$$
1 / 2 \mathrm{~N}_{2}+9 / 10 \mathrm{CO}_{2}+1 / 10 \mathrm{NH}_{4}+1 / 5 \mathrm{H}_{2} \mathrm{O}+1 / 10 \mathrm{HCO}_{3}^{-}+\mathrm{OH}^{-}
$$

In the last equation, the organic pollution in raw wastewater is represented by the formula $\mathrm{C}_{10} \mathrm{H}_{19} \mathrm{O}_{3} \mathrm{~N}$.

Other non-toxic compounds such as ethanol have been examined as electron donors for denitrification. Because an electron donor is added to allow denitrification, extreme care must be taken to avoid adding excess substrate. This bleed-through can lead to excess carbonaceous oxygen demand on the receiving body of water or in the case of denitrification of drinking water, initiating biological regrowth in the distribution system and associated health problems.

Most of the experience with denitrification to treat drinking water has been in Europe. Perhaps the earliest work was performed in England treating surface water supplies (Gregory and Sheiham, 1981). Both acetate and methanol were tested as electron donors for both fluidized bed and filter type systems (Gauntlett and Zabel, 1981). Acetate actually fostered a higher reaction rate than methanol, but a tenacious slime formed and caused difficulties with the inlet distribution system. Nitrate accumulation also occurred under certain operating conditions. Accurate enough control of methanol addition to prevent bleed-through was another problem encountered.

Ethanol is used as the electron donor in the BIODENT process marketed for drinking water treatment in France. A full-scale plant $\left(1,600 \mathrm{~m}^{3} / \mathrm{d}\right)$ has been operating in Eragny since August, 1983 (Philipot et. a‥, 1985). The system consists of a denitrification filter coupled with an aerated packed media filter for polishing, suspended solids removal and removal of any excess ethanol. Control of biomass by periodic backwashing was found to be essential for reliable nitrate removal, and helped reduce the amount of ethanol bleed-through. 
A summary of the full-scale denitrification systems operational in Europe is presented in Table 2-4. Fullscale denitrification studies have generally produced a water free of pathogens and objectionable taste and odors. There are, however, concerns about the biological denitrification for drinking water treatment that include developing monitoring techniques to help ensure no bleed-through of the added electron donor occurs. Even when the organic carton source is removed aerobically in a post treatment step, the chance of producing soluble microbial products (SMP) due to growth related or endogenous (cell decay) metabolism is increased. Although it appears that SMP will likely be less than $1.0 \mathrm{mg} / \mathrm{L}$ TOC, the effect of this on water quality as a result of producing chlorination by-products is unclear (Bouwer and Crowe, 1988). An improved method to control substrate addition, to allow sufficient denitrification to occur and prevent accumulation of nitrite while eliminating or significantly reducing the amount of excess organic carbon (electron donor) supplied to the system, would improve the reliability and cost effectiveness of denitrification processes.

During the past decade considerable research has been directed towards developing in-situ denitrification near the extraction well. A full-scale operation has been installed in Vsetaty Czechoslovakia (Janda et. al. . 1988). Nitrates of 20 to $24 \mathrm{mg} \mathrm{NO}_{3}{ }^{-} \mathrm{N} / \mathrm{L}$ were reduced to 11 to $16 \mathrm{mg} \mathrm{NO}_{3}{ }^{-}-\mathrm{N} / \mathrm{L}$. Several problems were reported including the induction of anaerobic conditions near the injection wells jue to the high local ethanol concentrations with resultant $\mathrm{H}_{2} \mathrm{~S}$ production and odor at the collection well and breakthrough of cthanoi to the collection well. Similarly, problems of poor nitrate reduction and inpairment of flow from the injection. wells due to gas build-up and a decrease in permeability was observed in a full-scale demonstration in Israel (Mercado ㅌ. al.. 1988). Probably the most advanced in-situ design for denitrification is the Nitredox process that is being used full-tcale $\left(5,200 \mathrm{M}^{3} / \mathrm{d}\right.$ ) in Bisamberg. Austria (Braester and Martinell, 1988). A combination of 16 reduction wells and 8 oxidation wells (radius of 18 anj 10 meters around the central collection well, respectively) were used to reduce nitrate levels from 23 to between 5 and $6 \mathrm{mg} \mathrm{NO}{ }_{3}^{-}-\mathrm{N} / \mathrm{L}$. Precipitation of reduced iron and manganese is a benefit also realized by this process.

As required for the above ground treatment of extracted water, improved monitoring for process control is a serious need for in-situ denitrification. 


\subsection{TRACE GASES ASSOCIATED WITH NITROGEN REMOVAL PROCESSES}

The fact that nitrous $\left(\mathrm{N}_{2} \mathrm{O}\right)$ and nitric (NO) oxides are released during nitrification and denitrification processes thas been observed for some ìme.

Bremmer and Blackmer (1978) provided evidence that $\mathrm{N}_{2} \mathrm{O}$ was released to the atmosphere via nitrification of fertilizer nitrogen. From 0.04 to 0.45 percent of the $\mathrm{N}$ added was released as $\mathrm{N}_{2} \mathrm{O}$. Addition of a nitrification inhibitor, nitrapyrin, reduced $\mathrm{N}_{2} \mathrm{O}$ emissions by several orders of magnitude. Lischultz and co-workers (1981) examined the production of $\mathrm{N}_{2} \mathrm{O}$ and $\mathrm{NO}$ by an ammonium oxidizing bacteria, Nitrosomonas europea, at different oxygen tensions. Yields of both $\mathrm{NO}$ and $\mathrm{N}_{2} \mathrm{O}$ rose markedly at $\mathrm{O}_{2}$ concentrations of 2 percent $(v / v)$ or less. Goreau et. al. (1980) observed that reduced concentrations of dissolved oxygen (range 0.18 to $7.0 \mathrm{mg} / \mathrm{L}$ ) resulted in increased production of $\mathrm{N}_{2} \mathrm{O}$ and decreased production of nitrate by a marine ammonium oxidizer. The amount of $\mathrm{N}_{2} \mathrm{O}$ increased from 0.1 to 0.5 of the total ammonium oxidized to ca. 10 percent of the total ammonium when the D.O. was reduced to 0.18 $\mathrm{mg} / \mathrm{L}$. Aulakh and co-workers (1984) reported from 0.1 to 0.8 percent of ammonium fertilizer applied to aerobic soils was recovered as $\mathrm{N}_{2} \mathrm{O}$.

Nogita and co-workers (1981) reported that $\mathrm{N}_{2} \mathrm{O}$ was evolved from a laboratory-scale activated sludge system operated as a single stage to achieve both BOD removal and nitrification. They observed that the amount of $\mathrm{N}_{2} \mathrm{O}$ released along the length of the plug flow reactor decreased as the ammonium level decreased (and nitrate level increased). They postulated that $\mathrm{N}_{2} \mathrm{O}$ was formed as the result of denitrification within the flocs. The $\mathrm{N}_{2} \mathrm{O}$ production and nitrogen loading rates were reported to correlate well. The $\mathrm{N}_{2} \mathrm{O}$ formation rate was related to the product of COD and nitrate loading.

Keeney et al. (1985) observed that high $\mathrm{CO}_{2}$ levels (50 jercent $\mathrm{v} / \mathrm{v}$ or greater) increased the amr $\mathrm{r}$ of $\mathrm{N}_{2} \mathrm{O}$ produced via nitrification in soil. It is unclear, however, whether this was due to reduced $\mathrm{O}$. $:$ vels, creation of anaerobic microniches $r r$ other effects. Robertson and Tiedje (1987) observed $t$. ther biological sources, in addition to normal nitrification and denitrification production, of $\mathrm{N}_{2} \mathrm{O}$ were, resent in aerobic soils. Fungi were suggested as a possible source. The source was non-abiotic and was not inhibited by an acetylene block that does inhibit both bacterial nitrification an denitrification (Parlain et. al., 1984). 
Table 2.4. Full- and Pilot-Scale Plants for Denitrification of Drinking Water

\begin{tabular}{|c|c|c|c|c|}
\hline Process & Location & $\begin{array}{c}\text { Influent } \\
\left(\mathrm{mg} \mathrm{NO}_{\mathbf{3}}-\mathrm{N} / \mathbf{1}\right)\end{array}$ & $\begin{array}{c}\text { Reduction } \\
\%\end{array}$ & Reference \\
\hline \multicolumn{5}{|c|}{ Biological Filters } \\
\hline - Full Scale & Eragny, France & 13.5 & 50 & $\begin{array}{l}\text { Philipot et. al. } \\
\text { (1983 a) }\end{array}$ \\
\hline - Full Scale & Eragny, France & 37.9 & 84 & $\begin{array}{l}\text { Philipot et. al. } \\
(1982 \text { b) }\end{array}$ \\
\hline - Pilot Scale & Great Britain & $\mathrm{NR}^{*}$ & $78-100$ & $\begin{array}{l}\text { Richard et. al. } \\
\text { (1983) }\end{array}$ \\
\hline - Pilot Scale & Nebraska, USA & 22.5 & 100 & $\begin{array}{l}\text { Dehab et. al. } \\
\text { (1988) }\end{array}$ \\
\hline \multicolumn{5}{|l|}{ Fluidized Bed } \\
\hline - Pilot Scale & Bucklesham GB & 14 & 100 & Cross et. al. (1985) \\
\hline - Full Scale & Stevenage GB & 15 & 63 & Hall et. al. (1986) \\
\hline \multicolumn{5}{|c|}{ Rotating Biological Contactor } \\
\hline - Full Scale & $\begin{array}{l}\text { La Verne, CA, } \\
\text { USA }\end{array}$ & $60-80$ & $91-93$ & Hansen (1987) \\
\hline \multicolumn{5}{|c|}{ Soil Aquifer Treatment } \\
\hline - Full Scale & $\begin{array}{l}\text { Castricum, The } \\
\text { Netherlands }\end{array}$ & 22.5 & 72 & Piet et. al. (1985) \\
\hline - Full Scale & $\begin{array}{l}\text { Rhine River, } \\
\text { Germany }\end{array}$ & $\mathrm{NR}^{*}$ & 75 & Kubmaul (1979) \\
\hline - Full Scale & Bocholt, Germany & 20 & $\mathrm{NR}^{*}$ & $\begin{array}{l}\text { Barrenstein et. al } \\
\text { (1986) }\end{array}$ \\
\hline \multicolumn{5}{|c|}{ Autotrophic Denitrification } \\
\hline - Pilot Scale & France & 18.1 & $90-100$ & $\begin{array}{l}\text { Blecon et. al. } \\
\text { (1983) }\end{array}$ \\
\hline - Pilot Scale & Eragny, France & $109-168$ & $78-85$ & $\begin{array}{l}\text { Philipot et. al. } \\
\text { (1983 a) }\end{array}$ \\
\hline - Pilot Scale & $\begin{array}{l}\text { Braunschweig, } \\
\text { Germany }\end{array}$ & 22.5 & 100 & Vorlop et. al. \\
\hline
\end{tabular}

NOTE:

a) Bouwer et. al. 1988 + additional information

* NR - Not Reported. 
Anderson and Levine (1986) reported that $\mathrm{N}_{2} \mathrm{O}$ production in soils was primarily associated with denitrification while NO production was primarily associated with nitrification. Belach and Tiedje (1981) modeled $\mathrm{NO}$ and $\mathrm{N}_{2} \mathrm{O}$ accumulation using Michaelis-Menton equations assuming that the kinetics of degradation of the intermediates $\mathrm{NO}$ and $\mathrm{N}_{2} \mathrm{O}$ conirolled their accumulation. They also reported that as the oxygen partial pressure was increased, it inhibited overall denitrification rates and $\mathrm{N}_{\mathbf{2}}$ production but the $\mathrm{N}_{2} \mathrm{O} / \mathrm{N}_{2}$ ratio increased substantially. Nielsen and co-workers (1990), using a microsensor to measure $\mathrm{N}_{2} \mathrm{O}$ and D.O. within a biofilm, reported that $\mathrm{N}_{2} \mathrm{O}$ flux rates decreased as the D.O. level was increased from nil to several ppm.

In summation, although the trace gases $\mathrm{N}_{2} \mathrm{O}$ and $\mathrm{NO}$ have been observed produced during nitrification and denitrification, no attempt has been made to relate the concentrations of these gases from an energetics or thermodynamic approach. This is the focus of this work. 


\section{SECTION 3}

\section{MATERIALS AND METHODS}

\subsection{MEDIA}

The same media was used for both batch and continuous denitrification and nitrification experiments. To preclude precipitation, and growth in the media, six separate stock solutions (solutions A-F) were prepared.

Solution A - Phosphate Buffer. A phosphate buffer solution was prepared using $\mathrm{KH}_{2} \mathrm{PO}_{4}(8.5 \mathrm{~g})$, $\mathrm{K}_{2} \mathrm{HPO}_{4}(21.75 \mathrm{~g}), \mathrm{Na}_{2} \mathrm{HPO}_{4} \cdot 7 \mathrm{H}_{2} \mathrm{O}(33.4 \mathrm{~g})$ and $\mathrm{NH}_{4} \mathrm{Cl}(1.7 \mathrm{~g})$ per liter of distilled water.

Solution B - Magnesium Sulfate. A stock magnesium sulfate solution was made using $22.5 \mathrm{~g}$ of $\mathrm{MgSO}_{4} \cdot 7 \mathrm{H}_{2} \mathrm{O}$ per liter of distilled water.

Solution C - Calcium Chloride. A stock calcium chloride solution was prepared by dissolving $27.5 \mathrm{~g}$ of $\mathrm{CaCl}_{2}$ (anhydrous) in 1.0 liter of distilled water.

Solution D - Trace Minerals. A trace mineral solution was prepared by dissolving $\mathrm{MnSO}_{4} \cdot \mathrm{H}_{2} \mathrm{O}$ $(0.07 \mathrm{~g})$, $\mathrm{CuSO}_{4} \cdot 5 \mathrm{H}_{2} \mathrm{O}(0.02 \mathrm{~g}), \mathrm{Na}_{2} \mathrm{MoO}_{4} \cdot 2 \mathrm{H}_{2} \mathrm{O}(0.002 \mathrm{~g}), \quad \mathrm{ZnSO}_{4} \cdot 7 \mathrm{H}_{2} \mathrm{O}(0.07 \mathrm{~g})$ and $\mathrm{CoCl}_{2} 6 \mathrm{H}_{2} \mathrm{O}(0.002 \mathrm{~g})$ in $100 \mathrm{ml}$ of distilled water.

Solution E - Iron Solution. A stock solution of ferrous iron was prepared by dissolving $0.2 \mathrm{~g}$ $\mathrm{FeSO}_{4} \cdot 7 \mathrm{H}_{2} \mathrm{O}$ in one liter of distilled water.

Solution F - Bicarbonate Buffer. A buffer solution was prepared by dissolving $50 \mathrm{~g}$ of $\mathrm{NaHCO}_{3}$ in one liter of distilled water.

The medium used for the chemostat and other experiments was made by combining the solution in the proportions below (Table 3-1).

Table 3-1. Composition of Media Used in Nitrification and Denitrification Tests

\begin{tabular}{||c|c|}
\hline \multicolumn{1}{|c|}{ Component } & mL/Liter of Feed \\
\hline Solution A & 50.0 \\
Solution B & 1.0 \\
Solution C & 1.0 \\
Solution D & 0.1 \\
Solution E & 1.0 \\
Solution F & 10.0 \\
\hline
\end{tabular}




\subsection{NITRIFYING INOCULUM PREPARATION}

Retum Activated Sludge (RAS) was obtained from the Amherst, MA POTW. This treatment plant was completely nitrifying an influent ammonium concentration of ca. $15 \mathrm{mgN} / \mathrm{L}$ when the RAS was obtained. This culture was then placed into a batch-feed, continuously aerated, two-liter reactor. Once daily the airflow to this reactor was stopped, and the sludge allowed to settle for 15 minutes. One liter of the supernatant was decanted and replaced with fresh media amended with $\mathrm{NH}_{4} \mathrm{Cl}$ to attain a starting ammonium concentration of $100 \mathrm{mg} \mathrm{N} / \mathrm{L}$. The reactor was operated for three weeks before removing solids for inoculation of the nitrification serum bottle assay.

\subsection{NITRIFICATION SERUM BOTTLE ASSAY}

Amber, $250 \mathrm{~mL}$ serum bottles were filled to a total of $100 \mathrm{~mL}$ of media, solutions and inoculum. Ammonium and nitrate starting concentrations were adjusted to the starting concentrations shown in Table 3-2 by adding concentrated solutions of $\mathrm{NH}_{4} \mathrm{Cl}(1 \mathrm{~g} / \mathrm{L})$ and $\mathrm{NaNO}_{2}(0.4 \mathrm{~g} / \mathrm{L})$. All samples were run in duplicate. A teflon coated stir bar was added to each serum bottle, which was then capped with butyl rubber septa and sealed using plastic screw-type caps. The botules were then placed on gang pulley magnetic mixers located in a $25^{\circ} \mathrm{C}$ constant temperature incubator (Hickey and Switzenbaum, 1987). After 48 hours, the headspace of each sample was assayed for $\mathrm{N}_{2} \mathrm{O}$ (double injections of the headspace from each sample). The samples were then uncapped and the aqueous phase immediately assayed for $\mathrm{pH}$, nitrate, nitrite, ammonium, and dissolved oxygen.

Table 3-2. Starting Conditions for Nitrification Serum Bottle Assay

\begin{tabular}{|c|c|c|}
\hline SAMPLE & $\mathrm{NH}_{4} \cdot \mathrm{N}(\mathrm{mg} / \mathrm{L})$ & $\mathrm{NO}_{2}-\mathrm{N}(\mathrm{mg} / \mathrm{L})$ \\
\hline $1 \mathrm{~A}$ & 15 & 0 \\
$\mathrm{~B}$ & 15 & 0 \\
\hline $2 \mathrm{~A}$ & 20 & 0 \\
$\mathrm{~B}$ & 20 & 0 \\
\hline 3 A & 25 & 0 \\
$\mathrm{~B}$ & 25 & 2 \\
\hline A A & 20 & 2 \\
B & 20 & 4 \\
\hline S A & 20 & 4 \\
B & 20 & 8 \\
\hline A & 20 & 8 \\
\hline B & 20 & 16 \\
\hline A & 20 & 16 \\
\hline
\end{tabular}

1) Starting $\mathrm{pH}$ was 7.5

2) Inoculum for each sample was $1 \mathrm{~mL}$ of ca. $2 \mathrm{~g} / \mathrm{L}$ slurry taken from a batch nitrification reactor operated for 3 weeks prior to initiating the assay. 


\subsection{DENITRIFYING INOCULUM PREPARATION}

Sludge was obtained from the final clarifier waste activated sludge line at the Amherst, MA POTW. This seed material was placed into three-liter working volume all-glass batch reactors. The reactors were initially started using a 10 percent inoculum of slv'ge $(300 \mathrm{ml})$ and $2,700 \mathrm{ml}$ of the basal feed described in Table 3-1. This feed was amended with $2.85 \mathrm{ml}$ of nitric acid (71 percent) and either $1.5 \mathrm{ml}$ of methanol or $6.24 \mathrm{~g}$ of sodium acetate for the methanol and acetate denitrifying cultures, respectively. The resultant mixed cultures were transferred to a new batch reactor ca. every two days using a 10 percent inoculum of the existing cultures. The cultures, developed over an approximate two month period, were then used in batch and chemostat studies.

\subsection{DENITRIFICATION BATCH ASSAYS}

The three-liter batch reactors were constructed to be gas tight. Gas production was not monitored, but a visual gauge of the rate of gas production was obtained by venting (bubbling) the gas produced through a liquid trap, which imposed a headspace pressure of approximately one inch of water column. The reactors were equipped with a septa port and a 15 gauge syringe attached to a teflon syringe valve placed to allow direct sampling the reactor aqueous phase. A port sealed with a butyl rubber septa, located at the top of the reactor, was used to sample the gaseous headspace. The reactors were maintained completely mixed using teflon coated stir bars driven by magnetic mixers (Coming). A one-quarter inch thick piece of polystyrene foam insulation was placed between the mixers and reactors to prevent heat transfer from the stir plates to the reactors. The reactors were operated at room temperature for all experimental runs (ca. $20-22^{\circ} \mathrm{C}$ ).

Batch assays were conducted with the acetate-using denitrifying cultures only. All tests were conducted using the basal media presented in Table 3-1. This was amended with $2.85 \mathrm{ml}$ of nitric acid (71 percent) and from 4.14 to $6.24 \mathrm{~g}$ of sodium acetate depending on whether the experiments were conducted using electron donor rich (excess acetate) or lean (insufficient acetate) conditions. A 10 percent inoculum of the acetate-using culture was used for each assay. After introduction of the acetate and nitrate (as nitric acid), denitrification, as evidenced by gas production, started without a significant lag period.

\subsection{DENITRIFICATION CHEMOSTAT EXPERIMENTS}

A three-liter working volume chemostat was constructed from plexiglass. As with the batch reactors, this vessel was equipped with ports to allow both gas and liquid sampling via syringes. Intemal baffles were added to the reactor to prevent formation of a vortex due to mixing. Mixing was supplied via a teflon coated stir bar driven by a magnetic mixer. 
For the experiments, the chemostat was initially filled with the contents of a batch reactor $(3,000 \mathrm{ml})$ which was immediately sealed and purged with $\mathrm{N}_{2}$ gas to remove oxygen. Forward flow to the reactor was then started. A flow rate of $1.25 \mathrm{ml} / \mathrm{min}$. was supplied from a reservoir containing the basal medium as shown in Table 3-1, amended with $\mathrm{HNO}_{3}$ to achieve a nitrate- $\mathrm{N}$ concentration of $200 \mathrm{mg} / \mathrm{l}$ and either methanol or sodium acetate at the desired starting concentrations. The hydraulic retention time at 1.25 $\mathrm{ml} / \mathrm{min}$. was 1.25 days. A masterflex tubing pump (Cole Parmer) equipped with a No. 13 pump head was used to deliver the feed to the reactor. An inverted $U$ was used on the effluent port of the chemostat to maintain a liquid seal in the reactor.

The chemostat was then generally operated for four days before initiating an experiment. The $\mathrm{pH}$ of the chemostat system equilibrated at ca. 7.8 (7.6-7.9) for all experiments except where otherwise indicated.

During the hydraulic overload experiment, the hydraulic residence time was reduced to 0.28 days by increasing the flow rate to $6.27 \mathrm{ml} / \mathrm{min}$.

\subsection{ANALYSES}

\subsection{1 $\quad \mathrm{N}_{2} \mathrm{O}$}

Nitrous Oxide was measured using a Varian 3700 gas chromatograph equipped with an electron capture detector using the method of Kaspar and Tiedje (1980). Based on the seven sequential injections of a 102 ppm $\mathrm{N}_{2}$ standard (Scientic Gas Co.) this method had a relative standard deviation of 0.21 percent.

\section{$3.7 .2 \underline{\mathrm{H}_{2}}$}

Hydrogen was analyzed chromatographically using a mercury oxide catalyst and photospectrometric detector (Hickey et al. 1987).

\subsubsection{Ammonium}

Ammonium was analyzed via the specific ion electrode method (Standard Methods, 16th Edition Method $417 \mathrm{~F})$.

\subsubsection{Dissolved Oxygen}

Dissolved oxygen was analyzed according to the Membrane Electrode Method (Standard Methods, 16th Edition, Section 421F). 


\subsubsection{Nitrate}

Nitrate was analyzed using several modifications of the Cadmium Reduction Method (Standard Methods, 16th Edition, Section 418C). Nitrite was analyzed according to Standard Methods, 16th Edition (Section 419).

\subsubsection{Solids, $\mathrm{pH}$ and COD}

Suspended solids were determined according to Section 209C; pH according to Section 423; and COD according to Section 508C, respectively, of Standard Methods, 16th Edition. 


\section{SECTION 4}

\section{RESULTS OF NITRIFICATION EXPERIMENTS}

\subsection{INTRODUCTION}

Nitrification is the conversion of ammonium to nitrate. This is a two-step process that involves two separate groups of bacteria. The first group converts ammonium to nitrite, while the second group converts the nitrite to nitrate. It is during the first step in the overall nitrification process that trace gases nitric (NO) and nitrous $\left(\mathrm{N}_{2} \mathrm{O}\right)$ oxides are formed. The energetics of ammonium oxidation to nitrite is presented in Table 4-1. As can be seen, considerably more energy is available to the organisms for conversion of ammonium to nitrous oxide $\left(\mathrm{N}_{2} \mathrm{O}\right)$ than the subsequent conversion of $\mathrm{N}_{2} \mathrm{O}$ to nitric oxide (NO) and nitrite. The nitrite formed is subsequently oxidized by a second group of organisms to nitrate $\left(\mathrm{NO}_{3}{ }^{-}\right)$.

Table 4-1. Thermodynamics of Ammonium Oxidation*

\begin{tabular}{|c|c|}
\hline & $\begin{array}{r}\text { Free Energy } \\
\Delta \mathrm{G}^{\circ} \\
(\mathrm{KJ} / \mathrm{mole}) \\
\end{array}$ \\
\hline \multicolumn{2}{|l|}{ Overall Reaction } \\
\hline $\mathrm{NH}_{4}^{+}+3 / 2 \mathrm{O}_{2} \quad \cdots>\mathrm{NO}_{2}^{-}+2 \mathrm{H}^{+}+\mathrm{H}_{2} \mathrm{O}$ & -274.75 \\
\hline \multicolumn{2}{|l|}{ Individual Steps } \\
\hline$\cdots 1 / 2 \mathrm{~N}_{2} \mathrm{O}+\mathrm{H}^{+}+3 / 2 \mathrm{H}_{2} \mathrm{O}$ & -212.09 \\
\hline $1 / 2 \mathrm{~N}_{2} \mathrm{O}+1 / 4 \mathrm{O}_{2}$ & -17.62 \\
\hline $\mathrm{NO}+1 / 4 \mathrm{O}_{2}+1 / 2 \mathrm{H}_{2} \mathrm{O}$ & -45.04 \\
\hline
\end{tabular}

NOTE:

a) After Thauer et. a‥ 1977

\subsection{BATCH SERUM BOTTLE TESTING}

A batch assay was conducted using different starting concentrations of ammonium and nitrite under conditions that would allow close to equilibrium to be attained between the liquid and gaseous phases (i.e., low biomass concentration and rapid mixing). Results of analysis of the different samples after 48 hours 
of incubation are presented in Table 4-2. As can be seen, increasing nitrite concentrations resulted in increased $\mathrm{N}_{2} \mathrm{O}$ concentrations in the gaseous headspace. When nitrite concentrations ranged from 1.05 to $1.25 \mathrm{mg} \mathrm{N} / \mathrm{L}, \mathrm{N}_{2} \mathrm{O}$ concentrations ranged from 3.7 to $5.2 \mathrm{ppm}$. An increase in nitrite- $\mathrm{N}$ to $2.1 \mathrm{mg} / \mathrm{L}$ resulted in a slight increase in $\mathrm{N}_{2} \mathrm{O}$ to 6.3 . At $11.3 \mathrm{mg} \mathrm{NO}_{2}{ }^{-} \mathrm{N} / \mathrm{L}$, the $\mathrm{N}_{2} \mathrm{O}$ level reached $21.3 \mathrm{ppm}$. It appeared that gaseous $\mathrm{N}_{2} \mathrm{O}$ concentrations did correlate quite well with $\mathrm{NO}_{2}^{-}-\mathrm{N}$ levels.

Table 4-2. Results of Batch Serum Bottle Testing for $\mathrm{N}_{2} \mathrm{O}$ Production During Nitrification

\begin{tabular}{|c|c|c|c|}
\hline $\begin{array}{c}\mathrm{NH}_{4}^{+}-\mathrm{N} \\
(\mathrm{mg} / \mathrm{L})\end{array}$ & $\begin{array}{c}\mathrm{NO}_{2}^{-}-\mathrm{N} \\
(\mathrm{mg} / \mathrm{L})\end{array}$ & $\begin{array}{c}\mathrm{N}_{2} \mathrm{O} \\
(\mathrm{ppm})\end{array}$ & $\begin{array}{c}\mathrm{pH} \\
(\mathrm{s.u} .)\end{array}$ \\
\hline 12.5 & 1.25 & 3.7 & 7.44 \\
17.9 & 1.15 & 4.3 & 7.43 \\
20.8 & 1.20 & 3.8 & 7.44 \\
16.7 & 1.05 & 5.2 & 7.44 \\
17.7 & 2.10 & 6.3 & 7.47 \\
14.8 & 7.0 & 7.1 & 7.53 \\
15.3 & 11.3 & 21.3 & 7.55 \\
\hline
\end{tabular}

\subsection{THERMODYNAMIC ANALYSIS}

An attempt was made to determine if a thermodynamic based relationship, similar to the one previously developed for $\mathrm{CO}$ in anaerobic systems (Hickey and Switzenbaum, 1990), could be developed for $\mathrm{N}_{2} \mathrm{O}$ in nitrification systems. The free energies were calculated according to equations 4-1 and 4-2. Results of calculating the available free energies based on the pH, D.O., equal to saturation, $9.2 \mathrm{mg} / \mathrm{L}$ for all samples, and nitrogen species concentrations are presented in Table 4-3.

The free energy associated with the oxidation of $\mathrm{N}_{2} \mathrm{O}$ to nitrite was observed to be consistently 25 percent of the total free energy available for conversion of ammonium to nitrite.

$$
\begin{aligned}
& \Delta \mathrm{G}_{1}=-172.2+5.7 \log \frac{\left[\mathrm{N}_{2} \mathrm{O}\right]^{1 / 2}}{\left[\mathrm{NH}_{4}^{+}\right]\left[\mathrm{O}_{2}\right]}-5.7 \mathrm{pH} \\
& \Delta \mathrm{G}_{2}=-22.8+5.7 \log \frac{\left[\mathrm{NO}_{2}\right]}{\left[\mathrm{N}_{2} \mathrm{O}^{1 / 2}\left[\mathrm{O}_{2}\right]^{1 / 2}\right.}-5.7 \mathrm{pH}
\end{aligned}
$$


Table 4-3. Free Energy Calculations for Serum Bottle Nitrification Assay

\begin{tabular}{||c|c|c|c|c||}
\hline \multirow{3}{*}{$\begin{array}{c}\text { Sample } \\
\text { No. }\end{array}$} & \multicolumn{4}{|c||}{ Free Energy (KJ/Mole Reaction) } \\
\cline { 2 - 5 } & $\mathrm{NH}_{4}^{+}->\mathrm{NO}_{2}{ }_{\mathrm{T}}$ & $\mathrm{NH}_{4}^{+}-\rightarrow 1 / 2 \mathrm{~N}_{2} \mathrm{O}$ & $\begin{array}{c}\Delta \mathrm{G}_{2} \\
1 / 2 \mathrm{~N}_{2} \mathrm{O} \rightarrow \mathrm{NO}_{2}{ }^{-}\end{array}$ & $\Delta \mathrm{G}_{2} / \Delta \mathrm{G}_{\mathrm{T}}$ \\
\hline 1 & -279.7 & -208.8 & -70.9 & 0.253 \\
\hline 2 & -280.8 & -209.5 & -71.3 & 0.254 \\
\hline 3 & -280.9 & -210.0 & -70.9 & 0.252 \\
\hline 4 & -280.7 & -209.1 & -71.6 & 0.255 \\
\hline 5 & -279.6 & -209.2 & -70.4 & 0.252 \\
\hline 6 & -276.9 & -209.0 & -67.9 & 0.245 \\
\hline 7 & -276.0 & -207.8 & -68.2 & 0.247 \\
\hline
\end{tabular}

Substituting $3 \Delta \mathrm{G}_{2}=\Delta \mathrm{G}_{1}$ and $4 \Delta \mathrm{G}_{2}=\Delta \mathrm{G}_{\mathrm{T}}$, equations 4-1 and 4-2 can be manipulated to yield the following equation:

$$
\mathrm{pH}-9.1=1 / 2 \log \frac{\left[\mathrm{NO}_{2}^{-}\right]^{3}\left[\mathrm{NH}_{2}^{+}\right]}{\left[\mathrm{O}_{2}\right]\left[\mathrm{N}_{2} \mathrm{O}\right]^{1 / 2}}
$$

This equation can be used to determine the effect of changes in concentrations of other variables such as $\mathrm{O}_{2}, \mathrm{NO}_{2}^{-}$and $\mathrm{NH}_{4}^{+}$levels on $\mathrm{N}_{2} \mathrm{O}$ concentrations. For example, as the dissolved oxygen concentration is increased, $\mathrm{N}_{2} \mathrm{O}$ concentrations will decrease according to the $1 / 2$ power, assuming all the other variables remain constant. These effects are summarized in Table 4-4.

A sensitivity analysis was performed using $\mathrm{N}_{2} \mathrm{O}$ concentrations of 1,10 and 100 times the measured concentration to determine the impact on the actual free energies available. This was done to determine whether a more elaborate predictive equation could be made for relating $\mathrm{NH}_{4}^{+}$concentrations with $\mathrm{N}_{2} \mathrm{O}$ levels. Results are presented in Table 4-5. As can be seen, a two order of magnitude change in $\mathrm{N}_{2} \mathrm{O}$ results in a minor change in calculated available free energy values. A sensitive predictive equation, therefore, cannot be constructed. Nevertheless, the results generated are quite useful. For example, if a nitrification system is maintained at constant D.O. and pH levels, rises in either $\mathrm{NH}_{4}^{+}$or $\mathrm{NO}_{2}^{-}$would cause the $\mathrm{N}_{2} \mathrm{O}$ levels to rise. Since accumulation of either $\mathrm{NH}_{4}^{+}$or $\mathrm{NO}_{2}^{-}$indicates a decrease in process efficiency, $\mathrm{N}_{2} \mathrm{O}$ has the potential to be used as a reliable indicator of process upset. 
Table 4-4. Effect of Changes in Other System Variables on Equilibrium $\mathrm{N}_{2} \mathrm{O}$ Concentration

\begin{tabular}{|c|c|c|c|}
\hline Variable & Change & $\mathrm{N}_{2} \mathrm{O}$ Change & Power \\
\hline $\mathrm{O}_{2}$ & $\star$ & $\bullet$ & $1 / 2$ \\
$\mathrm{NO}_{2}{ }^{-}$ & $\star$ & $\star$ & $3 / 2$ \\
$\mathrm{NH}_{4}{ }^{+}$ & $\star$ & $\star$ & $1 / 2$ \\
\hline
\end{tabular}

As is evident in reviewing Table $4-4, \mathrm{~N}_{2} \mathrm{O}$ response is especially sensitive to changes in nitrite concentrations. Since nitrite normally accumulates when there are problems. This suggests that monitoring nitrification processes for $\mathrm{N}_{2} \mathrm{O}$ may hold promise. 


\begin{tabular}{|c|c|c|}
\hline & 8 & 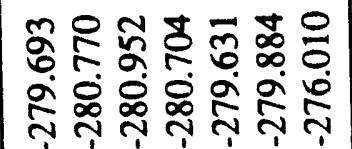 \\
\hline \multirow{3}{*}{ ס્ } & 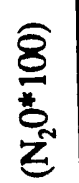 & 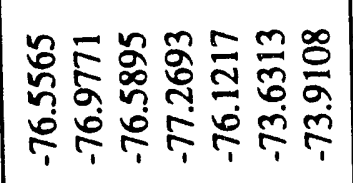 \\
\hline & 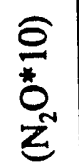 & 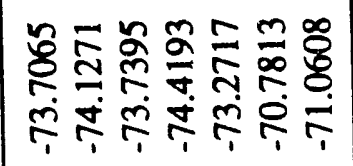 \\
\hline & o & 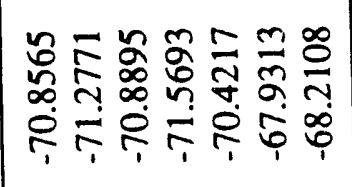 \\
\hline \multirow{3}{*}{ 5 } & $\begin{array}{l}\bar{\delta} \\
\text { o } \\
\text { ż } \\
z\end{array}$ & 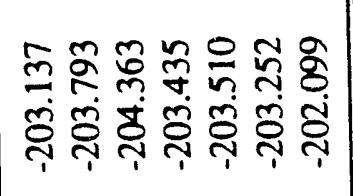 \\
\hline & $\begin{array}{l}\frac{\partial}{\partial} \\
\text { क् } \\
z\end{array}$ & 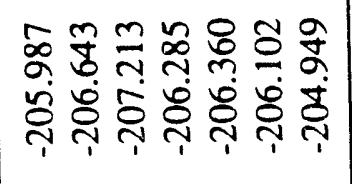 \\
\hline & o & 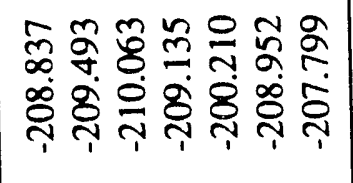 \\
\hline & 宫苾 & 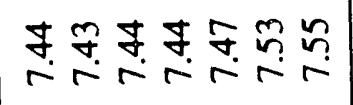 \\
\hline & 을 & $\dot{m} \stackrel{m}{\rightarrow} \stackrel{\infty}{m} \underset{n}{n} \dot{b}=\frac{m}{\sim}$ \\
\hline & 官曷 & $\begin{array}{l}80 \\
0\end{array}$ \\
\hline & 芝罗 & 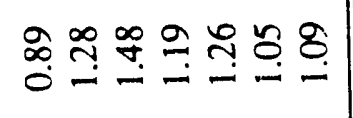 \\
\hline
\end{tabular}




\section{SECTION 5}

\section{RESULTS OF DENITRIFICATION STUDIES}

The response of both batch and chemostat systems using different electron donors (acetate and methanol) for electron donor excess, nitrate excess conditions, and an organic overload was examined during this study. The response of $\mathrm{N}_{2} \mathrm{O}$ and $\mathrm{H}_{2}$ (during later assays) to these different conditions is described below.

\subsection{BATCH ASSAY USING ACETATE AS THE ELECTRON DONOR}

Batch assays using acetate as the electron donor were conducted in two-liter mixed, sealed vessels.

For the initial assay, acetate was added at less than the stoichiometric amounts required to permit the reduction of all the nitrate added. The results shown in Figure 5-1 indicate that during this experimental run, the conversion si nitrate to nitrite was quite rapid; essentially complete conversion was observed within seven hours. Nitrite increased almost linearly during this period. Slight accumulation of $\mathrm{N}_{2} \mathrm{O}$ (from ca. 1.0 to $3.0 \mathrm{ppm}(\mathrm{v} / \mathrm{v})$ ) was concurrently observed. After seven hours incubation, the concentration of nitrite began to decrease with a concurrent increase in $\mathrm{N}_{2} \mathrm{O}$, which reached a peak level of ca. $86 \mathrm{ppm}$ after 840 minutes of incubation (nitrite $=3.570 \mathrm{ug} \mathrm{N} / \mathrm{L}$ ). After one complete day, the nitrite level had declined to 1.570 ug $\mathrm{N} / \mathrm{L}$ while $\mathrm{N}_{2} \mathrm{O}$ had decreased slightly to $72.2 \mathrm{ppm}$. The lack of sufficient acetate obviously slowed the overall rates of the various denitrification reactions. The final $\mathrm{pH}$ was observed to be 8.8. Whether this in pan accounted for denitrification occurring as a sequence of reactions rather than concurrently, is unknown.

A second assay (Run 2) was conducted using an acctate to nitrite ratio of $4.5 / 1$, or more than sufficient to completely reduce all of the added nitrate. During the course of this assay, nitrite accumulatio: was slight, peaking at 735 ug N/L after 150 minutes (Table 5-1). The concentration of $\mathrm{N}_{2} \mathrm{O}$ increased from an initial value of zero to a high of $3.1 \mathrm{ppm}$ and then decreased, thereafter, back to less than $0.5 \mathrm{ppm}$.

A third run, at a lower $\mathrm{pH}$, was conducted with an added acetate/nitrate ratio of 3.5 . However, the innculum also had some nitrate. There was likely insufficient acetate for this run as during run 1. Just as observed during run 1, denitrification occurred as a series of sequential steps (Table 5-2). As nitrate was reduced, nitrite accumulated and as nitrite was reduced, $\mathrm{N}_{2} \mathrm{O}$ accumulated. The final $\mathrm{pH}$ was 7.8 for this run. 

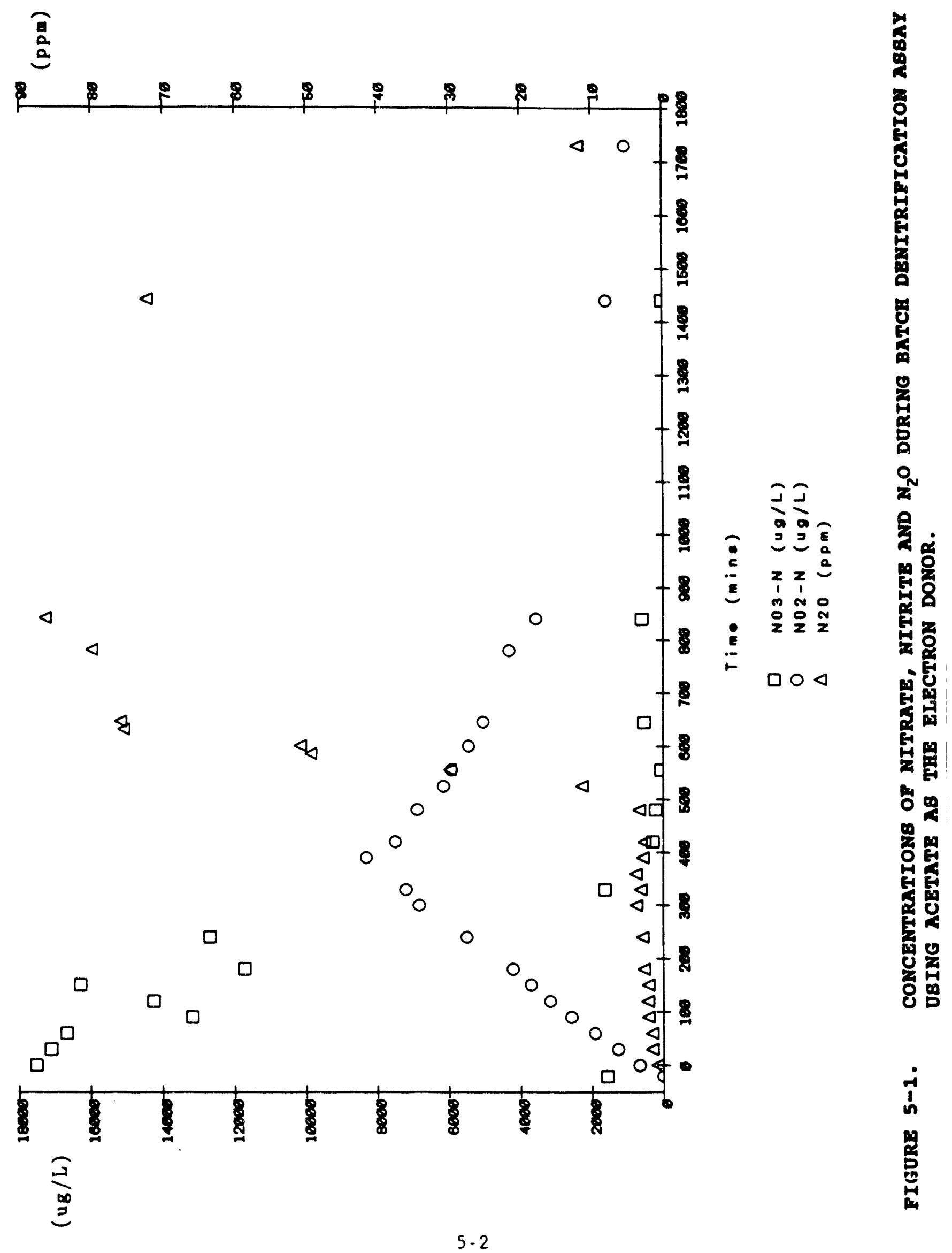
Table 5-1. Concentrations of Nitrogen Species During Batch Denitrification Assay (Run 2) Using Acetate as the Electron Donor

\begin{tabular}{|c|c|c|c|}
\hline $\begin{array}{c}\text { Time } \\
\text { (min.) }\end{array}$ & $\begin{array}{c}\mathrm{NO}_{3}-\mathrm{N} \\
(\mathrm{ug} / \mathrm{L})\end{array}$ & $\begin{array}{c}\mathrm{NO}_{2}-\mathrm{N} \\
(\mathrm{ug} / \mathrm{L})\end{array}$ & $\begin{array}{c}\mathrm{N}_{2} \mathrm{O} \\
(\mathrm{ppm})\end{array}$ \\
\hline-10 & 4210 & 25 & -- \\
0 & 19120 & 410 & 0 \\
30 & 17000 & 220 & 1.22 \\
60 & -- & -- & 1.55 \\
90 & 11360 & 500 & 1.95 \\
125 & -- & -- & 2.40 \\
150 & 7560 & 735 & 3.10 \\
240 & 4590 & 25 & 2.02 \\
510 & -- & -- & 0.68 \\
\hline
\end{tabular}

A second assay under acetate rich conditions (acetate/nitrate ratio $=4.5$ ) was conducted (Run 4). The inoculum added was from a batch reactor also supplied with excess acetate. Just as observed during run 2, the reduction of nitrate was accompanied with concurrent increases in nitrite and $\mathrm{N}_{2} \mathrm{O}$ (Table 5-3). After the nitrate and nitrite concentrations decreased to low concentrations, the $\mathrm{N}_{2} \mathrm{O}$ level also declined. By 300 minutes the $\mathrm{N}_{2} \mathrm{O}$ was observed to be below the detection limit of ca. $0.3 \mathrm{ppm}$.

The concentration of $\mathrm{H}_{2}$ in the gas phase was also tracked during run 4. rjuring the initial three hours, the $\mathrm{H}_{2}$ concentration remained approximately constant varying between 22 and $38 \mathrm{ppm}$. After essentially all of the nitrate, nitrite and $\mathrm{N}_{2} \mathrm{O}$ were consumed, however, the $\mathrm{H}_{2}$ concentration rose rapidly peaking at ca. $145 \mathrm{ppm}$ after 390 minutes. At the 400 minute mark a pulse of nitrate, sufficient to increase the reactor concentration to $10 \mathrm{mg} / \mathrm{L}$, was applied to the system. The $\mathrm{H}_{2}$ concentration began to precipitously decrease reaching $28 \mathrm{ppm}$ by the 510 minute mark (Figure 5-2). This indicates that $\mathrm{H}_{2}$ was consumed and could be used for denitrification by the acetate using mixed culture developed.

A final assay (Run 5) was conducted using an acetate/nitrate ratio of 3.6. During this run, nitrite accumulated as nitrate was reduced. $\mathrm{N}_{2} \mathrm{O}$ also increased rapidly during the initial 150 minutes. When nitrite reduction dramatically slowed, presumably due to depletion of the acetate, $\mathrm{N}_{2} \mathrm{O}$ concentrations increased rapidly reaching a peak of $280 \mathrm{ppm}$ after 330 minutes (Table 5-4). A pulse of acetate sufficient to add $30 \mathrm{mg} / \mathrm{L}$ to the reactor was added at this time. The concentration of $\mathrm{N}_{2} \mathrm{O}$ decreased to ca. $146 \mathrm{ppm}$ during the ensuing 30 minutes and nitrite was completely consumed. The $\mathrm{N}_{2} \mathrm{O}$ level continued to decrease reaching $9 \mathrm{ppm}$ by 450 minutes. 


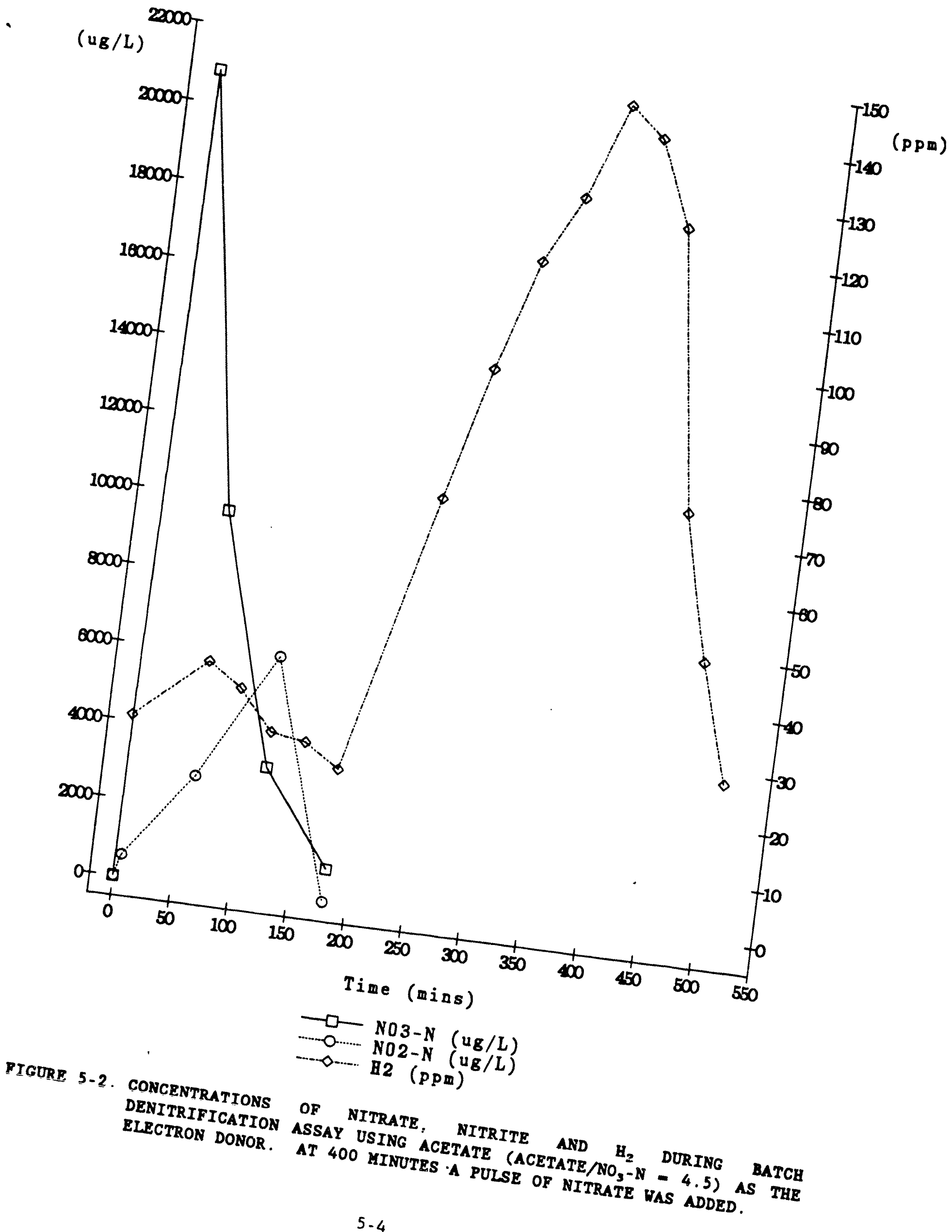


Table 5-2. Concentration of Nitrogen Species During Batch Denitrification Assay (Run 3) Using Acetate as the Electron Donor

\begin{tabular}{|c|c|c|c|}
\hline $\begin{array}{c}\text { Time } \\
\text { (min.) }\end{array}$ & $\begin{array}{c}\mathrm{NO}_{3}-\mathrm{N} \\
(\mathrm{ug} / \mathrm{L})\end{array}$ & $\begin{array}{c}\mathrm{NO}_{2}-\mathrm{N} \\
(\mathrm{ug} / \mathrm{L})\end{array}$ & $\begin{array}{c}\mathrm{N}_{2} \mathrm{O} \\
(\mathrm{ppm})\end{array}$ \\
\hline 0 & 9390 & 0 & $\mathrm{BDL}^{*}$ \\
40 & -- & - & 1.1 \\
60 & -- & 0 & 2.3 \\
90 & -- & - & 4.4 \\
120 & 22200 & 0 & 4.0 \\
150 & -- & - & 4.0 \\
180 & 20740 & 260 & - \\
210 & -- & - & 7.4 \\
240 & 18100 & 790 & 7.5 \\
270 & -- & -- & 9.3 \\
300 & 14830 & 1400 & 9.4 \\
360 & 4470 & 2230 & 9.4 \\
390 & -- & -- & 9.3 \\
420 & 9300 & 3090 & 8.4 \\
480 & -- & 4450 & 8.2 \\
510 & -- & -- &.- \\
540 & 2420 & 5250 &.- .2 \\
570 & -- & -- & 60.6 \\
600 & 4620 & 5250 & 75.5 \\
625 & -- & -- & \\
\hline
\end{tabular}

* BDL - Below Detection Limit

Table 5-3. Concentration of Nitrogen Species During Batch Denitrification Assay (Run 4) Using Acetate as the Electron Donor

\begin{tabular}{|c|c|c|c|}
\hline $\begin{array}{l}\text { Time } \\
\text { (nin) }\end{array}$ & $\begin{array}{c}\mathrm{NO}_{3}-\mathrm{N} \\
(\mathrm{ug} / \mathrm{L})\end{array}$ & $\begin{array}{l}\mathrm{NO}_{2}-\mathrm{N} \\
\text { (ug/L) }\end{array}$ & $\begin{array}{c}\mathrm{N}_{2} \mathrm{O} \\
(\mathrm{ppm})\end{array}$ \\
\hline $0^{\circ}$ & $\mathrm{TR}^{*}$ & 0 & $\mathrm{BDL}^{* *}$ \\
\hline 5 & 20,830 & 560 & -. \\
\hline 30 & -. & -. & -- \\
\hline 60 & 9,630 & 2,780 & 4.9 \\
\hline 90 & -- & -. & 5.5 \\
\hline 120 & 3,230 & 6,090 & 7.7 \\
\hline 150 & -- & -- & 9.0 \\
\hline 180 & 840 & 0 & 6.0 \\
\hline 240 & -. & -. & 1.6 \\
\hline 270 & -. & $\ldots$ & 0.6 \\
\hline 300 & -. & .. & $\mathrm{BDL}^{* *}$ \\
\hline
\end{tabular}

* TR - Trace

** BDL - Below Detection Limit 
Table 5-4. Concentration of Nitrogen Species During Batch Denitrification Assay (Run 5) Using Acetate as the Electron Donor

\begin{tabular}{|c|c|c|c|}
\hline $\begin{array}{c}\text { Time } \\
(\min )\end{array}$ & $\begin{array}{c}\mathrm{NO}_{3}-\mathrm{N} \\
(\mathrm{ug} / \mathrm{L})\end{array}$ & $\begin{array}{c}\mathrm{NO}_{2}-\mathrm{N} \\
(\mathrm{ug} / \mathrm{L})\end{array}$ & $\begin{array}{c}\mathrm{N}_{2} \mathrm{O} \\
(\mathrm{ppm})\end{array}$ \\
\hline-15 & 3570 & 0 &.- \\
0 & 20250 & 120 & 0.0 \\
30 & -- & -- & 2.1 \\
60 & 8970 & 2690 & 4.9 \\
95 & -- & -- & 6.7 \\
120 & 2580 & 6195 & 24.7 \\
150 & 2360 & 6460 & 47.8 \\
180 & 2600 & 5510 & -- \\
210 & -- & -- & 50.6 \\
240 & 1020 & 3750 & 56.8 \\
270 & -- & -- & 115.0 \\
300 & 0 & 3750 & 193.6 \\
330 & -- & 2470 & 280.5 \\
360 & 270 & 50 & 146.6 \\
390 & -- & -- & 55.5 \\
420 & -- & -- & -.0 \\
450 & -- & & 9.0 \\
\hline
\end{tabular}

The concentration of hydrogen, which was below the $1.0 \mathrm{ppm}$ detection limit during the initial 330 minutes of the assay, increased to $19 \mathrm{ppm}$ by 390 minutes and $52 \mathrm{ppm}$ by 450 minutes. As before, when excess acetate was present the hydrogen concentration rose markedly.

A comparison of the results from Runs 4 and 5 is graphically illustrated in Figure 5-3. During both runs the starting $\mathrm{pH}$, and nitrate levels were the same (ca. 7.8 and $20,000 \mathrm{ug} /$, respectively). Whether excess acetate was supplied (filled triangles) or not (open triangles), nitrate concentrations decreased rapidly at approximately the same rate with a concurrent increase in nitrite during the initial 120 minutes. There was little accumulation of $\mathrm{N}_{2} \mathrm{O}$ in the acetate rich system while $\mathrm{N}_{2} \mathrm{O}$ accumulaied to ca. $25 \mathrm{ppm}$ in the acetate lean (excess nitrate) system. In the acetate lean system, the subsequent conversion of nitrite was slow and $\mathrm{N}_{2} \mathrm{O}$ continued to rise. Some residual nitrate was also observed. This was not observed for the acetate rich system in which accumulated nitrite was rapidly reduced without significant accumulation of $\mathrm{N}_{2} \mathrm{O}$. From these results it appears that $\mathrm{N}_{2} \mathrm{O}$ accumulation is a good indicator of having insufficient electron donor, in this case acetate, present. Addition of more acetate to the acetate lean system resulted in the rapid reduction of nitrite and $\mathrm{N}_{2} \mathrm{O}$. 

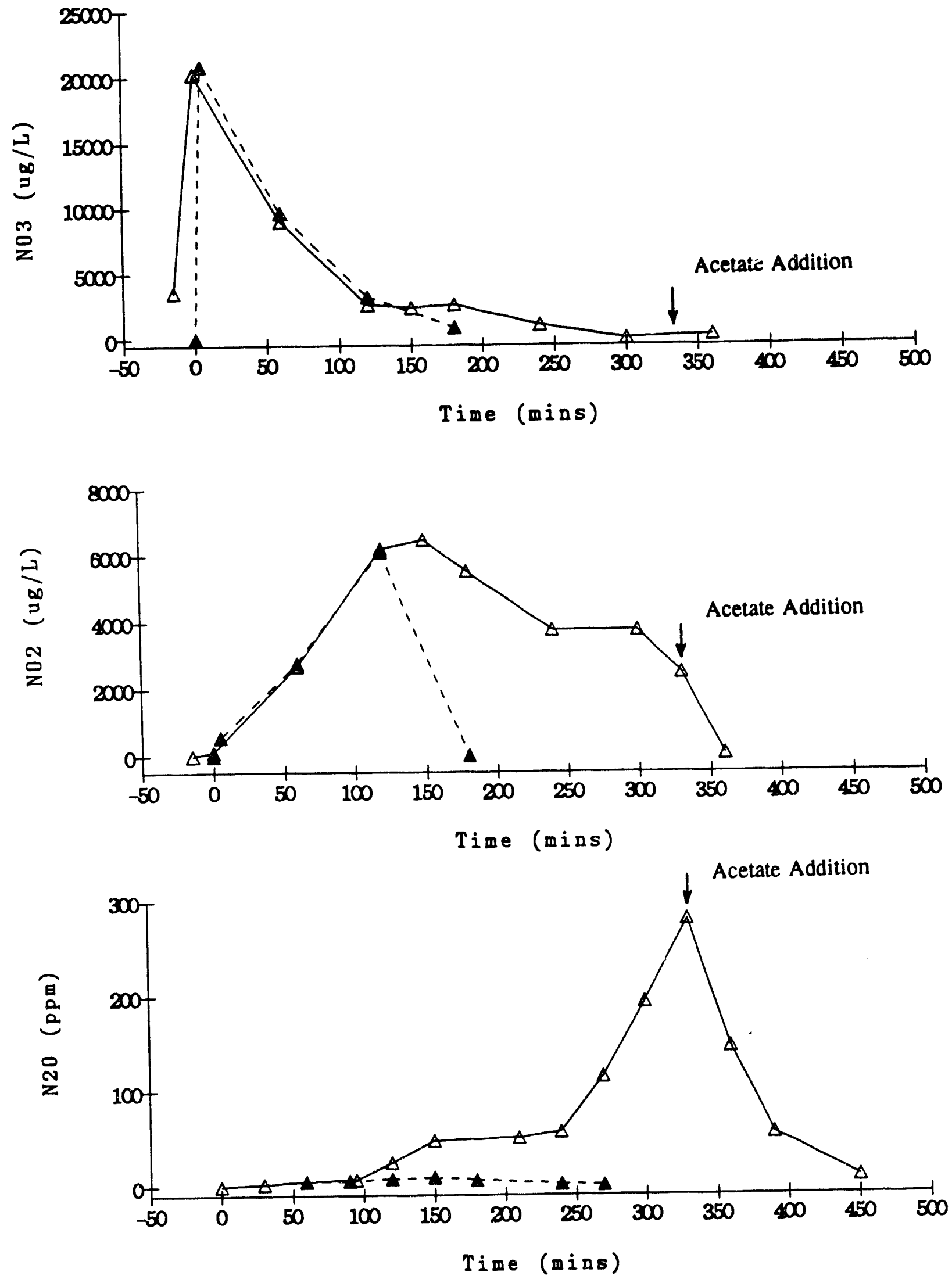

FIGURE 5-3. COMPARISON OF NITRATE CONVERSION, NITRITE AND NITROUS OXIDE ACCUNULATION PATTERNS DURING EXPERIRENTAL RUN 4 (EXCESS ACETATE FILIED TRIANGLES) AND RUN 5 (EXCESS NITRATE - OPEN TRIANGLES). 
Another observation was that $\mathrm{N}_{2} \mathrm{O}$ accumulation appeared to be related to nitrite and not nitrate conversion. To test this, an experimental run was conducted using nitrite in the feed in place of nitrate.

An acetate to nitrite ratio of 7.5 was used for Run 6. As shown in Table 5-5, with sufficient acetate present, nitrite was reduced rapidly and little $\mathrm{N}_{2} \mathrm{O}$ accurnulation was observed. It appears that $\mathrm{N}_{2} \mathrm{O}$ accumulation is related to nitrite reduction only when an insufficient amount of electron donor (acetate) is present.

Table 5-5. Concentrations of Nitrite and $\mathrm{N}_{2} \mathrm{O}$ During Reduction of Nitrite Using Acetate as an Electron Donor (Run 6)

\begin{tabular}{|c|c|c|}
\hline $\begin{array}{c}\text { Time } \\
\text { (mins) }\end{array}$ & $\begin{array}{c}\mathrm{NO}_{2}-\mathrm{N} \\
(\mathrm{ug} / \mathrm{L})\end{array}$ & $\begin{array}{c}\mathrm{N}_{2} \mathrm{O} \\
(\mathrm{ppm})\end{array}$ \\
\hline 0 & $\mathrm{BDL}^{*}$ & $\mathrm{BDL}^{*}$ \\
20 & 5300 & - \\
60 & 3650 & 0.7 \\
90 & -- & 0.9 \\
120 & 3080 & 1.4 \\
180 & 1760 & 1.3 \\
240 & 870 & 1.2 \\
270 & 230 & 1.4 \\
300 & $\mathrm{BDL}$ & 0.0 \\
\hline
\end{tabular}

* BDL - Below Detection Limit

\subsection{CHEMOSTAT STUDIES USING ACETATE AS THE ELECTRON DONOR}

The feed to an acetate-fed chemostat (acetate/nitrate ratio $=4.5$ ) was suddenly switched to a nitrate only feed $(t=0)$. At this time, no nitrate, nitrite or $\mathrm{N}_{2} \mathrm{O}$ were observed in the chemostat aqueous or gaseous phases. The concentration of $\mathrm{H}_{2}$ was $40 \mathrm{ppm}$ whicis, based on the batch assay results, indicates excess acetate. Unknown interferences in the COD test prevented getting clear information on the acetate concentration.

After switching to the nitrate only feed, nitrite, but not nitrate, began to immediately accumulate. The concentration of $\mathrm{H}_{2}$ began to decline and $\mathrm{N}_{2} \mathrm{O}$ began to increase. Within one hour, the $\mathrm{H}_{2}$ level decreased to less than $10 \mathrm{ppm}$ while $\mathrm{N}_{2} \mathrm{O}$ increased to more than $100 \mathrm{ppm}$. Nitrate also began to be detected in the effluent (ca. $2000 \mathrm{ug} / \mathrm{L}$ ) during this period. The gaseous $\mathrm{CO}_{2}$ level remained at ca. 6.0 percent throughout 
the entire experimental run. Within three hours the $\mathrm{N}_{2} \mathrm{O}$ concentration exceeded $1000 \mathrm{ppm}$ and nitrite levels had increased to ca. 47,000 ug/L as shown in Figure 5-4.

A second experimental run was conducted using the same scenario. The chemostat had been supplied with a feed containing excess acetate ovemight which produced a starting $\mathrm{H}_{2}$ concentration of ca. $76 \mathrm{ppm}$. A $200 \mathrm{mg} \mathrm{N} / \mathrm{L}$ nitrate only feed was started at time zero. During the initial four hours, no nitrate, nitrite or $\mathrm{N}_{2} \mathrm{O}$ accumulation was observed, although the $\mathrm{H}_{2}$ concentration decreased to ca. $40 \mathrm{ppm}$. To hasten results, the strength of the nitrate feed was increased from 200 to $500 \mathrm{mg} / \mathrm{L}$ after 270 minutes. Within one hour, nitrate and nitrite accumulated to 2000 and 1000 ug N/L, respectively. The $\mathrm{N}_{2} \mathrm{O}$ concentration initially increased to ca. $5 \mathrm{ppm}$ and after nitrate feed change rapidly increased during the next 30 minutes to more than $100 \mathrm{ppm}$ as the nitrate concentration increased to $5000 \mathrm{ug} \mathrm{N} / \mathrm{L}$ (Figure 5-5). The $\mathrm{H}_{2}$ level continued to decline. Surprisingly, nitrite did not accumulate rapidly as it had during the prior chemostat and batch studies. The reason for this is unknown.

\subsection{CHEMOSTAT STUDIES USING METHANOL AS THE ELECTRON DONOR FOR DENITRIFICATION}

A methanol-fed chemostat was started and brought to steady-state at slightly deficient electron donor $(\mathrm{MeOH})$ feed levels. This resulted in a starting concentration of nitrate, nitrite and $\mathrm{N}_{2} \mathrm{O}$ of 3,470 ug N/L, 80 ug N/L, and $8.2 \mathrm{ppm}$, respectively. A feed with a sufficient methanol/nitrate ratio (3/1) was started (200 $\mathrm{mg} \mathrm{NO}_{3}^{-}-\mathrm{N} / \mathrm{L}$ ) at time zero.

Nitrate and $\mathrm{N}_{2} \mathrm{O}$ concentrations immediately began to decrease as seen in Table 5-6. Within two hours effluent nitrate and $\mathrm{N}_{2} \mathrm{O}$ concentrations were observed to be $540 \mathrm{ugN} / \mathrm{L}$ and $4.8 \mathrm{ppm}$, respectively. During the next hour, the concentrations of both decreased to or below their respective detection limits.

A second experimental run was conducted where a system receiving a sufficient amount of methanol was switched to a nitrate only $(200 \mathrm{mg} / \mathrm{L})$ feed at time zero. After 2 hours, nitrate began to accumulate and $\mathrm{N}_{2} \mathrm{O}$ simultaneously increased. Within 30 minutes the $\mathrm{N}_{2} \mathrm{O}$ level was above 7.0 ppm while nitrate had risen to $2000 \mathrm{ug} / \mathrm{L}$ as shown in Figure 5-6. Nitrate concentration continued to increase during the ensuing several hours while $\mathrm{N}_{2} \mathrm{O}$ leveled off at ca. $12 \mathrm{ppm}$ after three hours. Hydrogen concentrations decreased slightly during the entire experiment. 

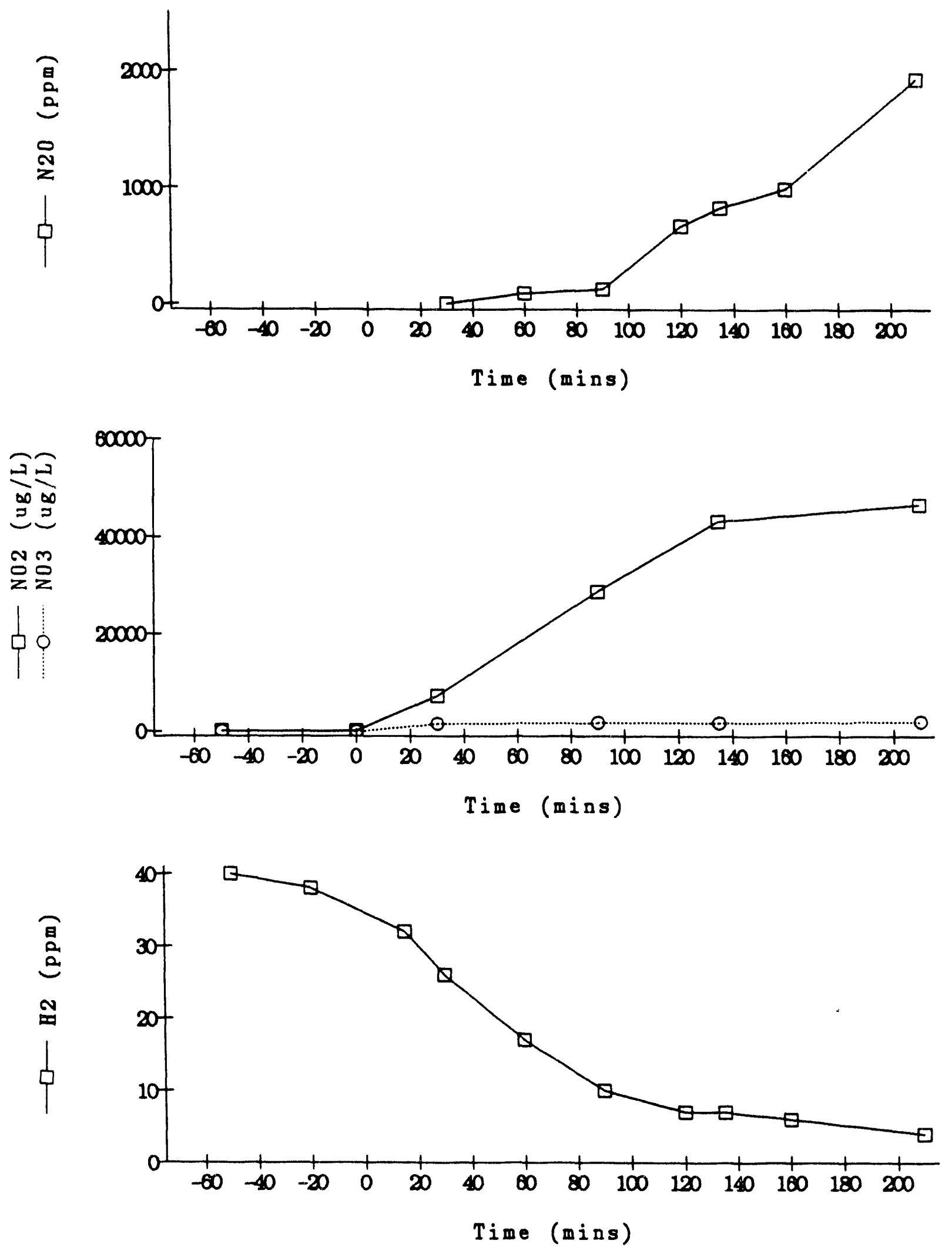

FIGURE 5-4. CONCENTRATIONS OF NITRATE, NITRITE, $\mathrm{N}_{2} \mathrm{O}$ AND $\mathrm{H}_{2}$ DURING DENITRIFICATION OSING ACETATE AS THE ELECTRON DONOR (ACETATE/NO, $-N=4.5$ ) IN A CHEMOSTAT. AT TIRE $t=0$, THE FEED WAS SWITCHED TO $200 \mathrm{mg} / \mathrm{L}$ NITRATE ONLY. 

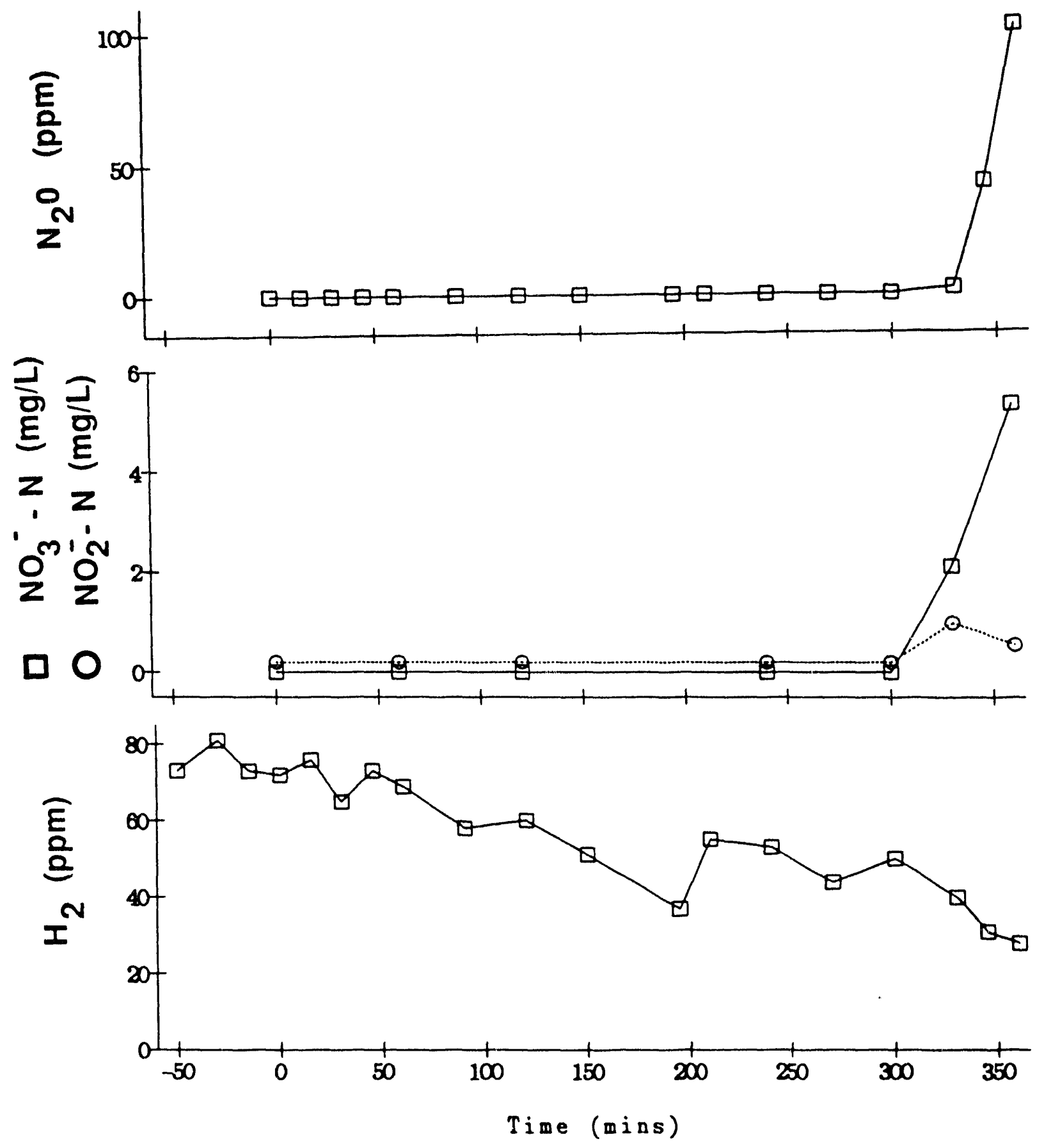

FIGURE 5-5. CONCENTRATIONS OF NITRATE, NITRITE, $\mathrm{N}_{2} \mathrm{O}$ AND $\mathrm{H}_{2}$ IN AN ACETATE-FED (EXCESS ACETATE) DENITRIFYING CHEMOSTAT. AT TIME $t=0$, FEED WAS SWITCHED TO A $200 \mathrm{mg} \mathrm{NO}_{3}-\mathrm{N} / \mathrm{L}$ ONLY. AT TIME $t=270$ MINUTES, THE FEED WAS CHANGED TO $500 \mathrm{mg} \mathrm{NO}_{3}-\mathrm{N} / \mathrm{L}$. 
Table 5-6. Summary of Chemostat Experiment Using Methanol as Electron Donor

\begin{tabular}{|c|c|c|c|c||}
\hline $\begin{array}{c}\text { Time } \\
(\mathrm{min})\end{array}$ & $\begin{array}{c}\mathrm{NO}_{3}-\mathrm{N} \\
(\mathrm{ug} / \mathrm{L})\end{array}$ & $\begin{array}{c}\mathrm{NO}_{2}-\mathrm{N} \\
(\mathrm{ug} / \mathrm{L})\end{array}$ & $\begin{array}{c}\mathrm{N}_{2} \mathrm{O} \\
(\mathrm{ppm})\end{array}$ & $\begin{array}{c}\mathrm{H}_{2} \\
(\mathrm{ppm})\end{array}$ \\
\hline 0 & 3470 & 80 & 8.2 & 2 \\
30 & -- & -- & 7.4 & 2 \\
60 & 2170 & 80 & 6.8 & 3 \\
90 & -- & -- & 5.8 & 2 \\
120 & 540 & 90 & 4.8 & 3 \\
155 & -- & -- & $\mathrm{BDL}^{*}$ & 1 \\
180 & 10 & 30 & 1 \\
\hline
\end{tabular}

\section{* BDL - Below Detection Limit}

To discern how a methanol-fed chemostat would respond to an organic overload, the HRT of a reactor operating using a feed with a methanol $/ \mathrm{NO}_{3}-\mathrm{N}$ ratio of $3 / 1$, was reduced from 1.25 to 0.28 days. The nitrate concentration began to increase almost immediately, reaching $4070 \mathrm{ug} / \mathrm{L}$ within $30 \mathrm{minutes}$. The $\mathrm{N}_{2} \mathrm{O}$ concentration concurrently increased to over $5 \mathrm{ppm}$ (Figure 5-7). The concentration of both continued to increase for 90 minutes (peak nitrate and $\mathrm{N}_{2} \mathrm{O}$ concentrations of $13240 \mathrm{ug} / \mathrm{L}$ and ca. $11 \mathrm{ppm}$, respectively). At this time, the HRT was reduced back to 1.25 days. Nitrate and $\mathrm{N}_{2} \mathrm{O}$ both decreased back to near non-detect by the 180 and 240 minute marks of the experiment, respectively.

Nitrite concentrations remained quite low throughout the experiment, increasing from a baseline of 25-30 $\mathrm{ug} / \mathrm{L}$ to a peak concentration of $370 \mathrm{ug} / \mathrm{L}$ after 60 minutes. No $\mathrm{H}_{2}$ was detected in the headspace during this experiment.

An experiment with a methanol feed $(\mathrm{MeOH} /$ nitrate) ratio of 3.5 was started, where at time zero, the normal feed was replaced with a nitrate only $(200 \mathrm{mg} / \mathrm{L})$ feed. After 120 minutes the nitrate concentration began to rise reaching $8290 \mathrm{ug} / \mathrm{L}$ by 300 minutes. The $\mathrm{N}_{2} \mathrm{O}$ level had risen to ca. $7 \mathrm{ppm}$ by this time while the $\mathrm{H}_{2}$ level decreased from 7 to $1 \mathrm{ppm}$ as seen in Figure 5-8. At this time the chemostat feed solution was spiked with methanol to attain a feed methanol concentration of $8000 \mathrm{mg} / \mathrm{L}$. Nitrate and $\mathrm{N}_{2} \mathrm{O}$ levels decreased to near non-detect levels during the ensuing 60 minutes while the $\mathrm{H}_{2}$ concentration increased slightly from 1 to ca. $6 \mathrm{ppm}$. 

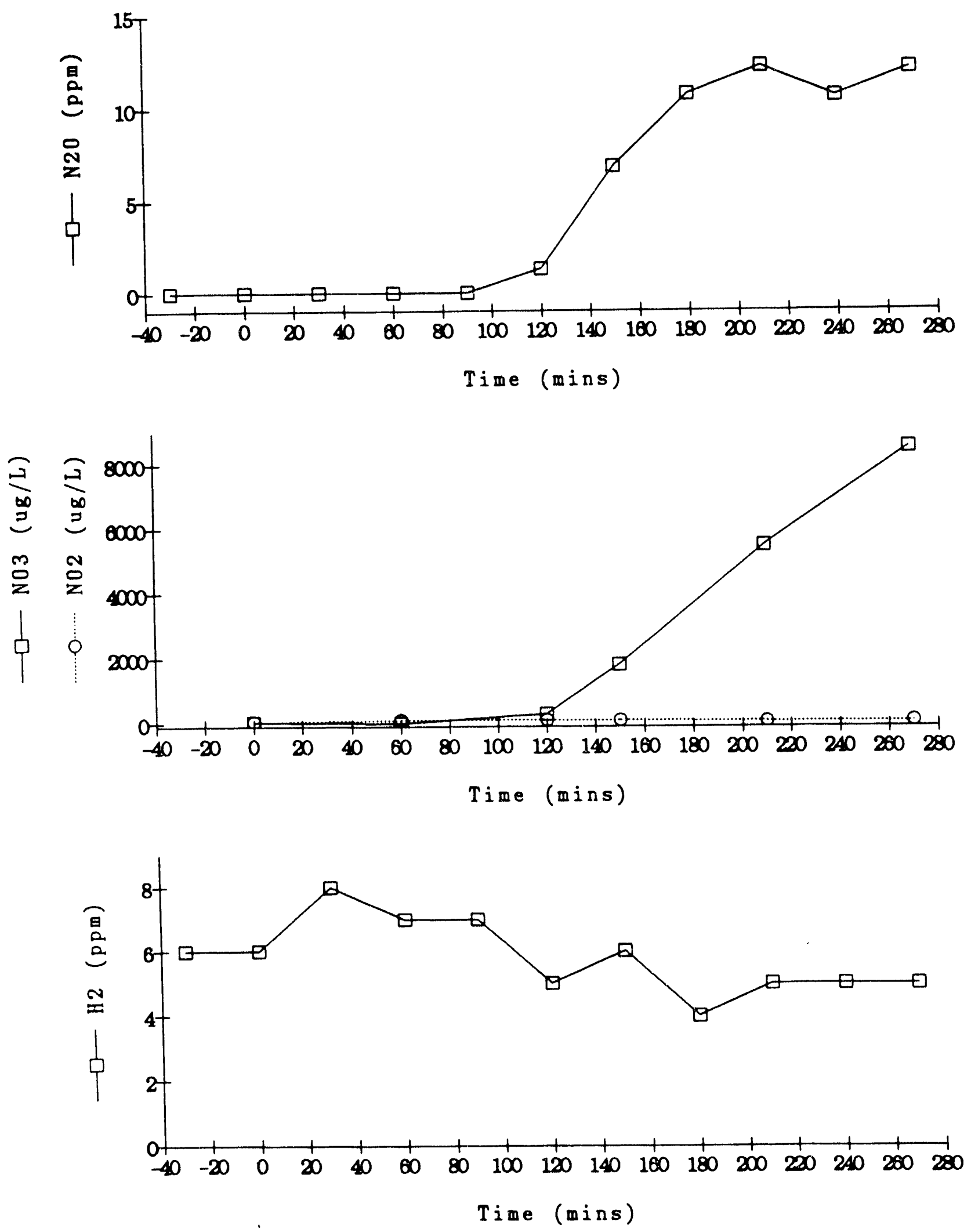

PIGURE 5-6. CONCENTRATIONS OF NITRATE, NITRITE $\mathrm{N}_{2} \mathrm{O}$ AND $\mathrm{H}_{2}$ IN $A$ NETHANOL-PED CHEMOSTAT (METHANOL/NO $3-N=3 / 1$ ). AT TIME ZERO, A FEED CONTAINING $200 \mathrm{mg}_{8} \mathrm{NO}_{3}-\mathrm{N} / \mathrm{L}$ ONLY WAS USED TO REPLACE THE NORMAL FEED. 

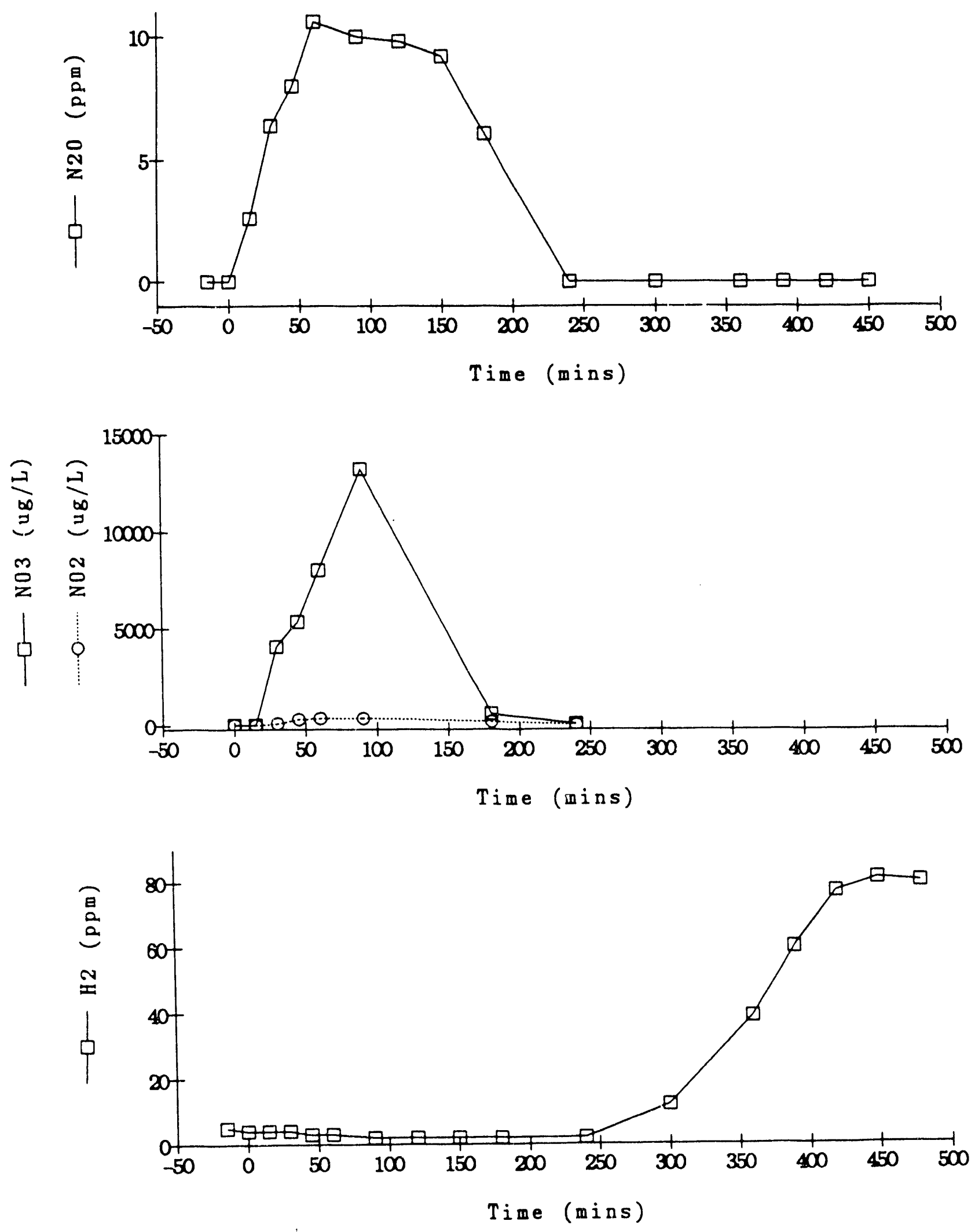

FIGURE 5-7. EFFECT OF REDUCING THE HRT OF A METHANOL-FED CHEMOSTAT (METHANOL/NITRATE-N $=3 / 1$ ) FROM 1.25 TO 0.28 DAYS $(t=0)$. AT $t=90$ MINUTES, THE HRT WAS INCREASED BACK TO 1.25 DAYS. 

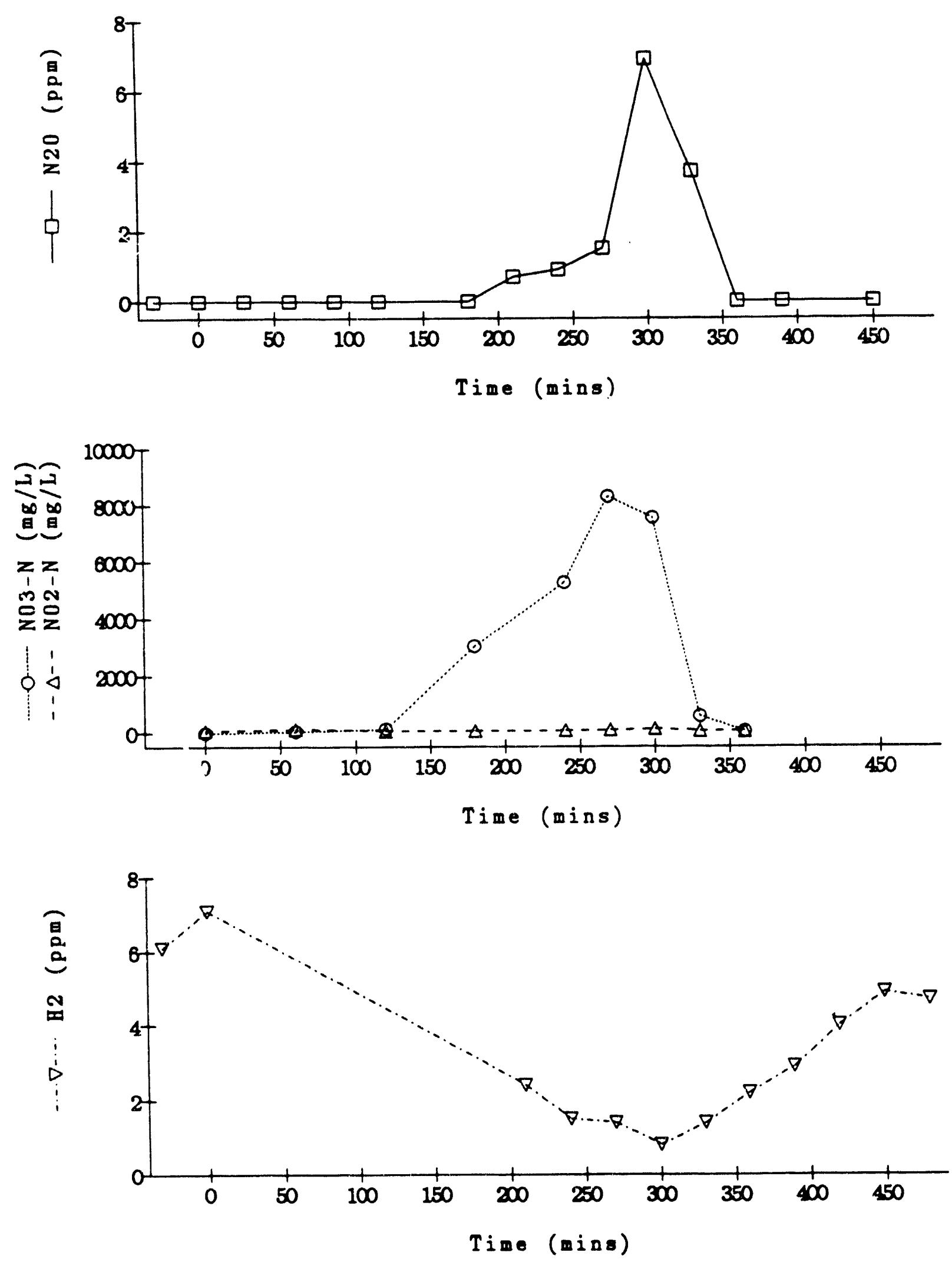

FIGURE 5-8. CONCENTRATIONS OF NITRATE, NITRITE, $\mathrm{N}_{2} \mathrm{O}$ AND $\mathrm{H}_{2}$ IN A YETHANOL-FED (METHANOL/NO, $-\mathrm{N}=3.5 / 1$ ) CHEMOSTAT. AT TIME ZERO, THE FEED WAS SWTMCHED TO A NTTRATE ONLY (200 mGN/L) EEED. AT $t=60$ YINTITES THE FEED SOLUTION WAS SPIKED WITH KETHANOL TO OBTAIN A CONCENTRATION OF Ca $8000 \mathrm{mg} / \mathrm{L}$. 
A final identical experiment was cor.Jucter except nitrate was replaced with nitrite in the feed. At time zero, a $200 \mathrm{mg} / \mathrm{l}$, nitrite only feed was used to replace the feed with nitrite and methanol. After 6 hours, no change was observed. At this time, the HRT was reduced to ca. 0.62 days and the nitrite feed concentration increased to $1000 \mathrm{mg} \mathrm{N} / \mathrm{L}$. Within the next hour, the nitrite concentration increased to 4900 ug $\mathrm{N} / \mathrm{L}$ while the $\mathrm{N}_{2} \mathrm{O}$ concentration increased to $80 \mathrm{ppm}$ as illustrated in Figure 5-9.

This result demonstrates that $\mathrm{N}_{2} \mathrm{O}$ will accumulate during accumulation of nitrite as well as nitrate, as was previously observed when acetate was used as the electron donor.

\subsection{SUMMARY}

A series of batch assays was conducted using acetate as the electron donor for denitrification. During these experiments, acetate was supplied at both stochiometric (or greater) amounts as well as at dosages less than required to completely reduce the amount of nitrate added (generally $200 \mathrm{mgN} / \mathrm{L}$ ). When stochiometric or greater amounts of acetate were added, an amount of nitrite accumulated during the initial 120 to 150 minutes at between 4 and 30 percent of the initial nitrate concentration (runs 2 and 4 , respectively). Nitrite then decreased rapidly to $20 \mathrm{ugN} / \mathrm{L}$ or less. Nitrous oxide $\left(\mathrm{N}_{2} \mathrm{O}\right)$ accumulated slightly, reaching peak concentrations of 3.1 and $9.0 \mathrm{ppm}(\mathrm{v} / \mathrm{v})$ for runs 2 and 4 , respectively. During these experiments it was clear that nitrate reduction was rapid and accumulation of nitrite was due to the inability of the inocula to tumover nitrite as quickly. During experiments, when acetate was added at less than the amount required to completely reduce the starting nitrate concentration (Runs 1, 3 and 5), observed results were different. During the initial hour or so, nitrate was rapidly reduced and nitrite accumulated at rates equal to or greater than observed before. Nitrite continued to remain at elevated levels and $\mathrm{N}_{2} \mathrm{O}$ started to accumulate, especially under the lowest acetate/nitrate- $\mathrm{N}$ ratio of 3.6 (Run 5), to much greater levels. Juring run $5, \mathrm{~N}_{2} \mathrm{O}$ levels reached ca. $25 \mathrm{ppm}$ by 2 hours incubation. In ail cases $\mathrm{N}_{2} \mathrm{O}$ concentrations eventually reached levels approaching or exceeding $100 \mathrm{ppm}$.

Based on the observations it was postulated that $\mathrm{N}_{2} \mathrm{O}$ might indicate insufficient electron donors were present. Chemostat studies were initiated to examine this under more realistic conditions.

During chemostat experiments using acetate as the electron donor, acetate was added at excess amounts before initiating the experiments. At time equal to zero, the feed was switched to nitrate only (200 $\mathrm{mgN} / \mathrm{L}$ ). Two different results were observed. During the first assay, nitrite accumulated within 30 minutes. The headspace concentration of $\mathrm{N}_{2} \mathrm{O}$ concurrently increased exceeding $100 \mathrm{ppm}$ within one hour. 


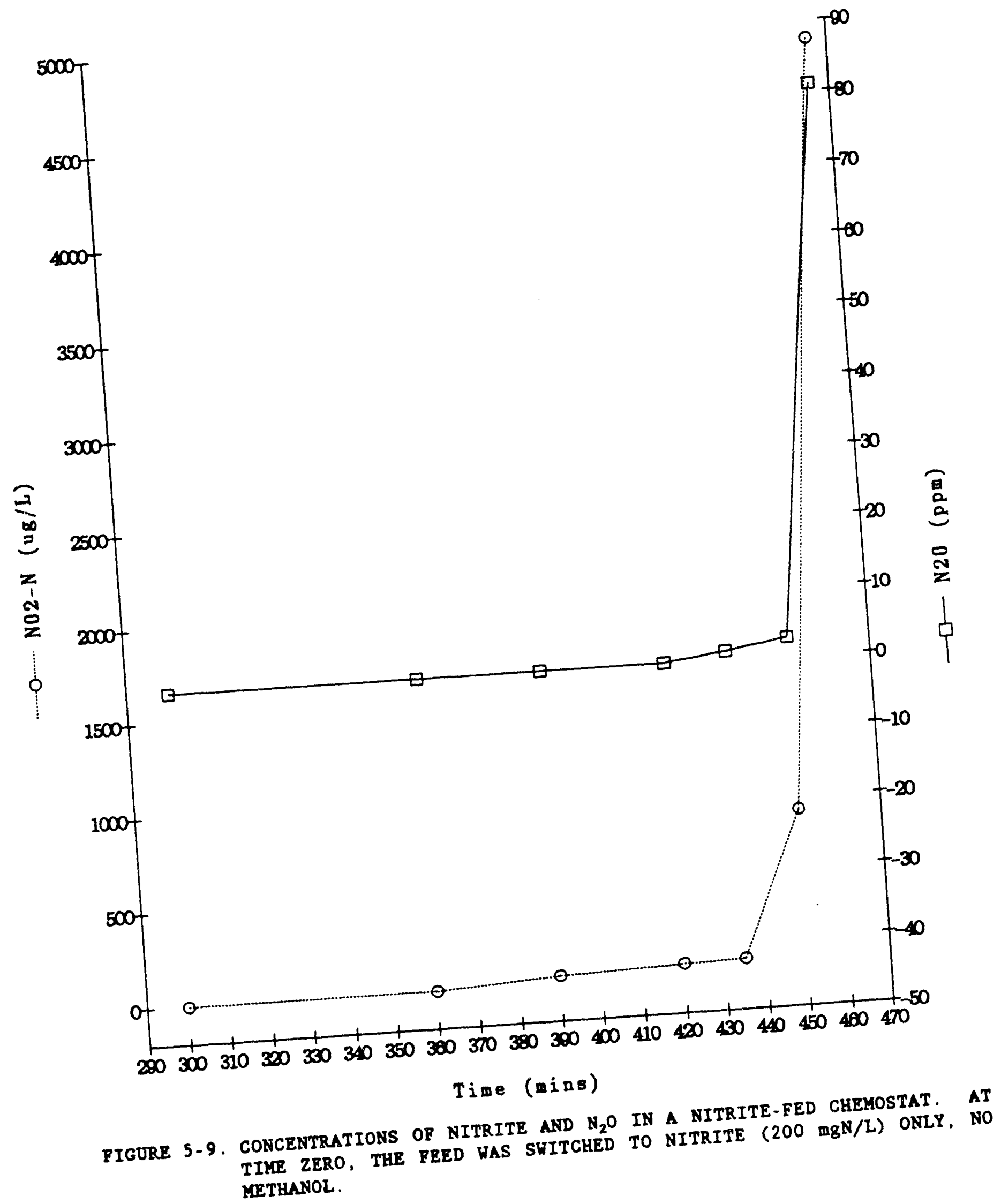


Nitrate did increase slightly but not to the extent of nitrite. Hydrogen, which had been present at $40 \mathrm{ppm}$ at the start of the assay decreased rapidly to below $10 \mathrm{ppm}$ within the initial 120 minutes. Essentially, chemostat results were as anticipated based on the batch assay results.

The second experiment was different only in that nitrate rather than nitrite accumulated to a greater extent. This may have been, in part, related to the higher nitrate feed level ( 500 versus $200 \mathrm{mgN} / \mathrm{L}$ ) used for the second experiment. The common phenomenon of $\mathrm{N}_{2} \mathrm{O}$ rapidly increasing when insufficient electron donor was present was observed for both experimental trials. In both cases, the concentration of $\mathrm{H}_{2}$ simultaneously decreased with increasing $\mathrm{N}_{2} \mathrm{O}$.

A series of experimental runs (chemostat) were conducted using methanol as the electron donor. During experiments when systems operating under electron donor sufficient conditions were suddenly supplied with a feed containing nitrate only (to establish electron donor "poor" conditions), nitrate concentrations began to increase. This was mirrored by a concurrent rapid increase in $\mathrm{N}_{2} \mathrm{O}$ concentrations (Figures 5-6 and 5-8). With methanol as the electron donor, significant nitrite accumulation was not observed during electron donor "lean" conditions. The concentration decreased concurrent with the increase in $\mathrm{N}_{2} \mathrm{O}$. It should be noted that $\mathrm{N}_{2} \mathrm{O}$ concentrations were much less $(<10 \mathrm{ppm})$ than the level observed when acetate (excess amounts) was used as the electron donor.

If a sufficient amount of methanol was then added to the chemostat, nitrate quickly decreased to below detection limits. This was mirrored by a rapid decline in $\mathrm{N}_{2} \mathrm{O}$ levels (Figure 5-8) and increase in $\mathrm{H}_{2} \mathrm{O}$ concentration.

The effect of an organic overload was also examined. In this case, as the HRT of the chemostat was reduced from 1.25 to 0.28 days, with a concurrent increase in applied organic loading rate, $\mathrm{N}_{2} \mathrm{O}$ accumulation was observed within minutes and actually preceded the observed accumulation of nitrate. When the HRT (and organic load) was reduced to the prior conditions, nitrate and $\mathrm{N}_{2} \mathrm{O}$ concurrently declined. Finally, since nitrite did not accumulate to any significant extent during the experimental trials using methanol as the electron donor, a system was operated with a nitrite only feed. In this case, the accumulation of nitrite was tracked by a concurrent increase in $\mathrm{N}_{2} \mathrm{O}$ levels. 
All results indicate that $\mathrm{N}_{2} \mathrm{O}$ is an extremely sensitive and reliable indicator of a build-up of either nitrate or nitrite in denitrifying systems, regardless of the cause; i.e., insufficient electron donor present or organic (hydraulic) overload.

The response of $\mathrm{H}_{2}$ was the inverse of the response of $\mathrm{N}_{2} \mathrm{O}$. At times when excess electron donor was present, $\mathrm{H}_{2}$ increased. Under electron donor "poor" or "lean" conditions, the $\mathrm{H}_{2}$ concentration decreased. This trend was consistent regardless of which electron donor, acetate or methanol, was used. The concentrations that hydrogen reached appeared to be related to the electron donor used. Lower $\mathbf{H}_{2}$ concentrations were observed with methanol, the more energy rich electron donor. 


\section{SECTION 6}

\section{CONCLUSIONS}

The concentration of $\mathrm{N}_{2} \mathrm{O}$ was observed to predictably respond to changes in the environmental conditions (i.e., substrate concentrations) for both nitrification and denitrification systems. The specific conclusions that can be drawn from this work are detailed below.

\subsection{NITRIFICATION}

- Batch test results indicated an increase in ammonium concentration resulted in only a slight increase in $\mathrm{N}_{2} \mathrm{O}$ concentrations over the small concentration range examined $\left(12.5-20.8 \mathrm{mg} \mathrm{NH}_{4}^{+}-\mathrm{N} / \mathrm{L}\right)$.

- An increase in nitrite concentration, by contrast, resulted in a significant increase in $\mathrm{N}_{2} \mathrm{O}$.

- Free energy calculations, based on dividing the oxidation of ammonium to nitrite into two half-cell reactions, representing the production and consumption of $\mathrm{N}_{2} \mathrm{O}$, revealed that an essentially constant free energy was associated with each reaction over the range of conditions studied.

- Based on this observation, it can be predicted that increases in nitrite and ammonium will result in increases in $\mathrm{N}_{2} \mathrm{O}$ to the $3 / 2$ and $1 / 2$ power, respectively.

- $\quad \mathrm{N}_{2} \mathrm{O}$ should, therefore, be a relatively sensitive indicator of nitrite accumulation, a phenomenon that normally accompanies loss of efficiency of nitrification.

\subsection{DENITRIFICATION}

- Rapid accurnulation of $\mathrm{N}_{2} \mathrm{O}$ was observed whenever insufficient electron donor (either methanol or acetate) was present in both batch and continuous flow chemostat experiments.

- A rapid increase in $\mathrm{N}_{2} \mathrm{O}$ was also observed when flow (and load) to a chemostat reactor was increased ca. five-fold.

- Based on the above, $\mathrm{N}_{2} \mathrm{O}$ appears to be a sensitive indicator of electron donor deficiency. This means that monitoring $\mathrm{N}_{2} \mathrm{O}$ would be a useful method to ensure that sufficient electron donor is fed to a denitrification system.

- When acetate was used as an electron donor, it was observed that $\mathrm{H}_{2}$ accumulated when excess donor was supplied.

- This same phenomenon was also observed for cases when methanol was used as the electron donor, but to a much lesser extent. 
- Based on these results, it appears that $\mathrm{H}_{2}$ would be useful for monitoring to ensure acetate is not fed to excess in a denitrification system but may not be effective for methanol.

- In an acetate-fed, denitrification system the simultaneous monitoring of $\mathrm{N}_{2} \mathrm{O}$ and $\mathrm{H}_{2}$ would appear to have promise as inputs to a control system to ensure the correct amount of acetate is always supplied.

- Continued efforts in developing a control system based on monitoring both $\mathrm{N}_{2} \mathrm{O}$ and $\mathrm{H}_{2}$ for denitrification systems appear worthwhile. 


\section{SECTION 7}

\section{REFERENCES}

1. The Nation's Water Resources 1975-2000, Volume 1: Summary, U.S. Water Resources Council, U.S. Government Printing Office (1979).

2. Bouwer, E.J., and P.C. Crowe, "Biological Processes in Drinking Water Treatment." Jour. AWWA, 80:82-93 (1988).

3. Geldreich, E.E., H.D. Nash, D.J. Reasoner, and R.H. Taylor, "The Necessity of Controlling Bacterial Populations in Potable Waters: Community Water Supply." Jour. AWWA, 64:596-602 (1972).

4. Camper, A.K., M.W. LeChevallier, S.C. Broadway, G.A. McFeters, "Bacteria Associated with Granular Activated Carbon Particles in Drinking Water." Appl. Environ. Microbiol., 52(3):434438 (1986).

5. Olsen, B.H., "Assessment and Implication of Bacterial Regrowth in Water Distribution Systems." EPA-600/52-82-072 (Sept. 1982).

6. Cullimore, D.R., and A.E. Meann, "The Identification, Cultivation, and Control of Iron Bacteria in Groundwater." P. 219-261 in F.A. Skinner and J.A. Skewar (ed.) Aquatic Microbiology, Academic Press, Inc., London (1977).

7. Goodall, J.B., "Biological Removal of Ammonia." In Oxidation Techniques in Drinking Water Treatment (Edited by Kuhn W. and Sontheimer H.), pp. 586-596. EPA-570/9-79-020 (1979).

8. Houel, N., A. Deguin, J.P. Moulinot, Y. Richard, M. Rizet, and J. Sibony, "3. Elimination des formes azotees dans les eaux alimentaires." Techniques et Sciences Municipales - 1'Eau 77th year, no. 6: 292-300 (1982).

9. Rittmann, B.E., and L. Snoeying, "Achieving Biologically Stable Drinking Water." Jour. AWWA, 76(10): 106-114 (1984).

10. Committee Report: "Microbiological Considerations for Drinking Water Regulation Revisions." Jour. AWWA, 79:(5) 81-88 (1987).

11. Larson, T.E., "Deterioration of Water Quality in Distribution Systems." Jour. AWWA, 58(10):1307 (1966).

12. Larson, T.E., "Bacteria, Corrosion, and Red Water." Jour. AWWA, 31 (7):1186 (1939).

13. Gauntlett, R.B., "Removal of Ammonia and Nitrate in the Treatment of Potable Water." In Biological Fluidized Bed Treatment of Water and Wastewater (Edited by Cooper P.F. and Atkinson B.), pp. 48-58. Ellis Horwood Limited, Chichester, England (1981). 
14. National Academy of Science and National Academy of Engineering. Water Quality Criteria 1972. Rept. by Com. on Water Quality at request of USEPA, Washington, D.C. (1972).

15. Ehreth, D.J. and E. Barth. Control of Nitrogen in Wastewater Effluents, USEPA (Revised, March 1974).

16. Bablon, G.P., C. Ventresque and R.B. Aim, "Developing a sand-GAC filter to achieve high-rate biological filtration," Jour. AWWA $\underline{80}$ (12):47-53 (1988).

17. Bablon, G.P., C. Ventresque, F. Damez, and M.C. Hascoet, "Removal of Organic Matter by Means of Combined Ozonation/BAC Filtration, A Reality on an Industrial Scale at the Choisy-le-Roi Treatment Plant." Presented at the AWWA Annual Conference, Denver, Colorado, June 22-26. 1986, pp. 1705-1713 (1986).

18. Bourbigot, M.M., R. Lheritier, and M. Benezet-Toulze, "Traitement biologique de l'eau potable. Mesure de l'activite bacterienne dans les materiaux filtrants." Techniques et Sciences Municipales - l'Eau 76th year, 12:639-648 (1981).

19. Jekel, M., "Biological Treatment of Surface Waters in Activated Carbon Filters." Water Resources Center, KIWA, and Engler Bunte Institute joint meeting, Karlsnuhe, FRG. (1977).

20. Bourbigot, M.M., "Filtration biologique sur charbon actif utilise en second etage de traitement. Etude en station pilote." Trib. Cebedeau 448(34):13i-1443 (1981).

21. Rice, R.G., L. Gomella, and G.W. Miller, "Rouen, France, Water Treatment Plant: good organics and ammonia removal with no need to regenerate carbon beds." Civil Engineering 48(5):76-82 (1978).

22. Short, C.S., "Removal of Ammonia from River Water - 2." Technical Report TR3. Medmenham, Water Research Centre (1975).

23. Milliner, R., et at., "Biological pretreatment at Tewkesbury. Water Treatment and Examination. 21(4):318-326 (1972).

24. Melbourne, J.D., "River Trent Treatment," Technical Report TR74, Medmenham, Water Research Centre, 136 pp (1978).

25. Richard, Y., "Biological Methods for the Treatment of Ground Water." Oxidation Techniques in Drinking Water Treatment (Edited by Kuhn W. and Sontheimer H.), pp. 560-585. EPA-570/9-79020 (1979).

26. Sibony, J., "Techniques et procedes d'elimination des produits azotes dans les eaux potables." Pollutec Conferences Techniques Intermationales pp. 1-16 (1982).

27. Kubmaul, H., "Purifying Action of the Ground in the Treatment of Drinking Water." In Oxidation Techniques in Drinking Water Treatment (Edited by Kuhn W. and Sontheimer, H.), pp. 597-607. EPA-570/9-79-020 (1979). 
28. Wilderer, P.A., U. Forstner, and O.R. Kuntschik 1985, "The Role of River Bank Filtration along the Rhine for Municipal and Industrial Water Supply." In Artificial Recharge of Groundwater (Edited by Asano T.), pp. 509-527. Butterworth Publishers, Boston, MA.

29. Pye, V.I., R. Patrick and J. Quarles, Groundwater Contamination in the United States. University of Pennsylvania Press, Philadelphia, PA (1983).

30. Asano, T., Artificial Recharge of Groundwater. (Editor) Butterwork Publishers, Boston, MA (1985).

31. Clifford, D. and C.C. Lin, "Ion Exchange and Membrane Processes for Nitrate Removal from Water." pp. 14-23 in Proc. of the ASCE Specialty Conf. on Environ. Engineering, July 6-8 (1983).

32. Dahab, M.F. and Y.W. Lee, "Nitrate Removal from Water Supplies using Biological Denitrification." Jour. Water Poll. ConL. Fed., 60(9):1670-1674 (1988).

33. Gregory, R., and I. Sheiham, "Biological Fluidized-Bed Denitrification of Surface Water." The Economics of a Remedy for Nitrate in Drinking Water. Biological Fluidized Bed Treatment of Water and Wastewater (P.F. Cooper and B. Atkins, editors). Ellis Horwood, Chichester, England (1981).

34. Gauntlett, R. and T.F. Zabel, "Biological Denitrification for Potble Water Treatment." Water Services pp. 17-18 (1981).

35. Philipot, J.M., F. Chaffange, and O. Pascal, "Biological Nitrate Removal: Assessment of one year's operation of the Erangy plant," Wat. Supply, 3:93-98 (1985).

36. Kurh M., I.J. Dunn, and J. R. Bourne, "Biological denitrification of drinking water using autotrophic organisms with $\mathrm{H}_{2}$ in a fluidized bed biofilm reactor." Biotechnol. Bioeng., 29:493501 (1987).

37. Houel, N., et al., "Elimination des Formes Azotees dans les Eaux Alimentaires." Tecinniques et Sciences Municipales - 1-Eau, $\underline{6}: 292$ (1982).

38. Blecon, G., et al., "Procede de Denitrification Biologique Autotrophe par Thiobacillus denitrificans on Sulfur-Maerl." Revue francaises des Science de l'Eau 2:3:267 (1983).

39. Schippers, J.C., et al., "Removal of Nitrate by Slow Sulphur-Limestone Filtration." Aqua, $5: 274$ (1987).

40. Van der Hoek, J.P., A.B. Griffioen and A. Klapwijk, "Biological Regeneration of Nitrate-loaded anion-exchange resins by denitrifying bacteria." J. Chem. Tech. Biotechnol., 43:213-222 (1988).

41. Van der Hoek, J.P., P.J.M. Van der Ven and A. Klapwijk, "Combined ion exchange/biological denitrification for ntirate removal from groundwater under different process conditions." Water Res., 22(6) 679-684 (1988). 
42. Chalupa, M., "Problems in Czechoslovakia regarding methods of removal of nitrates from drinking water." Water Sci. Technol., 20(3):211-213 (1988).

43. Vorlop, K.D., U. Dirks, J. Schmidt, and T. Tacke, "Process of nitrate removal in drinking 'water with immobilized cells - and metals - catalysts with hydrogen." Preprint of unpublished data.

44. Janda, V., J. Rudovsky, J. Wanner, and K. Marha. "In-situ denitrification of drinking water." Water Sci. Technol., 20(30):215-219 (1988).

45. Mercado, A., M. Libhaber, and M.I.M. Soares, "In-situ biological groundwater denitrification: Concepts and preliminary field tests." Water Sci. Technol., 20(3):197-209 (1988).

46. Braester, C. and R. Martinell, "The Vyredox and Nitredox Methods of In-Situ Treatment of Groundwater." Water Sci. Technol., 20(3):144-163 (1988).

47. Barrenstein, A., U. Kramer and P. Obermann, "Unterirdische aufberitung von nitratreichen grundwasser durch infiltration mit aufbereitetem abwasser odoer methanreichem erdgas." Wasser, 106:99-116 (1986).

48. Piet, G.J., and B.C.J. Zoeteman, "Band and dume filtration of surface water in the Netherlands in Artificial Recharge of Groundwater, pp. 529-540 (T. Asano, et.) Butterworth Pub., Boston, MA (1985).

49. Jeris, J.S., R.W. Owens, R. Hickey, and F. Flood, "Biological Fluidized-Bed Treatment for COD and Nitrogen Removal." J. Wat. Pollut. Control Fed. 49(5):816-831 (1977).

50. Rittmann, B.E., "Comparative Performance of Biofilm Reactor Types." Biotechnol. \& Bioeng. 24:1341-1370 (1982).

51. Solt, G., Nitrate removal: A compromise solution. Water Qual. Int1. 1 1:29-30 (1987).

52. Nielsen, L.P., Christensen, P.B., Revsbeck, N.P. and Sorensen, J., "Denitrification and oxygen respiration in biofilms studied with a microsensor for nitrous oxide and oxygen." Microb. Ecol., 19:63-72 (1990).

53. Aulakh, M.S., Rennie, D.A. and Paul, E.A., "Acetylene and $\mathrm{N}^{-}$serve effects upon $\mathrm{N}_{2} \mathrm{O}$ emissions from $\mathrm{NH}_{4}{ }^{+}$and $\mathrm{NO}_{3}{ }^{-}$treated soils under aerobic and anaerobic conditions." Soil Biol. Biochem., 16:351-356 (1984).

54. Martikainen, P.J., "Nitrous oxide emission associated with autotrophic ammonium oxidation in acid coniferous forest soils." Appl. Environ. Microbiol., 50:1519-1525 (1985).

55. Remde, A., Slemr, F. and Conrad, R., "Microbial production and uptake of nitric oxide in soil." FEMS Microbial. Ecol., 62: $221-230$ (1989).

56. Kaspar, H.F. and Tiedje, J.M., "Response of electron capture detector to hydrogen, oxygen, nitrogen, carbon dioxide, nitric oxide and nitroash oxide." J. of Chromotog... 193:142-147 (1980). 
57. Betlach, M.R. and Tiedje, J.M., "Kinetic explanation for the accumulation of nitrite, nitric oxide and nitrous oxide during bacterial denitrification." Appl. Environ. Microbiol., 42:1074-1084 (1981).

58. Andersen, I.C. and Levine, J.S., "Relative roles of nitric and nitrous oxide production by nitrifiers, denitrifiers and ntirate respirers." Appl. Environ. Microbiol., 51:938-945 (1986).

59. Goreau, T.J., et al., "Production of $\mathrm{NO}_{2}^{-}$and $\mathrm{N}_{2} \mathrm{O}$ by nitrifying bacteria at reduced concentrations of oxygen." Appl. Environ. Microbial. 40:526-532 (1980).

60. Kaplan, W.A., et al., "Nitrous oxide in fresh water systems: an estimate for the yield of atmospheric $\mathrm{N}_{2} \mathrm{O}$ ) associated with disposal of human wastes." Page oph., 116:423-429 (1978).

61. Parkin, T.B., Kaspar, H.F., Sexstone, A.J. and Tiedje, J.M., "A gas-flow soil core method to measure field dentrification rates." Soil Biol. Biochem., 16:323-330 (1984).

62. Keeney, D.R., Sahrawat, K.L and Adams, S.S., "Carbon dioxide concentration in soil: effects on nitrification, denitrification, and associated nitrous oxide production." Soil Biol. Biochem.. 17:571-573 (1985).

63. Nogita, S., Saito, Y. and Kuge, T., "A new indicator of the activated sludge process-nitrous oxide." Water Sci. Technol., 13:199-204 (1981).

64. Lischultz, F., et al., "Production of $\mathrm{NO}$ and $\mathrm{N}_{2} \mathrm{O}$ by soil nitrifying bacteria," Nature, 294:641-643 (1981).

65. USEPA, Methods for Chemical Analysis of Water and Wastes, (1979).

66. Hickey, R.F. and Switzenbaum, M.S., "The Behavior of Carbon Monoxide as a Trace Component of Methanogenesis in Anaerobic Digester Gases on Methanogenesis from Acetate," Environmental Sci. Technol., 24:1642-1648 (1990).

67. Hickey, R.F. and Switzenbaum, M.S., "The Response and Utility of Hydrogen and Carbon Monoxide as Process Indicators of Anaerobic Digesters Subject to Organic and Hydraulic Overloads," Res. J. Wat. Pollut. Cont. Fed., 63:129-140 (1991).

68. Hickey, R.F., Vanderwielen, J. and Switzenbaum, M.S., "The Effect of Organic Toxicants on Methane Production and Hydrogen Gas Levels During the Anaerobic Digestion of Waste Activated Sludge," Wat. Res., 11:1417-1427 (1987). 

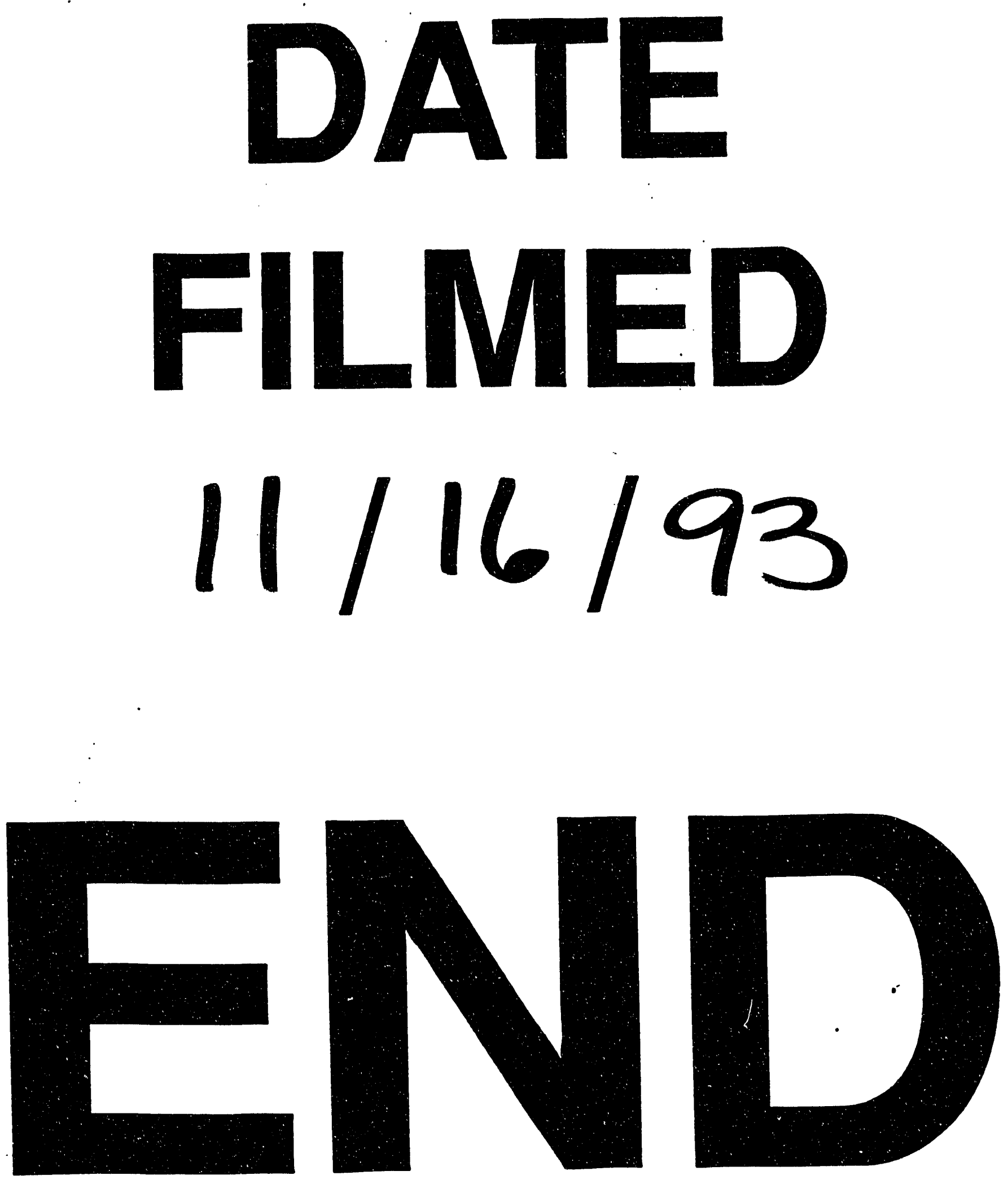
